Advances in Retrofit and Testing of Reinforced Concrete Shear Walls: Part 1 - Seismic Retrofit of Deficient Walls with Fibre-Reinforced Polymer Sheets Part 2 - Building Scale Performance Evaluation using Hybrid Simulation

by

Joshua Edward Woods

\begin{abstract}
A thesis submitted to the
Faculty of Graduate and Postdoctoral Affairs

in partial fulfillment of the requirements for the degree of
\end{abstract}

Doctor of Philosophy

Ottawa-Carleton Institute for Civil and Environmental Engineering

Department of Civil and Environmental Engineering

Carleton University

Ottawa, Ontario

December, 2018

Joshua Edward Woods, 2018 
The undersigned hereby recommends to the

Faculty of Graduate and Postdoctoral Affairs

acceptance of the thesis

\title{
Advances in Retrofit and Testing of Reinforced Concrete Shear Walls: Part 1 - Seismic Retrofit of Deficient Walls with Fibre-Reinforced Polymer Sheets Part 2 - Building Scale Performance Evaluation using Hybrid Simulation
}

\author{
submitted by Joshua Edward Woods \\ in partial fulfillment of the requirements for the degree of \\ Doctor of Philosophy
}

Dr. David Lau, Thesis Supervisor

Dr. Jeffery Erochko, Thesis Co-supervisor

Dr. Yasser Hassan, Department Chair

Ottawa-Carleton Institute for Civil and Environmental Engineering

Department of Civil and Environmental Engineering

Carleton University

December, 2018 


\section{Abstract}

Reinforced concrete (RC) shear walls are a common lateral load resisting structural system widely used in seismically active regions. Despite the advancements in modern seismic design provisions used around the world, there is still a large inventory of existing RC shear wall structures designed and constructed based on older, less stringent design guidelines. The first study presented in this thesis describes the results of an experimental study on the effectiveness of using externally bonded carbon fibre-reinforced polymer (CFRP) sheets to enhance the seismic performance of non-ductile reinforced concrete $(\mathrm{RC})$ shear wall specimens representative of low aspect ratio walls. Nine $2 / 3$ scale RC shear wall specimens were designed to replicate structural deficiencies commonly found in older RC shear wall structures constructed during the 1960s and 1970s. The non-ductile reinforcement details include insufficient shear reinforcement, lack of boundary elements, lap splices within the potential plastic hinge region, and low concrete compressive strength. The wall specimens were repaired or strengthened using vertical and horizontal CFRP sheets and then cyclically tested to simulate seismic loading. A tube anchor system was used to transfer the tensile force carried by the CFRP sheet to the foundation of the specimens. Experimental results demonstrate that the CFRP retrofitting system is able to restore the original strength and initial stiffness of severely damage specimens and significantly increase the flexural strength, ductility, and energy dissipation capacity in strengthening applications.

The second study presented in this thesis examines the use of hybrid simulation to capture the earthquake response of a multi-storey RC shear wall structure. Hybrid simulation combines the strengths of analytical modelling and experimental testing to analyze the response of a com-

plete structural system. In this approach, hybrid simulation is able to capture the exact nonlinear behaviour of complex structural components or subassemblies through testing while at the same 
time supplementing it with simulation of the rest of the structure through computer modelling to capture the global system response of an entire structural system. Compared with traditional component level seismic testing, hybrid simulation is able to include the effects of mass and damping at the system level, resulting in a more realistic representation of the loading conditions and thus determination of the system structural response. Despite considerable interest in the development and implementation of hybrid testing, there have been relatively few studies on the use of hybrid simulation to study the behaviour of RC structures. This is because of the inherent high stiffness in most RC structures, which presents unique control challenges on the experimental side of the hybrid simulation because a small target displacement typically corresponds to a very large increment in force. Consequently, this study examines the feasibility of using hybrid testing to study the earthquake response of a multi-storey RC shear wall structure. The experimentally tested shear wall is taken from a three-story prototype structure. In the hybrid simulation, the first story shear wall is experimentally tested at $2 / 5$ of its original scale and the remaining two stories of the shear wall are modelled analytically using multi-layered shell elements in OpenSees. Results of the study demonstrate that it is feasible to use hybrid simulation to capture the seismic response of stiff RC shear wall structures. 


\section{Organization of Thesis}

This thesis is divided into two parts. Part I, entitled Seismic Retrofit of Deficient Walls with Fibre-Reinforced Polymer Sheets examines the effectiveness of using externally bonded carbon-fibre reinforced polymer (CFRP) sheet to improve the in-plane seismic performance of deficient squat reinforced concrete (RC) shear walls tested un in-plane cyclic load. Chapters of Part I include Introduction, Background, Experimental Program, Tube Anchor Optimization and Design, Results and Discussion, and Conclusions and Future Research.

Part II, entitled Building Scale Performance Evaluation using Hybrid Simulation describes a study on the use of hybrid simulation to study the seismic response of a multi-storey RC shear wall structure. The study focuses on the feasibility of using hybrid to study the earthquake response of a full-scale RC shear wall structure as well as examining some of the challenges surrounding hybrid testing of stiff structural components. Chapters of Part II include the Introduction, Background, Prototype RC Structure, Analytical and Hybrid Models, Experimental Program, Results and Discussion, and Conclusions and Future Research. 


\section{Acknowledgments}

First and foremost, I would like to thank my supervisor Professor David Lau, who has been a tremendous teacher, mentor, and friend throughout my graduate studies at Carleton University. To my co-supervisor, Professor Jeffrey Erochko, I will be forever grateful for your constant support and guidance throughout this work. To the other professors who have supported me along the way, including Professor John Gales and Professor Carlos Cruz-Noguez, I am thankful for your support, mentorship, and the opportunities you have given me over the course of my studies.

I would like to thank Professors Tony Yang, Murat Saatcioglu, Heng Khoo, and Xin Wang for taking time out of their very busy schedules to review this thesis and provide valuable feedback.

It has been quite a journey since first setting foot on the campus of Carleton University in the fall semester of 2008. After a little more than 10 years, Carleton has become a second home to me and I will always be thankful for the many friends I have made along the way. I would like to extend my sincerest thanks to the staff in the Structures Laboratory at Carleton University including Stanley Conley, Jason Arnott, Pierre Trudel, Marie Tudoret, Kenneth Akhiwu, and Stephen Vickers. During many years of experimental work, you have become like a second family to me and I will be forever grateful for the many laughs we shared (which were often a result of practical jokes). To the graduate students who dedicated their time to lend a helping hand in the laboratory, including Ahmed Hassan, Sean Miller, Eytan Fiszman, Jeff Salmon, Seth Gatien, and Said Said, I could not have completed this work without your help.

Finally, I would like to extend a heartfelt thanks to my dearest friend Pedram Mortazavi and my family for their support throughout this work. Specifically, my fiancée Marie, my parents Kelly and Paul, and my brother Sheldon. 


\title{
Table of Contents
}

\begin{abstract}
$\quad$ iii
Acknowledgments $\quad$ vi

Table of Contents vii

List of Tables $\quad$ xi

List of Figures xiii

Nomenclature $\quad x x$

I Seismic Retrofit of Deficient Walls with Fibre-Reinforced Polymer Sheets 1

\section{Chapter 1. Introduction}

1.1 Motivation ..................... 2

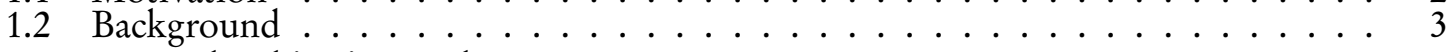

1.3 Research Objectives and Scope . . . . . . . . . . . . . . . . . . 3
\end{abstract}

\section{Chapter 2. Background}

2.1 Failure Modes in RC Shear Walls . . . . . . . . . . . . . . . . . 6

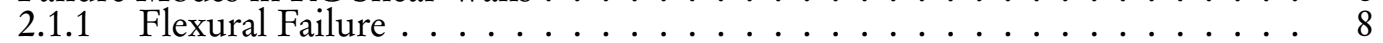

2.1.2 Diagonal Tension Shear Failure $\ldots \ldots \ldots \ldots \ldots \ldots \ldots$

2.1.3 Diagonal Compression Failure . . . . . . . . . . . . . . . . 9

2.1 .4 Sliding Shear Failure . . . . . . . . . . . . . . . . . . . . 10

2.1.5 Lap Splice Failure Modes . . . . . . . . . . . . . . . . . . . . . . . 10

2.2 Structural Testing of FRP-Strengthened Shear Walls $\ldots \ldots \ldots \ldots \ldots \ldots$

2.3 Anchorage in FRP Retrofitted Structures . . . . . . . . . . . . . . . . 21

2.3.1 Mechanical Anchor System . . . . . . . . . . . . . . . . 24

\section{Chapter 3. Experimental Program}

3.1 Methodology . . . . . . . . . . . . . . . . . . . 30

3.2 Shear Wall Specimens . . . . . . . . . . . . . . . . . . . . . . 30

3.3 Material Properties . . . . . . . . . . . . . . . . . . . . 31

3.4 Design of Plain RC Shear Walls $\ldots \ldots \ldots \ldots \ldots \ldots \ldots$

$3.4 .1 \quad$ Flexural Design . . . . . . . . . . . . . . . . . . 35

3.4.2 Shear Design . . . . . . . . . . . . . . . . . . . . . . 37

3.5 Design of FRP-strengthened RC Shear Walls . . . . . . . . . . . . . 40

3.5.1 Flexural Design with FRP Reinforcement . . . . . . . . . . . . 40

3.5.2 Shear Design with FRP Reinforcement . . . . . . . . . . . . . 42 
3.6 Experimental Test Setup . . . . . . . . . . . . . . . . . . . . . 45

3.6 .1 Loading Protocol . . . . . . . . . . . . . . . . . . . . 47

3.6 .2 Instrumentation . . . . . . . . . . . . . . . . . 47

\section{Chapter 4. Anchor System Optimization}

4.1 Analytical Modelling of Mechanical Anchor Systems . . . . . . . . . . . . . . 56

4.1 .1 Model Descriptions . . . . . . . . . . . . . . . . . 56

4.1 .2 Model Results . . . . . . . . . . . . . . . . . . . . . . 59

4.2 Tube Anchor Optimization . . . . . . . . . . . . . . . . . . 62

4.2.1 Tube Anchor Design Methdology . . . . . . . . . . . . . . . . 64

4.3 Tube Anchor Design . . . . . . . . . . . . . . . . . 66

4.4 FRP Anchor System . . . . . . . . . . . . . . . . . 67

\section{Chapter 5. Results and Discussion}

5.1 Control Walls without FRP . . . . . . . . . . . . . . . . . . . 69

5.2 Repaired Wall with FRP Sheets … . . . . . . . . . . . . . . . . . . 80

5.3 Strengthened Walls with FRP Sheets . . . . . . . . . . . . . . . . . . . . . 94

5.4 Overall Response Comparison . . . . . . . . . . . . . . . . . . . . . . 103

5.5 Influence of Aspect Ratio . . . . . . . . . . . . . . . . . . . . . . 111

5.6 Comparison with Code Equations . . . . . . . . . . . . . . . . . . 114

5.7 Comparison of FRP and Tube Anchor System Performance . . . . . . . . . . . 119

5.8 Finite Element Modelling of CFRP Retrofitted Walls . . . . . . . . . . . . . . . . 124

\section{Chapter 6. Conclusions and Future Research}

6.1 Conclusions . . . . . . . . . . . . . . . . . . . . . . 128

6.2 Recommendations for Future Research . . . . . . . . . . . . . . . . . . . . . . . . 130

\section{Building Scale Performance Evaluation using Hybrid Simulation 131}

\section{Chapter 1. Introduction}

1.1 Motivation . . . . . . . . . . . . . . . . . . . . . 132

1.2 Methodology . . . . . . . . . . . . . . . . . . . 135

1.3 Research Objectives and Scope . . . . . . . . . . . . . . . . . 136

\section{Chapter 2. Background}

2.1 Structural Testing of RC Structures . . . . . . . . . . . . . . . . . . . 139

2.2 Hybrid Simulation . . . . . . . . . . . . . . . . . . . . . 141

2.2.1 History of Hybrid Testing . . . . . . . . . . . . . . . . . . . . 141

2.2.2 Conducting a Hybrid Test . . . . . . . . . . . . . . . . . . . . . . 143

2.2.3 Integration Schemes for Hybrid Testing . . . . . . . . . . . . . . . . . 144

2.2.4 Continuity of Loading in Hybrid Testing . . . . . . . . . . . . . . . . 160

2.2.5 Applications of Hybrid Testing . . . . . . . . . . . . . . . . . 162

2.2.6 Force-based Hybrid Simulation . . . . . . . . . . . . . . . . . 175

\section{Chapter 3. Prototype RC Structure}

3.1 Preliminary Design . . . . . . . . . . . . . . . . . . . 178

3.2 Seismic Design . . . . . . . . . . . . . . . . . . . . . . 182

3.2.1 Equivalent Static Force Procedure . . . . . . . . . . . . . . . . . 182

3.2.2 Response Spectrum Analysis . . . . . . . . . . . . . . . . . . . . . 186 
3.3 Design of Gravity Load Resisting Structural System ～. . . . . . . . . . . . . . 189

3.4 Design of Lateral Load Resisting Structural System . . . . . . . . . . . . . . . . 190

3.4.1 Shear Wall Flexural Design . . . . . . . . . . . . . . . . . . . . . . 190

3.4.2 Shear Wall Shear Design . . . . . . . . . . . . . . . . . . . . . 195

3.5 Scaling of Prototype Structure . . . . . . . . . . . . . . . . 198

\section{Chapter 4. Analytical and Hybrid Models}

4.1 Introduction . . . . . . . . . . . . . . . . . . . . . . . . 203

4.2 Model Validation . . . . . . . . . . . . . . . . . . 205

4.2.1 Multi-Layer 3D Shell Element . . . . . . . . . . . . . . . 207

4.2.2 Multi-Layer 2D Shell Element . . . . . . . . . . . . . . . . . . . 213

4.3 Finite Element Modeling of Prototype Wall . . . . . . . . . . . . . . . . . . . . 216

4.4 Model Sensitivity Analysis . . . . . . . . . . . . . . . . . . . . . 220

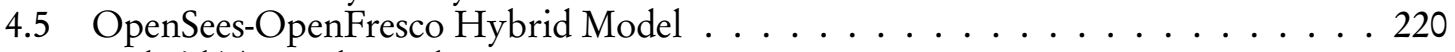

4.6 Hybrid Test Rehearsals . . . . . . . . . . . . . . . . . . . . . . . . . . . . . 229

\section{Chapter 5. Experimental Program}

5.1 Experimental Objectives . . . . . . . . . . . . . . . . . . . 232

5.2 Test Specimens . . . . . . . . . . . . . . . . . . . . . . . 232

5.3 Material Properties . . . . . . . . . . . . . . . . . . . 236

5.4 Experimental Test Setup . . . . . . . . . . . . . . . . . . 238

5.4.1 Hydraulic Control and Data Acquisition . . . . . . . . . . . . . . 241

5.4.2 High-Precision Encoders for Hybrid Simulation . . . . . . . . . . . . . . . 244

5.4 .3 MTS DOF Control Software . . . . . . . . . . . . . . . . . . . 248

5.4 .4 Instrumentation . . . . . . . . . . . . . . . . . . 251

5.4.5 Applied Earthquake Ground Motions . . . . . . . . . . . . . . . . 256

\section{Chapter 6. Results and Discussion}

6.1 Introduction . . . . . . . . . . . . . . . . . . . . . . . . . 264

6.2 Preliminary Testing of Steel Column Assembly . . . . . . . . . . . . . . . . . . . . . . 264

6.3 Hybrid Simulation of Wall $1 \ldots \ldots$. . . . . . . . . . . . . . . 269

6.3 .1 Initial Stiffness of W1 . . . . . . . . . . . . . . . . . . . 269

6.3.2 Local Hybrid Simulation Results . . . . . . . . . . . . . . . . . . 272

6.3.3 Global Hybrid Simulation Results . . . . . . . . . . . . . . . . . . . 288

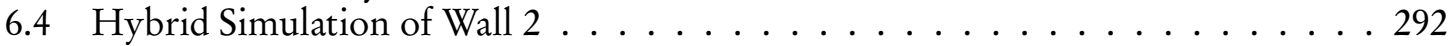

6.4.1 Initial Stiffness of W2 . . . . . . . . . . . . . . . . . . . . . . . . . 292

6.4.2 Local Hybrid Simulation Results . . . . . . . . . . . . . . . . . . . . . 294

6.4.3 Global Hybrid Simulation Results . . . . . . . . . . . . . . . . . . . . . . 309

6.5 Comparison of Hybrid Simulation Results . . . . . . . . . . . . . . . . . . . 313

6.6 Comparison of Hybrid and Fully Analytical Results . . . . . . . . . . . . . . . . . 325

6.6.1 Wall Specimen W1 . . . . . . . . . . . . . . . . . . 325

6.6 .2 Wall Specimen W2 . . . . . . . . . . . . . . . 331

\section{Chapter 7. Conclusions and Future Research}

7.1 Conclusions . . . . . . . . . . . . . . . . . 336

7.2 Recommendations for Future Research . . . . . . . . . . . . . . 338

$\begin{array}{ll}\text { List of References } & 340\end{array}$

Appendix A. Hybrid Simulation Results Summary 


\section{Appendix B. Additional Design Details}

B.1 Design Loads . . . . . . . . . . . . . . . . . . . . . . . . . . . . . 379

B.2 Equivalent Static Force Procedure . . . . . . . . . . . . . . . . 380

B.3 Response Spectrum Analysis . . . . . . . . . . . . . . . . . . . . . 383

B.4 Design of Gravity Load Resisting System . . . . . . . . . . . . . . . . . . 385

B.5 Experimental Test Setup Design . . . . . . . . . . . . . . . . . . 388

B.5.1 Rigid Loading Beam . . . . . . . . . . . . . . . . . . 388

B.5.2 Steel Column Assembly Design . . . . . . . . . . . . . . . . . . . 389

B.5.3 RBS Detail Design . . . . . . . . . . . . . . . . . 391

\section{Appendix C. Drawings}




\section{List of Tables}

\section{Part I: Seismic Retrofit of Deficient Walls with FRP Sheets}

3.1 Shear wall specimen geometry, aspect ratio, and reinforcement ratios . . . . . . 33

3.2 Steel reinforcement material properties . . . . . . . . . . . . . . 34

3.3 Concrete and CFRP material properties . . . . . . . . . . . . . . . 35

3.4 Design flexural and shear strengths of control walls . . . . . . . . . . . . . . 40

3.5 Design flexural and shear strengths of retrofitted walls . . . . . . . . . . 45

4.1 Material properties for the mechanical and FRP anchor systems . . . . . . . 68

5.1 Control wall results . . . . . . . . . . . . . . . . . . 71

5.2 Repaired wall results . . . . . . . . . . . . . . . . . . 83

5.3 Strengthened wall results . . . . . . . . . . . . . . . . . 95

5.4 Shear wall response comparison with respect to control . . . . . . . . . . . . . 104

5.5 Comparison between strength prediction equations and experimental results . . . 117

\section{Part II: Building Scale Performance Evaluation using Hybrid Simulation}

3.1 Shear wall design forces from equivalent static force procedure . . . . . . . . . . 185

3.2 Modal properties of prototype RC shear wall structure . . . . . . . . . . . . . 187

3.3 Shear wall design forces from response spectrum analysis . . . . . . . . . . . . . 189

3.4 Shear wall flexural design properties . . . . . . . . . . . . . . . . . . . . . . . . . . . . . . . . . . . .

3.5 Shear wall shear design properties . . . . . . . . . . . . . . . . 200

3.6 Prototype structure scaling factors . . . . . . . . . . . . . . . . . . . . 201

3.7 Scaled shear wall design properties . . . . . . . . . . . . . . . . . . . . . . . 201

4.1 Shear wall material properties (from Hiotakis, 2004) . . . . . . . . . . . . . 204

4.2 Model analysis times under varying dynamic analysis parameters . . . . . . . 221

5.1 Average steel reinforcement material properties . . . . . . . . . . . . . . . . 237

5.2 Concrete material properties . . . . . . . . . . . . . . . 237

5.3 MTS 793 PID tuning parameters for 3-DOF station setup . . . . . . . . . . 251

5.4 Hybrid simulation ground motion sequence . . . . . . . . . . . . . 259

5.5 Hybrid simulation earthquake ground motion sequence . . . . . . . . . . . 261

6.1 Shear wall W1 lateral, axial, and rotational initial stiffness . . . . . . . . . . . 272

6.2 W1 critical structural response parameters . . . . . . . . . . . . . . . . 284

6.3 W1 structural response parameters . . . . . . . . . . . . . . . . . 287

6.4 W1 global structural response parameters (part 1 of 2 ) . . . . . . . . . . . . 290

6.5 W1 global structural response parameters (part 2 of 2$) \ldots \ldots . \ldots 291$

6.6 W2 Initial stiffness and yield displacement . . . . . . . . . . . . . . . . . . 292 
6.7 W2 critical structural response parameters . . . . . . . . . . . . . . . . . 310

6.8 W2 structural response parameters (part 1 of 2$) \ldots \ldots . \ldots . \ldots . \ldots 311$

6.9 W2 structural response parameters (part 2 of 2 ) . . . . . . . . . . . . . . 312

6.10 W2 global structural response parameters (part 1 of 2 ) . . . . . . . . . . . . 315

6.11 W2 global structural response parameters (part 2 of 2 ) . . . . . . . . . . 316

B.1 Specified dead and live loads . . . . . . . . . . . . . . . . . 381

B.2 Specified wind loads . . . . . . . . . . . . . . . . . . 381

B.3 Uniform hazard spectrum for Victoria, British Columbia . . . . . . . . . . . . . 382

B.4 Seismic weight of prototype structure . . . . . . . . . . . . . . . . . . . . . . . . . . . . 383

B.5 Equivalent static force procedure results . . . . . . . . . . . . . . . . 384

B.6 Response spectrum analysis results . . . . . . . . . . . . . 386

B.7 Dimensions of the rigid steel loading beam . . . . . . . . . . . . . 388

B.8 Moment capacity and shear strength of the rigid loading beam . . . . . . . . . 389

B.9 Dimensions of the rigid steel column . . . . . . . . . . . . . . . . . . . . . . . . . . . . . . . .

B.10 Design dimensions for the RBS detail . . . . . . . . . . . . . . . . . . . . . . . . . . . . . . . . . . . . . . .

B.11 Bolted end plate details for RBS connection . . . . . . . . . . . . . . . . 393 


\section{List of Figures}

\section{Part I: Seismic Retrofit of Deficient Walls with FRP Sheets}

2.1 Shear wall failure modes: (a) flexural failure; (b) diagonal tension shear failure; (c) diagonal compression shear failure; (d) sliding shear failure . . . . . . . . . . . 11

2.2 (a) Lap splice mechanical behaviour; (b) lap splice splitting failure . . . . . . . . . 13

2.3 Hysteretic response behaviour comparison for original and rehabilitated shear wall specimens (from Antoniades et al., 2005) . . . . . . . . . . . . . . 15

2.4 (a) Shear wall and test setup; (b) sixth storey moment-rotation hysteretic response behaviour at $200 \%$ earthquake ground motion intensity (from El-Sokkary et al.,

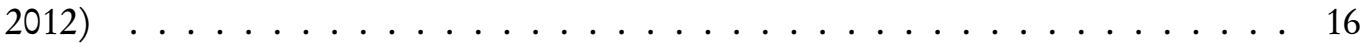

2.5 (a) Lap splice failure; Shear wall hysteretic response behaviour: (b) control wall;

(c) retrofitted wall(from Paterson and Mitchell, 2003) . . . . . . . . . . . 17

2.6 (a) Retrofitting scheme; Shear wall hysteretic response behaviour: (b) control wall; (c) retrofitted wall (from Layssi et al., 2012) . . . . . . . . . . . . . . . 18

2.7 Force-deformation response behaviours for control (CW), repaired (RW), and strengthened (SW) shear wall specimens (from Cruz-Noguez et al., 2014b) . . . . 19

2.8 Anchorage requirements in FRP retrofit of RC beams and shear walls . . . . . . . 22

2.9 Common anchor systems used in FRP sheet retrofit applications . . . . . . . . 24

2.10 Failure progression of steel angle anchor . . . . . . . . . . . . . . 26

2.11 Failure progression of steel tube anchor . . . . . . . . . . . . . . 27

2.12 Anchor performance comparison: (a) hysteretic response; (b) force-deformation response (Lombard et al., 2000; Hiotakis et al., 2004 and Cruz-Noguez et al., 2014b) 29

3.1 Steel reinforcement detailing: (a) series 1; (b) series 2; (c) series 3; (d) series 4 (36 $d_{b}$

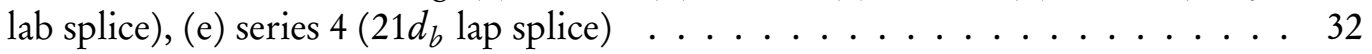

3.2 Material test results: (a) concrete; (b) steel; (c) SCH-41 Tyfo CFRP . . . . . . . . 36

3.3 CFRP reinforcement detailing: (a) series 1; (b) series 2; (c) series 3; (d) series 4 $\left(36 d_{b}\right.$ lab splice), (e) series $4\left(21 d_{b}\right.$ lap splice $) \ldots \ldots \ldots \ldots . \ldots \ldots$

3.4 Shear wall experimental test setup . . . . . . . . . . . . . . . . 46

3.5 Shear wall in-plane cyclic loading protocol . . . . . . . . . . . . 48

3.6 Steel strain gauge configuration for each shear wall series . . . . . . . . . . . . . . 49

3.7 LVDT configuration for each shear wall series . . . . . . . . . . . . . . . . 50

3.8 Test setup for the digital image correlation $\ldots \ldots \ldots \ldots$

3.9 ImPro Stereo graphical user interface $\ldots \ldots \ldots \ldots \ldots$

3.10 Region of interest: (a) CW4a; (b) RW4a; (c) CW4b; (d) RW4b . . . . . . . . . . 55 
4.1 (a) Anchor geometries; (b) anchor cross-sections (Lombard et al., 2000; Hiotakis et al., 2004) . . . . . . . . . . . . . . . . . . . . 57

4.2 (a) Idealized FRP stress profiles; (b) cross-section pressure distributions . . . . . . 58

4.3 Angle anchor: (a) analytical modeling results; (b) experimental observations (Lombard et al., 2000) . . . . . . . . . . . . . . . . 61

4.4 Tube anchor: (a) analytical model results; (b) experimental observations . . . . . 61

4.5 Tube anchor optimization: (a) finite element model; (b) optimization results . . . 63

4.6 Effect of anchor tube flexibility on vertical FRP stress distribution . . . . . . . 64

4.7 Design methodology example for FRP retrofitted RC shear wall . . . . . . . . . . 66

4.8 Tube anchor system design details . . . . . . . . . . . . . . . 67

4.9 FRP anchor system design details . . . . . . . . . . . . . . . . 68

5.1 Hysteretic response behaviour and concrete crack distributions for control walls:

(a) CW1; (b) CW2; (c) CW3; (d) CW4a (e) CW4b . . . . . . . . . . . . . . 72

5.2 Damage to shear wall specimens CW1 and CW2 . . . . . . . . . . 73

5.3 Wall specimen CW1 steel strain profiles: (a) vertical; (b) horizontal . . . . . . . . 73

5.4 Wall specimen CW2 steel strain profiles: (a) vertical; (b) horizontal . . . . . . . . 73

5.5 Wall specimen CW3 steel strain profiles: (a) vertical; (b) horizontal . . . . . . . . 74

5.6 Damage to shear wall specimens: (a) CW4a; (b) CW4b . . . . . . . . . . . . 76

5.7 Wall specimen CW4a lap splice strain: (a) bottom lapped bar; (b) top lapped bar . 77

5.8 Specimen CW4a horizontal steel strain distributions: (a) vertical; (b) horizontal . 77

5.9 Concrete Crack distributions for wall specimen CW4a at select peak cycles . . . . 78

5.10 Specimen CW4b lap splice strain: (a) bottom lapped bar; (b) top lapped bar . . . 79

5.11 Wall specimen CW4b horizontal steel strain distributions: (a) top; (b) bottom . . 80

5.12 Concrete crack distributions for specimen CW4b at select peak cycles . . . . . . . 80

5.13 Horizontal cracking up to lap splice height in specimen CW4b . . . . . . . . 81

5.14 Repair procedure: (a) crack epoxy injection; (b) surface preparation; (c) vertical CFRP layers; $(\mathrm{d})$ horizontal CFRP layers . . . . . . . . . . . . . . . 82

5.15 Hysteretic response and FRP-concrete debonding patterns for repaired walls: (a) RW1; (b) RW2; (c) RW3; (d) RW4a (e) RW4b . . . . . . . . . . . . . . . . . . 84

5.16 Specimen RW1 flexural steel reinforcement hysteretic response . . . . . . . . . 85

5.17 Specimen RW2 CFRP strain profiles: (a) vertical; (b) horizontal . . . . . . . . . . 87

5.18 Specimen RW3 steel/CFRP strain profiles: (a) vertical steel; (b) horizontal CFRP 88

5.19 Damage to repaired walls with lap splices: (a) RW4a; (b) RW4b . . . . . . . . . . 89

5.20 Comparison of vertical strain in top and bottom lapped bars: (a) CW4a; (b) RW4a 90

5.21 CFRP surface strain fields for specimen RW4a at select peak cycles . . . . . . . 91

5.22 Specimen RW4a horizontal CFRP strain profiles . . . . . . . . . . . . . . 91

5.23 Observation of horizontal cracks above lap splice for RW4a . . . . . . . . . . . 92

5.24 CFRP surface strain fields for specimen CW4b at select peak cycles . . . . . . . 93

5.25 Relative displacement analysis at lap splice height for RW4b . . . . . . . . . . . 93

5.26 Hysteretic behaviour and concrete crack distributions for the strengthened walls:

(a) SW1; (b) SW2a; (c) SW2b (d) SW3 . . . . . . . . . . . . . . . . . . . 96

5.27 Damage to strengthened wall specimen SW1 f . . . . . . . . . . . . . 97

5.28 Specimen SW1 steel strain profiles: vertical (left) and horizontal (right) . . . . . . 97

5.29 Damage to strengthened wall specimens SW2a and SW2b . . . . . . . . . . . . 99

5.30 Vertical and horizontal steel strain profiles: (a) SW2a; (b) SW2b . . . . . . . . . . 101 
5.31 Vertical and horizontal CFRP strain profiles: (a) SW2a; (b) SW2b . . . . . . . . . 101

5.32 Specimen SW3: (a)Vertical steel strain profiles; (b)Horizontal CFRP/steel strain . 103

5.33 Specimen SW3: (a) vertical steel strain profiles; (b) horizontal CFRP/steel strain . 103

5.34 Damage to the foundation of wall specimen SW3 . . . . . . . . . . . . . . 104

5.35 Control and repaired hysteretic responses: (a)W1; (b)W2; (c)W3; (d)W4a; (e) W4b 105

5.36 Control versus strengthened hysteretic response comparison: (a)SW1/CW1;

(b)SW2a/CW2 \& SW2b/CW2; (c)SW3/CW3 . . . . . . . . . . . . . 106

5.37 Comparison of the hysteretic response behaviour of specimens SW2a and SW2b . 109

5.38 Illustration of flexure, shear, and sliding shear displacement in RC shear wall . . . 112

5.39 Flexure, shear, and sliding shear displacements: (a) CW1; (b) CW2; (c) CW3 . . . 113

5.40 Flexure, shear, and sliding shear displacements: (a) W1; (b) W2 specimens . . . . 115

5.41 Displacement contribution time-histories: (a) CW1; (b) RW2; (c) SW2a; (d) SW2b 115

5.42 Specimen SW2a strain profiles: (a) vertical CFRP; (b) horizontal CFRP . . . . . 120

5.43 Specimen SW2b strain profiles: (a) vertical CFRP; (b) horizontal CFRP . . . . . 121

5.44 Anchor damage at ultimate: (a) SW2a (tube anchor); (b) SW2b (FRP fan anchor) 123

5.45 Force-displacement response comparison: (a) strengthened wall; (b) repaired wall

(From Cruz-Noguez et al., 2014c) . . . . . . . . . . . . . . . . . . . 126

5.46 Force-displacement response comparison: (a) SW1; (b) SW2a (From Hassan, 2015) 127

\section{Part II: Building Scale Performance Evaluation using Hybrid Simulation}

2.1 Characteristics of structural test methods in earthquake engineering . . . . . . . 139

2.2 Hybrid simulation command-feedback process . . . . . . . . . . . . . . 145

2.3 Implicit Newmark method with fixed iterations and equal displacement increments (adapted from Schellenberg et al., 2009) . . . . . . . . . . . . . . . 152

2.4 Generalized-alpha-OS method (adapted from Schellenberg et al., 2009) . . . . . . 158

2.5 Ramp-and-hold versus predictor-corrector control strategies (adapted from Schellenberg et al., 2009) . . . . . . . . . . . . . . . . . . . 163

2.6 Three-storey steel structure and actuator placement (from Molina et al., 1999) . . 166

2.7 Reinforced concrete structure and actuator placement (from Negro et al., 2004) . 167

2.8 Reinforced concrete frame and numerical model (from Saouma et al., 2013) . . . . 169

2.9 Typical RC shear wall test specimen (from Whyte and Stojadinovic, 2013b) . . . 169

2.10 Steel rocking frame hybrid test setup (from Eatherton and Hajjar, 2010) . . . . 171

2.11 Damping device real-time hybrid test setup (from Karavasilis et al., 2011) . . . . . 173

3.1 Plan view of the 3 -storey prototype RC structure . . . . . . . . . . . . . . . . 180

3.2 Elevation view of the 3 -storey prototype RC structure . . . . . . . . . . . 181

3.3 Calculation of $B_{x}$ and $B_{y}$ for torsional sensitivity $\ldots \ldots \ldots \ldots \ldots$

3.4 Modal response of prototype RC shear wall structure . . . . . . . . . . . . . . 188

3.5 Bending moment capacity and shear strength envelopes . . . . . . . . . . . 194

3.6 Full-scale shear wall design . . . . . . . . . . . . . . . . . . . . . . 199

3.7 Scaled shear wall design . . . . . . . . . . . . . . . . . . . . . . . . . . . . . . . . . . . . . . . . . . . . . . . . . .

4.1 Typical shear wall specimen tested by Hiotakis (2004) . . . . . . . . . . . . 205

4.2 Beam-column model of Hiotakis (2004) shear wall . . . . . . . . . . . . . . 206

4.3 Material constitutive relationships: (a) steel; (b) concrete . . . . . . . . . . . . . . 207

4.4 Shear wall hysteretic response behaviour comparison: (a) Hiotakis (2004) experimental results; (b) OpenSees fibre model . . . . . . . . . . . . . . . . 208 
4.5 Multi-layered shell element coordinate system and element layers . . . . . . . . 210

4.6 Shear wall finite element models: 96, 270, 1080, and 1920 elements . . . . . . . . 211

4.7 (a) mesh sensitivity; (b) hysteretic response comparison with Hiotakis (2004) . . 212

4.8 (a) mesh sensitivity; (b) hysteretic response comparison with Hiotakis (2004) . . 215

4.9 Influence of $A_{p}$ and $B_{n}$ on the shear wall finite element model . . . . . . . 216

4.10 Finite element model of prototype RC shear wall . . . . . . . . . . . . . . 218

4.11 Sample earthquake time-history for prototype shear wall model . . . . . . . . . 219

4.12 Model sensitivity: (a) time-step (Newmark $\beta$ Method), (b) convergence tolerance;

(c) time-step (Newmark HS Method); (d) number of fixed iterations . . . . . . . 221

4.13 OpenFresco software architecture for local hybrid test (adapted from Schellenberg et al., 2007) . . . . . . . . . . . . . . . . . . 223

4.14 Coordinate transformation for OpenFresco experimental element . . . . . . . . 224

4.15 Coordinate transformation between global and actuator DOFs . . . . . . . 227

4.16 Combined OpenSees-OpenFresco hybrid shear wall model . . . . . . . . . . . . 230

4.17 Comparison between fully analytical and substrcutured hybrid shear wall models 231

5.1 Shear wall specimens geometry and steel reinforcement detailing . . . . . . . . 234

5.2 Reaction block geometry and steel reinforcement detailing . . . . . . . . . . 235

5.3 Steel column assembly geometry and design details . . . . . . . . . . . . 236

5.4 Material testing results: (a) concrete; (b) steel . . . . . . . . . . . . . . . . . 237

5.5 3D Experimental test setup for shear wall hybrid simulation . . . . . . . . . . 239

5.6 2D Experimental test setup for shear wall hybrid simulation . . . . . . . . . . . 239

5.7 Shear wall hybrid test setup in the laboratory . . . . . . . . . . . . . . . . 240

5.8 3D Experimental test setup for steel column assembly hybrid simulation . . . . . 242

5.9 Steel column assembly experimental test setup illustration . . . . . . . . . . . 243

5.10 Steel column assembly experimental setup in the laboratory . . . . . . . . . . 243

5.11 Architecture of the hybrid simulation setup at Carleton University . . . . . . . . 245

5.12 Potential deformation mechanisms in the experimental test setup . . . . . . . 246

5.13 High-precision encoder components and assembled device . . . . . . . . . . . . 247

5.14 (a) Command displacement; absolute displacement error: (b) $2 \mathrm{~mm} / \mathrm{s}$; (c) $8 \mathrm{~mm} / \mathrm{s}$;

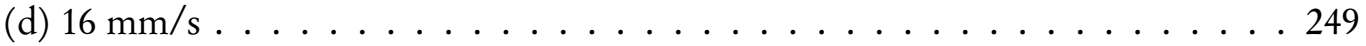

$5.153 \mathrm{D}$ actuator configuration in MTS DOF control software . . . . . . . . . . 252

5.16 Displacement command/feedback and error: (a) X; (b) Y; and (c) RZ . . . . . . . 253

5.17 LVDT and strain gauge locations for steel column hybrid test . . . . . . . . . 254

5.18 LVDT and strain gauge locations for RC shear wall hybrid test . . . . . . . . . . . 255

5.19 Image correlation: (a) setup; (b) result (adapted from Woods and Lau, 2016) . . 256

5.20 Hybrid testing station at Carleton University . . . . . . . . . . . . . . . 257

5.21 Earthquake records: (a) Northridge (1994); (b) Kobe (1995); (c) Loma Prieta (1989) 258

5.22 W1 hybrid test: (a) earthquake ground motion sequence; (b) response spectra . . 261

5.23 W2 hybrid test: (a) earthquake ground motion sequence and response spectra; (b) supplementary earthquake records and response spectra . . . . . . . . . 263

6.1 Initial stiffness of steel column assembly: (a) axial stiffness; (b) lateral stiffness . . 265

6.2 Model of column assembly test setup: (a) model mesh; (b) displacement results . . 266

6.3 Steel column friction model: (a) cyclic displacement time history; (b) comparison of theoretical and measured hysteretic responses (c) theoretical elastic stiffness; (c) friction model; . . . . . . . . . . . . . . . . . 267 
6.4 Friction characterization: (a) X/Y direction; (b) RZ direction . . . . . . . . . . 268

6.5 Hybrid simulation results from the steel column subassembly . . . . . . . . . . . 270

6.6 Shear wall W1 lateral, axial, and rotational initial stiffness . . . . . . . . . . . 273

6.7 W1 Northridge 20\%: Displacement and rotation command/feedback response . . 275

6.8 W1 Northridge 20\%: Comparison between actuator and encoder displacements . 275

6.9 W1 Northridge 50\%: (a) moment-rotation hysteresis; (b) maximum vertical strain

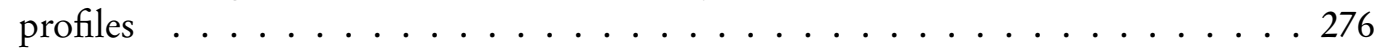

6.10 Image capture experimental test setup . . . . . . . . . . . . . . . . . 278

6.11 W1 Northridge 50\%: $e_{x y}$ surface strain field and crack distribution . . . . . . . . 278

6.12 W1 Northridge 50\%: (a) unfiltered Y-force; (b) filtered Y-force . . . . . . . . . . . 279

6.13 W1 Northridge 100\%: (a) top displacement history; (b) moment-rotation hysteresis 279

6.14 W1 Northridge 100\%: strain profiles in the vertical reinforcement . . . . . . . . . 280

6.15 W1 Northridge 200\%: (a) top displacement history; (b) moment-rotation hysteresis 282

6.16 W1 Northridge 200\%: maximum crack width distributions . . . . . . . . . . . . 283

6.17 W1 Northridge 300\%: (a) X displacement and hysteresis; (b) Z rotation and hysteresis . . . . . . . . . . . . . . . . . . . 284

6.18 W1 Northridge $300 \%$ : vertical strain profiles . . . . . . . . . . . . . 285

6.19 Damage to shear wall W1 following the hybrid simulations . . . . . . . . . 286

6.20 Comparison between analyzed and marked crack distributions . . . . . . . . . 286

6.21 Comparison of global shear wall response values . . . . . . . . . . . . . . 289

6.22 Shear wall W2 lateral, axial, and rotational initial stiffness . . . . . . . . . . . 293

6.23 W2 Northridge 20\%: displacement and rotation command/feedback response . . 296

6.24 Comparison of $\mathrm{X}$ displacement and $\mathrm{Z}$ rotation error for specimens W1 and W2 . 296

6.25 W2 Northridge 100\%: (a) force-displacement and (b) moment-rotation hysteretic responses . . . . . . . . . . . . . . . . . . . . . . 297

6.26 W2 Northridge 100\%: strain profiles: (a) vertical steel reinforcement at wall base; (b) horizontal steel reinforcement over wall height . . . . . . . . . . . . . . 298

6.27 W2 Northridge 100\%: maximum crack width distribution . . . . . . . . . . . . 298

6.28 W2 Northridge 200\%: (a) moment rotation hysteresis; (b) vertical steel strain profile at wall base . . . . . . . . . . . . . . . . . . . . 299

6.29 W2 Northridge 300\%: (a) X displacement time history and force displacement hysteresis; (b) Z rotation time history and moment rotation hysteresis . . . . . . 301

6.30 Northridge 100\%: response comparison for initial and aftershock earthquakes . . 302

6.31 W2 Kobe 50\%: (a) X displacement and shear force time histories; (b) Z rotation and moment-rotation hysteresis . . . . . . . . . . . . . . 303

6.32 W2 Kobe 50\%: maximum crack width distributions . . . . . . . . . . . . 303

6.33 W2 Loma Prieta 150\%: (a) Z moment rotation hysteresis; (b) maximum vertical steel reinforcement strain profiles at shear wall base . . . . . . . . . . . . . . 304

6.34 W2 Loma Prieta 150\%: concrete crack distribution . . . . . . . . . . . . . . . 305

6.35 Comparison of $\mathrm{X}$ displacement and $\mathrm{Z}$ rotation error for specimen $\mathrm{W} 2 \ldots . . . .306$

6.36 Comparison of gravity load (Y-force) response during Northridge $20 \%$ and Loma Prieta $150 \%$ hybrid tests . . . . . . . . . . . . . . . . . 307

6.37 (a) lateral displacement and force time histories; (b) force displacement and moment rotation hysteretic responses . . . . . . . . . . . . . . . . 308 
6.38 Maximum measured strain values: (a) vertical steel reinforcement at wall base and mid-height; (b) horizontal steel reinforcement over wall height . . . . . . . . . . 308

6.39 Damage to shear wall W2 following the cyclic test . . . . . . . . . . . . . . . 309

6.40 Comparison between analyzed and marked crack distributions . . . . . . . . . 310

6.41 Comparison of global shear wall response values . . . . . . . . . . . . . . . 314

6.42 Northridge 20\% global response: (a) first storey displacement time history; (b) maximum displacement profile; (c) maximum overturning moment profile . . . . 317

6.43 Northridge $100 \%$ local response: (a) X displacement and Z rotation time histories; (b) force displacement and moment rotation hysteresis . . . . . . . . . . . . . 319

6.44 Northridge 100\% local response: (a) X displacement and force time histories; (b) maximum inter-storey drift, shear, and moment distributions . . . . . . . . . . 320

6.45 Northridge 200\% local response: (a) X displacement and Z rotation time histories; (b) force displacement and moment rotation hysteresis . . . . . . . . . . . . . 321

6.46 Northridge $200 \%$ local response: (a) X displacement and $\mathrm{Z}$ moment time histories; (b) maximum inter-storey drift, shear, and moment distributions . . . . . . . . 322

6.47 Northridge $300 \%$ local response: (a) X displacement and Z rotation time histories; (b) force displacement and moment rotation hysteresis . . . . . . . . . . . . . 324

6.48 Comparison between marked cracked patterns for wall specimens W1 and W2 . . 324

6.49 Northridge 20\%: comparison between fully analytical and hybrid test results . . . 327

6.50 Northridge 50\%: comparison between fully analytical elastic wall model and hybrid test results . . . . . . . . . . . . . . . . . . . . . 327

6.51 Northridge 50\%: comparison between fully analytical nonlinear wall model and hybrid test results . . . . . . . . . . . . . . . . . . . 328

6.52 Northridge 100\%: comparison between fully analytical and hybrid test results . . 329

6.53 Northridge 200\%: comparison between fully analytical and hybrid test results . . 329

6.54 Comparison between fully analytical and hybrid test results: (a) Northridge $300 \%$

(b) Northridge $100 \%, 200 \%$, and $300 \%$ earthquake sequence . . . . . . . . . 331

6.55 Northridge 20\%: comparison between fully analytical and hybrid test results . . . 332

6.56 Northridge 200\%: comparison between fully analytical and hybrid test results . . 333

6.57 Northridge 300\%: comparison between fully analytical and hybrid test results . . 335

\section{Appendix A: Hybrid Simulation Results Summary}

A.1 W1 Hybrid Simulation: Northridge 20\% . . . . . . . . . . . . . . . . . . . . . . 355

A.2 W1 Hybrid Simulation: Northridge 50\% . . . . . . . . . . . . . . . . . . . . . . . 356

A.3 W1 Hybrid Simulation: Northridge 100\% . . . . . . . . . . . . . . . . 357

A.4 W1 Hybrid Simulation: Northridge 200\% . . . . . . . . . . . . . . . . . 358

A.5 W1 Hybrid Simulation: Northridge $300 \%$. . . . . . . . . . . . . . . 359

A.6 W2 Hybrid Simulation: Northridge 20\% . . . . . . . . . . . . . . . . 360

A.7 W2 Hybrid Simulation: Northridge 100\% . . . . . . . . . . . . . . . . 361

A.8 W2 Hybrid Simulation: Northridge 200\% . . . . . . . . . . . . . . . . . . . . 362

A.9 W2 Hybrid Simulation: Northridge 300\% . . . . . . . . . . . . . . . . 363

A.10 W2 Hybrid Simulation: Northridge 100\% Aftershock . . . . . . . . . . . . . 364

A.11 W2 Hybrid Simulation: Kobe 50\% . . . . . . . . . . . . . . . . 365

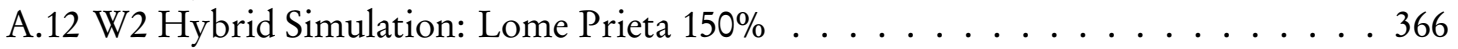

A.13 W1 global shear wall response: Northridge $20 \% \ldots$. . . . . . . . . . . . . 367 
A.14 W1 global shear wall response: Northridge $50 \%$. . . . . . . . . . . . . . 368

A.15 W1 global shear wall response: Northridge $100 \%$. . . . . . . . . . . . . . . . . . . . . . . . 369

A.16 W1 global shear wall response: Northridge $200 \%$. . . . . . . . . . . . . . . . . . . . . . . . . . . . . . . .

A.17 W1 global shear wall response: Northridge $300 \%$. . . . . . . . . . . . . . . . . . . 371

A.18 W2 global shear wall response: Northridge 20\% . . . . . . . . . . . . . . . . 372

A.19 W2 global shear wall response compared with W1: Northridge 100\% . . . . . . . 373

A.20 W2 global shear wall response compared with W1: Northridge 200\% . . . . . . . 374

A.21 W2 global shear wall response compared with W1: Northridge 300\% . . . . . . 375

A.22 W2 global shear wall response: Northridge 100\% . . . . . . . . . . . . . . . . . 376

A.23 W2 global shear wall response: Kobe $50 \%$. . . . . . . . . . . . . . . . . . . . . 377

A.24 W2 global shear wall response: Loma Prieta $150 \% \ldots 378$ 


\section{Nomenclature}

The following symbols are used in this thesis:

$A_{c v}=$ net area of cross-section resisting shear $\left(\mathrm{mm}^{2}\right)$

$A_{e}=$ effective shear wall area, taken as $90 \%$ of the total shear wall area $\left(\mathrm{mm}^{2}\right)$;

$A_{g}=$ gross area of the structural element $\left(\mathrm{mm}^{2}\right)$;

$A_{w}=$ cross-sectional area of the wall $\left(\mathrm{mm}^{2}\right)$;

$A_{v}=$ area of horizontal steel reinforcement within a distance of $\mathrm{s}\left(\mathrm{mm}^{2}\right)$;

$B_{x}=$ torsional sensitivity factor;

$c=$ depth to the neutral axis from extreme fibre in compression $(\mathrm{mm})$;

$d=$ distance from extreme fiber in comp. to centroid of tensile reinforcement $(\mathrm{mm})$;

$D_{n x}=$ total building width in direction under consideration $(\mathrm{mm})$;

$d_{v}=$ effective shear depth, taken as greater of $0.9 \mathrm{~d}$ or $0.72 \mathrm{~h}(\mathrm{~mm})$;

$d_{x e, a v g}=$ average of four corner displacements under torsional loading $(\mathrm{mm})$;

$d_{x e, \max }=$ maximum of four corner displacements under torsional loading $(\mathrm{mm})$;

$E_{c}=$ concrete modulus of elasticity $(M P a)$;

$E_{s}=$ steel modulus of elasticity $(M P a)$;

$e_{x}=$ eccentricity between centre of mass and rigidity $(\mathrm{mm})$;

$F_{a}=$ site response modification factor for low-period structures $\left(T_{a}<1 s\right)$;

$f_{c}^{\prime}=$ 28-day concrete compressive strength $(M P a)$;

$F_{t}=$ concentrated force applied to top storey of structure $(k N)$;

$f_{y}=$ yield stress of the steel reinforcement $(M P a)$;

$F_{x}=$ seismic force applied at level $x(k N)$;

$h_{s}=$ height of the building storey under consideration $(\mathrm{mm})$;

$h_{n}=$ total building height $(\mathrm{mm})$;

$h_{w}=$ height of the wall $(\mathrm{mm})$;

$I_{e}=$ earthquake importance factor;

$I_{g}=$ gross moment of inertia of the cross-section $\left(\mathrm{mm}^{4}\right)$;

$L_{p}=$ plastic hinge length $(\mathrm{mm}) ;$

$l_{w}=$ length of the wall $(\mathrm{mm})$;

$M_{f}=$ factored applied moment $(N-m m)$;

$M_{r}=$ factored moment resistance $(k N-m m)$;

$M_{v}=$ higher mode factor, taken as 1.0 for $T_{a} \leq 1 \mathrm{~s}$; 


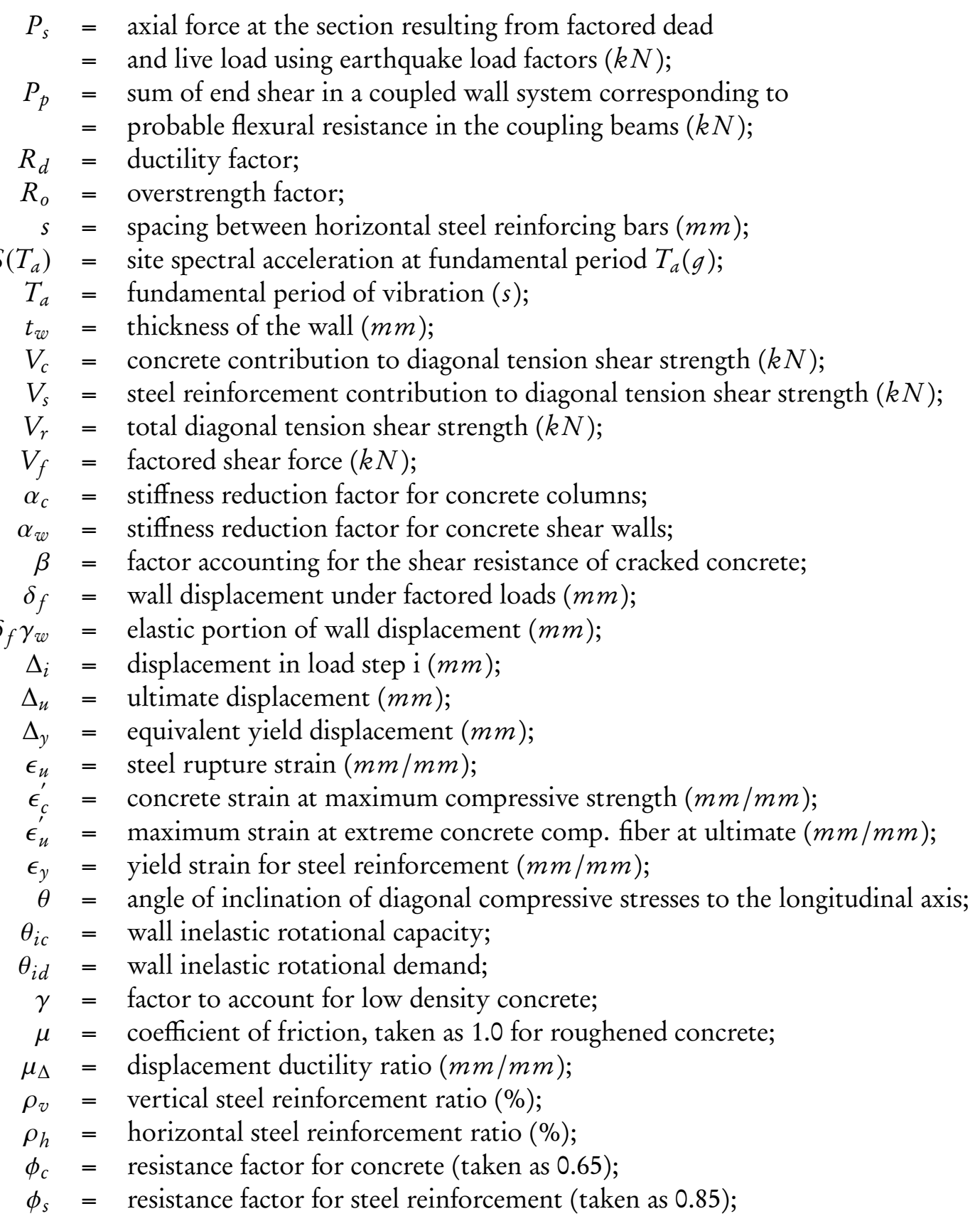




\section{Part I}

Seismic Retrofit of Deficient Walls with Fibre-Reinforced Polymer Sheets 


\section{Chapter 1. Introduction}

\subsection{Motivation}

Research over the past several decades has resulted in better understanding of the seismic behavior and significant improvements in the seismic performance of reinforced concrete (RC) shear wall structures. These advancements are reflected in the seismic design provisions in current design standards for RC structures in North America (ACI 318-14; CSA A23.3-14). Despite these improvements in earthquake resistant design of RC structures, there is still a large inventory of existing RC shear wall structures designed and constructed based on older less-stringent design guidelines (ACI 318-68; CSA A23.3-77) that are in need of repair or strengthening. Previous studies and observations during recent earthquakes (Turkey, 1999; Chile, 2010; New Zealand, 2011) have shown that these older RC shear wall structures are susceptible to severe damage under moderate to large earthquakes due to insufficient shear reinforcement, little or no concentration of vertical steel reinforcement or additional confinement of the concrete at the two ends of the shear wall, and the presence of lap splices within the plastic hinge region (Saatcioglu et al., 2001; Kam et al., 2011; Wallace, 2015). These deficiencies can result in a lack of shear strength, ductility, and energy dissipation capacity, which lead to poor seismic performance. Consequently, there is a need for an effective means to mitigate the seismic risk associated with these older existing RC shear wall structures. 


\subsection{Background}

A practical alternative to conventional retrofitting techniques is the use of externallybonded fibre-reinforced polymer (FRP) sheets. The majority of research to date on seismic retrofit of RC shear walls using FRP has focused on the use of FRP jackets to improve shear strength, energy dissipation capacity, and confinement of the boundary elements in shear wall specimens (Antoniades et al., 2003; Paterson and Mitchell, 2003; Ghobarah and Khalil, 2004; Elnady, 2008). The common approach to improve confinement in each of these studies is achieved by wrapping the FRP sheets around the ends of the shear wall, however, there are often cases when the ends of a RC shear wall are not exposed or inaccessible, making it impossible to apply the FRP sheets around the wall. Common examples include flanged and L-shaped shear walls in need of repair or strengthening. In such cases, additional effective concrete confinement cannot be provided by wrapping the FRP sheets around the ends of the wall without creating some separation between the wall and the adjacent member. The process of exposing the ends of the shear wall can also cause significant disruption to the occupants and operation of the facility and affect the torsional stiffness of the structural element. The present study also recognizes that flexural strengthening of RC shear walls may be required in addition to shear strengthening to meet current design standards, something that has not been addressed in previous studies for shear dominant walls. Past studies by Lombard et al. (2000), Hiotakis (2004), and Cruz-Noguez et al. (2014b) at Carleton University have investigated the use of carbon fibre-reinforced polymer (CFRP) sheets to increase

or restore the flexural strength of RC shear walls using externally bonded CFRP sheets applied to the faces of RC shear wall specimens, however, these studies focus on the retrofit of wall specimens designed according to modern design standards (CSA A23.3-94) and do not address the deficiencies in older nonductile RC shear walls susceptible to brittle shear failure.

\subsection{Research Objectives and Scope}

This study expands on previous research conducted by Lombard et al. (2000), Hiotakis (2004), and Cruz-Noguez et al. (2014b) to investigate the potential for using externally bonded 
CFRP sheets to enhance the seismic performance of shear wall specimens representative of those designed according to older RC design standards used in North America (ACI 318-68; CSA A23.377). Nine RC shear wall specimens expected to exhibit brittle shear behavior due to the deficiencies present in their design are constructed and tested. The shear wall specimens have three different height-to-length aspect ratios $(1.2,0.85$, and 0.65$)$, to study the effect this has on the cyclic behaviour of the shear wall specimens as well as the effectiveness of the CFRP retrofitting system in shear walls with small aspect ratios, commonly referred to as squat walls. The deficiencies in the design of the shear wall specimens are representative of those typically found in older construction practices including insufficient shear reinforcement, no additional concrete confinement or concentration of vertical steel reinforcement near the ends of the wall, presence of lap splices of the vertical steel reinforcement within the plastic hinge region, and a low concrete strength.

In an attempt to improve the seismic performance of the shear wall specimens, they are strengthened or repaired with unidirectional CFRP sheets applied in the vertical and horizontal directions and then cyclically tested to failure. In the present study, the CFRP sheets are not wrapped around the ends of the wall specimens to represent conditions encountered in the field application of FRP sheets applied only to the two wall panel surfaces, and thus to understand the effectiveness of the FRP retrofitting system without additional confinement at the two ends. The ability of the CFRP retrofitting system to improve the seismic performance of the wall specimens is assessed in terms of in-plane strength, ductility, and energy dissipation capacity. In addition, the performance and effectiveness of two different anchor systems used to transfer the tensile forces carried by the CFRP to the foundations of the specimens are also evaluated and a detailed design methodology for the application of a steel tube anchor system first implemented by Hiotakis (2004) is developed.

The objectives of the shear wall experimental tests are:

- Study the behaviour of RC shear walls detailed with deficiencies found in older less-stringent design standards, including insufficient shear reinforcement, a lack of confinement in the shear wall boundary elements, and lap splices of the longitudinal reinforcement within the 
plastic hinge region;

- Determine the effectiveness of using vertical and horizontal externally bonded FRP sheets to increase the in-plane flexural and shear strengths of non-ductile RC shear walls;

- Investigate the influence of aspect ratio on the behaviour of the wall specimens and effectiveness of the FRP retrofit;

- Compare the flexural and shear strengths of the wall specimens to strength prediction equations from a range of design standards as well as those from the literature to assess their ability to predict the strength of shear wall specimens with a range of aspect ratios;

- Study the performance of two different anchor systems (tube anchor, FRP anchors) used for the transfer of load carried by the FRP sheets to adjacent supporting structural members which also prevent premature debonding to allow utilization of a larger portion of the ultimate capacity of the FRP composite material; and

- Develop a comprehensive design methodology for the application of the steel tube anchor system in retrofitting applications of RC structures. 


\section{Chapter 2. Background}

\subsection{Failure Modes in RC Shear Walls}

The research presented in parts I and II of this thesis are motivated by the need to further examine the behaviour and improve the seismic performance of $\mathrm{RC}$ shear wall structures. Prior to in-depth discussion and analysis on the behaviour of RC shear walls, it is important to understand the parameters that influence their in-plane behaviour under earthquake ground motion and their potential modes of failure, both of which are discussed in detail in this section.

The design of civil engineering structures to remain elastic throughout the entire duration of a moderate to large earthquake event cannot be feasibly achieved, both from an aesthetic perspective because of the number and size of structural members that would be required, but also from an economic point of view of high cost-to-benefit ratio for ordinary structures. To overcome these limitations in seismically active regions around the world, modern seismic design standards permit specific structural elements to experience inelasticity at designated locations, commonly referred to as plastic hinging. Allowing plastic hinges to form in designated structural elements reduces the total seismic force on a structure. Adjacent structural elements to those that are expected to yield are designed at force levels higher than those associated with the yielding elements to ensure that plastic hinges form only at the desired locations throughout a structure. This design philosophy is commonly known as capacity-based design.

Modern seismic design standards allow engineers to detail RC shear walls as yielding elements such that they experience inelastic behavior during a major seismic event and act as a fuse, 
which dissipates seismic energy and protects surrounding structural elements from sustaining major damage. Reinforced concrete shear walls are typically detailed such that they form a plastic hinge at their base. The region over which the plastic hinge forms at the base of a RC shear wall is commonly referred to as the plastic hinge region. In the design of a RC shear wall structure, the engineer must ensure that the shear walls have sufficient stiffness, strength, and ductility to survive a major earthquake and prevent loss of life; this is the primary design objective in building codes used throughout the world. Past understanding led many engineers to believe that RC shear walls were too brittle in nature to resist seismically induced lateral loads (Fintel, 1995), however, through proper detailing of longitudinal and transverse reinforcement, a ductile response and sufficient energy dissipation capacity can be achieved. In tall slender walls, with a height-to-length aspect ratio larger than $2\left(h_{w} / l_{w}>2\right)$, this design goal can be easily achieved as walls with this aspect ratio behave like a beam and deform predominantly in flexure. Structural elements which deform predominantly in flexure have higher displacement ductilities and exhibit a gradual loss in strength up to failure. Alternatively, shear walls with a height-to-length aspect ratio less than $2\left(h_{w} / l_{w}<2\right)$ tend to exhibit a combination of flexure and shear behavior. Structural elements that behave predominantly in shear exhibit small displacement ductilities and fail in a sudden and brittle manner. When the aspect ratio is less than 1 , the shear wall is commonly referred to as a squat wall and will exhibit a shear dominated response, which can potentially lead to undesirable brittle failure modes that need to be considered in the shear wall design. In general, there are four commonly recognized failure modes for conventional RC shear walls subject to in-plane loading:

- Flexural failure;

- Diagonal tension shear failure;

- Diagonal compression failure; and

- Sliding shear failure;

In some cases, a shear wall may have lap splices of the vertical steel reinforcement located within the potential plastic hinge region typically at the base of the wall where the moment is maximum. In these scenarios, lap splice bond failure should also be considered as a fifth failure mode. Each 
of these failure modes are discussed in more detail in the following subsections.

\subsubsection{Flexural Failure}

Flexural failure refers to yielding of the vertical steel reinforcement in the plastic hinge region, typically along the base of a wall. This is the most desirable mode of failure in RC shear walls because it occurs in a ductile manner. This ductile flexural failure mode serves as an important source of energy dissipation in the seismic response of RC shear wall structures. To achieve a ductile flexural failure, the shear capacity of the wall must be larger than the load associated with yielding of the vertical steel reinforcement. In shear walls with aspect ratios larger than 2 $\left(h_{w} / l_{w}>2\right)$, flexural failure is achieved through proper detailing of vertical and horizontal steel reinforcement, resulting in significant non-linearity in the vertical reinforcing steel, as illustrated in Fig. 2.1a. Yielding of vertical steel reinforcement often coincides with the formation of a plastic hinge, typically at the base of the wall, however, in low aspect ratio walls $\left(h_{w} / l_{w} \leq 2\right)$, achieving a flexurally ductile failure is more difficult because of the high shear stresses in these types of walls at their full flexural capacity. In some cases, it can be difficult to provide sufficient shear reinforcement to eliminate the potential for eventual shear related failure. This is acceptable if the potential for shear failure is recognized when determining the ductility demand on the wall system. With this in mind, it is important to understand the three predominant modes of shear failure, which are discussed in the following sections.

\subsubsection{Diagonal Tension Shear Failure}

Walls with insufficient shear reinforcement in the form of horizontal steel stirrups are typically susceptible to diagonal tension shear failure. During the in-plane loading of a RC shear wall, diagonal cracks form in the concrete in the principle tensile stress direction. At the location of these cracks, horizontal steel reinforcement is responsible for transferring shear stresses across the cracks in the concrete. Under multiple reversed loading cycles, such as those during an earthquake, the horizontal steel reinforcement is subject to increasing inelastic deformations that can lead to rupture of the horizontal steel reinforcement in tension if there is not enough horizontal

Joshua Woods, Department of Civil and Environmental Engineering, Carleton University 
steel reinforcement to transfer the shear stress across a crack. After the horizontal steel reinforcement exceeds its ultimate tensile strain and ruptures, the shear stresses are no longer transferred across diagonal cracks in the concrete leading to failure of the shear wall in a sudden and brittle manner. As shown in Fig. 2.1b, diagonal tension shear failure is often characterized by a clear diagonal failure plane, which forms from one corner of the shear wall to the other. Diagonal tension shear failure is considered an undesirable mode of failure because of its brittle nature that causes a sudden loss in load carrying capacity, potentially leading to structural collapse. Parameters that influence the tendency for a RC shear wall to experience diagonal tension shear failure include the amount of axial load on the wall and the aspect ratio of the wall. As discussed previously, shear walls with a low aspect ratio experience high shear stresses, and thus are more susceptible to diagonal tension shear failure if adequate shear reinforcement is not provided.

\subsubsection{Diagonal Compression Failure}

Alternatively, when shear walls subject to high shear stresses are detailed with an adequate amount of transverse steel reinforcement to prevent diagonal tension shear failure, concrete crushing may occur in the bottom corners of the wall, or the toes, which is commonly referred to as diagonal compression. Under reversed cyclic load, high compressive forces develop along the shear wall compression strut (opposite to principle tensile stress direction), causing localized crushing in the toes of the shear wall. Degradation of the concrete in the toes of a shear wall under load reversals can result in instability, illustrated in Fig. 2.1c, because of a lack of confinement around the ends of a shear wall. In addition, if there is inadequate confinement of the vertical steel reinforcement in the boundary elements near the ends of a wall, this can lead to buckling of the vertical steel reinforcement and a sudden loss in load carrying capacity. After concrete crushing is initiated in the toes of a shear wall, it often spreads over the entire length of the wall under subsequent load reversals. Diagonal compression failure can be avoided by ensuring that the maximum shear stress in the concrete at the corresponding flexural strength does not exceed the ultimate diagonal compressive stress of the concrete through adequate concrete confinement and concrete compressive strength.

Joshua Woods, Department of Civil and Environmental Engineering, Carleton University 


\subsubsection{Sliding Shear Failure}

When adequate horizontal reinforcement is provided and the shear stresses in the wall are limited to less than the ultimate diagonal compressive stress of the concrete, the tendency for a shear wall to fail in diagonal tension and diagonal compression can be controlled. In such cases, flexural steel reinforcement is expected to yield within the plastic hinge region, typically located at the base of the wall. As a result of yielding along the base of the shear wall, the flexural steel reinforcement will experience large inelastic deformations causing a horizontal crack in the concrete along the base of the wall. This forms a horizontal shear plane that experiences a sliding action under multiple load reversals. The shear force is transferred along the horizontal shear path through friction between the concrete surfaces and dowel action of the flexural reinforcement. As illustrated in Fig. 2.1d, sliding of the wall along the shear plane results in a significant loss in stiffness, particularly at low-load levels, leading to what is referred to as "pinching" in the hysteretic response behaviour and a loss in energy dissipation capacity. Under further load reversals, the bond along vertical reinforcing bars as well as the aggregate interlock along the horizontal shear path will degrade, ultimately leading to kinking of the vertical bars (illustrated in Fig. 2.1d) and failure of the wall specimen.

\subsubsection{Lap Splice Failure Modes}

Prior to the introduction of modern seismic design provisions, older design guidelines permitted the use of lap splices in critical locations where significant inelastic deformations were anticipated, commonly referred to as the plastic hinge region. In doing so, the presence of lap splices of vertical steel reinforcement in the critical plastic hinge region of a shear wall introduces alternative modes of failure in addition to those discussed in the previous sections. Provisions in the ACI 318-68 American design standard permitted lap splices in RC walls such that they met the minimum lap length requirements of 24,30 , and 36 times the bar diameter $\left(d_{b}\right)$ for $275 \mathrm{MPa}$, $345 \mathrm{MPa}$, and $415 \mathrm{MPa}$ steel reinforcement, respectively. Alternatively, modern design standards compute required lap splice lengths based on the size of the bar being lapped, and the lap length 


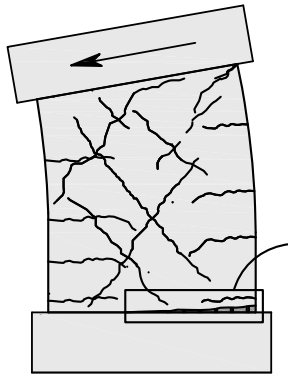

(a)

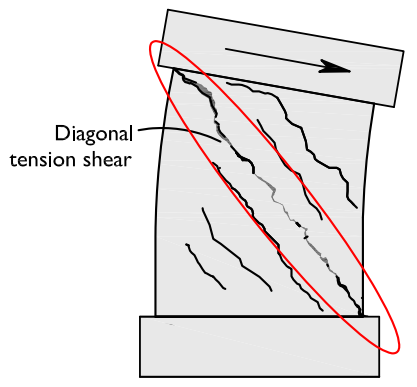

(b)

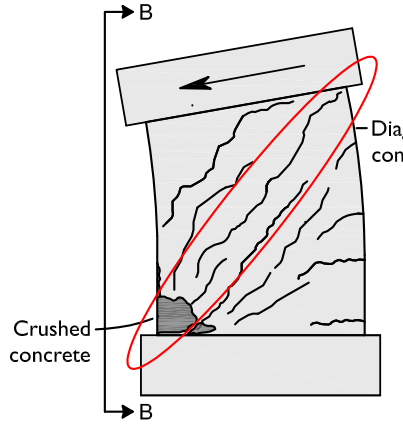

(c)

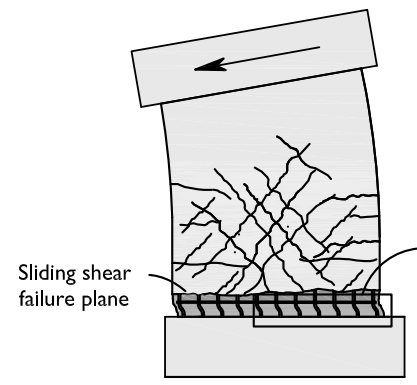

(d)
Flexurally ductile

failure mode

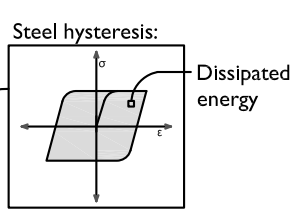

[From Hiotakis (2004)]

Diagonal shear failure plane

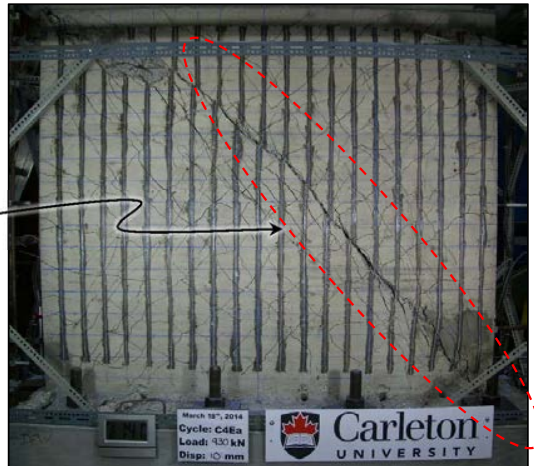

[From Woods et al. (20|5)]
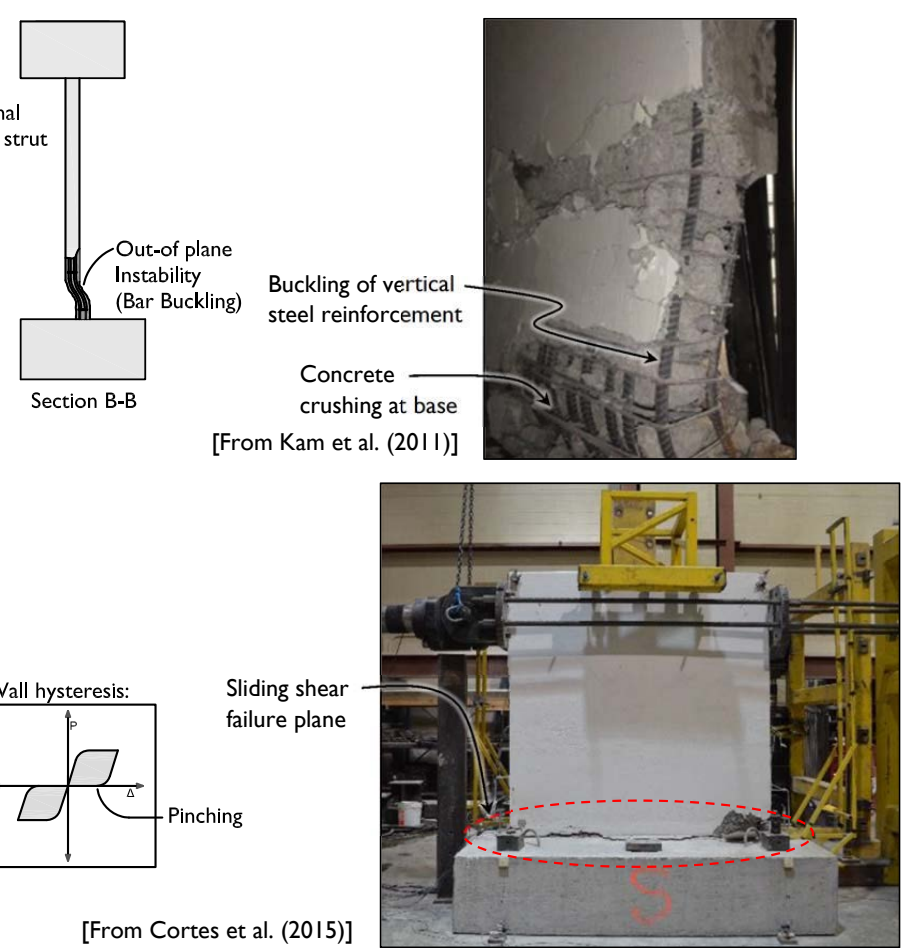

Figure 2.1 Shear wall failure modes: (a) flexural failure; (b) diagonal tension shear failure; (c) diagonal compression shear failure; (d) sliding shear failure 
required to reach yield in the lapped bars. In CSA A23.3-04, the simplified development length $\left(l_{d}\right)$ of deformed bars in tension for walls having a clear spacing between bars less than $2 d_{b}$ is computed using Eq. 2.1:

$$
l_{d}=0.6 k_{1} k_{2} k_{3} k_{4} \frac{f_{y}}{\sqrt{f_{c}^{\prime}}} d_{b} \leq 300 \mathrm{~mm}
$$

where $k_{1}$ through $k_{4}$ are modification factors to account for epoxy coated rebar, concrete density, and the size of the reinforcing bar, $f_{y}$ is the yield stress of the lapped steel reinforcement, $f_{c}^{\prime}$ is the 28-day compressive strength of the concrete, and $d_{b}$ is the diameter of the reinforcing bar being lapped. Using this approach, a standard 20M reinforcing bar for comparative purposes has a require lap length of 42 bar diameters $(840 \mathrm{~mm})$, greater than the recommended 36 times the bar diameter recommended in older design provisions.

Past test results on RC beams, column, and walls with insufficient lap splice length have demonstrated that lap splices located in plastic hinge zones have a tendency to slip when the compressive strain in the concrete at the base of the wall exceeds 0.002 (Priestley and Paulay, 1992), which crushes the concrete surrounding the lap splice resulting in slippage between lapped bars in tension under subsequent load reversals. Slippage between bars occurs as a result of degradation of the mechanical and chemical bonds that are formed between the steel rebar and concrete substrate after casting. The chemical bond relates to the adhesion between the two materials and the mechanical bond relates to the friction and interlocking mechanism between the ribs on the deformed steel reinforcing bar and the concrete keys formed when casting the concrete around the lap splice. As the lap splice is subject to load, bond stress forms between the concrete keys and the ribs of the bar as well as between the ribs of the two lapped bars.

Once relative slip between the two bars is initiated, two possible failure modes are considered: (1) pullout failure of the surrounding concrete or (2) splitting failure, caused by cracks in the concrete along the longitudinal direction of the lap splice. The tendency to experience one failure mode versus another depends on whether or not the splice is adequately confined. Confinement could result from the presence of transverse hoop reinforcement or the presence of flexural or shear reinforcement. If adequate confinement is provided, relative movement between the bars 
is restrained. A failure plane develops around the outside surface of the bar as the concrete keys around the bar are crushed, forming a failure surface, or sleeve, slightly larger than the diameter of the bar. In poorly confined concrete, splitting failure typically occurs when splitting cracks form around the lap splice. As illustrated in Fig. 2.2, when slipping occurs between two bars in a lap splice, splitting cracks oriented along the length of the lap splice form in the concrete. When sufficient confinement is not provided, the splitting cracks widen, and the ability of the bar to to maintain their load carrying capacity decreases. Failure occurs when the two bars slip relative to one-another.

In a RC shear wall, once splitting failure occurs between lapped bars they are no longer in contact with each other, which results in an incompatibility in transferring the lateral load and the wall specimen is not able to achieve its full ultimate moment capacity. In these cases, the strength of the wall is governed by the residual capacity of the lap splice. In addition to confinement, other factors that can influence the behaviour of lapped steel reinforcement include the vertical reinforcement ratio, spacing between lapped bars, the amount of concrete cover, and the presence of axial load (Priestley and Paulay, 1992).
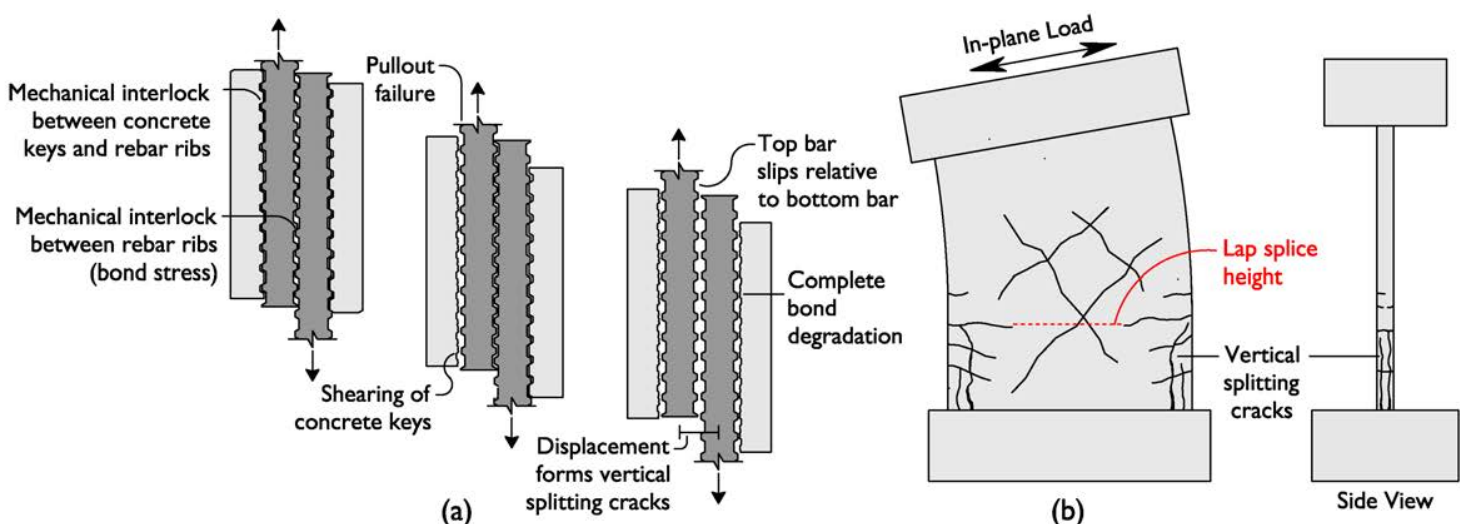

Figure 2.2 (a) Lap splice mechanical behaviour; (b) lap splice splitting failure 


\subsection{Structural Testing of FRP-Strengthened Shear Walls}

The majority of research to date on seismic retrofit of RC shear walls using externally bonded fibre-reinforced polymer (FRP) sheets has focused on the use of FRP jackets to improve shear strength, energy dissipation capacity, and confinement of the boundary elements in shear wall specimens (Antoniades et al., 2003; Paterson and Mitchell, 2003; Ghobarah and Khalil, 2004; Elnady, 2008).

A study by Antoniades et al. (2003) and Antoniades et al. (2005) focused on the use of externally bonded carbon fibre-reinforced polymer (CFRP) sheets to improve the seismic performance of shear wall specimens designed according to modern design standards, with sufficient shear reinforcement and adequate confinement of the boundary elements and the ends of the shear wall. The test specimens were low aspect ratio shear wall specimens $\left(h_{w} / l_{w}=1.0\right)$, in full conformity with modern European seismic design detailing for RC shear walls (Eurocode 8). The wall specimens were tested in a rehabilitation scenario, in which the wall specimens were initially loaded to failure and subsequently repaired using FRP sheets. The study applied horizontal Ushaped CFRP wraps around the wall specimens in an attempt to restore the strength of the walls. Figure 2.3 shows the force-deformation hysteretic response behaviour for the original and rehabilitated shear wall specimens. The study concluded that the use of CFRP sheets was able to restore the in-plane strength and the majority of the energy dissipation capacity in severely damaged RC shear walls. In addition, the authors also found that introducing adequately anchored strips of CFRP in the vertical direction, as flexural reinforcement, was an effective method of restoring the energy dissipation capacity in the damaged wall specimens.

Ghobarah and Khalil (2004) extended the work of Antoniades et al. (2003) and conducted an experimental investigation to improve the ductility, energy dissipation capacity, and prevent brittle shear failure in poorly detailed low aspect ratio shear wall specimens $\left(h_{w} / l_{w}=1.0\right)$ without lap splices. The study found that the control wall specimens without FRP sheets failed prematurely in diagonal tension shear failure. The authors found that the application of externally bonded CFRP sheets at a 45 degree angle prevented premature shear failure, and improved the 


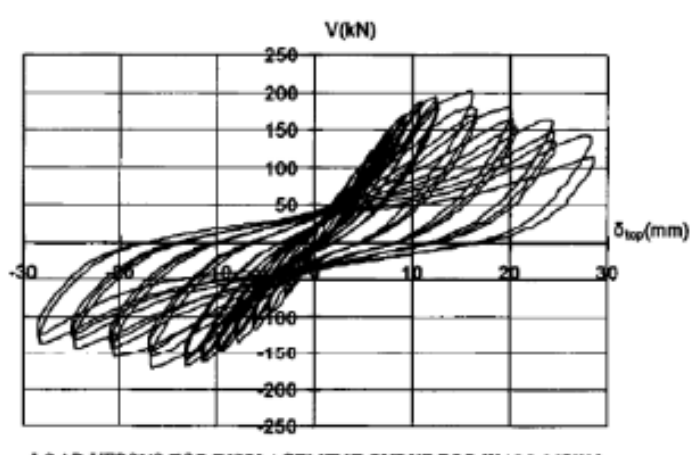

LOAD VERSUS TOP DISPLACEMENT CURVE FOR WALL MSW6

Original specimen

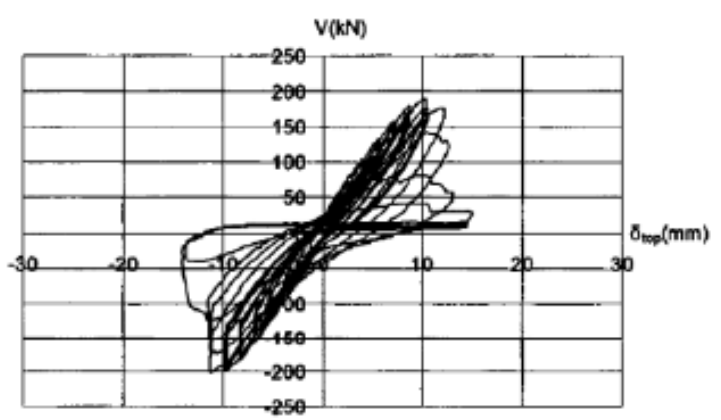

LOAD VERSUS TOP DISPLACEMENT CURVE FOR WALL. FRPMSW6

Repaired and strengthened specimen

Figure 2.3 Hysteretic response behaviour comparison for original and rehabilitated shear wall specimens (from Antoniades et al., 2005)

ductility and energy dissipation capacity of the wall specimens in both repair and strengthening applications. The study also found that the use of steel anchors through the wall performed better compared to CFRP anchors because of their ability to carry a higher load before yielding in tension.

More recently, a study by El-Sokkary et al. (2012) investigated the seismic behaviour of two 8-storey cantilevered RC shear walls rehabilitated using CFRP sheets and subjected to base excitations using a shake table. The study aimed to investigate the effect of higher modes on the behaviour of multi-storey shear walls as well as the performance of a CFRP retrofitting strategy. Figure 2.4 shows a typical 8-storey shear wall specimen and the results of the FRP retrofit. The unretrofitted wall specimens demonstrated significant inelastic deformations and formation of a plastic hinge at the first and sixth storeys. Following the initial test, the walls were rehabilitated with vertical and horizontal CFRP sheets wrapped around the wall at the sixth storey, and only horizontal CFRP sheets at the first-storey, to prevent increasing the strengh and stiffness of the first storey shear wall. Vertical CFRP sheets at the sixth storey were anchored through the floor slab using vertically oriented CFRP fan anchors. Results of the CFRP retrofit showed that the rotations and strains in the flexural reinforcement at the sixth storey shear wall were significantly reduced, even at $200 \%$ ground motion intensity level. The authors recommend that special attention be taken to detailing FRP fan anchors when used for flexural rehabilitation. 

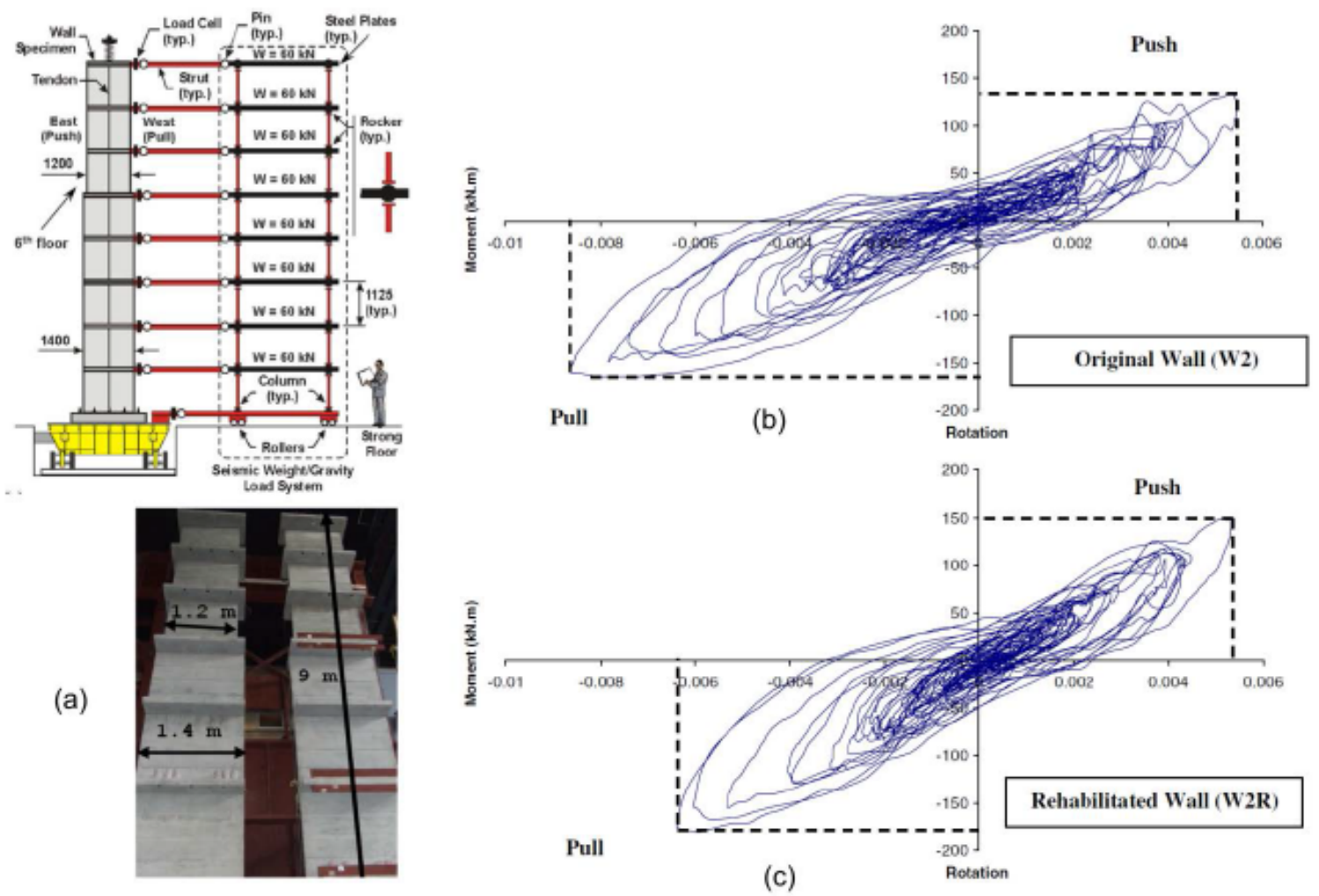

Figure 2.4 (a) Shear wall and test setup; (b) sixth storey moment-rotation hysteretic response behaviour at $200 \%$ earthquake ground motion intensity (from El-Sokkary et al., 2012) 
Past research on the use of CFRP sheets to improve the seismic performance of RC members with lap splices has been largely limited to RC beams and columns. In RC shear walls, the few studies have focused on the use of CFRP jackets to improve the shear strength and confinement of the boundary elements in flexurally dominant shear walls with aspect ratios larger than 2.5. In RC shear walls with adequate lap splice length, Paterson and Mitchell (2003) studied the use of two retrofitting strategies to improve the seismic performance of deficient flexural RC shear walls $\left(h_{w} / l_{w}=2.7 \& 3.2\right)$ with adequate lap splice length, namely a RC collar and CFRP wrap with headed bars. Both retrofitting techniques were successful in improving the ductility and energy dissipation capacity in flexurally dominant shear walls with lap splices of the longitudinal reinforcement in the plastic hinge region. The addition of the concrete collar shifted the height of the plastic hinge above the lap splice region, preventing brittle failure of the lap splice. In the wall specimen retrofitted with CFRP w rap and headed bars, the results showed an improvement in the confinement of the wall boundary elements and a reduction in the shear stress in the horizontal reinforcing steel. Figure 2.5 shows the lap splice splitting cracks in the test of the control wall, as well as a comparison of the force versus deformation response of the control and retrofitted wall specimen with CFRP sheets and headed bars.

A study by Layssi et al. (2012) expanded on previous research by Paterson and Mitchell (2003) and studied the effectiveness of using CFRP wraps for flexural walls $\left(h_{w} / l_{w}=2.7\right)$ with insufficient lap splice length in addition to poor confinement of the boundary elements and shear

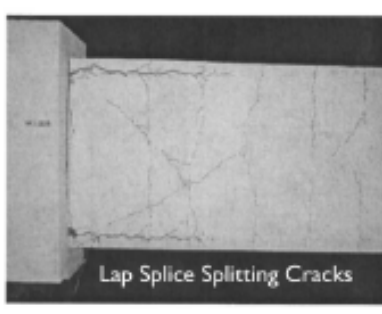

(a)

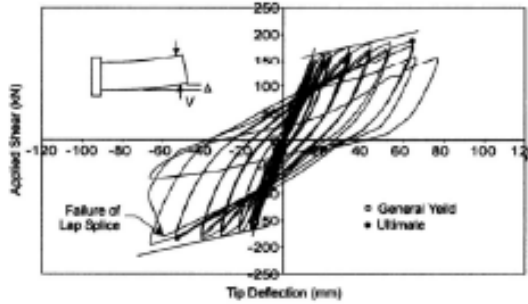

(b)

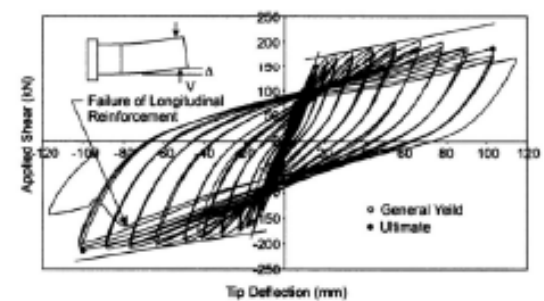

(c)

Figure 2.5 (a) Lap splice failure; Shear wall hysteretic response behaviour: (b) control wall; (c) retrofitted wall(from Paterson and Mitchell, 2003) 
deficiencies (Layssi et al., 2012). The proposed rehabilitation strategy in this study included externally bonded CFRP sheets wrapped around the wall and did not include headed bars or a concrete collar in an attempt to improve the practicality of the retrofitting scheme and ease of construction. In the control walls with lap splices, side-splitting failure of the lap splice cause by separation of the lapped bars and brittle failure in the lap splice region occurred before the vertical steel reinforcement yielded. Figure 2.6 shows the retrofitting scheme and hysteretic response behaviour of a control and strengthened wall specimen. The use of CFRP jackets in the strengthened walls was successful in delaying failure of the lap splice. Some yielding in the flexural reinforcement allowed the wall to reach the a ductility level of 2.0 and improved the energy dissipation capacity of the wall specimens.

The common approach to improve the seismic response of the wall specimens in each of these studies is achieved by wrapping the FRP sheets around the ends of the shear wall, however, there are often cases when the ends of a $\mathrm{RC}$ shear wall are not exposed or inaccessible, making it impossible to apply the FRP sheets around the wall. Common examples include flanged, Cshaped, or L-shaped shear walls in need of repair or strengthening. In such cases, additional confinement that is effective cannot be provided by wrapping the FRP sheets around the ends of the wall without creating some separation between the wall and the adjacent member. The process of exposing the ends of the shear wall would cause significant disruption to the occupants and operation of the facility. The present study also recognizes that flexural strengthening of RC

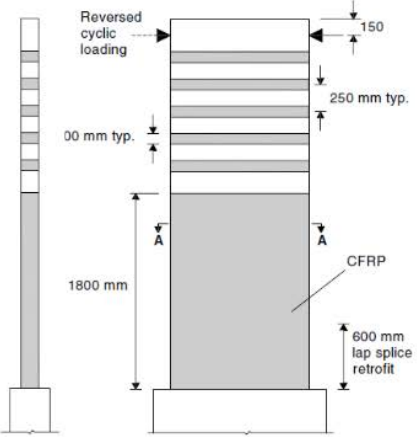

(a)

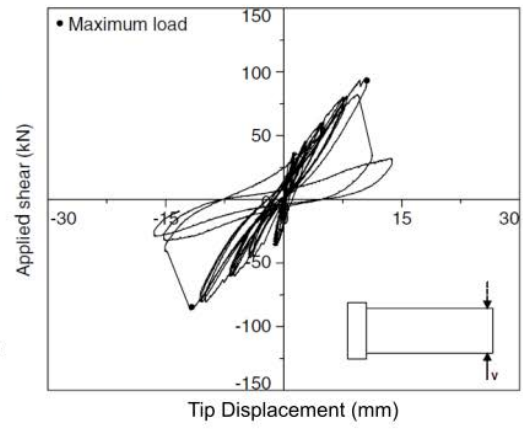

(b)

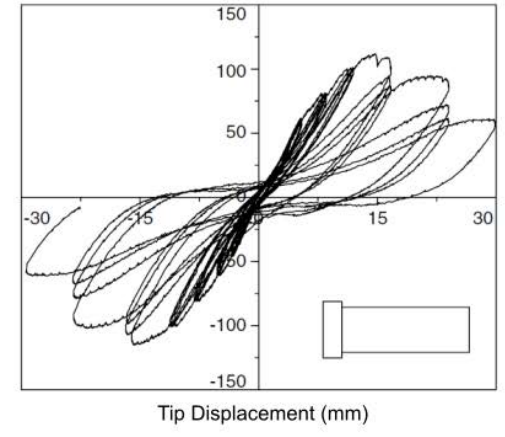

(c)

Figure 2.6 (a) Retrofitting scheme; Shear wall hysteretic response behaviour: (b) control wall;

(c) retrofitted wall (from Layssi et al., 2012) 
shear walls may be required in addition to shear strengthening to meet current design standards, something that has not been addressed in previous studies for shear dominant walls.

Past studies at Carleton University by Lombard et al. (2000), Hiotakis (2004), and CruzNoguez et al. (2014b) have investigated the use of CFRP sheets to increase or restore the flexural strength of RC shear walls using externally bonded CFRP sheets applied to only the faces of the shear wall. The experimental programs conducted by Lombard et al. (2000) and Hiotakis (2004) were divided into two phases $(1 \& 2)$ based on the type of anchor system used, namely, a steel angle anchor and a steel tube anchor. The study by Cruz-Noguez et al. (2014b) examined in greater depth the results of the experimental work presented by Lombard et al. (2000); Hiotakis (2004), making detailed comparisons between the observed failure mechanisms, FRP-concrete debonding progressions, and force-deformation response behaviour. Figure 2.7 shows the experimental results of the CFRP retrofitting scheme for both phases of the study. The study found that in repair applications, the CFRP retrofitting system was able to recover most of the initial elastic stiffness and increased the maximum flexural capacity of the seismically damaged walls. In strengthening applications, for walls without pre-existing damage, the system was effective in significantly increasing the stiffness and ultimate flexural capacity of the wall specimens. The study also found that the significant improvements in ductility and energy dissipation capacity could be achieved when vertical and horizontal CFRP plies were used together, as opposed to using only vertical or only horizontal CFRP sheets.

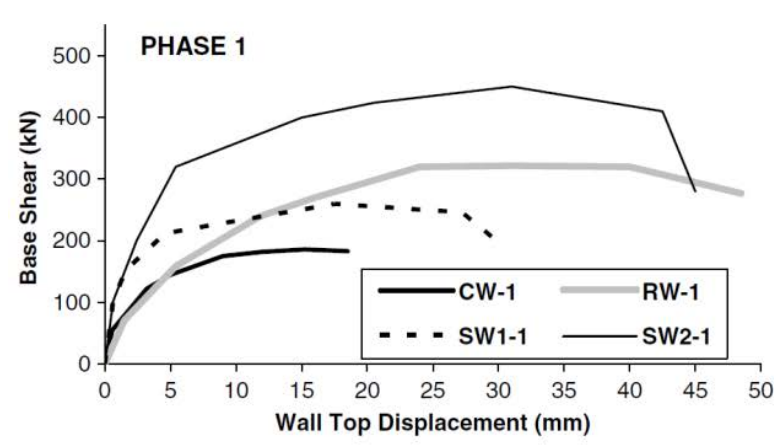

(a)

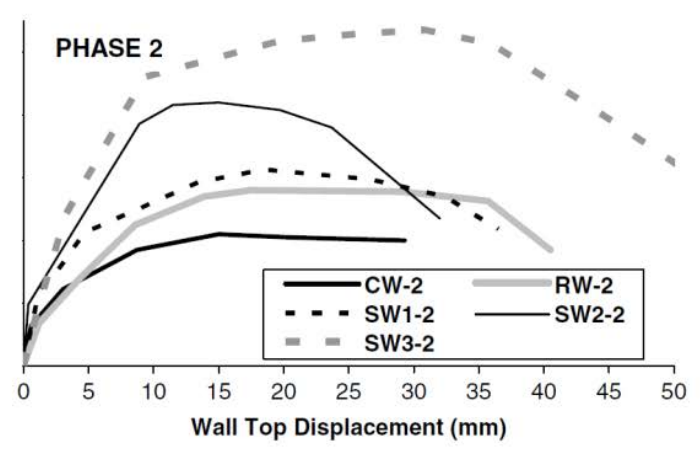

(b)

Figure 2.7 Force-deformation response behaviours for control (CW), repaired (RW), and strengthened (SW) shear wall specimens (from Cruz-Noguez et al., 2014b) 
The studies by Lombard et al. (2000), Hiotakis (2004), and Cruz-Noguez et al. (2014b) focused on the retrofit of wall specimens designed according to 'modern' design standards (CSA A23.3-94) and do not address the deficiencies in older non-ductile RC shear walls susceptible to brittle shear failure or the influence that aspect ratio has on the performance of the CFRP retrofitting strategy. This study expands on previous research and investigates the potential for using externally bonded CFRP sheets to enhance the seismic performance of shear walls representative of those designed according to older design standards (ACI 318-68; CSA A23.3-77).

In this study, nine RC shear wall specimens representative of low aspect ratio shear walls, which are particularly susceptible to brittle shear failures, are tested under in-plane reversed static cyclic loading. Of the nine shear wall specimens, two match the aspect ratio of the walls tested by Lombard et al. (2000) and Hiotakis (2004) $\left(h_{w} / l_{w}=1.2\right)$ to allow for comparison of the performance of the CFRP retrofitting system between studies. Results from the these wall specimens were originally presented in Woods (2014), but are described again in more detail in this thesis to provide a complete set of experimental results for the entire study and allow for comparison between the experimental results from each series of wall specimens. The additional seven wall specimens have lower aspect ratios $(0.85 \& 0.65)$ to better understand the performance of the CFRP retrofitting system in non-ductile squat shear walls. The wall specimens are expected to exhibit brittle shear behavior due to insufficient shear reinforcement and no additional concrete confinement or concentration of vertical steel reinforcement near the ends of the shear wall. Test results from these seven wall specimens are presented for the first time in Part 1 of this thesis. The wall specimens are strengthened or repaired with unidirectional CFRP sheets applied in the vertical and/or horizontal directions. In the present study, the CFRP sheets are not wrapped around the ends of the specimens to represent the field application of the FRP sheets applied only to the two wall panel surfaces, and thus to understand the effectiveness of the FRP retrofitting system without additional confinement at the two ends. The performance of the CFRP retrofitting system is assessed in terms of in-plane strength, ductility, and energy dissipation capacity. The performance and effectiveness of the tube anchor system used to transfer the tensile forces carried by the CFRP to the foundations of the specimens are also evaluated. 
In addition, a finite element modelling approach for the tube anchor system was also originally presented in Woods (2014). This modelling approach is once again described herein to provide context for the parametric study, which is based on the same finite element modelling approach and used to optimize the performance of the tube anchor system and develop a design methodology for its application in FRP retrofitting scenarios, both of which are original works in this study.

\subsection{Anchorage in FRP Retrofitted Structures}

The design of an anchor system and its role in the FRP retrofitting system depends on the application. For example, in flexurally strengthened RC beams, the FRP sheets must be provided with the appropriate development length to ensure they are capable of reaching their design stress prior to debonding. In this retrofit scenario, the maximum stress in the FRP typically occurs at midspan of the beam and the orientation of the FRP fibres coincides with the direction in which the development length can be provided. In this application, although an anchor system can reduce the required development length and improve the performance of the FRP retrofit by delaying FRP-concrete debonding, particularly at the termination of the FRP sheet, it is not a requirement for increasing the flexural strength of the retrofitted beam because sufficient length is available over which the stress in the FRP sheet can be developed.

In the shear strengthening of a RC beam, which is illustrated in Fig. 2.8, the vertically oriented FRP fibres meet the adjacent supporting floor slab at a 90 degree angle. Although the maximum stress occurs at mid-depth of the beam section, the depth of the beam is often not sufficient to fully develop the stress in the FRP sheet and an anchor system should be provided to develop the stress in the FRP sheet and improve the shear strength of the retrofitted beam.

Alternatively, in RC shear walls flexurally strengthened with vertical FRP layers, the location of maximum stress in the FRP layers is at the base of the wall, where the wall connects with the adjacent supporting structural element (floor slab), typically at an angle of 90 degrees (Fig. 2.8). In this case, development length cannot be relied upon to transfer the load carried by the vertical FRP sheets to the adjacent supporting structural element and an anchor system must 
be provided to increase the flexural strength of the retrofitted wall. Strictly speaking, an anchor is only required when the maximum stress in the FRP sheet occurs at the intersection between two structural elements. In this scenario, anchorage is required to ensure the FRP contributes to the load carrying capacity of the member, however, the implementation of an adequately designed anchor in all three applications can prevent premature separation of the FRP sheet from the concrete substrate. By preventing premature FRP-concrete debonding, this allows the FRP material to achieve comparatively higher levels of stress and utilize a greater portion of its high strength capacity, increasing the efficiency of the FRP retrofit.

As proposed by Grelle and Sneed (2011), anchor systems are capable of achieving effective performance through one or more of the following design principles, depending on the intended application of the anchor: (1) reducing the required development length; (2) resisting tensile forces developed within the FRP; or (3) transferring the full force carried by the FRP laminate to an adjacent structural element. With respect to design principle (1), a technique that researchers have used in the past to increase the available development length is through the use of a U-shaped anchor. U-shaped anchors aim to increase the capacity of FRP retrofitted RC members and prevent premature debonding by increasing the FRP-to-concrete bond length at the end of the FRP sheet. A groove is formed in the concrete on the face of the adjacent supporting structural element into which the FRP sheet is placed (Fig. 2.9). After forming the FRP sheet around the groove it is filled with an epoxy resin. In some cases, a steel or FRP bar is placed inside the groove after placing the FRP sheet to increase the ease of installation. Although U-shaped anchors have been shown
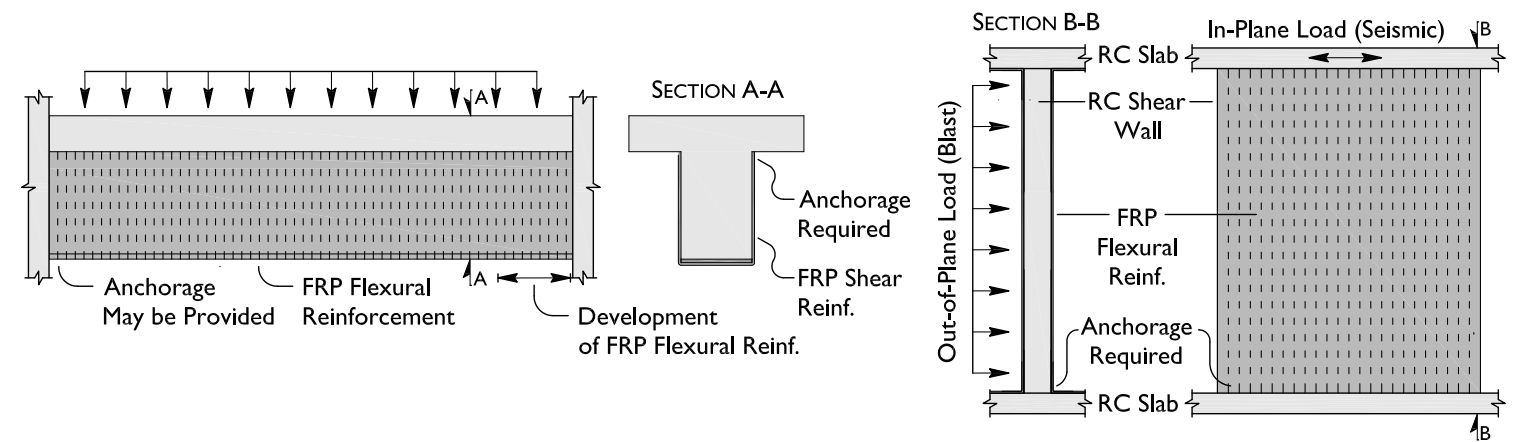

Figure 2.8 Anchorage requirements in FRP retrofit of RC beams and shear walls 
to delay premature debonding in both static and blast tests compared with when no anchorage is provided (Khalifa et al., 1999; Ceroni et al., 2008; Carney and Myers, 2003), their effectiveness in increasing the load carrying capacity of the retrofitted member is limited and results have shown their use does not prevent premature debonding from occurring. In addition, failure of these types of anchor systems are brittle and non-ductile once the FRP material begins to peel from the concrete groove (Kalfat et al., 2013). Because of these concerns, the U-shaped anchor is not discussed further in detail. The remaining commonly employed existing anchor systems utilize design principles (2) and (3) and are generally grouped into two categories: FRP and mechanical anchor systems. Figure 2.9 illustrates an anchor system from each of these two categories in addition to the previously discussed U-shaped anchor implemented in the strengthening of a RC shear wall with vertical FRP layers.

Fiber-reinforced polymer (FRP) anchors, which are sometimes referred to as spike or fan anchors, are fabricated from many strands of carbon or glass fibres. At one end, the fibres are splayed over the FRP sheet and embedded in the composite matrix. At the other end, a dowel is formed by bundling the composite fibres together, which is inserted into a hole in the adjacent structural element. Although it has been shown under static and blast loading that fan anchors are capable of increasing the capacity of FRP retrofitted RC members significantly, the failure of fan anchors are sudden and brittle upon rupture of the composite material (Zhang and Smith, 2012; Lam and Teng, 2001; Jacques, 2011); a failure mode that should typically to be avoided if the member may be loaded beyond its design load during an extreme loading condition, such as an earthquake.

Alternatively, mechanical anchor systems are designed to transfer the forces carried by the FRP laminate to an adjacent structural element using a steel section. Common steel sections used for mechanical anchors include steel plates and angles. The steel section is bolted at the end of the FRP sheet, which is typically located at the interface between the retrofitted member and the adjacent supporting structural element. Because of ease of construction, the use of a steel mechanical anchor is an attractive alternative to FRP fan anchors because it can be fabricated by non-specialized personnel and are available in an array of material grades and sizes depending 

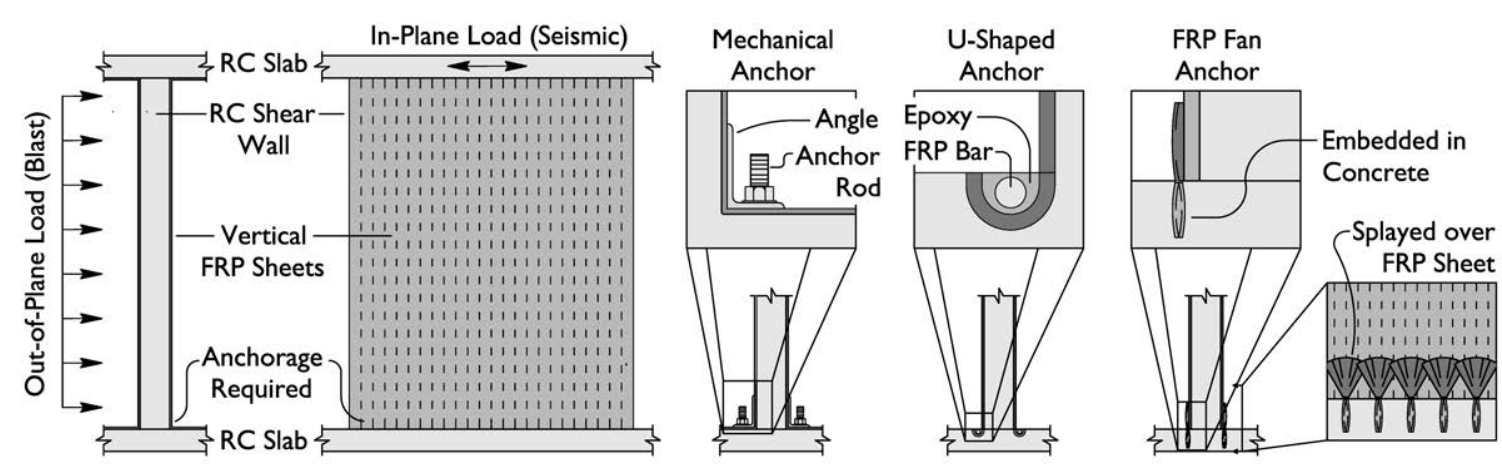

Figure 2.9 Common anchor systems used in FRP sheet retrofit applications

on the application. Steel mechanical anchors can also be designed such that the FRP retrofitting system fails in a ductile manner by detailing the steel anchor as a fuse that activates after the retrofitted member reaches the design load, eliminating the potential for undesirable sudden and brittle failure modes. This is particularly important for FRP retrofitted elements subjected to extreme loading. In this scenario, the structural element could be overloaded and the ductile steel mechanical anchor system acts as a fuse, preventing sudden and brittle failure. Past tests on FRP retrofitted RC beams and walls under in-plane cyclic load and out-of-plane blast loads by Kanakubo et al. (2000); Hall et al. (2002); Hiotakis et al. (2004) and Ehsani and Peña (2009) have demonstrated that a well-designed mechanical anchor can eliminate the potential for premature debonding and allow the designer to take advantage of a greater portion of the high strength capacity of the FRP material. Experimental investigations have also shown that steel mechanical anchorage systems generally provide higher anchorage strength when compared with FRP fan and U-shaped anchor systems (Kalfat et al., 2013).

\subsubsection{Mechanical Anchor System}

Mechanical anchor systems are designed to increase the capacity of FRP repair and strengthening systems through the use of a structural section at the termination of an FRP sheet. The most common structural sections used in anchorage applications include steel plates, angles and tubes. In most cases, a steel structural section is bolted into adjacent supporting structural elements to transfer tensile stresses from the FRP sheets. For construction purposes, the use of steel 
sections as an FRP anchor is an attractive option because the anchor can be readily fabricated.

Previous studies by Lombard (1999) and Hiotakis (2004) have shown that the type of anchor system used to transfer the force carried by the FRP to the supporting members can have a significant impact on the effectiveness of the FRP repair or strengthening system. The steel angle anchor system used by Lombard (1999) is predisposed to premature debonding of the FRP sheets due to rotation of its flanges. The flange rotation occurs because of the eccentricity in the load path of the forces transferred from the FRP sheet to the wall foundation (Lombard et al., 2000; Hiotakis et al., 2004; Cruz-Noguez et al., 2014b). This ultimately leads to the premature debonding and fracture of the FRP fibres before the FRP sheet can reach its ultimate tensile capacity. To overcome the shortcomings of the steel angle anchor system, Hiotakis et al. (2004) developed a tube anchor system for the transfer of the load carried by the FRP sheet to the foundation of a specimen. The test results by Hiotakis et al. (2004) show that by using the tube anchor system the FRP sheet is able to reach its ultimate tensile strength and ruptures, allowing the wall specimen to attain a higher lateral load carrying capacity.

\subsubsection{Steel Angle Anchor}

A common mechanical anchor is a steel angle anchor fabricated from an off-the-shelf steel angle. The steel angle is placed along the end of the FRP sheet and bolted to the adjacent structural element. Figure 2.9 shows a typical angle anchor installation at the base of a RC shear wall strengthened with vertical FRP sheets. The angle anchor is designed to transfer the force carried by the FRP sheet through the flange of the angle, which is epoxy-bonded to the FRP sheet and fixed to the adjacent supporting structural element using steel anchor bolts. The angle anchor has been studied in the flexural strengthening of RC shear walls and the shear strengthening of RC beams by Lombard et al. (2000) and Kanakubo et al. (2000), respectively. In the study by Lombard et al. (2000), a RC shear wall is flexural strengthened for in-plane seismic load using vertically oriented carbon fibre-reinforced polymer (CFRP) sheets. In this application, the vertical CFRP sheets need to be anchored at the base of the shear wall so that they contribute to the lateral load carrying capacity of the wall. Observations by Lombard et al. (2000) found that the steel 
angle anchor actually causes early debonding of the CFRP sheets from the concrete wall before the CFRP material reaches its rupture strength. Figure 2.10 shows a typical failure progression of the steel angle anchor. Failure of the steel angle anchor occurs because of an eccentricity between the force carried by the FRP sheet and the tie-down reactions of the anchoring bolts that secure the anchor to the adjacent structural element. As the FRP sheet is loaded in tension, the eccentricity between these two forces causes a moment, which results in rotation of the angle flange, referred to as a 'prying' action. During the cyclic response of the wall, the prying action pulls the FRP sheet away from the surface of the concrete wall, causing premature debonding. When the load is reversed, the debonded FRP sheet buckles in compression and fractures along the top edge of the vertical flange, breaking the hardened composite matrix leaving a sharp edge which then cuts the FRP fibres. This behavior effectively reduces the load carrying capacity of the retrofitted RC member because the FRP sheet ruptures prior to reaching its ultimate capacity in tension, reducing the efficiency of the retrofitting system.

\subsubsection{Steel Tube Anchor}

Motivated by the observed prying action of the steel angle anchor, Hiotakis et al. (2004) developed a steel tube anchor that prevents premature debonding by concentrically transferring the forces carried by the FRP sheet to the adjacent supporting structural element. During the installation of the tube anchor, the FRP sheet is wrapped around a steel tube and connected to the adjacent structural element using steel anchor rods installed at a 45 degree angle. Figure 2.11 shows the design and failure progression for the steel tube anchor. The design of the tube anchor
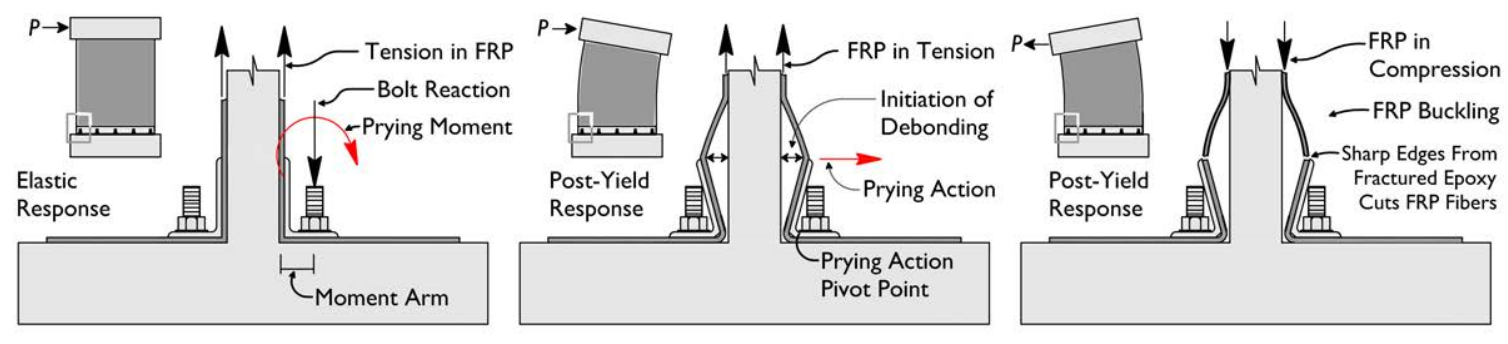

Figure 2.10 Failure progression of steel angle anchor 
is based on the pulley principle: as the FRP sheet is loaded in tension, the force carried by the FRP sheet is equated by the tension in the horizontal portion of the FRP sheet, which must be provided with the appropriate development length. By wrapping the CFRP sheet around the tube and placing the anchor bolts in the direction of the resultant load, the eccentricity between the force carried by the FRP sheet and the tie-down reaction from the anchor bolts is eliminated. Even after FRP debonding is initiated above the tube anchor, the lack of sharp edges or stress concentrations at the base of the wall prevents the FRP sheet from fracturing when it buckles in compression. This means that if the load reverses, which occurs during an earthquake, the FRP is able to continue carrying load in tension even after FRP-concrete debonding occurs until the FRP sheet ruptures at its ultimate tensile strength. This behavior allows the retrofitted member to achieve a higher load carrying capacity and greater efficiency when compared with the steel angle anchor, utilizing a larger portion of the high-strength capacity of the FRP material.

As a proof of concept of the tube anchor, Hiotakis et al. (2004) conducted an experiment on a flexurally strengthened shear wall specimen using vertical CFRP sheets. The shear wall specimens had the same geometry, were tested under the same cyclic loading protocol, and had identical steel/CFRP reinforcement ratios to the wall tested by Lombard et al. (2000). The difference between the two shear wall specimens was the anchor system. Additional details on both experimental programs are available in Lombard et al. (2000), Hiotakis et al. (2004) and Cruz-Noguez et al. (2014b). Figure 2.12 shows the improvement in the seismic performance of the FRP retrofit by comparing the hysteretic response and load-displacement curves for two shear wall specimens.

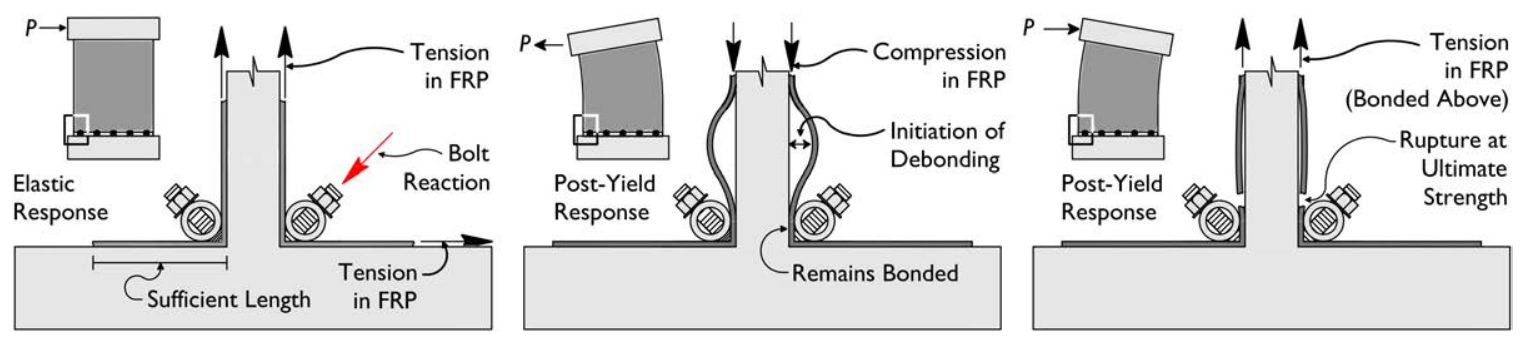

Figure 2.11 Failure progression of steel tube anchor 
As shown in the load-displacement curves (Fig. 2.12b), the behavior of the two shear wall specimens in the elastic range are similar, with only minor differences in the initial stiffness, yield load, and yield displacement attributed to the in-situ material properties of the concrete, steel, and CFRP and the placement of the vertical steel reinforcement inside the wall. The post-yield response of the shear wall specimens show that significant improvements in terms of strength, ductility and energy dissipation capacity can be achieved when using the tube anchor compared with the angle anchor. Experimental observations also indicate that the FRP sheet was able to reach its ultimate tensile strength, maximizing the efficiency of the retrofitting system. Although experimental results by Hiotakis et al. (2004) suggest that the tube anchor system improved the efficiency of the FRP retrofit, this application of the tube anchor was a proof of concept, and thus was significantly over-designed. In this study, detailed finite element models are developed to better understand the performance of both anchor systems and develop a tube anchor design methodology. 


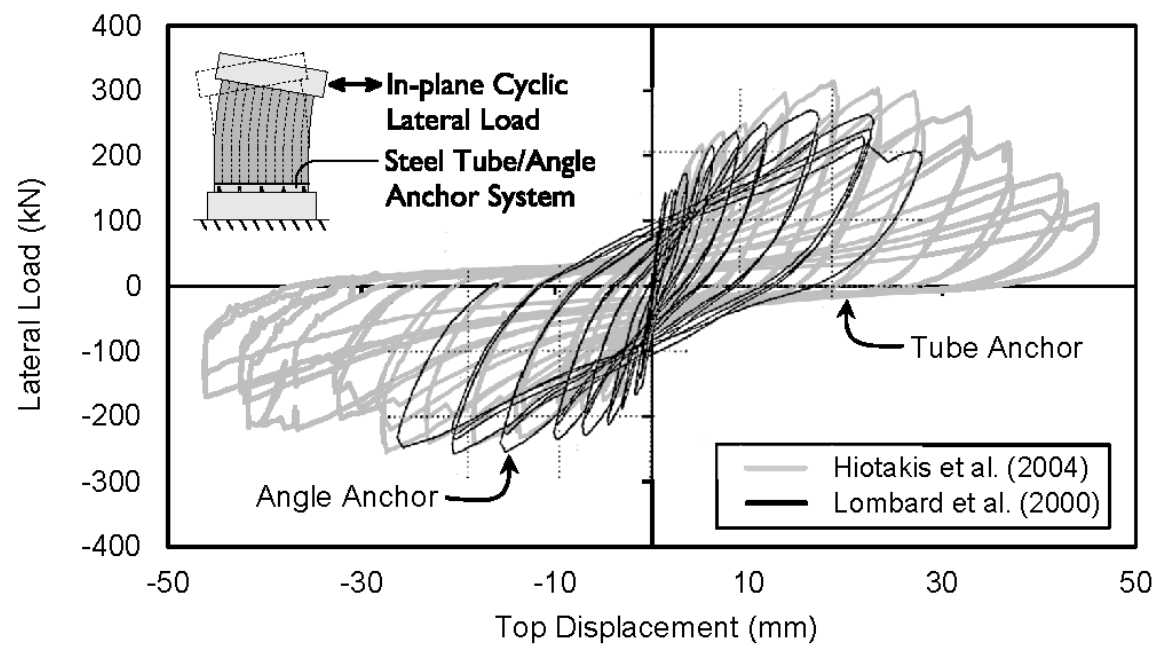

Figure 2.12 Anchor performance comparison: (a) hysteretic response; (b) force-deformation response (Lombard et al., 2000; Hiotakis et al., 2004 and Cruz-Noguez et al., 2014b) 


\section{Chapter 3. Experimental Program}

\subsection{Methodology}

The shear wall test specimens in this experimental program include nine cantilevered RC squat walls designed according to the ACI 318-68 (ACI Committee 318 1968) building code, a standard that is comparable to the CSA A23.3-77 (CSA A23.3 1977) Canadian RC design standard. The specimens have height-to-length aspect ratios $\left(h_{w} / l_{w}\right)$ of $1.2,0.85$, and 0.65 , respectively. In addition to aspect ratios matching those tested by Hiotakis (2004) and Lombard et al. (2000), this study includes shear walls with a larger variation in the aspect ratio, specifically smaller aspect ratios, whose behaviour is more shear dominated and thus more susceptible to brittle shear failures. The design deficiencies in the wall specimens are commonly found in older RC shear wall structures constructed during the 1960s and 1970s including insufficient shear reinforcement, lack of boundary elements, lap splices within the potential plastic hinge region, and low concrete compressive strength. Consequently, the wall specimens tested without CFRP sheets failed in a brittle, shear dominant manner.

\subsection{Shear Wall Specimens}

Fig. 3.1 illustrates the geometry and steel reinforcement detailing for the 5 different shear wall types tested ((a) through (e)). There are nine shear wall specimens in total: 2 type (a), 3 type (b), 2 type (c) and one of types (d) and (e). The wall specimens are separated into four series (1 to 4) based on their height-to-length $\left(h_{w} / l_{w}\right)$ aspect ratio and steel reinforcement detailing. Table 3.1 shows the geometry, aspect ratio, and horizontal and vertical steel reinforcement ratios, and CFRP 
retrofitting configurations for the RC shear wall specimens. Wall specimens in series 1 have an aspect ratio of 1.2, and represent moderate aspect ratio walls that exhibit a combination of flexural and shear response behaviour. Alternatively, specimens in series 2, 3, and 4 have aspect ratios of $0.85,0.65$, and 0.65 , respectively; representing low aspect ratio squat walls whose response are heavily shear dominated. Series 4 wall specimens have lap splices of the vertical steel reinforcement in the plastic hinge region. The RC shear wall specimens with an aspect ratio of 0.65 (Series 3 and 4) are expected to exhibit shear critical behaviour, where the large majority of deformation comes as a result of shear deformation leading to high shear stresses under the application of in-plane lateral load.

Of the nine shear wall test specimens, there are four control specimens, identified as CW1 through CW4, which are first tested to failure without externally bonded CFRP reinforcement. Following the initial test, they are repaired using externally bonded CFRP sheets and subsequently tested again, changing their designations to RW1 through RW4. The ability of the CFRP repair system to restore the initial stiffness of the wall and increase the shear and flexural strengths, ductility, and energy dissipation capacity of the repaired wall specimens is evaluated. This part of the research provides insight on using the CFRP sheets in repair applications for walls that have experienced damage during an earthquake. The remaining series 1, 2, and 3 shear wall specimens are strengthened with CFRP sheets prior to testing, which is referred to as strengthening, and are designated SW1 through SW3. The performance of the strengthened specimens are compared with their respective control wall to evaluate the effectiveness of using externally bonded CFRP sheets as a retrofitting strategy for deficient RC shear wall structures that are identified as being susceptible to damage.

\subsection{Material Properties}

Table 3.2 shows the material properties of the steel reinforcement and Table 3.3 shows the material properties for the concrete, dry CFRP fibre, and cured CFRP laminate (CFRP and 


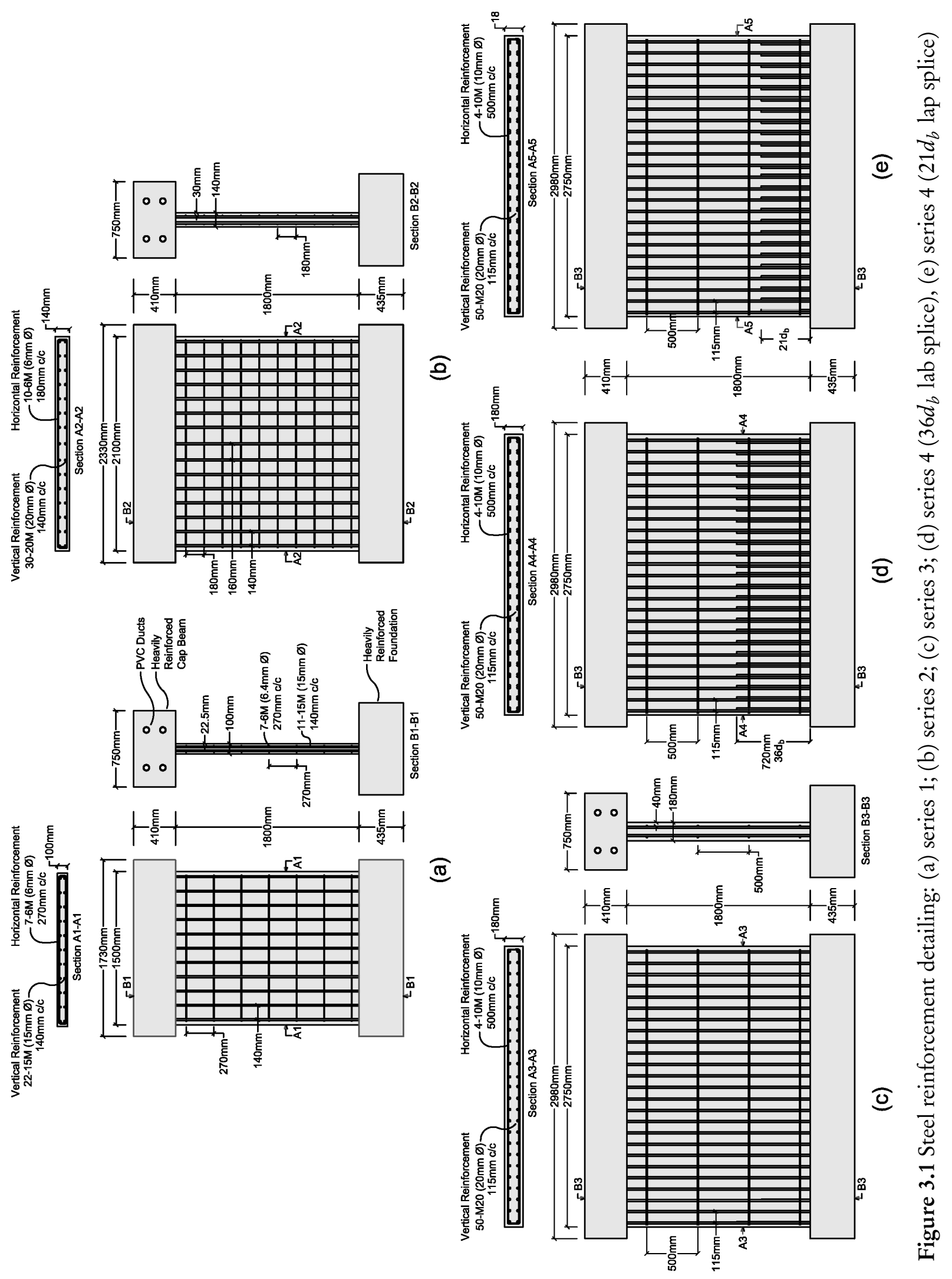




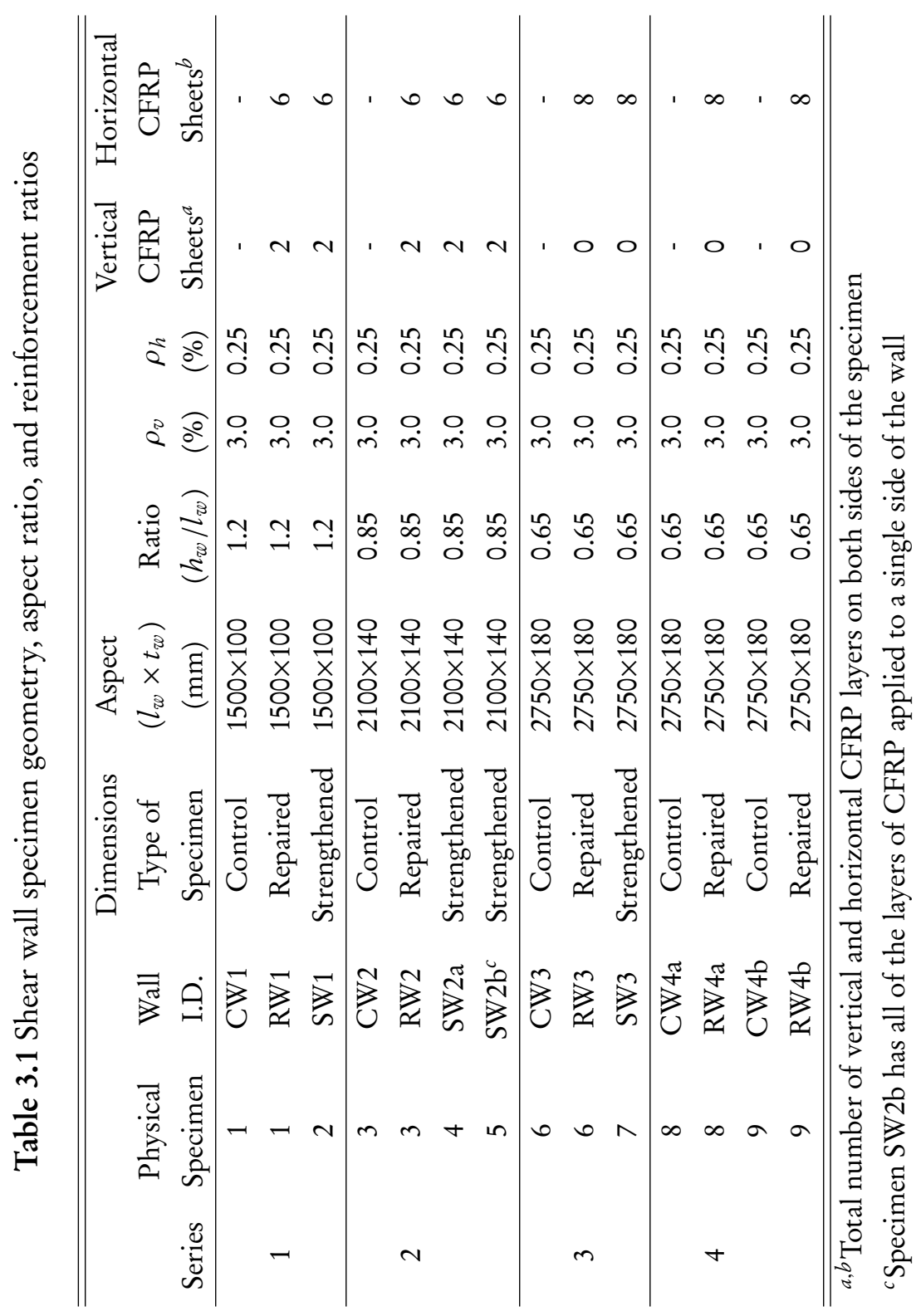

Joshua Woods, Department of Civil and Environmental Engineering, Carleton University 
epoxy). The shear wall specimens are constructed with a low strength concrete typical of construction during the 1960s and 1970s. The wall specimens were cast in three stages: (1) foundation, (2) wall panel, and (3) cap beam. The surface of the foundation was roughened before casting the wall panel to increase the sliding shear strength at the interface between the wall panel and foundation. High-strength concrete was specified in the design of the foundation and cap beam to avoid cracking and spalling in these elements before failure of the wall specimens. Figure 5.4 shows the results of material testing conducted on the steel, concrete, and CFRP used in this study. The concrete strength of the test specimens was determined according to cylinder compression tests and results for the wall panels range between $15 \mathrm{MPa}$ and $25 \mathrm{MPa}$. The 28-day concrete compressive strength for each stage of pouring (foundation, wall panel, and cap beam) are reported in Table 3.3. Grade 400 reinforcing steel was used for the vertical and horizontal steel reinforcement whose strength was determined according to an average of three coupon tests for each size of steel reinforcing bar. The CFRP sheets used in this study were Tyfo SCH-41 and the epoxy was Tyfo S saturant. The properties of the cured CFRP laminate were determined by performing tension tests on CFRP coupons, following the guidelines in ASTM D3039 (ASTM (American Society for Testing and Materials), 2013).

\subsection{Design of Plain RC Shear Walls}

This section describes the procedures used to design the control wall specimens, including detailing of the vertical and horizontal steel reinforcement. In addition, a detailed analysis of

Table 3.2 Steel reinforcement material properties

\begin{tabular}{lcccc}
\hline \hline \multicolumn{1}{c}{ Property } & 6M Bars & 10M Bars & 15M Bars & 20M Bars \\
\hline$f_{y}(\mathrm{MPa})$ & 415 & 440 & 452 & 480 \\
$E_{s}(\mathrm{GPa})$ & 160 & 170 & 188 & 208 \\
$\epsilon_{y}(\mathrm{~mm} / \mathrm{mm})$ & 0.0026 & 0.0026 & 0.0024 & 0.0023 \\
$\epsilon_{u}(\mathrm{~mm} / \mathrm{mm})$ & 0.012 & 0.141 & 0.115 & 0.137 \\
\hline \hline
\end{tabular}

Note: steel properties based on average of 3 coupon tests 
Table 3.3 Concrete and CFRP material properties

\begin{tabular}{|c|c|c|c|c|c|c|c|c|c|}
\hline \multirow{2}{*}{\multicolumn{2}{|c|}{ Foundation Blocks }} & \multirow{2}{*}{\multicolumn{2}{|c|}{$\frac{\text { Concrete Properties }}{\text { Wall Panels }}$}} & & & \multicolumn{4}{|c|}{ CFRP Properties } \\
\hline & & & & \multicolumn{2}{|c|}{ Cap Beams } & \multicolumn{2}{|c|}{ Dry Fibre } & \multicolumn{2}{|c|}{ Cured Laminate } \\
\hline$f_{c}^{\prime}(\mathrm{MPa})$ & 73.4 & $f_{c}^{\prime}(\mathrm{MPa})$ & 19.1 & $f_{c}^{\prime}(\mathrm{MPa})$ & 64.8 & $f_{f}(\mathrm{MPa})$ & 4000 & $\left.f_{f}\right)(\mathrm{MPa})$ & 931 \\
\hline$E_{c}(G P a)$ & 35.8 & $E_{c}(\mathrm{GPa})$ & 19.2 & $E_{c}(\mathrm{GPa})$ & 39.4 & $E_{f}(\mathrm{GPa})$ & 230 & $E_{f}(\mathrm{GPa})$ & 83.8 \\
\hline$\epsilon_{c}^{\prime}$ & 0.0028 & $\epsilon_{c}^{\prime}$ & 0.0018 & $\epsilon_{c}^{\prime}$ & 0.0019 & $\epsilon_{f u}$ & 0.017 & $\epsilon_{f u}$ & 0.01 \\
\hline$\epsilon_{u}^{\prime}$ & - & $\epsilon_{u}^{\prime}$ & 0.0042 & $\epsilon_{u}^{\prime}$ & - & $t_{f}(\mathrm{~mm})$ & 0.11 & $t_{f}(\mathrm{~mm})$ & 0.012 \\
\hline
\end{tabular}

Notes: concrete and FRP laminate test results are the average of 3 cylinder/coupon tests; and CFRP dry fibre properties are based on tabulated values from the manufacturer.

their flexural and shear strength was conducted using older and modern design standards as well as a number of empirical relationships available in the literature. The combined shear-flexure interaction in squat RC shear walls with aspect ratios less than 2 makes it harder to predict their strength and failure mechanism compared with flexurally dominant RC shear walls. Comparing the experimental results with those from design equations will provide some information on the ability of these design expressions to predict the strength of squat reinforced concrete shear walls. Table 3.4 summarizes the results for the shear and flexural strengths of the wall specimens without CFRP reinforcement using a number of techniques described in the following subsections.

\subsubsection{Flexural Design}

The ACI 318-68 and CSA A23.3-77 design standards for RC structures had no explicit requirement for the concentration of vertical steel in the ends of the wall or any requirements for additional confinement in these regions. The vertical reinforcement ratio in all of the wall specimens is $3 \%$, which is much greater than the minimum vertical steel reinforcement ratio of $0.15 \%$ required by the ACI 318-68 and CSA A23.3-77 design standards. The vertical reinforcement ratio is selected such that the failure mode of the control specimens is governed by shear. It is noted that this is an unrealistically high vertical steel reinforcement ratio and such a high vertical reinforcement ratio would be rarely encountered in actual practice, however, the goal was to ensure that response and failure mode of the control wall specimens would be dominated by shear. Although even $1 / 4$ of the vertical steel reinforcement would still likely result in a shear dominant 


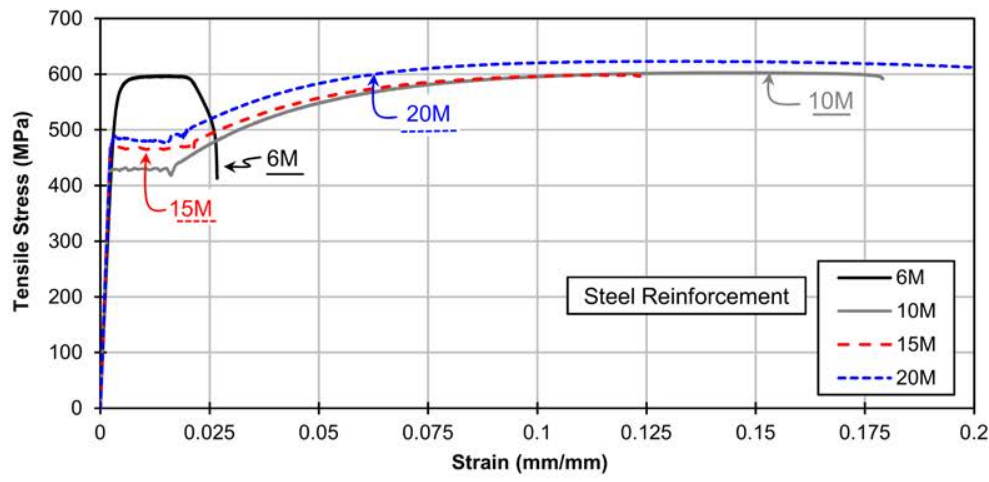

(a)

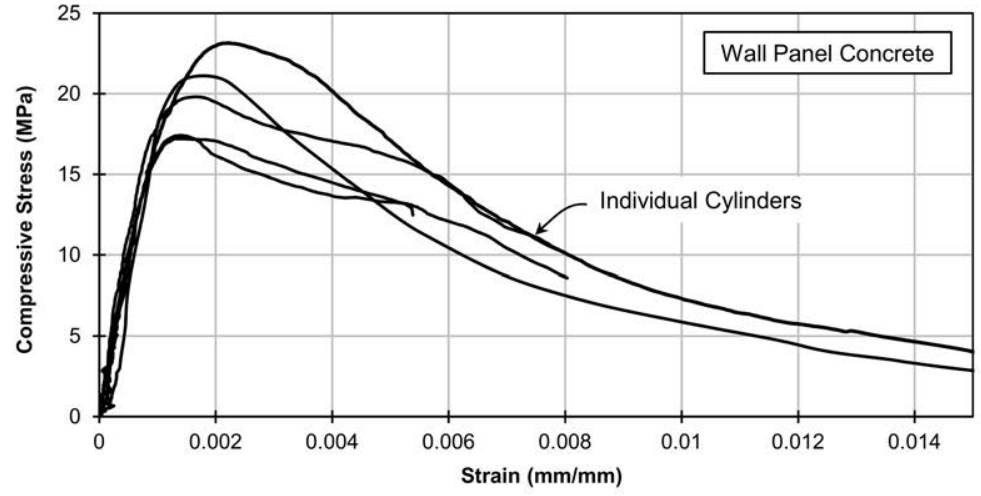

(b)

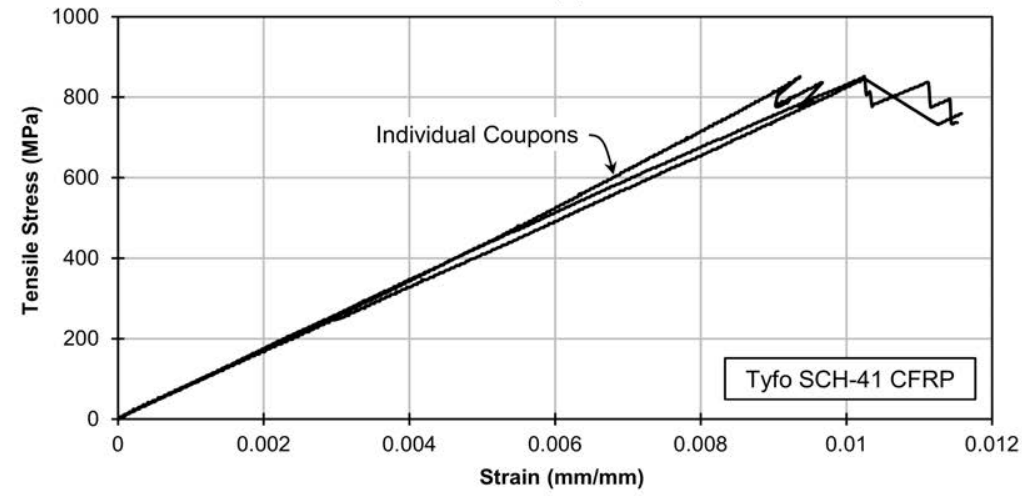

(c)

Figure 3.2 Material test results: (a) concrete; (b) steel; (c) SCH-41 Tyfo CFRP

response, the use of such a large vertical steel reinforcement ratio represented a worst-case scenario for the FRP retrofit, in which the contribution from flexure to the total response of the control wall specimens would be at a minimum. Thus, the behaviour of these specimens would provide insight on the effectiveness of using externally bonded FRP sheets to avoid brittle shear failure 
and to promote a more ductile response.

To determine the flexural strength of RC shear walls, the ACI 318-14 and CSA A23.3-14 design standards for reinforced concrete structures in the United States and Canada, respectively, recommend using a strain-compatibility analysis, assuming plane sections remain plane and perfect bond between steel and concrete materials. Although the wall specimens in this study are considered squat shear walls and tend to behave as deep beams, which violates the plane-sections hypothesis, it is assumed that a when the shear wall reaches its flexural capacity a large number of reinforcing bars along the base of the wall will have reached their yield stress and any errors in the strain calculations will not have a large significance on the flexural strength. The flexural strength calculations are based on a maximum usable compressive strain in the concrete of 0.0035 , as recommended by the CSA A23.3-04 design standard.

\subsubsection{Shear Design}

The wall specimens in this study are detailed with the minimum horizontal steel reinforcement ratio of $0.25 \%$ as prescribed by both the ACI 318-68 and CSA A23.3-77 design standards. The strength against diagonal tension shear failure $\left(V_{r}\right)$ is determined according to the ACI 318-68 design standard, which takes into account the contributions to the shear strength of the wall from the concrete and steel reinforcement:

$$
V_{r}=\left(\frac{n A_{v} f_{y h} d}{S}\right) \cot \theta+\left(0.16 \sqrt{f_{c}^{\prime}}+17 \frac{\rho_{v} V_{u} d}{M_{u}}\right) A_{w}
$$

where $n$ is the number of reinforcing bars per layer; $S$ is the spacing of the horizontal steel reinforcing bars; $A_{v}$ is the area of horizontal reinforcement within a distance of $S ; f_{y h}$ is the yield stress of the horizontal reinforcement; $d$ is the distance from the extreme fibre in compression to the centroid of the tensile steel reinforcement; $\theta$ is the assumed angle of the diagonal crack, conservatively taken as $45 \mathrm{deg} ; f_{c}^{\prime}$ is the 28-day compressive strength of the concrete; $\rho_{v}$ is the vertical steel reinforcement ratio; $V_{u}$ is the shear applied at the section; $M_{u}$ is the moment applied at the section; and $A_{w}$ is the area of the shear wall.

Because the wall specimens are expected to experience a brittle shear failure, a more refined 
estimation of their diagonal tension shear strength is computed using four different modern approaches in addition to the method in the ACI 318-68 design standard. Results from the shear strength prediction equations are compared with experimental results to assess the validity of current shear design methods for squat RC shear walls.

The second approach used to determine the diagonal tension shear strength of the wall specimens $\left(V_{r}\right)$ is a semi-empirical equation based on the modified truss analogy used for the seismic design of RC shear walls in the ACI 318-05 design standard computed as:

$$
V_{r}=\left(\alpha_{c} \sqrt{f_{c}^{\prime}}+\rho_{h} f_{y h}\right) A_{w}
$$

where $\alpha_{c}$ is an aspect ratio coefficient, taken as 0.25 for walls with a height-to-length aspect ratio $\left(h_{w} / l_{w}\right)$ less than 1.5; and $\rho_{h}$ is the horizontal steel reinforcement ratio. The third approach is the general shear design method based on the modified compression field theory in the CSA A23.3-04 design standard, the shear strength $\left(V_{r}\right)$ can be computed as:

$$
V_{r}=\left[\beta \sqrt{f_{c}^{\prime}} t_{w} d+\left(\frac{A_{v} f_{y h} d}{S}\right) \cot \theta\right] A_{w}
$$

where $\beta$ is a factor accounting for the shear resistance of cracked concrete; and $t_{w}$ is the thickness of the shear wall. The fourth and fifth approaches are empirical approaches proposed by Wiradinata (1985) and Wood (1990), respectively, which take into account the increase in shear strength observed in walls with aspect ratios less than 1.5. The approach by Wood (1990) is a friction-based model developed for squat walls. Wood (1990) recommended that the shear strength of low-rise wall be computed as:

$$
V_{r}=\frac{\sqrt{f_{c}^{\prime}}}{2} A_{w} \leq \frac{5 \sqrt{f_{c}^{\prime}}}{6} A_{w}
$$

where $\frac{5 \sqrt{f_{c}^{\prime}}}{6} A_{w}$ is a limit to prevent web crushing (diagonal compression shear failure). In addition, Wood (1990) recommends that for walls with large amount of vertical reinforcement, a better estimation of the nominal shear strength against diagonal tension shear failure $\left(V_{r}\right)$ can be 
computed as:

$$
V_{r}=\frac{A_{v f} f_{y v}}{4 A_{c v}} A_{w}
$$

where $A_{c v}$ is the effective area of the concrete section $\left(t_{w} \times l_{w}\right)$. The final approach used to compute the diagonal tension shear capacity of low-rise squat walls is a method proposed by Wiradinata (1985), which combines the contributions from the horizontal steel reinforcement $\left(V_{s}\right)$ and concrete $\left(V_{c}\right)$ to obtain the total diagonal tension shear strength $\left(V_{r}\right)$ as:

$$
V_{r}=\left[\left(0.5-\frac{h_{w}}{6 l_{w}}\right) A_{e} \sqrt{f_{c}^{\prime}}+\left(\frac{A_{v} f_{y h} d}{S}\right) \cot \theta\right]
$$

where $A_{e}$ is the effective area of the concrete section $\left(0.9 A_{w}\right)$. The average of all five approaches predicts the onset of diagonal tension shear of $280 \mathrm{kN}, 550 \mathrm{kN}$, and $1000 \mathrm{kN}$ for series 1 through 4 wall specimens, respectively. Series 3 and 4 wall specimens have the same overall dimensions and steel reinforcement ratios, and as a result have equal shear strengths, which is assumed to not be adversely affected by the presence of the lapped vertical steel reinforcement in the plastic hinge region. In addition to the strength against diagonal tension shear failure, the strength of the wall specimens against sliding shear failure $\left(V_{s l}\right)$ is computed according to CSA A23.3-04:

$$
V_{s l}=\left[c+\mu\left(\frac{A_{s} f_{y}}{t_{w} d}\right)\right] A_{w} \leq 0.25 f_{c}^{\prime} A_{w}
$$

where $\mathrm{c}$ and $\mu$ are the cohesion and friction parameters, taken as $\mathrm{c}=0.5 \mathrm{MPa}$ and $\mu=1.00$ as recommended by the CSA A23.3-04 design standard. Results from the average strength against sliding shear for each shear wall series is $778 \mathrm{kN}, 1470 \mathrm{kN}$, and $2750 \mathrm{kN}$ for series 1 through 4 wall specimens, respectively, determined according to the CSA A23.3-04 design standard. The average capacity against diagonal compression failure or web crushing is determined according to the ACI 318-05 design standard:

$$
V_{r}=0.83 \sqrt{f_{c}^{\prime}} A_{w}
$$

which results in diagonal compression capacities of $567 \mathrm{kN}, 1040 \mathrm{kN}$ and $2475 \mathrm{kN}$ for series 1 through 4 wall specimens, respectively. Table 3.4 summarizes the results from the design strength 
equations. Design strength calculations confirm that as anticipated, because of the deficiencies in the shear wall design and the shear dominant nature of low aspect ratio walls, diagonal tension shear is the controlling mode of failure for the control wall specimens without CFRP sheets.

\subsection{Design of FRP-strengthened RC Shear Walls}

\subsubsection{Flexural Design with FRP Reinforcement}

One of the principle objectives of this study is to investigate the potential for using CFRP sheets to increase the in-plane flexural strength of a RC shear wall that meets the minimum design requirements of older design standards, specifically, the ACI 318-68 and CSA A23.3-77 design standards. Figure 3.3 illustrates the CFRP reinforcement details for the shear wall specimens in this study. To improve the flexural strength of the series 1 and 2 wall specimens, a single layer of vertically oriented unidirectional CFRP laminate is applied on each side of the wall specimen and anchored to the concrete foundation. Although all of the wall specimens have horizontal CFRP sheets to increase their shear strength, vertically oriented CFRP sheets are not applied to the series 3 and 4 shear wall specimens because the resulting increase in flexural strength would result in an uneconomical level of shear strengthening (horizontal CFRP layers) to accompany the increase in flexural strength to ensure a flexural ductile mode of failure.

When determining the design strength of FRP-strengthened RC elements, it is commonly

Table 3.4 Design flexural and shear strengths of control walls

\begin{tabular}{|c|c|c|c|c|c|c|c|c|}
\hline \multirow{3}{*}{$\begin{array}{l}\text { Wall } \\
\text { Series }\end{array}$} & \multirow{3}{*}{$\begin{array}{c}\text { Flexural } \\
\text { Strength } \\
(\mathrm{kN})\end{array}$} & \multirow{3}{*}{$\begin{array}{c}\text { ACI } \\
318-68\end{array}$} & \multicolumn{3}{|c|}{ Diagonal Tension Capacity $(\mathrm{kN})$} & \multirow{3}{*}{$\begin{array}{l}\text { Wood } \\
\text { Model }\end{array}$} & \multirow{3}{*}{$\begin{array}{c}\text { Diagonal } \\
\text { Comp. } \\
(\mathrm{kN})\end{array}$} & \multirow{3}{*}{$\begin{array}{c}\text { Sliding } \\
\text { Shear } \\
(\mathrm{kN})\end{array}$} \\
\hline & & & $\mathrm{ACI}$ & CSA & Wiridinata & & & \\
\hline & & & $318-05$ & A23.3-04 & Model & & & \\
\hline W1 & 516 & 239 & 325 & 194 & 302 & 350 & 818 & 581 \\
\hline W2 & 1370 & 512 & 637 & 404 & 678 & 686 & 1602 & 1139 \\
\hline W3 & 3100 & 1016 & 1192 & 812 & 1300 & 1284 & 2998 & 2131 \\
\hline W4 & 3100 & 1016 & 1192 & 812 & 1300 & 1284 & 2998 & 2131 \\
\hline
\end{tabular}

Note: $f_{c}^{\prime}=21.8 \mathrm{MPa}$, the average of all 22 concrete cylinder tests. 


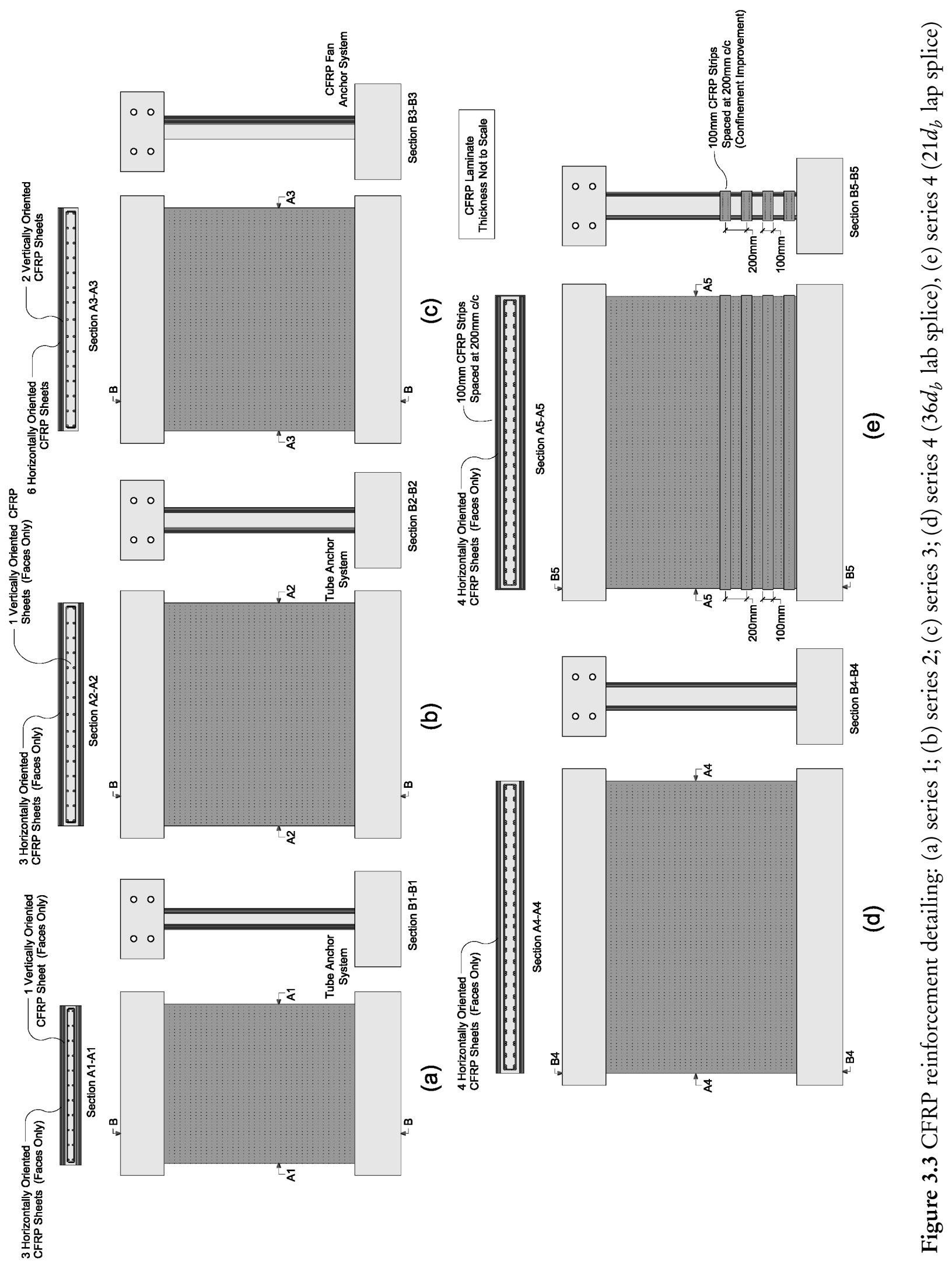


recognized that separation or debonding between the FRP laminate and the concrete substrate contributes to the failure of the strengthened member (Teng et al., 2002; Lombard et al., 2000; Cruz-Noguez et al., 2014b). In such cases, debonding of the FRP laminate occurs before the material reaches its ultimate tensile strength, reducing the ability to utilize the full strength capacity of the FRP material and resulting in a lower capacity for the strengthened member (Teng et al., 2002; Cruz-Noguez et al., 2014b). To account for the effect of FRP-concrete debonding and avoid overestimating the capacity of the strengthened member, the ACI 440.2-08 and CSA S806-02 design standards for the application of externally bonded FRP sheets limit the effective strain in the FRP laminate to the strain level at which FRP-concrete debonding can occur (ACI, 2008; CSA, 2002). When considering the effects of debonding in flexural FRP reinforcement for RC shear walls, the CSA S806-02 design standard specifies a strain limit of 0.007. The limiting debonding strain for surface bonded flexural FRP reinforcement $\left(\epsilon_{f d}\right)$ specified in the ACI 440.2-08 design standard is determined using Equation 3.10:

$$
\epsilon_{f d}=0.41 \sqrt{f_{c}^{\prime} / n_{s} E_{f} t_{f}} \leq 0.9 \epsilon_{f u}
$$

where $E_{f}$ is the tensile modulus of the FRP; $n_{s}$ is the total number of FRP sheets applied on both sides of the member; and $t_{f}$ is the thickness of a single FRP sheet. For a single layer of vertical FRP on each side of the wall, Eq. 3.10 results in an average limiting strain of 0.0082 for the wall specimens in this study. Table 3.5 shows the flexural strength of the wall specimens in this study repaired or strengthened with vertical CFRP reinforcement. The flexural strength of the wall specimens is calculated using a strain compatibility analysis based on a maximum usable compressive strain in the concrete of 0.003 and conservatively placing an upper bound on the strain in the vertical CFRP to less than the debonding strain limit of 0.007 recommended by the CSA S806-02 design standard.

\subsubsection{Shear Design with FRP Reinforcement}

For the design of the FRP retrofitting system, the capacity of the wall specimens against diagonal tension shear failure before retrofit must be increased beyond its flexural capacity to 
ensure the retrofitted specimen will fail in a flexural ductile manner. An increase in the wall specimens capacity against diagonal tension shear failure can be achieved through the addition of horizontally oriented unidirectional CFRP sheets. The total diagonal tension shear capacity of the strengthened specimens $\left(V_{r, f}\right)$ is the sum of the contributions from the concrete $\left(V_{c}\right)$, horizontal reinforcing steel $\left(V_{s}\right)$, and the horizontal FRP layers $\left(V_{f}\right)$.

In the repaired walls, there is a smaller contribution from the concrete and horizontal steel reinforcement to the total diagonal tension shear capacity because of the damage sustained by the control wall during the initial test. However, the diagonal cracks in the concrete are repaired prior to the application of the CFRP sheets, which restores a portion of the concrete contribution to the shear strength. Taking this into consideration, it is recognized that the true strength against diagonal tension shear failure lies somewhere between the shear strength of the horizontal CFRP layers and the sum of the contributions from the horizontal CFRP layers, horizontal steel reinforcement and repaired concrete. The capacity against diagonal tension shear failure for both cases (with and without horizontal steel reinforcement and concrete contributions) are presented in Table 3.5 to provide a range for the estimated diagonal tension shear capacity of the wall specimens. The observed contribution of the concrete and horizontal steel reinforcement to the diagonal tension shear strength of the repaired specimens will be discussed with the experimental results.

Similar to the behaviour of the vertical FRP layers discussed earlier, the debonding or separation of the horizontal FRP layers from the concrete substrate can prevent the horizontal CFRP from utilizing its full tensile capacity. To avoid overestimating the diagonal tension shear strength of the repaired and strengthened wall specimens, the maximum strain in the horizontal FRP sheets is limited to the strain level at which FRP-concrete debonding can occur. In the CSA S806-02 design standard, the limiting strain in the horizontal FRP is taken as 0.004, while the ACI 440.2-08 design standard recommends the limiting strain for the horizontal FRP can be calculated using Equation 3.11:

$$
\epsilon_{f l}=\kappa_{v} \epsilon_{f u} \leq 0.004
$$


where $\kappa_{v}$ is a bond reduction coefficient that depends on the concrete strength; the type of wrapping scheme; and the stiffness of the FRP laminate. Using the specified ultimate tensile strain of the CFRP material used in this study and an assumption of three horizontally oriented CFRP layers on each side of the wall specimen results in $\kappa_{v}=0.131$ and a limiting debonding strain in the horizontal CFRP of $\epsilon_{f l}=0.0022$, however, a limiting debonding strain of 0.004 is used in the design of the horizontal FRP because the CSA standard explicitly defines the limit for walls, whereas the limit from the ACI standard is based on tests from RC beams. The contribution to the diagonal tension shear capacity from the horizontal CFRP layers $\left(V_{f}\right)$ is calculated according to the CSA S806-02 design standard, which is the same method described in the ACI 440.2-08 design standard but the value for the limiting debonding strain for the horizontal CFRP layers is different. The CSA S806-02 design standard recommends $V_{f}$ be calculated using Equation 3.12:

$$
V_{f}=m n_{s} t_{f} f_{f} d
$$

where $m$ is the number of wall faces reinforced with FRP; $d$ is the effective length of the wall taken as $0.8 l_{w} ; f_{f}$ is the design stress in the FRP taken as $\epsilon_{f l} E_{f}$; where $\epsilon_{f l}$ is the limiting debonding strain in the horizontal CFRP layers (0.004). Table 3.5 shows the resulting FRP contribution to the shear strength and total diagonal tension shear capacity of each repaired and strengthened specimen. Although the application of horizontal FRP layers is successful in increasing the capacity against diagonal tension shear failure, it does not contribute to increasing the capacity against sliding shear or diagonal compression shear failure. Therefore, the limit on the maximum number of horizontal CFRP sheets that can be applied to the wall specimens is governed by the upper bound given by the lesser of the capacity against sliding shear or diagonal compression shear failure. In this study, as evident from strength estimations shown in Table 3.5, flexural failure is the governing mode of failure in the CFRP repaired and strengthened wall specimens because of the addition of the horizontal CFRP layers. In all of the retrofitted wall specimens, the additional shear strength against diagonal tension shear failure provided by the horizontal CFRP sheets ensures that the shear demand associated with the flexural strength of the walls is achieved to allow ductile response behaviour up to ultimate failure under cyclic loading. Flexural and shear 
strengths from the experiments will be compared with the strength predictions in Table 3.5 to assess the ability of current FRP design standards for the application of externally bonded FRP reinforcement to predict the strength and governing mode of failure in low aspect ratio RC shear walls.

\subsection{Experimental Test Setup}

Figure 3.4 shows the experimental test setup. The wall specimens were secured to the laboratory strong floor and a hydraulic actuator applied the reversed cyclic load sequence to the top of the wall specimens. The actuator is connected between the wall specimen and reaction frame using two hinges. This experimental setup ensures that no moments are created at the top of the wall specimen and that it behaves as a cantilevered wall. A lateral restraint system prevents outof-plane displacements of the wall specimen. Axial load is not applied to the wall specimens. The presence of axial load on the wall specimens would effect the performance of the FRP retrofitting system, by delaying yielding of the vertical steel reinforcement resulting in a larger shear demand on the wall specimen at its flexural capacity. However, the goal of the present study is to understand the behaviour of the retrofitting system in shear critical low aspect ratio wall specimens without the influence of additional parameters, such as axial load. This will allow for comparison

Table 3.5 Design flexural and shear strengths of retrofitted walls

\begin{tabular}{lccccc}
\hline \hline & $\begin{array}{c}\text { Flexural } \\
\text { Strength } \\
(\text { Plain RC })\end{array}$ & $\begin{array}{c}\text { Flexural } \\
\text { Strength } \\
(\text { with FRP })\end{array}$ & $\begin{array}{c}\text { Diagonal Tension } \\
\text { Shear Strength } \\
(\text { Plain RC) }\end{array}$ & $\begin{array}{c}\text { FRP Contribution } \\
\text { to Diagonal Tension } \\
\text { Shear Strength } \\
\left(V_{r}\right)^{a}(\mathrm{kN})\end{array}$ & $\begin{array}{c}\text { Diagonal Tension } \\
\text { Shear Strength } \\
(\text { with FRP) }\end{array}$ \\
Series & $(\mathrm{kN})$ & $(\mathrm{kN})$ & 286 & 775 & 1060 \\
\hline W1 & 510 & 600 & 589 & 1090 & 1630 \\
W2 & 1477 & 1510 & 1121 & 2030 & 3150 \\
W3 & 3100 & - & 1121 & 2030 & 3150 \\
W4 & 3100 & - & $\left(V_{r}\right)$ & $(\mathrm{kN})$ \\
\hline \hline
\end{tabular}

${ }^{a} V_{r}$ is the average shear strength from the five methods discussed in the control wall design; and Note: $f_{c}^{\prime}=21.8 \mathrm{MPa}$, for all strengths, the average of all 22 concrete cylinder tests. 

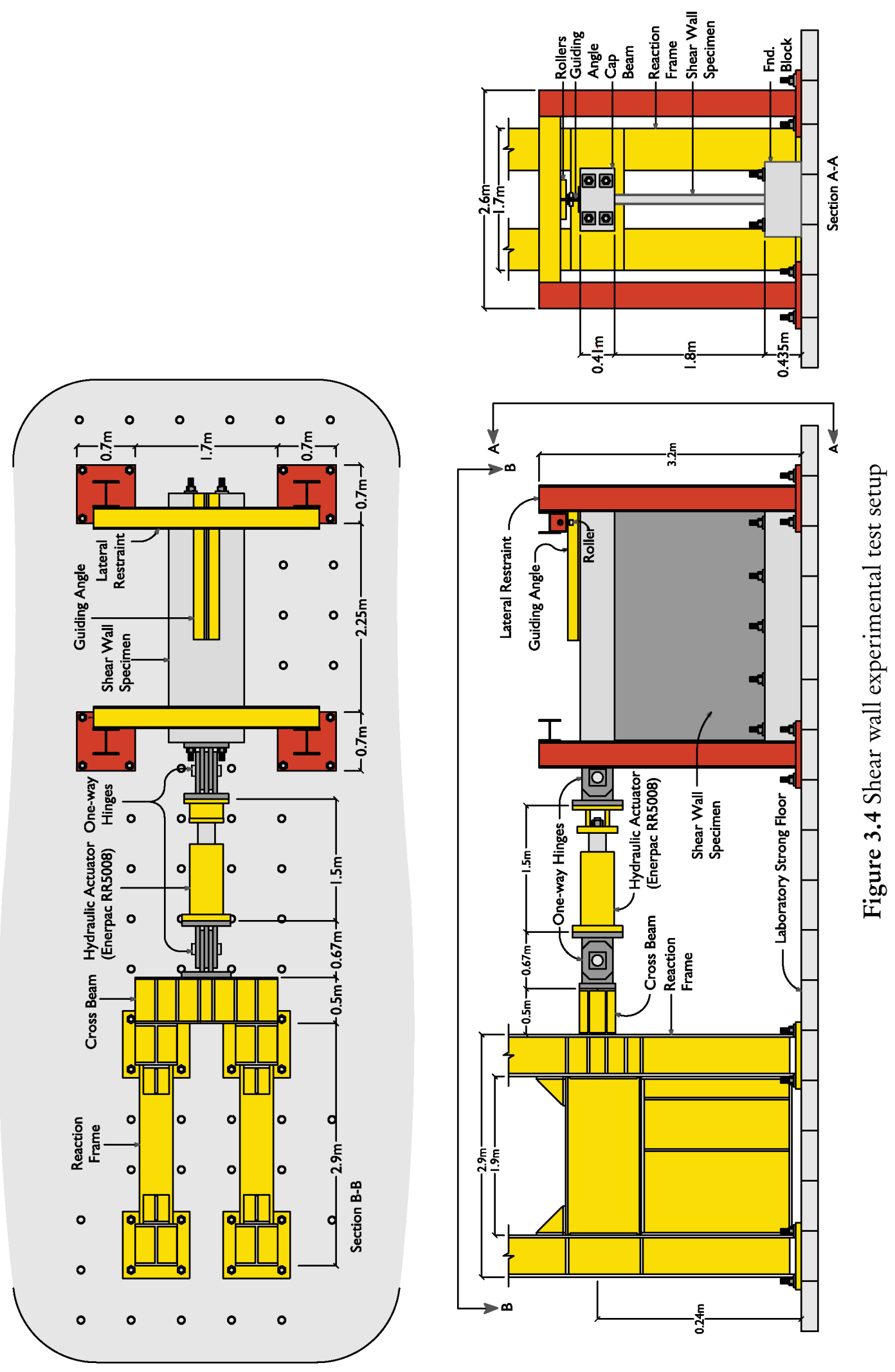
with future studies considering the effect of axial load.

\subsubsection{Loading Protocol}

To simulate the drift demand and damage effects on a $\mathrm{RC}$ shear wall during a major seismic event, the wall specimens were tested under quasi-static cyclic load up to failure. Figure 3.5 shows the cyclic lateral load sequence used in this study. Each of the specimens is first tested in load control, applying load cycles at $25 \%, 50 \%, 75 \%$ and $100 \%$ of the estimated yield load (determined from strain-compatibility analysis). The test is then continued in displacement control by increasing the target displacement ductility level gradually up to failure. Each load cycle is repeated twice to account for the softening effects of repeated load cycles on the strength and stiffness of the shear wall specimens. The wall specimens in this study are not tested under axial load to avoid introducing the additional complexities associated with the presence of axial load and shear interaction to the shear dominated behaviour under investigation. Omitting axial load also allows for comparison with past test results which did not include the effects of axial load. Future studies will aim to determine the influence of axial load on the performance of the retrofitting system, although it is expected that the application of the CFRP sheets would not significantly affect the axial load capacity of the shear wall specimens.

\subsubsection{Instrumentation}

The lateral load applied to the top of the wall specimens is measured using two pressure transducers for push and pull load directions. At the end of each load cycle, the test is stopped to record the progression of crack distributions in the concrete and FRP debonding patterns. Each of the wall specimens has approximately 30 strain gauges, as illustrated in Figure 3.6, to measure the strains along the horizontal and vertical steel reinforcement at the base, mid-height, and top of the wall specimen. The strain gauges are used to determine the propagation of yielding throughout the steel reinforcement inside each test specimen. Additional steel strain gauges are installed along the length of the lapped bars in series 4 wall specimens with lap splices of the vertical steel reinforcement to estimate the bond capacity of the lapped vertical steel reinforcing 


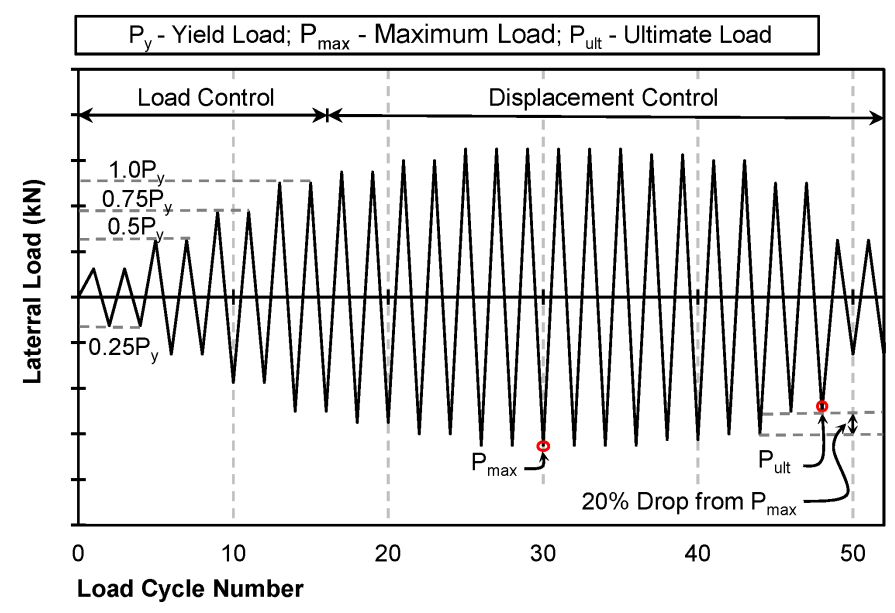

Figure 3.5 Shear wall in-plane cyclic loading protocol

bars.

The displacements of the wall specimen are measured using between 16 and 20 linear potentiometers (LPs), depending on the series of wall specimen. All of the displacements are measured relative to the shear wall foundation block using a specially constructed instrumentation frame. The locations of the LPs for each series of wall specimen are illustrated in Figure 3.7. Lateral displacements and rotations of the wall specimen are measured at the base, mid-height, and top of the wall panel. Shear deformations are measured using diagonally mounted LPs on the face of the wall specimen. Out-of-plane displacement is monitored by two LPs attached to the cap beam on top of the shear wall specimen. The measured displacements are used to analyze the deformation mechanisms of each wall specimen and separate the total top displacement into contributions from shear and flexural behaviour. The displacement contributions from shear and flexural deformations are important factors for understanding the response behaviour and deformation mechanisms for comparing the influence of aspect ratio on the response of the wall specimens as well as the effectiveness of the retrofitting strategy in its ability to shift the behaviour of a wall specimen to a more flexural dominant response. 

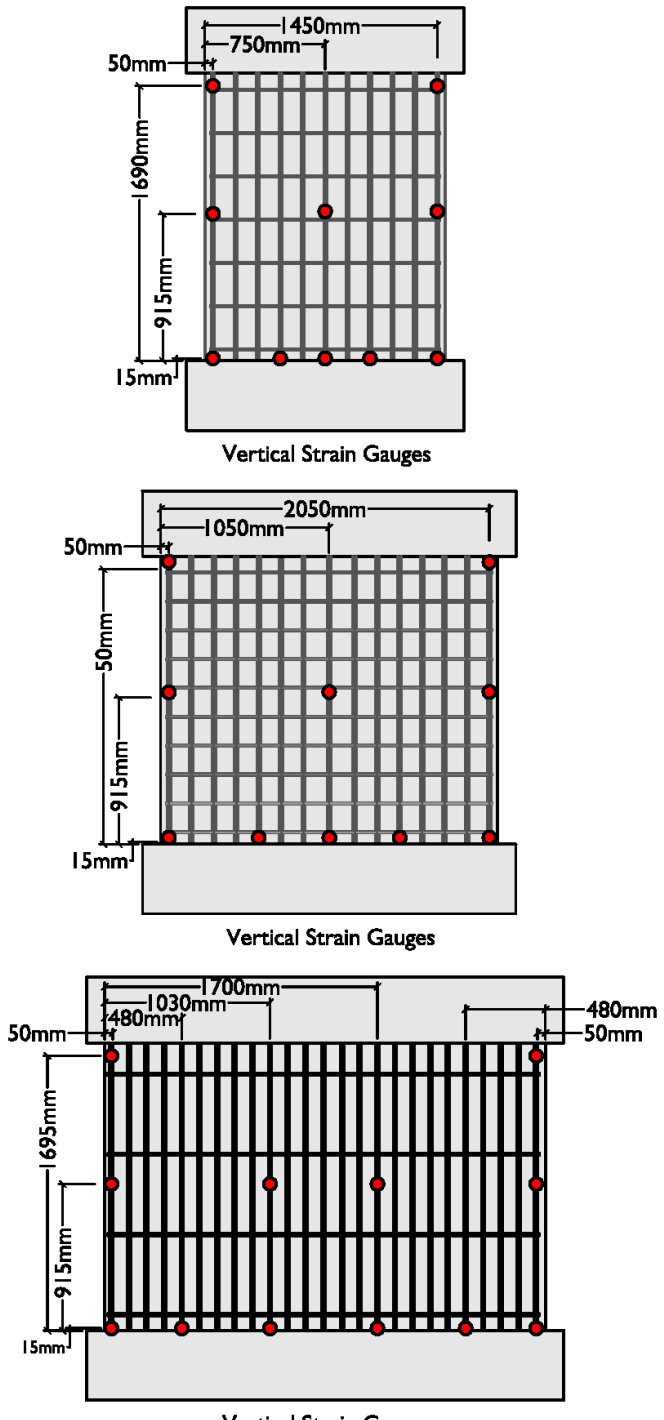

Vertical Strain Gauges

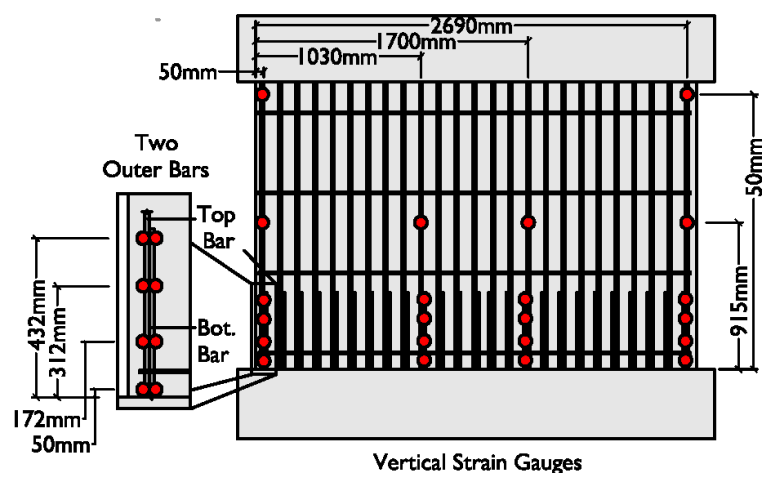

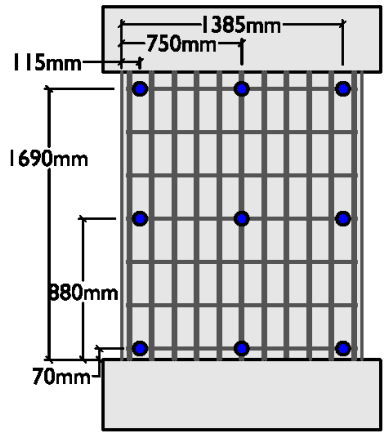
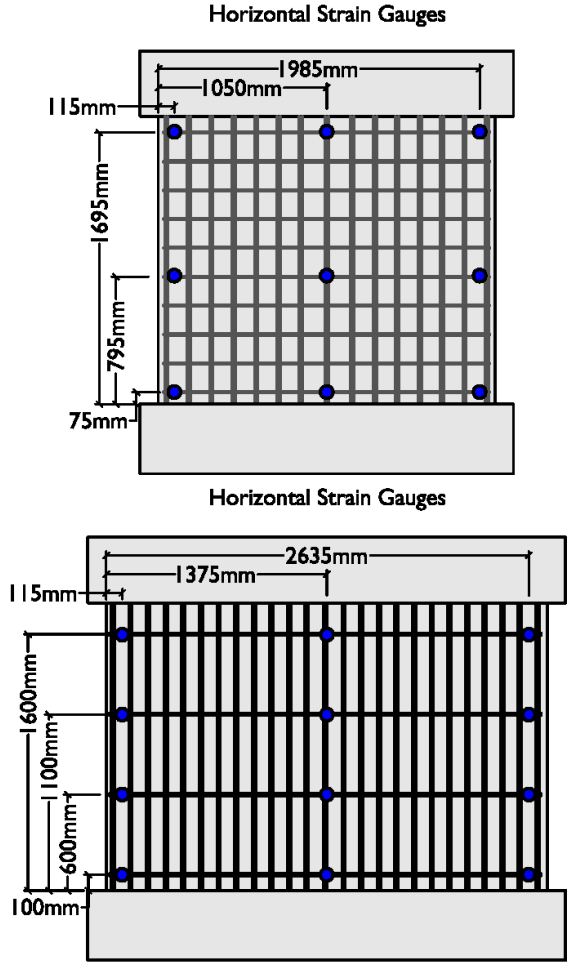

Horizontal Strain Gauges

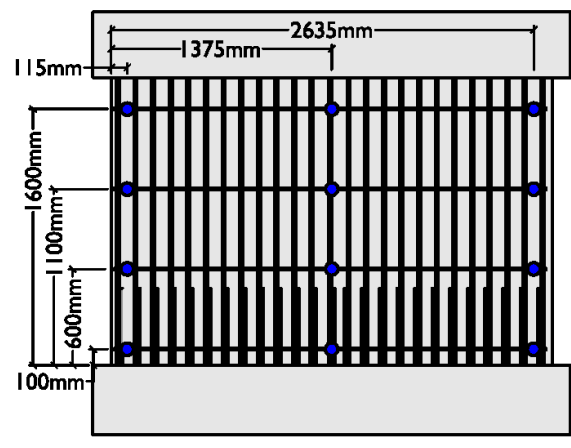

Horizontal Strain Gauges

Figure 3.6 Steel strain gauge configuration for each shear wall series 

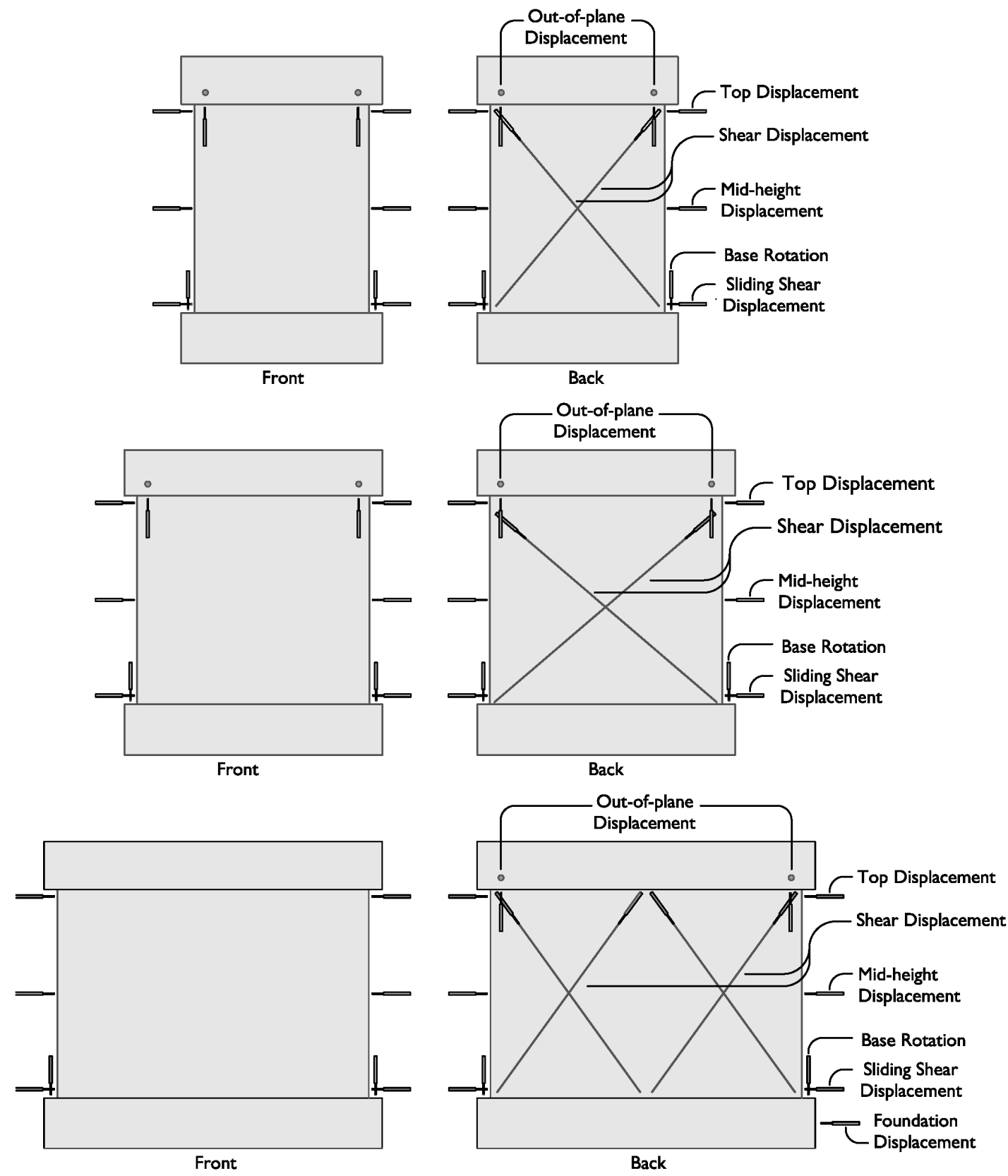

Figure 3.7 LVDT configuration for each shear wall series 


\subsubsection{Digital Image Correlation}

Experimental tests conducted on reinforced concrete elements are typically concerned with measuring crack distributions over the surface of the concrete as well as displacement and strain at critical locations. Traditionally, crack distributions in RC structural elements during experimental testing are recorded by manually marking visible cracks in the concrete. This technique can be time consuming and potentially dangerous, as users are required to approach a damaged test specimen under load. Furthermore, testing of elements under earthquake loading often requires the load to be applied continuously without stopping to prevent relaxation of the test specimen, making it difficult to take detailed measurements such as crack widths during testing. Displacement and strain are conventionally measured using electrical strain and displacement gauges located at critical locations on the test specimen. Although these gauges are effective in capturing the desired response parameters, the critical locations for these sensors may not always be obvious, especially in large-scale structural tests. The use of such techniques can also be time consuming and expensive, requiring significant investment in the cost of the sensors, time for their installation, and large data acquisition systems to collect the data.

In an attempt to overcome these challenges, this study uses an image analysis method capable of measuring crack distributions, displacement and strain fields in structural engineering experiments in the series 4 shear wall tests (CW4 \& RW4). The technique uses high-definition photographs taken at various stages throughout the experiment. The images are post-processed using a freely available software tool called ImPro Stereo (Yang et al., 2014). Using ImPro Stereo, photos of the damaged specimen are compared with baseline photos taken at the beginning of the test to determine the displacement and strain fields over a specific area of interest on the surface of the test specimen. Past studies have employed this image analysis technique to monitor crack widths and measure strain and displacement fields in structural experiments on a RC shear wall and the tubular surface of a RC column (Yang et al., 2012, 2015). In both of these studies, the monitored measurement region represented a small section of the total surface of the structural element. In this study, the image analysis method is employed to capture the displacement and strain distribution over the entire face of the RC shear wall specimen, which represents a significantly 
larger measurement region when compared with past experiments. The use of image analysis in this application provides a simple, safe, and cost effective method for measuring displacement and strain fields in addition to monitoring crack widths in structural experiments on RC elements. The following section briefly describes the steps involved in the image analysis setup.

Before the experiment is conducted, there are preliminary steps that must be completed to ensure that the results of the image-based analysis are accurate. These steps include preparing the surface of the test specimen, mounting two high-definition cameras and taking a number of calibration photos. Surface preparation consists of painting or marking the surface of the test specimen to ensure that the measurement region, referred to as the region of interest (ROI), has enough unique characteristics to detect movement. The common method for surface preparation is a speckle pattern technique, which applies tiny speckles over the surface of the ROI. These speckles form unique identifiers that can be used to track the movement in the ROI during loading. Figure 3.8 shows the outline of a typical ROI and the speckle pattern on the surface of the wall.

After applying the speckle pattern to the surface of the specimen, the two cameras are set in place next to one another and focused on the ROI, as shown in Fig. 3.8. Using this image analysis method, the cameras are not required to be placed directly perpendicular to the ROI, but should be positioned such that the ROI is located within the centre portion of the camera view. This will ensure the ROI remains within the camera view throughout the entire test and the higher distortion effects close to the edges of the camera view can be avoided. Throughout the duration of the test, the cameras should remain fixed, avoiding any slight movements, which would decrease the accuracy of the measurement system. Prior to testing, pairs of calibration photos are used to provide information on each camera in addition to the position of each camera relative to the ROI and relative to one another. An object with a checkerboard pattern (Fig. 3.8) is placed in the ROI during the calibration photos. Because the ROI in this study is very large, a series of 12 calibration photos are taken with the checkerboard located in different positions around the ROI. Once the test begins, photos are taken incrementally over the course of the experiment. As discussed previously, it is important that the cameras remain firmly fixed throughout the test and that the photos from both cameras are taken simultaneously to ensure the cameras are capturing 


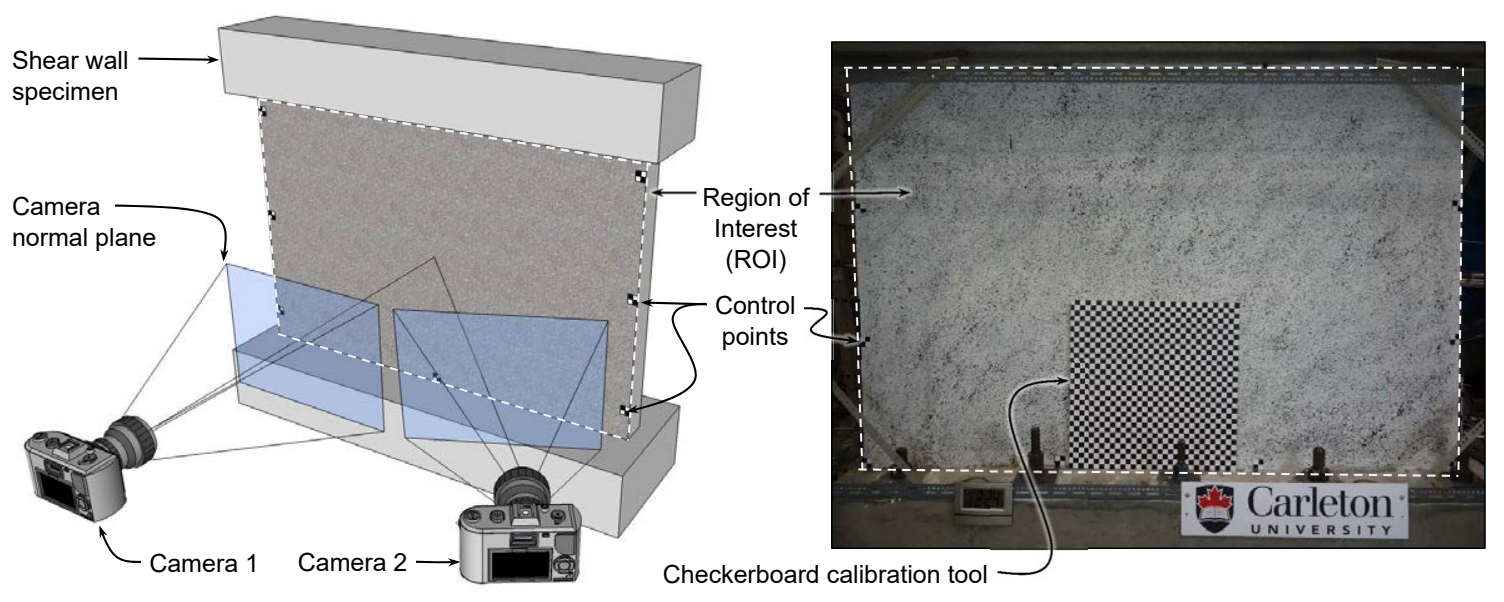

(a)

(b)

Figure 3.8 Test setup for the digital image correlation

the state of the specimen at the same instant. A remote connected to both cameras ensures both photos are taken simultaneously and a clock within the ROI is used to match the test photos with other measured parameters (eg. load, displacement, etc...). It is also important to ensure that the apertures, exposure time, lighting conditions, and focal length remain constant throughout the test to avoid distorting the results.

Once the preliminary steps are complete and the photos of the specimen during the test are collected, the images are analyzed using ImPro stereo. The ImPro Stereo software used to analyze the photos is freely available and uses a MATLAB based program, Bouguets Camera Calibration Toolbox, and OpenCV library to conduct the image analysis (Bouguet, 2008; Vision, 2016). Figure 3.9 shows the user interface for ImPro Stereo. The steps to image analysis include stereo calibration, control point positioning, metric image rectification, surface displacement analysis, and visualization. Stereo calibration is conducted using the previous calibration photos and Bouguets Camera Calibration Toolbox (Bouguet, 2008). The purpose of the calibration is to estimate the intrinsic parameters of the cameras (eg. lens focus and distortion factors) in addition to extrinsic parameters including the coordinate transformation between cameras. The calibration parameters of the cameras and a stereo triangulation technique are used to identify the $3 \mathrm{D}$ locations of the control points on the surface of the ROI. In this study, three control points are manually selected in the ImPro Stereo user interface for the first set of images to completely define the 
three axes of the measurement region. In the subsequent photos of the deformed test specimen the control points are automatically located using template matching. The surface of the ROI is then converted to a plane image; removing the effects of perspective and image distortion using a process known as metric rectification. The displacement fields for the deformed test specimen are then determined by analyzing the rectified images of the deformed specimen relative to the initial photos.

Each of these steps is carried out to capture the crack patterns, displacement, and strain fields for the series 4 wall specimens with lap splices (CW4a, RW4a, CW4b, and RW4b). Figure 3.10 shows the ROI for each of the four shear wall tests. Results from the image analysis method are discussed in Chapter 5 of Part I. The results are used to get a better understanding of the behaviour of deficient squat RC shear walls with lap splices, evaluate the performance of the retrofitting system, and examine the effectiveness of using the ImPro Stereo to study a large measurement region. 


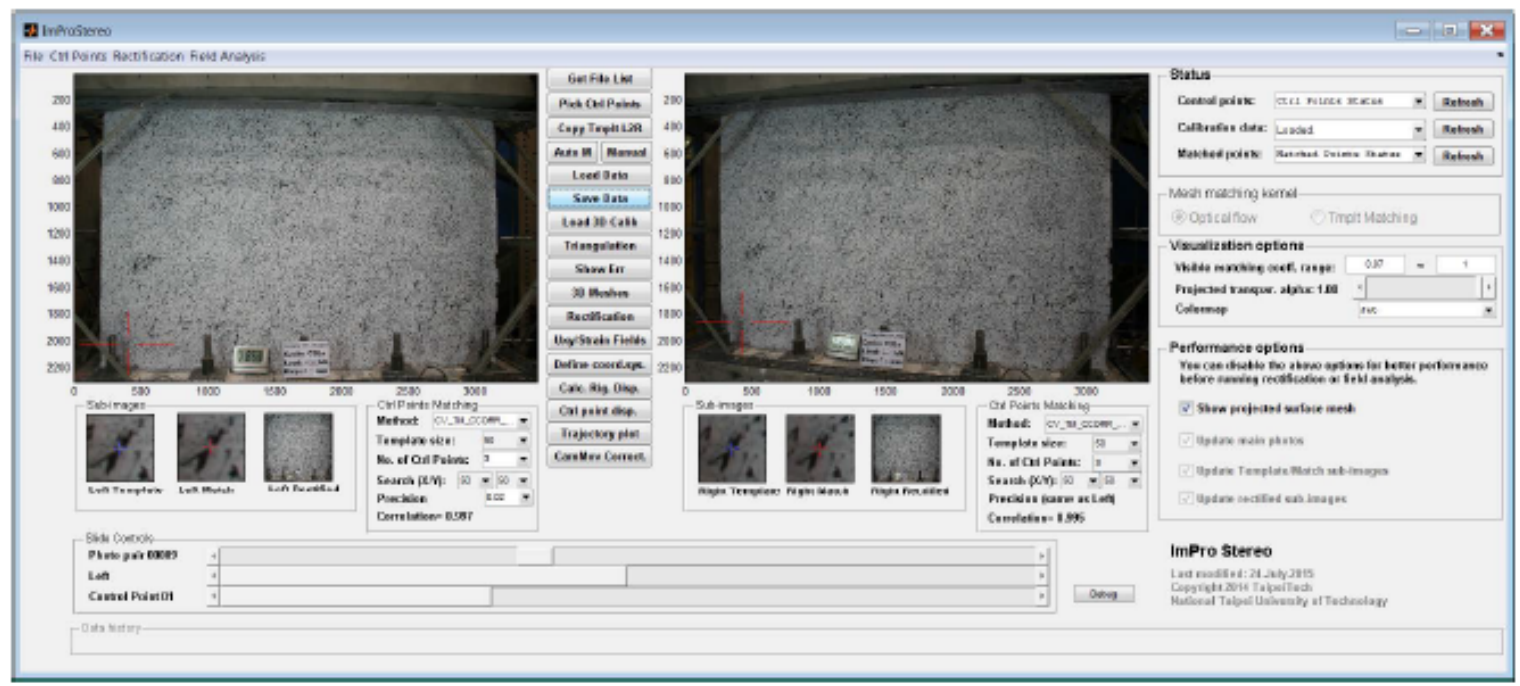

Figure 3.9 ImPro Stereo graphical user interface

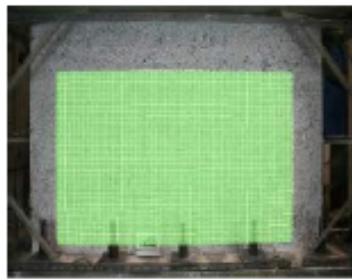

(a)

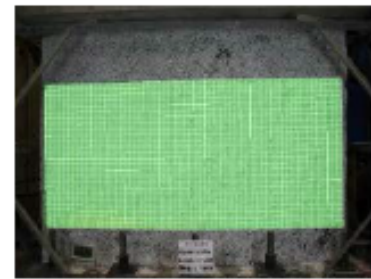

(b)

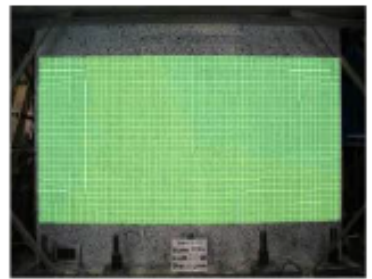

(c)

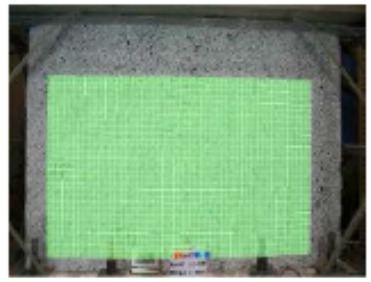

(d)

Figure 3.10 Region of interest: (a) CW4a; (b) RW4a; (c) CW4b; (d) RW4b 


\section{Chapter 4. Anchor System Optimization}

\subsection{Analytical Modelling of Mechanical Anchor Systems}

Prior to conducting the cyclic tests on the shear wall test specimens, a significant amount of research was conducted on the design and optimization of the tube anchor system used to anchor the vertical CFRP layers. Past experimental results by Lombard et al. (2000) and Hiotakis et al. (2004) have demonstrated the steel tube anchor system improved the efficiency of the FRP retrofit when compared with the steel angle anchor system, however, this application of the tube anchor was a proof of concept, and thus was significantly over-designed. To verify the observed performance of the steel angle anchor and tube anchor systems, detailed finite element models are developed. In the case of the tube anchor system, the developed finite element model is used to develop a tube anchor design methodology, devised to improve the efficiency of the tube anchor system and design the tube anchors implemented in this study on the seismic retrofit of deficient RC shear walls retrofitted with externally bonded CFRP sheets.

\subsubsection{Model Descriptions}

Prior to modelling the anchor systems for the current experimental program, finite element models of the steel angle and steel tube anchor systems developed by Lombard et al. (2000) and Hiotakis et al. (2004) were modelled to compare with the observed experimental results and verify the proposed numerical modelling procedure. Both anchors were modeled using the finite element software ABAQUS (Dassault Systemes, 2013). Figure 4.1 shows the geometry of the steel angle and tube anchors tested by Lombard et al. (2000) and Hiotakis et al. (2004), respectively. The 
angle anchor is constructed of a $\mathrm{L} 150 \times 100 \times 10 \mathrm{~mm}$ grade $350 \mathrm{~W}$ steel angle section. Five $31.75 \mathrm{~mm}$ (1.25 in) drop-in expansion anchors are used to connect the angle to the foundation of the shear wall test specimen. Alternatively, the tube anchor is constructed of a $76.2 \mathrm{~mm}$ ( 3 in) heavy-walled steel pipe, which has a wall thickness of $12.7 \mathrm{~mm}$ ( $0.5 \mathrm{in})$. Five $38.1 \mathrm{~mm}$ (1.5 in) epoxy adhesive anchors are installed at a 45 degree angle to connect the steel tube to the foundation of the shear wall test specimen.

The angle anchor is modeled using 8-node solid brick elements (CD3D8R). Because of the complex geometry of the tube anchor, it is modeled in using 10-node quadratic tetrahedral solid 3D stress elements (C3D10). The mesh size for both models is optimized using a mesh sensitivity analysis to balance model and computational efficiency with accuracy of results. Both steel materials are modeled using a bilinear stress-strain response, assuming a steel yield strain of 0.002 and a constant yield plateau at a stress of $400 \mathrm{MPa}$. Boundary conditions applied to both models include full fixity at the base of the wall, assuming that sufficient embedment length into the concrete foundation is provided to prevent pullout of the anchor rods. In addition, the base of the steel angle and tube are restrained from displacing in the direction of the concrete foundation, which in this experiment acts as the supporting structural element.

The load applied to the anchor systems is based on an idealized loading condition for an in-plane laterally loaded RC shear wall flexurally strengthened using vertically oriented externally bonded FRP sheets. Figure 4.2a shows the idealized load distribution on the shear wall under inplane or out-of-plane seismic load. Under out-of-plane load, it is assumed that the tensile stress
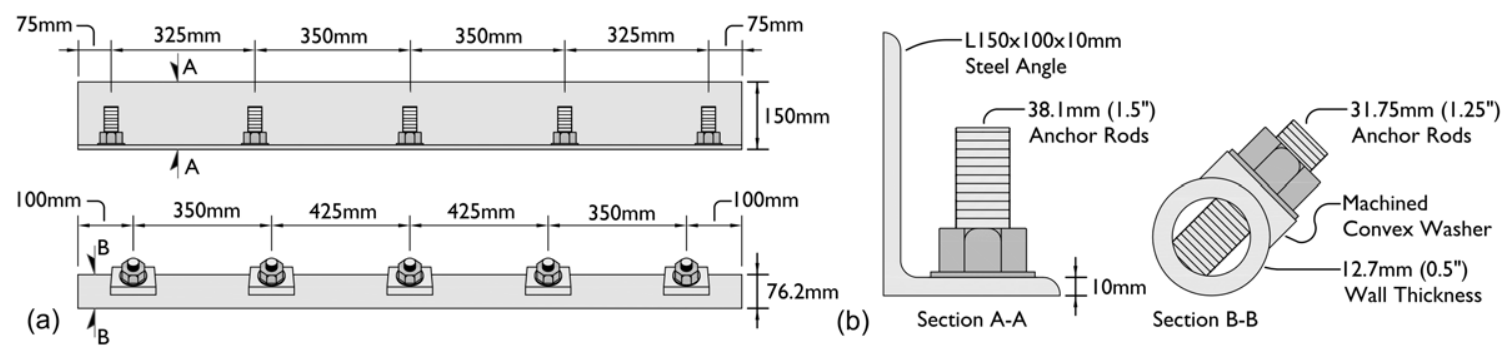

Figure 4.1 (a) Anchor geometries; (b) anchor cross-sections (Lombard et al., 2000; Hiotakis et al., 2004) 
carried by the vertical FRP laminate is constant over the length of the wall up to the ultimate tensile strength of the FRP laminate. Under in-plane lateral load (as was the case in studies by Lombard et al. (2000) and Hiotakis et al. (2004)), it is assumed that the tensile stress carried by the vertical FRP laminate varies linearly up to the neutral axis of the shear wall. Analytical and experimental results by Hassan et al. (2013) and Woods et al. (2016) have shown that a linear distribution of stress in the FRP sheet is a good assumption up to the maximum load carrying capacity of the shear wall and that the neutral axis typically lies somewhere between $70 \%$ and $85 \%$ of the shear wall length, depending on the aspect ratio of the wall, which is the ratio of its height to its length. It is assumed that minor variations in the load distribution would not significantly impact the behavior of the anchor system and a conservative neutral axis depth of $85 \%$ of the shear wall length was assumed for the analytical study. For a shear wall length of $1500 \mathrm{~mm}$ in the studies by Hiotakis et al. (2004) and Lombard et al. (2000), this results in an assumed neutral axis depth of $0.85 l_{w}=0.851500 \mathrm{~mm}=1275 \mathrm{~mm}$.

For both anchor systems, the stress carried by the vertical FRP sheets is transferred to the adjacent structural element through several steel anchor bolts installed along the length of the anchor. Between anchor bolts, the steel section is responsible for resisting the stresses carried by the vertical FRP layers. Figure $4.2 \mathrm{~b}$ shows the resulting pressure distribution over the crosssection of each anchor. The pressure is applied to each respective anchor over the area of steel in contact with the vertical FRP sheet. For the steel angle anchor, the maximum shear stress acting

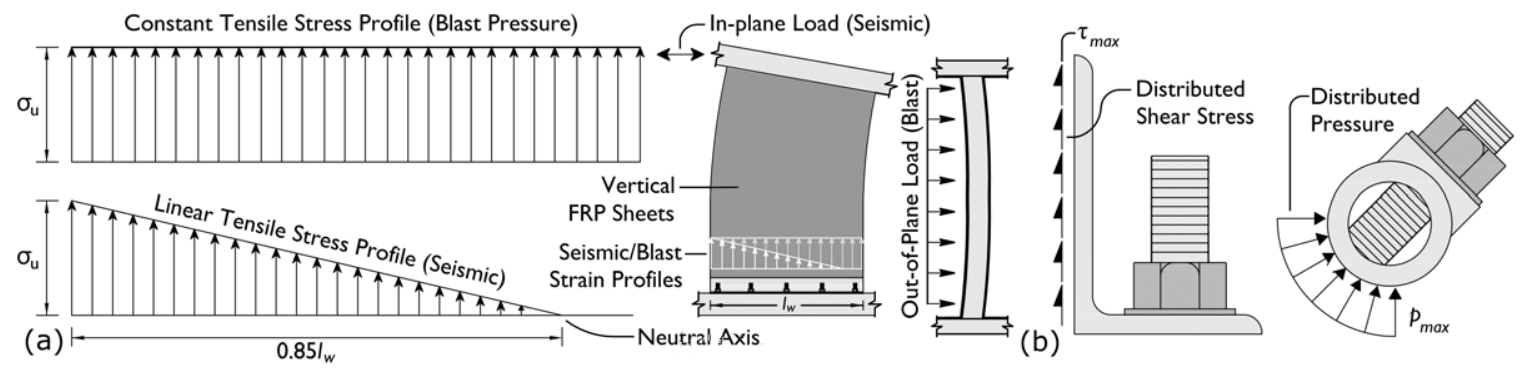

Figure 4.2 (a) Idealized FRP stress profiles; (b) cross-section pressure distributions 
on the flange of the steel angle $\left(\tau_{\max }\right)$ can be determined using Eq. 4.13:

$$
\tau_{\max }=\frac{\sigma_{u} t_{s} n_{s}}{d_{f}}
$$

where $\sigma_{u}$ is the ultimate tensile capacity of the FRP composite material; $t_{s}$ is the thickness of a single layer of vertically oriented FRP; $n_{s}$ is the total number of vertically oriented FRP sheets supported by the anchor; and $d_{f}$ is the height of the flange in contact with the vertical FRP. For a single layer of vertically oriented CFRP in the study by Lombard et al. (2000), using the net fibre area properties of the FRP that are available $\left(t_{s}=0.11 \mathrm{~mm}, \sigma_{u}=3480 \mathrm{MPa}\right)$, this results in $\tau_{\max }=2.55 \mathrm{MPa}$ applied as a linearly decreasing distributed shear stress up to the neutral axis of the shear wall specimen. Alternatively, for the tube anchor system, the maximum pressure acting over the base of the tube $\left(p_{\max }\right)$ can be determined using Eq. 4.14:

$$
p_{\max }=\frac{\sigma_{u} t_{s} n_{s}}{r_{o}}
$$

where $r_{o}$ is the outer radius of the steel tube. This results in $p_{\max }=13.86 \mathrm{MPa}$ for a single layer of vertically oriented FRP composite in the study by Hiotakis et al. (2004), once again using the net fibre area properties of the CFRP that are available for that study $\left(t_{s}=0.11 \mathrm{~mm}, \sigma_{u}=4800 \mathrm{MPa}\right)$. The calculated pressure is applied as a linearly decreasing pressure over the area of steel in contact with the vertical FRP sheet up to the neutral axis of the shear wall specimen.

\subsubsection{Model Results}

The results from the finite element models are used to not only validate the observed behavior from experimental studies by Lombard et al. (2000) and Hiotakis et al. (2004) but also to further study the performance and failure mechanisms of both anchor systems. Figure 4.3 compares the deformed angle anchor from the finite element model with experimental observations. The analytical results confirm that the eccentricity between the force applied to the flange of the steel angle from the vertical CFRP sheet and the reaction force from the anchor bolts causes a prying action or rotation of the angle. Measurement from the test suggest that the maximum 
flange separation from the wall was approximately $20 \mathrm{~mm}$. The analytical model predicts an ultimate displacement of $19.6 \mathrm{~mm}$ at $60 \%$ of the ultimate tensile capacity of the CFRP sheet at the edge of the wall. Unfortunately, there is no experimental data on the maximum strain achieved in the vertical CFRP sheet during tests conducted by Lombard et al. (2000). Nonetheless, analytical results do confirm that the rotation of the angle flange occurs before the FRP sheet approaches its ultimate tensile capacity, which caused the decrease in load carrying capacity of the shear wall with the angle anchor compared to the wall specimen with the tube anchor as shown in Fig. 2.12. More detrimentally, the initiation of premature debonding caused by the angle flange rotation causes the CFRP to buckle in compression and rupture, leading to a loss in ductility because the CFRP sheet can no longer contribute to the lateral load carrying capacity of the shear wall.

Analytical results from the tube anchor finite element model also correlate well with experimental observations from Hiotakis et al. (2004). Figure 4.3 shows the deformed shape of the finite element model under the maximum load from the vertical CFRP sheet and compares it with the observed tube anchor behaviour during the experimental test. During the test, it was noted that the tube anchor experienced very little displacement and no visible inelastic deformation under the applied load from a single sheet of CFRP. Comparing with the finite element model, the maximum tube displacement in the outermost tube anchor bay is $0.3 \mathrm{~mm}$, which would not have been visible during the test. In addition, the maximum stress in the steel tube from the analytical model is approximately $40 \%$ of the yield strength of the steel material and the two outermost anchor bolts experience a maximum stress of $258 \mathrm{MPa}$ ( $58 \%$ of their yield strength). Once again, there is no experimental data available on the maximum strain in the vertical CFRP sheet during the tests conducted by Hiotakis et al. (2004). However, visual evidence from the test suggests that the vertical CFRP sheets were able to reach their ultimate tensile strength and rupture at the edges of the shear wall, shown in Fig. 4.4b. Thus, analytical and experimental results suggest that the tube anchor remained elastic during the test, even up to the ultimate tensile strength of the CFRP sheet.

Joshua Woods, Department of Civil and Environmental Engineering, Carleton University 

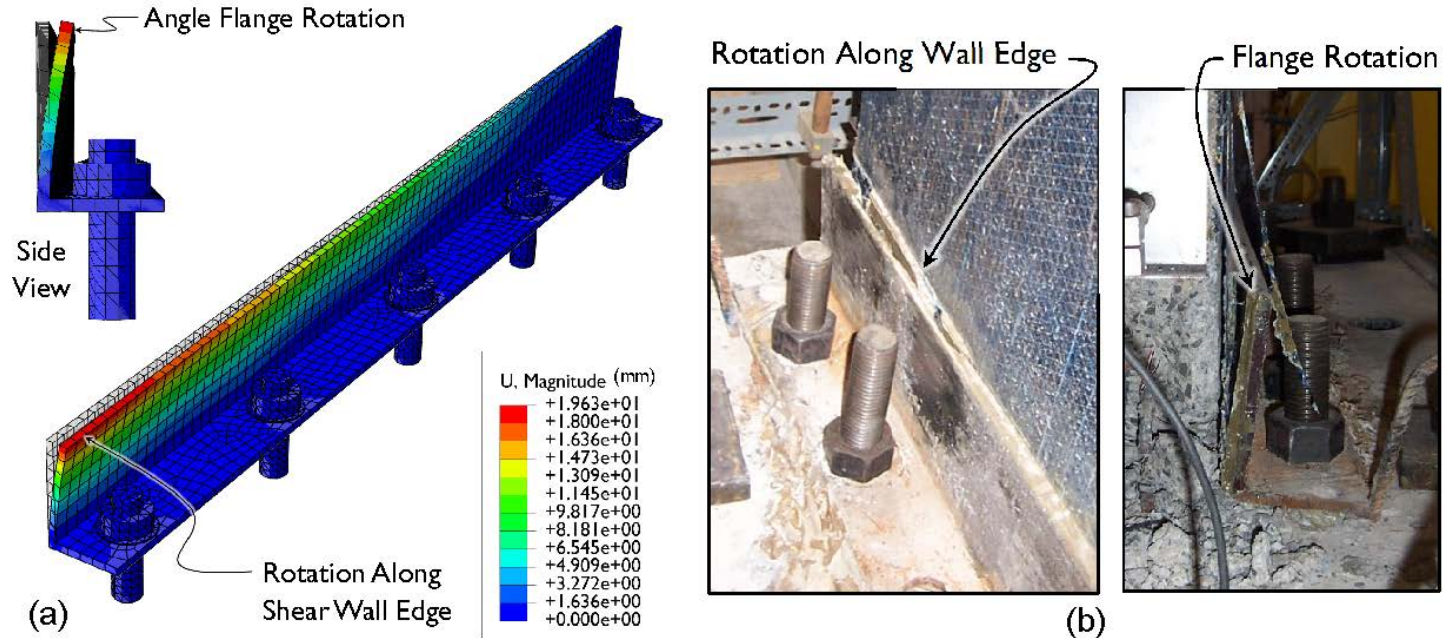

(b)

Figure 4.3 Angle anchor: (a) analytical modeling results; (b) experimental observations (Lombard et al., 2000)
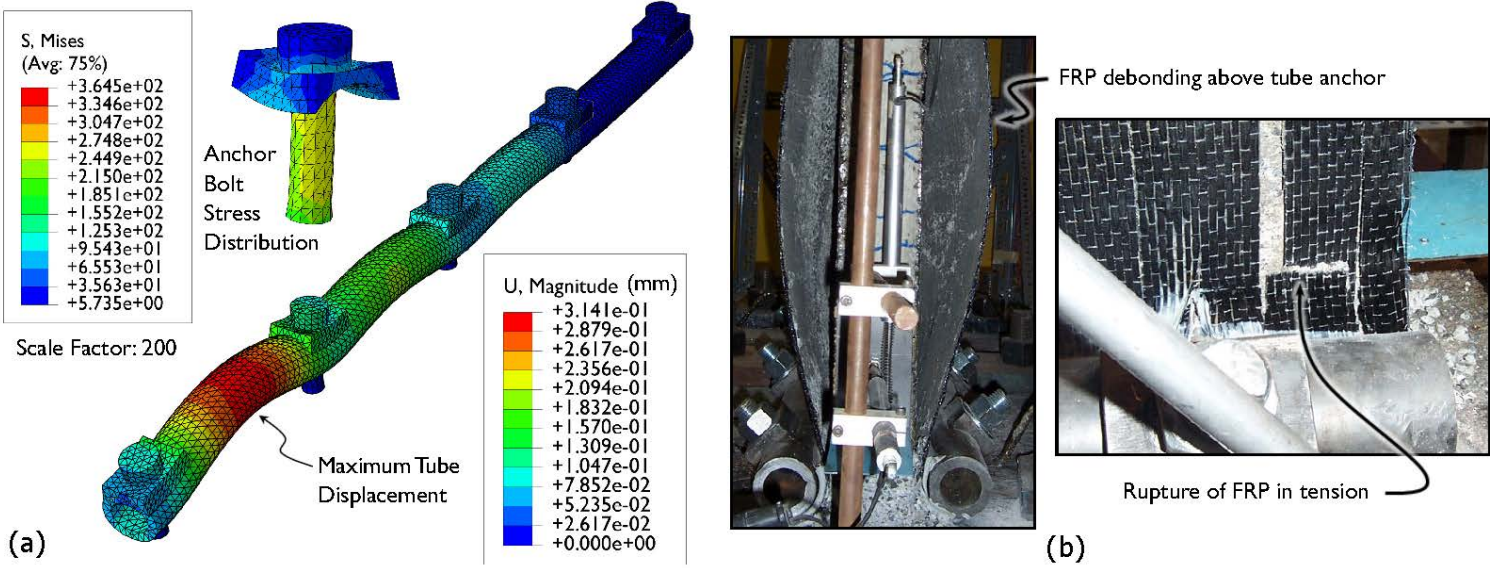

Figure 4.4 Tube anchor: (a) analytical model results; (b) experimental observations 


\subsection{Tube Anchor Optimization}

Experimental results have demonstrated the effectiveness of the tube anchor system in preventing premature debonding and allowing a large portion of the high strength capacity of the FRP sheets to be utilized. Experimental observations and analytical results also suggest that the tube anchor system employed by Hiotakis et al. (2004) was over-designed. The tube anchor design tested by Hiotakis et al. (2004) was a feasibility and proof-of-concept test, thus it is expected that the tube anchor design could be optimized in future implementation. Through the use of the previously discussed finite element modeling approach, a parametric study was conducted using different tube geometry and anchor bolt spacing. The goal of the parametric study is to improve the cost-efficiency of the tube anchor while maintaining the same level of performance. To reduce the size of the finite element model and improve its computational efficiency, only a single section of the tube anchor was modeled, assuming once again full fixity at the base of the wall and that each anchor bay (between anchor rods) acts independently from one-another. This reduction in model size also makes the results applicable to a wide range of anchor designs, because in most applications the design of the tube anchor will be governed by the section of anchor which is under the highest load from the FRP sheet. Using the results of this parametric study, a designer is able to select an appropriate sized tube anchor for a specific anchor bay length. Figure 4.5a shows typical single-bay anchor model in this study. Six different tube diameters ranging from $38.1 \mathrm{~mm}$ ( $1.5 \mathrm{in})$ to $101.6 \mathrm{~mm}$ ( 4 in) at $12.7 \mathrm{~mm}$ (0.5 in) increments and three different wall thicknesses of $6.35 \mathrm{~mm}(0.25 \mathrm{in}), 12.7 \mathrm{~mm}$ (0.5 in) and alternative solid steel rods are also considered. In addition, three different anchor bay lengths of $100 \mathrm{~mm}, 325 \mathrm{~mm}$, and $500 \mathrm{~mm}$ measured centerto-center between anchor rods are studied. The models are subjected to a uniformly distributed pressure equivalent to the ultimate tensile capacity of a single sheet of vertically oriented FRP reinforcement (Eq. 4.14).

Figure 4.5a shows the deformed shape and Von Mises stress distribution for a typical anchor model. As anticipated, the highest displacement occurs in the center of the tube while the highest steel stresses are in the anchor bolts. Figure $4.5 \mathrm{~b}$ shows the maximum tube displacements for 


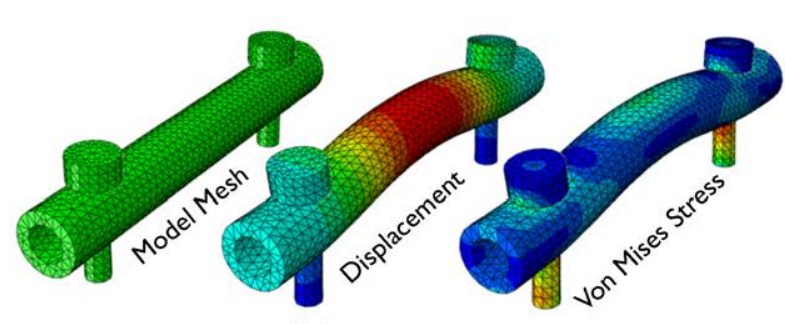

(a)

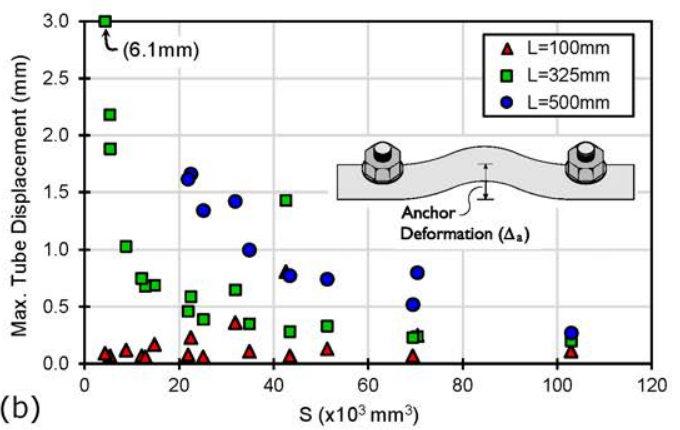

Figure 4.5 Tube anchor optimization: (a) finite element model; (b) optimization results

all of the anchor models plotted against the steel tube section modulus $(S=I / y)$. The three different anchor bay lengths are indicated by the shape and colour of the markers in the graph. Results from the parametric study show that small stocky tube sections tend to perform better than large thin tubes because of the tendency of the large tubes to collapse under external pressure, causing warping of the round section. In addition, sections with an unsupported length of $500 \mathrm{~mm}$ between anchor bolts and a section modulus less than 20 experience significant yielding under the design load and are not shown on the graph. This suggests that unsupported lengths of $500 \mathrm{~mm}$ or greater should not be used where the distribution of pressure applied to the anchor is the largest.

Selection of an appropriate tube size in the design of the tube anchor is assumed to be based on a maximum allowable displacement of the steel tube. This design parameter is quantified by analyzing the effects of the flexibility of the tube anchor on the stress profile in the FRP sheet. Figure 4.6 illustrates a single bay of the tube anchor under a linear stress profile from the FRP sheet. As the tube deforms, the FRP sheet relaxes between anchor rods, which results in a decrease in stress at mid-span, however, the stress between the anchor rods redistributes, resulting in an increase in stress at the locations of the anchor rods. As the tube continues to deform, higher stresses will ultimately lead to premature rupture of the FRP material at the anchor bolt locations. Based on this design assumption, the displacement of the tube can be related to a loss in potential load carrying capacity of the FRP sheet, or efficiency of the tube anchor. The efficiency $\left(\eta_{\text {anchor }}\right)$ of a particular tube anchor system can be computed using Eq. 4.15:

$$
\eta_{\text {anchor }}=1-\frac{\Delta_{a} E_{f}}{h \sigma_{u}}
$$


where $\Delta_{a}$ is the maximum tube displacement; $E_{f}$ is the modulus of elasticity of the FRP composite material; and $h$ is the height of the FRP sheet being anchored, from the location of the anchor to the opposite end of the FRP sheet. Using Eq. 4.15 and the analytical displacement results shown in Fig. 4.5 an optimal tube size can be selected by the designer based on the specific application and the desired efficiency of the anchor system.

\subsubsection{Tube Anchor Design Methdology}

Experimental results discussed in this paper have shown that effective anchorage of vertical FRP sheets can contribute to an increase in strength, ductility, and energy dissipation capacity specifically in FRP retrofitted RC shear walls. These results are also reflected in modern design standards for the application of externally bonded FRP composites, which recognize that anchorage is an important aspect of an FRP retrofit (CSA, 2002; ACI, 2008), however, these standards do not provide any guidance on the analysis or design of such a system. To overcome these limitations, a simple set of design steps are presented to assist in the implementation of the tube anchor in FRP retrofit applications. The design methodology is summarized in the following steps.

1. Determine the demands on the structural element that is to be strengthened (new structures) or retrofitted (existing structures) with FRP sheets. For a shear wall, this includes the axial load, lateral shear force, and overturning moment at the top of the shear wall;

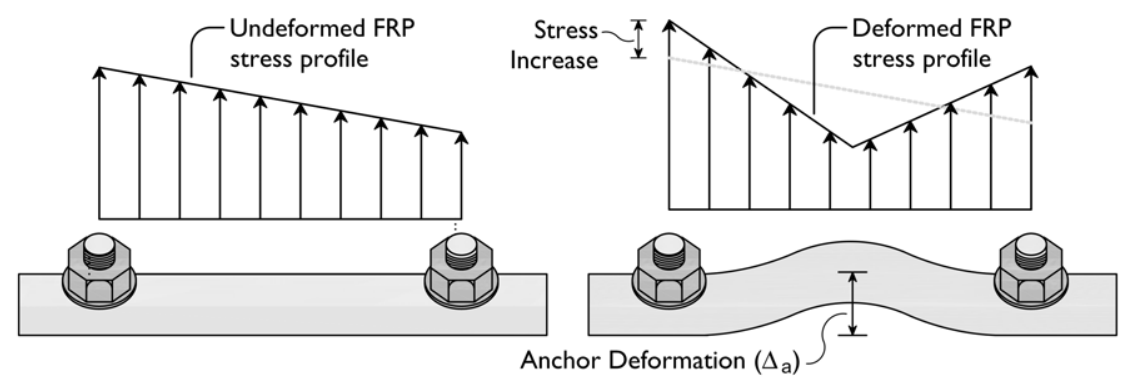

Figure 4.6 Effect of anchor tube flexibility on vertical FRP stress distribution 
2. For new construction, establish the load sharing between the steel reinforcement and FRP sheets so that the number of FRP sheets required for flexural/shear strength can be determined. In retrofit applications, the capacity of the existing member must be assessed and the number of FRP layers required is then based on the shear/flexural strengths required to meet the demands;

3. Based on the material properties of the FRP composite and number of layers requiring anchorage, calculate the total resultant load that must be transferred to an adjacent structural element based on the idealized ultimate stress distribution in the FRP sheet. For in-plane loading, relevant for lateral wind or earthquake loading, this stress distribution is assumed to vary linearly up to the neutral axis of the retrofitted element. For RC shear walls, the distance to the neutral axis can be approximated by using $75-85 \%$ of the shear wall length. A constant stress distribution or point loading may be more appropriate for out-of-plane loading;

4. Determine the minimum number of anchor rods that are required to transfer the resultant load from the FRP sheet(s) to the adjacent structural element using the desired size and available embedment depth according the manufacturers recommendations;

5. Evenly distribute the anchor rods along the length of the structural element to determine the maximum unsupported length of the steel tube. For cases where the stress distribution in the FRP sheet varies along the elements length, anchor bolt spacing can be optimized such that they are more closely spaced in regions under higher stress;

6. Calculate the maximum allowable displacement of the anchor tube using Eq. 4.15 based on the desired efficiency of the anchor system; and

7. Select an appropriately sized steel tube section to meet the allowable displacement criteria. This can be determined through finite element simulations, experimental testing, or the simplified design chart shown in Fig. $4.5 \mathrm{~b}$ if a single sheet of CFRP is used.

The list of steps presented above provides a simple design methodology for the tube anchor 
that can be used as an iterative procedure to determine the most efficient and economical tube anchor design. Figure 4.7 illustrates an application of the listed design steps in the FRP retrofit of a RC shear wall using externally bonded FRP sheets.

\subsection{Tube Anchor Design}

In this study, the tube anchor system developed by Hiotakis et al. (2004) is optimized using finite element analysis to improve the cost-efficiency of the tube anchor and simplify its fabrication process (Woods, 2014). A parametric study has been conducted to determine the optimal tube size and anchor bolt spacing. The optimized tube anchor design has a shorter fabrication time and uses less material when compared to the system as originally proposed by Hiotakis (2004) (Lau and Woods, 2017). Figure 4.8 shows the design details of the steel tube anchor system for series 1 and 2 wall specimens. Note that because there are no vertical CFRP layers in the series 3 and 4 wall specimens; anchorage of the vertical CFRP sheets is not required. The properties and geometry of the steel tube and the anchor rods are the same for series 1 and 2 wall specimens. The tube anchor is designed for the full ultimate tensile strength of the FRP material, to avoid premature failure of the anchor system before the specimens reach their ultimate capacity.

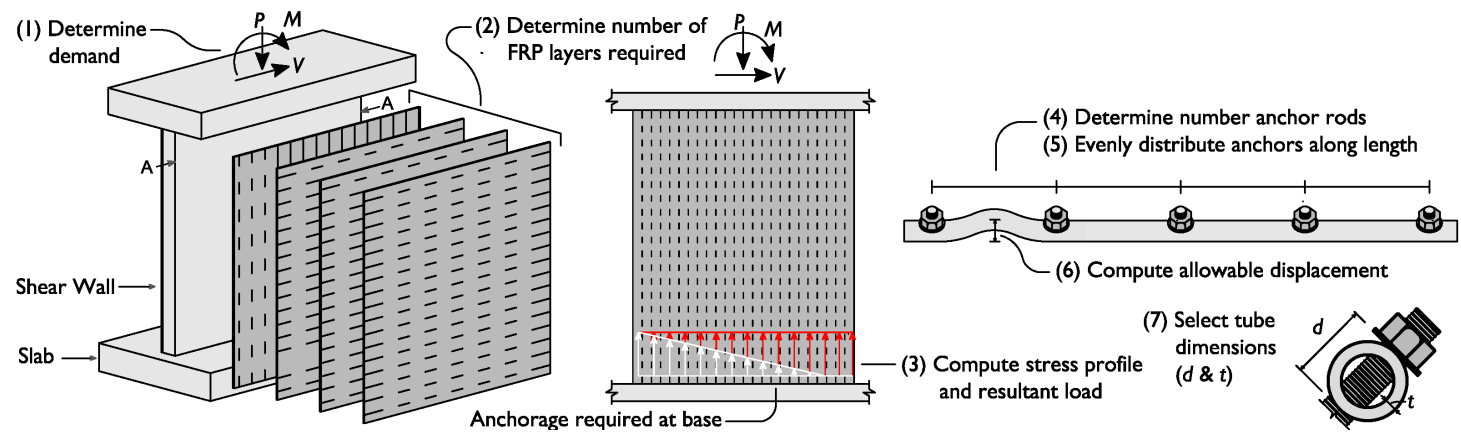

Figure 4.7 Design methodology example for FRP retrofitted RC shear wall 


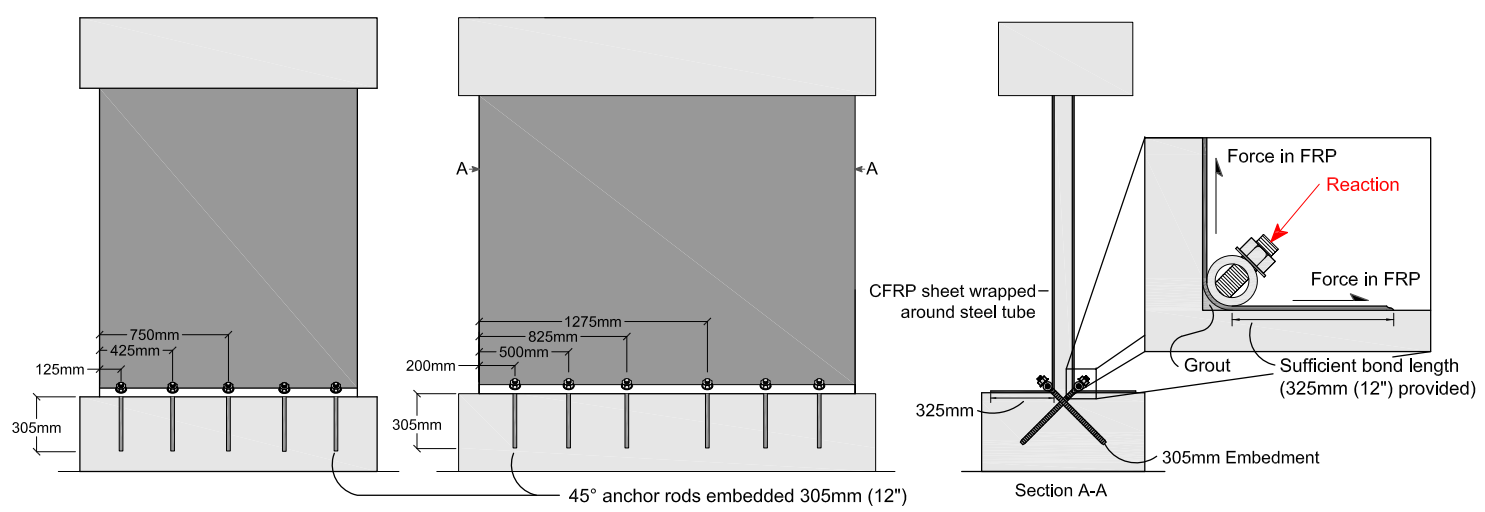

Figure 4.8 Tube anchor system design details

\subsection{FRP Anchor System}

The second type of anchor investigated in this study is a system consisting of FRP anchors, referred to in some cases as spike or fan anchors. Figure 4.9 shows the dimensions and layout of the fan anchors, which are utilized in the cyclic testing of shear wall specimen SW2b. The FRP anchor system design includes a combination of carbon and glass FRP anchors, the properties for which are shown in Table 4.1. In addition to a different anchor system, specimen SW2b also has all of the CFRP layers applied to a single side of the specimen. This will allow for comparison with specimen SW2a to get a better understanding of the effect of the distribution of the CFRP layers and the influence of the anchor system on the overall performance of the FRP retrofitting system. In specimen SW2b anchor system design, 13-510 mm long CFRP anchors were embedded into the foundation of the wall specimen to transfer the load carried by the two vertical CFRP layers to the concrete foundation. To prevent premature debonding of the six horizontal CFRP layers, 11-150 mm GFRP anchors were isntalled along each edge of the wall. 


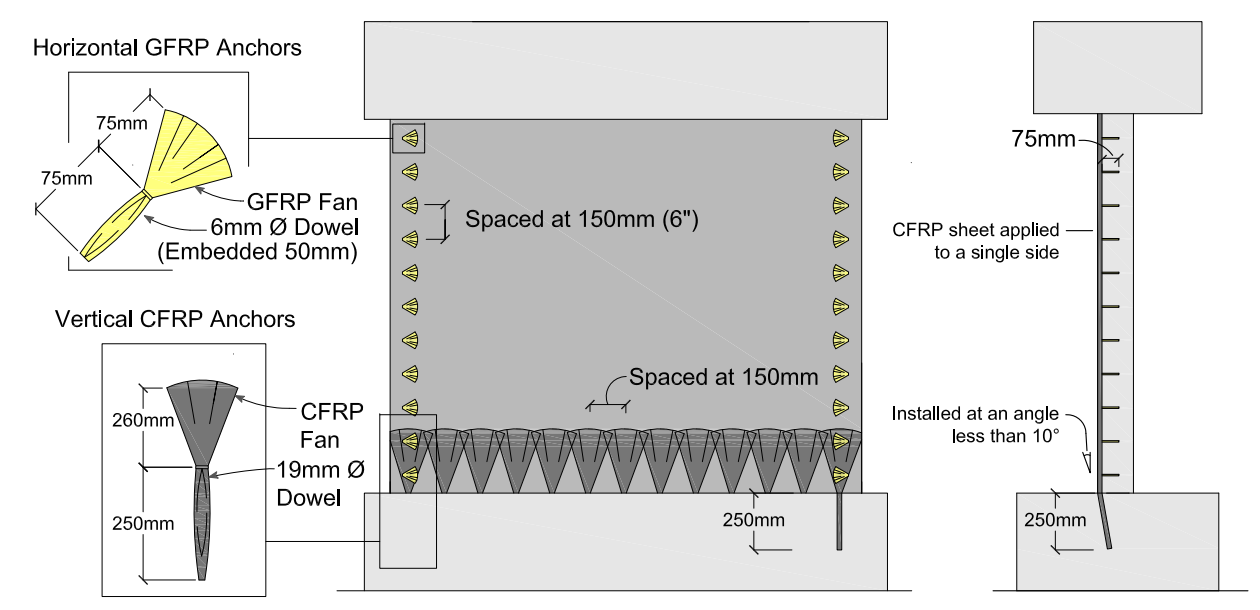

Figure 4.9 FRP anchor system design details

Table 4.1 Material properties for the mechanical and FRP anchor systems

\begin{tabular}{lccccccc}
\hline \hline & \multicolumn{3}{c}{ Tube Anchor } & \multicolumn{3}{c}{ CFRP/GFRP Anchors } \\
\cline { 2 - 6 } \multicolumn{2}{c}{ Steel Tube } & \multicolumn{2}{c}{ Anchor Rods } & \multicolumn{2}{c}{ CFRP Anchors } & \multicolumn{2}{c}{ GFRP Anchors } \\
\hline Material & ASTM & Material & ASTM & & Tyfo & Tyfo \\
Grade & A513 & Grade & A193 & Type & SCH & Type & SEH \\
$f_{y}(\mathrm{MPa})$ & 590 & $f_{y}(\mathrm{MPa})$ & 724 & $f_{u}(\mathrm{MPa})$ & 986 & $f_{u}(\mathrm{MPa})$ & 575 \\
$f_{u}(\mathrm{MPa})$ & 667 & $f_{u}(\mathrm{MPa})$ & 862 & $E_{t}(\mathrm{GPa})$ & 95.8 & $E_{t}(\mathrm{MPa})$ & 26.1 \\
$\epsilon_{u}$ & 0.322 & $\epsilon_{u}$ & 0.16 & $\epsilon_{u}$ & 0.012 & $\epsilon_{u}$ & 0.022 \\
\hline \hline
\end{tabular}

Note: gross laminate properties (fibre + epoxy) are shown for the CFRP/GFRP anchors. 


\section{Chapter 5. Results and Discussion}

\subsection{Control Walls without FRP}

The control specimens were tested first to assess the seismic performance of shear walls with the common deficiencies found in walls designed according to obsolete design standards and to serve as a baseline for later comparison with the CFRP repaired and strengthened specimens. Table 5.1 shows the results for the control wall specimens, including the initial stiffness, yield load, maximum load, ultimate drift achieved, displacement ductility, and energy dissipation capacity. Figure 5.1 shows the hysteretic response behaviour and crack distribution for each control wall specimen. The displacement ductility $\left(\mu_{\Delta}\right)$ of the wall specimens is calculated using Eq. 5.16:

$$
\mu_{\Delta}=\frac{\Delta}{\Delta_{y}}
$$

where $\Delta$ is the maximum displacement of the test specimen at a particular load cycle and $\Delta_{y}$ is the yield displacement of the wall specimen. The ultimate displacement ductility $\left(\mu_{\Delta}\right)$ is computed using the same equation, but with the ultimate displacement $\left(\Delta_{u}\right)$ in the numerator, which is defined by the displacement when the lateral load carrying capacity of the test specimen drops $20 \%$ from the maximum load reached during the test. It should be noted that the measured yield displacement in the control wall specimens is not based on yielding of the vertical steel reinforcement, as would traditionally be the case in most typical shear wall specimens. This is because the vertical steel reinforcement did not yield during testing of the control wall specimens, predominantly because of their large vertical steel reinforcement ratio (3\%). Alternatively, nonlinearity in the response of the shear wall specimens, which was used to establish the yield displacement, 
is a result of the shear dominant nature of the wall specimens. Their shear dominant nature and the deficiencies in their design resulted in the formation of large diagonal cracks in the concrete and sliding along the shear wall base producing nonlinearity in the shear wall response.

Test results for specimens CW1 and CW2 demonstrate the brittle nature of the control wall specimens, as the results show significant damage of up to $40 \%$ reduction of the maximum capacity following the test. This exceeded the target $20 \%$ drop from the maximum capacity, but could not be controlled because of the brittle response of the shear wall specimens. Failure of CW1 and CW2 occured in a sudden and brittle diagonal tension shear failure at lateral drifts of $0.74 \%$ and $0.54 \%$, respectively. Figure 5.2 shows the diagonal tension shear failure planes for wall specimens CW1 and CW2. Rupture of the horizontal steel reinforcement leading to diagonal tension shear failure occurred at lateral loads of $341 \mathrm{kN}$ and $1020 \mathrm{kN}$ for CW1 and CW2, respectively.

Figures 5.3 and 5.4 show the horizontal steel strains along the height of the shear wall specimens at increasing levels of lateral load. The horizontal steel reaches its rupture strain $(0.012 \mu \epsilon)$ in the centre of the wall, where significant diagonal cracking is observed. Figure 5.3 and Fig. 5.4 also show the vertical steel strain profiles at the base of the wall for specimens CW1 and CW2 at increasing levels of lateral load. Results show that yielding only occurs in the vertical steel reinforcing bars located closest to the edges of the specimens. The average displacement ductility of specimens CW1 and CW2 at their ultimate load is 1.20 and 1.25, respectively. The lack of yielding along the vertical reinforcement and very little concrete crushing in the toes of the walls contributes to their observed lack of ductility. The hysteretic response behaviour of specimens CW1 and CW2 (Fig. 5.1) show minimal ductility and energy dissipation capacity, characterized by tight, closely spaced hysteretic loops.

Among the larger shear wall specimens with an aspect ratio of 0.65 , control wall specimen CW3 was tested first, which had no lap splices of the vertical flexural steel reinforcement. At the beginning of the test, the shear dominant nature of the specimen was clearly evident from the formation of large diagonal cracks in the centre of the wall. As the test continued, the diagonal 


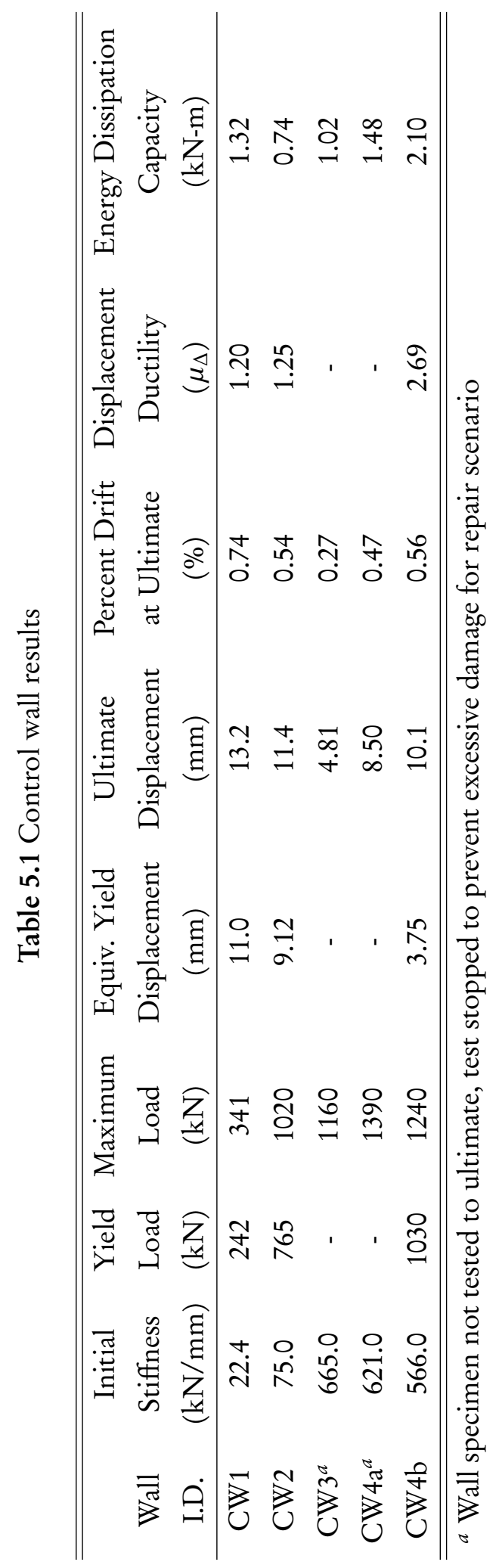


(a)
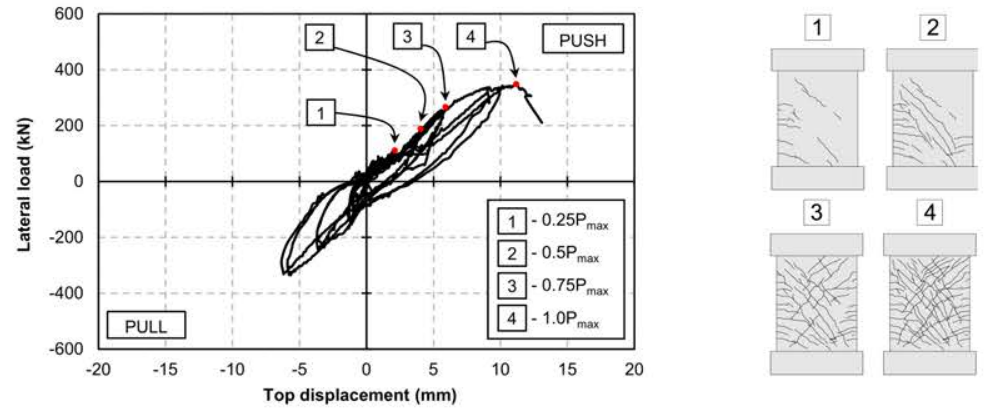

(b)
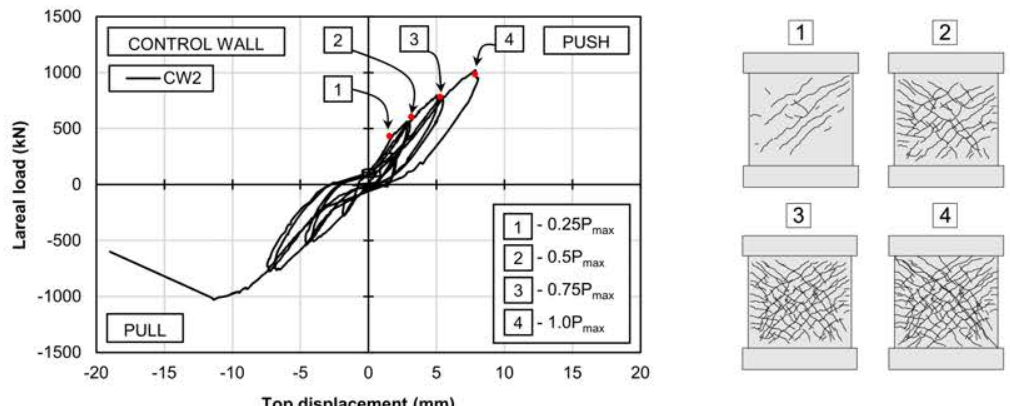

(c)
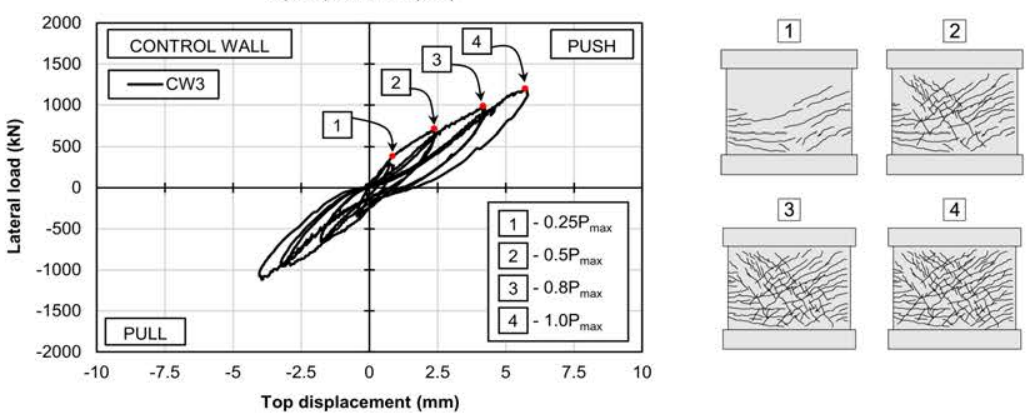

3

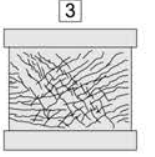

4
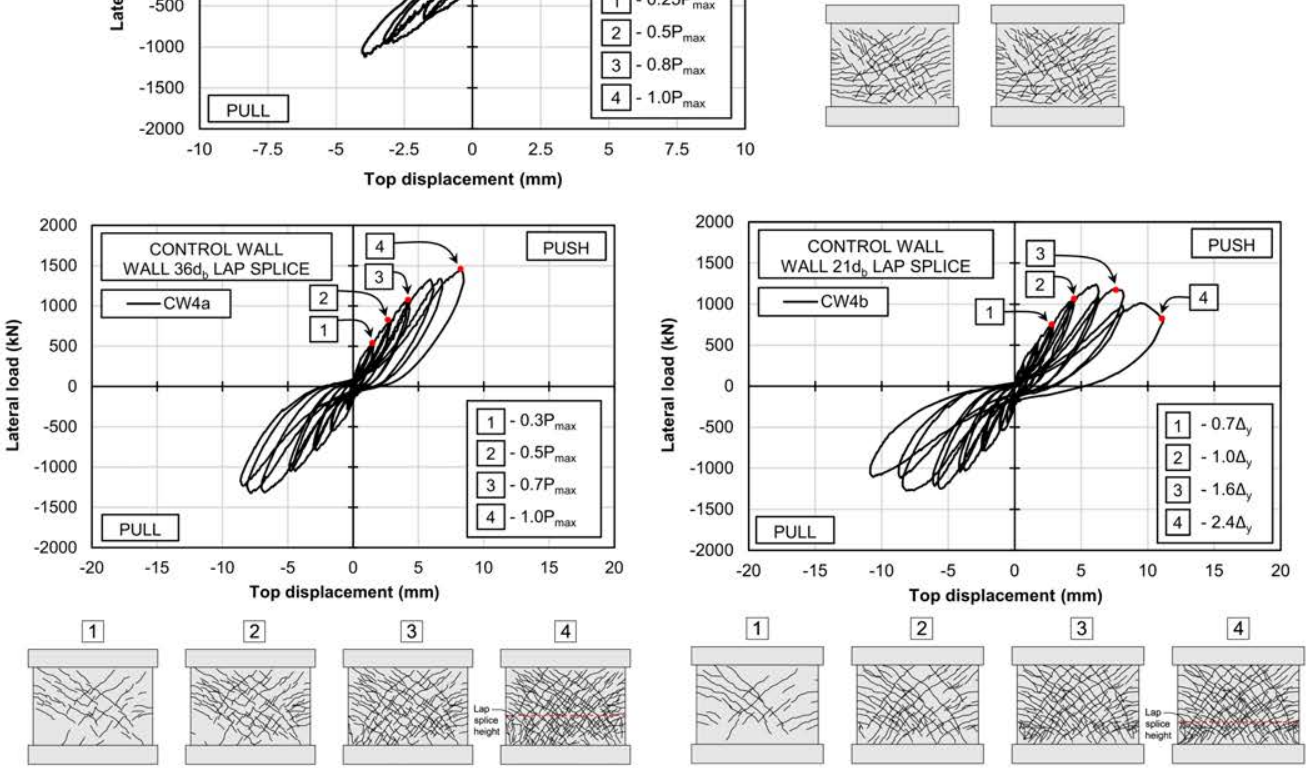

(d)

(e)

Figure 5.1 Hysteretic response behaviour and concrete crack distributions for control walls: (a) CW1; (b) CW2; (c) CW3; (d) CW4a (e) CW4b 


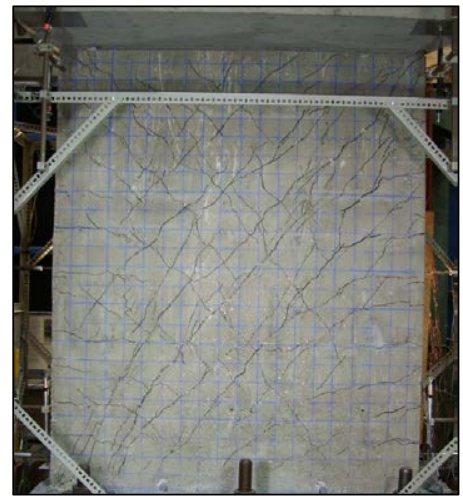

(a) CW1

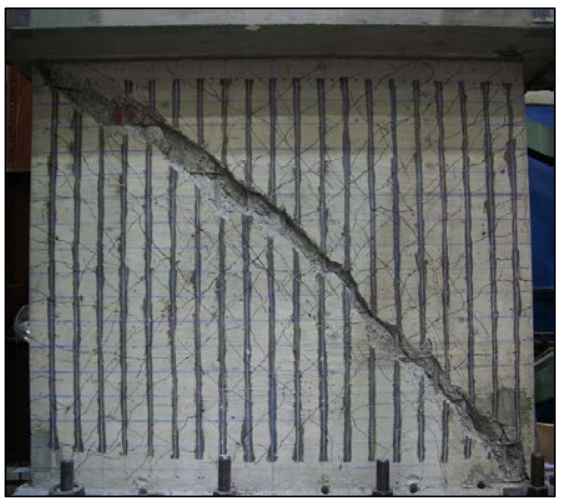

(b) CW2

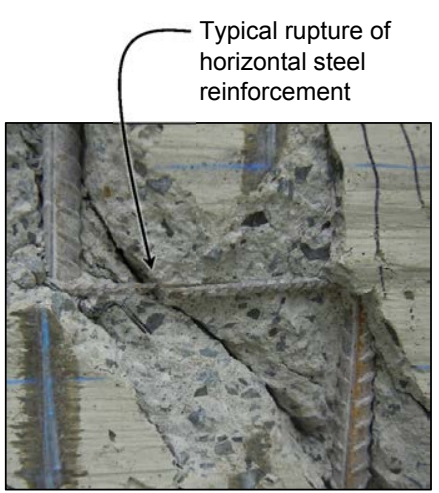
horizontal steel reinforcement

Figure 5.2 Damage to shear wall specimens CW1 and CW2
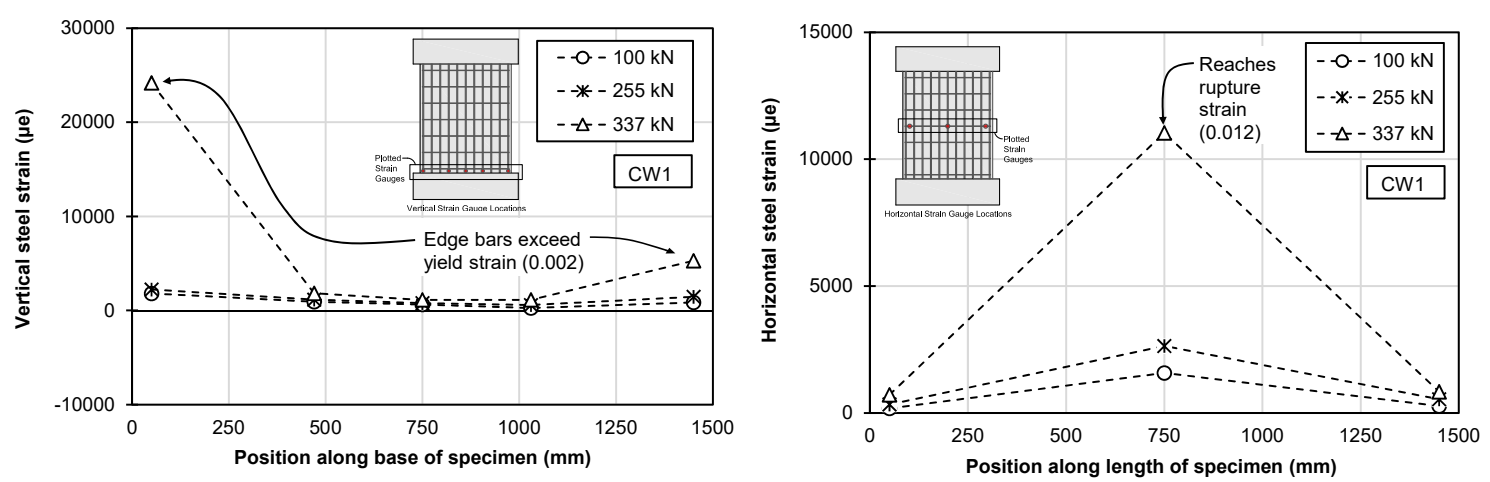

Figure 5.3 Wall specimen CW1 steel strain profiles: (a) vertical; (b) horizontal

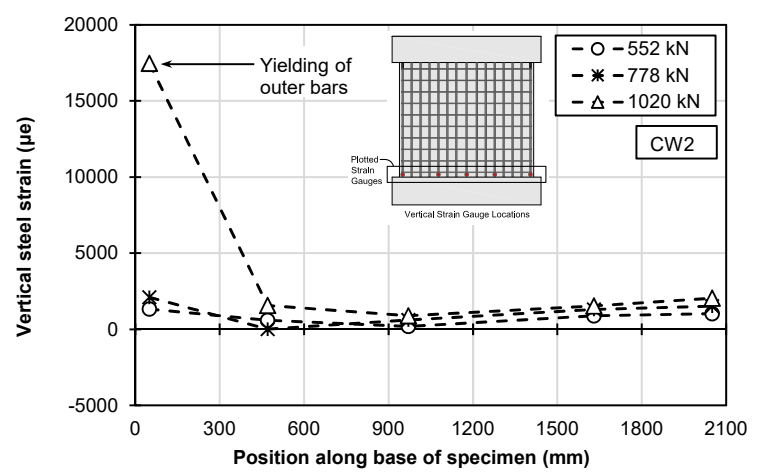

(a)

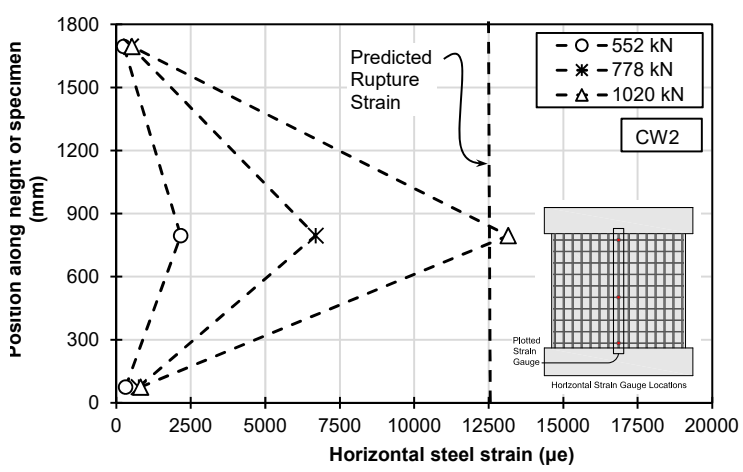

(b)

Figure 5.4 Wall specimen CW2 steel strain profiles: (a) vertical; (b) horizontal 
cracks in the concrete dilated and extended from one corner of the wall specimen to the other in an $\mathrm{x}$ configuration. Figure 5.1 shows the crack distribution at the end of the test. The concrete crack distribution shows extensive diagonal cracks in the concrete and the formation of a wide crack near the centre of the specimen. Analysis of the strain profiles in the horizontal steel reinforcement shown in Fig. 5.5 show that at the maximum load, the horizontal steel reinforcement yields in the centre of the wall specimen, at the location of the large observed crack, prior to yielding in the vertical steel reinforcement. Past tests conducted on wall specimens CW1 and CW2 showed that an increase in load after the horizontal steel reinforcement had yielded lead to significant losses in load carrying capacity (up to 40\%), leaving the wall with little to no residual shear capacity. Taking this into consideration, testing of specimen CW3 was stopped at a lateral drift of $0.27 \%$ to ensure that a more practical repair scenario could be achieved in comparison with wall specimens CW1 and CW2, however, it was later determined that it was the lack of ductility in the $6 \mathrm{M}$ horizontal steel reinforcement used in the construction of specimens CW1 and CW2 which contributed to their extremely brittle failure, such that testing of specimen CW3 could have continued past first yielding in the horizontal steel reinforcement. Nonetheless, the hysteretic response for specimen $\mathrm{CW} 3$ (Fig. 5.1) shows the minimal ductility and energy dissipation capacity comparable to specimens CW1 and CW2 exhibited by wall specimen CW3 because the flexural steel reinforcement did not yield prior to stopping the test.

Specimen CW4a, which had a $36 d_{b}$ lap splice length $(720 \mathrm{~mm})$, showed very similar behaviour in terms of deformation mechanisms and concrete crack distributions in comparison

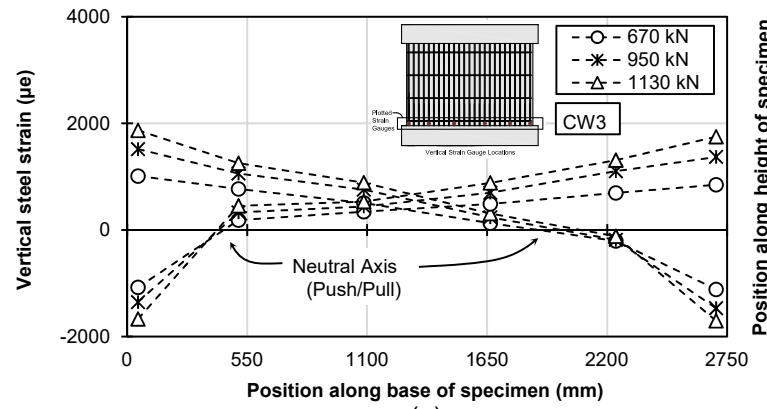

(a)

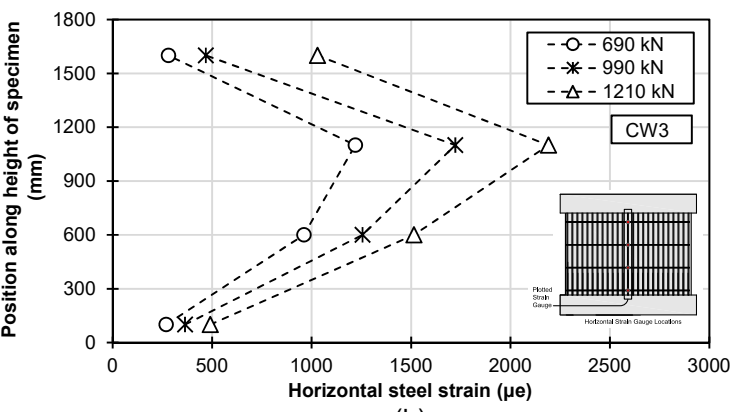

(b)

Figure 5.5 Wall specimen CW3 steel strain profiles: (a) vertical; (b) horizontal 
with specimen CW3 with the exception that specimen CW4a had very few horizontally oriented cracks in the concrete during the test when compared with wall specimen CW3. Horizontal cracks are commonly associated with flexural behaviour and thus are an indication that flexural behavior is not as predominant in specimen CW4a because of the lapped flexural steel reinforcement. As shown in Fig. 5.6, the first vertical splitting crack along the length of the lap splice was observed at a load of $1040 \mathrm{kN}$ and lateral drift of $0.25 \%$. Figure 5.7 shows the strain profiles along the length of the top and bottom lapped bars at the edge of the wall specimen. The results show that early on during the test, strain values are comparable in the top and bottom bars, and that strains between the lapped bars are predominantly transferred close to the top of the top lapped bars, and then the strains are more evenly distributed in the bottom lapped bars. It is also noted that at lateral load levels of $1340 \mathrm{kN}$ the strain in the lapped bars drops in comparison with the previous load cycle, suggesting slippage between lapped bars. This behaviour was confirmed by visual observation of widening vertical splitting cracks at the ends of the wall during the test.

Figure 5.8 shows the strain distributions in the horizontal steel reinforcement along the height and length of wall specimen CW4a. In contrast to specimen CW3, testing was continued past the point of first yielding of the horizontal steel reinforcement in an attempt to achieve yielding in the flexural steel reinforcement, however, this was not achieved because of slippage between lapped bars at the ends of the wall, shown in Fig. 5.6, which prevented the vertical steel reinforcement from yielding. After reaching a maximum load of $1340 \mathrm{kN}$, a wide diagonal crack formed from one corner of the specimen to the other at $0.5 \%$ lateral drift (Fig. 5.6). Upon observation of this wide diagonal crack, the test was stopped to prevent brittle diagonal tension shear failure from causing irrecoverable damage to the wall specimen. The hysteretic response of specimen CW4a (Fig. 5.1) shows higher deformability compared to specimen CW3 because of the additional test cycles past the point of first yielding in the horizontal steel reinforcement and the additional energy dissipated from slippage between the lapped bars.

The observed performance of specimen CW4a was also verified through post-processing of the high-definition images taken during the test. The images were processed using the freely 


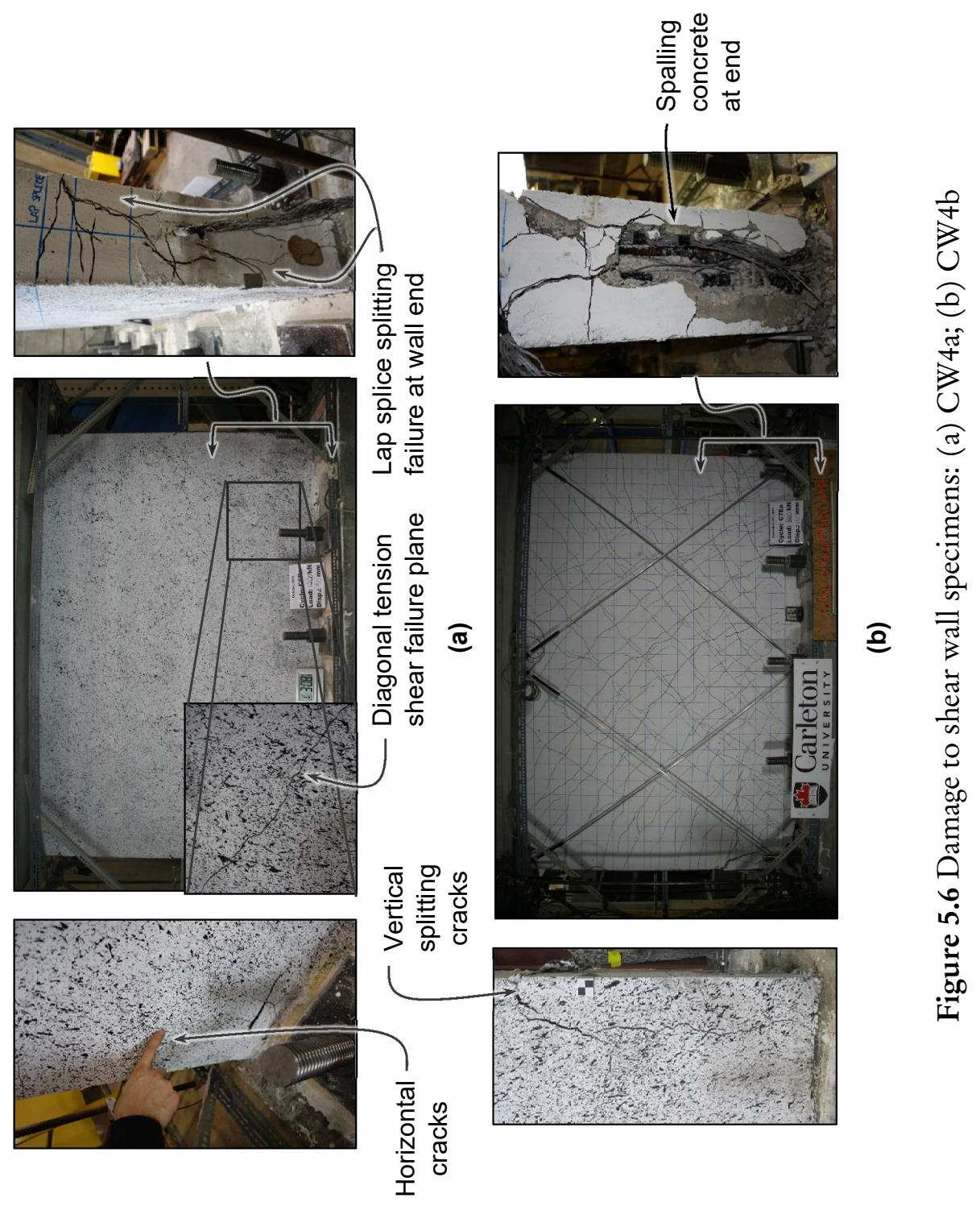




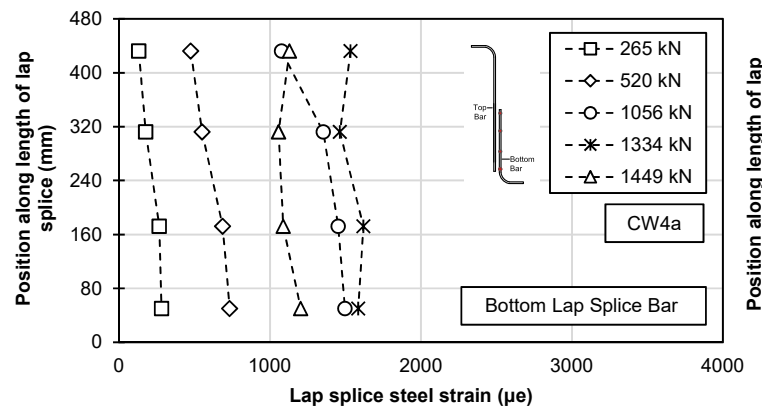

(a)

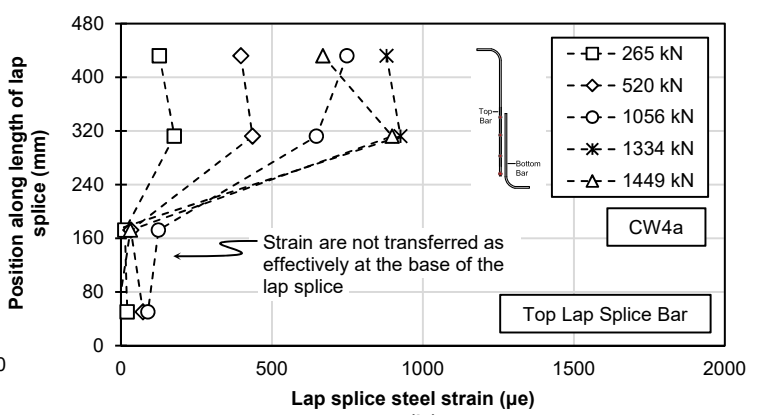

(b)

Figure 5.7 Wall specimen CW4a lap splice strain: (a) bottom lapped bar; (b) top lapped bar

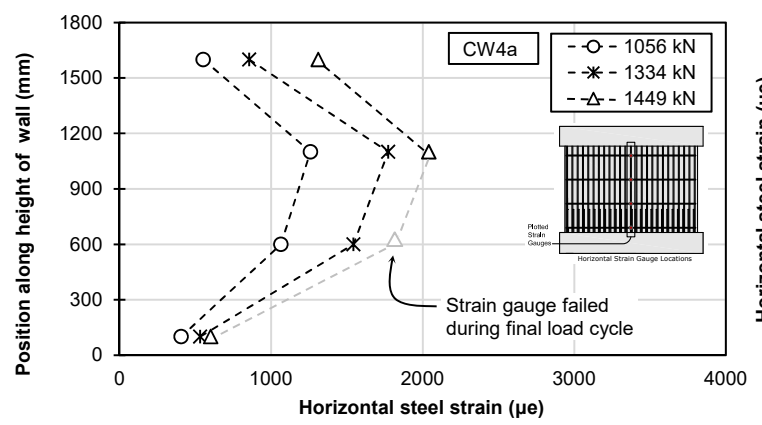

(a)

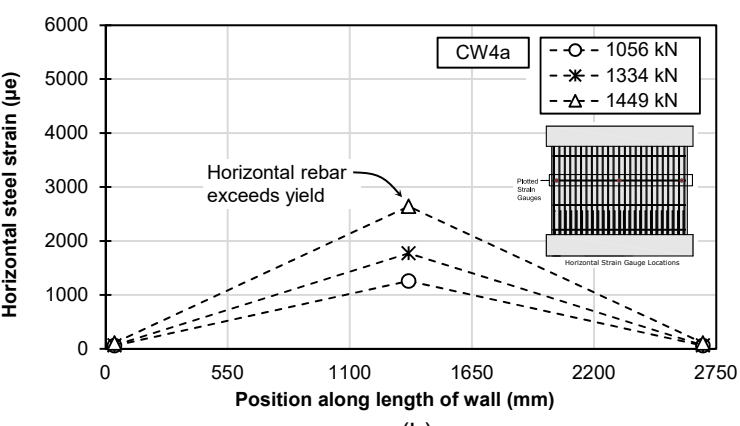

(b)

Figure 5.8 Specimen CW4a horizontal steel strain distributions: (a) vertical; (b) horizontal 
available software tool ImPro Streo. Results from the digital image correlation (DIC) software provides information related to the crack distributions and displacement and strain fields for each load stage throughout the test. Figure 5.9 shows the DIC results for the crack distribution in the concrete for selected cycle peaks. The shade of the crack in the DIC results represents the relative width of the crack opening. The results clearly show the significant presence of diagonal cracks in the concrete throughout the test. The results also show that the maximum diagonal crack width during the final load cycle in the pull direction is approximately $1.8 \mathrm{~mm}$. These results demonstrate the effectiveness of using ImPro Stereo to track concrete crack distributions throughout a test, without the need to manually mark the cracks using a pen or stop the test.

In contrast with wall specimen CW4a, specimen CW4b, which had a $21 d_{b}$ lap splice length $(420 \mathrm{~mm})$, sustained significant damage to the lap splice region during the test, including wide splitting cracks along the length of the lap splices near the ends of the shear wall. These cracks developed in the concrete before the flexural reinforcement yielded and before the formation of a wide diagonal shear crack. Figure 5.6 shows the damage sustained by wall specimen CW4b during the test. A horizontal crack at the top of the lap splice was first observed at a lateral load of $500 \mathrm{kN}$ and a lateral drift of $0.1 \%$. Vertical splitting cracks along the length of the lap splice at the ends of the wall were not observed until the specimen reached $0.23 \%$ lateral drift and a lateral load of $1000 \mathrm{kN}$. At $0.56 \%$ lateral drift, relative slipping occurred in the lapped bars at the two ends of the wall which lead to the formation of a horizontal shear plane at the top of the lap splice, $420 \mathrm{~mm}$

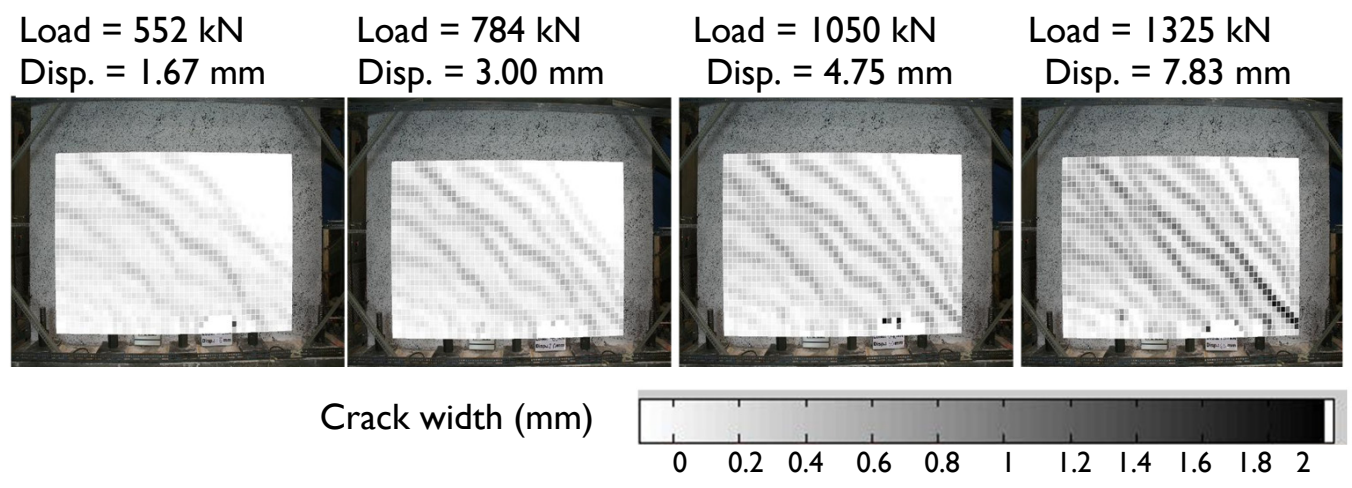

Figure 5.9 Concrete Crack distributions for wall specimen $\mathrm{CW} 4 \mathrm{a}$ at select peak cycles Cycles: C2Ra, C3Rb, C4Rb, C5Rb 
from the base of the specimen, at which point the test was stopped to prevent excessive damage to the lap splice region.

Figure 5.10 shows the vertical strain in the lapped steel bars at the ends of the shear wall at increasing levels of lateral load. In general, the results show that the maximum vertical strain in the lapped bars were much lower in comparison with wall specimen CW4b. The top and bottom bars have comparable strain distributions, indicating that the lap splice is still able to develop some bond stress, however, the vertical steel reinforcement was not able to sustain significant tensile strains, suggesting that the provided lap splice length is not sufficient to transfer compressive forces between lapped bars. At the maximum load, a significant drop in vertical strain shown in (Fig. 5.10) corresponds to the formation of the horizontal shear plane at the top of the lap splice region. Figure 5.11 shows the strain profiles in the horizontal steel reinforcement layers at the top and bottom of the wall specimen, respectively. Results show that at the maximum load, the strain in the top layer of horizontal steel reinforcement exceeded the yield strain in the centre of the wall. Comparatively, the strain values were lower in the bottom layer of horizontal steel reinforcement at the maximum load, below the horizontal shear plane which formed along the top of the lap splice.

Figure 5.12 shows the crack distribution results for wall specimen CW4a from the ImPro Stereo DIC software. The results show that in a similar manner to specimen CW4a, there were a significant number of diagonal cracks in the concrete, however, the formation of horizontal cracks

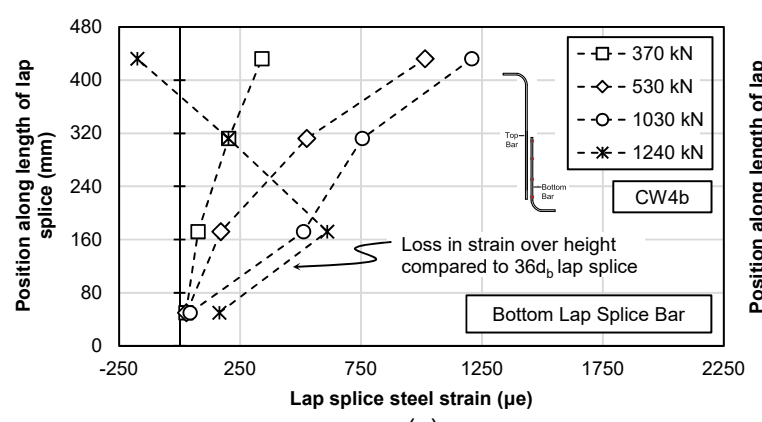

(a)

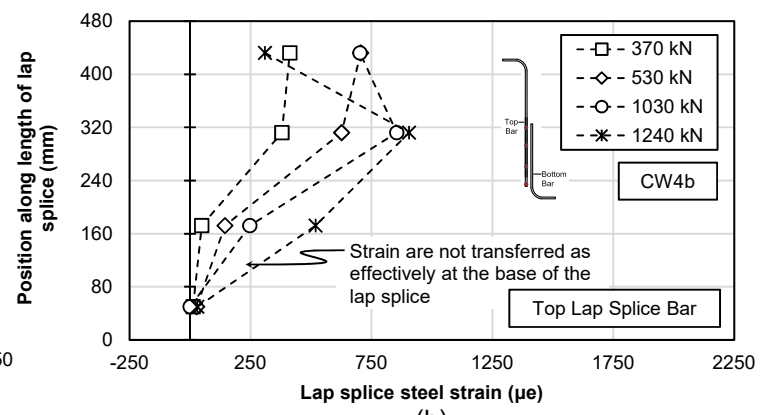

(b)

Figure 5.10 Specimen CW4b lap splice strain: (a) bottom lapped bar; (b) top lapped bar 


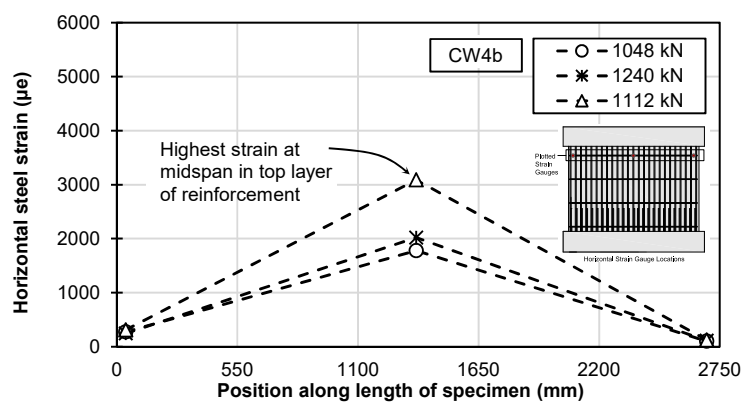

(a)

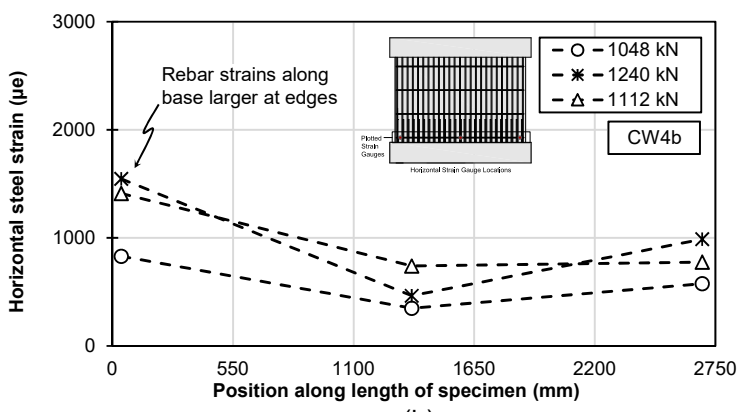

(b)

Figure 5.11 Wall specimen CW4b horizontal steel strain distributions: (a) top; (b) bottom

at the height of the lap splice are also shown to form early on during the test. Figure 5.13 shows the horizontal cracks at the edges of the wall by extracting the first subplot from Fig. 5.12 and narrowing the range of the colour-map to make the cracks clear. These cracks are an indication of the degradation of the interlocking bond between lapped bars, which occurs significantly earlier in specimen $\mathrm{CW} 4 \mathrm{~b}$ than those observed in specimen $\mathrm{CW} 4 \mathrm{a}$. This confirms the observed results that the $21 d_{b}$ lap splice length not sufficient to effectively transfer the forces between the lapped bars.

\subsection{Repaired Wall with FRP Sheets}

Following the initial tests, the control wall specimens were repaired using a combination of epoxy resin, patching mortar, and CFRP composite sheets. Figure 5.14 shows the repair sequence for a typical wall specimen in this study. First, loose concrete was removed and significant cracks

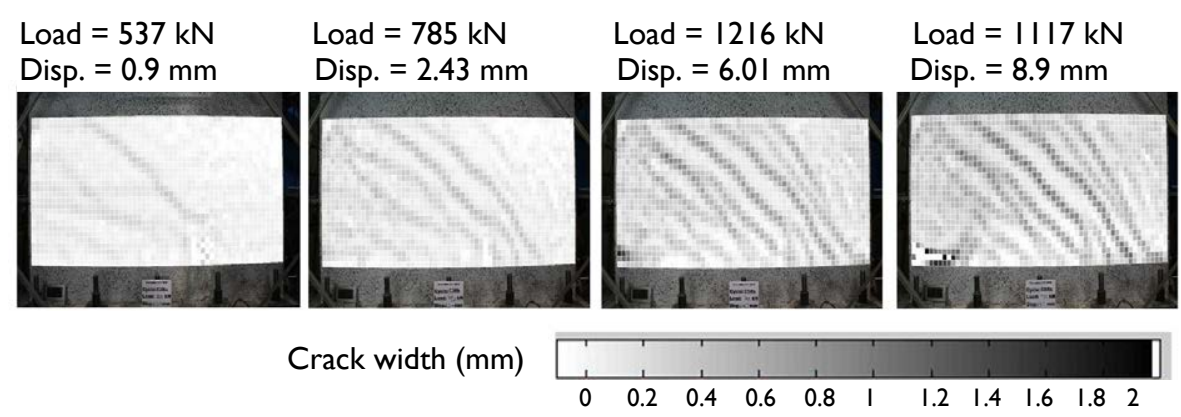

Figure 5.12 Concrete crack distributions for specimen CW4b at select peak cycles 


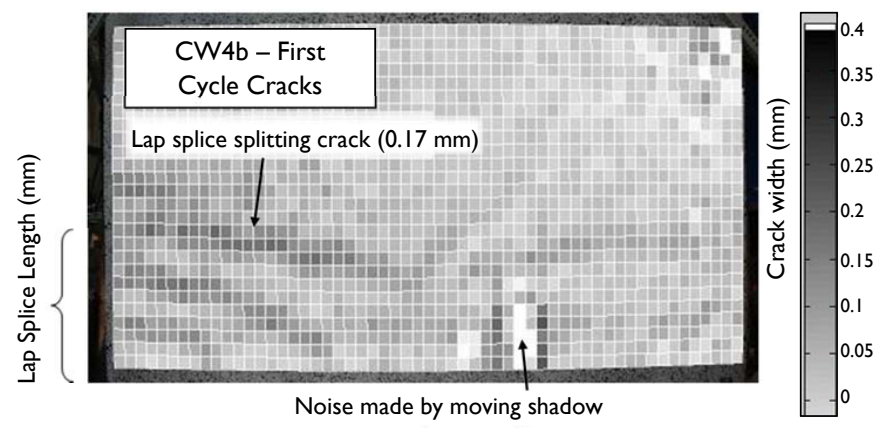

Figure 5.13 Horizontal cracking up to lap splice height in specimen CW4b

$(0.25 \mathrm{~mm}-5 \mathrm{~mm})$ were injected with a low viscosity epoxy. A thicker epoxy was then used to seal any remaining larger cracks. Finally, a thin coat of patching mortar was applied over the surface of the wall specimen to smooth the concrete surface to allow for the application of the CFRP sheets. In contrast to previous studies (Antoniades et al., 2003; Paterson and Mitchell, 2003; Ghobarah and Khalil, 2004; Elnady, 2008), the CFRP sheets are not wrapped around the wall specimens to simulate the scenario in the field when the sides of a RC shear wall may not be accessible. This is an attempt to make the FRP retrofitting system more practical and less disruptive when compared to wrapping the CFRP sheets around the wall, while still improving the seismic performance of the shear wall. The only exception to this is in the CFRP retrofitting scheme for specimen CW4b, which has additional confining CFRP strips wrapped around the wall specimen, $100 \mathrm{~mm}$ in width spaced at $200 \mathrm{~mm}$ on centre over the height of the lap splice (Fig. 3.3).

Structural response parameters for the repaired wall specimens are shown in Table 5.2 and Fig. 5.15 shows the hysteretic response behaviour of the rehabilitated walls and their respective CFRP debonding progressions.

In wall specimen RW1, the test results showed that performance of the wall specimen could not be significantly improved because of the damage sustained by the specimens during the initial test, however, the retrofitting system was able to restore the specimens to its original state, with some minimal improvements in ductility and energy dissipation capacity. The restoration of the initial stiffness in specimen RW1 is attributed to the rehabilitation of the diagonal cracks 


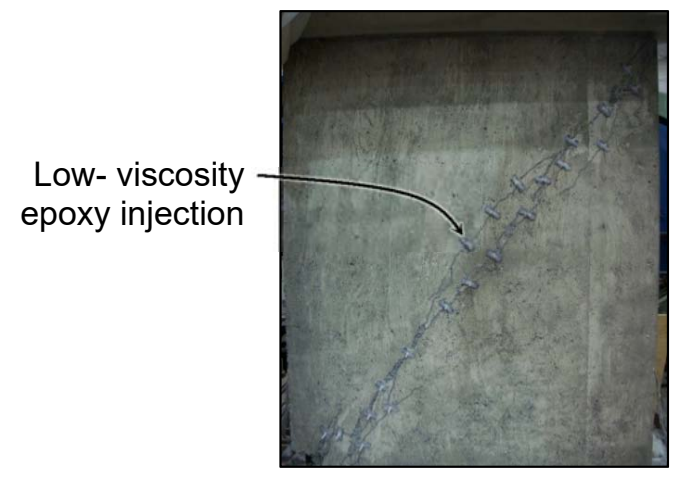

(a)

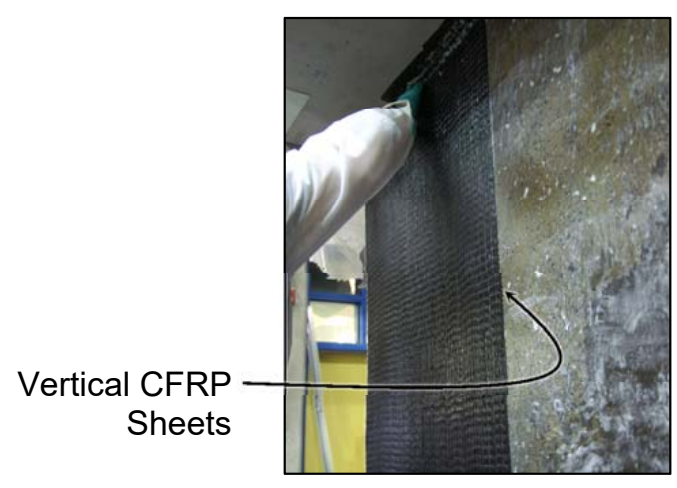

(c)

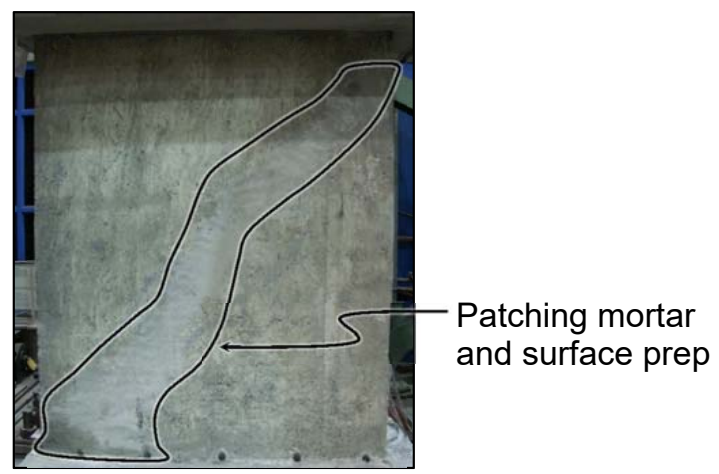

(b)

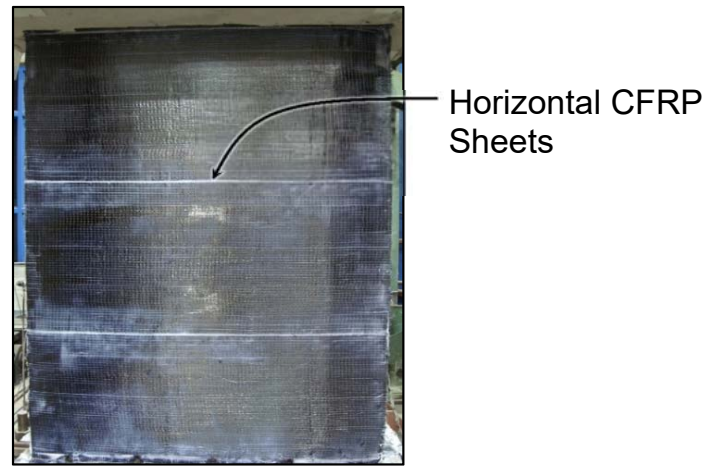

(d)

Figure 5.14 Repair procedure: (a) crack epoxy injection; (b) surface preparation; (c) vertical CFRP layers; (d) horizontal CFRP layers 


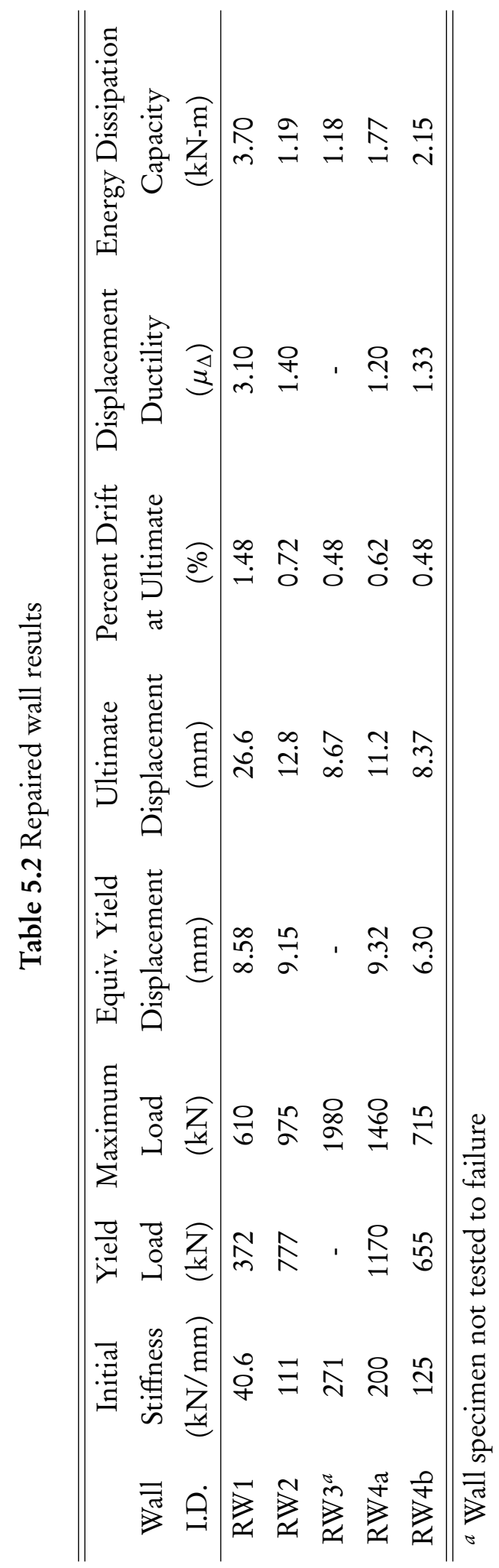


(a)
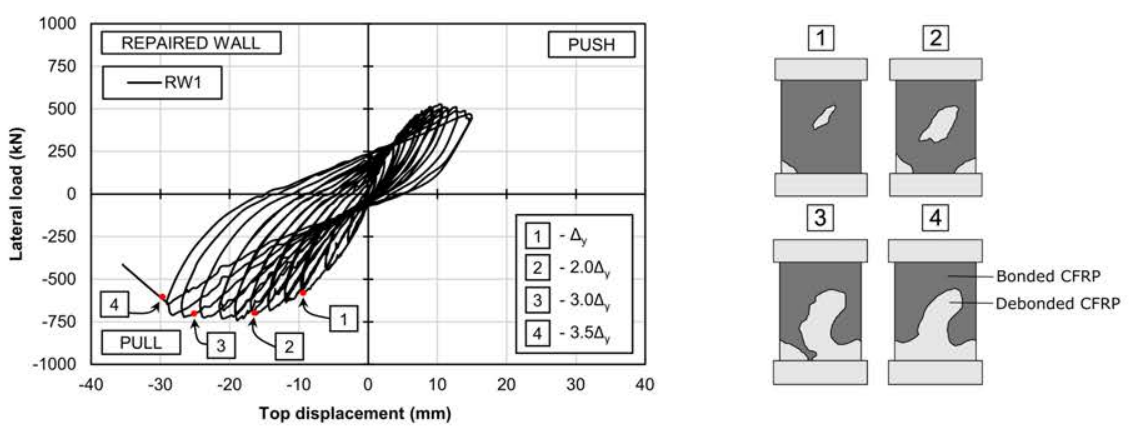

(b)
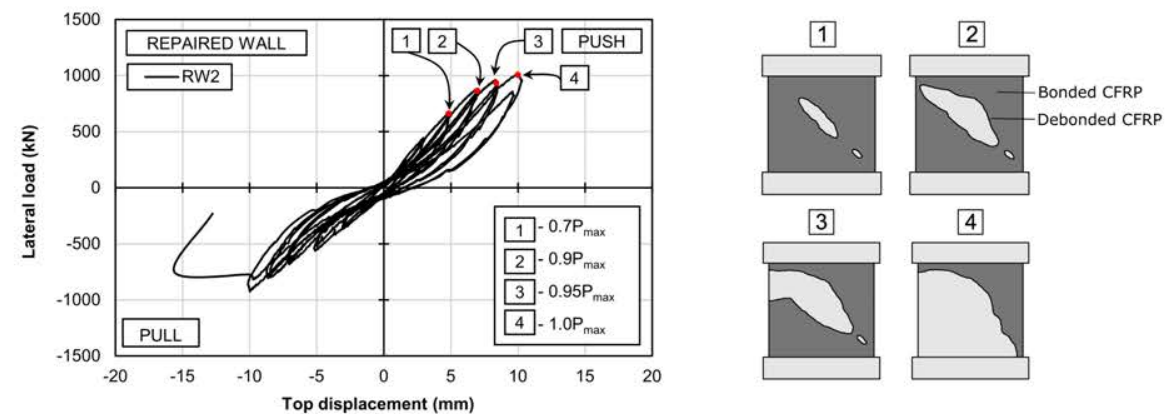
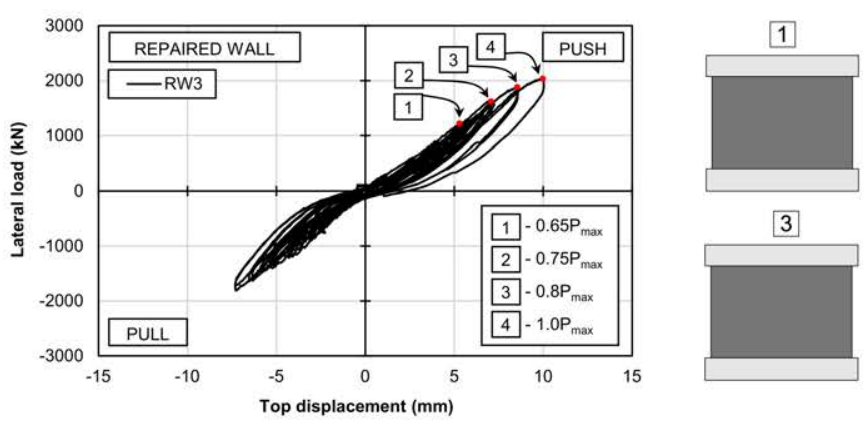

3
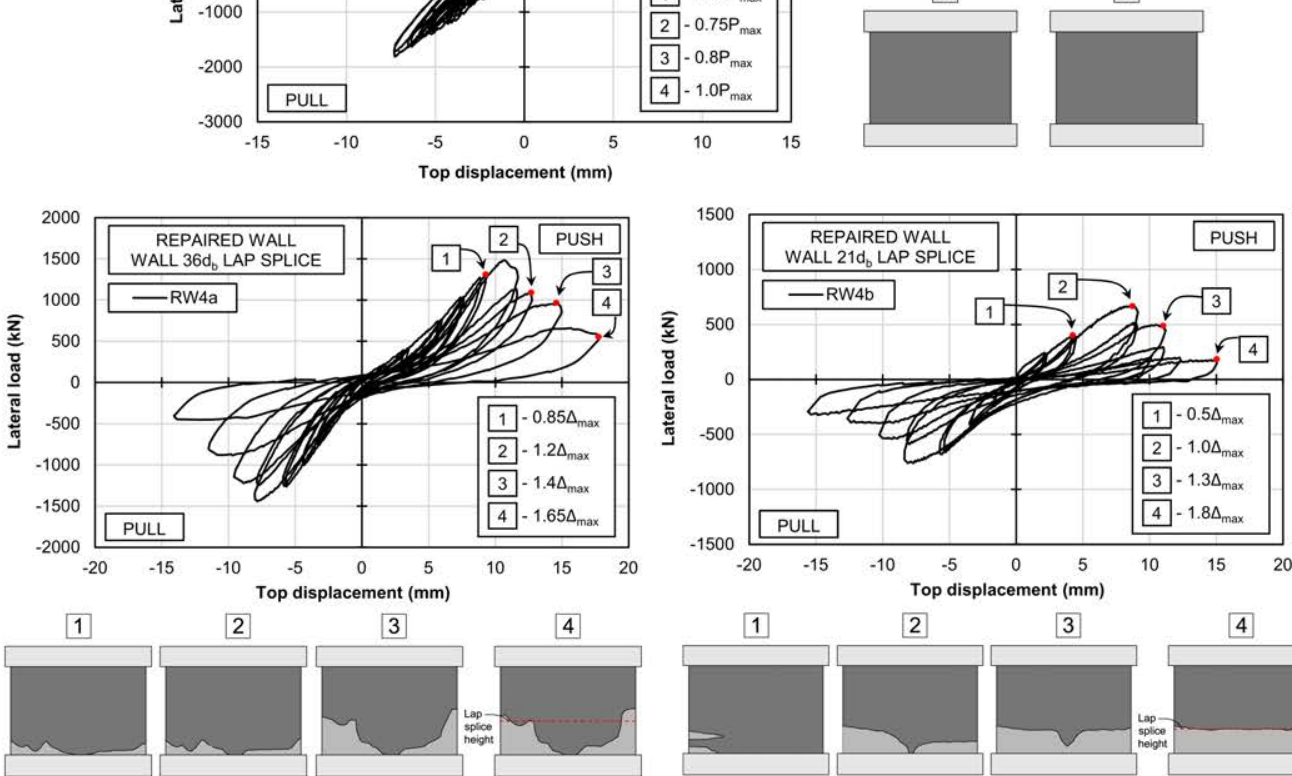

(d)

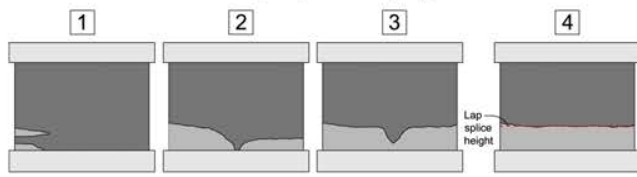

(e)

Figure 5.15 Hysteretic response and FRP-concrete debonding patterns for repaired walls: (a) RW1; (b) RW2; (c) RW3; (d) RW4a (e) RW4b 
formed during the initial test and the application of the vertical CFRP sheets. Debonding of the CFRP laminate in wall specimen RW1 first occurs along the diagonal of the specimen, around the previously formed diagonal failure plane at very low drift ratios ranging from $0.2 \%$ to $0.38 \%$. At higher levels of lateral drift $(0.57 \%-0.88 \%)$, debonding initiates at the toes of specimen RW1. Debonding in these regions occurs because of concrete crushing beneath the CFRP laminate, which spreads towards the center of the wall specimens under multiple load reversals. Figure 5.16 shows the hysteretic response of the vertical steel reinforcement in specimen RW1 at the edges of the wall specimen. The results show that the additional shear strength provided by the horizontal CFRP layers allows significant yielding of the vertical steel reinforcement at the base of the wall prior to shear failure. Significant yielding of the vertical steel reinforcement results in a flexural ductile failure of wall specimen RW1. A lack of confinement in the boundary elements of specimen RW1 after significant crusing in the toes of the wall specimen in combination with the high compressive forces required in resisting the overturning moment of the applied lateral load resulted in buckling of the vertical steel reinforcement, which ultimately lead to a large drop in load carrying capacity at a lateral drift of $1.48 \%$. The asymmetric hysteretic response of specimen RW1 is attributed to the significant amount of damage the wall sustained in one direction (push) compared to the other (pull). Nonetheless, the hysteretic behaviour of specimen RW1 shows an improvement in the displacement ductility, which was 3.1 compared with 1.2 in its original state (CW1) and energy dissipation capacity of the wall.
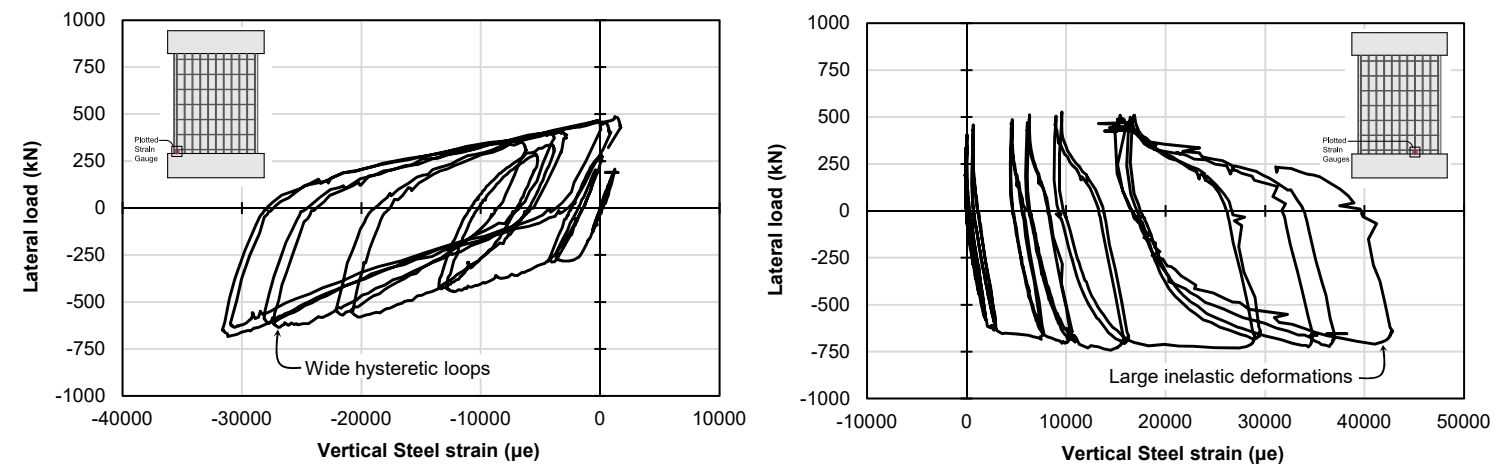

Figure 5.16 Specimen RW1 flexural steel reinforcement hysteretic response 
In contrast with wall specimen RW1, failure of wall specimen RW2 occurs as a result of significant CFRP debonding around the diagonal crack formed during the initial test. Ultimately, the CFRP sheets were unable to prevent the diagonal failure plane formed during the original test from reopening, resulting in failure of specimen RW 2 at $0.72 \%$ drift. The retrofitting system is able to restore the initial stiffness and lateral load carrying capacity of specimen CW 2 to $96 \%$ of its original state, despite the severe damage sustained by the specimen during the initial test. Asymmetry in the hysteretic behaviour of specimen RW2 is not observed because the wall failed before the displacement controlled portion of the loading protocol. Although a significant improvement in the seismic performance could not be achieved in specimen RW2, the CFRP retrofitting system is able to restore the specimen close to its original state with very minor improvements in ductility and energy dissipation capacity. In contrast with wall specimen RW1, wall specimen RW2 exhibits some concrete crushing in the toes of the specimen at its ultimate load, but has comparatively less yielding in the vertical steel reinforcement, resulting in a slightly higher displacement ductility of 1.41 for specimen RW2 compared with 1.25 for specimen CW2.

Figure 5.17 shows the strain profiles in the vertical and horizontal CFRP layers at increasing levels of lateral load. Results show that the vertical strain profile in the CFRP sheet remains approximately linear at increasing levels of lateral load. The results show that the neutral axis is located at approximately $75 \%$ of the shear wall length, which is consistent with the assumptions made during the design of the tube anchor. Asymmetry in the horizontal CFRP strain distribution is attributed to CFRP debonding in these regions during the test. The maximum vertical and horizontal strain of $1900 \mu \epsilon$ and $1000 \mu \epsilon$, respectively, are significantly less than the limiting vertical and horizontal debonding strains of $7000 \mu \epsilon$ and $4000 \mu \epsilon$ recommended by CSA S806-02, respectively. This is attributed both to the pre-existing damage sustained by the wall specimen prior to testing and the shear dominant nature of shear walls with aspect ratios less than 2.0.

In specimen RW3, the application of the CFRP sheets is not able to completely restore the initial stiffness of the wall, but significant improvements in the lateral load carrying capacity and lateral drift capacity are observed. Specimen RW3 shows very little visible damage and no debonding or separation of the CFRP sheets from the concrete substrate at the maximum load 


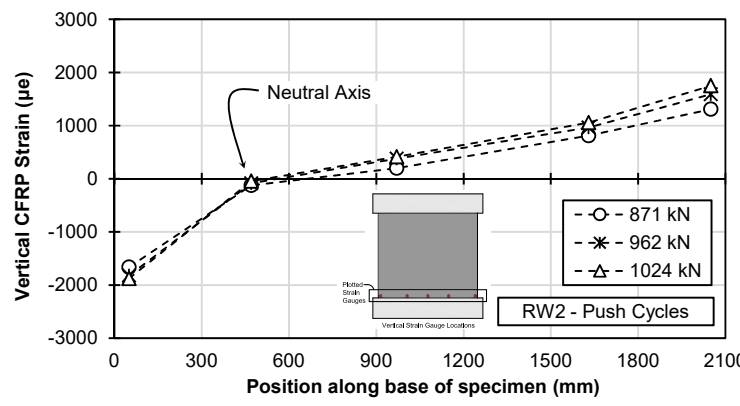

(a)

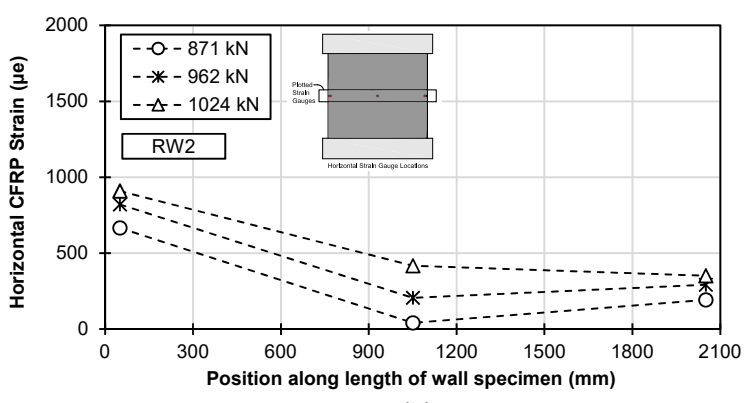

(b)

Figure 5.17 Specimen RW2 CFRP strain profiles: (a) vertical; (b) horizontal

carrying capacity. Unfortunately, reaching the ultimate load carrying capacity of specimen RW3 could not be achieved because of premature failure in the wall specimen foundation, which is responsible for transferring the load from the wall to the laboratory strong floor. Nonetheless, specimen RW3 exhibits significant strength increases of 170\% when compared with the control wall and did not show significant signs of distress following failure of the shear wall foundation.

Figure 5.18a shows the strain profile in the vertical steel reinforcement for specimen RW3 along the base of the shear wall at increasing levels of lateral load. The results show that at the maximum load, the first two layers of flexure steel reinforcement in specimen RW3 yield. In addition, the first layer of steel reinforcement in compression also yields at the opposite end of the wall. Figure 5.18b shows the strain profiles for the vertical strain in the vertical steel reinforcement at the base of the wall and the horizontal strain in the CFRP at mid-height. Results demonstrate that up to the maximum load carrying capacity, the strains in the vertical steel reinforcement are well above the steel yield strain at both ends of the shear wall while the strains in the horizontal CFRP reinforcement remain below $1000 \mu \epsilon$, well below the measured rupture strain of the CFRP material. These results suggest that the addition of the horizontal CFRP sheets prevented the horizontal steel reinforcement from yielding and prevented brittle diagonal tension shear failure, which was observed in tests on the control wall specimens. The test results demonstrate that in a squat wall with a very low aspect ratio $\left(h_{w} / l_{w}=0.65\right)$ without lap splices, the application of horizontal CFRP sheets is capable of improving the lateral load carrying capacity of the shear wall, eliminating the potential for brittle diagonal tension shear failure, and allowing the flexural 


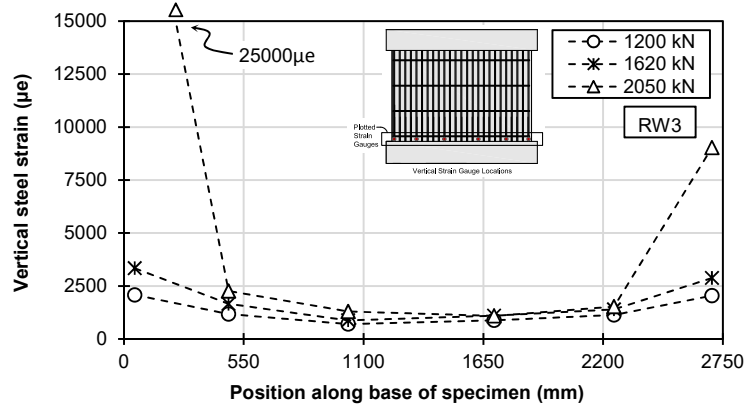

(a)

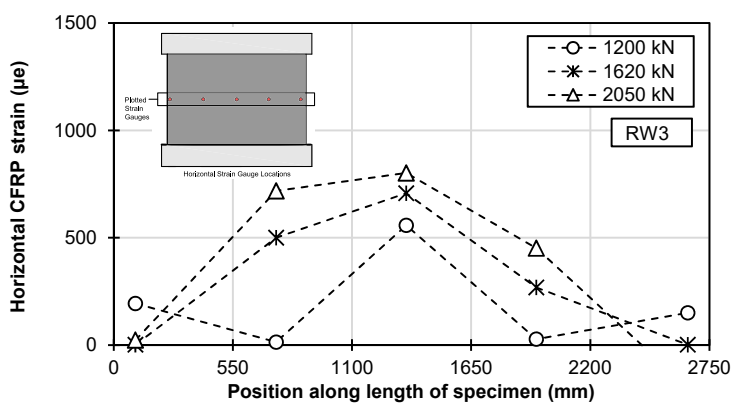

(b)

Figure 5.18 Specimen RW3 steel/CFRP strain profiles: (a) vertical steel; (b) horizontal CFRP

steel reinforcement at the two ends of the shear wall to yield.

There is a notable contrast in the performance of wall specimens RW4a and RW4b with lap splices compared to wall specimen RW3 without lap splices (Fig. 5.15). In wall specimen RW4a, which had a lap splice length of $36 d_{b}$, the application of the horizontal CFRP sheets is successful in restoring the in-plane load carrying capacity of the wall specimen, but is not able to completely restore the initial in-plane stiffness. Figure 5.19 shows the damage sustained by wall specimen RW4a during the test. As shown in Fig. 5.19, at an average load of $1020 \mathrm{kN}$, the vertical lap splice cracks formed during the previous test of wall specimen CW4a reopen. Specimen RW4a reaches a maximum average load carrying capacity of $1460 \mathrm{kN}$ at an average lateral drift of $0.51 \%$. At this stage during the test, splitting cracks along the length of the lap splices at the ends of the wall and a small sliding shear crack along the base of the wall are visible. Under subsequent load reversals, degradation of the strength and stiffness of the wall (Fig. 5.15) occurs as the lap splice failure results in a large gap at the base of the wall and a rocking motion as the wall slides back and forth on its foundation.

Figure 5.20 shows the vertical strain profiles at the maximum load in the top and bottom lap spliced bars along the length of the wall for specimen CW4a and RW4a, respectively. Comparing the strain profiles in specimen CW4a and RW4a, these results show that the application of the CFRP sheets is effective in restoring the performance of the lap splice. In addition, the strains on the compressive side are higher in specimen RW4a, indicating improved performance 


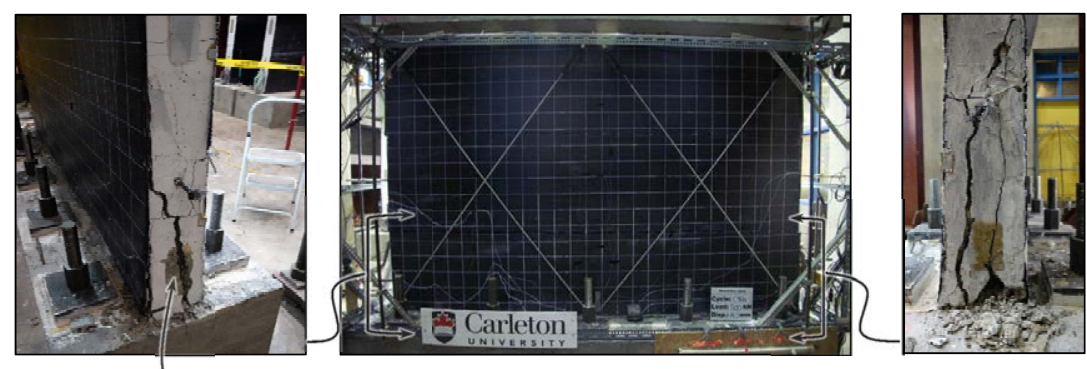

(a)

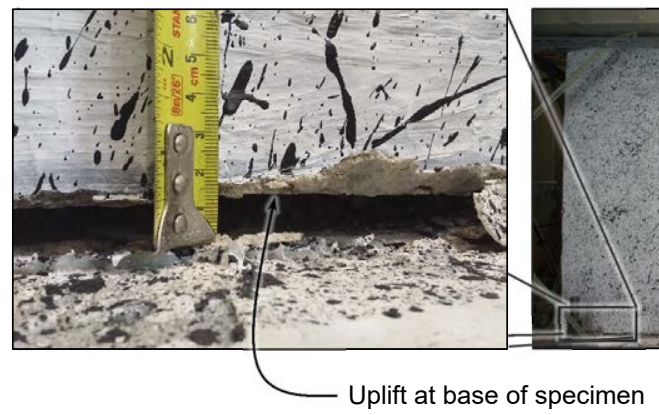

Figure 5.19 Damage to repaired walls with lap splices: (a) RW4a; (b) RW4b

due to the presence of the CFRP layers. In specimen RW4a, no debonding of the CFRP laminate from the concrete substrate is observed until the specimen enters the post-peak range. As shown in Fig. 5.15, the CFRP laminate separates from the concrete and debonds up to the top of the lap splice $(720 \mathrm{~mm}$ from the base of the wall) at the two ends of the wall. Even without providing additional concrete confinement in the boundary elements at the two ends of the wall, the repaired wall specimen has marginally higher deformability and improved energy dissipation capacity compared to the control wall.

The image analysis results for specimens RW4a represent the deformation of the surface of the CFRP sheet rather than the concrete surface of the wall. In this case, the cracks in the original $\mathrm{RC}$ wall are covered by the CFRP sheets and cannot be captured in the photos or analyzed directly using the DIC technique. As a result, no shear cracks are observed in the digital image correlation results, however, the image analysis technique is able to capture the two-dimensional spatial strain distribution in the CFRP sheet throughout each test. Figure 5.21 shows the horizontal shear strain field $\left(e_{x y}\right)$ for specimen RW4a at the peak of selected load cycles. These results can be 
compared with discrete measurement results from electrical strain gauges mounted on the surface of the CFRP sheets, shown in Fig. 5.22. Generally, results between the image analysis technique and conventional electrical strain gauges agree, and show that the horizontal strain on the surface of the CFRP sheet is generally the highest close to the centre of the wall, reaching a maximum horizontal strain of approximately $800 \mu \epsilon$ according to the image analysis results. Results also show a drop in horizontal strain at the height of the lap splice on the right side of the specimen after the wall reaches its ultimate load. In addition, two horizontal cracks at the top of the lap splice $(720 \mathrm{~mm}$ from the base of the wall) are captured in the image analysis results. The horizontal crack width can be determined using the $e_{y y}$ (vertical) strain field estimation.

Figure 5.23a shows the $e_{y y}$ strain field for the final load cycle in the push direction, and shows the wide horizontal crack at the right side of the wall. The crack is estimated to be $0.5 \mathrm{~mm}$ wide using the vertical displacement field $\left(u_{y y}\right)$. This crack is equivalent to the width of one half of a pixel (as 1 pixel $=0.955 \mathrm{~mm}$ in the image analysis results). Figure $5.23 \mathrm{~b}$ shows a similar horizontal crack at the left side of the wall which forms during the final load cycle in the opposite loading direction.

In contrast with specimen RW4a, the capacity of specimen RW4b which had a shorter lap splice length of $21 d_{b}$, could not be restored to its original state as a result of the insufficient lap splice length and the level of damage sustained by the wall during the initial test. Even with the application of the additional CFRP strips wrapped around the wall over the lap splice length, it

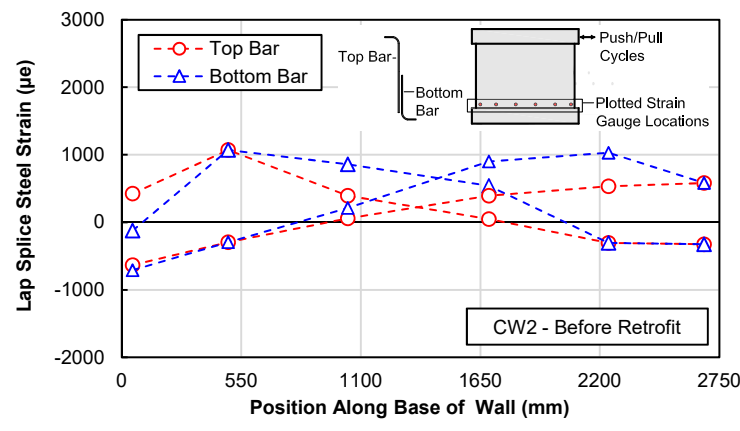

(a)

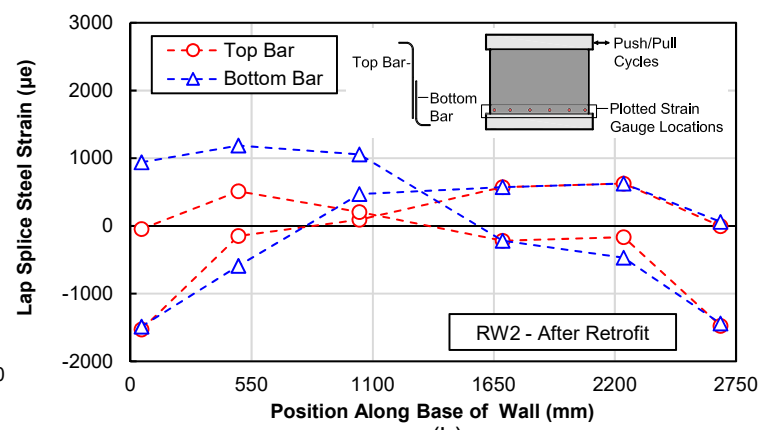

(b)

Figure 5.20 Comparison of vertical strain in top and bottom lapped bars: (a) CW4a; (b) RW4a 


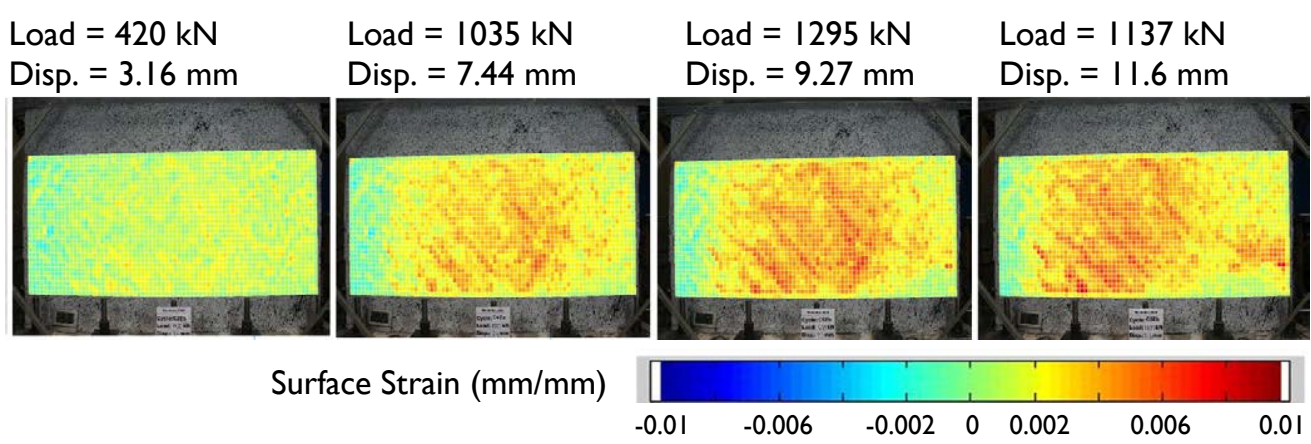

Figure 5.21 CFRP surface strain fields for specimen RW4a at select peak cycles

was not enough to prevent the lap splice from reopening. The initial stiffness of the wall specimen Cycles: C2Eb, C4Ea, C5Eb, C6Eb was $22 \%$ of the original stiffness of specimen $\mathrm{CW} 4 \mathrm{~b}$, once again because no effort was made to restore the damaged bond between lapped bars following the initial test. As illustrated in Fig. 5.19, a horizontal shear plane forms at the top of the lap splice, $420 \mathrm{~mm}$ from the base of the specimen between the CFRP confining strips. The CFRP sheets have no strength perpendicular to the primary fiber direction, so it is unable to prevent the concrete from splitting at the height of the lap splice. As shown in Fig. 5.19, which is similar to what was observed during tests on specimen RW4a, an approximately $15 \mathrm{~mm}$ wide gap forms at the base of the wall between the wall panel and the foundation block. During the post-peak cycles, this gap opens and closes as the wall rocks back-and-forth causing significant strength and stiffness degradation that are evident in the hysteretic response (Fig. 5.15). The specimen reaches a maximum load carrying capacity of $715 \mathrm{kN}$ and an ultimate lateral drift of $0.6 \%$. The maximum load carrying capacity is $60 \%$ of the maximum capacity of specimen CW4b. Experimental results from specimen RW4b with

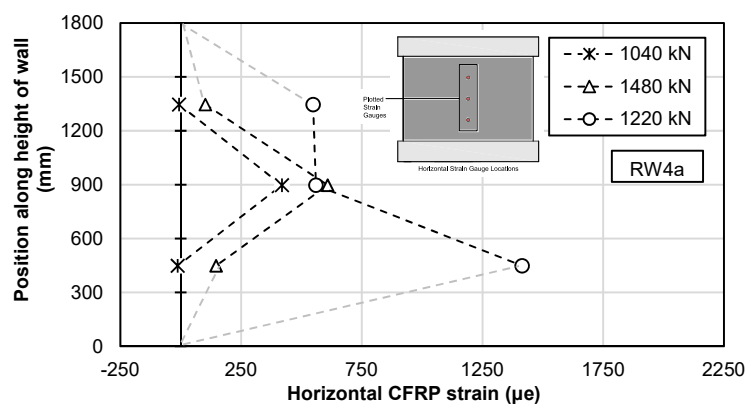

(a)

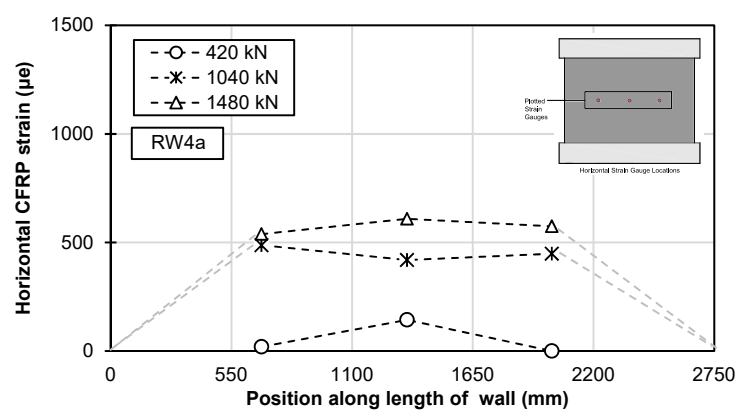

(b)

Figure 5.22 Specimen RW4a horizontal CFRP strain profiles 
insufficient lap splice length show that more intrusive measures, such as welding of lapped bars, or some means to provide adequate confinement to the lap splice region are required to restore the performance of the wall specimen. To add confining pressure to the lap splice, a complete CFRP wrap over the height of the lap splice or the addition of headed bars and a concrete collar could improve the residual capacity of the lap splice.

Figure 5.24 shows the shear strain field for specimen RW4b at selected peak cycles. Results show that in a similar manner to specimen RW4a, a wide horizontal crack forms at the height of the lap splice ( $420 \mathrm{~mm}$ from the base of the wall for specimen RW4b). This crack is clearly shown in the shear strain field $\left(e_{x x}\right)$ because there is a relative horizontal slip across the two sides of the crack. Results also show that this slippage occurs in one direction (red line) during the push cycles and subsequently in the other during the pull cycles as specimen RW4b rocked back and forth at the height of the shear plane, which coincides with the top of the lap splice. Figure 5.25 shows displacement field for the final load cycle, which clearly identifies the location of the shear plane and the large discrepancy in displacement on the two sides of the crack. The results show that the majority of the displacement occurs above the shear plane, which is determined to open to a maximum width of approximately $11.4 \mathrm{~mm}$. The relative horizontal slip on either side of the crack can also be estimated using the displacement field results and reaches a maximum of $3.3 \mathrm{~mm}$ during the final load cycle.

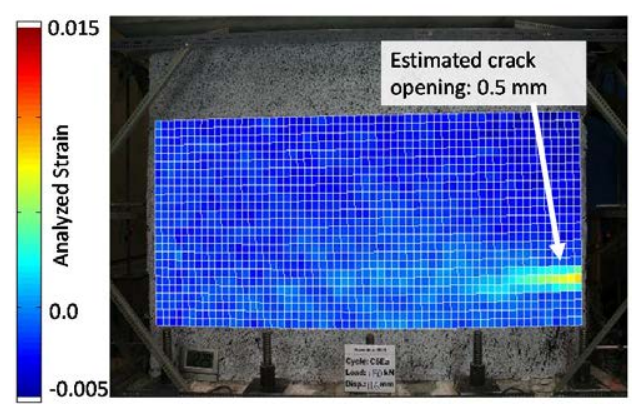

(a)

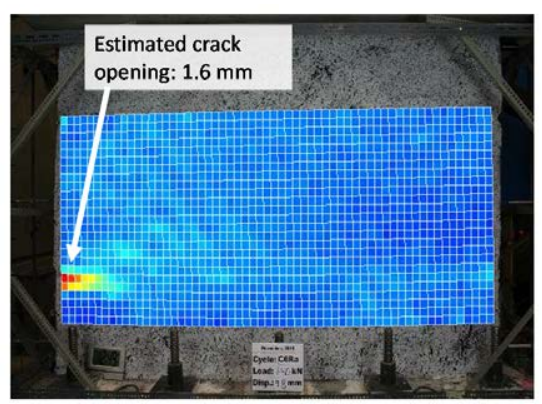

(b)

Figure 5.23 Observation of horizontal cracks above lap splice for RW4a 


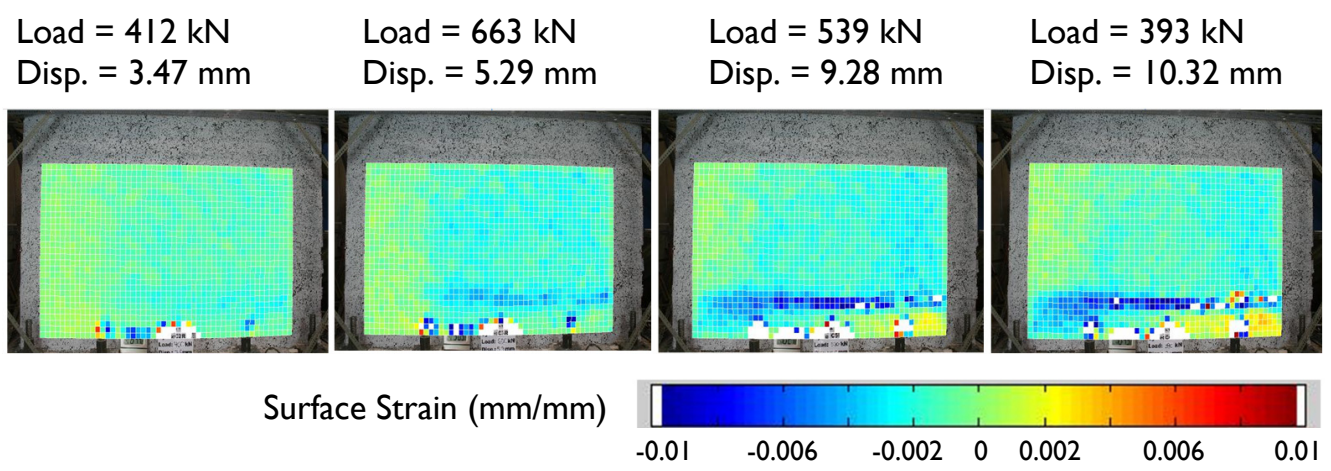

Cycles: C2Rb, C3Ra, C5RafFifRere 5.24 CFRP surface strain fields for specimen CW4b at select peak cycles

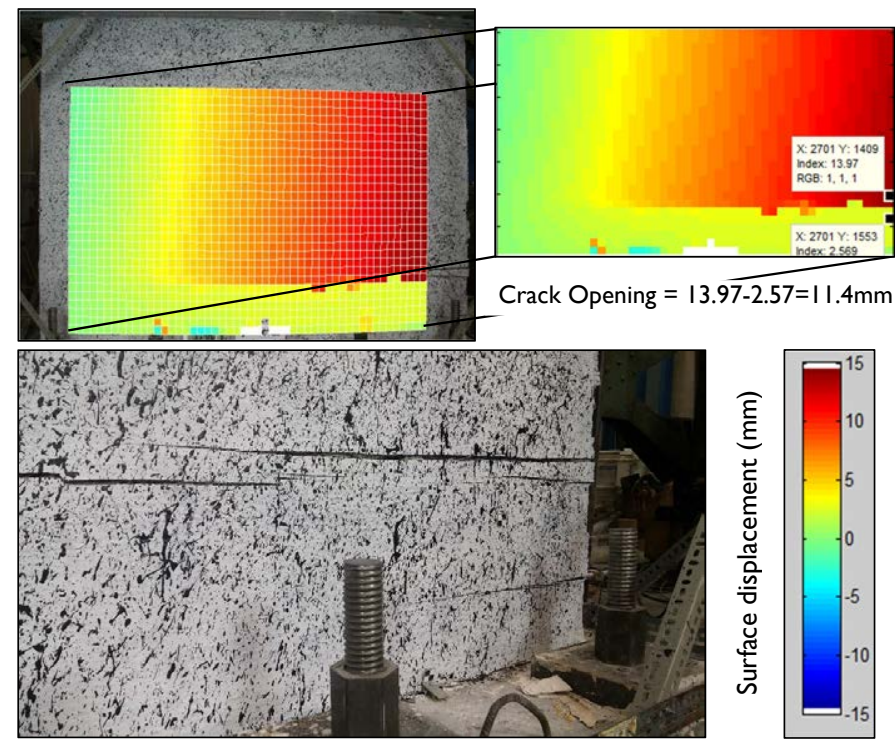

Figure 5.25 Relative displacement analysis at lap splice height for RW4b 


\subsection{Strengthened Walls with FRP Sheets}

The CFRP sheets are applied to the strengthened specimens in the same manner as the repaired wall specimens. Structural response parameters for the strengthened wall specimens are shown in Table 5.3 and Fig. 5.26 shows the hysteretic performance of the rehabilitated walls and their CFRP debonding progression.

Specimen SW1 exhibits a very ductile response up to failure, especially when considering the non-ductile details in its original design. Figure 5.27 shows the damage sustained by wall specimen SW1 during the test. Debonding of the CFRP from the concrete substrate first occurs in the toes of the specimen at drift ratios of $0.82 \%$ to $0.88 \%$, which are higher levels of drift when compared to when debonding is first observed in wall specimen RW1. Debonding along the base of specimens SW1 (Fig. 5.27), is again attributed to the opening of large cracks and crushing of the concrete at the base. Specimen SW1 experiences significant concrete crushing and vertical steel reinforcement yielding along the base of the wall after it reached a maximum load of $633 \mathrm{kN}$.

Figure 5.28 shows the vertical and horizontal steel strain profiles at increasing levels of lateral load throughout the test. The results show that significant yielding occurred throughout the vertical steel reinforcement, contributing to the observed ductile response. In addition, due to the addition of the horizontal CFRP layers, the strain in the horizontal steel reinforcement is shown to remain well below the predicted rupture strain throughout the test, eliminating the potential for diagonal tension shear failure. As a result of the extensive yielding throughout the vertical steel reinforcement at the base, specimen SW1 achieves a displacement ductility of 4.1, which is a significant improvement compared to the control wall. Similar to the behaviour observed in specimen RW1, a lack of confinement in the boundary elements after crushing of the concrete in the toes of the specimen and large compressive forces in the boundary elements leads to out-of-plane buckling of the vertical reinforcement and a drop in lateral load carrying capacity (Fig. 5.27). The hysteretic response for specimen SW1, as shown in Fig. 5.26, shows good energy dissipation capacity, characterized by wide hysteretic loops. In contrast to specimen RW1, the 


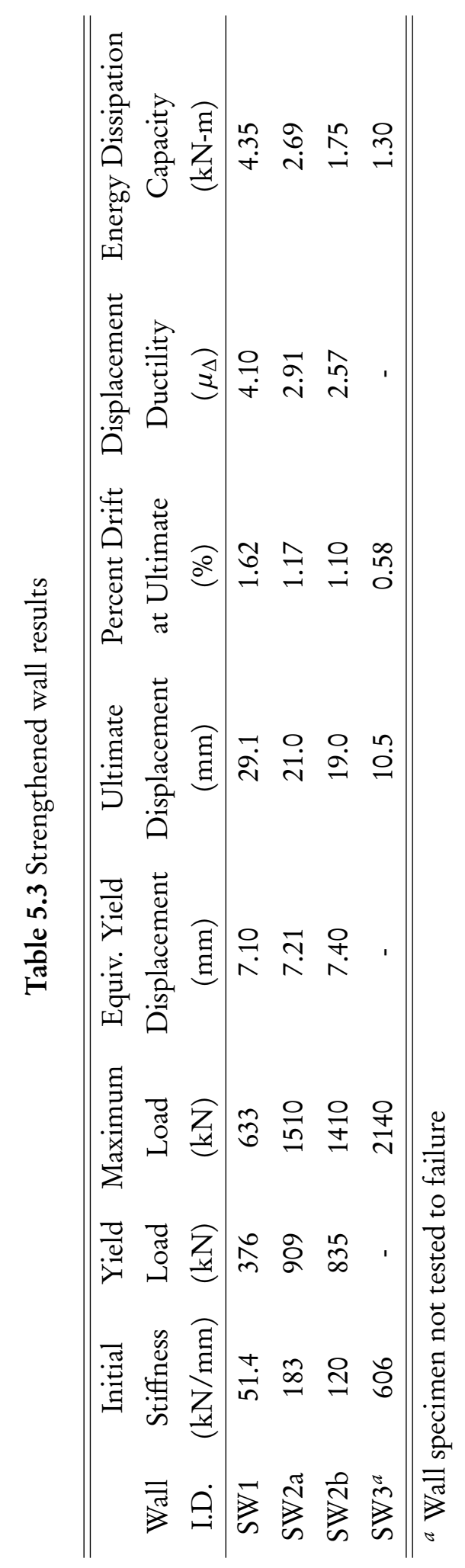


(a)

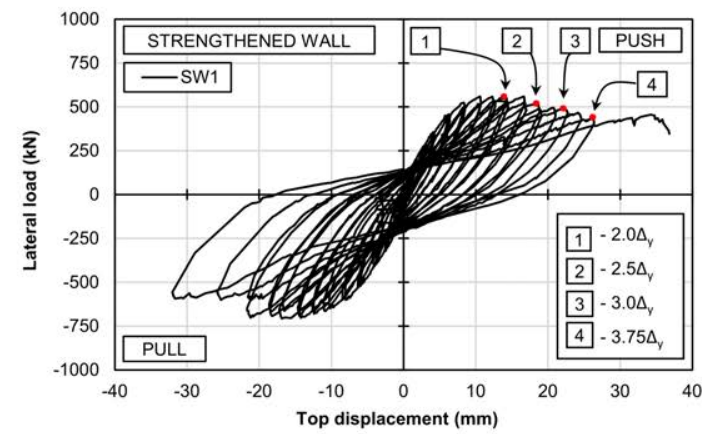

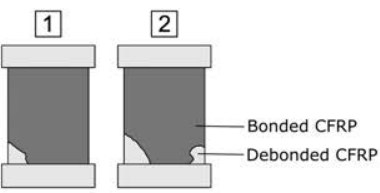

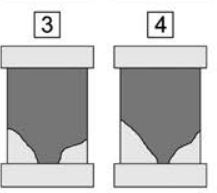

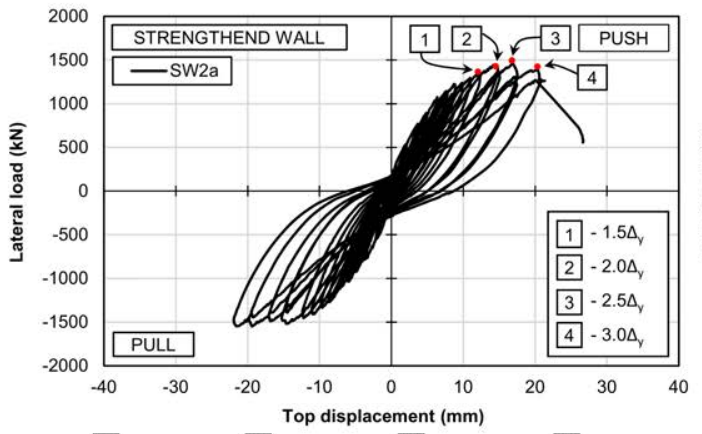
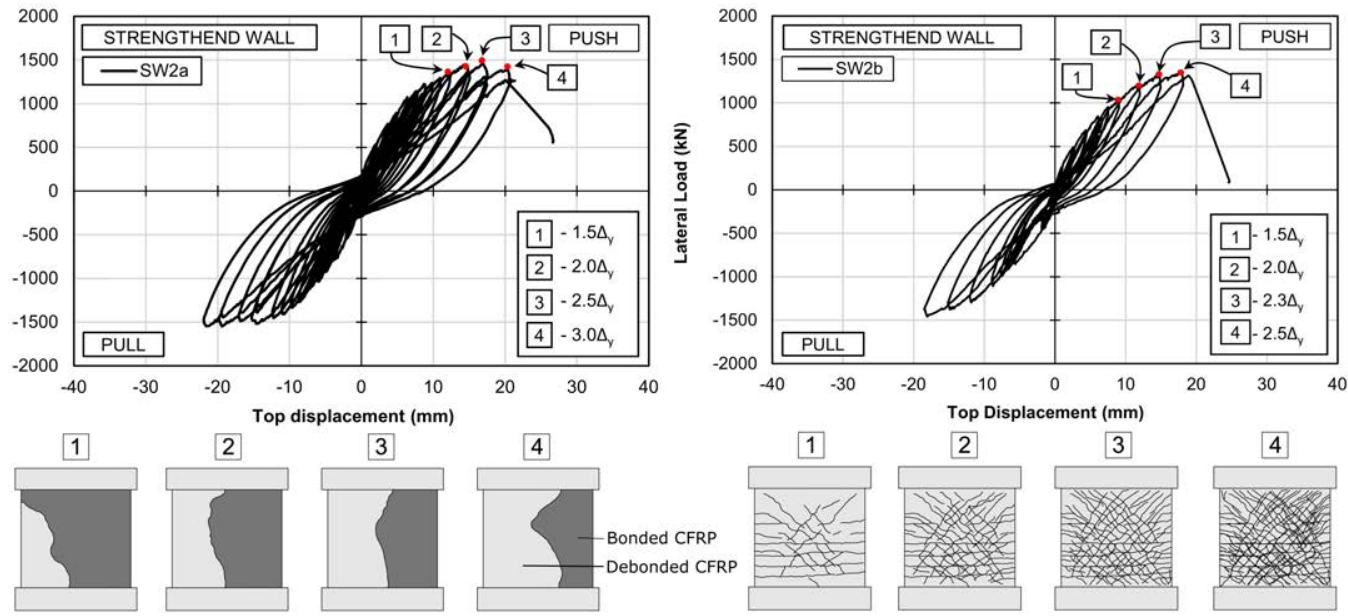
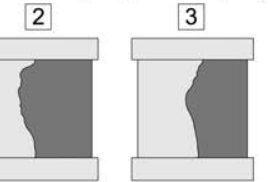

(b)
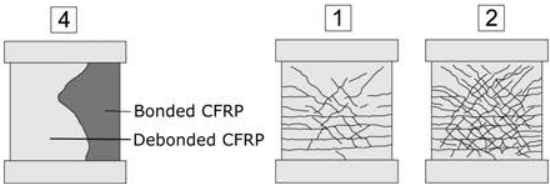

(c)

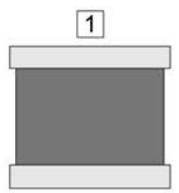

3
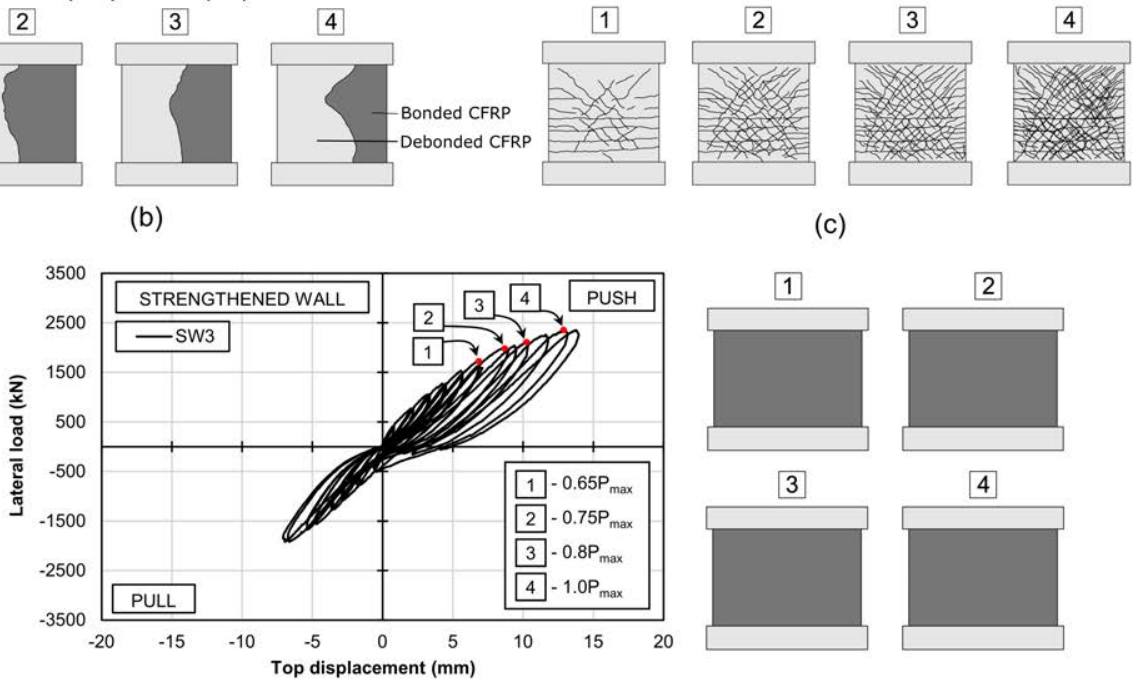

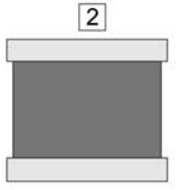

4

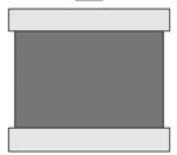

Figure 5.26 Hysteretic behaviour and concrete crack distributions for the strengthened walls: (a) SW1; (b) SW2a; (c) SW2b (d) SW3 


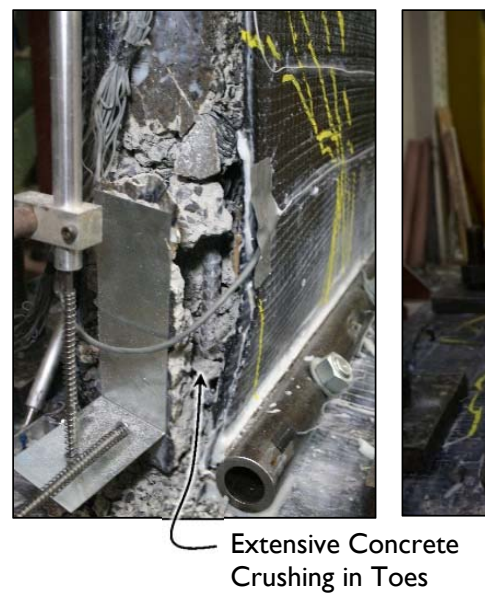

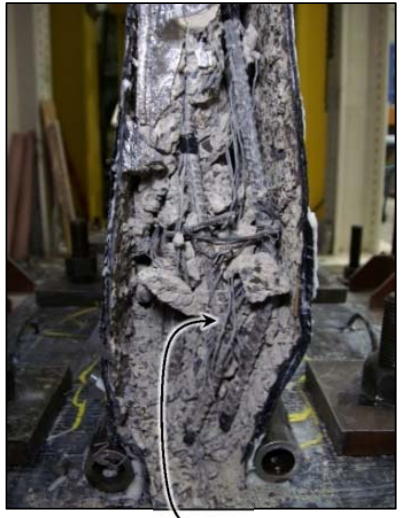

Crushing in Toes
Buckling of Vertical Steel Reinforcement

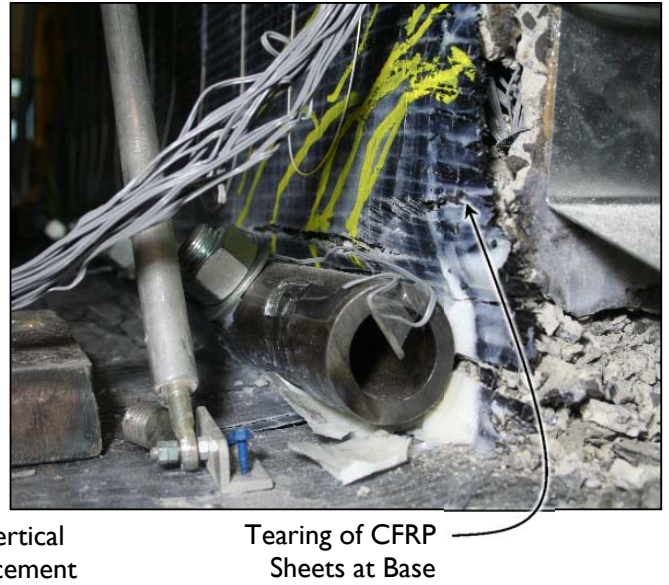

Sheets at Base

Figure 5.27 Damage to strengthened wall specimen SW1

hysteretic response of specimen SW1 is symmetric, which is attributed to the lack of pre-existing damage to the wall specimen.

Wall specimens SW2a and SW2b perform in a very similar manner in terms of forcedeformation characteristics and observed failure mechanism. The differences between the specimens include the type of anchor system and the distribution of the CFRP sheets. The influence of these parameters on the performance of the specimen are discussed in the following section. Figure 5.29 shows the damage sustained by specimens SW2a and SW2b during the test. The onset of CFRP-concrete debonding in specimen SW2a occurs at a lateral drift of approximately $0.88 \%$, which once again is significantly higher when compared with the repaired wall specimen (RW2); attributed to the lack of pre-existing damage to the strengthened shear wall specimen. Debonding
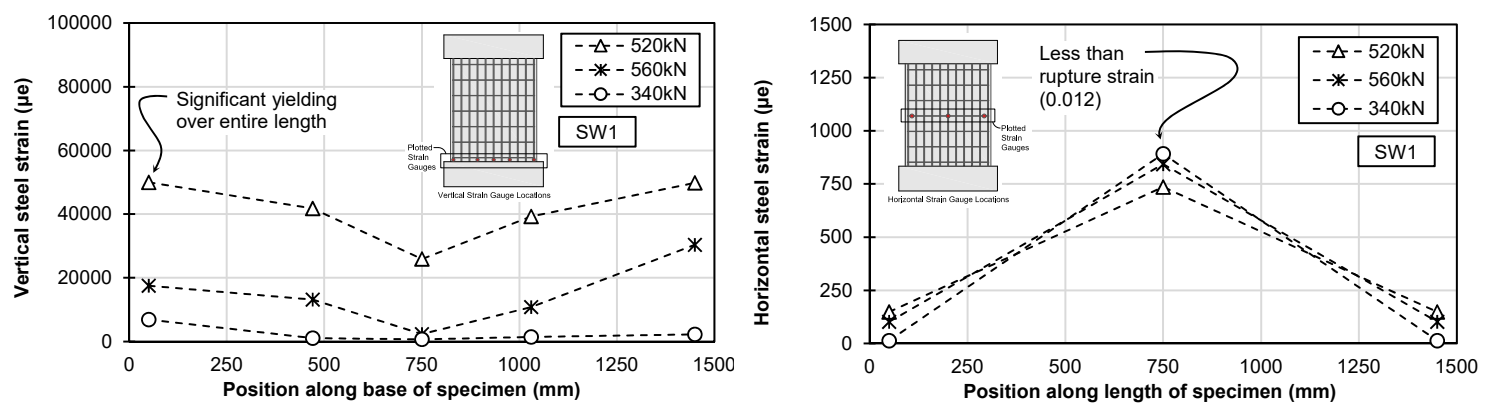

Figure 5.28 Specimen SW1 steel strain profiles: vertical (left) and horizontal (right) 
in specimen SW2a first occurs in the toes of the wall, which is due to the opening of flexural cracks in the concrete. In contrast, wall specimen SW2b does not experience any visible debonding prior to failure, which is attributed to the GFRP fan anchors placed along the edges of the wall. This result suggests that the application of additional anchorage along the edges of the shear wall prevents premature debonding of the CFRP laminate from the concrete substrate. In specimen SW2b, one side of the concrete surface was exposed during the test because all of the CFRP layers were applied to a single side of the wall. This allows for visual monitoring of the concrete crack distribution throughout the test (Fig. 5.26). It is interesting to note that horizontal flexural cracks along the height of the wall were observed before the formation of diagonal cracks in the concrete. This once again indicates a shift in behaviour of the shear wall to a more flexurally dominant behaviour compared with the control wall specimens. Strengthened wall specimens SW2a and SW2b reach their ultimate flexural capacity at a lateral load of $1505 \mathrm{kN}$ and $1405 \mathrm{kN}$, respectively.

Figure 5.30 shows the vertical and horizontal steel strain profiles at increasing levels of lateral load for wall specimens SW2a and SW2b. The results are very comparable between shear wall specimens in terms of horizontal and vertical steel strain distributions. Both wall specimens experience significant yielding in the vertical steel reinforcement while the strain in the horizontal steel reinforcement remains well below the predicted rupture strength. The increase in shear strength provided by the horizontal CFRP prevents premature diagonal tension shear failure and allows yielding of vertical steel reinforcing bars to spread toward the interior of the wall. The additional shear strength provided by the horizontal CFRP also results in lower strains in the horizontal reinforcing steel when compared to control wall specimen CW2. In addition to strains in the steel reinforcement, Figure 5.31 shows the vertical and horizontal CFRP strain profiles for wall specimens SW2a and SW2b. The results show that in specimen SW2a, the vertical CFRP strain profiles remain approximately linear on the tension side and reach a maximum tensile strain of approximately $1800 \mu \epsilon$. The vertical strain profiles for specimen SW2b show a drop in strain near the edge of the wall specimen, which is attributed to some slippage of the vertical CFRP anchors from the concrete foundation near the ends of the shear wall, which was visually observed 

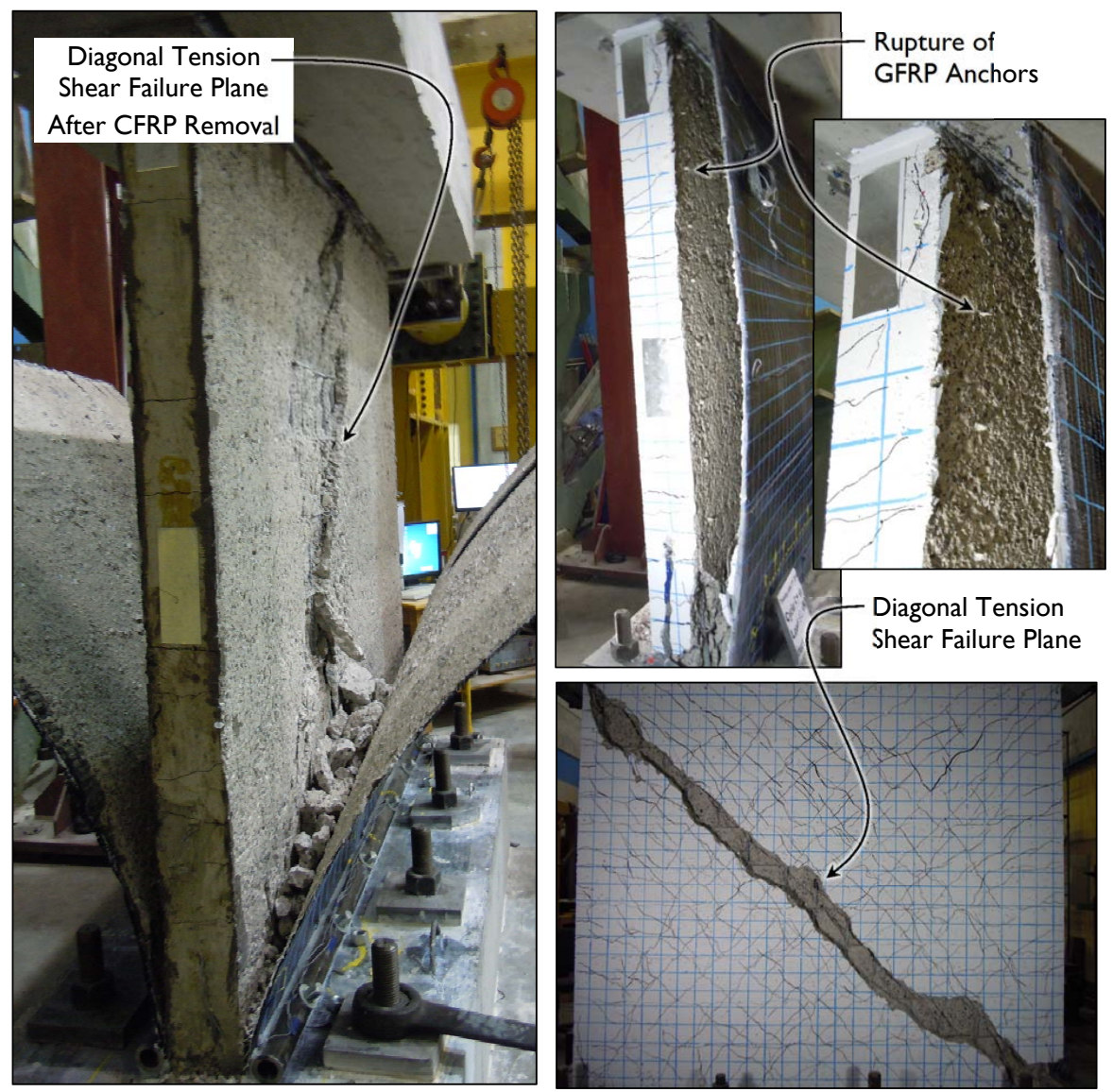

Figure 5.29 Damage to strengthened wall specimens SW2a and SW2b 
during testing. Comparing the horizontal strain profiles for wall specimens SW2a and SW2b, results show that debonding near the ends of specimen SW2b resulted in a loss in strain near the edges of the wall. In specimen SW2b, which used GFRP anchors to secure the CFRP laminate at the wall ends, shows a comparatively even distribution in horizontal strain along the length of the wall, once again confirming that the presence of anchorage at the ends of the wall is beneficial in preventing premature debonding of the CFRP laminate along the wall edge and improved the ability of the horizontal CFRP layers to carry larger strains.

The hysteretic responses for specimens SW2a and SW2b (Fig. 5.26) show good energy dissipation capacity and a ductile response of both wall specimens up to failure. The improved performance translated into improved ultimate displacement ductilities of 2.91 and 2.57 for specimens SW2a and SW2b compared with control specimen CW2, respectively. At maximum lateral drifts of $1.16 \%$ and $1.05 \%$ for SW2a and SW2b, respectively, both strengthened wall specimens fail in diagonal tension shear, after debonding of the CFRP from the concrete substrate, however, it is important to note that failure of both wall specimens occurs after achieving their flexural capacity. In specimen SW2a, debonding between the CFRP sheets and concrete substrate begins along the edge of the wall and propagates toward the interior. Debonding initiates in a similar region for specimen SW2b, but occurs later in the test after rupture of the GFRP anchors along the edge of the wall. Following rupture of the GFRP anchors, rapid delamination of the CFRP sheets from the concrete substrate and results in the formation of a large diagonal failure plane. In this mode of failure, once the CFRP laminate separates from the concrete substrate, the shear wall is no longer able to transfer the large shear stresses with only the horizontal steel reinforcement, leading to rupture of the horizontal steel reinforcement and failure of the wall specimens, as shown in Fig. 5.29.

The strengthened wall specimen SW3, which has an aspect ratio of 0.65 and has no lap splices of the vertical steel reinforcement has very similar cyclic performance when compared with specimen RW3. No debonding of the CFRP sheets from the concrete substrate was noted during testing of specimen SW3. Figure 5.32 shows the vertical and horizontal strain profiles in 

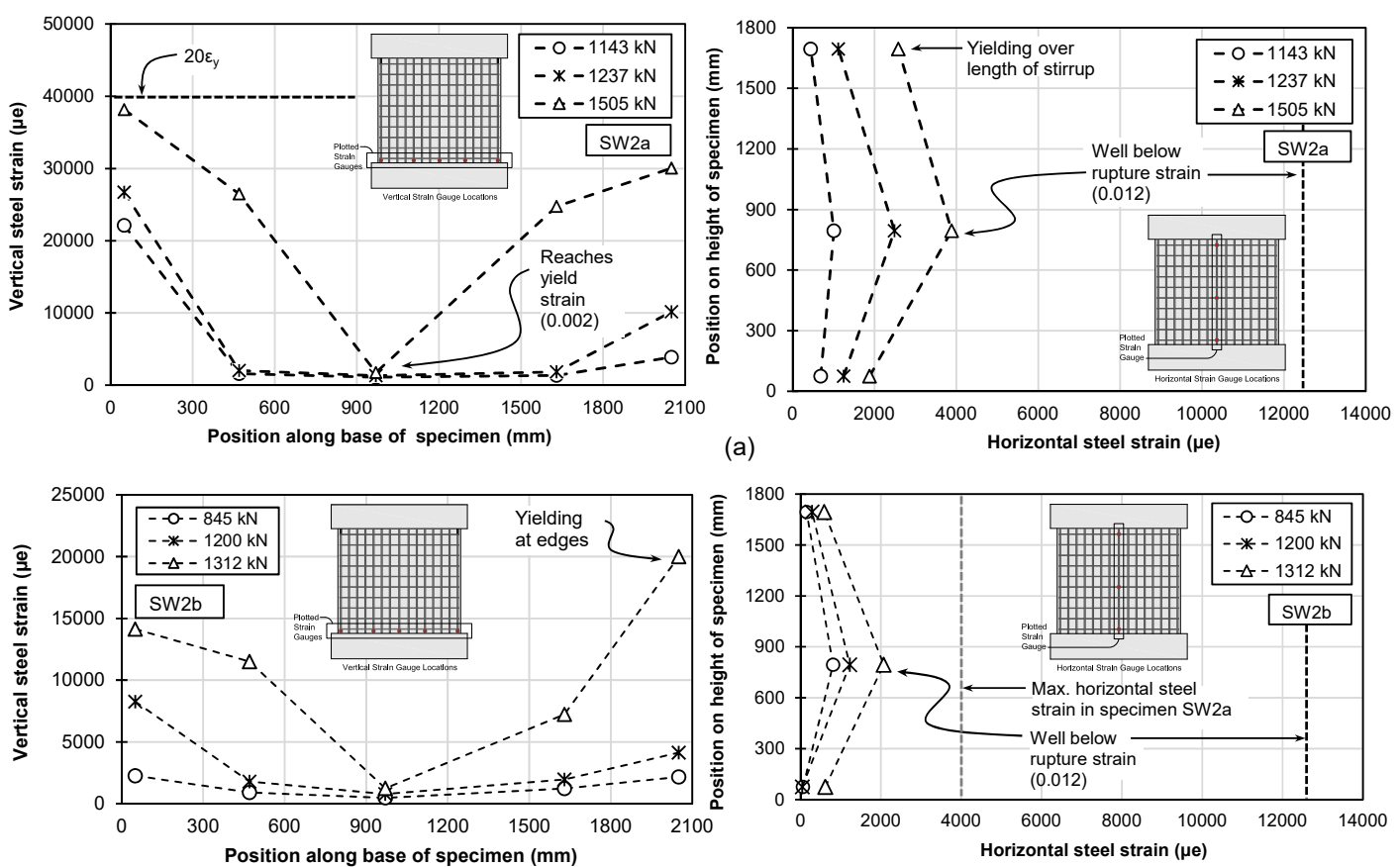

(b)

Figure 5.30 Vertical and horizontal steel strain profiles: (a) SW2a; (b) SW2b
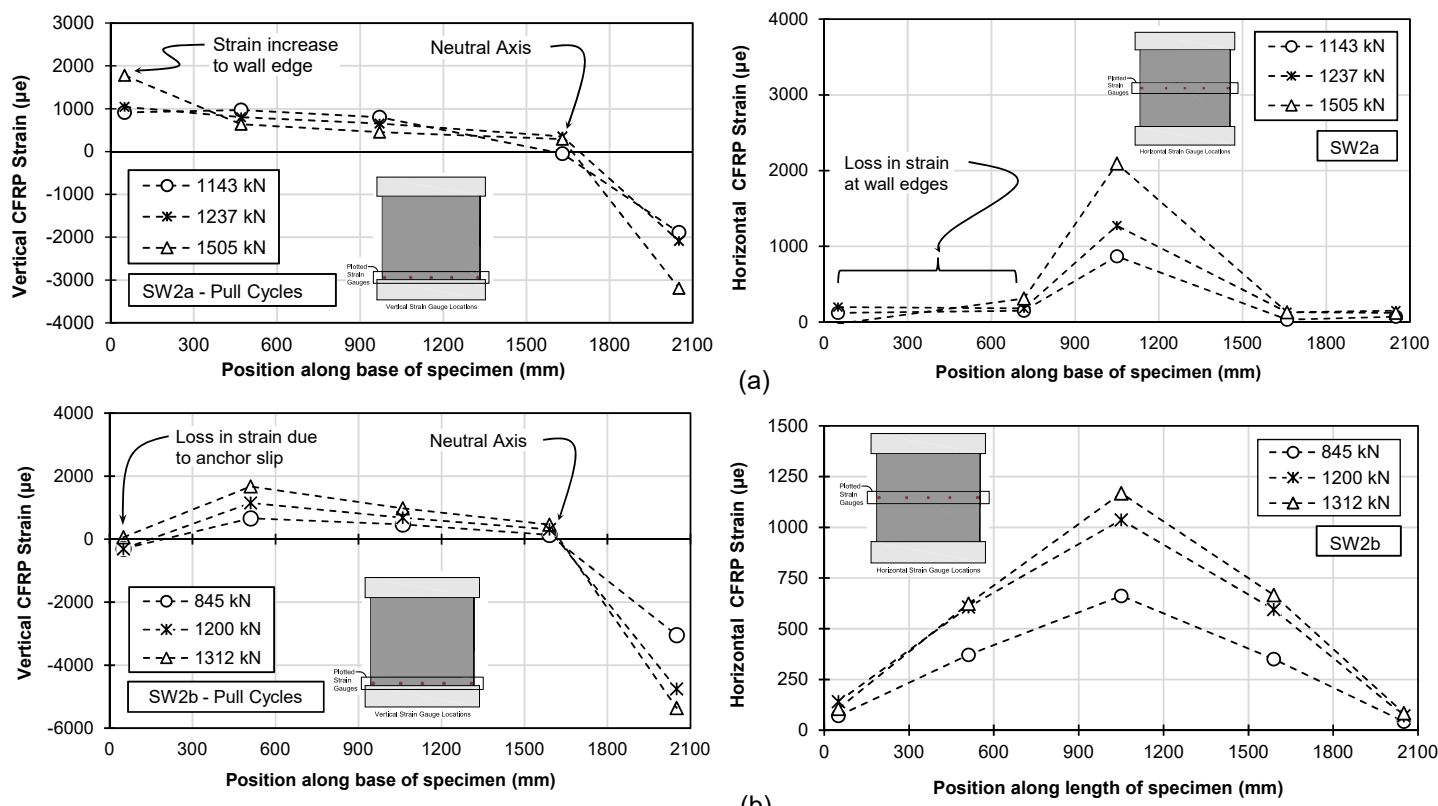

(b)

Figure 5.31 Vertical and horizontal CFRP strain profiles: (a) SW2a; (b) SW2b 
the steel reinforcement at increasing levels of lateral load. The results show that at a maximum lateral load of $2355 \mathrm{kN}$, the first two layers of vertical steel reinforcement exceed their yield strain. In addition, the first layer of vertical steel reinforcement in compression yields at the opposite end of the wall specimen.

Figure 5.33 shows the horizontal strain profiles on the surface of the horizontal CFRP layers as well as the horizontal strain hysteretic behaviour in the centre of the wall for the horizontal steel and CFRP reinforcement for specimen SW3. Results demonstrate that up to the maximum load carrying capacity, the strains in the steel and CFRP reinforcement are only approximately $50 \%$ of those recorded at the same location in the control specimen CW3. Unfortunately, in a similar manner to specimen RW3, the ultimate load carrying capacity of SW3 could not be achieved because of premature failure in the wall foundation, which transfers the loads from the wall to the laboratory strong floor. Figure 5.34 shows the damage to the foundation of specimen SW3 during the test. The observed damage includes two large splitting cracks down the centre of the foundation block at its ends and diagonal shear cracks along the length of the foundation. Nonetheless, specimen SW3 exhibits a significant strength increase of 184\%, when compared with specimen CW3. Figure 5.26 shows the hysteretic response behaviour for specimen SW3. The results show that the wall specimen remains approximately linear, with only minor nonlinearity observed in its hysteretic response behaviour, which may be attributed to damage in the wall specimen foundation, and not damage to the wall specimen itself. Asymmetry in the hysteretic behaviour is attributed to the loading protocol, which was limited to applying increasing force in the push direction as a result of damage to the shear wall foundation, which prevented the application of displacements larger than $7.5 \mathrm{~mm}$ in the pull direction.

Joshua Woods, Department of Civil and Environmental Engineering, Carleton University 


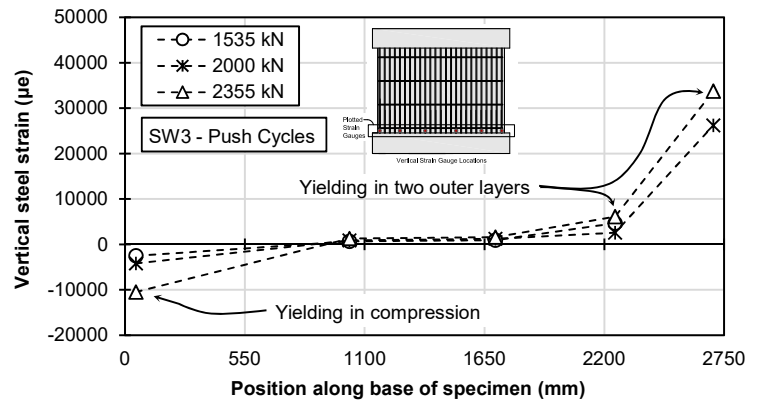

(a)

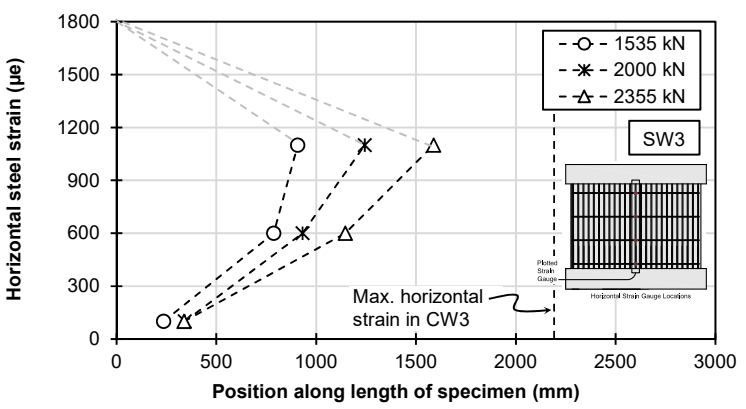

(b)

Figure 5.32 Specimen SW3: (a)Vertical steel strain profiles; (b)Horizontal CFRP/steel strain

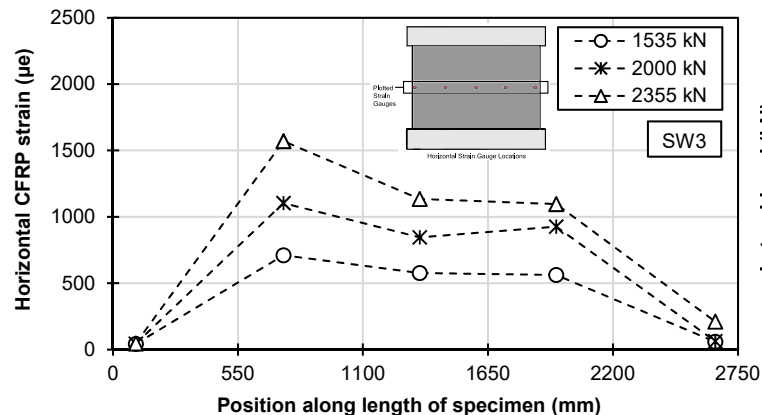

(a)

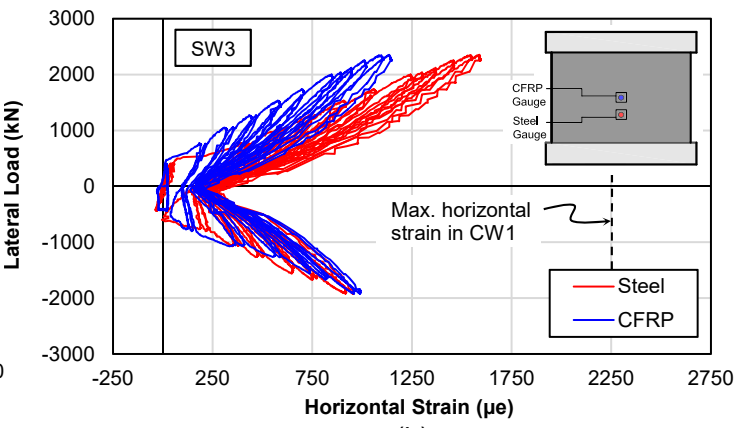

(b)

Figure 5.33 Specimen SW3: (a) vertical steel strain profiles; (b) horizontal CFRP/steel strain

\subsection{Overall Response Comparison}

Table 5.4 compares important structural response parameters of strengthened and repaired wall specimens and Figures 5.35 and 5.36 compares the hysteretic response behaviour of the repaired and strengthened wall specimens with their respective control wall to evaluate the effectiveness of the CFRP retrotting scheme in improving the seismic performance of deficient RC shear wall specimens. The seismic performance of the wall specimens is evaluated in terms of their initial stiffness, in-plane strength, ductility, and energy dissipation capacity.

In general, the performance of the repair procedure varies depending on the level of damage sustained by the control wall, aspect ratio of the wall specimen, and steel reinforcement detailing. The test results demonstrate that the application of the CFRP sheets was able to completely 


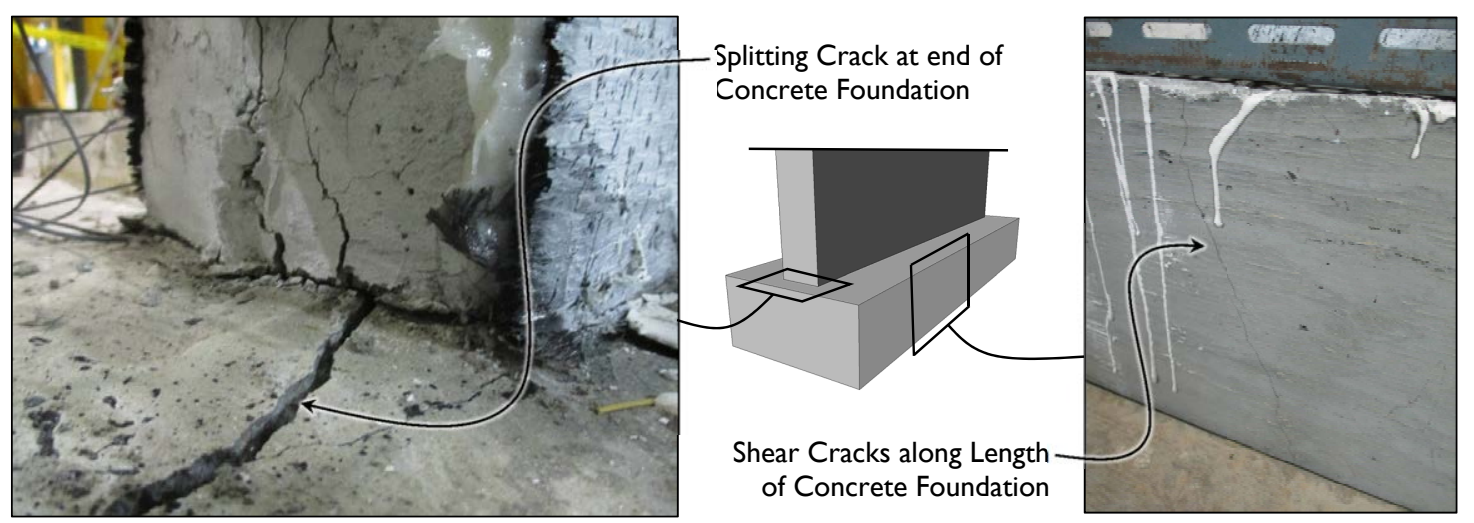

Figure 5.34 Damage to the foundation of wall specimen SW3

Table 5.4 Shear wall response comparison with respect to control

\begin{tabular}{|c|c|c|c|c|c|}
\hline $\begin{array}{l}\text { Wall } \\
\text { I.D. }\end{array}$ & $\begin{array}{c}\text { Initial } \\
\text { Stiffness } \\
\qquad\left(K_{i}\right)\end{array}$ & $\begin{array}{l}\text { Maximum } \\
\text { Load } \\
\left(P_{\max }\right)\end{array}$ & $\begin{array}{l}\text { Maximum } \\
\text { Deflection } \\
\left(\Delta_{\max }\right)\end{array}$ & $\begin{array}{c}\text { Displacement } \\
\text { Ductility } \\
\left(\mu_{\Delta}\right)\end{array}$ & $\begin{array}{c}\text { Normalized } \\
\text { Energy Dissipation } \\
\text { Capacity }\left(E_{n}\right)\end{array}$ \\
\hline CW1 & 1.00 & 1.00 & 1.00 & 1.20 & 1.00 \\
\hline RW1 & 1.81 & 1.79 & 2.02 & 3.10 & 3.55 \\
\hline SW1 & 2.29 & 1.86 & 2.27 & 4.10 & 6.00 \\
\hline CW2 & 1.00 & 1.00 & 1.00 & 1.25 & 1.00 \\
\hline RW2 & 1.48 & 0.96 & 1.12 & 1.40 & 2.27 \\
\hline SW2a & 2.44 & 1.15 & 1.84 & 2.91 & 3.67 \\
\hline SW $2 b$ & 1.60 & 1.38 & 1.67 & 2.57 & 2.36 \\
\hline $\mathrm{CW}^{a}{ }^{a}$ & 1.00 & 1.00 & 1.00 & - & 1.00 \\
\hline $\mathrm{RW}{ }^{a}$ & 0.41 & 1.71 & 1.80 & - & 1.16 \\
\hline $\mathrm{SW} 3^{a}$ & 0.91 & 1.84 & 2.18 & - & 1.27 \\
\hline CW4a & 1.00 & 1.00 & 1.00 & - & 1.00 \\
\hline RW4a & 0.32 & 1.05 & 1.32 & 1.20 & 1.20 \\
\hline $\mathrm{CW} 4 \mathrm{~b}$ & 1.00 & 1.00 & 1.00 & 2.69 & 1.00 \\
\hline RW 4b & 0.22 & 0.58 & 1.05 & 1.33 & 1.02 \\
\hline
\end{tabular}

${ }^{a}$ Wall specimen not tested to failure because of premature foundation failure 
(a)

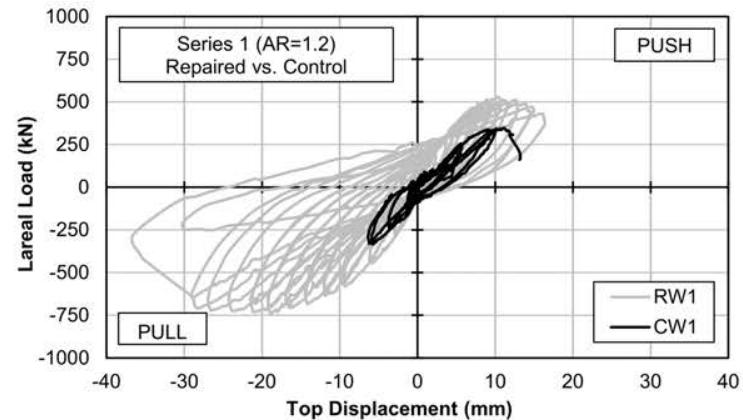

(b)
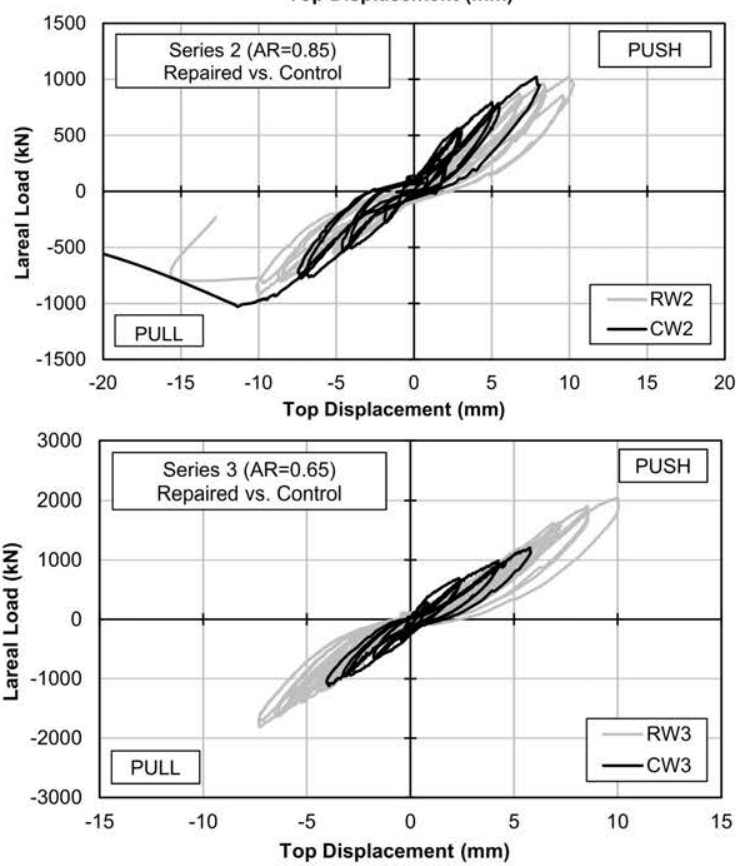

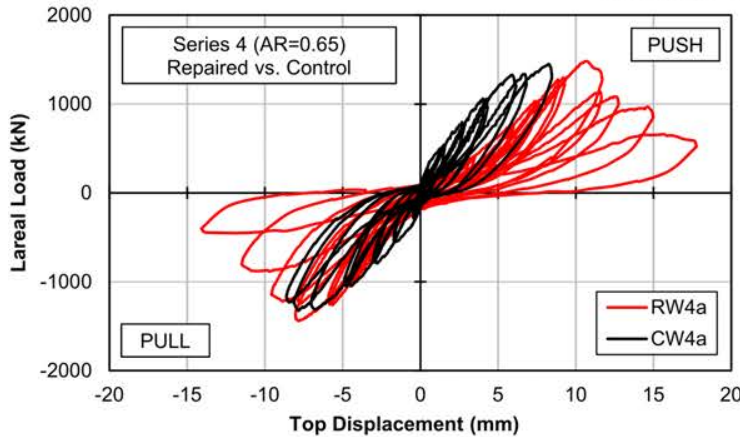

(d)

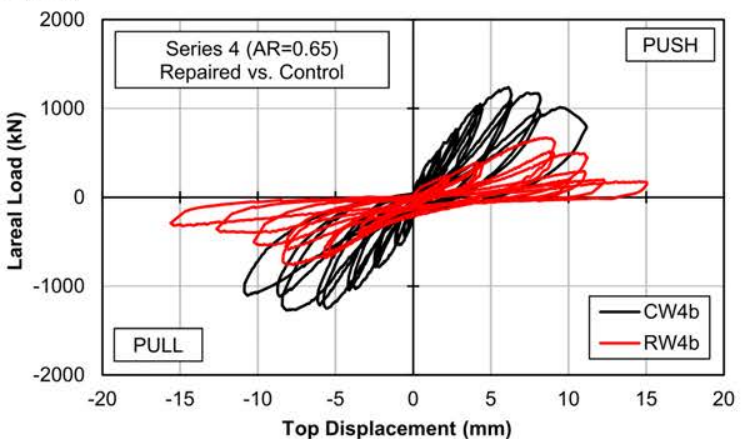

(e)

Figure 5.35 Control and repaired hysteretic responses: (a)W1; (b)W2; (c)W3; (d)W4a; (e) W4b 


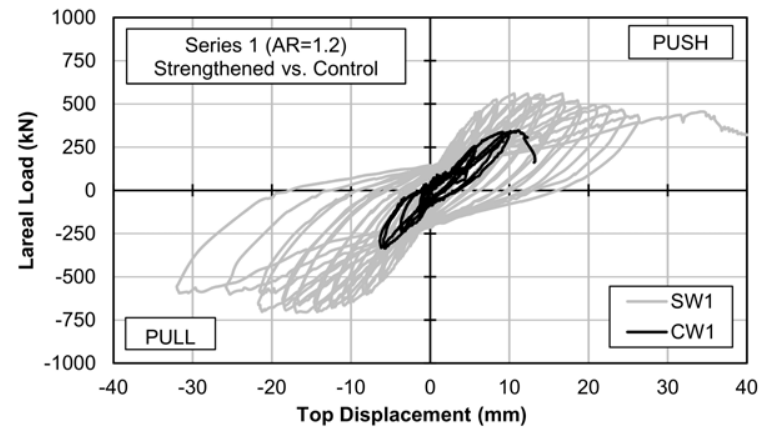

(a)
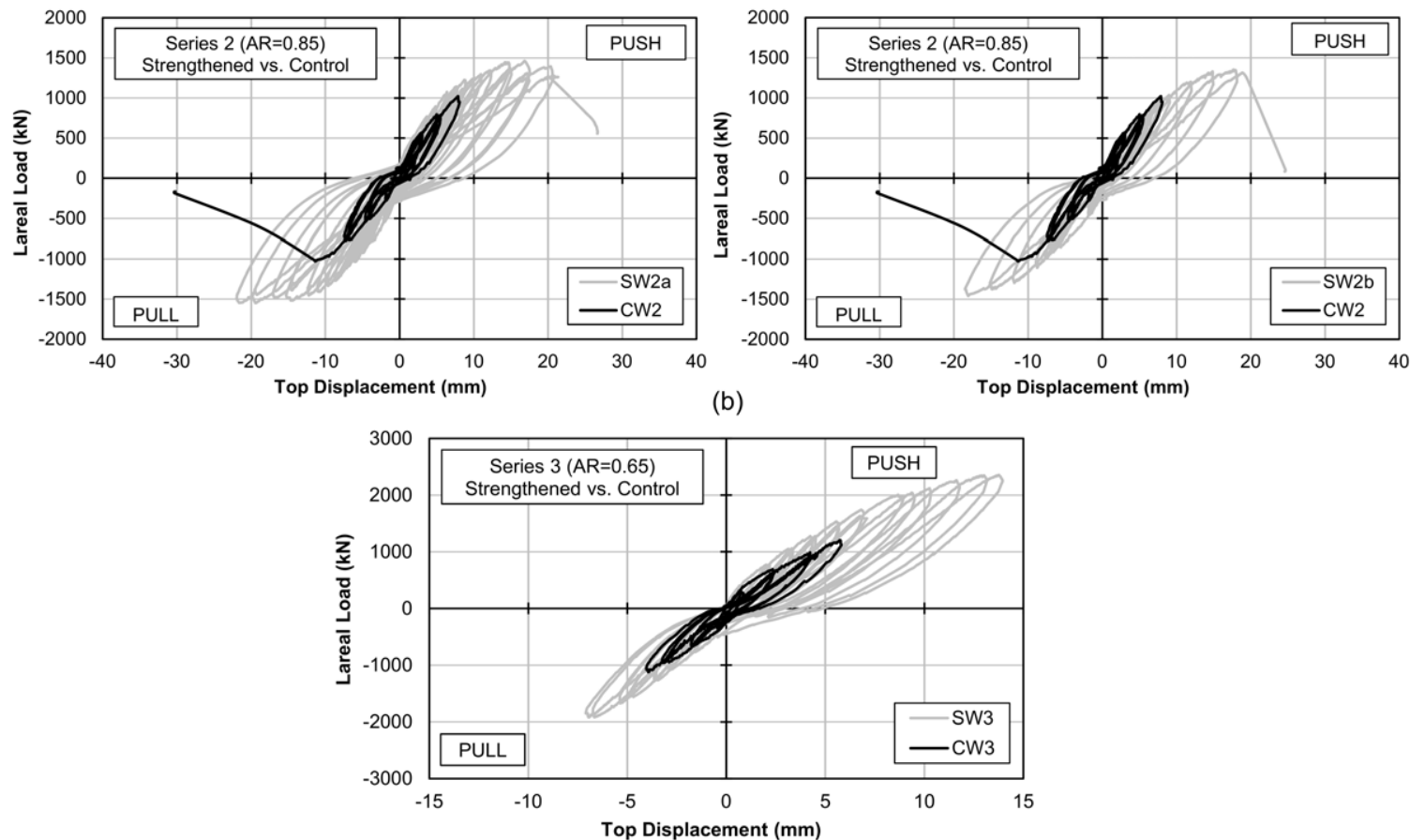

(c)

Figure 5.36 Control versus strengthened hysteretic response comparison: (a)SW1/CW1; (b)SW2a/CW2 \& SW2b/CW2; (c)SW3/CW3 
restore and increase the initial stiffness of specimen RW1. The increase in initial stiffness of the specimen is attributed to the application of vertical CFRP layers, which have been shown in previous studies by Lombard et al. (2000), Hiotakis (2004), and Cruz-Noguez et al. (2014b) to improve the initial stiffness of comparable shear wall specimens. Wall specimen RW1 also shows an improvement in in-plane strength and energy dissipation capacity compared to wall specimen CW1, however, because of the assymetric hysteretic response behaviour exhibited by specimen RW1, the improvements in seismic performance was non-uniform depending on the direction of loading. Nonetheless, the average in-plane strength of wall specimen RW1 was 1.79 times larger than the strength of specimen CW1 while the displacement and energy dissipation capacities were over 2 and 3.5 times larger, demonstrating satisfactory performance of the retrofitting strategy.

Comparatively, the CFRP rehabilitation strategy was not as effective in specimen RW2, which showed only modest improvements in in-plane strength, lateral displacement capacity, and cummulative energy dissipation capacity. This is attributed to the severity of the damage sustained by the wall specimen during the initial test, which left the wall specimen with virtually no shear strength contributions from the horizontal steel reinforcement or concrete. Even so, the retrofitting scheme increased the in-plane stiffness by 1.5 times and restored the in-plane strength of the wall specimen. These results demonstrate the potential for more significant improvements in seismic performance when the CFRP retrofitting system is applied to wall specimens with less severe pre-existing damage. This was verified during testing of specimen RW3, in which the initial test on specimen CW3 was stopped before the onset of yielding in the horizontal steel reinforcement. Specimen RW3 had approximately 1.7 and 1.8 times the original in-plane strength and deformability compared to the control wall specimen, respectively. Because specimen RW3 was not tested to failure, the ultimate load and displacement are likely even larger than those observed during testing. The initial stiffness of specimen RW3 was only $41 \%$ of the original stiffness of specimen CW3. The inability to restore the initial stiffness of the wall specimen is attributed to the fact that no vertical CFRP layers were applied to specimen RW3; once again suggesting that it is the application of vertical CFRP layers that contributes to improving the initial stiffness of the retrofitted wall specimens. 
There is a stark contrast in the performance of the CFRP retrofitting technique in the two shear wall specimens with lap splices. In specimen RW4a, which had a $36 d_{b}$ lap splice length, the rehabilitation strategy restores the in-plane strength and lateral displacement capacity of wall specimen CW4a without any attempt to restore the bond between lapped bars or providing additional confinement near the base of the wall. In specimen RW4b, which had a $21 d_{b}$ lap splice length, the rehabilitation is not able to restore the in-plane strength of the wall specimen because of the damage sustained by the wall during the initial test. Once again the initial stiffness of the wall specimens with lap splices were not restored because no vertical CFRP layers were applied. The results show that in shear walls with inadequate lap splice length, more intrusive repair strategies that attempt to restore the bond between lapped steel bars are required to improve the seismic performance of the wall specimen. Potential intrusive rehabilitation strategies could include welding of the lapped bars, additional confining reinforcement around the lap splice region, or the addition of a concrete collar, however, in squat wall specimens with low aspect ratios the addition of a concrete collar around the lap splice may promote the sliding shear failure mode observed during test RW4b, which may not significantly improve the seismic performance of the shear wall.

All of the strengthened wall specimens show significant improvements in seismic performance compared with their respective control wall specimens. Specimen SW1, which had the highest aspect ratio $\left(h_{w} / l_{w}=1.2\right)$ exhibits significant increases in initial stiffness, in-plane strength, ultimate displacement, and large improvements in energy dissipation capacity compared to specimen CW1. The improvements in seismic performance are evident from the comparison of the hysteretic response behaviour between specimens CW1 and SW1. Specimens SW2a and SW2b also show improvements in initial stiffness, in-plane strength, lateral displacement capacity and energy dissipation capacity. The improvements in these parameters are not as significant as specimen SW1 because of its higher aspect ratio, which resulted in a more ductile flexurally dominant behaviour. Nonetheless, the CFRP retrofitting system improved the seismic performance and hysteretic response behaviour of specimens SW2a and SW2b. As noted in Table 3.1, wall specimen SW2a had all of the vertical and horizontal CFRP layers distributed evenly on either side of the wall. Alternatively, wall specimen SW2b had the same number of layers as SW2a, but 
the layers were all installed on a single side of the shear wall. This allows for a comparison between the two specimens to get a better understanding of the effect of CFRP sheet distribution on the in-plane behaviour of the shear wall. Based on the test results, the effect of placing all of the CFRP layers on a single side of the wall specimen did not have a significant impact on the ultimate load carrying capacity or the lateral drift capacity, which differ by only $8 \%$ and $10 \%$, respectively. The hysteretic response behaviour of the two specimens, shown in Fig. 5.37, shows very similar behaviour, including the initial stiffness, yield load, and post-peak response. However, restraining the out-of-plane deformations of the wall specimens could have influenced the results of the comparison because it eliminates the potential for torsional sensitivity caused by applying all of the CFRP sheets on a single side of the wall.

Specimen SW3, which had the lowest aspect ratio among the strengthened test specimens also showed improvements in seismic performance. Specimen SW3, which was not tested to failure because of premature failure in the shear wall foundation, had a lateral load carrying capacity 1.84 times larger than the control wall and exhibited a $27 \%$ increase in energy dissipation capacity. Strain values from the horizontal reinforcement, shown in Fig. 5.32, show that the maximum strain in the horizontal reinforcement in specimen SW3 was approximately $2 / 3$ of maximum strain recorded in specimen $\mathrm{CW} 3$ at almost twice the lateral load, demonstrating the load sharing between the horizontal CFRP sheets and the horizontal steel reinforcement and the potential for a larger increase in load carrying capacity if the foundation had not failed prematurely. It is once again noted that the initial stiffness of wall specimen SW3 compared to specimen CW3 is slightly

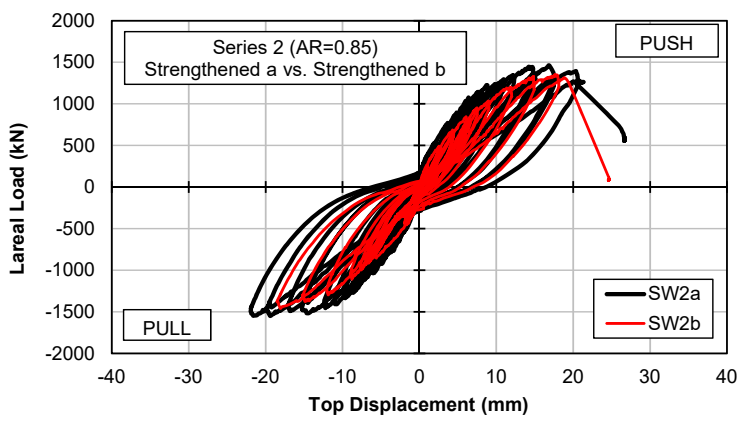

Figure 5.37 Comparison of the hysteretic response behaviour of specimens SW2a and SW2b 
lower, because wall specimen SW3 did not have any vertical CFRP layers. The slight decrease in initial stiffness compared to specimen CW3 is attributed to the variability between test specimens and the placement of the vertical steel reinforcement inside the wall, which has been shown in previous studies to have a significant impact on the initial stiffness of a wall specimen (Cruz-Noguez et al., 2014b).

Results from the digital image correlation software ImPro Stereo were shown to be valuable tool in assessing the performance of the CFRP retrofitting system. Specifically, the ability to produce detailed crack distributions following a test with the ability to zoom in on the results in a particular region of interest. This showcases the dynamic capabilities of using ImPro Stereo to analyze experimental results after a test is complete, without the need to stop the test or a priori knowledge of measurement location. In CFRP retrofitted wall specimens, the ImPro Stereo software was able to track displacement and strain distribution on the surface of the CFRP layers which were used to construct a complete two dimensional surface strain field, something that is not feasible using conventional measurement technologies. Through the use of the image analysis technique, accurate deformation measurements, such as the vertical and horizontal slip that occurred at the height of the shear plane in specimen SW4b could be analyzed in detail, which could not be determined using conventional sensors without a priori knowledge of the location in which the slip was going to occur. The use of ImPro Stereo allows the location and magnitude of deformations in critical areas to be post-processed and studied in more detail after the test was complete. The results show great potential for the use of these technologies in structure engineering experiments.

Ultimately, the CFRP retrofitting system is shown to be able to successfully restore the performance of a severely damaged wall to its original state in repair applications, with minor improvements in terms of strength, ductility, and energy dissipation capacity. The effectiveness of the retrofitting strategy in repair applications depends on the level of damage sustained by the original wall specimen and the design detailing deficiencies which may not be addressed by the minimally disruptive retrofitting solution presented here, which require more intrusive retrofitting strategies. In strengthening applications, the CFRP retrofitting system shows great potential for 
improving the seismic performance of shear dominant RC squat walls. Results show significant improvements in terms of in-plane strength, ductility and energy dissipation capacity as well as the potential for an increase in in-plane stiffness or strength if vertical CFRP layers are applied.

\subsection{Influence of Aspect Ratio}

It is commonly recognized that RC shear walls with aspect ratios larger than 2 have predominantly flexurally dominant behaviour, shear walls with aspect ratios between 1.5 and 2 experience a combined shear-flexure response, and those with aspect ratios less than 1.5 would generally be considered shear dominant walls. The implications of this behaviour on the steel reinforcement detailing and expected failure modes is well documented for plain RC shear walls, however, the influence that aspect ratio has on the performance of CFRP retrofitted concrete shear walls is not as well understood. Thus, one of the parameters studied in this experimental program was the influence of aspect ratio $\left(h_{w} / l_{w}\right)$, specifically in shear-flexure dominated and shear dominated wall specimens, on the behaviour and observed failure mechanisms in repaired and strengthened RC shear walls using externally bonded CFRP sheets.

In this study, three different height-to-length aspect ratios and their influence on the performance of a CFRP retrofitting system were investigated. Aspect ratios of 1.2, 0.85, and 0.65 were selected to represent a range of shear dominant behaviours. Results from the control wall specimens without CFRP sheets demonstrated the potential for brittle non-ductile diagonal tension shear failure in squat RC shear walls when adequate horizontal steel reinforcement was not provided. All of the control specimens tested in this study exhibited significant diagonal cracking, illustrated in Fig. 5.1, prior to the formation of horizontal flexural cracks in the concrete or concrete crushing along their base, which are typically indicative of flexural behaviour.

The influence of aspect ratio is also evident when studying the performance of the CFRP retrofit for wall specimens with decreasing aspect ratios. Although in all of the strengthened wall specimens the retrofitting strategy was effective in improving the in-plane strength of the wall specimens, as the aspect ratio decreased, the ductility of the wall specimens also decreased. This is a result of the higher shear stresses that must be transfered in wall specimens with lower 
aspect ratios because of their comparatively higher flexural strength, which results in a lower margin between the flexural and shear strengths of the shear wall. This results in earlier onset of shear failure following flexural failure in the wall specimens, reducing their ductility and energy dissipation capacity and reducing the overall effectiveness of the CFRP retrofit.

To quantify the observed shift in behaviour of the control wall specimens from a shear dominant to a more flexurally dominant behaviour, the total top displacement of each control wall specimen is divided into contributions from flexure, shear and sliding shear displacement. Figure 5.38 illustrates the three displacement components that contribute to the total top displacement. Flexural displacements were captured using vertically oriented LVDT's to measure the rotation of the top and bottom of the specimen. Diagonally oriented LVDTs were used to monitor the shear deformation of the specimens and horizontally oriented LVDTs at the base of the wall were used to measure sliding between the wall panel and the shear wall foundation.

Figure 5.39 shows the results of the displacement analysis for the control walls at the cracking, yield, and ultimate loads. The results clearly show the influence of aspect ratio on the response of the shear wall specimens. Wall specimen CW1 $\left(h_{w} / l_{w}=1.2\right)$, has approximately equal contributions from flexure and shear to its total response, suggesting that its response behaviour lies between flexurally dominant and shear dominant. Comparatively, wall specimen CW2 $\left(h_{w} / l_{w}=0.85\right)$ shows an increase in shear deformation to the total displacement response.

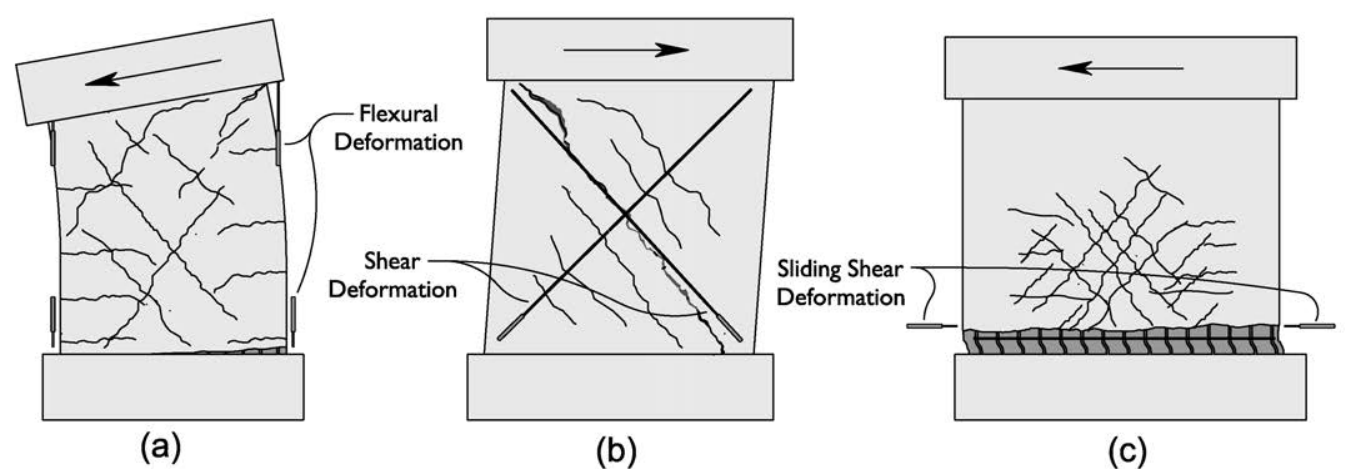

Figure 5.38 Illustration of flexure, shear, and sliding shear displacement in RC shear wall 
At the yield load, shear deformation contributes to more than $65 \%$ of the total response of specimen CW2. Specimen CW3 $\left(h_{w} / l_{w}=0.65\right)$ also shows a shift toward more shear dominant behaviour, but emphasizes significance of sliding shear displacement in the total response of wall specimens with very low aspect ratios, contributing to more than $35 \%$ of the total response at the ultimate load. These results demonstrate the shift in behaviour as the aspect ratio of a wall decreases and the role that the deformation mechanisms play in the observed failure mode and seismic performance of the wall specimens.

To examine the influence that the application of the CFRP layers has on the deformation mechanisms during the tests, Figure 5.40 shows the flexure, shear, and sliding shear displacement contributions to the total lateral displacement of the series 1 and 2 control, repaired, and strengthened wall specimens. For the repaired wall specimens, shear deformation contributes to over $70 \%$ of the total top displacement, which is larger than the shear displacement contributions in the control and strengthened wall specimens. This is attributed to the reopening of the diagonal failure plane formed during the initial control tests. Once the previously formed failure plane reopens and the CFRP sheets debond from the concrete substrate, which was observed in both tests, the opening and closing of this crack during subsequent load cycles dominates the response. Alternatively, the results from both strenghtened wall specimens show a clear shift to a more flexurally dominant behaviour, as the flexural deformation makes up approximately $60 \%$ and $30 \%$ of the total displacement in specimens SW1 and SW2b, respectively. This indicates a shift to a more flexurally dominant behaviour when compared with the control wall, as the strengthened
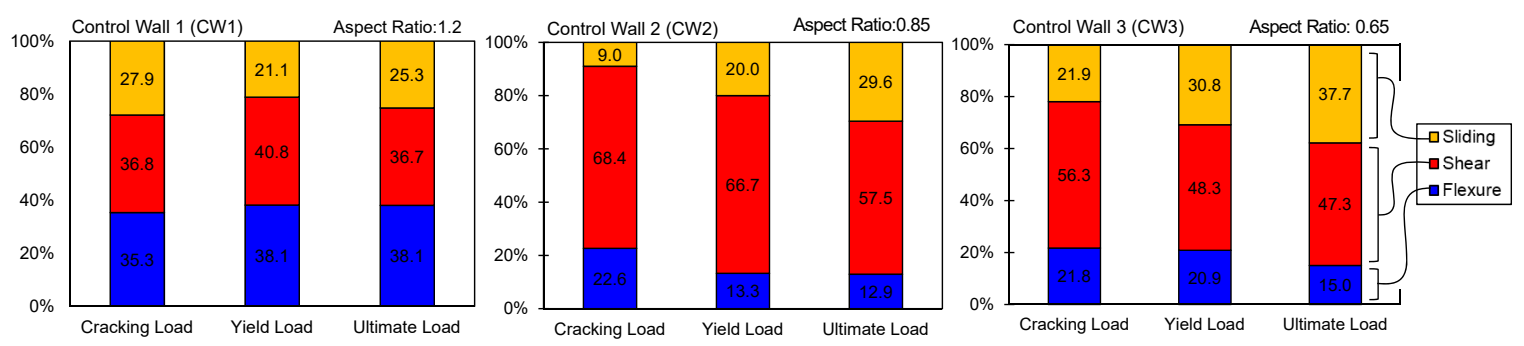

Figure 5.39 Flexure, shear, and sliding shear displacements: (a) CW1; (b) CW2; (c) CW3 
wall specimens experience more yielding in the vertical steel reinforcement and concrete crushing at the base of the wall prior to failure. This shift in behaviour translates into an increase in displacement ductility and energy dissipation capacity in both strengthened walls.

Figure 5.41 shows the time-history of the displacement contributions from flexure, shear, and sliding shear deformation. The results confirm once again that for specimen CW2 and RW2, shear deformation makes up a significant component of the total displacement of the wall specimens. Wall specimens SW2a and SW2b show very comparable deformation distributions, with flexural deformation making up a large part of the total response, particularly close to the end of the test after the vertical steel reinforcement yields in tension. It is also noted that there is a larger contribution from sliding shear deformation near the end of tests conducted on SW2a and SW2b because of the number of load cycles and load reversals applied to these specimens, which degrades the bond along the base of the wall forming a sliding shear plane.

Ultimately, analysis of the deformation contributions from flexure, shear, and sliding shear to the total top displacement effectively quantifies the observed shift in behaviour among the wall specimens and the effect of decreasing aspect ratio on the response of the wall specimens. Results demonstrate that for walls whose aspect ratio is less than 1.0 , shear deformations contribute to more than $50 \%$ of the total response of the wall while for specimens whose aspect ratios is less than 1.0, sliding shear deformations has a more critical behaviour. In the repaired wall specimens, shear deformation are shown to dominate the response as a result of reopening of diagonal cracks formed during the initial test. The strengthened specimens have a larger contribution from flexural displacement to the total displacement, but as the aspect ratio increases this contribution decreases substantially, which reduces the effectiveness of the CFRP retrofitting strategy.

\subsection{Comparison with Code Equations}

Experimental results have demonstrated that the response of low aspect ratio shear walls strengthened with FRP sheets is a combination of shear and flexural responses. This makes the behavior of low aspect ratio walls less predictable compared to flexural walls because of the mixed 

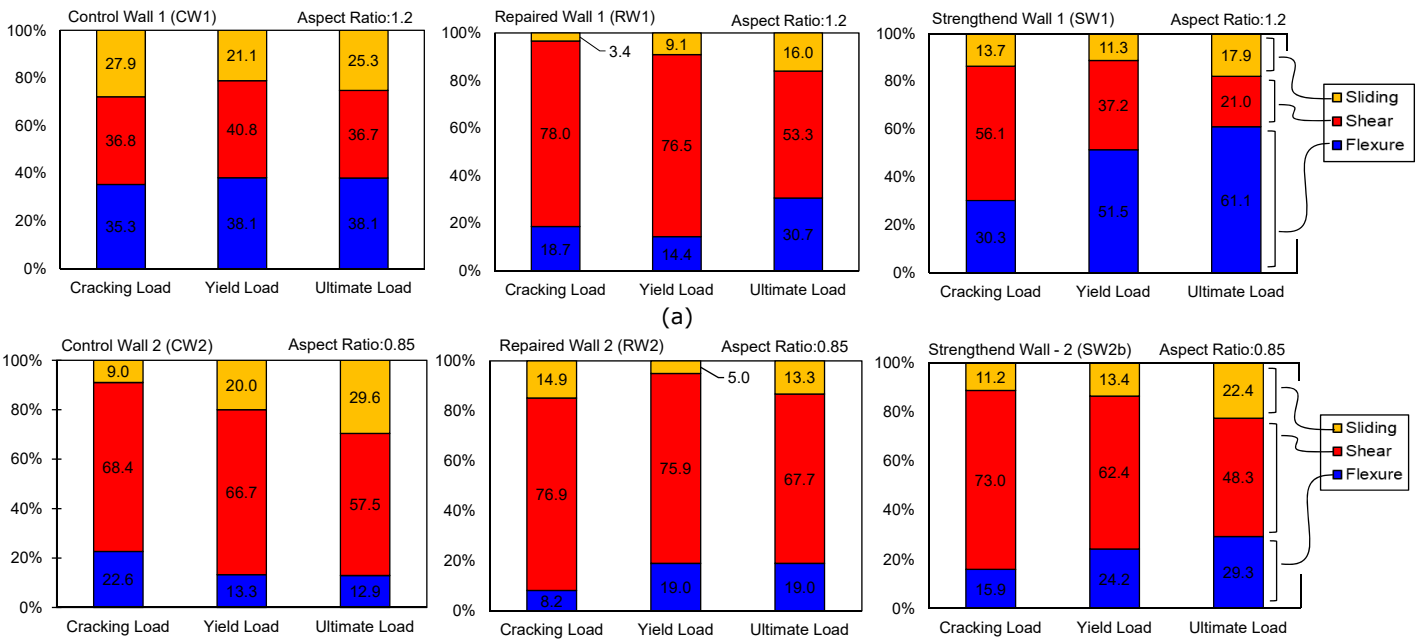

(b)

Figure 5.40 Flexure, shear, and sliding shear displacements: (a) W1; (b) W2 specimens
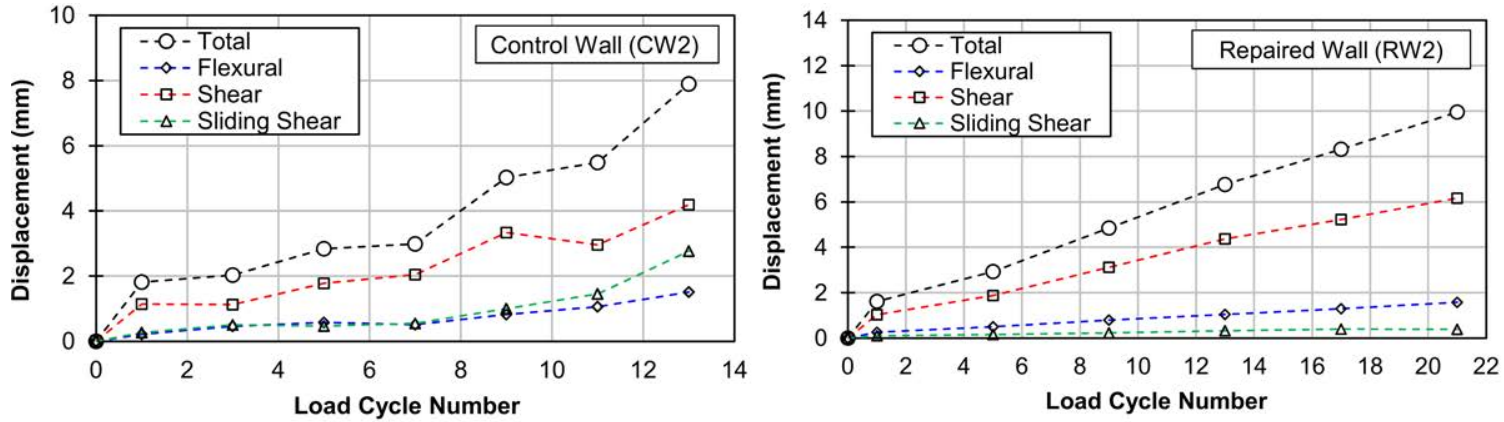

(a)

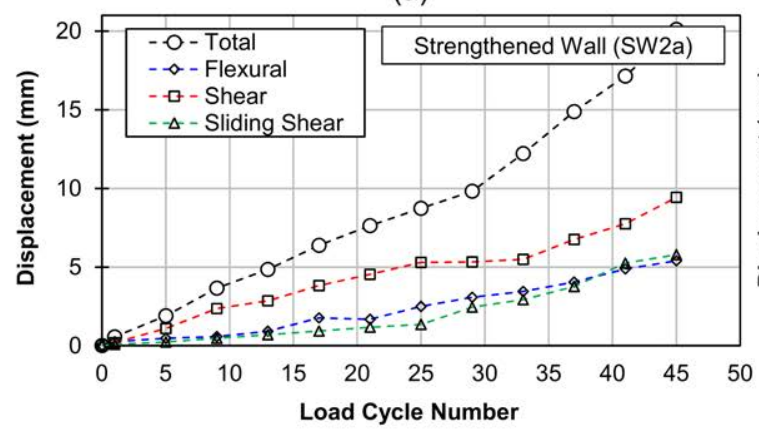

(c)

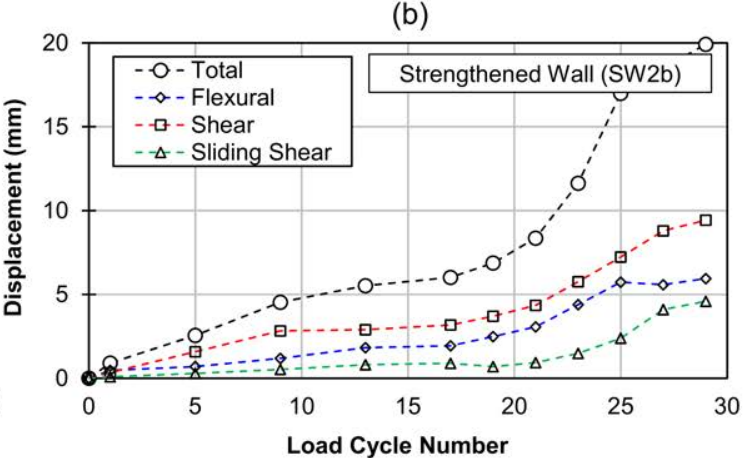

(d)

Figure 5.41 Displacement contribution time-histories: (a) CW1; (b) RW2; (c) SW2a; (d) SW2b 
mode of failure under cyclic loading (Salonikios et al., 1999). To assess the validity of current design procedures for low aspect ratio RC shear walls repaired or strengthened with externally bonded FRP reinforcement, the design equations used to predict the maximum strength and governing mode of failure of the specimens are compared with experimental results. Table 5.5 presents the summary of the results. The design strength against diagonal tension shear failure is the average of the 5 approaches previously presented (ACI 318-68, ACI 318-05, CSA A23.3-04, Wiridinata (1985), and Wood (1990)). The results show that the current design methods are capable of predicting the governing failure mode of diagonal tension shear failure in the control shear wall specimens. The design equations predict the control specimens failing in diagonal tension shear well before reaching their flexural strength, which is confirmed by steel strain profiles measured along the base of the specimens. Results show that for specimen CW1, Wood's model (1990) provides the best estimate of the diagonal tension shear strength of the specimen, with only $2.8 \%$ difference between predicted and measured results. For specimen $\mathrm{CW} 2$, the design equations are able to predict the onset of diagonal tension shear as the governing mode of failure, but all approaches significantly underestimate the shear strength. The models developed by Wood (1990) and Wiradinata (1985) have the closest predicted failure load compared to those measured during the test, but the models still underestimate the experimental result by approximately $45 \%$. Results show that all five approaches are conservative for wall specimens with aspect ratios less than 1.0. However, for specimens with aspect ratios equal to 1.2, the design equations yield a better prediction of the diagonal tension shear strength of the wall. Models developed specifically for walls with aspect ratios less than 1.5 by Wood (1990) and Wiradinata (1985) provide a more accurate prediction of the diagonal tension shear strength when compared with the design standard procedures.

For specimen RW1, the design equations successfully predict the onset of diagonal compression failure as the governing mode of failure. The predicted diagonal compression capacity of $583 \mathrm{kN}$ correlates well with the average lateral load carrying capacity of $610 \mathrm{kN}$ ( $2.8 \%$ difference). For specimen RW2, design equations once again predict the onset of diagonal compression failure first at $995 \mathrm{kN}$. However, the wall specimen fails in diagonal tension shear at $975 \mathrm{kN}$ due to the 


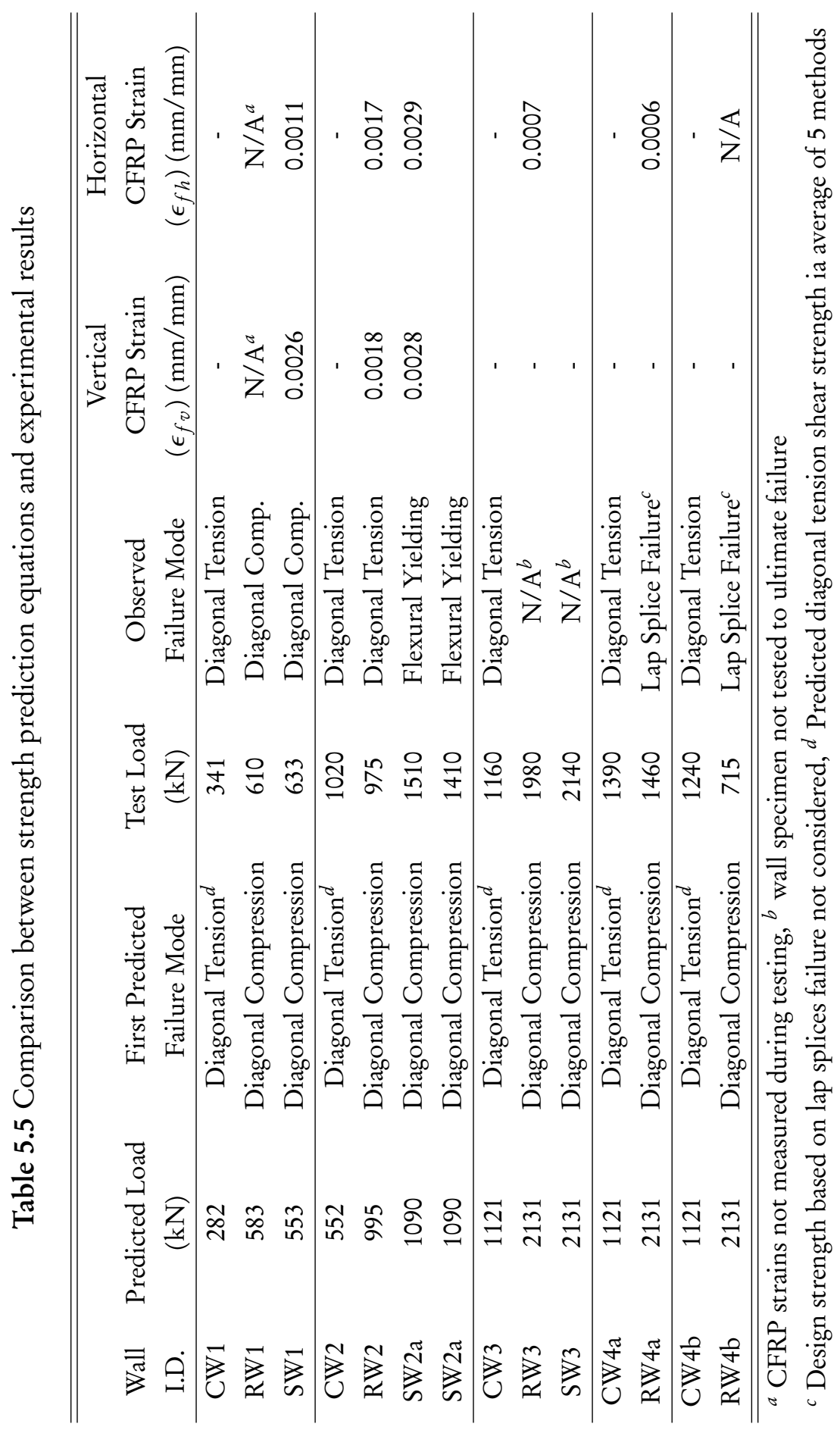


reopening of the previously formed diagonal failure plane. If the contribution of the concrete and horizontal steel reinforcement is ignored when estimating the capacity of specimen RW2, which represents the case where the repaired concrete and remaining horizontal steel reinforcement did not contribute to the diagonal tension shear capacity of the repaired specimen, then the predicted failure load in diagonal tension shear is $1090 \mathrm{kN}$. This suggests that the extensive damage sustained by specimen CW2 during the initial test left the specimen with very little shear strength prior to being repaired using FRP sheets. Nonetheless, the specimen did experience some concrete crushing along its base, which is an indication of diagonal compression failure, suggesting that the design equation gives an accurate prediction of the load for the onset of diagonal compression.

For specimen SW1, design equations correctly identify the governing mode of failure as diagonal compression failure and flexural yielding, predicting a maximum load of $553 \mathrm{kN}$ and $593 \mathrm{kN}$, respectively for the two modes of failure. Although diagonal compression is predicted to occur first, both occur simultaneously during the test. Specimen SW1 reaches an ultimate load of $630 \mathrm{kN}$, which is within $11.3 \%$ and $5.9 \%$ of the predicted strengths against diagonal compression and flexural yielding respectively. For specimen SW2, the design equations predict failure of the specimen in diagonal compression at a lateral load of $1090 \mathrm{kN}$. Although the specimen does experience some crushing along the base, the specimen surpasses the predicted diagonal compression failure load and reaches its flexural capacity at $1510 \mathrm{kN}$, which is within $2 \%$ of the predicted flexural strength from the sectional analysis $(1540 \mathrm{kN})$. Results also show that the sliding shear strength, which is predicted to occur at a lateral load of $1300 \mathrm{kN}$ for specimen SW2, is also underestimated. These results suggest that the code procedures for computing diagonal compression and sliding shear capacity for walls with aspect ratios less than 1.0 are conservative, and the actual strength against these two modes of failure could be significantly higher.

Table 5.5 also shows the maximum strains in the vertical and horizontal CFRP for each specimen. Strain in the CFRP is not measured during testing of specimen RW1. In all cases, the maximum vertical strain in the CFRP is less than the limiting debonding strain of 0.007 and 0.0081 recommended by the CSA S806-02 and ACI 440.2-08 design standards respectively. This is attributed to failure of the specimens in other modes, such as diagonal compression failure or 
diagonal tension shear failure, before the CFRP is able to reach the limiting strain value. Results suggest that limiting debonding strain values for vertical CFRP layers in low aspect ratio walls may be much lower because of the walls inherent shear dominant behaviour. The debonding strain limits for the horizontal CFRP of 0.004 and 0.002 from the CSA 806-02 and ACI 440.2-08, respectively are closer to the maximum horizontal strain measured during testing. ACI 440.2-08 limits the maximum strain in the horizontal CFRP to 0.0022, which is close to the maximum strain measured in the horizontal CFRP during testing of 0.0029 . These results indicate that the number of horizontal CFRP layers applied to the member does have an impact on the limiting debonding strain in the horizontal CFRP, something which is taken into consideration in the ACI 440.2-08 design standard, but is not taken into consideration in CSA S806-02, which assumes a constant limiting debonding strain of 0.004 in horizontal CFRP layers.

In the series 3 and 4 wall specimens, it is more difficult to compare the code prediction equations with the observed behaviour because the wall specimens were either not tested to failure, or lap splice failure occurred before the onset of flexural yielding, diagonal tension shear failure, diagonal compression shear failure, or sliding shear failure. In control wall specimens CW3, CW4a, and CW4b, the average of the prediction equations does a good job at approximating the diagonal tension shear capacity of the shear wall. Wall specimens RW3 and SW3 were not tested to failure because of premature failure in their foundation blocks, so it is not possible to compare the predicted/observed design strength and failure mode. For specimens RW4a and RW4b, failure of the bond between lapped steel reinforcement occurred before the onset of one of the 4 considered failure modes. Nonetheless, the results demonstrate that the considered methods do a reasonable job at predicting the diagonal tension shear strength of squat RC shear walls.

\subsection{Comparison of FRP and Tube Anchor System Performance}

Figure 5.26 compares the hysteretic response behaviour for walls SW2a and SW2b. Results show that at the global response level, both wall specimens perform comparably, with only minor differences in strength, ductility, and energy dissipation capacity. The initial stiffness of wall SW2a is $394 \mathrm{kN} / \mathrm{mm}$ compared with $360 \mathrm{kN} / \mathrm{mm}$ for wall SW2b. The $9 \%$ difference in initial 
in-plane stiffness is not attributed to the different anchor systems but instead the distribution of the vertical FRP sheets on each side of the wall as well as the precise placement of the vertical flexural steel reinforcement inside the wall, which Cruz-Noguez et al. (2014b) has noted can have an impact on the initial in-plane stiffness of a RC shear wall. Wall SW2a reaches a maximum average lateral load carrying capacity of $1500 \mathrm{kN}$ and ultimate drift of $1.2 \%$. In comparison, wall SW $2 \mathrm{~b}$ has a maximum load carrying capacity of $1400 \mathrm{kN}$ and an ultimate drift of $1.05 \%$. In terms of displacement ductility, which is taken as the ratio of the ultimate displacement to the yield displacement, wall W1 has a slightly higher displacement ductility of 2.91, compared to 2.57 for wall specimen W2. However, because wall specimen W2 had all of the FRP layers applied to a single side of the wall, it is difficult to differentiate the influence of each anchor system on the global response behaviour of each wall specimen.

A good measure for comparison of the performance of each anchor is the FRP strain profiles for the vertical CFRP sheet along the base of the wall. Figure 5.42 and 5.43 show the vertical and horizontal CFRP strain profiles at three comparable lateral load levels for each wall. Note that similar behaviour is observed in the opposite load direction, so only one load direction is shown for simplicity. Five strain gages installed directly on the vertical FRP layer $100 \mathrm{~mm}$ above the foundation along the length of each wall are used to monitor the strains in the vertical CFRP reinforcement. For wall SW2a, the results show a decrease in strain near the edge of the wall in tension. This strain loss is attributed to a wide sliding shear crack that developed along the

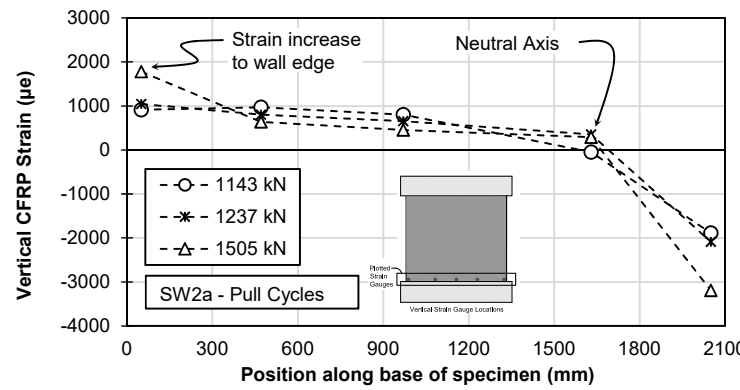

(a)

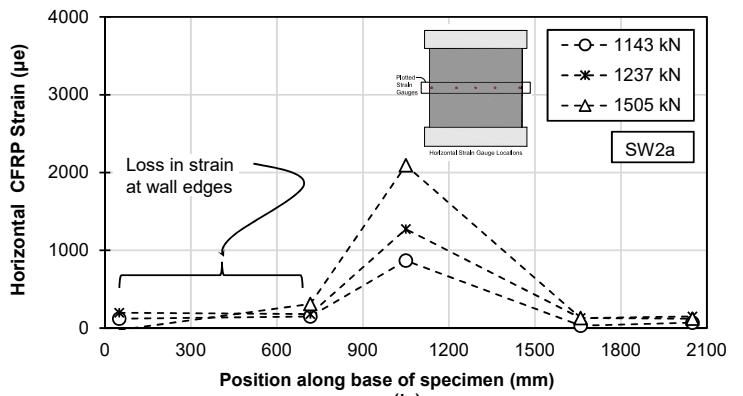

(b)

Figure 5.42 Specimen SW2a strain profiles: (a) vertical CFRP; (b) horizontal CFRP 


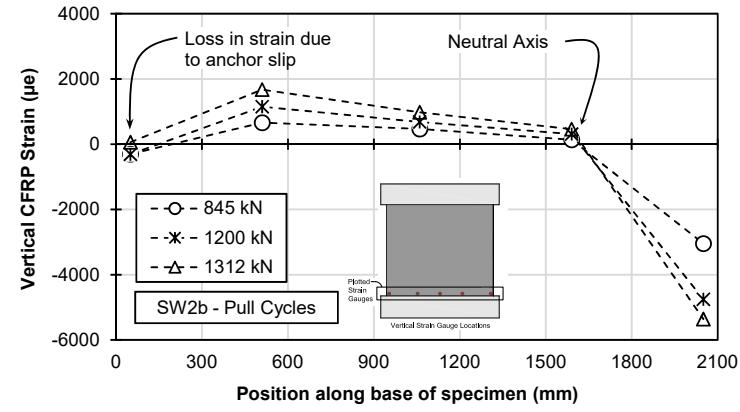

(a)

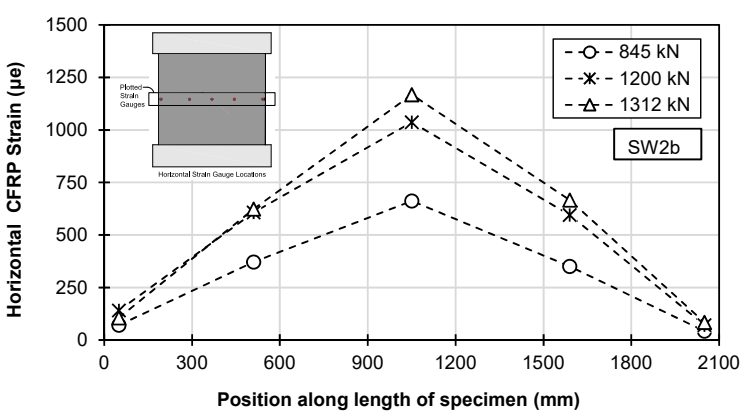

(b)

Figure 5.43 Specimen SW2b strain profiles: (a) vertical CFRP; (b) horizontal CFRP

base of the wall, which causes uplift of the CFRP sheet on the top of the foundation, resulting in debonding of the CFRP sheets from the concrete substrate near the edges of the shear wall. These behaviors resulted in relaxation of the CFRP sheets close to the edges of the wall specimen. Figure 5.44a shows the sliding shear crack beneath the tube anchor and debonding between the FRP laminate and the concrete foundation. Away from the edge of the wall, the crack in the concrete at the wall-foundation interface is not as wide and does not cause relaxation of the vertical CFRP sheet, resulting in higher strain levels towards the interior of the wall.

In wall SW2b, a similar loss in strain near the edge of the wall is observed, however, in this case the loss in strain is attributed to slippage between the bundle of CFRP fibers and the concrete foundation. During testing of wall W2, small slippage between the bundles of CFRP fibers and the concrete foundation was observed, particularly close to the edge of the wall in tension during each load cycle. When under tension, the vertical fan anchors made a distinct "popping" noise as the bundled CFRP fibers slipped from the concrete hole, contributing to the loss in strain carried by the vertical CFRP sheets at these locations. At the ultimate load, the CFRP laminate and vertical anchors at the edges of the wall began to separate from the concrete substrate, as shown in Fig. 5.44b, which could also have contributed to the loss in strain in these regions. Comparing the strain profiles in the two anchor systems away from the edge of the shear wall, tensile strains in wall SW2a are comparatively higher, reaching a maximum tensile strains of $3000 \mu \epsilon$ compared to $1630 \mu \epsilon$ for the wall SW2b. This is once again attributed to the fact that some level of slip occurs between the fan anchor dowel and concrete hole depending on the tensile force carried by 
the CFRP sheets at that location, resulting in the lower tensile strains compared to wall SW2a. However, the vertical compressive strains in wall SW2b are much higher compared to wall SW2a, reaching maximum compressive strains of $5360 \mu \epsilon$ and $2890 \mu \epsilon$, respectively. This is attributed to the fact that there were 8 layers of CFRP on only one side of wall SW2b, in addition to the fan anchors which were placed between vertical CFRP sheets. This created a much thicker layered composite section that was capable of achieving higher compressive strains prior to debonding and buckling of the FRP sheets in compression. The strain profiles in figures 5.42a and 5.43b also show the location of the neutral axis for each shear wall specimen, which is very similar in both cases and is located at approximately $75 \%$ of the shear wall length. This lies within the suggested limits from past results discussed previously and confirms that the assumed neutral axis location of $0.85 l_{w}$ used in the design methodology for the tube anchor system was a conservative assumption for this study.

Finally, it should be noted that the maximum vertical tensile strain observed in the CFRP of walls SW2a and SW2b are much lower than the ultimate tensile strain of the CFRP laminate used in this study $\left(\epsilon_{u}=1.0 \%\right)$. This is due to the fact that walls W1 and W2 are squat shear wall specimens, which are typically characterized as having an aspect ratio $\left(h_{w} / l_{w}\right)$ less than 1.5 . The aspect ratio for walls SW2a and SW2b is 0.85 , which leads to high shear stresses causing failure before significant yielding of the flexural steel reinforcement and the development of large strains in the vertical CFRP composite. Both walls reached their yield loads, characterized by yielding of the vertical flexural reinforcement but failed in shear shortly thereafter. Figure 5.29 shows the damage sustained by both walls at failure and the FRP debonding progression for both tests. In wall SW2a, FRP-concrete debonding occurs progressively, starting at the edges of the wall, and the debonded area becomes larger with each load cycle until failure. In wall SW2b, a similar final FRP-concrete debonding pattern is noted, but the separation of the CFRP sheet from the concrete substrate and the shear failure of the wall occurs suddenly, after brittle failure of the horizontal GFRP fan anchors along the edge of the wall (Fig. 5.29b). This shows that the presence of FRP anchorage can influence the failure mechanisms and observed failure pattern of an FRP retrofitted RC member, something that should be taken into consideration during 

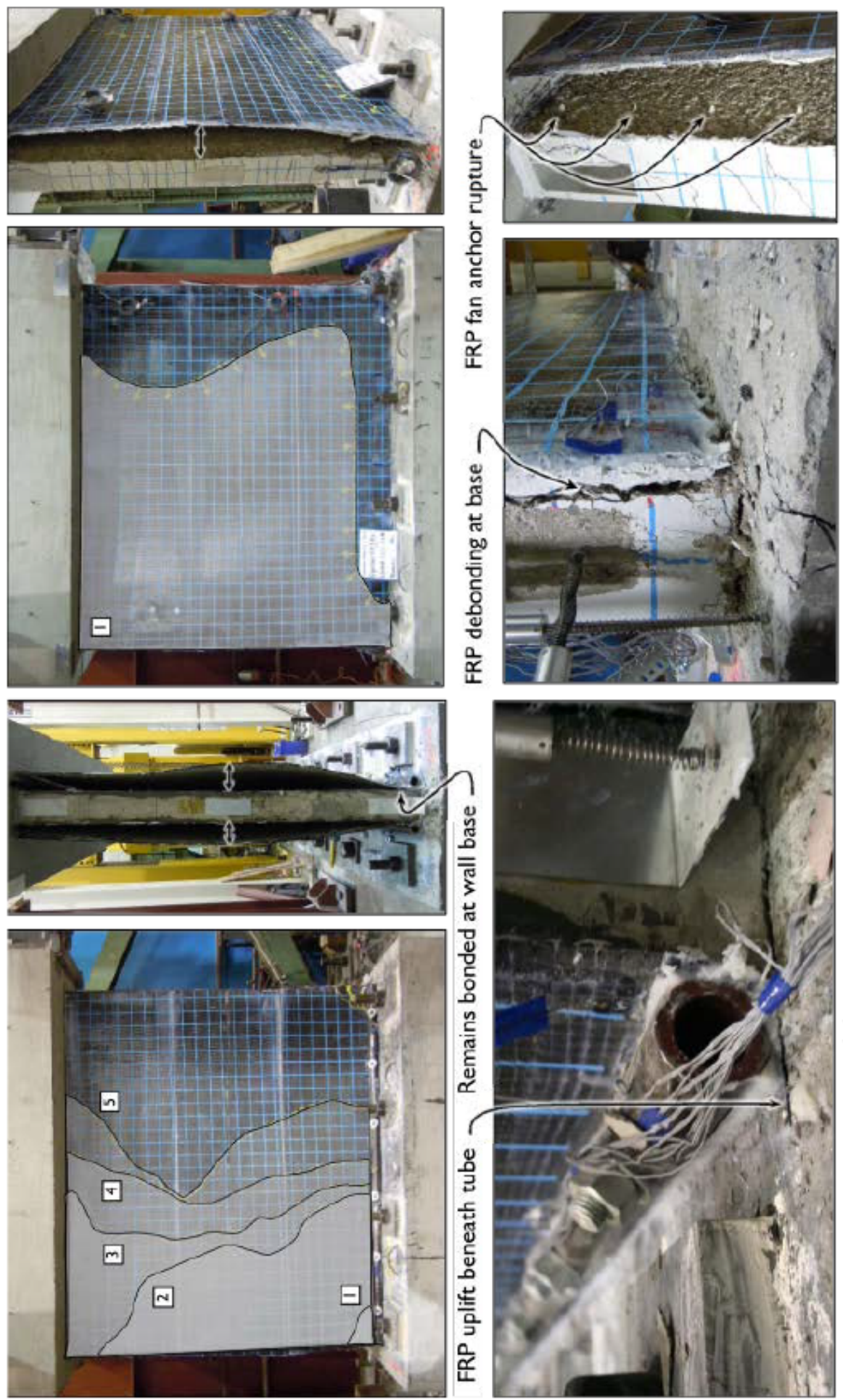

ปี 
the design of the FRP retrofit to ensure a brittle failure mode is not unintentionally introduced. Future experimental studies will test the tube anchor system in a flexurally dominant shear wall element and other RC members (beams, columns, and slabs) to get a better understanding of the behavior of the tube anchor at the ultimate tensile strength of the vertical FRP sheets and in other potential applications. Nonetheless, both anchor systems were shown to be effective in providing anchorage of the vertical CFRP sheets to increase the flexural capacity of the walls when compared to control shear walls tested without CFRP sheets. The design procedure for the tube anchor system is also shown to be successful in reaching optimal design of the tube anchor without the need to conduct detailed finite element simulations on the specific tube geometry and anchor bolt spacing.

\subsection{Finite Element Modelling of CFRP Retrofitted Walls}

Much of the research presented and discussed in Part 1 of this thesis has focused on experimental results from a series of tests conducted on deficient RC shear walls retrofitted with externally bonded CFRP sheets. During this experimental program, a parallel study has focused on the development of a robust finite element modelling approach for FRP retrofitted RC shear walls that is used to validate the observed experimental results. The following section provides a brief overview of this parallel research program to provide completeness to this work.

The finite element modelling approach developed to predict the force-deformation response of the deficient RC shear walls specimens tested in this study is based on the originally developed modelling approach by Cruz-Noguez et al. (2014c). This approach used the finite element software Vector2, developed at the University of Toronto, to analyze the cyclic response of the wall specimens with modern design details tested by Hiotakis (2004) and Lombard et al. (2000). The Vector2 finite element modelling software has been specifically designed for modelling RC structures, and is based on the modified compression field theory (MCFT) formulated by Vecchio and Collins (1986a). In this formulation, the concrete is modelled as an orthotropic material with smeared rotating cracks. The shear wall specimens are modelled using four node rectangular elements with smeared steel reinforcement, while the CFRP sheets are modelled using discrete truss 
elements.

To capture the interaction between the concrete and the CFRP laminate, which ultimately leads to debonding or separation of the CFRP sheet from the concrete substrate, two spring elements linking the CFRP reinforcement to the concrete substrate were defined in orthogonal directions. In this approach, the horizontal spring element represents the shear behaviour of the CFRP-concrete interaction and a longitudinal spring represents the normal behaviour. Each of these springs was assigned a bond-slip relationship. To capture debonding, when the crack width in the adjacent concrete element reaches the critical bond stress value, the bond strength is set to zero, effectively separating the FRP truss from the concrete element. To optimize this process, a subroutine was created to monitor crack widths in the concrete elements throughout the analysis and track the debonding progression during the cyclic loading. Additional information on the aforementioned modelling approach, bond-slip models, and debonding subroutine are available in Hassan et al. (2013) and Cruz-Noguez et al. (2014a,c).

Results of the study by Cruz-Noguez et al. (2014c) found that this modelling approach was effective in predicting the cyclic response of FRP-strengthened RC shear walls designed according to what would be considered modern seismic design standards. Figure 5.45 compares the forcedisplacement response of a strengthened shear wall specimen tested by Hiotakis (2004) (SW1-1) with the finite element modelling results from Cruz-Noguez et al. (2014c). The results show that the finite element model does a good job at predicting the strength, ductility, and energy dissipation capacity of the strengthened wall specimen. Figure 5.45 also shows the results from a repaired wall specimen (RW1-1). The results show that in repaired walls, the analytical model is able to capture the ultimate strength of the repaired wall specimen but is not able to accurately capture the complete force-deformation response. Nonetheless, the study ultimately demonstrated the effectiveness of the proposed modelling approach and its ability to accurately capture the nonlinear response of FRP-retrofitted RC shear walls.

Based on the developed modelling approach by Cruz-Noguez et al. (2014c), Hassan (2015) extended the approach such that it was applicable for modelling the deficient RC shear walls tested in Part 1 of this thesis, however, some additional development and modification to the original 


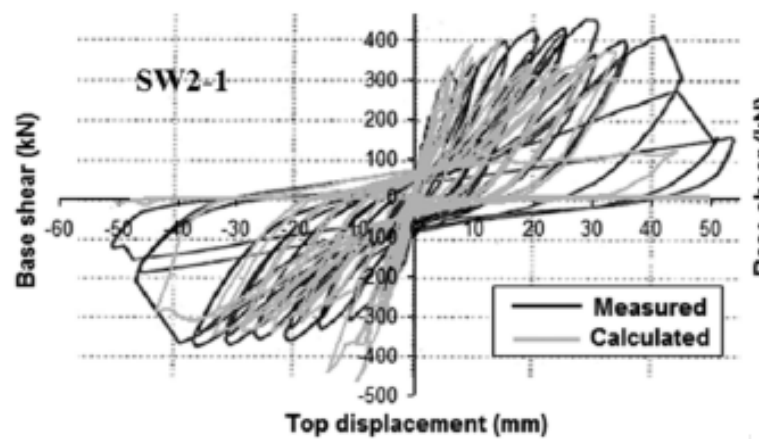

(a)

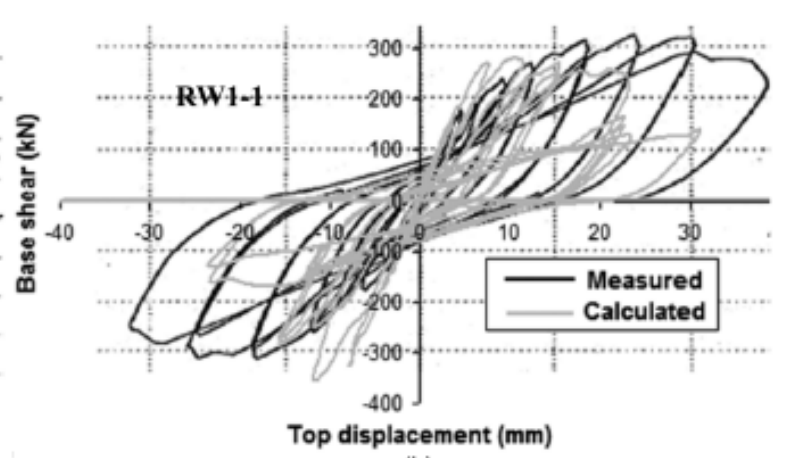

(b)

Figure 5.45 Force-displacement response comparison: (a) strengthened wall; (b) repaired wall (From Cruz-Noguez et al., 2014c)

subroutine was required because of the shear dominant response of the deficient RC shear walls tested in this study. Hassan (2015) found that because of the presence of many diagonally oriented shear cracks in the response of the deficient RC shear wall specimens, the original FRP-concrete debonding subroutine developed by Cruz-Noguez et al. (2014c) was no longer accurate because it did not account for the presence of diagonally oriented cracks in the concrete. Hassan (2015) found that because of the presence of diagonally oriented cracks in the concrete, compared with many horizontally oriented flexural cracks observed during testing of the walls tested by Hiotakis (2004) and Lombard et al. (2000), the FRP-concrete interaction was two-dimensional, and thus the properties of the link elements that connected the concrete and FRP together were modified to account for this two-dimensional debonding behaviour. Additional information related to the development of the two-dimensional debonding subroutine can be found in Hassan (2015); Hassan et al. (2015) and Hassan and Lau (2016).

Figure 5.46 compares the response of wall specimens SW1 and SW2a in this study with the finite element modelling results produced by Hassan (2015). The results demonstrate that the finite element model with the two-dimensional debonding subroutine does a good job at predicting the strength and cyclic behaviour of the FRP-strengthened deficient RC shear walls. The models were also found to be able to accurately predict the failure mechanisms in all of the tested walls, including brittle diagonal tension shear failure in the control wall specimens and diagonal 
compression shear failure and FRP-concrete debonding in the strengthened wall specimens. Additional modelling results can be found in Hassan (2015); Hassan et al. (2015) and Hassan and Lau (2016).

The parallel finite element studies aimed at replicating the experimental results presented in Part 1 of this thesis are still ongoing. Finite element models are being developed to capture the response of the Series 3 and 4 squat wall specimens, including developing a modelling approach that is able to capture the bond-slip response of RC shear walls with lap splices. Some additional information on these ongoing studies can be found in Shaheen (2014).

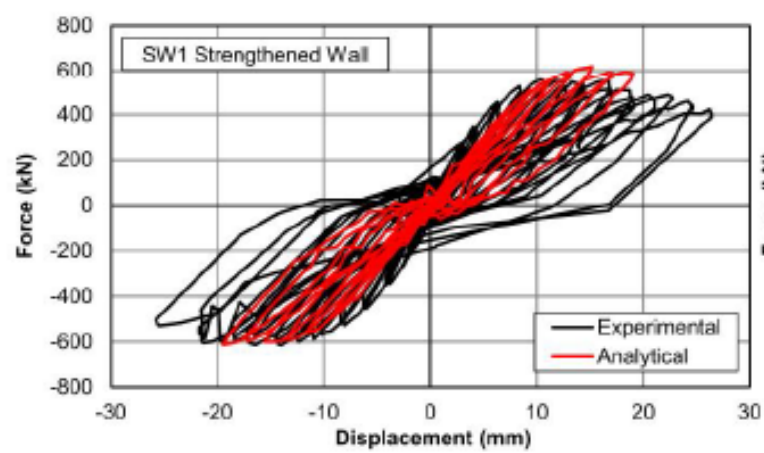

(a)

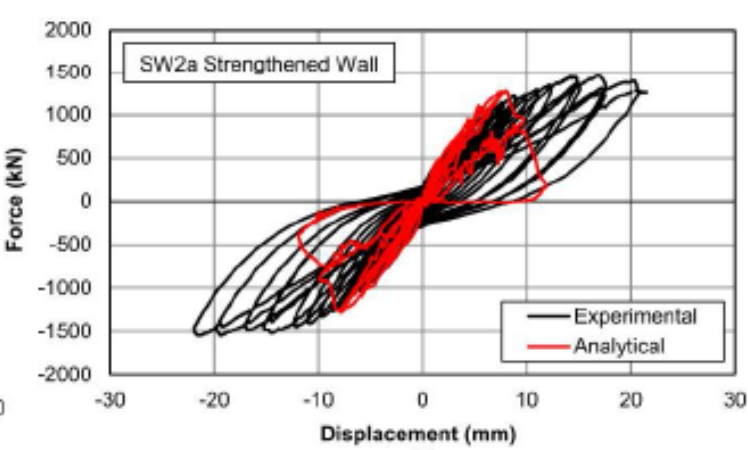

(b)

Figure 5.46 Force-displacement response comparison: (a) SW1; (b) SW2a (From Hassan, 2015) 


\section{Chapter 6. Conclusions and Future Research}

\subsection{Conclusions}

Part I of this thesis presented results related to the performance of RC shear walls designed according to older design standards, specifically CSA A23.3-77 and ACI 318-68. To improve the performance of wall specimens with the common deficiencies from older design standards, an innovative CFRP retrofitting system was investigated. The system consists of uni-directional externally bonded CFRP sheets applied in the vertical and horizontal directions to increase the flexural and shear capacity of the specimens. The following conclusions can be made from this experimental research program:

1. Reinforced concrete shear walls with common structural deficiencies from CSA A23.3-77 and ACI 318-68, including insufficient shear reinforcement, poor confinement of the boundary elements, and lap splices of the vertical steel reinforcement are susceptible to sudden and brittle diagonal tension shear failure under in-plane loading. Walls with these deficiencies have poor seismic performance, including insufficient ductility and energy dissipation capacity;

2. In strengthening applications, for walls without pre-existing damage, results of the CFRP retrofitting system proved that it is capable of preventing premature diagonal tension shear failure and improving the seismic performance of wall specimens, including improving the in-plane load carrying capacity, ductility, and energy dissipation capacity; 
3. In repair applications, for shear walls with pre-existing cyclic damage, the CFRP retrofitting system was shown to be effective in, at a minimum, restoring the in-plane strength and lateral displacement capacity of the wall specimen to its original state, even in severely damaged walls. In all of the repaired wall specimens, premature debonding of the CFRP laminate from the concrete substrate due to the opening of pre-existing cracks in the concrete continues to be a limiting factor in the response of the specimen;

4. In applications for walls with lap splices of the vertical steel reinforcement near the base of the wall, the experimental results showed that particular attention should be paid to assessing the damage to the lap splice bond, which has a significant impact on the effectiveness of the CFRP retrofit. In scenarios where there is significant damage to the lap splice region, more instrusive retrofitting scenarios that attempt to restore the bond between bars is required;

5. The shear strength of a wall specimen can be significantly improved even without wrapping the CFRP sheet around the shear wall, significantly reducing the intrusiveness of the retrofitting strategy for walls that may be difficult to access or impossible to provide additional effective confinement at their ends, such as a C-shaped, T-shaped, or L-shaped shear wall. The application of vertical CFRP layers helps to restore the initial in-plane stiffness in damaged wall specimens and increase the initial in-plane stiffness in strengthening applications;

6. Experimental and analytical results demonstrate effective performance of the steel tube anchor system in preventing premature debonding of the CFRP sheets along the base of the wall and allowing the vertical CFRP layers to carry stresses and contribute to the in-plane load carrying capacity of the wall, even after the CFRP sheet debonds at the base of the wall;

7. The described design methodology for the steel tube anchor system provides a simple and effective design tool that can be used to design effective CFRP anchor systems, something that is not available in current design standards; and 
8. The performance of an alternative FRP fan anchor system is also shown to be effective in delaying premature debonding of the CFRP layers from the concrete substrate. Specifically, anchor placed along the edges of the shear wall to anchor the horizontal CFRP layers are shown to delay debonding and allow the horizontal CFRP sheet to carry larger strain, thus stresses.

\subsection{Recommendations for Future Research}

This research has focused on a small section of a large research area on the performance of RC structures retrofitting with advanced composite materials. The following include several topics/initiatives suggested for further investigation:

1. Testing of multi-storey shear walls detailed according to modern and older design standards and repaired or strengthened using externally bonded FRP sheets;

2. Research the potential effects of the out-of-plane deformation response of RC shear walls to seismic excitation on the in-plane stiffness of the wall;

3. Testing of deficient FRP strengthened and repaired RC shear walls including the effects of axial load, simulating the gravity load of the structure supported by the shear wall and investigated the effects this has on the seismic performance and observed failure modes of the shear wall;

4. Application of the tube anchor system in the seismic retrofit of circular columns and curved walls as well as other RC elements including beams and slabs;

5. Develop simple design charts for the tube anchor system, such that the designer can easily select an appropriately sized tube without the need to conduct detailed finite element analysis or experimental testing; and

6. Study the performance of FRP-retrofitted RC shear walls under the effects of one or more alternative hazards, including blast, impact, and fire. This research could provide insight into the adoption of a performance based design approach for FRP retrofitted RC structures. 


\section{Part II}

\section{Building Scale Performance Evaluation using Hybrid Simulation}




\section{Chapter 1. Introduction}

\subsection{Motivation}

Reinforced concrete (RC) shear walls are a lateral load resisting structural system predominantly used to resist wind and earthquake loads. The construction of cast-in-place RC shear walls began in the 1960s and 1970s, at a time of intense construction following the end of the second world war (Fintel, 1995). During this time, the lack of proper analytical tools to predict the behaviour of RC shear wall structures under lateral loads led to poor performance of these types of structures during moderate to large earthquakes. Consequently, it was a common perception at the time that the $\mathrm{RC}$ moment resisting frame was a superior alternative to shear wall buildings because of their inherent flexibility and ductility, which would reduce seismic forces on a structure. The reason for such poor performance of shear wall buildings during the time was because of their inherent high stiffness, causing them to attract higher seismic forces during an earthquake when compared with a RC moment frame. Without the appropriate levels of ductility and energy dissipation capacity provided by proper detailing of longitudinal and transverse steel reinforcement, this led to brittle behaviour of RC shear wall buildings during earthquakes throughout the 1960s (Chile, 1960; Macedonia, 1963; Venezuela, 1967) (Fintel, 1995). However, research over the past five decades on the seismic behaviour and performance of RC shear wall structures has shed light on their ability to resist earthquake loads and provide a dependable ductile response when adequate detailing of horizontal and vertical steel reinforcement are provided. 
More recently, with the development of advanced computer simulation techniques, experimental testing to study the nonlinear behaviour of RC shear walls, and the collection of earthquake ground motion records, engineers have established a better understanding of how shear wall buildings behave during earthquakes. These advancements in the seismic design of RC structures are reflected in the current design standards for RC structures used in North America (ACI 31814; CSA A23.3-14) (ACI, 2014; CSA, 2014a). The performance objective adopted in these design standards for earthquake resistance is life safety: preventing loss of life and injury of a structure's occupants during a design earthquake. In the case of a moderate to major earthquake, RC shear wall structures are expected to be damaged and behave inelastically, relying on ductility and energy dissipation through yielding of vertical steel reinforcement and crushing of concrete to prevent structural collapse. The recent development of displacement-based or performance-based seismic design has led to the adoption of separate performance objectives for different hazard levels of earthquake events, in addition to life safety. For small to medium earthquakes expected to occur once or multiple times during the service life of a structure, additional basic objectives such as limiting damage and reducing economic loss are also considered. In most-cases, four basic performance levels are defined: fully operational, operational, life safe, and near collapse (FEMA, 2000). The transparent definition of these four objectives gives flexibility to the owner of a structure to choose how their building will preform during an earthquake and its functionality afterwards.

To meet the multiple performance objectives of performance-based seismic design, engineers are required to have detailed and accurate computer models to predict the nonlinear behaviour of RC shear wall structures under earthquake loads. Modern computer simulation programs allow engineers to model complex structures under the load effects of an earthquake, however, it remains a challenge to accurately capture the nonlinear behaviour of critical elements analytically. These critical elements are those in a structure that are expected to be damaged during an earthquake and exhibit highly nonlinear behaviour to dissipate seismic energy.

Traditionally, experimental testing has been used as a tool to capture the inelastic behaviour of critical structural components. The results of such a test provides detailed information on the inelastic behaviour of a structural element, including its ductility, energy dissipation capacity, 
and local failure mechanisms. With respect to RC shear walls, their seismic behaviour has been studied extensively through the use of reversed cyclic testing, in which a RC shear wall is subjected to the load and/or drift demands associated with an earthquake. Although these techniques are sufficient to determine the ductility and energy dissipation characteristics of a single structural element, it fails to capture the global response of the complete structural system.

Alternatively, large shaking table tests have been conducted on full-scale or near full-scale RC shear walls and RC shear wall buildings to study their global performance (Panagiotou et al., 2010; Fleischman et al., 2015; Astroza et al., 2016; Ghorbanirenani et al., 2011). Although these test are effective in determining the seismic behaviour and response of RC shear walls and RC shear wall structures, these tests are often limited to a single configuration of a structure and require significant investment of time and money both in terms of the experimental test specimen as well as the facility in which the test is conducted.

Recently, an experimental technique known as hybrid testing has gained significant traction in the research community. The hybrid test method essentially bridges the gap between quasistatic testing and shaking table tests. It combines physical testing in a laboratory with numerical simulation on a computer to capture the response of large-scale structures in an economically feasible manner. Elements of a structure that are expected to behave in a linear, predictable manner are modelled numerically on a computer, while critical elements that are expected to exhibit highly nonlinear behaviour and are difficult to capture analytically are experimentally tested in a laboratory. The innovative aspect of the hybrid test method is the relationship between the analytical model and experimental test, which are required to interact with one another at each time-step for the duration of the earthquake ground motion. In this approach, the hybrid testing method provides an ideal balance between the efficiency of a computational model and the accuracy of an experimental test. Hybrid test results provide more accurate information on the global performance of a structure when compared to purely numerical finite element analysis because the exact nonlinear response of the critical structural components is captured in the lab. Hybrid testing is also more efficient when compared with full-scale shaking table tests because only a portion of the structure is physically tested in the lab. In addition, the hybrid test method also 
provides information related to the local response of the critical structural element that would have traditionally been obtained during conventional component-level experimental testing, such as ductility, energy dissipation capacity, and failure mechanisms. Ultimately, hybrid testing is an important tool in improving our understanding of the seismic behaviour of large-scale civil engineering structures. The long-term goal is to use hybrid simulation in the development of more accurate numerical models, which can in turn be used to assist design engineers in determining if a particular structure will be able to meet the multiple objectives associated with performance based seismic design.

\subsection{Methodology}

Experimental and numerical research on the seismic performance of a prototype RC shear wall building has been conducted to study its dynamic behaviour under earthquake loads. The prototype structure is a three-storey RC shear wall building designed according to modern seismic design standards (ACI 318-14; CSA A23.3-14) (ACI, 2014; CSA, 2014a). The novelty in this research stems from the fact that although the hybrid testing technique is not new, much of the work to date on hybrid testing has focused on the development of control algorithms, numerical integration schemes, and inter-ware platforms. Hybrid testing has been used in limited applications to study the performance of flexible steel and concrete structures, but there has been comparatively less research related to the use of hybrid simulation in stiff structures, more specifically, RC shear wall structures. This is in part because of the challenges stiff structural systems present on the control side of the hybrid simulation, which is a specific area of focus in this study. In addition, the majority of the hybrid simulations conducted to date have used discretized beam column elements to model the analytical substructure. This study focuses on the use of shell elements in the analytical model of the RC shear wall structure. The research objectives and scope at the onset of this hybrid simulation project are described in the following section. 


\subsection{Research Objectives and Scope}

The main objective of this research is to develop and assess the feasibility of using hybrid simulation as a tool to study the seismic performance of RC shear wall structures. Other objectives of the research include:

- Develop an accurate and efficient numerical modelling technique for RC shear walls using the earthquake engineering simulation software OpenSees;

- Combine the developed numerical modelling approach with the hybrid simulation software OpenFresco to allow for the hybrid simulation of a multi-storey RC shear wall;

- Investigate and develop solutions to the challenges surrounding the use of hybrid simulation in stiff structural systems;

- Implement and asses the ability of modern control software to control multiple degrees-offreedom in experimental testing;

- Study the seismic behaviour of RC shear walls designed according to modern design standards under varying earthquake intensity using hybrid simulation;

- Assess the feasibility of using hybrid simulation to capture the global response of a RC shear wall structure; 


\section{Chapter 2. Background}

Reinforced concrete (RC) shear walls are one of the most commonly employed lateral load resisting structural systems in seismically active regions around the world. Despite initial presumptions that RC shear wall structures behaved in a brittle manner during earthquakes, advances in understanding the seismic behaviour of RC shear walls through experimental testing and analytical simulation have shown that RC shear wall structures can provide a dependable ductile response. Following a major earthquake event, modern building codes (NBCC 2010; CSA A23.314) for RC structures ensure that the life-safety of the occupants of a structure is preserved. Recent earthquake events around the world (Christchurch, 2011 and Tohoku, 2011) have demonstrated the effectiveness of modern seismic design standards for RC structures in preventing structural collapse and meeting the life-safety performance objective. However, despite the ability of modern seismic design standards to meet the life-safety performance objective, they do not ensure that a structure will remain operationally functional following an earthquake. The loss of operational functionality following an earthquake can lead to unexpected economic losses, something that is not taken into consideration during the initial design of the structure. More recently, there has been a shift in the seismic design philosophy towards designing more resilient infrastructure, which are able to not only survive a moderate earthquake without reaching the collapse state, but also remain operationally functional. This methodology is reflected in the performance based seismic design (PBSD) philosophy. Performance based seismic design provides a means to more reliably predict seismic risk and permits building owners to make a financial decision to obtain a higher level of performance, thereby reducing the risk and potential losses following an earthquake and increasing the resiliency of a structure (SEAOC, 1995; ATC, 1996; FEMA, 1997); and 
FEMA (2000).

A consequence of the shift towards the adoption of a PBSD is that engineers need more accurate computer simulation models to ensure that a specific performance target is met based on the objectives outlined by the client. This poses a significant challenge for practicing engineers, as the use of modern computer simulation tools to accurately predict the inelastic dynamic response of complex structural systems is not trivial. In cases where there is some uncertainty related to the nonlinear behaviour of a structural element, experimental testing provides reliability and accuracy that cannot be established using computer simulation, no matter how complex the modelling tool.

With respect to RC shear walls, experimental testing has been extensively used in the past to characterize and better understand their seismic behaviour and develop modelling approaches to predict their nonlinear behaviour. Much of this research has used reversed quasi-static cyclic testing of single-storey RC shear wall elements (Salonikios et al., 1999; Lombard, 1999; Palermo et al., 2002; Massone and Wallace, 2004; Hiotakis, 2004; Kuang and Ho, 2008; Cruz-Noguez et al., 2014b; Woods et al., 2016) to characterize the nonlinear behaviour of RC shear walls. Although the results from these studies have led to a better understanding of the performance of RC shear walls, the applicability of these results to full-scale structural systems under actual ground motion records is not well understood, resulting in a need to study the global response of multi-storey shear wall systems under actual earthquake ground motion records.

The hybrid simulation technique presented herein provides an economically feasible means for examining the seismic response of complex structural systems using a combination of physical and analytical substructures. Specific components of the structural system that are expected to exhibit a predictable response (ie. those which are expected to remain elastic or near-elastic) under earthquake ground motions are modelled analytically while structural elements that are expected to experience significant nonlinearity are tested experimentally. The following section outlines the background, evolution, and major developments in the establishment of hybrid testing as a globally accessible test method.

Joshua Woods, Department of Civil and Environmental Engineering, Carleton University 


\subsection{Structural Testing of RC Structures}

To date, there have been a wide variety of experimental tests conducted to examine the seismic performance of RC shear wall structures (Oesterle et al., 1976, 1979; Lefas et al., 1990; Pilakoutas and Elnashai, 1995; Salonikios et al., 1999; Lombard et al., 2000; Palermo et al., 2002; Hiotakis, 2004; Massone and Wallace, 2004; Adebar et al., 2007; Kuang and Ho, 2008; Panagiotou et al., 2010; Lowes et al., 2012; Woods et al., 2016). These tests range in complexity from the most cost efficient small-scale pseudo-static tests on individual shear wall components to very costly shaking table tests on full-scale multi-storey RC shear wall structures. Figure 2.1 compares and contrasts some of the benefits and drawbacks of the commonly employed testing techniques used in earthquake engineering.

The most basic experimental test used to establish the seismic performance of a RC shear wall at the component level is a quasi-static reversed cyclic test. Quasi-static reversed cyclic tests involve the application of a predetermined load or displacement sequence to a structural element that is assumed to be representative of the load and/or displacement that the structural element would experience during an earthquake. This testing technique captures the nonlinear behaviour of the structural element and quantifies its important structural response parameters, such as its ductility and energy dissipation capacity, and failure mechanisms. However, the demands imposed

\begin{tabular}{|c|c|c|c|}
\cline { 2 - 4 } \multicolumn{1}{c|}{} & Quasi-Static Test & Shaking Table Test & Hybrid Simulation \\
\hline Dynamics & No & Yes & Yes \\
\hline $\begin{array}{c}\text { Strain Rate } \\
\text { Effects }\end{array}$ & No & Yes & $\begin{array}{c}\text { Yes } \\
\text { (If at Real-Time) }\end{array}$ \\
\hline $\begin{array}{c}\text { Large } \\
\text { or } \\
\text { Full-Scale }\end{array}$ & Yes & $\begin{array}{c}\text { Limited by Shake Table } \\
\text { Capacity and Cost }\end{array}$ & Yes \\
\hline
\end{tabular}

Figure 2.1 Characteristics of structural test methods in earthquake engineering 
on the test specimen, although well-intentioned, may not be directly related to the observed demands during an earthquake, leading to the potential for under or over-testing of the structural element (Schellenberg et al., 2009). Furthermore, the application of a quasi-static cyclic loading does not account for the constantly changing load distributions the structure experiences during an earthquake, does not account for strain-rate effects, and does not provide any information pertaining to the global response of a structure during an earthquake.

Alternatively, shaking table tests account for some of the shortcomings associated with the quasi-static test method, including its ability to closely match the boundary conditions, accelerations, and velocities a structure experiences during an earthquake. Shaking table tests are able to provide realistic results on how structural systems and elements will behave under specific ground motions, including inertial and energy dissipation capacity, yielding mechanisms, failure mechanisms, and strain-rate effects. However, because there are practical limitations on the size and capacity of most shaking tables, researchers are often forced to test only a single structural configuration at a smaller scale, which again raises the same questions around the applicability of the results when compared with what is expected during an actual earthquake. For large-scale shaking tables, such as the shake table at the University of California San Diego or E-Defense in Japan, which are capable of testing full-scale structures, the investment in terms of time for construction and high cost raises questions about its practicality.

The following section discusses the hybrid simulation test method, a structural testing method that bridges the gap between quasi-static experimental tests and shaking table tests. The hybrid test method combines the benefits of component-level testing with the capabilities of modern finite element analytical tools to study full-scale civil engineering structures. Combining the benefits of experimental testing and analytical modelling, hybrid simulation provides a means to study a dynamic response of a full-scale structure, potentially including the effects of strain-rate if the hybrid test is conducted in real-time, in a cost effective and accurate manner. 


\subsection{Hybrid Simulation}

\subsubsection{History of Hybrid Testing}

The concept of hybrid testing was first introduced by Takanashi et al. (1975) as an alternative to shake table testing. During this time, researchers were examining different available methods for capturing the dynamic behaviour of structural elements and systems. Takanashi et al. (1975) referred to the testing technique as "online testing" because of its potential to be used for geographically distributed testing, where more than one physical test specimen is tested at one or more laboratories around the world. At that time, there were limitations on the wide-spread adoption of hybrid simulation as a test method because of the lack of fast and reliable testing and computational hardware (Schellenberg et al., 2009). Over the past three decades, hybrid simulation has evolved into a practical and viable alternative to shaking table tests given the advancement in computational capabilities and experimental testing equipment (Mahin and Shing, 1985; Takanashi and Nakashima, 1987; Mahin et al., 1989; Shing et al., 1996; Magonette and Negro, 1998).

One of the biggest initial challenges in the implementation of hybrid testing was the delay between the application of the displacement on the physical test specimen and the time required to complete the next time-step in the numerical simulation. This waiting time, which is referred to as "ramp-and-hold" loading would result in relaxation of the test specimen and diminished the applicability of the results for a dynamic test. However, the ramp-and-hold loading method was quickly replaced with continuous command signal generation to provide improved results by reducing the relaxation of the test specimen during the "hold" sequence of testing. Continuous testing techniques have since been implemented in real-time environments to ensure that the commands sent to the actuators in the laboratory are updated at deterministic rates. The concept of continuous testing is discussed in more detail later in this chapter. Continuous testing is particularly important for applications of real-time hybrid testing, in which the load is applied and the next step of the numerical integration is completed real-time. Techniques for the implementation of real-time hybrid testing have been extensively studied in the past but will not be

Joshua Woods, Department of Civil and Environmental Engineering, Carleton University 
described in detail or employed here (Nakashima et al., 1992; Darby et al., 1999; Horiuchi et al., 1999; Nakashima and Masaoka, 1999; Darby et al., 2001; Shing et al., 2002). The hybrid test method has been further extended to geographically distributed hybrid testing through advances in communication technologies (Tsai et al., 2003; Mosqueda et al., 2006; Stojadinovic et al., 2006; Chang, 2008), allowing researchers to test more than one physical specimen in more than one laboratory around the world, but geographically distributed hybrid testing is also not implemented or discussed in detail here.

In the early 2000s, researchers using the hybrid testing method recognized a need for a common framework for adapting different finite element modelling softwares into the hybrid simulation, conducting tests in different laboratories, and using different laboratory equipment (hydraulic controllers, data acquisition systems etc...). This led to the development of the Opensource Framework for Experimental Setup and Control, commonly referred to as OpenFresco, developed by researchers at the University of California at Berkeley (Schellenberg et al., 2007). OpenFresco acts as an inter-ware between the analytical models developed in different finite element softwares (e.g. OpenSees, LS-Dyna, Abaqus, Simulink etc...) and the physical testing equipment in the laboratory, which are manufactured by a wide range of companies. The development of OpenFresco and other comparable inter-wares for hybrid simulation such as UISimCor provide the means to employ a variety of commercial finite element softwares together with laboratory control and data acquisition systems seamlessly (Schellenberg et al., 2007; Kwon et al., 2005).

The development of transparent frameworks for hybrid simulation (OpenFresco \& UISimCor) that are capable of integrating different finite element softwares and facilitating geographically distributed hybrid tests consisting of multiple sub-assemblies with different laboratory hardware has led to the world-wide adoption of the hybrid simulation technique. One exemplary example of the collaboration hybrid simulation can foster between institutions was the George E. Brown Network for Earthquake Engineering Simulation (NEES), which was comprised of 15 advanced earthquake engineering test facilities located throughout the United States. Today, a tremendous amount of research associated with experimental errors, modelling errors, explicit 
integration schemes, and substructuring/coupling techniques have improved the accuracy and efficiency of hybrid simulation (Chang, 2002; Mosqueda et al., 2007; Schellenberg et al., 2007, 2008; Kim et al., 2011). A comprehensive state-of-the-art review of developments in the accuracy and stability of software, hardware, explicit integration techniques and the developments of distributed hybrid testing and real-time hybrid testing has been described by McCrum and Williams (2016) and will be briefly described in the following sections.

\subsubsection{Conducting a Hybrid Test}

To conduct a hybrid simulation, the first requirement is a substructuring approach by which a structure is separated into experimental and analytical substructures. Next, a discretized model of the analytical substructure to be analyzed on a computer under the effects of static and/or dynamic loading must be developed. The equation of motion for a multi-degree of freedom hybrid model is discretized in time and is shown in Eq. 2.17:

$$
\mathbf{M} \ddot{\mathbf{U}}_{n}+\mathbf{C} \dot{\mathbf{U}}_{n}+\mathbf{P}_{r}\left(\mathbf{U}_{n}, \dot{\mathbf{U}}_{n}\right)=-\mathbf{M} \lambda \ddot{\mathbf{U}}_{g, n}
$$

where $\mathbf{M}$ and $\mathbf{C}$ are the mass and damping matrices for the structure, respectively, $\mathbf{P}_{r}\left(\mathbf{U}_{n}, \dot{\mathbf{U}}_{n}\right)$ are the element resisting forces (dependent on displacement and velocity), $\mathbf{U}_{i}, \dot{\mathbf{U}}_{i}, \ddot{\mathbf{U}}_{i}$ are the displacement, velocity, and acceleration vectors at the element degrees-of-freedom (DOFs) at time $t_{i}, \ddot{\mathbf{U}}_{g, i}$ is the ground acceleration vector at time $t_{i}$, and $\lambda$ is the influence vector that relates the DOF at the ground excitation to the hybrid model DOFs. Figure 2.2 illustrates the process and steps for conducting a hybrid simulation. An earthquake acceleration time series at discrete times is the input for the finite element model. At each discrete time step, a numerical integration technique is used to solve the equation of motion for the structure and obtain a target displacement vector for imposing the load demands to the physical substructure. Through the use of a controller in the laboratory, static or dynamic hydraulic actuators apply one or more target displacements to the physical substructure and the data-acquisition system comprising of several different measurement instruments including transducers and load cells record the restoring forces and achieved displacements. The restoring forces are measured and fed back to the finite element software and 
into the equation of motion of the structure. The equation of motion is solved again at the next time step and this process is repeated throughout the duration of the ground motion record.

The advantages of using the hybrid simulation method in contrast to quasi-static and shaking table testing is that hybrid simulation provides an opportunity to better understand the behaviour of an entire structural system without the financial, space, and equipment limitations associated with full-scale testing. In addition, analytical modelling uncertainties are reduced because the highly-nonlinear structural elements are physically tested in the laboratory. An added benefit of the hybrid simulation technique is that more than one physical test can be conducted in one or more laboratories around the world, capitalizing on the unique capabilities in each of the facilities. If the hybrid simulation is conducted at real-time it also has the potential to capture material strain rate effects.

Over the past decade, there has been a tremendous amount of research into the challenges associated with hybrid simulation, and as a result, the development of advanced hybrid control softwares and error compensation schemes has led to excellent performance of the technique when compared with conventional shaking table tests (Lin and Christenson, 2009; Lamarche et al., 2010; Saouma et al., 2013). One significant difference between a purely analytical finite element model and a hybrid simulation is the numerical integration scheme that is used to solve the equations of motion and obtain the response of a structure. Because the tangent stiffness of the experimental specimen cannot be easily obtained, this presents a challenge for conventional numerical integration techniques that typically iterate to find a solution. To overcome these challenges, there has been a tremendous amount of research into numerical integration techniques designed specifically for hybrid simulation over the past three decades, some of which are highlighted in the next section.

\subsubsection{Integration Schemes for Hybrid Testing}

Hybrid simulation uses substructuring techniques to separate the numerical and physical portions of a structure whose responses are connected together through one or more of their 


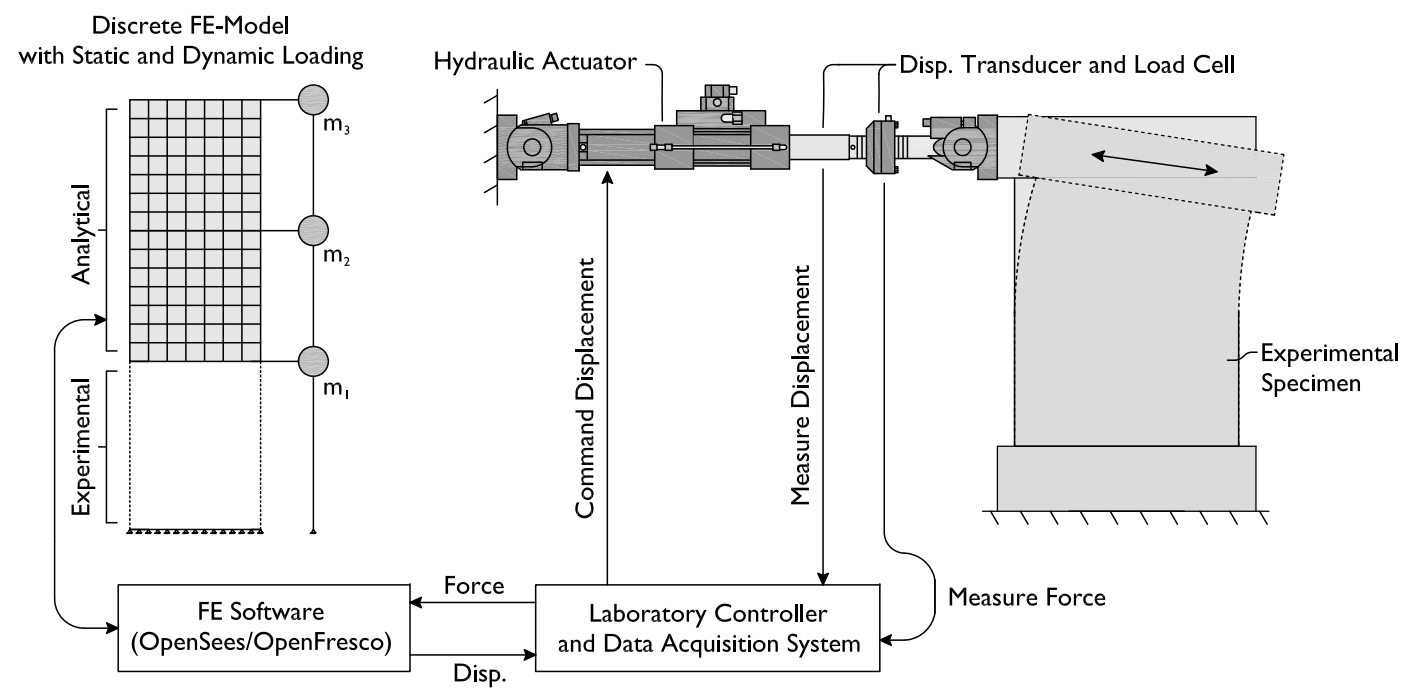

Figure 2.2 Hybrid simulation command-feedback process

common degrees-of-freedom (DOFs). It is in terms of these common DOFs that a set of equations of motion are assembled for the structural system. These second-order ordinary differential equations of motion are discretized in time and an incremental approach is utilized to solve for the response of the system for the entire duration of loading. In this approach, dynamic equilibrium, including the effects of inertia and damping, is satisfied at each time step. In the case of a structure subjected to an earthquake ground motion, the time-step often corresponds to or is less than the time-step interval of the ground motion record, which are spaced at equal time intervals for the duration of the ground acceleration record. Therefore, given the solution of the displacements and velocities at the degrees-of-freedom for a structure $\left(\mathbf{U}_{i}\right.$ and $\left.\dot{\mathbf{U}}_{i}\right)$ at a particular instant in time $\left(t_{i}\right)$, it is the goal of the numerical integration scheme to find the appropriate displacement increment $(\Delta \mathbf{U})$ so that the system remains in equilibrium for $\mathbf{U}_{i}+\Delta \mathbf{U}$ at time $t_{i}+\Delta t$, where $\Delta t$ corresponds to the length of the previously discussed time-step. To find these solutions there are a number of different strategies that can be employed but most often the series of differential equations is solved directly through the use a of numerical integration scheme. Over the past 50 years, a large number of integration schemes have been developed. Some of the most widely used strategies will be described in detail here and include explicit, implicit and operator splitting numerical integration schemes which have been developed specifically for adaptation to hybrid 
simulation.

The difference between explicit and implicit integration schemes lies in their dependence on themselves. That is, for explicit integration schemes, the solution at the next time step can be solely expressed by the current state of the system and the solution of one or more previously determined states of the system. Typically, explicit integration schemes are conditionally stable, meaning that a suitably small time step must be selected to ensure the accuracy of the method. This critical time step is referred to as the stability limit and is typically related to the natural period of vibration of a particular structure. The advantage of explicit integration schemes is that they are usually easy to implement, computationally efficient, and fast in execution. However, the restrictions on the stability limit or minimum time step can often cause problems, especially in very stiff systems, which results in a very high natural frequency and requires a very small time step for stability. Explicit schemes are also unstable or cannot be applied to systems with a singular mass matrix, or a massless DOF, which is often the case when the mass at rotational DOF are ignored. Alternatively, implicit integration schemes use the state of the system at the next time step $\left(t_{i+1}\right)$ to find the solution to the equation of motion. In most cases, implicit methods demand more computational time because they require iteration to converge on an accurate solution. However, their main advantage lies in the fact that in some cases implicit integration schemes can be unconditionally stable, meaning that the method is stable for any selected time step, which is particularly useful for very stiff systems. The disadvantages of implicit methods are their reliance on iterations to find the correct solution. They can also be computationally expensive, because they require the solution to a system of linear equations involving the Jacobian for one or more iterations, which is a very computationally expensive procedure.

In addition to whether the integration scheme is explicit or implicit, another important characteristic that must be considered for hybrid simulation is the number of steps or calls the methods are required to make to the physical substructure to solve for the state of the system at the next time step. In explicit integration schemes this is not a major concern, because the methods directly solve for the state of the system at the next time step without requiring any information from the current step. However, for implicit integration schemes that require a number 
of steps, this can lead to errors in the loads applied to the specimen and requires the tangent stiffness matrix for the experimental element, which can be very difficult to determine from physical experimental setups. In addition, the displacement increments during iteration for an implicit integration scheme generally decrease as the technique converges to the correct solution, causing non-continuous movement of the hydraulic actuators that are applying the target displacement to the physical specimen.

To overcome these challenges with implicit integration schemes, researchers have proposed fixed iteration implicit integration schemes and implicit predictor-corrector integration schemes which predict a solution using an explicit expression and then correct it one or more times using an implicit expression. Ultimately, the integrator selected depends on the problem and its application. In this study the numerical integration scheme needs to be applicable for hybrid simulation, which comes with a number of special considerations and requirements that have been described by Schellenberg et al. (2009), who recommends that the numerical integration scheme:

- Be of order $p \geq 2$ accurate; meaning that numerical errors are kept to a minimum;

- Make as few function calls as possible to avoid having to apply displacement and measure forces in the experimental test setup many times, which can be time consuming and introduce additional errors;

- In cases where the mass matrix is singular (DOFs with zero mass), the method should be unconditionally stable and fully implicit;

- The parts of the Jacobian representing the experimental element stiffness should remain constant to avoid having to determine the tangent stiffness matrix for the experimental element, which can be challenging depending on the experimental setup;

- Provide some adjustable numerical damping to suppress the excitation of higher modes due to experimental errors influencing the numerical solutions; and

- Calculate uniform displacement increments as either increasing or decreasing within a single time step to avoid overshooting the correct target displacement in the laboratory while also applying smooth continuous loading.

In light of these requirements, two popular numerical integration schemes (implicit and 
partially explicit) that have been developed specifically for hybrid simulation are discussed, chiefly those that have been incorporated into the OpenSees simulation framework and are applicable for hybrid simulation; including an implicit Newmark fixed iteration method (INM-HS) and a generalized Alpha-OS method ( $\mathrm{G} \alpha$-OS); both modified from the original methods for hybrid simulation by Schellenberg et al. (2009). These are two of the most widely adopted integration methods for hybrid simulation. Strictly explicit integration schemes are not discussed further here because they are not applicable for systems with singular mass matrices, which is the case for the analytical model in this study.

\subsubsection{Implicit Newmark Fixed Iteration Integration Scheme}

In 1959, Newmark developed a numerical integration technique that is commonly referred to as Newmark's $\beta$ method (Newmark, 1959). This is one of the most common and most widely recognized numerical integration schemes for purely analytical structural dynamics problems. In this method, the displacements and velocities at the next time step $\left(\mathbf{U}_{i+1}\right.$ and $\left.\dot{\mathbf{U}}_{i+1}\right)$ are given by Eq. 2.18a and Eq. 2.18b, respectively:

$$
\begin{gathered}
\mathbf{U}_{i+1}=\mathbf{U}_{i}+\Delta t \dot{\mathbf{U}}_{i}+\Delta t^{2}\left(\frac{1}{2}-\beta\right) \ddot{\mathbf{U}}_{i}+\Delta t^{2} \beta \ddot{\mathbf{U}}_{i+1} \\
\dot{\mathbf{U}}_{i+1}=\dot{\mathbf{U}}_{i}+\Delta t(1-\gamma) \ddot{\mathbf{U}}_{i}+\Delta t \gamma \ddot{\mathbf{U}}_{n+1}
\end{gathered}
$$

where $\gamma$ and $\beta$ represent the assumed variation in the acceleration over the time step. Their selection has an impact on the stability and accuracy of the method. For example, for values of 0.5 and 0.25 for $\gamma$ and $\beta$, respectively, this results in the average acceleration method, which is commonly used in these types of problems. The Newmark method was first used for hybrid simulation by Mahin and Williams (1980), who proposed an explicit Newmark method that would be straightforward to implement in hybrid simulation because it did not require iterations. However, because of the stability conditions for very stiff structures, whose highest period of vibration would require a very small time step, this integration scheme is no longer commonly used in hybrid simulation. The implicit Newmark method is suitable for a purely analytical problem, but 
the method must be adjusted for hybrid simulation to include the physical portion of the substructure and ensure a fixed number of iterations with equal displacement increments are used for each time step. The final requirement ensures that smooth and continuous loading is applied to the test specimen. To do so, the expressions shown in Eq. 2.18a and Eq. 2.18b are reformulated to obtain expressions for the velocity $\left(\dot{\mathbf{U}}_{i+1}\right)$ and acceleration $\left(\ddot{\mathbf{U}}_{i+1}\right)$ at time $t_{i+1}$, shown in Eq. $2.19 \mathrm{a}$ and Eq. 2.19b:

$$
\begin{gathered}
\dot{\mathbf{U}}_{i+1}=\frac{\gamma}{\Delta t \beta}\left(\mathbf{U}_{i+1}-\mathbf{U}_{i}\right)-\left(\frac{\gamma}{\beta}-1\right) \dot{\mathbf{U}}_{i}-\Delta t\left(\frac{\gamma}{2 \beta}-1\right) \ddot{\mathbf{U}}_{i} \\
\ddot{\mathbf{U}}_{i+1}=\frac{1}{\Delta t^{2} \beta}\left(\mathbf{U}_{i+1}-\mathbf{U}_{i}\right)-\frac{1}{\Delta t \beta} \dot{\mathbf{U}}_{i}-\left(\frac{1}{2 \beta}-1\right) \ddot{\mathbf{U}}_{i}
\end{gathered}
$$

The resulting velocity and accelerations obtained from these equations are then substituted into the following equation of motion (Eq. 2.20):

$$
\mathbf{F}\left(\mathbf{U}_{i+1}\right)=\mathbf{M} \ddot{\mathbf{U}}_{i+1}+\mathbf{C} \dot{\mathbf{U}}_{i+1}+\mathbf{P}_{r}\left(\mathbf{U}_{i+1}\right)-\mathbf{P}_{i+1}=0
$$

where $\mathbf{P}_{r}$ are the element resisting forces for the physical and analytical substructures, $\mathbf{P}$ are external forces, and $\mathrm{F}$ is the residual force vector (also referred to as the unbalanced force vector). The system of nonlinear equations for the unknown displacements are determined using an iterative Newton-Raphson algorithm. In this case, an expression for the effective stiffness matrix and force vector for a particular iteration $(k)$ take the form shown in Eq. 2.21a and Eq. 2.21b:

$$
\begin{gathered}
\mathbf{K}_{e f f}^{(k)}=c_{3} \mathbf{M}+c_{2} \mathbf{C}+c_{1} \mathbf{K}_{t}\left(\mathbf{U}_{i+1}^{(k)}\right) \\
\mathbf{P}_{e f f}^{(k)}=-\mathbf{M} \ddot{\mathbf{U}}_{i+1}^{(k)}-\mathbf{C} \dot{\mathbf{U}}_{i+1}^{(k)}-\mathbf{P}_{r}\left(\mathbf{U}_{i+1}^{(k)}\right)+\mathbf{P}_{i+1}
\end{gathered}
$$

where $c_{1}=1, c_{2}=\gamma /(\Delta t \beta)$, and $c_{3}=1 /\left(\Delta t^{2} \beta\right)$. This method is a predictor-multi-corrector method in that predictors are used to determine the initial displacement $\left(\mathbf{U}_{i+1}^{(k=1)}\right)$, velocity $\left(\dot{\mathbf{U}}_{i+1}^{(k=1)}\right)$, and acceleration $\left(\ddot{\mathbf{U}}_{i+1}^{(k=1)}\right)$ vectors to start the Newton-Raphson iterations, which are 
shown in Eq. 2.22a to Eq. 2.22c:

$$
\begin{gathered}
\mathbf{U}_{i+1}^{(k=1)}=\mathbf{U}_{i} \\
\dot{\mathbf{U}}_{i+1}^{(k=1)}=-\left(\frac{\gamma}{\beta}-1\right) \dot{\mathbf{U}}_{i}-\Delta t\left(\frac{\gamma}{2 \beta}-1\right) \ddot{\mathbf{U}}_{i} \\
\ddot{\mathbf{U}}_{i+1}^{(k=1)}=-\frac{1}{\Delta t \beta} \dot{\mathbf{U}}_{i}-\left(\frac{1}{2 \beta}-1\right) \ddot{\mathbf{U}}_{i}
\end{gathered}
$$

This algorithm will only converge if the intial predicted displacement vector is close to the actual solution, so the displacement vector at the initial time $t_{i}$ is used as a trial displacement vector, which is then corrected using Eq. 2.23a to Eq. 2.23c and the process is iterated until convergence is achieved.

$$
\begin{aligned}
& \mathbf{U}_{i+1}^{(k+1)}=\mathbf{U}_{i+1}^{(k)}+c_{1} \Delta \mathbf{U}^{(k)} \\
& \dot{\mathbf{U}}_{i+1}^{(k+1)}=\dot{\mathbf{U}}_{i+1}^{(k)}+c_{2} \Delta \mathbf{U}^{(k)} \\
& \ddot{\mathbf{U}}_{i+1}^{(k+1)}=\ddot{\mathbf{U}}_{i+1}^{(k)}+c_{3} \Delta \mathbf{U}^{(k)}
\end{aligned}
$$

where $\Delta \mathbf{U}^{(k)}$ is the displacement increment from the previous time-step $\left(\mathbf{U}_{i+1}^{(k)}-\mathbf{U}_{i}^{(k)}\right)$. To measure convergence, a number of techniques have been recommended by Schellenberg et al. (2009), which include using the vector norm of the displacement increments, effective/unbalanced force vector, or an energy measure, all of which are available and can be easily implemented in the OpenSees framework. The implicit Newmark method previously discussed is stable such that the selected time step $(\Delta t)$ meets the requirements shown in Eq. 2.24:

$$
\frac{\Delta t}{T_{n}} \leq \frac{(\gamma-0.5) \xi+\sqrt{0.5 \gamma-\beta+(\gamma-0.5)^{2} \xi^{2}}}{(\gamma-2 \beta) \pi}
$$

where $T_{n}$ is the natural period of the structure and $\xi$ is the damping ratio. To meet the requirement for continuous loading of the test specimen, a slightly modified version of the previously described technique has been implemented in OpenSees by Schellenberg et al. (2009). This technique uses a fixed number of iterations during the Newton-Raphson solving scheme. Therefore, regardless of the state of the convergence test, the iterative process is terminated following the specified 
number of constant iterations. To overcome the challenge of changing displacement increments during each iteration, trial displacements $\left(\mathbf{U}_{i+1}^{(k+1)}\right)$ are not used directly to update the model or applied to the physical substructure, but rather are reduced using interpolation functions. The interpolation functions are not described in detail here but are available in Schellenberg et al. (2009). The modified corrector equations are shown in Eq. 2.25a to Eq. 2.25c:

$$
\begin{aligned}
& \mathbf{U}_{i+1}^{(k+1)}=\mathbf{U}_{i+1}^{(k)}+c_{1} \Delta \mathbf{U}_{\text {scaled }}^{(k)} \\
& \dot{\mathbf{U}}_{i+1}^{(k+1)}=\dot{\mathbf{U}}_{i+1}^{(k)}+c_{2} \Delta \mathbf{U}_{\text {scaled }}^{(k)} \\
& \ddot{\mathbf{U}}_{i+1}^{(k+1)}=\ddot{\mathbf{U}}_{i+1}^{(k)}+c_{3} \Delta \mathbf{U}_{\text {scaled }}^{(k)}
\end{aligned}
$$

where scaled displacement increment $\left(\Delta \mathbf{U}_{\text {scaled }}^{k}\right)$ is determined according to the maximum number of iterations $\left(k_{\max }\right)$ specified by the user for each time step. This resulting integration method produces uniform displacement increments for each iteration, making it applicable for maintaining smooth continuous loading in hybrid simulation. This method has been the work of many authors including Dorka and Heiland (1991); Shing et al. (1991); Zhong (2005) and has been implemented into OpenSees for use with OpenFresco by Schellenberg et al. (2009). Figure 2.3 summarizes the Implicit Newmark Fixed Iteration integration scheme for hybrid simulation. 
Intialize Step:

$$
\begin{aligned}
& \mathbf{U}_{o}, \dot{\mathbf{U}}_{o}, \ddot{\mathbf{U}}_{o} \\
& \mathbf{K}_{\mathrm{eff}}=c_{3} \mathbf{M}+c_{2} \mathbf{C}+c_{1} \mathbf{K}_{i} \text { (Case 1: Initial Stiffness) }
\end{aligned}
$$

Analyze:

$$
\text { for } \begin{aligned}
(i=0, i<N, i++)(\text { for } N \text { time steps }) \\
\mathbf{U}_{i+1}^{(k=1)}=\mathbf{U}_{i} \\
\dot{\mathbf{U}}_{i+1}^{(k=1)}=-\left(\frac{\gamma}{\beta}-1\right) \dot{\mathbf{U}}_{i}-\Delta t\left(\frac{\gamma}{2 \beta}-1\right) \ddot{\mathbf{U}}_{i} \\
\ddot{\mathbf{U}}_{i+1}^{(k=1)}=-\frac{1}{\Delta t \beta} \dot{\mathbf{U}}_{i}-\left(\frac{1}{2 \beta}-1\right) \ddot{\mathbf{U}}_{i} \\
\mathbf{P}_{\text {eff }}^{(k=1)}=\mathbf{P}_{i+1}-\mathbf{P}_{o, i+1}-\mathbf{P}_{r}\left(\mathbf{U}_{i+1}^{(k=1)}\right)-\mathbf{M} \ddot{\mathbf{U}}_{i+1}^{(k=1)}-\mathbf{C U}_{i+1}^{(k=1)}
\end{aligned}
$$

do

$$
\begin{aligned}
& \mathbf{K}_{\text {eff }}^{(k)}=c_{3} \mathbf{M}+c 2 \mathbf{C}+c 1 \mathbf{K}_{m}\left(\mathbf{U}_{i+1}^{(k)}\right) \text { (case 2: mixed stiffness) } \\
& \Delta \mathbf{U}^{(k)}=\text { solve }\left(\mathbf{K}_{e f f}^{(k)} \Delta \mathbf{U}^{(k)}=\mathbf{P}_{e f f}^{(k)}\right) \text { (Newton-Raphson algorithm) } \\
& \Delta \mathbf{U}_{\text {scaled }}^{(k)}=\mathbf{U}_{i+1}^{k-1}+\sum_{j=i+1-n}^{i+1} \mathbf{U}_{j} L_{n, j}\left(\frac{k}{k_{\text {max }}}\right) \\
& \mathbf{U}_{i+1}^{(k+1)}=\mathbf{U}_{i+1}^{(k)}+c_{1} \Delta \mathbf{U}_{\text {scaled }}^{(k)} \\
& \dot{\mathbf{U}}_{i+1}^{(k+1)}=\dot{\mathbf{U}}_{i+1}^{(k)}+c_{2} \Delta \mathbf{U}_{\text {scaled }}^{(k)} \\
& \ddot{\mathbf{U}}_{i+1}^{(k+1)}=\ddot{\mathbf{U}}_{i+1}^{(k)}+c_{3} \Delta \mathbf{U}_{\text {scaled }}^{(k)} \\
& \mathbf{P}_{\text {eff }}^{(k+1)}=\mathbf{P}_{i+1}-\mathbf{P}_{o, i+1}-\mathbf{P}_{r}\left(\mathbf{U}_{i+1}^{(k+1)}\right)-\mathbf{M U}_{i+1}^{(k+1)}-\mathbf{C U}_{i+1}^{(k+1)}
\end{aligned}
$$

while $\left(k<k_{\text {max }} ; k++\right)$ (where $k_{\text {max }}$ is the maximum number of iterations)

$$
\begin{aligned}
& \mathbf{K}_{\mathrm{eff}}=c_{3} \mathbf{M}+c_{2} \mathbf{C}+c_{1} \mathbf{K}_{m}\left(\mathbf{U}_{i+1}^{(k+1)}\right) \text { (case 2: mixed stiffness) } \\
& \mathbf{U}_{i+1}^{(k+1)}=\mathbf{U}_{i+1}^{(k)}+c_{1} \Delta \mathbf{U}^{(k)} \\
& \dot{\mathbf{U}}_{i+1}^{(k+1)}=\dot{\mathbf{U}}_{i+1}^{(k)}+c_{2} \Delta \mathbf{U}^{(k)} \\
& \ddot{\mathbf{U}}_{i+1}^{(k+1)}=\ddot{\mathbf{U}}_{i+1}^{(k)}+c_{3} \Delta \mathbf{U}^{(k)}
\end{aligned}
$$

end

Figure 2.3 Implicit Newmark method with fixed iterations and equal displacement increments (adapted from Schellenberg et al., 2009) 


\subsubsection{Generalized Alpha OS Integration Scheme}

In addition to the implicit Newmark fixed iteration integration scheme for hybrid simulation, a second popular integration scheme known as the generalized alpha-OS method is also presented herein. The method has also been incorporated into the OpenSees and OpenFresco frameworks by Schellenberg et al. (2009). The method is based on a combination of the generalized alpha methods developed by Hilber et al. (1977); Chung and Hulbert (1993) and the operator splitting method developed by Nakashima et al. (1990), each of which are described briefly below.

The family of alpha methods are unique in that they introduce numerical damping to suppress high-frequency response stemming from noise in the experimental element feedback loop. This is something that has not been addressed in the previous implicit Newmark method. The resulting integration scheme is one that improves the convergence of the method and prevents overshooting. In the alpha method, on which the generalized alpha-OS method is based, the weighted equations of motion are formulated between time steps $t_{i}$ and $t_{i+1}$ by introducing weighting parameters $\alpha_{m}$ and $\alpha_{f}$, for which the equation of motion is written as shown in Eq. 2.26:

$$
\mathbf{M} \ddot{\mathbf{U}}_{i+\alpha_{m}}+\mathbf{C} \dot{\mathbf{U}}_{i+\alpha_{f}}+\mathbf{P}_{r, i+\alpha_{f}}=\mathbf{P}_{i+\alpha_{f}}
$$

To determine the velocity $\left(\dot{\mathbf{U}}_{i+\alpha_{f}}\right)$ and acceleration $\left(\ddot{\mathbf{U}}_{i+\alpha_{m}}\right)$ between time steps, the following expressions are used with the weighting parameters as shown in Eq. 2.27a and Eq. 2.27b:

$$
\begin{aligned}
\dot{\mathbf{U}}_{i+\alpha_{f}} & =\left(1-\alpha_{f}\right) \dot{\mathbf{U}}_{i}+\alpha_{f} \dot{\mathbf{U}}_{i+1} \\
\ddot{\mathbf{U}}_{i+\alpha_{m}} & =\left(1-\alpha_{m}\right) \ddot{\mathbf{U}}_{i}+\alpha_{m} \ddot{\mathbf{U}}_{i+1}
\end{aligned}
$$

The internal resisting force $\left(\mathbf{P}_{r, i+\alpha_{f}}\right)$ (at a particular displacement increment) and external force vectors $\left(\mathbf{P}_{i+\alpha_{f}}\right)$ are expressed using the generalized trapezoidal rule as shown in Eq. 2.28a and 
Eq. 2.28b:

$$
\begin{gathered}
\mathbf{P}_{r, i+\alpha_{f}}=\left(1-\alpha_{f}\right) \mathbf{P}_{r}\left(\mathbf{U}_{i}\right)+\alpha_{f} \mathbf{P}_{r}\left(\mathbf{U}_{i+1}\right) \\
\mathbf{P}_{i+\alpha_{f}}=\left(1-\alpha_{f}\right) \mathbf{P}_{i}+\alpha_{f} \mathbf{P}_{i+1}
\end{gathered}
$$

In a manner very similar to the Newmark method, when substituted in the previously shown equation of motion in Eq. 2.26, this yields a system of nonlinear equations that uses an iterative Newton-Raphson procedure to solve for the response of the system. This yields an effective stiffness matrix $\left(\mathbf{K}_{e f f}^{(k)}\right)$ and effective unbalanced force vector $\left(\mathbf{P}_{e f f}^{(k)}\right)$ shown in Eq. 2.29a and Eq. 2.29b:

$$
\begin{gathered}
\mathbf{K}_{e f f}^{(k)}=\alpha_{m} c_{3} \mathbf{M}+\alpha_{f} c_{2} \mathbf{C}+\alpha_{f} c_{1} \mathbf{K}_{t}\left(\mathbf{U}_{i+1}^{(k)}\right) \\
\mathbf{P}_{e f f}^{(k)}=\mathbf{P}_{i+1}-M\left[\left(1-\alpha_{m}\right) \ddot{\mathbf{U}}_{i}+\alpha_{m} \ddot{\mathbf{U}}_{i+1}\right]-\mathbf{C}\left[\left(1-\alpha_{f}\right) \dot{\mathbf{U}}_{i}+\alpha_{f} \dot{\mathbf{U}}_{i+1}\right]-\mathbf{P}_{r, i+\alpha_{f}}\left(\mathbf{U}_{i+1}^{(k)}\right)
\end{gathered}
$$

where $c_{1}=1, c_{2}=\gamma /(\Delta t \beta)$, and $c_{3}=1 /\left(\Delta t^{2} \beta\right)$. This method is also a predictor-multicorrector method in that predictors are used to determine the initial displacement $\left(\mathbf{U}_{i+1}^{(k=1)}\right)$, velocity $\left(\dot{\mathbf{U}}_{i+1}^{(k=1)}\right)$, and acceleration $\left(\ddot{\mathbf{U}}_{i+1}^{(k=1)}\right)$ vectors to start the Newton-Raphson iterations, which are the same as those from the Newmark method shown in Eq. 2.22a to Eq. 2.22c, and are not presented again. In a similar manner to the Newmark method, the calculated displacement increments are scaled to ensure continuous loading and the process is repeated until the maximum number of iterations are reached. This method controls the numerical damping through the selection of the weighting parameters $\alpha_{m}$ and $\alpha_{f}$, which are based on an added parameter $\rho$ and can be computed using the proposed relationships by Chung and Hulbert (1993) shown in Eq. 2.30a 
to Eq. 2.30d:

$$
\begin{gathered}
\alpha_{m}=\frac{2-\rho}{1+\rho} \in[2.0,0.5] \\
\alpha_{f}=\frac{1}{1+\rho} \in[1.0,0.5] \\
\beta=\frac{1}{(1+\rho)^{2}} \in[1.0,0.25] \\
\gamma=0.5+\alpha_{m}-\alpha_{f} \in[1.5,0.5]
\end{gathered}
$$

The previously discussed alpha method is combined with an operator-splitting (OS) method proposed by Nakashima et al. (1990) to form what is referred to in OpenSees as the generalized alpha-OS method for hybrid simulation. In the OS method, the computation of the displacement, velocity, and acceleration at the next time step is divided into a predictor and a corrector phase. The Newmark equations are first used to solve for the displacements $\left(\mathbf{U}_{i+1}\right)$ and velocities $\left(\dot{\mathbf{U}}_{i+1}\right)$ at time step $t_{i+1}$ using the displacements at the previous time step according to Eq. 2.31a to Eq. $2.31 \mathrm{~b}$ :

$$
\begin{gathered}
\mathbf{U}_{i+1}=\mathbf{U}_{i}+\Delta t \dot{\mathbf{U}}_{i}+\frac{\Delta t^{2}}{2}(1-2 \beta) \ddot{\mathbf{U}}_{i+1}+\Delta t^{2} \beta \ddot{\mathbf{U}}_{i+1}=\tilde{\mathbf{U}}_{i+1}+\Delta t^{2} \beta \ddot{\mathbf{U}}_{i+1} \\
\dot{\mathbf{U}}_{i+1}=\dot{\mathbf{U}}_{i}+\Delta t(1-\gamma) \ddot{\mathbf{U}}_{i}+\Delta t \gamma \ddot{\mathbf{U}}_{i+1}=\tilde{\mathbf{U}}_{i}+1+\Delta t \gamma \ddot{\mathbf{U}}_{i+1}
\end{gathered}
$$

The main difference between the OS method and those previously discussed is that the vector of internal resisting forces $\left[\mathbf{P}_{r}\left(\mathbf{U}_{i+1}\right)\right]$ at time step $t_{i+1}$ is approximated using Eq. 2.32:

$$
\mathbf{P}_{r}\left(\mathbf{U}_{i+1}\right) \cong \mathbf{K}_{i} \mathbf{U}_{i+1}-\mathbf{K}_{i} \tilde{\mathbf{U}}_{i+1}+\mathbf{P}_{r}\left(\tilde{\mathbf{U}}_{i+1}\right)
$$

This OS technique assumes that the difference between the elastic and the nonlinear restoring forces at the predictor displacements $\left(\tilde{\mathbf{U}}_{i+\alpha_{f}}\right)$ is approximately equal to the difference between the elastic and nonlinear resisting forces at the new displacements $\left(\mathbf{U}_{i+\alpha_{f}}\right)$. Expressions for the effective stiffness matrix and force vector can be formed in a similar manner to the previously 
described Newmark method, which take the form shown in Eq. 2.33a and Eq. 2.33b:

$$
\begin{gathered}
\mathbf{K}_{e f f}^{(k)}=c_{3} \mathbf{M}+c_{2} \mathbf{C}+c_{1} \mathbf{K}_{1} \\
\mathbf{P}_{e f f}^{(k)}=\mathbf{P}_{i+1}-\mathbf{C} \tilde{\mathbf{U}}_{i+1}-\mathbf{P}_{r}\left(\tilde{\mathbf{U}}_{i+1}\right)
\end{gathered}
$$

where $c_{1}=1, c_{2}=\gamma /(\Delta t \beta)$, and $c_{3}=1 /\left(\Delta t^{2} \beta\right)$. This method is a predictor-one-corrector method, meaning only one force acquisition is required from the physical substructure per time step, which is particularly attractive in hybrid simulation because of the previously discussed implications associated with function calls to the experimental setup. Once the displacement increment vector $(\Delta \mathbf{U})$ is known, the displacements, velocities, and accelerations are corrected with Eq. 2.34a, Eq. 2.34b, and Eq. 2.34c:

$$
\begin{gathered}
\mathbf{U}_{i+1}=\tilde{\mathbf{U}}_{i+1}+c_{1} \Delta \mathbf{U} \\
\dot{\mathbf{U}}_{i+1}=\tilde{\mathbf{U}}_{i+1}+c_{2} \Delta \mathbf{U} \\
\ddot{\mathbf{U}}_{i+1}=c_{3} \Delta \mathbf{U}
\end{gathered}
$$

Ultimately the two previously discussed numerical integration schemes (alpha method and OS method) developed by Chung and Hulbert (1993) and Nakashima et al. (1990) were combined together by Schellenberg et al. (2009) to form what is referred to as the generalized alpha-OS method. The method is derived from the same equation of motion shown in Eq. 2.26 and the Newmark equations for the OS method shown in Eq. 2.31a to Eq. 2.31b. The internal resisting force on the left hand side of the equation of motion is approximated using Eq. 2.35:

$$
\mathbf{P}_{r, i+\alpha_{f}} \cong \mathbf{K}_{i} \mathbf{U}_{i+\alpha_{f}}-\left(\mathbf{K}_{i} \tilde{\mathbf{U}}_{i+\alpha_{f}}-\tilde{\mathbf{P}}_{r, i+\alpha_{f}}\right)
$$

After substituting the internal resisting force vector in the equation of motion, expressions for the effective stiffness matrix and force vector can be formed in a similar manner to the previously 
described Newmark method, which are shown in Eq. 2.36a and Eq. 2.36b:

$$
\begin{gathered}
\mathbf{K}_{e f f}=\alpha_{m} c_{3} \mathbf{M}+\alpha_{f} c_{2} \mathbf{C}+\alpha_{f} c_{1} \mathbf{K}_{i} \\
\mathbf{P}_{e f f}=\mathbf{P}_{i+\alpha_{f}}-\mathbf{M}\left(1-\alpha_{m}\right) \ddot{\mathbf{U}}_{i}-\mathbf{C}\left[\left(1-\alpha_{f}\right) \dot{\mathbf{U}}_{i}+\alpha_{f} \tilde{\mathbf{U}}_{i+1}\right]-\tilde{\mathbf{P}}_{r, i+\alpha_{f}}+\mathbf{K}_{i}\left(1-\alpha_{f}\right)\left(\mathbf{U}_{i}-\tilde{\mathbf{U}}_{i}\right)
\end{gathered}
$$

where $c_{1}=1, c_{2}=\gamma /(\Delta t \beta)$, and $c_{3}=1 /\left(\Delta t^{2} \beta\right)$. Once the displacement increment is known, the displacement, velocity, and acceleration response quantities are corrected with Eq. 2.37a to Eq. $2.37 \mathrm{c}$ in a very similar manner to that of the OS method previously discussed:

$$
\begin{gathered}
\mathbf{U}_{i+1}=\tilde{\mathbf{U}}_{i+1}+c_{1} \Delta \mathbf{U} \\
\dot{\mathbf{U}}_{i+1}=\tilde{\mathbf{U}}_{i+1}+c_{2} \Delta \mathbf{U} \\
\ddot{\mathbf{U}}_{i+1}=c_{3} \Delta \mathbf{U}
\end{gathered}
$$

In the same manner as the previously discussed alpha method, the amount of numerical damping can be adjusted through the use of the $\rho$ parameter. The benefits of this method include the fact that it is second-order accurate $(p \geq 2)$ for displacements and velocities, unconditionally stable, only requires the effective force vector once per time step, requires only the initial stiffness matrix for the experimental element, and provides the option of adding numerical damping to eliminate high frequency noise from the experimental equipment. The generalized alpha OS method is also not an iterative method. Therefore, the generalized alpha OS method meets all of the suggestions for a numerical integrator for hybrid simulation described by Schellenberg et al. (2009) earlier. However, because of the approximation used to express the internal resisting forces $\left(\mathbf{P}_{r, i+\alpha_{f}}\right)$, the algorithm does not produce accurate results for highly nonlinear systems (including geometric nonlinearities), but for structures that do not exhibit these tendencies, the generalized alpha OS method is a very attractive integration scheme for hybrid simulation. Figure 2.4 summarizes the Generalized Alpha-OS integration scheme for hybrid simulation. 


$$
\begin{aligned}
& \text { Intialize Step: } \\
& \mathbf{U}_{o}, \dot{\mathbf{U}}_{o}, \ddot{\mathbf{U}}_{o} \\
& \alpha_{m}=\frac{2-\rho}{1+\rho} \in[2.0,0.5] \text { (where } \rho \text { specifies numerical damping) } \\
& \alpha_{f}=\frac{1}{1+\rho} \in[1.0,0.5] \\
& \beta=\frac{1}{(1+\rho)^{2}} \in[1.0,0.25] \\
& \gamma=0.5+\alpha_{m}-\alpha_{f} \in[1.5,0.5] \\
& \mathbf{K}_{\mathrm{eff}}=\alpha_{m} c_{3} \mathbf{M}+\alpha_{f} c_{2} \mathbf{C}+\alpha_{f} c_{1} \mathbf{K}_{i} \text { (factorized storage) } \\
& \text { Analyze: } \\
& \text { for }(i=0, i<N, i++) \text { (for } N \text { time steps) } \\
& \tilde{\mathbf{U}}_{i+1}=\mathbf{U}_{i}+\Delta t \dot{\mathbf{U}}_{i}+\frac{\Delta t^{2}}{2}(1-2 \beta) \ddot{\mathbf{U}}_{i} \\
& \dot{\tilde{\mathbf{U}}}_{i+1}=\dot{\mathbf{U}}_{i}+\Delta t(1-\gamma) \ddot{\mathbf{U}}_{i} \\
& \mathbf{P}_{\text {eff }}=\mathbf{P}_{i+\alpha_{f}}-\mathbf{P}_{o, i+\alpha_{f}}-\tilde{\mathbf{P}}_{r, i+\alpha_{f}} \\
& -\mathbf{K}_{i}\left(1-\alpha_{f}\right)\left(\mathbf{U}_{i}-\tilde{\mathbf{U}}_{i}\right)-\mathbf{M}\left(1-\alpha_{m}\right) \ddot{\mathbf{U}}_{i}-\mathbf{C}\left[\left(1-\alpha_{f}\right) \dot{\mathbf{U}}_{i}+\alpha_{f} \dot{\tilde{\mathbf{U}}}_{i+1}\right] \\
& \Delta \mathbf{U}=\operatorname{solve}\left(\mathbf{K}_{\mathrm{eff}} \Delta \mathbf{U}=\mathbf{P}_{\mathrm{eff}}\right) \text { (Newton-Raphson algorithm) } \\
& \mathbf{U}_{i+1}=\tilde{\mathbf{U}}_{i+1}+c_{1} \Delta \mathbf{U} \\
& \dot{\mathbf{U}}_{i+1}=\dot{\tilde{\mathbf{U}}}_{i+1}+c_{2} \Delta \mathbf{U} \\
& \ddot{\mathbf{U}}_{i+1}=c_{3} \Delta \mathbf{U}
\end{aligned}
$$

Figure 2.4 Generalized-alpha-OS method (adapted from Schellenberg et al., 2009)

\subsubsection{Accuracy of Numerical Integration Schemes}

To compare the accuracy of the presented methods, Ramos et al. (2013) conducted a comprehensive assessment using a prototype two-storey steel frame model. Ramos et al. (2013) used several different time steps to get an understanding of when a specific numerical integration scheme might be better than another. The numerical integration schemes studied include the 
implicit Newmark fixed iteration scheme, the alpha method, the operator splitting method, and the generalized alpha OS method for hybrid simulation. Ramos et al. (2013) concluded that as the size of the time step decreases all of the methods converge to the same results. To get a better understanding of the accuracy of each method, the energy balance error (EBE) described by Filiatrault et al. (1992) was used as a measure of comparison between the accuracy of the methods. Results demonstrated that both the iterative Newmark method for hybrid simulation and the generalized alpha OS method produced very good results and significantly smaller energy balance errors when compared to the traditional operator splitting and alpha methods. In addition to the size of the time step, Ramos et al. (2013) also studied the number of fixed iterations required in the Newmark method to obtain a reasonable solution and suggest that 10 iterations are required, at most, for a typical analysis, which were in-line with previous observations by Zhong (2005).

In addition to EBE, computational time is also a very important factor for hybrid simulation. Generally, results of the study by Ramos et al. (2013) showed an exponential relationship between the size of the time step and the total computational time. Results also demonstrated that the generalized alpha OS method required significantly less computational time compared to the Newmark fixed iteration method because of the fact that the stiffness matrix of the system does not need to be inverted for each integration time step in non-iterative methods.

Finally, Ramos et al. (2013) also experimentally compared the accuracy of the two integration schemes for an actual hybrid simulation conducted on a steel column from a $2 \mathrm{D}$ frame in the SEESL lab at the University of Buffalo at Suny. While the column was designed to remain elastic, it was connected to a pin mounted between two plates at its base. Sacrificial steel coupons were inserted between the top and bottom plates, facilitating simple replacement and allowing repeatable nonlinear hybrid simulations. This experimental configuration exhibited a highly nonlinear response that was designed to challenge the abilities of the numerical integration schemes to provide accurate and reliable results (Ramos et al., 2013). The authors found that in general, both methods had similar levels of accuracy and remained stable for time steps up to 0.005 s. However, the Newmark fixed iteration method was shown to be superior to the generalized alpha OS method as it was more accurate up to the largest time step of 0.01 s with as little as 5 fixed iterations per 
time step, while the generalized alpha OS method lost some accuracy for time steps larger than 0.005 s. Ultimately, both methods will be considered in the following sections and selection of the appropriate numerical integration scheme will depend on its performance under the specific conditions for this study.

\subsubsection{Continuity of Loading in Hybrid Testing}

There are three broad components required to conduct an accurate hybrid simulation: (1) the finite element software (e.g. OpenSees), (2) the interware used to connect the finite element software with the laboratory equipment (e.g. OpenFresco), which are discussed in detail in Chapter 4 , and (3) the laboratory equipment itself. The laboratory equipment consisting of hydraulic controllers and data acquisition systems are critical components of hybrid simulation which represent a significant source of error for hybrid simulation results. McCrum and Williams (2016) divides the experimental errors in the laboratory into two categories: (1) systematic and (2) random errors. Random errors are those that come as a result of noise in the measurement devices (eg. load cells, displacement transducers etc...). Past studies have demonstrated that these errors are easy to compensate through the use of filters and their resulting effects have shown to be negligible in comparison to those associated with systematic errors (McCrum and Williams, 2016). Systematic errors are those introduced by the response of the actuators themselves, which can have a significant impact on the results of the hybrid simulation because of the subsequent effects on the dynamic response of the experimental element. These errors stem from the time required to compute the target displacement increment from the numerical integration scheme, which may not occur at the same sampling rate for the hydraulic actuator. For example, conventional actuators require a command every $0.1024 \mathrm{~s}$, but it typically takes much longer for the finite element model to converge and send a target displacement to the hydraulic controller. As a result, the hydraulic control system and the output from the finite element software run at different rates. Initially, this challenge was overcome through what was referred to as a "ramp-and-hold" procedure. This technique would hold the actuator in a fixed position until the target displacement was sent from 
the finite element software. Once the displacement command was received by the hydraulic controller, the actuator would ramp up to the target displacement. Although this technique works, the hold procedure has been shown to result in relaxation of the test specimen and uneven loading rates can affect the accuracy in determining the dynamic response of the experimental element (Thewalt and Mahin, 1995).

The challenge of synchronization between the hydraulic controller and the finite element software was a major challenge for early implementations of the hybrid simulation technique but has been overcome by the use of predictor-corrector control algorithms placed between the hydraulic control system and the finite element software. This predictor-corrector is not to be confused with the predictor-corrector methods described in relation to the numerical integration schemes. Figure 2.5 shows the basic principles behind the predictor-corrector algorithm and compares it with the traditional ramp and hold procedure. While the finite element software is solving for the target displacement during the next time step using a numerical integration scheme, the predictor-corrector algorithm is generating target displacement commands for the actuator at the appropriate rate. The algorithm uses a forward prediction method to approximate the next target displacement based on past results. After the numerical integration is complete and the actual target displacement is known, the algorithm switches from predictor to corrector mode and moves towards the actual target displacement. Note that the algorithm ensures that the displacement values are always given to the actuator at the appropriate sampling rate. In using this method, it allows the actuators to be in motion constantly, avoiding relaxation of the test specimen because of a lack of synchronization between the finite element software and laboratory controllers. Popular predictor-corrector techniques have been introduced by Nakashima and Masaoka (1999) and Schellenberg et al. (2009). The technique developed by Nakashima and Masaoka (1999) uses extrapolation and interpolation from displacement values at past integration points. Alternatively, Schellenberg et al. (2009) developed another predictor method based on velocity and acceleration. Schellenberg et al. (2009) recommends that predictor-corrector algorithms be based on velocities and accelerations when used in conjunction with iterative numerical integration schemes. More 
complicated algorithms that use three-loop hardware architecture have been developed by Campbell and Stojadinovic (1998) and Mosqueda (2003) to accommodate for the additional complication of distributed hybrid testing, but are not discussed in detail here.

In addition to errors associated with the synchronization between the numerical integration scheme utilized in the finite element software and the hydraulic controllers, there is an additional delay or time lag between the time when the displacement command is sent to the actuator and the time required to convert the hydraulic energy into force or movement. This delay has been reported in the literature to be between $5 \mathrm{~ms}$ and $10 \mathrm{~ms}$ (McCrum and Williams, 2016), which might not seem like a significant delay, but for applications of real-time hybrid simulation this can introduce additional challenges. In the past, these challenges have been overcome through the use of actuator delay compensation techniques which can be broadly grouped into constant delay compensation (Horiuchi et al., 1996; Nakashima and Masaoka, 1999; Darby et al., 2001; Wallace et al., 2005), adaptive compensation (Wagg and Stoten, 2001; Darby et al., 2002; Neild et al., 2005; Chen and Tsai, 2013; Chae et al., 2013), and model-based filtering (Carrion and Spencer, 2006). This study specifically focuses on pseudo-dynamic hybrid simulation, in which the test is conducted on an extended time scale, thus the effects of actuator delay are not as significant and the compensation techniques are not discussed in detail here.

\subsubsection{Applications of Hybrid Testing}

Although there has been a significant amount of work related to the development of the hybrid testing technique including: application frameworks, experimental error and delay compensation algorithms, explicit integration schemes, and substructuring/coupling techniques there has actually been very few applications of these developments to RC structures, particularly for RC shear wall structures. Much of the hybrid testing to-date has focused on the seismic performance of steel structures (Balendra et al., 1987; Shing et al., 1994; Thewalt and Mahin, 1995; Molina et al., 1999), limited work on RC structures (Negro et al., 2004; Saouma et al., 2013; Whyte and Stojadinovic, 2013b), and some comprehensive work on advanced structural systems such as advanced bracing systems (Tsai et al., 2008; Eatherton and Hajjar, 2010; Karavasilis et al., 2011; 


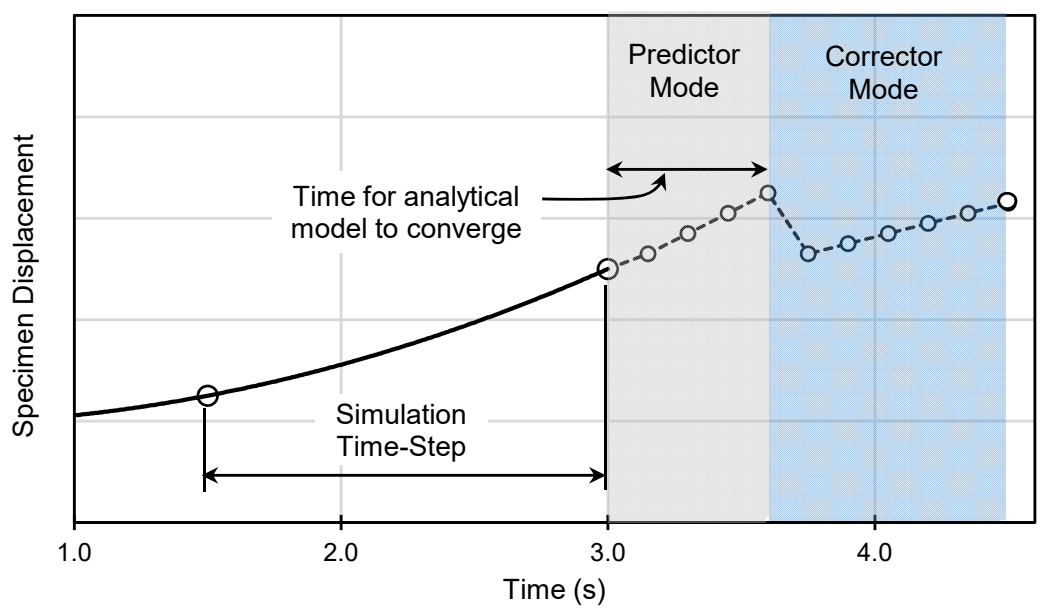

Figure 2.5 Ramp-and-hold versus predictor-corrector control strategies (adapted from Schellenberg et al., 2009)

Kammula et al., 2012; Seo et al., 2014; Dong et al., 2015) and base isolators (Molina et al., 2002; Abbiati et al., 2015; Sarebanha et al., 2017). The following sub-sections will further examine some specific case studies for hybrid simulations conducted in the past.

\subsubsection{Hybrid Testing of Steel Structures}

One of the earliest implementations of hybrid testing to better understand the seismic performance of steel structures was a study conducted by Balendra et al. (1987) who used pseudodynamic testing to examine the behaviour of a single-storey eccentrically braced frame. Specifically, the goal of using the pseudo-dynamic test method was to account for the effects of inherent damping in the structure. The single-storey frame was reduced to a single degree of freedom system and subject to a ground acceleration record. The study found that the pseudo-dynamic test method successfully accounted for the effects of inherent damping and was useful in determining the inelastic deformation characteristics of the shear link, which would have otherwise been difficult to capture using a purely analytical simulation.

Following Balendra et al. (1987), Shing et al. (1994) used the pseudo-dynamic test method and substructuring techniques to assess the performance of a three-storey concentrically braced 
frame under seismic loading. Specifically, the bottom storey of the three-storey frame was physically tested in the laboratory while the remaining two upper stories of the frame were modelled analytically. To perform the numerical integration in the finite element model, Shing et al. (1994) used an implicit time integration scheme based on the alpha method developed by Hilber et al. (1977). This integration scheme allowed the authors to adopt a simple substructuring method by retaining all degrees-of-freedom in the structural model while ignoring those with zero mass in the experimental substructure. In the experimental setup, the effects of overturning moment and axial load were ignored. The $1940 \mathrm{El}$ Centro ground motion record was applied to the structure in the analytical model. Results of the hybrid test correlated well with purely analytical simulations, verifying the nonlinear models used to capture the behaviour of the concentrically braced steel frame.

To extend the pseudo-dynamic test method outside of planar structures and understand its applicability to three-dimensional structures subjected to bi-directional ground motion, Thewalt and Mahin (1995) used hybrid simulation to get an understanding of the seismic behaviour of a scaled single storey structure with a rigid diaphragm and four steel columns. In particular, it was a goal of this study to compare the results with those conducted on a shaking table to validate the performance of the pseudo-dynamic test method. Three hydraulic actuators were used to control the three degrees-of-freedom in the response of the structure. Thewalt and Mahin (1995) cite some of the major challenges encountered during the pseudo-dynamic test, including displacement errors because of experimental geometry, feedback from the displacement transducers, force oscillation, and relaxation of the test specimen during "hold" periods. Ultimately, after compensating for these errors, the authors found that a shaking table can viably be replaced through the use of pseudo-dynamic testing if the aforementioned errors are controlled in the experimental system. Identification and classification of these errors by Thewalt and Mahin (1995) led to the implementation of different control strategies to improve the performance of the pseudo-dynamic test method.

Following the test by Thewalt and Mahin (1995), Molina et al. (1999) questioned the effect of scale on the performance of the pseudo-dynamic test method in the previously described study. 
So to account for the effect of additional degrees-of-freedom and the testing of very large and stiff structures, Molina et al. (1999) tested a full-scale three-storey steel building with composite concrete slabs. Four hydraulic actuators at each storey were used to apply bi-directions earthquake loading to the structure, including lateral displacements in each direction and rotation. Figure 2.6 shows the locations of the hydraulic actuators and the tested three-storey structure. While the experimental structure was constructed in the laboratory, the mass of the structure was modelled analytically. The explicit Newmark method, which is equivalent to the Central Difference algorithm was used to perform the step-by-step numerical integration, which the authors state required a small time step because of the conditional stability of the method. Ultimately, the authors concluded that the pseudo-dynamic test method was successful in determining the seismic behaviour of a real-size building subjected to bi-directional earthquake loading.

More recently, Lin et al. (2011) conducted a series of hybrid tests on a three-storey singlebay full-scale buckling-restrained braced frame $(\mathrm{BRBF})$ at the National Centre for Research on Earthquake Engineering (NCREE) in Taiwan. These hybrid tests verified the merits of the newly developed OpenFresco software in a collaboration between NCREE and UC Berkeley. The hybrid test employed a generic 3-DOF experimental element which was responsible for sending the displacement command and receiving the restoring force from the physical specimen in the lab. The study found that the OpenFresco hybrid control software worked well as a newly developed inter-ware and the test results demonstrated that hybrid simulation could be used as an effective tool to study the seismic response of a full-scale BRBF.

Although this subsection only touches the surface of the total number of hybrid simulations conducted on steel structures, it provides a brief overview of some of the major accomplishments during early implementation of the pseudo-dynamic test method to steel structures. In many cases, steel structures were used during early implementation of the hybrid test method because of their resilience and the potential for repeatability within the elastic range for many tests. This allowed researchers to focus on experimental errors within the method as opposed to strictly examining the performance of the structural system. These developments were a crucial step for modern applications of the hybrid test method to concrete structures and advanced structural 

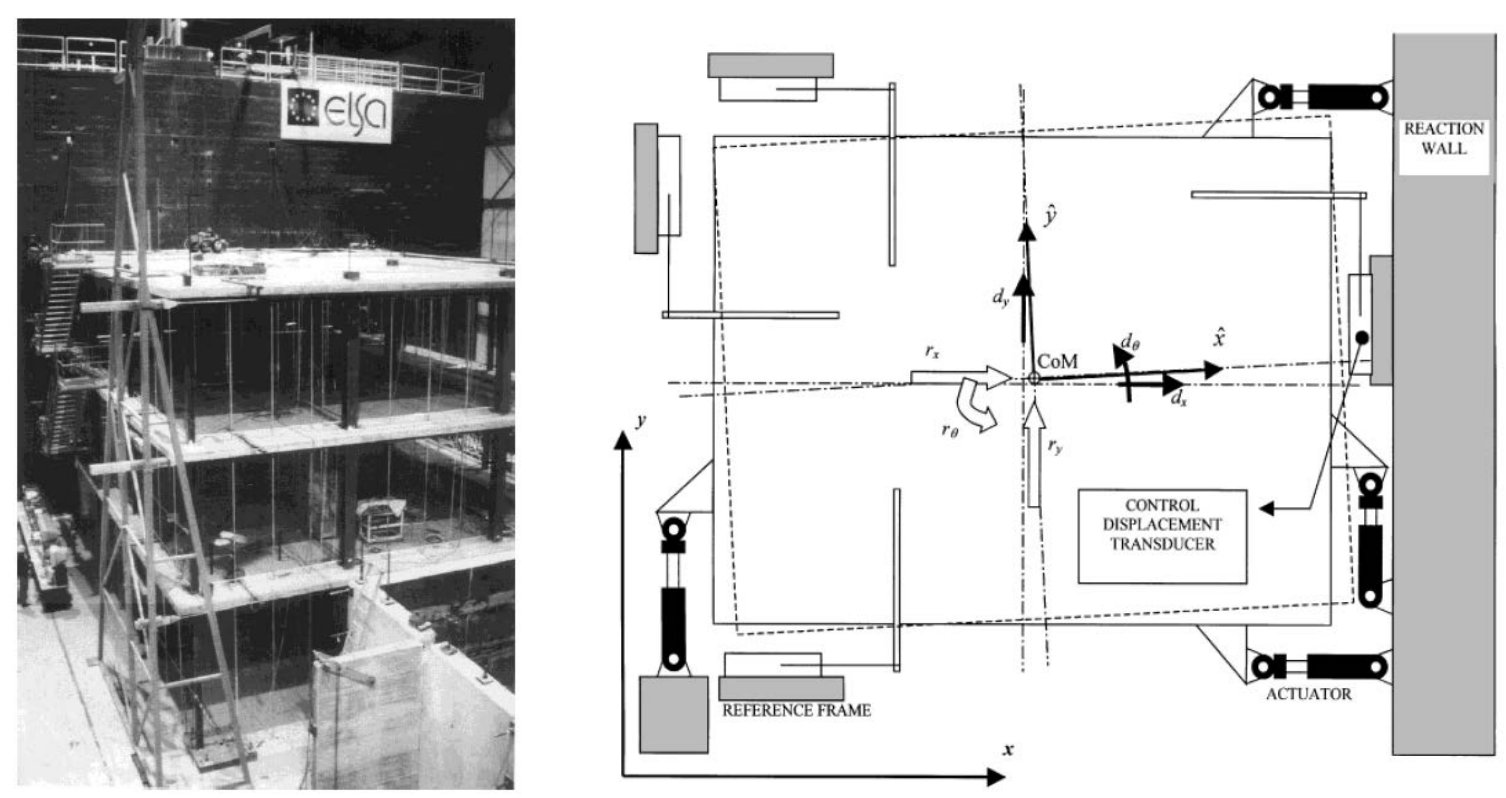

Figure 2.6 Three-storey steel structure and actuator placement (from Molina et al., 1999)

systems, which are discussed in the following subsections.

\subsubsection{Hybrid Testing of RC Structures}

In RC structures, one of the earliest implementations of hybrid testing is a study by Negro et al. (2004) and Jeong and Elnashai (2005), who conducted a pseudo-dynamic test on a full-scale 3-storey RC frame with a torsional eccentricity designed according to old European building codes for gravity load only. While the structure was tested physically in the laboratory, the mass and damping for the structure was modelled numerically and the restoring forces were experimentally measured. Figure 2.7 shows the full-scale concrete building and the placement of the actuators in plan-view in the Seismic Performance and Assessment and Rehabilitation (SPEAR) facility in Italy. The results of the study demonstrate the capabilities of using hybrid simulation to capture the torsional response of the overall structural system and observe the damage characteristics and failure mechanisms in the non-seismic columns.

A study by Charlet (2007) used the hybrid simulation test method, including OpenSees and OpenFresco, to study the gravity load collapse of a RC frame. Specifically, the physical 

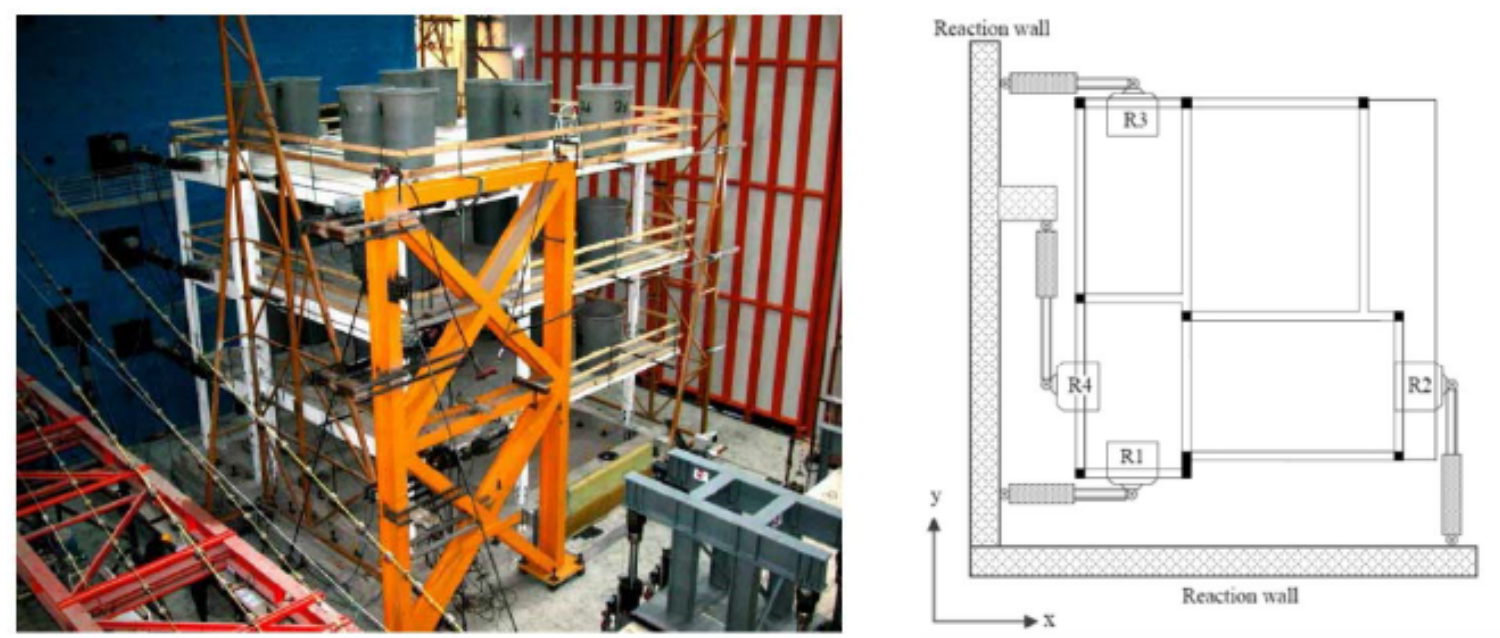

Figure 2.7 Reinforced concrete structure and actuator placement (from Negro et al., 2004)

test specimen consisted of a single shear-critical reinforced concrete column while the remaining components of the nonductile RC frame were modelled numerically in OpenSees. The physical test setup included three actuators used to apply the in-plane displacement, axial displacement, and rotation at the top of the concrete column. The hybrid test methodology used an iterative implicit integration scheme and a iterative predictor-corrector algorithm with mode-switch to enable the use of force control for the actuators in combination with a displacement-based finite element analysis engine. Results of the hybrid tests were compared with shake table test for a full-scale structure. Results showed that both the hybrid test results and shake table test produced comparable shear and axial load failure of the shear-critical column. However, the analytical model was not able to capture the residual drifts observed in the shake table tests.

Saouma et al. (2013) studied the use of real-time hybrid simulation (RTHS) to get a better understanding of the seismic performance of a three-storey, three-bay RC frame based on a design which had been previously tested on a shake table (Ghannoum and Moehle, 2012). As shown in Fig. 2.8, the concrete frame was modelled numerically using nonlinear beam column elements with the exception of one nonductile first-storey column, which was physically tested in the laboratory. Results of the tests demonstrated that RTHS can provide a cost-effective substitute to shake-table testing, giving comparable accuracy in results. The shake table test and RTHS 
both demonstrated very comparable resulting failure modes, demonstrating the capability of hybrid simulation to capture the same failure mechanisms that would be obsverved in an expensive, large-scale dynamic experimental test. Saouma et al. (2013) also concluded that the results of the test demonstrate the potential for RTHS to completely replace the far more expensive shaking table test.

Finally, Whyte and Stojadinovic (2013a,b) studied the response of two squat RC shear walls representative of the type of shear walls in an industrial nuclear facility under earthquake ground motion. Identical test specimens had previously been tested under static-cylic load at the State University of New York at Buffalo (SUNY Buffalo) by Rocks et al. (2011), which would allow for comparison of the response of the wall specimens under static cyclic and dynamic loading. Figure 2.9 shows the test setup for a typical squat shear wall specimen. The hybrid model included the physical test setup (Fig. 2.9) of the RC one-storey shear wall and the numerical portion of the model consisted of a large mass, modelled using OpenSees and OpenFresco. Because the shear wall specimen proved to be very stiff and required small displacement increments to obtain a smooth force response, the traditional displacement control strategies proved ineffective for testing such a stiff specimen. To overcome this challenge, researchers used a high precision and high resolution displacement encoder to measure the command displacement from the analytical model. Results demonstrated that the encoder worked well in ensuring a smooth force response for even small command displacements. Comparison of the results between the hybrid tests and the previously conducted static cyclic tests show very comparable results in terms of global force-deformation response and ultimate failure mode. However, the physical test setup in this study did not include the effects of vertical load and overturning moment on the performance of the wall specimen, which could have potentially influenced the observed behaviour during the hybrid simulation when compared with the static cyclic tests.

Joshua Woods, Department of Civil and Environmental Engineering, Carleton University 

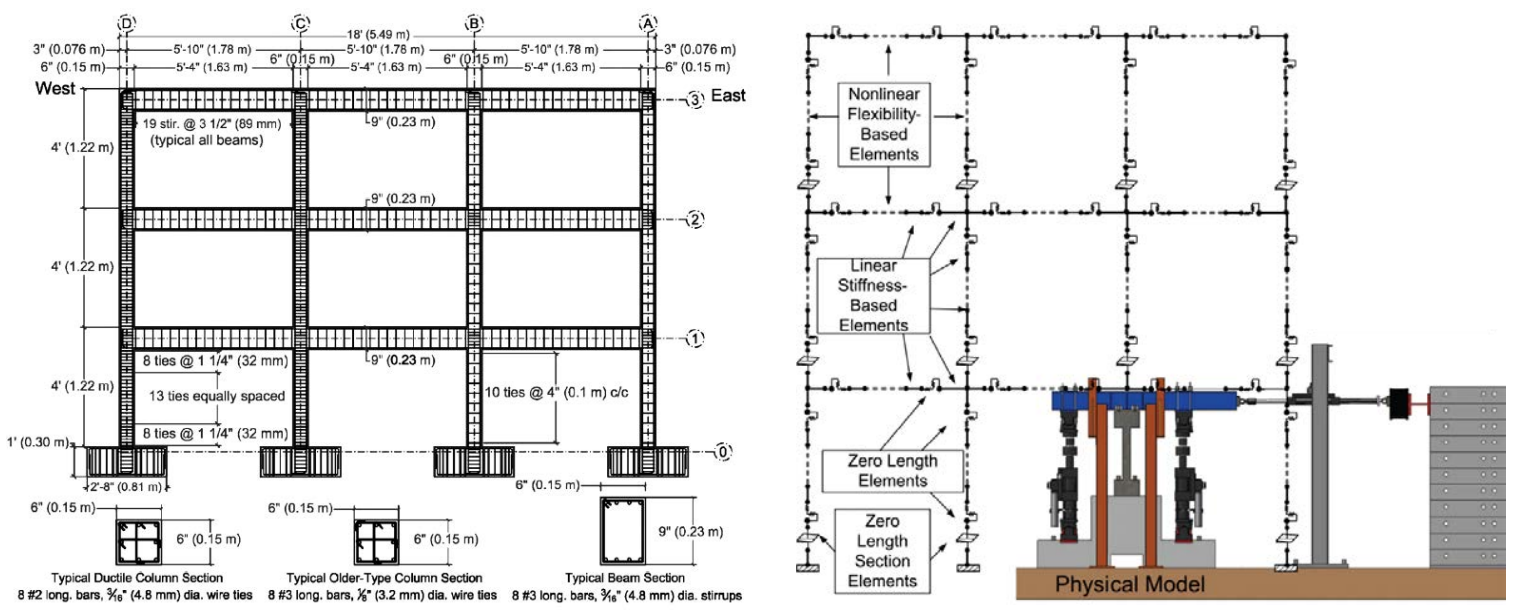

Figure 2.8 Reinforced concrete frame and numerical model (from Saouma et al., 2013)
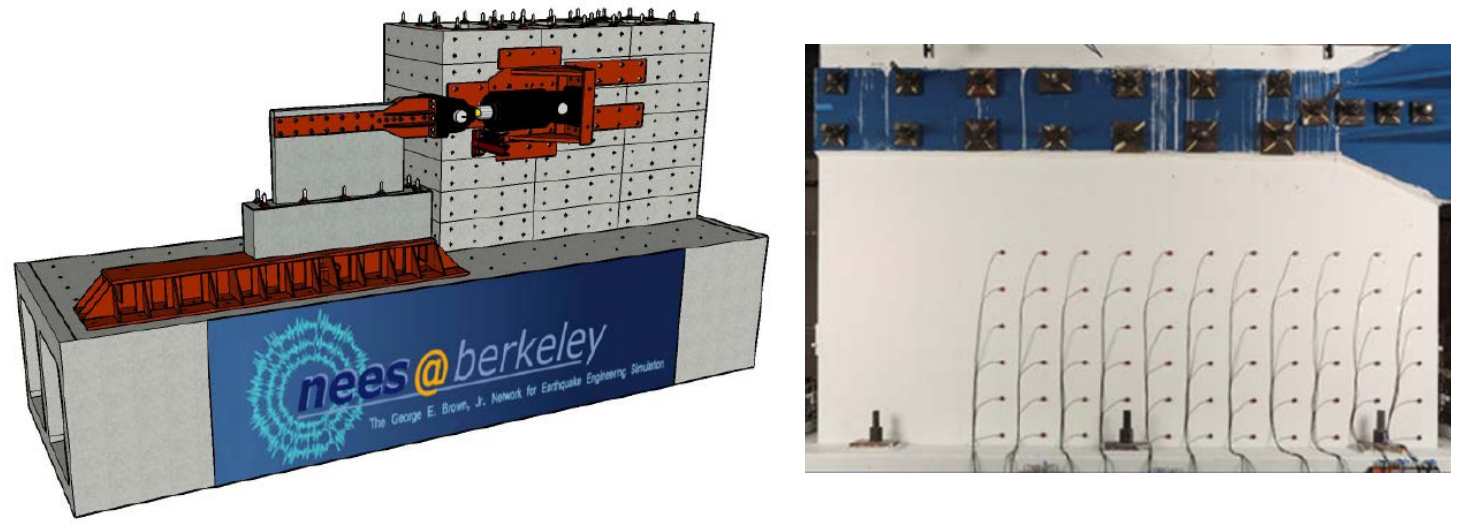

Figure 2.9 Typical RC shear wall test specimen (from Whyte and Stojadinovic, 2013b) 


\subsubsection{Hybrid Testing of Advanced Structural Systems}

In addition to studying the performance of conventional steel and RC structures, hybrid simulation also lends itself particularly well to the development and validation of advance structural systems. These systems include advanced braces and dampers, self-centering rocking structures, and base isolators. All of these systems are designed to improve the resilience of structures subjected to earthquake loads, ensuring that a structure is not only capable of achieving the lifesafety performance objective but also ensuring the structure remains operational and functional following a moderate to large seismic event. In addition, many of these systems rely on dynamic testing to provide realistic information on their performance which cannot be reproduced using traditional static test methods. As a result, some of these studies are conducted at or near real-time.

Eatherton and Hajjar (2010) validated the performance of a controlled rocking steel braced frame system using hybrid simulation. The tested frame specimen was a three-storey steel braced rocking frame, shown in Fig. 2.10, which was taken from a prototype structure designed in Los Angeles, California. The goal of using hybrid simulation was to demonstrate the performance of the system under real ground motions including the second-order (P-delta) effects from the gravity loads as well as the influence of the rest of the structure on the behaviour of the frame. The Pdelta effects and the effect of ambient building resistance were modelled analytically in OpenSees and were linked to the experimentally tested rocking frame using the UI-SimCor inter-ware. The frame was subjected to the 1995 Kobe earthquake record to allow for comparison between other tests conducted at the E-Defense shaking table test facility in Japan. The alpha-OS time stepping numerical integration scheme was employed. Figure 2.10 shows a schematic representation of the hybrid simulation test setup. Results once again demonstrated the ability of using hybrid simulation to validate seismic performance of an innovative steel rocking wall structure. The use of hybrid simulation allowed researchers to examine the influence of second order effects and resistance from the rest of the building analytically, without the need to construct a full-scale structure in the lab. 


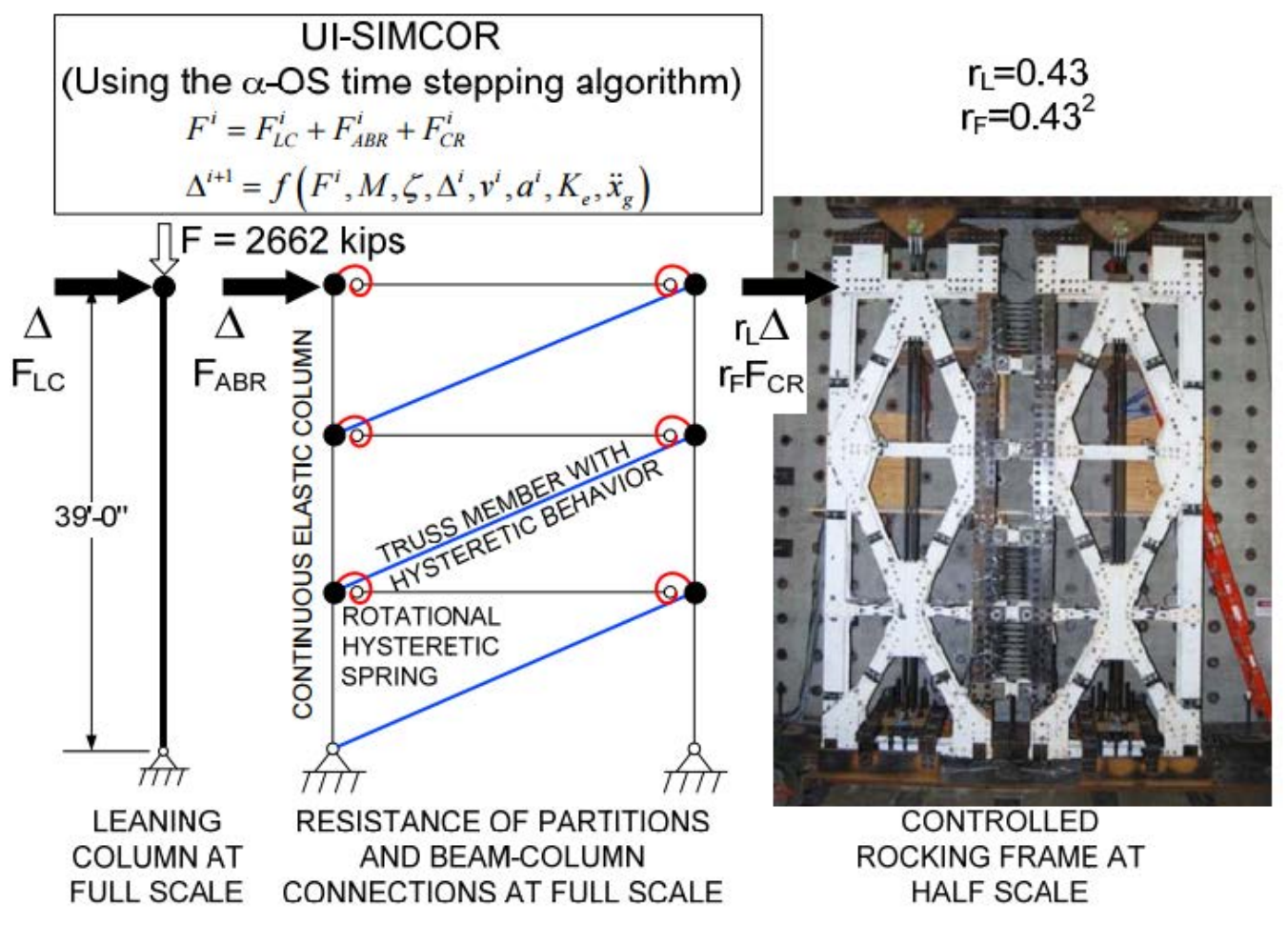

Figure 2.10 Steel rocking frame hybrid test setup (from Eatherton and Hajjar, 2010)

A study by Karavasilis et al. (2011) examined the use of large-scale real-time hybrid simulation to evaluate the effectiveness of supplemental damping devices for seismic hazard mitigation. In this case, because the damping devices proposed were rate-dependent, real-time testing was a necessity to accurately capture the performance of the dampers. In this study, the dampers were treated as experimental substructures while the structure was modelled analytically. The method allowed repeated testing of the structure subjected to different input ground motions without the need to repair the damaged structure after each test. Figure 2.11 shows the analytical and experimental substructures for the real-time hybrid simulation. A CR unconditionally stable explicit integration algorithm developed by Chen et al. (2009) was used in the study in addition to an adaptive inverse compensation (AIC) method developed by Chen and Ricles (2010) to compensate for actuator delay during the simulation. Results demonstrated that the structure designed with dampers required smaller structural sections compared to a conventional special moment resisting frame and had better seismic performance. Real-time hybrid simulation proved to be 
an effective tool in analyzing the effects of the dampers on the peak floor velocities, floor accelerations, and floor spectra accelerations, something that could not be feasibly achieved without testing a full-scale structure on a shaking table, which would be time consuming and expensive.

Another advanced structural bracing system was studied by Kammula et al. (2012) using the pseudo-dynamic test method. The study focused on a self-centering energy dissipative (SCED) brace developed to reduce residual drifts in steel structures following an earthquake. A single SCED brace in a steel frame formed the physical portion of the substructure while the remaining 6-storey prototype structure was modelled analytically in the finite element software OpenSees. The interware UI-SimCor developed by Kwon et al. (2005) was used to connect the analytical and experimental substructures and the alpha operator splitting scheme was selected as the numerical integration technique. Ultimately, hybrid simulation was shown to be a very effective tool in assessing the seismic performance of the SCED brace, allowing over 30 ground motions to be applied to the physical and analytical substructures. Given the number of tests that could be conducted on the physical specimen, results of the study were also used to develop seismic fragility functions of a structural system with SCED braces, demonstrating another unique capability of the hybrid simulation technique (Kammula et al., 2014).

Finally, a structural system that lends itself particularly well to hybrid simulation are base isolated systems. This is due to the fact that in order to accurately capture the response of these systems they should be tested at or near full scale and at a realistic loading rate, as the effects of scale and strain rate can have a pronounced impact on their behaviour. In addition, base isolated structures tend to remain at or near elastic throughout moderate earthquakes, because all of the deformation is concentrated in the isolators. The resulting system is one that lends itself to substructuring, where the isolation system is physically tested in the lab and the structure is modelled analytically. In a study by Molina et al. (2002), the experimentally tested isolating devices were $250 \mathrm{~mm}$ diameter rubber bearings, designed for application beneath a four-storey prototype structure. A hydraulic actuator was used to apply the lateral load to the rubber bearing, while an airbag was used to apply a constant axial load. However, the test was not conducted at real-time because of limitations associated with laboratory equipment. Instead, the hybrid test was conducted at a 

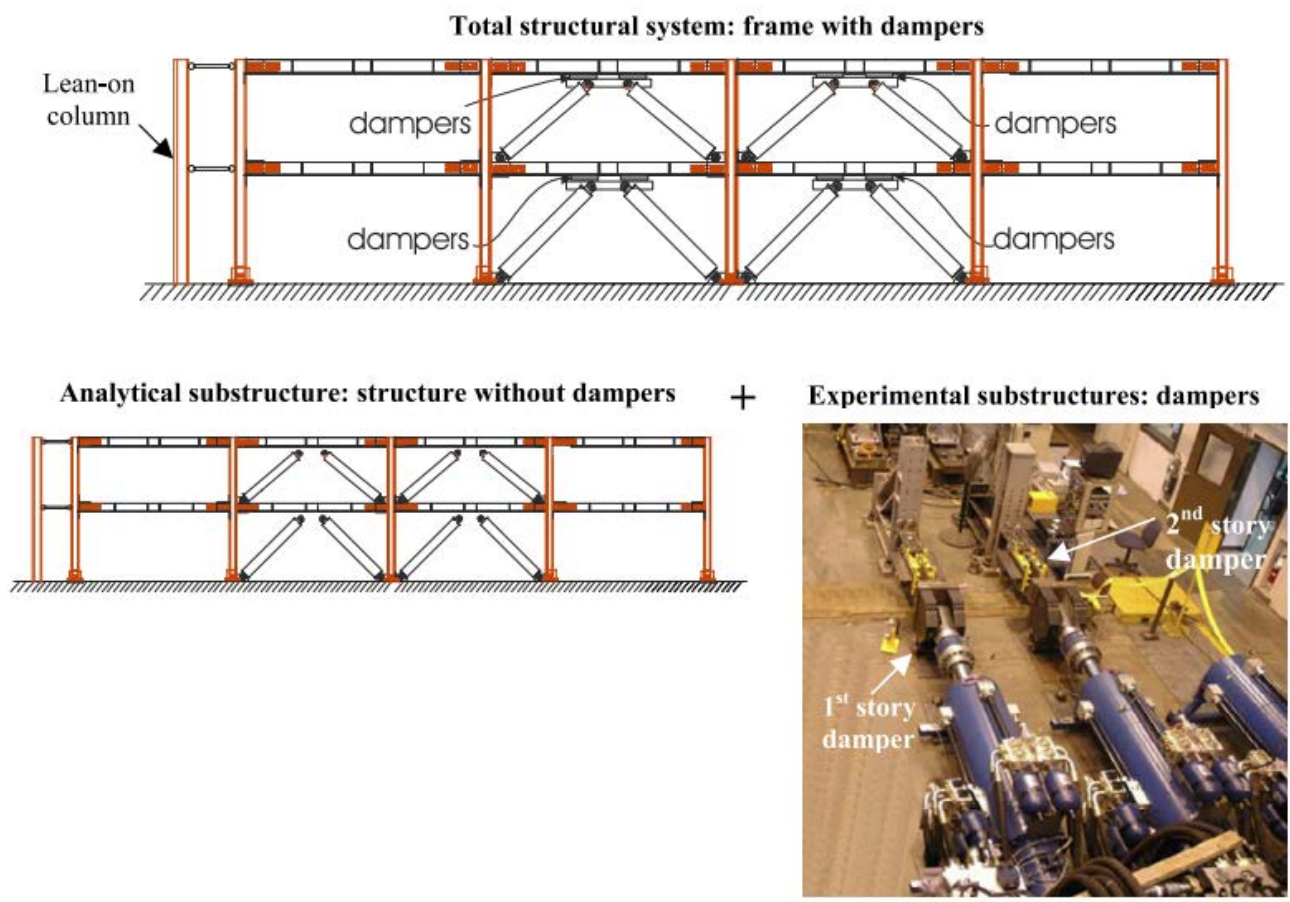

Figure 2.11 Damping device real-time hybrid test setup (from Karavasilis et al., 2011)

lower speed (10-30 times longer than the original record) and a compensation technique was used to capture the strain rate effects of the isolator. This compensation technique scaled the measured isolator load according to some characterizing tests that had been completed on the same rubber type as those tested in this study. Results demonstrated that the compensation scheme worked well in relieving the dependence on testing speed and the hybrid test method allowed the response of the whole system to be established, as opposed to the response of a single isolator.

Although Molina et al. (2002) established that the hybrid test method worked well for base isolated structures, developing an accurate compensation scheme for different sizes and types of isolators would be time consuming and costly. To better establish the seismic performance of full scale isolation bearings, Sarebanha et al. (2017) used the CALTRANS Seismic Response Modification Device (SRMD) test facility to conduct full-scale tests on isolation bearings. One of the unique features of this test facility is its ability to apply real-time 6-DOF earthquake loading to a full-scale bearing device, including lateral displacements of up to $1219 \mathrm{~mm}$ and axial loads up to $53400 \mathrm{kN}$. The test specimens included three $1.5 \mathrm{~m}$ diameter lead core rubber bearings and 
the prototype structure was a nuclear power plant. Because the analytical model had hundreds of nodes and thousands of degrees-of-freedom, the finite element software OpenSeesSP, a modified version of OpenSees that allows for simulations on high-performance computing platforms was utilized in this study. Results demonstrated that by using the parallel computing capabilities of OpenSeesSP, they had the ability to run up to 2.5 times faster than a conventional computer. The test also used a real-time predictor-corrector algorithm developed by Mosqueda et al. (2006) and Schellenberg et al. (2009) to account for actuator delay, which is crucial in a real-time hybrid simulation. Results of the study identified substantial vertical and horizontal coupling for the structure, which had a significant effect on the floor response spectra of the superstructure. It was also shown that it was essential to include vertical ground motion input to accurately predict floor response spectra near the vertical frequencies of the superstructure. In identifying these coupling effects, it emphasizes the valuable role that hybrid simulation can play in understanding the complete response of a system. These results could otherwise not have been established without conducting a full-scale test of the prototype structure on a shaking table, something that would be impossible at full-scale, given the size and weight of a typical nuclear power plant.

In this study, the work conducted by past researchers on the hybrid simulation of RC shear wall structures is extended and applied to a multi-storey RC shear wall structure. This includes application of the appropriate boundary conditions (axial load, shear, and overturning moment) to the experimentally tested shear wall and modelling additional storeys of the shear wall analytically. The goal is to assess the feasibility of using hybrid simulation to study the response of stiff RC shear wall structures and address some of the challenges surrounding the use of hybrid simulation to study stiff structural elements. Additionally, the hybrid test results will provide insight into the influence that the dynamic response of the global structural system has on the response of the experimental element, specifically if it has an impact on the observed ductility, energy dissipation capacity, and failure mechanisms when compared with traditional component-level testing. 


\subsubsection{Force-based Hybrid Simulation}

Although hybrid simulation has been used extensively to study the seismic response of comparatively flexible structural systems, it has not been used to study stiff structural systems because of the challenges stiff structural systems present in both the analytical and physical substructures of the hybrid simulation. These challenges include selection of an appropriate numerical integration scheme for stiff structural systems, small displacement increments that must be accurately applied in the laboratory, and stiff cross-coupling of hydraulic actuators controlled using displacement control for multi degree-of-freedom hybrid tests, which can lead to high force feedback (Bousias, 2014). One suggested approach to overcome these challenges is the adoption of a force-based hybrid simulation formulation, which uses a force command signal and displacement feedback as opposed to the traditional displacement command and force feedback approach that has been discussed in previous sections of this thesis. The following section outlines some of the developments in force-based hybrid simulation as well as some of its current limitations.

Past studies by Seible et al. (1996); Sivaselvan et al. (2008); Igarashi et al. (1990); Charlet (2007); Elkhoraibi and Mosalam (2007); Elkhoraibi (2007) and Kim (2009) have demonstrated that the incorporation of force-based hybrid simulation techniques can be effective in overcoming some of the previously discussed challenges when conducting hybrid tests on stiff structural systems. Researchers have found this can be accomplished if appropriate care is taken to ensure that the actuators are capable of applying the calculated forces with minimal error to the physical substructure and if an appropriate numerical integration scheme and solution algorithm based on a force-based formulation are employed (Bousias, 2014). Force-based numerical integration schemes for hybrid simulation have recently been developed and employed by Forouzan et al. (2017). The force-based hybrid method developed by Forouzan et al. (2017), referred to as the quadratic alpha method, is a non-iterative solution algorithm which satisfies force equilibrium at each time step. The method has been shown to be effective in a small-scale hybrid test with a single actuator used to control the physical substructure in the lab. The results demonstrate the feasibility of using the force-based framework with a physical specimen and are currently extending their research to include nonlinear structural systems as well as systems with multiple degrees-of-freedom. 
Although there are benefits in the use of a force-based hybrid simulation formulation for stiff structural elements, it also has some challenges of its own that must be overcome prior to its widespread use. One such example applies to systems with multiple degrees-of-freedom, which require multiple actuators to apply the command force to the physical specimen in the laboratory. In this type of test, the physical substructure receives the command signal (force or displacement) in terms of global degrees-of-freedom and must convert the command signal to actuator degrees-of-freedom (DOF) to apply the correct command forces or displacements to the physical substructure. In conventional displacement-based hybrid simulations, a transformation with a unique solution is used to convert the command displacements in global coordinates to actuator coordinates, however, for the case of command forces, no such unique solution exists. Another challenge of the force-based hybrid simulation approach occurs during the post-yield response of the physical substructure, during which the test specimen may experience some softening in its response. During this stage of the test, the hybrid control mode must be switched to a displacement-based formulation to ensure that the system does not become unstable.

To overcome the challenge of softening of the physical substructure during the hybrid test and the potential for instability when using a force-based hybrid simulation formulation, researchers have proposed mixed-mode hybrid simulation control techniques (Charlet, 2007; Nakata et al., 2007; Yang et al., 2017). One of the first implementations of a mixed mode control approach for hybrid simulation of stiff structural elements was conducted by Charlet (2007). The high axial stiffness of the concrete column tested in this study presented a challenge because of the small target displacements sent to the actuators controlling the gravity load during each time step. To overcome this challenge, an iterative algorithm was implemented in the hybrid control software to generate continuous force commands for the vertical actuators based on the displacement commands from OpenSees, the measured displacement to the test specimen, and effective stiffness of the test specimen. Although technically this is a mixed-mode hybrid test, the formulation of the hybrid test was still based on a displacement formulation, and does not provide a force-based hybrid simulation formulation for hybrid testing. 
Alternatively, Nakata et al. (2007) and Yang et al. (2017) have both proposed hybrid simulation formulations that are based on a force-based solution to the equations of motion, such that command forces are sent to the physical substructure directly without having been converted to a force based on a command displacement. However, the studies by Nakata et al. (2007) and Yang et al. (2017) have different interpretations of the mixed-mode control technique in that the method proposed by Yang et al. (2017) uses displacement based formulations for the flexible DOFs in a multi DOF system and uses force-based formulation for the stiff DOFs (typically axially), while the study by Nakata et al. (2007) uses a purely force-based approach until a change in stiffness of the structure is detected. In the method proposed by Yang et al. (2017), the switching criteria is based on the comparison of the tangent stiffness to the initial stiffness of the specimen. The algorithms have been applied to simple structural systems consisting of a single storey one-bay concentrically braced moment frame. Results of the study found that the proposed force-based method was superior to a displacement based approach for the stiff structural element tested.

In this study, the hydraulic equipment used to control the actuators in the laboratory is not able to switch control modes in the middle of a hybrid test and the method developed by Yang et al. (2017) is not yet readily available in the OpenFresco framework. As a result, a displacementbased formulation is used for the hybrid tests in this study. Nonetheless, future developments and implementation of the mixed force and displacement-based hybrid test approach show great potential for overcoming some of the major challenges associated with hybrid testing of stiff structural systems.

Joshua Woods, Department of Civil and Environmental Engineering, Carleton University 


\section{Chapter 3. Prototype RC Structure}

The goal of this research is to assess the feasibility in using hybrid simulation to capture the nonlinear response of a RC shear wall through a physical experimental test, while modelling the rest of the shear wall in a finite element program. The application of the hybrid simulation to a multi-storey RC shear wall structure using a combination of analytical and physical substructures has yet to be studied extensively in the field of earthquake engineering and structural testing. The majority of research to date conducted to study the seismic behaviour of RC shear walls has focused on component-level structural testing or large-scale shaking table testing. To facilitate the hybrid simulation of a multi-storey RC shear wall structure, a prototype building located in a region of high seismicity has been designed. The following chapter describes the design of the prototype RC shear wall building.

\subsection{Preliminary Design}

The preliminary design phase in this study includes the structural design of a prototype RC structure to determine realistic shear wall geometries and probable seismic design forces. The prototype RC shear wall structure is a three-storey office building in Victoria, British Columbia. The structure conforms to a FEMA type C2 concrete shear wall building assessed in FEMA 440 (FEMA, 2005): Improvement of Nonlinear Static Seismic Analysis Procedures. The FEMA type C2 building is a concrete shear wall structure in which the gravity loads are carried by concrete columns while the exterior and interior walls act as bearing and shear walls. The applicable standards used in the design and analysis of the prototype building include: 
- National Building Code of Canada, NBCC, 2010 (NRC, 2010)

- Design of Concrete Structures, CSA A23.3-14 (CSA, 2014a)

- Building Code Requirements for Reinforced Concrete, ACI 318-14 (ACI, 2014)

- Minimum Design Loads for Building and Other Structures, ASCE7-10 (ASCE, 2013)

The three-storey prototype RC shear wall building has a first storey height of $4.5 \mathrm{~m}$ while the remaining two storeys measure $3.5 \mathrm{~m}$ tall. This results in an overall building height of $11.5 \mathrm{~m}$. In plan, the structure measures $30 \mathrm{~m}$ x $50 \mathrm{~m}$, which includes four $7.5 \mathrm{~m}$ bays in the N-S direction and four $12.5 \mathrm{~m}$ bays in the E-W direction. As illustrated in Figures 3.1 and 3.2, the principle lateral load resisting structural system (LLRS) includes perimeter shear walls in addition to a centrally located concrete core. The shear walls are distributed in such a way that they create a torsionally stable configuration, meaning the centre of the mass and rigidity will be reasonably close together as recommended by Priestley and Paulay (1992). The structure is analyzed for purposes of design using the 3-dimensional structural analysis software ETABS (Computers and Structures Inc., 2015). The member sizes for the gravity-load resisting system (GLRS) are determined using the three-dimensional building model and the preliminary member sizes for the LLRS are determined using the equivalent static force design procedure as outlined in the NBCC, 2010 (NRC, 2010). The shear wall dimensions are refined using the iterative response spectrum analysis (RSA) and design procedure described in the NBCC, 2010 (NRC, 2010). The LLRS for the three-storey RC structure is a moderately ductile shear wall system, which has ductility, $R_{d}$, and overstrength, $R_{o}$, factors of 2.0 and 1.4, respectively (NRC, 2010). Appendix B provides an in-depth discussion and additional details of the iterative seismic design process, outlining the considerations and assumptions made during the overall design of the three-storey RC structure and detailed design of the shear wall elements.

As shown in Figures 3.1 and 3.2, the three-storey building has six perimeter and interior RC shear walls in the N-S direction and six RC shear walls along the perimeter of the building in the E-W direction. Ultimately, one of the six identical perimeter shear walls oriented in the N-S direction is used for the hybrid simulation because of the computational limitations associated 

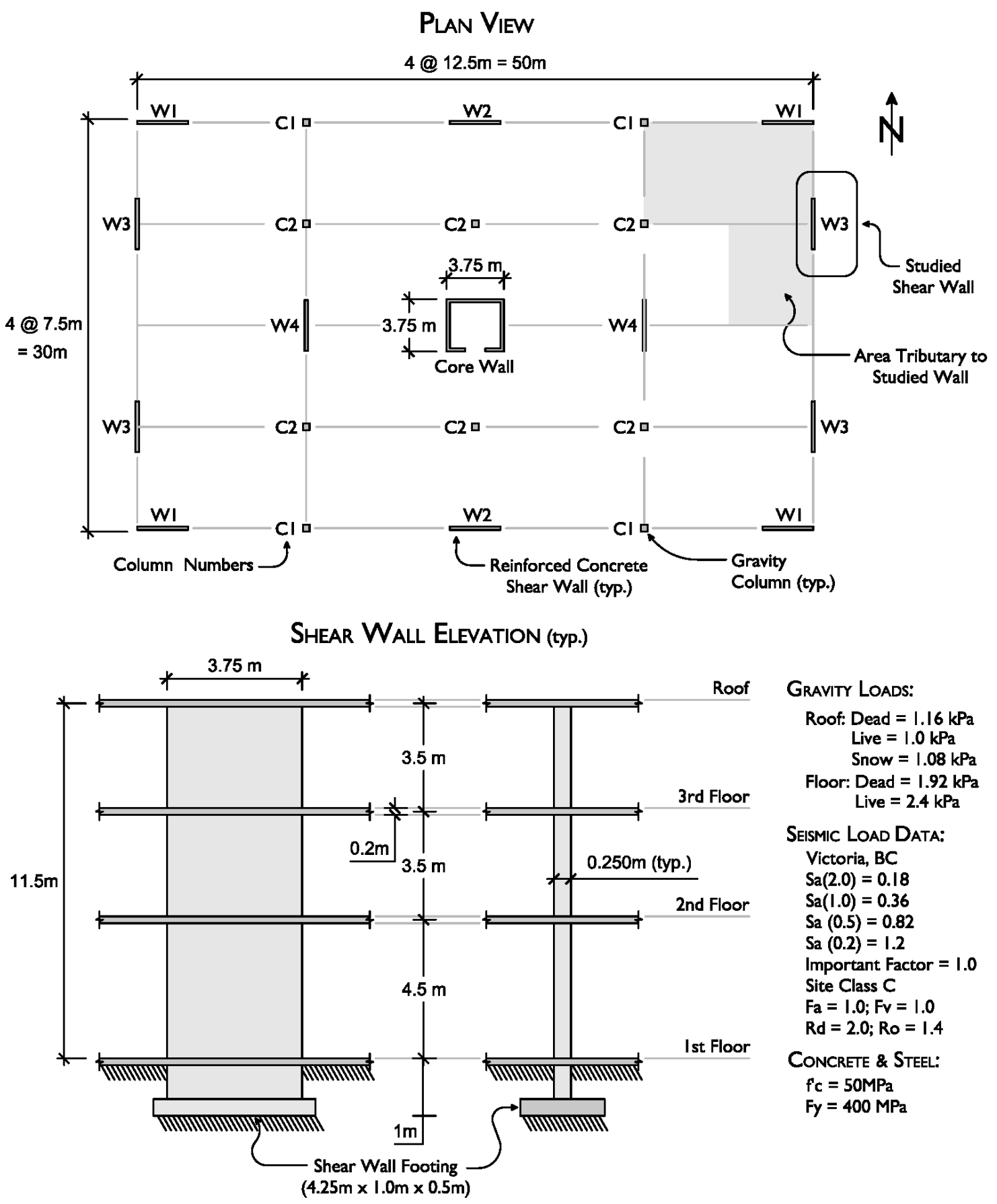

Figure 3.1 Plan view of the 3-storey prototype RC structure 


\section{Elevation View: N-S Direction}

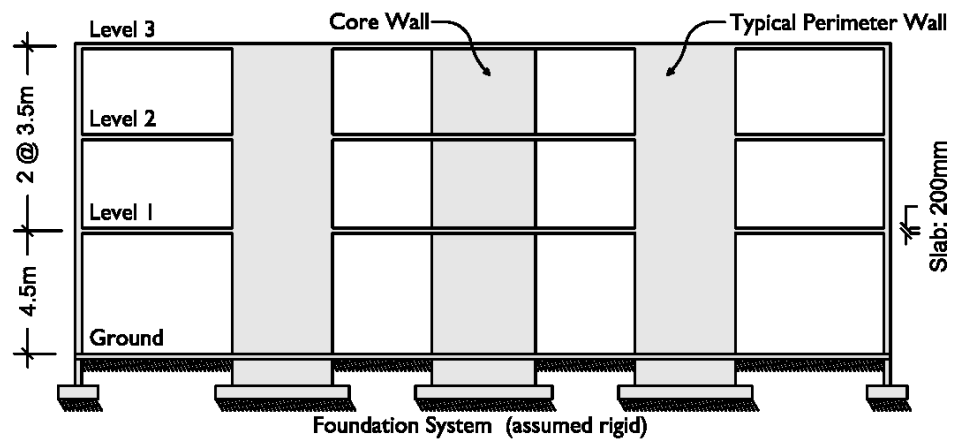

Elevation View: E-W Direction

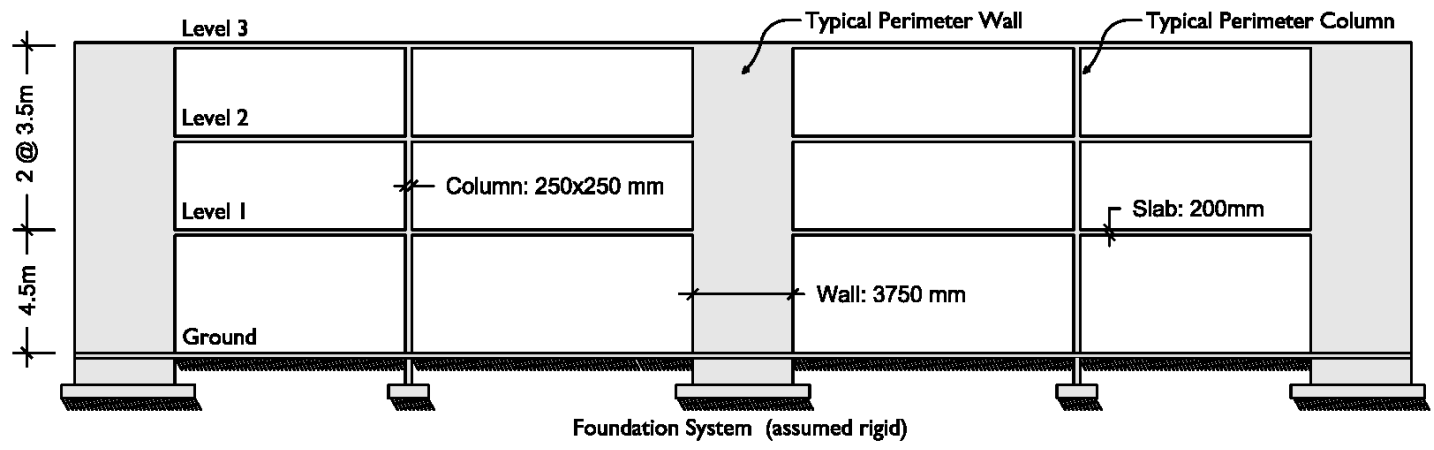

Figure 3.2 Elevation view of the 3-storey prototype RC structure 
with conducting nonlinear time-history analysis for the entire structure. During the design of the structure, walls perpendicular to the direction of lateral loading are assumed to have no lateral resistance, because their stiffness in comparison to the shear walls in the direction of the lateral load is negligible. The increased height of the first storey is selected to represent the larger storey height for commercial spaces typically integrated into the first storey of these types of structures. The gravity load design is completed for the structure based on the NBCC (2010) requirements, and includes allowance for structural components (e.g. slabs, columns, and walls) and non-structural components (e.g. roofing materials, electrical/mechanical, partitions etc...). The structure is designed as a typical office building of normal importance (importance factor $\left(I_{E}\right)$ of 1.0 for ultimate limit states design). The self-weight of the concrete slab is assumed to be $4.8 \mathrm{kPa}$ based on a uniform slab thickness of $200 \mathrm{~mm}$. The corresponding dead and live loads used in the design of the structure are listed in Appendix B. The design snow load is determined from the NBCC (2010) design requirements for Victoria, BC and results in an average distributed snow load over the surface of the roof of $1.08 \mathrm{kPa}$. The wind load analysis is conducted in accordance with NBCC 2010; however, because the structure is located in a highly seismic active region and the structure is short, the wind loads do not govern the design of the LLRS.

\subsection{Seismic Design}

\subsubsection{Equivalent Static Force Procedure}

The seismic design of the three-storey RC structure is initially carried out using the equivalent static force procedure as described in Part 4 of Division B of the NBCC (2010). Although a full dynamic analysis of the structure is required because of the high seismicity in the location of the building $\left(I_{e} F_{a} S_{a}(0.2)>0.35\right)$, results from the simplified equivalent static force procedure are used to establish the initial member sizes and serve as limitations on the results from the dynamic analysis in the following section. Spectral ground acceleration values taken from the NBCC (2010) are based on a $2 \%$ in 50 years probability of exceedence ( 2475 year return period). The code-based fundamental period of the structure $\left(T_{a}\right)$ is calculated according to NBCC (2010), 
which is shown in Eq. 3.38:

$$
T_{a}=0.05\left(h_{n}\right)^{3 / 4}
$$

where $h_{n}$ is the height of level "n" of the structure, which is taken as the total height of the structure, resulting in a code based period of $0.312 \mathrm{~s}$. The equivalent static force procedure approximates the elastic base shear $(V)$ and reduces it by a response modification factor $\left(R_{d} R_{o}\right)$ according to Eq. 3.39:

$$
V=\frac{S\left(T_{a}\right) M_{v} W I_{E}}{R_{d} R_{o}}<\frac{2}{3} \frac{S(0.2) M_{v} W I_{E}}{R_{d} R_{o}}
$$

where $W$ is the weight of the structure, which includes the dead loads acting on the structure and $25 \%$ of the roof snow load. The use of Eq. 3.39 results in a total seismic base shear of $9086 \mathrm{kN}$, which is governed by the upper bound of Eq. 3.39. This upper bound on Eq. 3.39 has been set in place to account for the relatively good observed performance of low-rise structures during recent earthquakes (NRC, 2010). The total base shear is distributed over the height of the building using Eq. 3.40:

$$
F_{x}=\frac{\left(V-F_{t}\right) W_{x} h_{x}}{\sum_{i=1}^{n} W_{i} h_{i}}
$$

where $W_{x} / W_{i}$ represent the weight that corresponds to level $\mathrm{x}$ or $\mathrm{i}, h_{x} / h_{i}$ is the height above ground of level x or $\mathrm{i}, n$ is the total number of levels in the building, and $F_{t}$ is an additional concentrated force applied to the top of the building to account for higher mode effects, which is taken as zero when the fundamental vibration period of the structure $\left(T_{a}\right)$ is less than $0.7 \mathrm{~s}$. The seismic weight, lateral force, and overturning moment for each level of the prototype building from the equivalent static force procedure can be found in appendix B. In addition to the distribution of the static base shear, structural irregularities must also be considered in the seismic design of the structure. Because the structure does not present irregularities associated with vertical stiffness, weight, geometry, in-plane strength, out-of-plane strength, weak storey, and the system is orthogonal, type 1-7 irregularities are not present in this building (NRC, 2010). In addition, given that 
the selected geometry of the structure is rectangular and symmetric, the centre of mass is expected to closely coincide with the centre of rigidity, which will result in minimal torsional sensitivity of the structure. However, torsional effect due to accidental eccentricity is considered as required by the design standard. Torsional sensitivity is defined by a factor $B_{x}$, which is calculated using Eq. 3.41:

$$
B_{x}=\frac{\delta_{x e, m a x}}{\delta_{x e, a v g}}
$$

where $\delta_{x e, \max }$ is the maximum displacement at extreme points of the storey under consideration, produced by the seismic force $(V)$ acting at 0.1 times the width of the building from the centre of mass in the direction under consideration, $\delta_{x e, a v g}$ is the average displacements at extreme points of the storey under consideration produced by the same force $\left[0.5\left(\delta_{x e, \min }+\delta_{x e, \max }\right)\right]$. Figure 3.3 shows the results of the evaluation of the $B_{x}$ factor for the top storey of the structure.

The results from the torsional sensitivity analysis confirm that in both directions, the $B_{x}$ factor remains below 1.7 for each storey in the structure. Because the structure is rectangular and symmetric and the torsional sensitivity factor is less than 1.7; the torsional moments $\left(T_{x}\right)$ about a vertical axis at each level throughout the building can be considered separately in each direction using Eq. 3.42 for each level in the structure:

$$
T_{x}=F_{x}\left(e_{x}+0.1 D_{n x}\right)
$$

where $F_{x}$ is the seismic force at the level under consideration, $e_{x}$ is the eccentricity between the centre of mass and rigidity, and $D_{n x}$ is the width of the building in the direction under consideration. The design shear force for each shear wall in each direction including the effects of torsion in the N-S and E-W directions are shown in Table 3.1. 

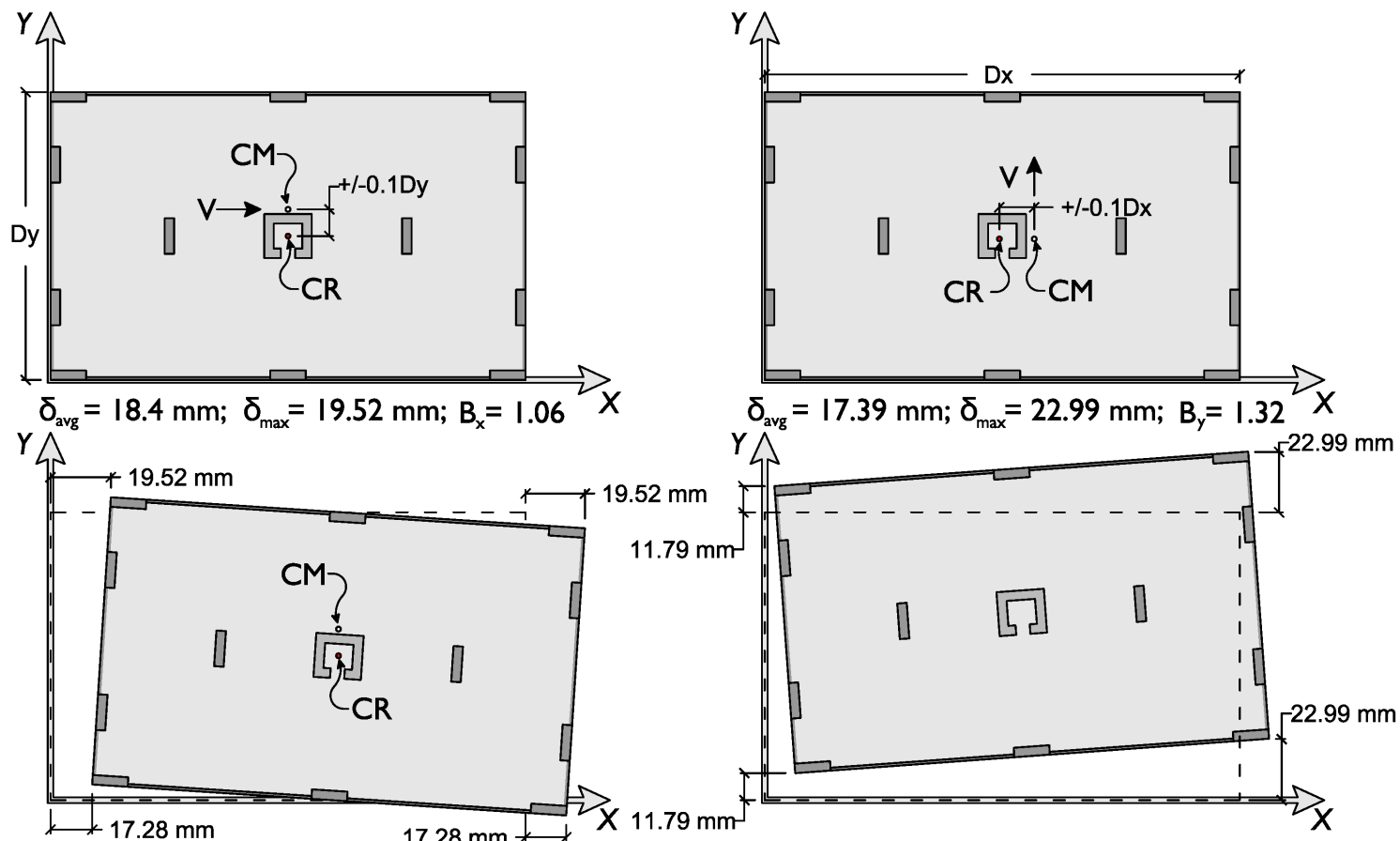

Figure 3.3 Calculation of $B_{x}$ and $B_{y}$ for torsional sensitivity

Table 3.1 Shear wall design forces from equivalent static force procedure

\begin{tabular}{ccccc}
\hline \hline Level & $\begin{array}{c}\text { Shear Force } \\
(\mathrm{kN})\end{array}$ & $\begin{array}{c}\text { Torsional Force } \\
(\mathrm{kN})\end{array}$ & $\begin{array}{c}\text { Design Force } \\
(\mathrm{kN})\end{array}$ & $\begin{array}{c}\text { Design Moment } \\
(\mathrm{kN}-\mathrm{m})\end{array}$ \\
\hline 3 (Roof) & 304 & \multicolumn{4}{c}{ N-S Direction } \\
2 & 650 & 217 & 422 & 1189 \\
1 & 1005 & 274 & 867 & 4069 \\
\hline & \multicolumn{5}{c}{ E-W Direction } \\
\hline 3 (Roof) & 298 & 46 & 344 & 9512 \\
2 & 745 & 85 & 830 & 4390 \\
1 & 1112 & 104 & 1216 & 10027 \\
\hline \hline
\end{tabular}




\subsubsection{Response Spectrum Analysis}

To optimize the design of the structure and get a better understanding of the dynamic performance of the building, a response spectrum analysis is conducted. It should be noted that a dynamic analysis of the structure is required according to the NBCC (2010) because the structure is located in Vicoria, BC, which is considered to have high seismicity $\left(I_{e} F_{a} S_{a}(0.2)>0.35\right)$. The response spectrum analysis method is an approximation for multi degree-of-freedom systems and gives an estimation of the maximum peak response without the need for conducting a nonlinear time history analysis. Response spectrum analysis is an iterative dynamic analysis method that uses a three-dimensional linearly elastic model of the structure to optimize the design (Filiatrault et al., 2013).

The results from the equivalent static force procedure discussed in the previous section are used as an initial condition for the construction of the three-dimensional model in ETABS (Computers and Structures Inc., 2015). In the dynamic analysis of RC structures, the linearly elastic finite element model must account for the reduction in element stiffness due to cracking of the concrete components, which is expected to occur under earthquake loading. The Canadian design standard for RC structures (CSA A23.3-14) recommends the following procedure for reducing the stiffness of structural elements (CSA, 2014a). The reduction factors $\left(\alpha_{w}\right.$ and $\left.\alpha_{c}\right)$ are applied to the gross moment of intertia $\left(I_{g}\right)$ or the shear area $\left(A_{v e}\right)$ of the structural element. This procedure assigns effective stiffness coefficients for columns and walls separately, denoted $\alpha_{w}$ and $\alpha_{c}$, respectively, which can be computed using Eq. 3.43 and Eq. 3.44:

$$
\begin{gathered}
\alpha_{w}=0.6+\left(\frac{P_{s}}{f_{c}^{\prime} A_{g}}\right) \leq 1.0 \\
\alpha_{c}=0.5+0.6\left(\frac{P_{s}}{f_{c}^{\prime} A_{g}}\right) \leq 1.0
\end{gathered}
$$

where $P_{s}$ is the axial force at the section resulting from factored dead and live load using earthquake load factors, $f_{c}^{\prime}$ is the compressive strength of the concrete, and $A_{g}$ is the gross area of the element. For simplicity, a conservative estimate of the effective stiffness of 0.6 is applied to the RC shear 
walls and 0.25 for the RC slabs in the model. After completion of the numerical model, a modal analysis is conducted to assess the dynamic behaviour of the structure. Table 3.2 shows the modal properties of the three-storey RC building, including mass participation ratios for the first six modes of vibration. Figure 3.4 shows the mode shapes and periods for each of the first six modes.

Results from the modal analysis indicate that the fundamental period of vibration of the structure $(0.410 \mathrm{~s})$ lies within the limits on the natural period of vibration assigned in the NBCC (2010) $\left(T_{a} \leq T \leq 2 * T_{a}\right)$. Following the modal analysis, the iterative response spectrum analysis method is conducted until the structure meets the requirements for strength and drift $\left(0.025 h_{s}\right)$. It is worth noting that the total design base shear $\left(V_{d}\right)$ remained above $80 \%$ of the static base shear described in the previous section, a lower limit set by the NBCC (2010) to avoid underestimating the total seismic base shear. The first 6 modes are considered in the response spectrum analysis, which accounts for greater than $\approx 95 \%$ mass participation in the two orthogonal directions and rotation. A complete set of results of the response spectrum analysis are presented in Appendix B. The SRSS method was used to combine the contribution from each mode in the analysis. The final seismic design forces for a typical shear wall in each direction (E-W and N-S) are shown in Table 3.3, including the design axial load, shear and moment.

Table 3.2 Modal properties of prototype RC shear wall structure

\begin{tabular}{ccccc}
\hline \hline & \multicolumn{4}{c}{ Mass Participation Ratios (\%) } \\
Mode & Period (s) & N-S & E-W & Rotation \\
\hline 1 & 0.410 & 75.7 & 0 & 0 \\
2 & 0.394 & 0 & 78.0 & 0 \\
3 & 0.341 & 0 & 0 & 74.7 \\
4 & 0.081 & 18.9 & 0 & 0 \\
5 & 0.076 & 0 & 19.3 & 0 \\
6 & 0.061 & 0 & 0 & 19.8 \\
\hline & Total (\%) & 94.6 & 97.3 & 94.5 \\
\hline \hline
\end{tabular}



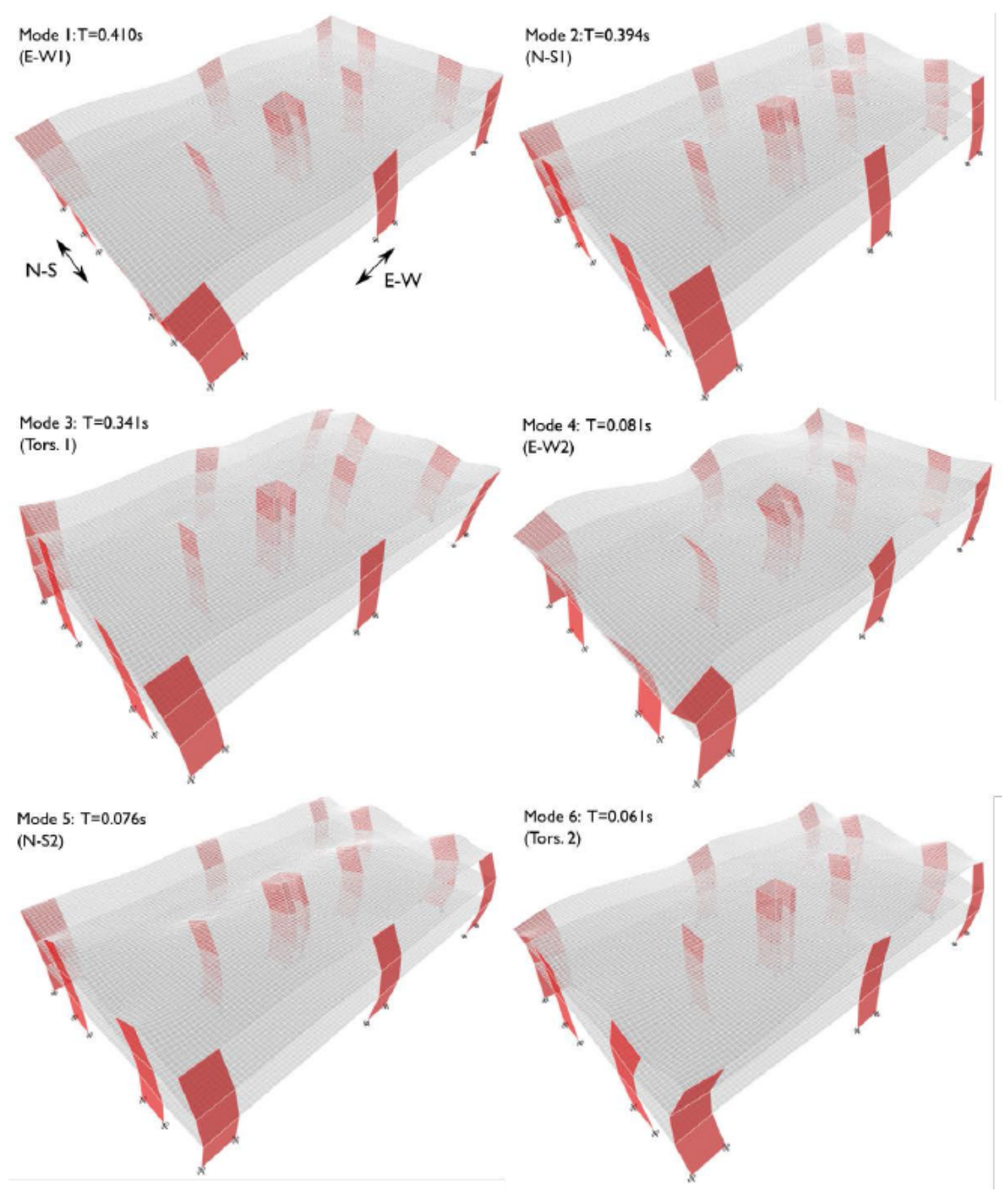

Figure 3.4 Modal response of prototype RC shear wall structure 
Table 3.3 Shear wall design forces from response spectrum analysis

\begin{tabular}{|c|c|c|c|}
\hline & \multicolumn{3}{|c|}{ E-W Direction } \\
\hline Level & $\begin{array}{l}\text { Storey Shear } \\
(\mathrm{kN})\end{array}$ & $\begin{array}{l}\text { Overturning Moment } \\
(\mathrm{kN}-\mathrm{m})\end{array}$ & $\begin{array}{l}\text { Axial Load } \\
(\mathrm{kN})\end{array}$ \\
\hline 3 (Roof) & 460 & 1466 & 152 \\
\hline 2 & 800 & 3905 & 379 \\
\hline \multirow[t]{2}{*}{1} & 1063 & 8255 & 595 \\
\hline & & N-S Direction & \\
\hline Level & $\begin{array}{l}\text { Storey Shear } \\
(\mathrm{kN})\end{array}$ & $\begin{array}{l}\text { Overturning Moment } \\
\qquad(\mathrm{kN}-\mathrm{m})\end{array}$ & $\begin{array}{c}\text { Displacement } \\
(\mathrm{mm})\end{array}$ \\
\hline 3 (Roof) & 415 & 1353 & 146 \\
\hline 2 & 697 & 3557 & 372 \\
\hline 1 & 990 & 7681 & 592 \\
\hline
\end{tabular}

\subsection{Design of Gravity Load Resisting Structural System}

While the RC shear walls are responsible for resisting the in-plane lateral loads due to wind and earthquake, modern design standards also recognize the importance of accounting for the deformation of the lateral load resisting system when designing the gravity load resisting system. This is a result of the fact that as the building deforms laterally during an earthquake or wind, the gravity load resisting system must also be capable of achieving the same level of deformability (Filiatrault et al., 2013). This is paramount for the structure to be able to meet the life-safety performance objective in modern seismic design standards used around the world. When designing the gravity load resisting system, the load combination shown in Eq. 3.45 applies for earthquake scenarios:

$$
1.0 D+1.0 E+0.5 L+0.25 S
$$

Under this load combination, gravity load carrying columns are required to have sufficient ductile rotational capacity to sustain the design inelastic rotations and cyclic demands. To do so, the design of gravity load carrying columns must consider the axial load demand, moment demand and shear demands and check if these demands exceed the column capacities under all gravity load 
combinations. The design of the RC slabs and columns were carried out using the appropriate clauses of CSA A23.3-14 but are not discussed in detail here. Additional details are provided in Appendix B.

\subsection{Design of Lateral Load Resisting Structural System}

Based on the forces from the ESFP and response spectrum analysis analysis techniques discussed in the previous section, the components of the seismic force resisting structural system are designed in accordance with CSA A23.3-14 (CSA, 2014a). The design of the shear wall elements includes both the flexural and shear strength requirements, each of which are discussed in the following two subsections.

\subsubsection{Shear Wall Flexural Design}

Moderately ductile shear wall design provisions are provided in the Canadian Design Standards for reinforced concrete structures CSA A23.3-14 (CSA, 2014a). A key parameter in the design of a $\mathrm{RC}$ shear wall is its aspect ratio, which is the ratio of the height of the wall to its length $\left(h_{w} / l_{w}\right)$. Walls with an aspect ratio of 2 or greater are generally considered flexurally dominant "slender" walls (CSA, 2014a). Walls with aspect ratios less than 2 are considered as "squat" walls, whose behaviour can be characterized by a combination of shear and flexural responses. Squat walls are required to meet additional design provisions to ensure a dependable seismic response. However, all of the wall specimens in the prototype structure have aspect ratios greater than 2, and are designed as flexurally dominant slender shear walls. The yielding mechanism in each wall is a plastic hinge intended to form at the base of the shear wall. Over the plastic hinge region, referred to as the plastic hinge length, specific detailing of horizontal and vertical steel reinforcement is required to ensure the wall will have sufficient rotational and shear capacity during a major seismic event. In CSA A23.3-14, the length of the plastic hinge $\left(L_{p}\right)$ is determined using Eq. 3.46:

$$
L_{p}=0.5 l_{w}+0.1 h_{w}
$$


where $l_{w}$ is the length of the longest wall in the direction of the building under consideration and $h_{w}$ is the total height of the wall (e.g. to the top of the building). Use of Eq. 3.46 results in a plastic hinge length of $3025 \mathrm{~mm}$ for a typical wall in the prototype structure, which is less than the height of the first storey of the structure. For regions above the plastic hinge length, following the principles of the capacity based seismic design methodology, the design overturning moment must be increased by the ratio of the factored moment capacity to the factored moment demand $\left(M_{r} / M_{f}\right)$ to ensure that yielding does not occur in adjacent storeys.

To avoid out-of-plane buckling of the shear walls in the structure under significant inelastic cyclic demands, the thickness of the wall $\left(t_{w}\right)$ for simple rectangular walls must be greater than one-twentieth of the unrestrained length of the wall $\left(l_{u}\right)$, which is typically the span between adjacent floor levels. For a first story height of $4500 \mathrm{~mm}$, this results in a minimum wall thickness of $225 \mathrm{~mm}$.

Over the length of the potential plastic hinge region $\left(L_{p}\right)$, the maximum spacing between vertical steel reinforcing bars is $300 \mathrm{~mm}$. Two curtains of distributed vertical and horizontal steel reinforcement are required when the in-plane factored shear force $\left(V_{f}\right)$ in the wall exceeds the limits shown in Eq. 3.47:

$$
V_{f}=0.18 \lambda \phi_{c} \sqrt{f_{c}^{\prime}} A_{c v}
$$

where $\lambda$ is a factor for lightweight aggregate, taken as 1.0 for this study, and $A_{c v}$ is the net area of the section resisting shear (ie. the web of the wall section). However, Filiatrault et al. (2013) recommends that regardless of the limit shown in Eq. 3.47 it is typically considered good practice, particularly in areas of high seismic activity as is the case for this prototype structure to use two curtains of vertical and horizontal steel reinforcement. For the boundary elements in the plastic hinge region, the minimum concentrated reinforcement ratio $\left(\rho_{l, c}\right)$ is given by $0.0015 t_{w} l_{w}$ at each end of the wall. Unique to the Canadian CSA A23.3 design standard, it prescribes further specifications based on assessment of the inelastic rotational demand on the plastic hinge and compares this to the inelastic rotational capacity of the wall. This represents a step towards performancebased seismic design because it relates to the ability of the wall to undergo lateral deformation 
(Filiatrault et al., 2013). The inelastic rotational demand $\left(\theta_{i d}\right)$ is determined using Eq. 3.48:

$$
\theta_{i d}=\left(\frac{\delta_{f} R_{o} R_{d}-\delta_{f} \gamma_{w}}{h_{w}-l_{w} / 2}\right) \geq 0.004
$$

where $\delta_{f} R_{d} R_{o}$ is the design displacement, $\delta_{f}$ is the displacement at the top of the wall under factored loads, and $\delta_{f} \gamma_{w}$ is the elastic portion of the displacement. The inelastic rotational capacity of a wall, $\theta_{i c}$ can be determined according to Eq. 3.49:

$$
\theta_{i c}=\left(\frac{\epsilon_{c u} l_{w}}{2 c}-0.002\right) \leq 0.025
$$

where $c$ is the depth to the neutral axis from the extreme fibre in compression and $\epsilon_{c u}$ is the ultimate concrete strain in compression, taken as 0.0035 , but can be increased to 0.014 if the compression region of the wall is confined as a ductile column. If the compression region of the wall is not detailed as a ductile column, but an increase in the ultimate concrete strain is required to meet the inelastic rotational demand, the column transverse reinforcement must be modified accordingly. Note that for moderately ductile walls, the ductility requirements in Eq. 3.48 and Eq. 3.49 can be considered satisfied if the depth to the neutral axis is less than $0.15 l_{w}=562.5 \mathrm{~mm}$, which is the case for the typical shear wall in this study. Regardless, the ductility demands and capacities are still computed.

Outside of the plastic hinge region, the distributed vertical steel reinforcement in the web must have a minimum spacing of $450 \mathrm{~mm}$ and the distributed reinforcement ratio in the vertical $\left(\rho_{l}\right)$ and horizontal $\left(\rho_{t}\right)$ directions must be larger than $0.25 \%$. The minimum concentrated reinforcement ratio $\left(\rho_{l, c}\right)$ is $0.001 t_{w} l_{w}$ at each end of the wall in the plastic hinge region for moderately ductile walls, however there are no additional requirements for concentrated vertical steel reinforcement outside the plastic hinge region in moderately ductile walls.

Based on these requirements, a typical RC shear wall flexural design is completed using a strain compatibility analysis and an ultimate concrete strain in compression of 0.0035 . The properties and design parameters for the design shear wall at each level in the structure are shown in Table 3.4. It is worth noting that the three-storey shear wall is designed to develop a plastic 
hinge yielding mechanism at its base, while the other two stories are capacity based designed to ensure they remain elastic throughout a significant seismic event. As described by Priestley and Paulay (1992), if the flexural strength of the wall specimen was to vary in accordance with the bending moment diagram, then plastic hinges could form with equal probability over the height of the wall. Furthermore, because the shear strength of the wall is lower within the plastic hinge region, special consideration to the shear design would be required over the entire height of the wall. Therefore, to ensure that the second and third storey walls have sufficient moment capacity to prevent the formation of a plastic hinge, the design forces obtained from the model are amplified by the wall over-strength factor $\left(M_{r} / M_{f}\right)$ for the first storey shear wall.

In addition, to account for strain hardening effects in the vertical steel reinforcement, the design moments are amplified by an additional 15\%. Priestley and Paulay (1992) recommend that vertical steel reinforcement be curtailed in such a way that it not give less than a linear variation in moment resistance over the height of the wall. This is illustrated in Fig. 3.5, which shows the moment due to static and lateral forces on wall, the assumed linear moment variation over the height of the wall, and the moment resistance over the height of the wall. Note that because the wall specimens are under axial compression, which also varies over the height of the wall, the required flexural reinforcement ratio is not proportional to the required bending moment capacity. If the flexural steel reinforcement ratio were to remain constant with height, the flexural resistance will reduce with height because the axial compression is smaller.

In addition to limitations on spacing of vertical steel reinforcement, there are also requirements on the ties to support longitudinal reinforcement. Generally, ties are provided in the form of horizontal steel reinforcement, which also provide shear strength. Outside of the plastic hinge region, if total area of distributed reinforcement is less than $0.005 \mathrm{Ag}$ and the bar size is $20 \mathrm{M}$ or smaller, the ties for distributed reinforcing steel need not be provided. Although this applied to the second and third storey shear walls, ties were provided for all levels in the form of horizontal steel reinforcement for shear strength regardless of the reinforcement ratio or bar size at that level. Inside the plastic hinge region, the minimum tie spacing requirement is the smallest of the 
Table 3.4 Shear wall flexural design properties

\begin{tabular}{lccc}
\hline \hline \multicolumn{1}{c}{ Parameter } & Level 1 & Level 2 & Level 3 \\
\hline$L_{p}(\mathrm{~mm})$ & 3025 & - & - \\
$c(\mathrm{~mm})$ & 305 & 228 & 117 \\
$\rho_{l} \%$ & 0.43 & 0.34 & 0.26 \\
$\rho_{l, c} \%$ & 0.32 & 0.13 & - \\
$\theta_{i d}$ & 0.0016 & 0.0211 & 0.0211 \\
$\theta_{i c}$ & 0.0017 & 0.0268 & 0.0543 \\
$M_{r}(\mathrm{kN}-\mathrm{m})$ & 8130 & 4778 & 2082 \\
$M_{f}(\mathrm{kN}-\mathrm{m})$ & 7681 & 3557 & 1353 \\
$M_{f, a m p}(\mathrm{kN}-\mathrm{m})$ & - & 4329 & 1647 \\
$M_{r} / M_{f}$ & 1.06 & 1.34 & 1.54 \\
\hline \hline
\end{tabular}

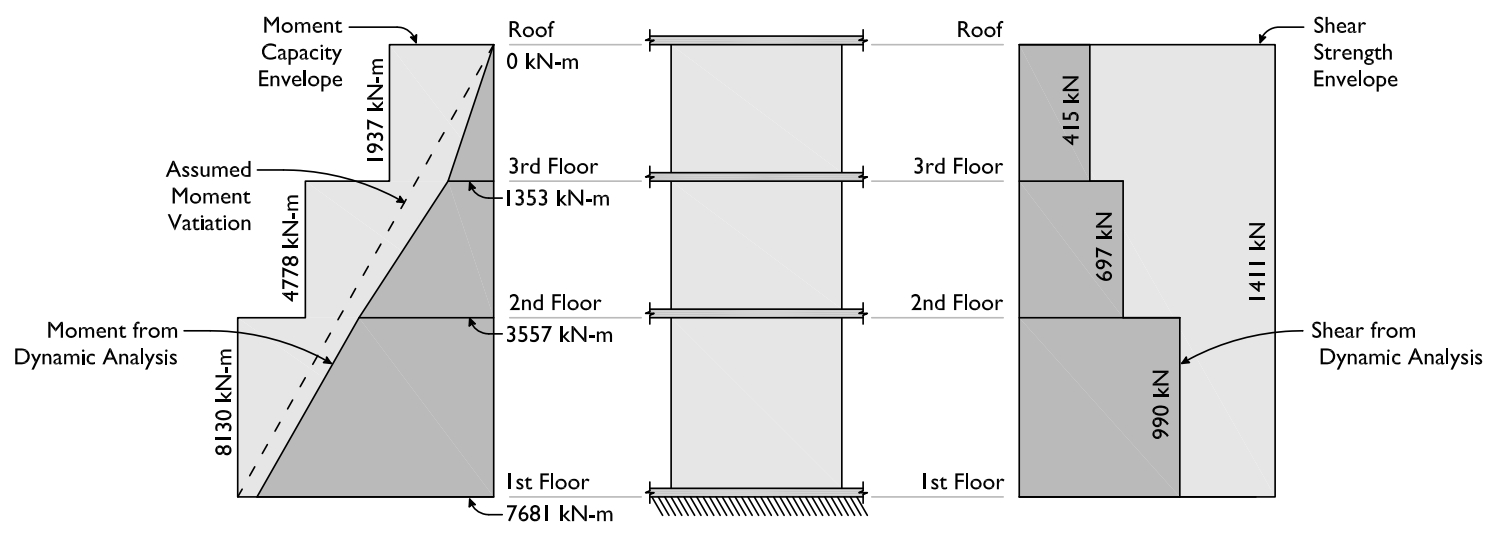

Figure 3.5 Bending moment capacity and shear strength envelopes 
following limits set by CSA A23.3-14:

- 16 times the minimum longitudinal bar diameter;

- 48 tie diameters;

- the least dimension of the compression member; and

- $300 \mathrm{~mm}$ in compressive members containing bundles of bars.

For the concentrated vertical steel reinforcement at the edges of the wall inside the plastic hinge region, there are additional restrictions on tie spacing, which is the smallest of the following limits:

- six longitudinal bar diameters;

- 24 tie diameters; and

- one-half the least dimensions of the member.

For the walls in this study, in addition to providing lateral support for the vertical steel reinforcement, the ties will also act as horizontal shear reinforcement. As a result, their maximum spacing is governed by the lesser of the spacing required for lateral support and shear strength, which is discussed in the following section.

\subsubsection{Shear Wall Shear Design}

The shear capacity of the shear walls in the prototype structure are determined according to Section 11 of CSA A23.3-14, which is based on the modified compression field theory developed by Vecchio and Collins (1986b) and Vecchio and Collins (1988). Note that in a similar manner to the capacity based design of the upper level shear walls, the shear resistance of the wall must account for the nominal moment capacity of the wall to ensure that the wall fails in flexure and avoids brittle shear related failures. As a result, the design shear forces are calculated by multiplying the shear force profile from the analysis by the flexural overstrength factor $\left(M_{r} / M_{f}\right)$ for the wall. As described in Chapter 2, the shear walls must be designed to avoid diagonal tension shear failure, diagonal compression shear failure and sliding shear failure. The shear capacity against diagonal tension shear failure $\left(V_{r}\right)$ is the sum of the shear strength contributions from the concrete 
$\left(V_{c}\right)$ and the horizontal steel reinforcement $\left(V_{s}\right)$, as shown in Eq. 3.50 (CSA, 2014a):

$$
V_{f} \leq V_{r}=V_{c}+V_{s}
$$

The concrete contribution to the shear strength $\left(V_{c}\right)$ is given by Eq. 3.51 (CSA, 2014a):

$$
V_{c}=\phi_{c} \lambda \beta \sqrt{f_{c}^{\prime}} b_{w} d_{v}
$$

where $\phi_{c}$ is the material reduction factor for concrete, taken as $0.65, \lambda$ is taken as 1.0 for normal weight concrete, $\beta$ is a factor that accounts for the shear resistance of cracked concrete, $b_{w}$ is the thickness of the wall, and $d_{v}$ is the effective shear depth, which has a minimum value of $0.8 l_{w}$ for walls. The contribution of the horizontal steel reinforcement to the shear strength $\left(V_{s}\right)$ is computing using Eq. 3.52 (CSA, 2014a):

$$
V_{s}=\frac{\phi_{s} A_{v} f_{y} d_{v} \cot \theta}{s}
$$

where $\phi_{s}$ is the material reduction factor for steel, taken as $0.85, A_{v}$ is the area of shear reinforcement within a distance $d_{v}$ along the member longitudinal axis, $\theta$ is the angle of inclination of diagonal compressive stresses to the longitudinal axis of the member, and $s$ is the shear reinforcement spacing. The value of the shear capacity of the wall is required to exceed:

- the shear corresponding to the probable moment capacity at the plastic hinge region; and

- the shear demand, with an upper bound set by the factored load combinations assuming $R_{d} R_{o}=1.3$.

This design methodology ensures that wall is capable of achieving its design flexural strength prior to failing in shear, allowing the wall to achieve the desired ductile mode of failure. The values of $\beta$ and $\theta$ for the plastic hinge region depend on the inelastic rotational demand (discussed in the previous section) and axial compression ratio on the shear wall and the following conditions apply (CSA, 2014a): For the value of $\beta$ :

- if $\theta_{i d} \geq 0.015$ then $\beta=0$ or;

Joshua Woods, Department of Civil and Environmental Engineering, Carleton University 
- if $\theta_{i d} \leq 0.005$ then $\beta=0.18$; and

- for $0.005<\theta_{i d}<0.015$ then linearly interpolate to obtain $\beta$.

For the value of $\theta$ :

- if $\left(P_{s}+P_{p}\right) \leq 0.1 f_{c}^{\prime} A_{g}$ then $\theta=45 \mathrm{deg}$; or

- if $\left(P_{s}+P_{p}\right) \geq 0.2 f_{c}^{\prime} A_{g}$ then $\theta \geq 35 \mathrm{deg}$; and

- for $0.1 f_{c}^{\prime} A_{g}<\left(P_{s}+P_{p}\right)<0.2 f_{c}^{\prime} A_{g}$ then linearly interpolate to obtain $\theta$.

where $P_{s}$ is the axial force at the section resulting from factored dead and live load using earthquake load factors and $P_{p}$ is the additional earthquake induced axial loads as a result of coupling shears for walls connected by coupling beams, which is not applicable in this study. Finally, the shear demand in the plastic hinge region is limited to prevent web crushing according to the inelastic rotational demand as follows:

- if $\theta_{i d} \geq 0.015$ then $V_{f} \leq 0.10 \phi_{c} f_{c}^{\prime} b_{w} d_{v}$;

- if $\theta_{i d} \leq 0.005$ then $V_{f} \leq 0.15 \phi_{c} f_{c}^{\prime} b_{w} d_{v}$; and

- for $0.015>\theta_{i d}>0.005$, then linearly interpolate to obtain $V_{f}$.

If the shear demand is higher than the limits presented above based on the inelastic rotational demand then the shear demand on the wall should be reduced by changing the dimensions of the wall or adding additional shear walls to the structure to reduce the demand. In addition to the diagonal tension shear capacity of the wall, its capacity against other failure modes associated with shear discussed in Chapter 2 of this work can be computed using the provisions of CSAA23.3-14 (CSA, 2014a). The capacity against sliding shear failure $\left(V_{s l}\right)$ can be computed using Eq. 3.53:

$$
V_{s l}=\left[c+\mu\left(\frac{A_{s} f_{y}}{t_{w} d}+\frac{N_{u}}{t_{w} l_{w}}\right)\right] A_{w} \leq 0.25 f_{c}^{\prime} A_{w}
$$

where $c$ is the cohesion stress along the interface between the base of the wall and the adjacent structural element, taken as $0.5 \mathrm{MPa}, \mu$ is the coefficient of friction between concrete surfaces, conservatively taken as 1.0 for concrete placed against hardened concrete whose surface has been cleaned and intentionally roughened according to CSA A23.3-14 (CSA, 2014a), and $N_{u}$ is the 
axial load acting on the shear wall. The capacity against diagonal compression $\left(V_{\text {comp }}\right)$ can be computed using Eq. 3.54:

$$
V_{\text {comp }}=0.83 \sqrt{f_{c}^{\prime}} A_{w}
$$

The final shear design parameters and capacities for a typical wall in the prototype structure are summarized in Table 3.5. Results show that the capacity of the wall against diagonal tension shear failure governs when compared to the other sliding and diagonal compression failures associated with shear. Note that because the minimum horizontal reinforcement ratio of $0.25 \%$ as prescribed by the CSA A23.3-14 design standard is sufficient to develop the required shear capacity at the base of the wall, the shear resistance does not change with respect to vertical location along the height of the wall. Figure 3.5 shows the final summary of the shear and moment capacity envelopes for a typical shear wall and Fig. 3.6 shows the steel reinforcement and cross-sectional details for a typical shear wall in the N-S direction of the prototype building.

\subsection{Scaling of Prototype Structure}

To meet the space requirements in the structural engineering laboratory at Carleton University and the force limits on the laboratory hydraulic actuators, the prototype structure designed in the previous section is scaled to $2 / 5(40 \%)$ of its original size. In the scaling procedure, it is assumed that all of the physical dimensions of the shear wall (ie. length, width, and thickness) are scaled to $40 \%$ of their original values. The scaling factor for length $\left(S_{L}\right)$ is taken as 0.4 . The modulus of elasticity for the concrete/steel in the structure is not scaled given that the stiffness of the material remains the same even at a smaller scale, therefore the scaling factor for the modulus of elasticity $\left(S_{E}\right)$ is taken as 1.0. The scaling factor for the acceleration $\left(S_{a}\right)$ is taken as 1.0 to apply full-scale accelerations to the model structure. Based on these three assumptions, the scaling factors for all of the other physical quantities in the model can be calculated and are shown in Table 3.6. To obtain the appropriate scaling factors for all of the other model quantities (e.g. force, moment, time, area etc...), a combination of $S_{L}, S_{E}$, and $S_{a}$ are used to obtain the correct units for 

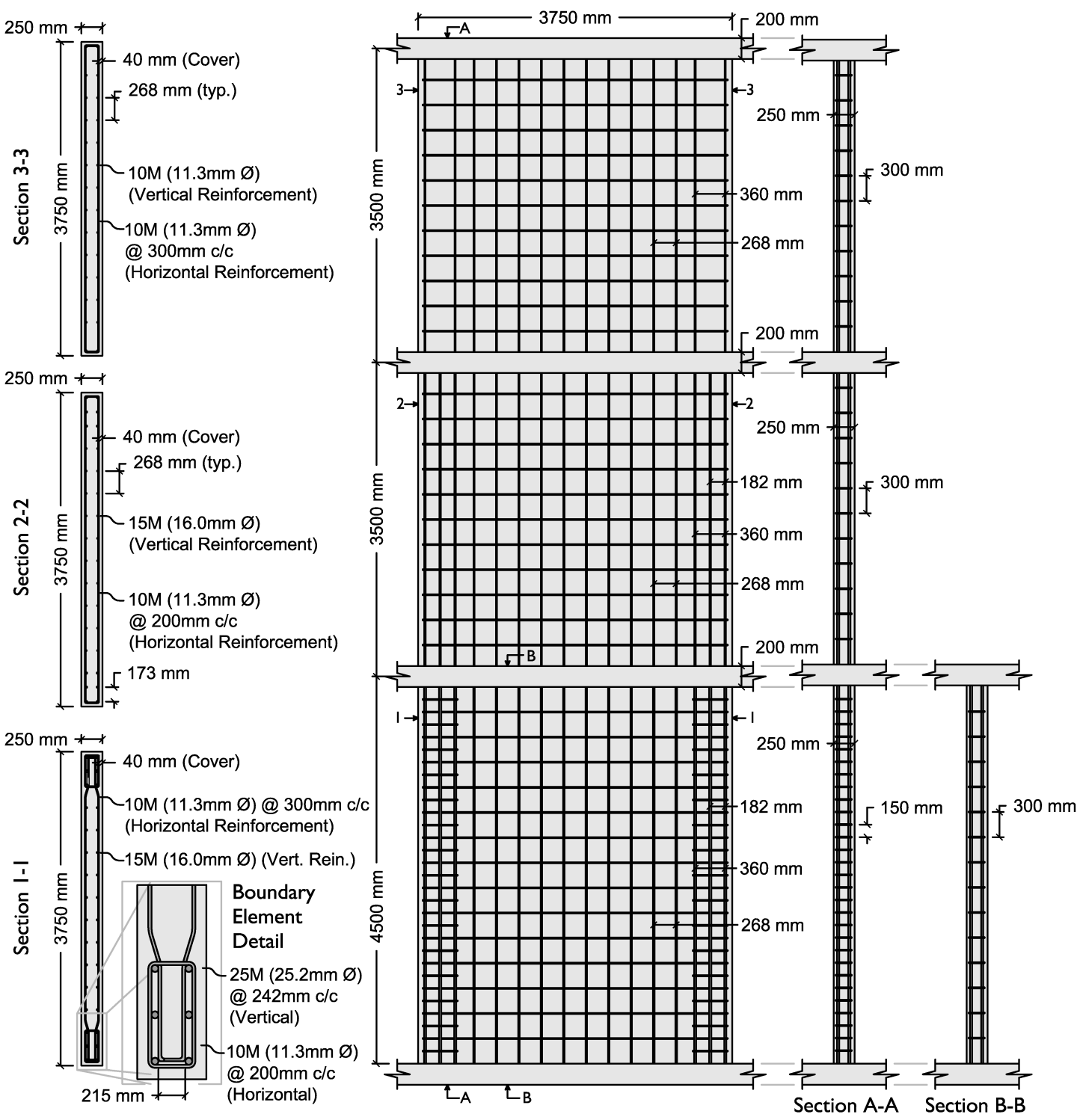

Figure 3.6 Full-scale shear wall design 
Table 3.5 Shear wall shear design properties

\begin{tabular}{lc}
\hline \hline Parameter & Design Value \\
\hline$f_{c}^{\prime}(\mathrm{MPa})$ & 50 \\
$d_{v}(\mathrm{~mm})$ & 3000 \\
$\rho_{h}(\%)$ & 0.25 \\
$s(\mathrm{~mm})$ & 300 \\
$\theta$ & $45 \mathrm{deg}$ \\
$\beta$ & 0.1 \\
$V_{f}(\mathrm{kN})$ & 990 \\
$V_{c}(\mathrm{kN})$ & 474 \\
$V_{s}(\mathrm{kN})$ & 937 \\
$V_{r}(\mathrm{kN})$ & 1411 \\
$V_{s l}(\mathrm{kN})$ & 4520 \\
$V_{c o m p}(\mathrm{kN})$ & 4950 \\
$V_{r} / V_{f}$ & 1.44 \\
\hline \hline
\end{tabular}

a particular response quantity. For example, to obtain the force scaling factor $\left(S_{F}\right)$, this would be equivalent to $S_{E}$ multiplied by $S_{L}^{2}$, resulting in units of $\mathrm{N} / \mathrm{m}^{2} \times \mathrm{m}^{2}=\mathrm{N}$. The mass scaling factor $\left(S_{M}\right)$ is obtained using Newton's second law, thus $S_{M}=S_{F} / S_{a}$, resulting in units of mass. Because the accelerations are left unscaled, the total time for the ground motion analysis is compressed. The time scaling factor can be determined as $S_{T}=\sqrt{S_{M} S_{L} / S_{F}}$, which gives the appropriate units of time $\left(\sqrt{\left(N s^{2} / m \times m\right) / N}=s\right)$. This procedure is repeated to obtain the scaling factors for all of the other response quantities, the formulas for which are also shown in Table 3.6.

The scaling factor of $2 / 5$ results in a reduced total building seismic weight (W) of $5483.5 \mathrm{kN}$ and resulting design axial load and shear force of $170 \mathrm{kN}, 158 \mathrm{kN}$ respectively. The scaled design moment at the base of the wall is $491.6 \mathrm{kN}-\mathrm{m}$ for the shear wall under consideration in the N-S direction (identified in Fig. 3.1). Table 3.7 shows the scaled dimensions, reinforcement ratios, and design capacities for a scaled experimental shear wall element and Fig. 3.7 illustrates a typical plan view and cross-sectional view of the shear wall. 
Table 3.6 Prototype structure scaling factors

\begin{tabular}{lcc}
\hline \hline \multicolumn{1}{c}{ Quantity } & Description & Scaling Factor \\
\hline Length & $S_{L}$ & 0.4000 \\
Elastic Modulus & $S_{E}$ & 1.0000 \\
Force & $S_{F}=S_{E} S_{L}^{2}$ & 0.1600 \\
Moment & $S_{M o}=S_{F} S_{L}$ & 0.0640 \\
Mass & $S_{M}$ & 0.1600 \\
Acceleration & $S_{a}=S_{F} / S_{M}$ & 1.0000 \\
Time & $S_{T}=\sqrt{S_{M} S_{L} / S_{F}}$ & 0.6324 \\
Area & $S_{A}=S_{L}^{2}$ & 0.1600 \\
Reinforcement Ratio & $S_{\rho}=S_{A} / S_{L}^{2}$ & 1.0000 \\
Section Modulus & $S_{Z}=S_{L}^{3}$ & 0.0640 \\
Moment of Inertia & $S_{I}=S_{L}^{4}$ & 0.0256 \\
\hline \hline
\end{tabular}

Table 3.7 Scaled shear wall design properties

\begin{tabular}{lc}
\hline \hline Parameter & Design Value \\
\hline$f_{c}^{\prime}(\mathrm{MPa})$ & 50 \\
$\rho_{l}(\%)$ & 0.40 \\
$\rho_{l, c}(\%)$ & 0.40 \\
$\rho_{h}(\%)$ & 0.28 \\
$\rho_{h, c}(\%)$ & 0.56 \\
$c(\mathrm{~mm})$ & 195 \\
$P / A_{g} f_{c}^{\prime}$ & 0.023 \\
$V_{r}(\mathrm{kN})$ & 193.6 \\
$V_{s l}(\mathrm{kN})$ & 1130 \\
$V_{c o m p}(\mathrm{kN})$ & 880 \\
$M_{r}(\mathrm{kN}-\mathrm{m})$ & 519.4 \\
\hline \hline
\end{tabular}



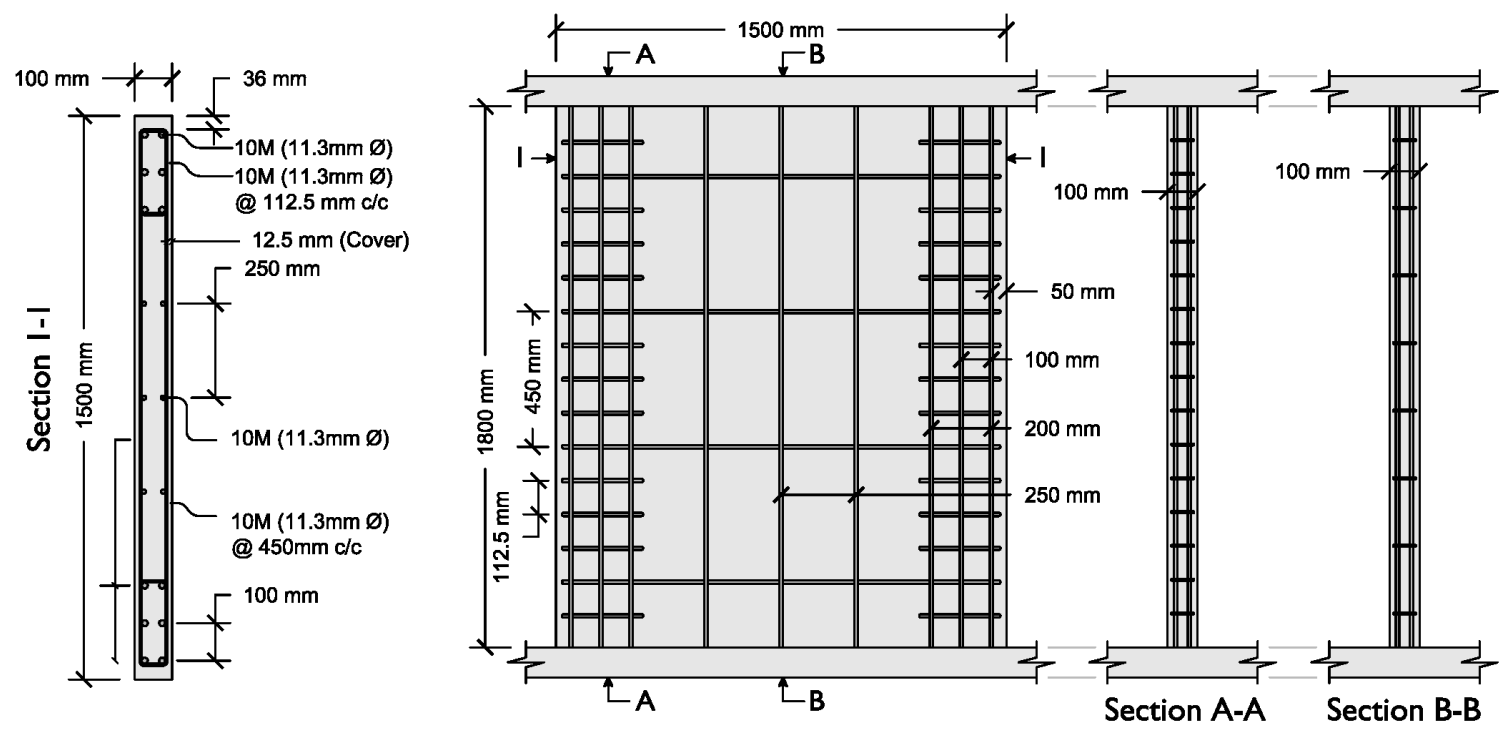

Figure 3.7 Scaled shear wall design 


\section{Chapter 4. Analytical and Hybrid Models}

\subsection{Introduction}

Hybrid simulation is based on the premise that by combining experimental testing and analytical modelling, this will result in a more realistic seismic load imposed on the experimental substructure and provide information on the response of the full-scale structural system during an earthquake. In this study, a number of reinforced concrete (RC) shear wall models have been developed in the Open System for Earthquake Engineering Simulation software, commonly referred to as OpenSees (McKenna et al., 2000). OpenSees is an object-oriented open-source finite element software framework sponsored by the Pacific Earthquake Engineering (PEER) centre that is primarily used for simulating the response of structural and geotechnical systems subject to earthquakes and other hazards (McKenna et al., 2000). OpenSees has numerous material models, elements, and powerful algorithms which have been shown to be able to accurately predict the nonlinear response of reinforced concrete elements under cyclic and dynamic loading. The open-source software allows researchers to build upon previously developed modelling algorithms and tools in subsequent research activities, thus facilitating further and new developments in expanding the capabilities of the OpenSees software platform. OpenSees is selected as the finite element modelling platform for this study because of its ability to be integrated seamlessly into the OpenFresco software for hybrid simulation, which acts as an interware connecting the finite element model with the control and data acquisition systems in a laboratory to facilitate hybrid simulation of structural and geotechnical systems (Schellenberg et al., 2007).

Prior to conducting a hybrid test, an accurate finite element model of the analytical portion 
of the structure is required. Although the analytical portion of the model is not expected to experience significant inelasticity, it is still useful to accurately capture the initial stiffness, cracking strength and post-yield response of the wall to ensure accurate results if unexpected nonlinearity occurs. To verify the accuracy of the finite element model, the developed modelling approaches are used to model a shear wall specimen with available experimental results from a past study on the cyclic behaviour of RC shear walls conducted by Hiotakis (2004). Figure 4.1 and Table 4.1 show the dimensions, steel reinforcement detailing and material properties used in the study by Hiotakis (2004). The specimen tested by Hiotakis (2004) is a $2 / 3$ scale cantilevered shear wall design according to "modern" seismic design standards (CSA A23.3-94). The shear wall had sufficient steel shear reinforcement to prevent premature shear failure and failed during experimental testing in a flexurally dominant manner; initiated through yielding of the vertical steel reinforcement at the base near the ends of the shear wall. The shear wall specimen had boundary elements in which additional concrete confinement was provided to promote a ductile response and prevent buckling of the vertical steel reinforcement after the initiation of concrete crushing at the base of the shear wall. The detailing of the steel reinforcement and material properties of the shear wall specimen tested by Hiotakis (2004) closely resembles the scaled wall specimen in this study, making it an excellent candidate for comparison purposes and development of a finite element model for the analytical substructure. The following subsection outlines the different modelling techniques in the development of the analytical substructure for the hybrid test and compares the analytical results with the experimental results from the RC shear wall specimen tested by

Table 4.1 Shear wall material properties (from Hiotakis, 2004)

\begin{tabular}{cccc}
\hline \hline \multicolumn{3}{c}{$\begin{array}{l}\text { Steel Rebar } \\
(10 \mathrm{M} \text { Bars })\end{array}$} & \multicolumn{2}{c}{ Concrete } \\
\hline Property & Value & Property & Value \\
\hline$f_{y}(\mathrm{MPa})$ & 452 & $f_{c}^{\prime}(\mathrm{MPa})$ & 35.9 \\
$E_{s}(\mathrm{GPa})$ & 200 & $E_{c}(\mathrm{GPa})$ & 19.2 \\
$\epsilon_{y}$ & 0.002 & $\epsilon_{c}^{\prime}$ & 0.002 \\
$\epsilon_{u}$ & 0.111 & $\epsilon_{u}^{\prime}$ & 0.01 \\
\hline \hline
\end{tabular}

Joshua Woods, Department of Civil and Environmental Engineering, Carleton University 


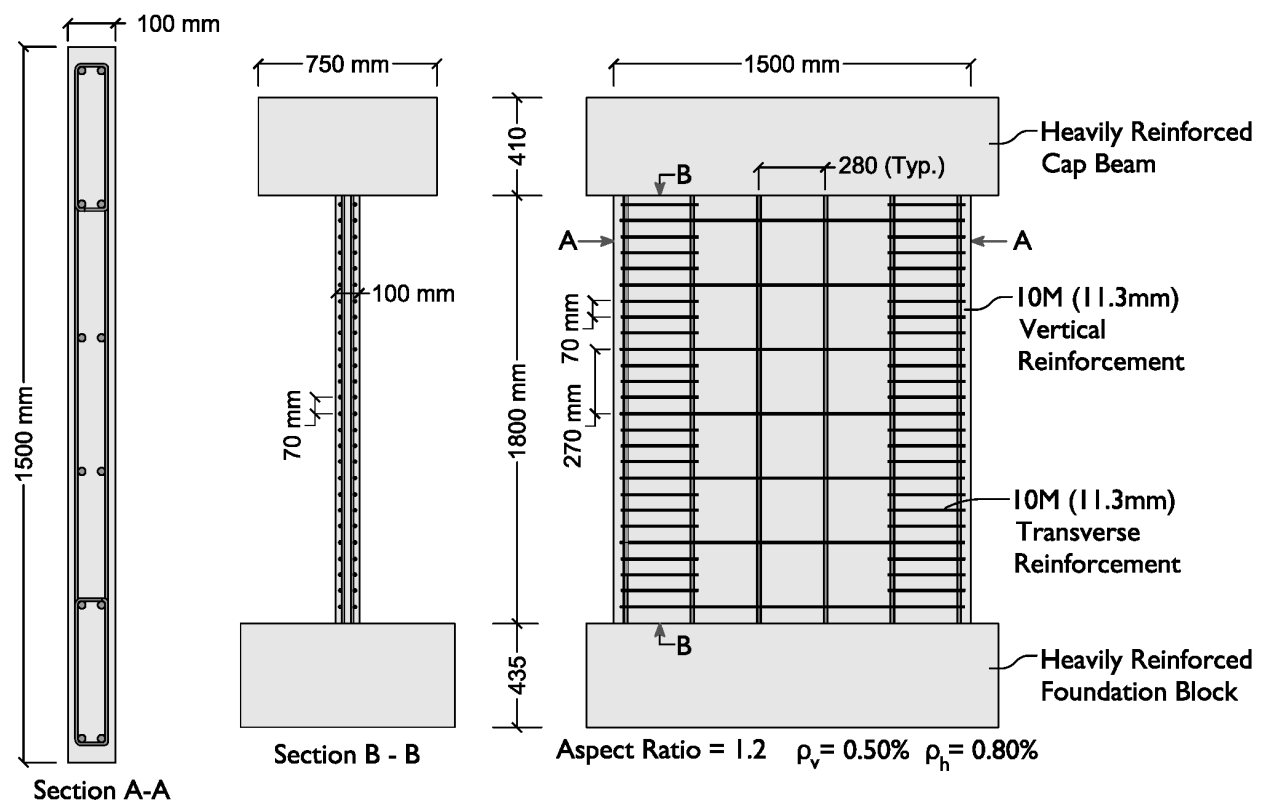

Figure 4.1 Typical shear wall specimen tested by Hiotakis (2004)

Hiotakis (2004).

\subsection{Model Validation}

The conventional finite element modeling approach used to capture the inelastic behaviour of RC elements in OpenSees has conventionally been based on the use of nonlinear beam column elements. The nonlinear beam column elements available in OpenSees include force or displacement-based elements with distributed plasticity. In this modelling approach, the RC section is subdivided into longitudinal fibers at discrete locations along the length of the element that are assigned uniaxial nonlinear constitutive relationships for concrete and reinforcing steel, as shown in Fig. 4.2. The behaviour of the section is determined implicitly through integration of the fibers, assuming that plane sections remain plane.

In the past, this modeling approach has been shown to yield accurate results in elements that behave predominantly in flexure, such as beams, columns, and very slender structural walls $\left(h_{w} / l_{w}>4.0\right)(\mathrm{Lu}$ et al., 2015). However, it is commonly recognized that the response of RC 

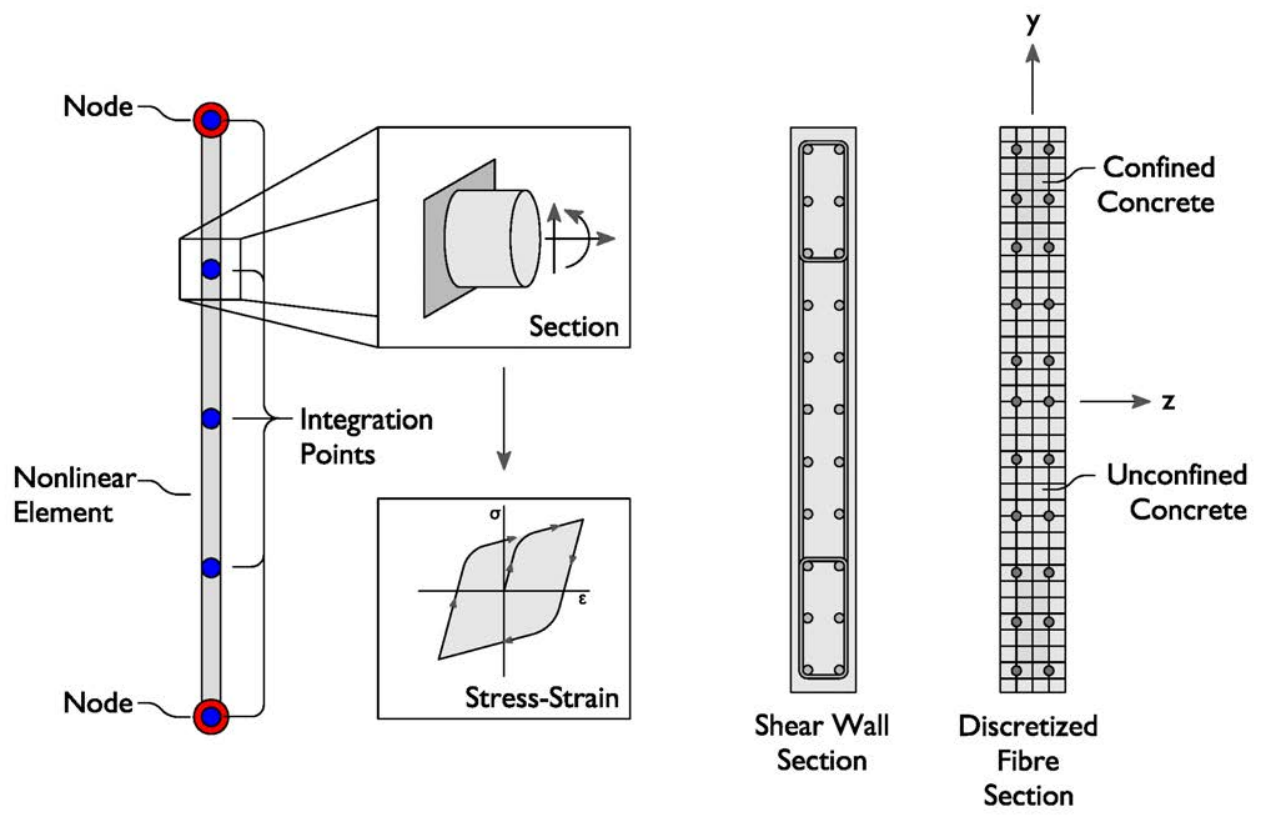

Figure 4.2 Beam-column model of Hiotakis (2004) shear wall

shear walls with aspect ratios $\left(h_{w} / l_{w}\right)$ less than 2 is a combination of a shear and flexural responses (Salonikios et al., 1999). To understand the deficiencies in the performance of the fibre section modelling approach in lower aspect ratio walls, the shear wall specimen tested by Hiotakis (2004) is modelled using a nonlinear beam column element with a fibre section model, as shown in Fig. 4.2. Figure 4.3 shows the constitutive models for steel and concrete used in the fibre section model. The steel reinforcement is modeled using a uniaxial constitutive nonlinear hysteretic material model (Steel02) proposed by Menegotto and Pinto (1973), which was modified by Filippou et al. (1983) to account for the effects of isotropic hardening of the material. The concrete is assigned a uniaxial constitutive hysteretic material model (Concrete02) which includes a linear tension softening branch (Yassin, 1994).

Following construction of the finite element model, the same cyclic lateral load sequence used by Hiotakis (2004) is applied to the top of the wall specimen. Figure 4.4 shows a comparison of the hysteretic response behaviour of the analytical model and experimental shear wall specimen. Analytical results show that the conventional beam-column modelling approach does a reasonable job of predicting the overall strength of the shear wall, but because it only considers 


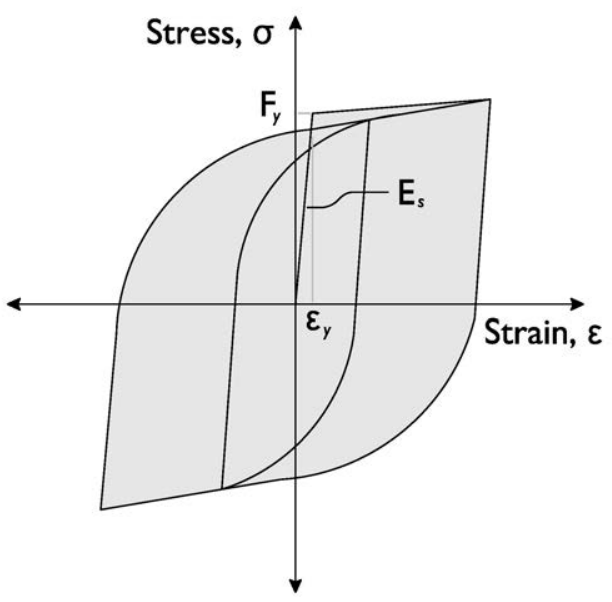

(a)

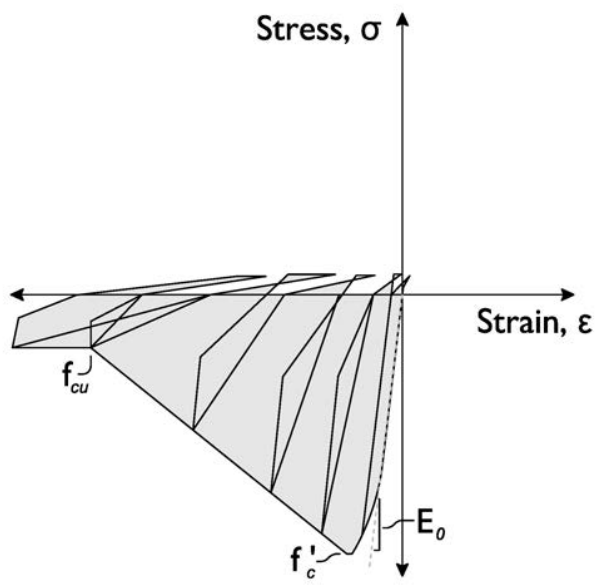

(b)

Figure 4.3 Material constitutive relationships: (a) steel; (b) concrete

the flexural response of the element and does not consider the interaction of shear and flexural behaviour, the model is not able to accurately capture the nonlinear hysteretic response of the wall. This is particularly evident in the unloading branch of the hysteretic response behaviour for the Hiotakis (2004) shear wall. The observed pinching behaviour is typically associated with the shear behaviour of a wall, which cannot be accurately captured in OpenSees using a nonlinear beam-column element and fibre section.

To overcome the limitations imposed by a fibre section beam-column model, the use of 2D shell elements is a viable alternative to capture the shear-flexure interaction observed in the hysteretic response behaviour of RC shear walls. In this study, two different modelling approaches using multi-layered shell elements in OpenSees have been employed to model the analytical substructure for the hybrid simulation, both of which are discussed in detail in the following subsections.

\subsubsection{Multi-Layer 3D Shell Element}

The first shell element modelling approach examined in this study uses a multi-layer shell element developed by Lu et al. (2015) that has recently been introduced into the OpenSees framework. The multi-layer shell formulation is an extension of the original ShellMITC4 element in 


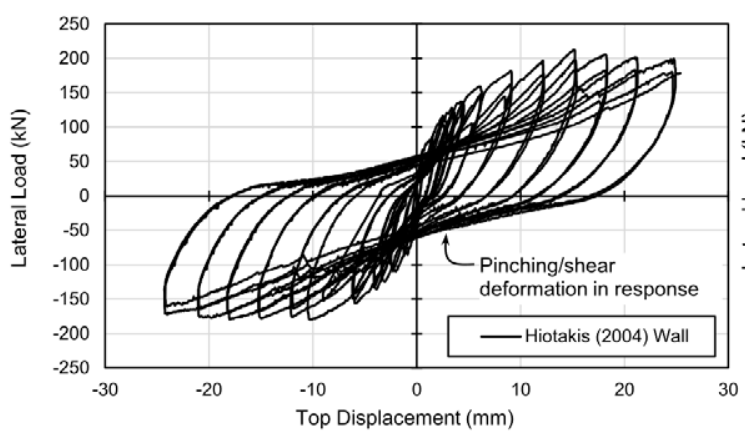

(a)

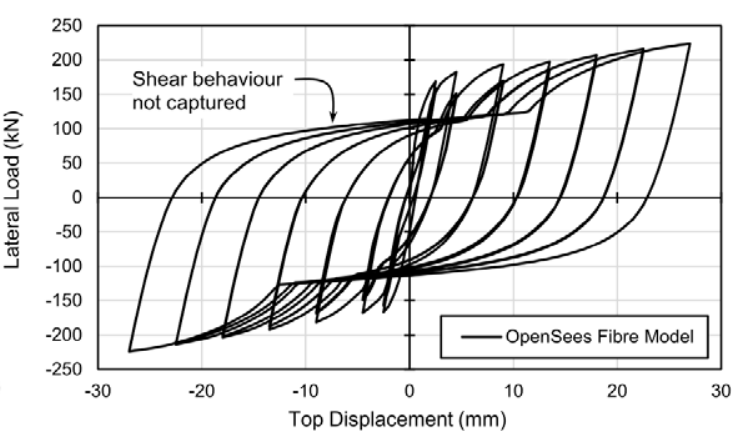

(b)

Figure 4.4 Shear wall hysteretic response behaviour comparison: (a) Hiotakis (2004) experimental results; (b) OpenSees fibre model

OpenSees, which is a 3D four node shell element based on theory proposed by Dvorkin and Bathe (1984). Figure 4.5 shows the coordinate system adopted by the four node shell element. The layered element reduces the complex three-dimensional behaviour of a RC shear wall into a two-dimensional shell by discretizing each layer of a wall into several layers that are assumed to be fully bonded together. The thickness and material properties for each layer in the element can be determined according to the geometry of the wall, horizontal and vertical steel reinforcement ratios, and the material properties of the confined/unconfined concrete and reinforcing steel. In the element definition, each layer of steel reinforcement is transformed into a thin sheet that is smeared over the entire surface of the shear wall. The thickness of the smeared reinforcement layer depends on the reinforcement ratio for that steel layer. The smeared reinforcement approach provides a more computationally efficient solution when compared with using discrete truss elements to model individual steel reinforcing bars in the concrete, while still providing accurate results, as shown by Cruz-Noguez et al. (2014c). The use of many concrete layers in the element also allows for confined and unconfined concrete in the wall to be modelled separately. For example, layered shell elements in the boundary elements would use a confined concrete model for the core concrete and an unconfined concrete model for the concrete cover. Figure 4.5 shows a typical distribution of the confined/unconfined concrete, horizontal reinforcing steel, and vertical reinforcing steel over the thickness of a shell element.

The axial strains and curvature at the middle of the element are initially calculated and the 
corresponding strains in each layer are obtained based on a plane-sections remain plane assumption (over the thickness of the element or wall). The stresses over each layer in the element are assumed to be consistent with those at the centre of the layer. Therefore, so long as the shear wall is divided into a sufficient number of layers, the multi-layer shell element is able to simulate the actual stress distribution over the thickness of the wall (for out-of-plane response). Lu et al. (2015) has demonstrated that the multi-layer shell element is suitable for capturing coupled in-plane/outof-plane bending as well as in-plane direct shear and coupled bending/shear behaviour, which is particularly important for RC shear walls.

This model is implemented in OpenSees through the use of a planar concrete constitutive model using the nDMaterial command in OpenSees and a PlaneStressUserMaterial. In this model, cracks in the concrete are assumed to occur once the principle tensile stress exceeds the specified concrete tensile strength. After cracking in the concrete occurs, the concrete is treated as an orthotropic material (Lu et al., 2015). The effect of aggregate interlock on the shear stiffness is incorporated into the model through the use of a shear retention factor, which reduces the shear modulus depending on the total cumulative plastic strain (Lu et al., 2015). The outof-plane shear modulus is defined through a separate nDmaterial in OpenSees and is assigned a PlateFromPlaneStress material.

The nonlinear behaviour of the steel reinforcement (including yielding and Bauschinger effects) are incorporated through a uniaxial material model. However, because the uniaxial material models cannot be assigned to the section layers directly, they must first be transformed into an $n$ Dmaterial using the PlateRebar command, which requires the orientation of the steel layer being defined. Vertical flexural steel reinforcement is assigned a layer direction of 0 degrees while horizontal steel reinforcement is assigned at 90 degrees.

Although the performance of the multi-layered shell element has been validated in a previous study by Lu et al. (2015), the applicability of using the element to model RC shear walls is once again validated by comparing with experimental results by Hiotakis (2004). To model the vertical and horizontal steel reinforcement (Fig. 4.1), the steel reinforcement ratios in different zones of the wall are assigned as uniformly distributed (smeared) reinforcement within individual 


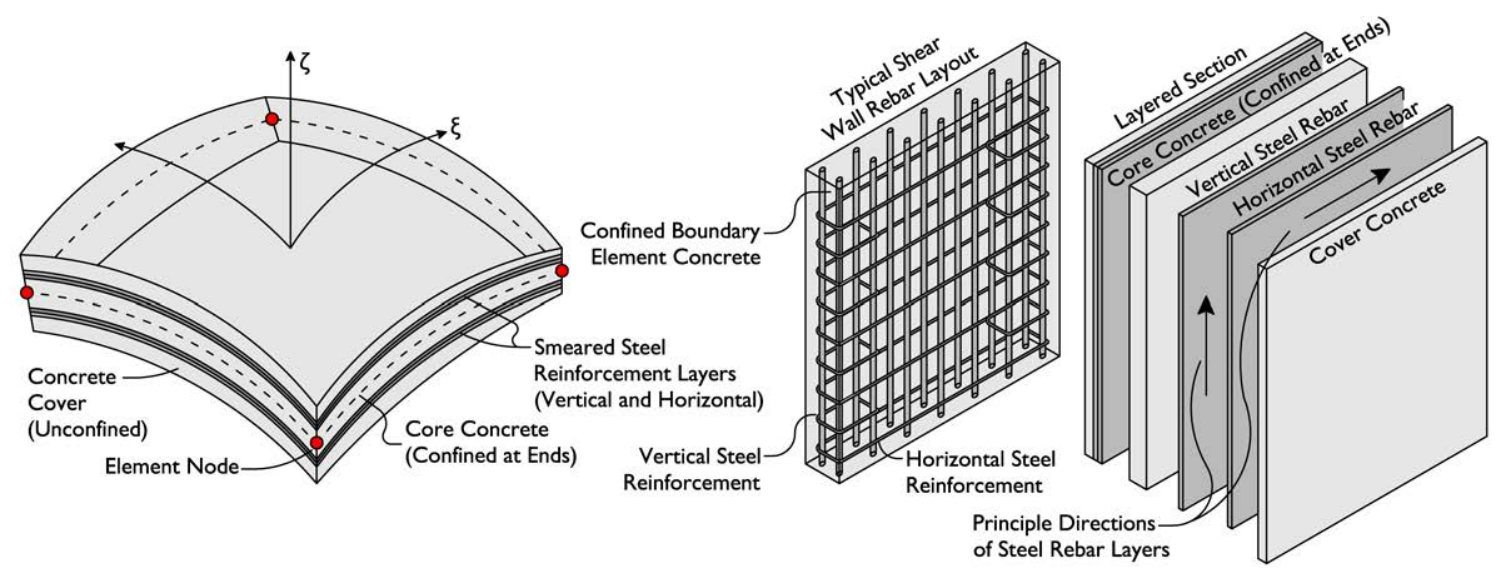

Figure 4.5 Multi-layered shell element coordinate system and element layers

layers of the multi-layered shell element. Smeared layers of steel reinforcement, confined, and unconfined concrete are assigned a uniaxial constitutive nonlinear hysteretic material model for steel and concrete discussed previously (Steel02 and Concrete02). One critical parameter that can significantly influence not only the accuracy of the finite element model but also its computational efficiency is the element size in the model. To investigate the stability and convergence of the finite element results, a mesh sensitivity analysis is conducted using four different shell element sizes. Figure 4.6 shows the discretized shear wall models used to validate the OpenSees modelling technique using the multi-layered shell element. Two different sets of concrete material properties are assigned to the confined boundary elements and the interior core elements, which are illustrated in Fig. 4.6. Shear wall geometry, steel reinforcement ratios and material properties for the concrete, vertical steel reinforcement, and horizontal steel reinforcement used in the analytical model matched those reported by Hiotakis (2004) and are shown in Table 4.1.

Figure 4.6 shows four different mesh sizes studied in the mesh sensitivity analysis, results for which are shown in Fig. 4.7. The finite element results demonstrate that satisfactory convergence is obtained with element sizes of $45 \times 50 \mathrm{~mm}$, resulting in a total of 1080 elements in the model. Based on the width of the wall specimen $(1.5 \mathrm{~m})$, this corresponds to an element size of $3.33 \%$ of the walls length, which will be used to determine an appropriate element size in larger shear 

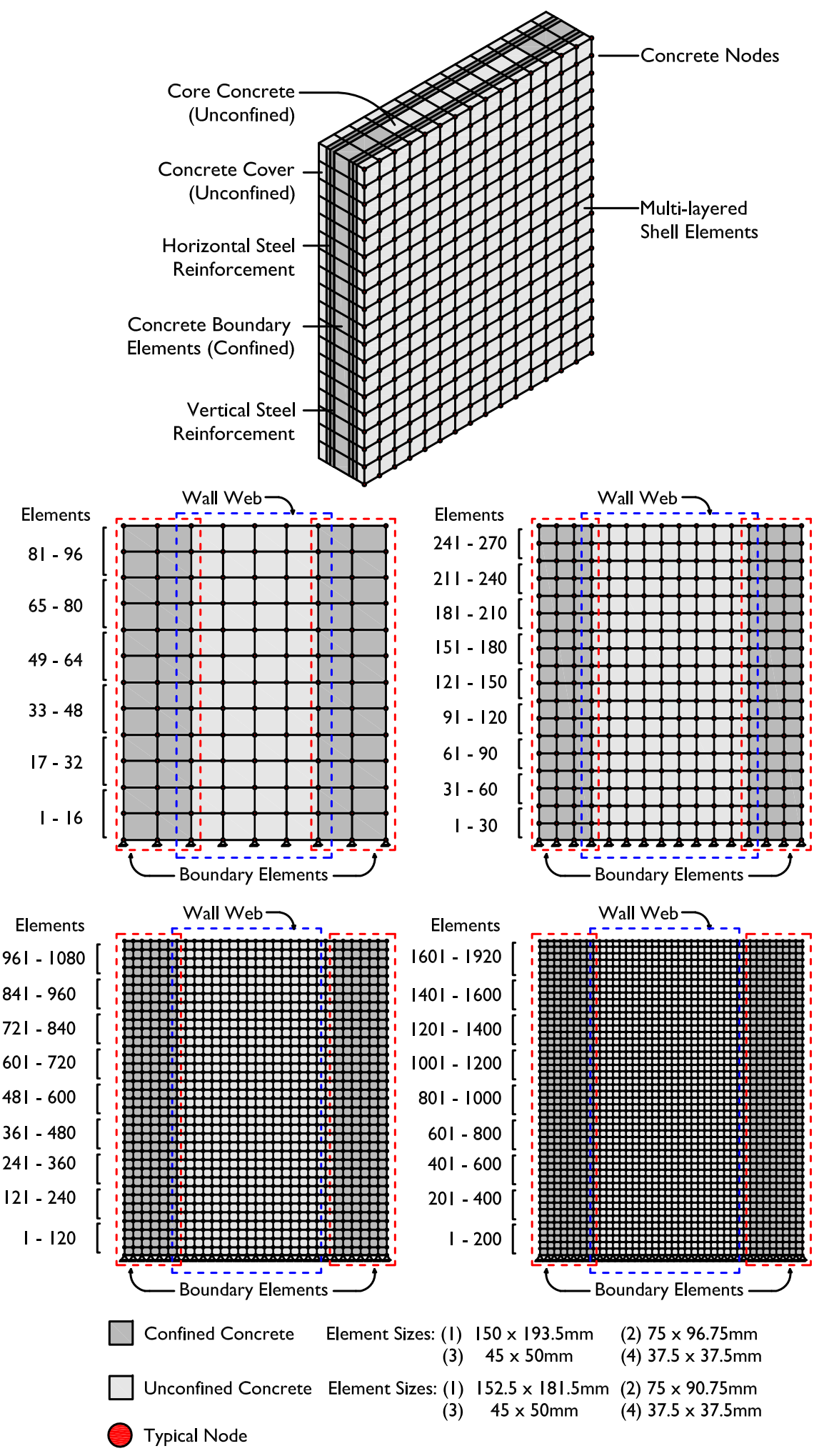

Figure 4.6 Shear wall finite element models: 96, 270, 1080, and 1920 elements 
wall models. Although the results from the 1920 element model are slightly improved when compared with experimental results from Hiotakis (2004), the additional computational time required (950\% increase) (42860s vs. 4496s) does not justify the slight improvement in overall correlation with the experimental results. For this reason, a mesh size of $45 \times 50 \mathrm{~mm}$ is recommended for this hybrid simulation study. In addition to the number of elements used in the model, a sensitivity analysis on the number of layers used in the multi-layered shell element is also studied. Because the stresses in each layers over the thickness of the element are assumed to be consistent with those at the centre of the layer, theoretically the number of subdivisions in each particular layer of material could influence the nonlinear performance of the element. However, because the shear wall is under in-plane loading and out-of-plane deformation is not expected, it is assumed that the model results would not be affected by the number of layers in each element in the model. Nonetheless, to verify the influence of the number of layers on the cyclic performance of the shear wall, three different numbers of concrete layers are used in the finite element model (12, 24, and 48 layers), which were evenly distributed over the shear wall cross-section. The results of the element layer sensitivity analysis confirm that increasing the number of subdivisions in the concrete layers has virtually no impact on the finite element modelling results, nor does it significant influence the computation time. As a result 12 layers is assumed satisfactory in the subsequent models.

Based on an appropriate mesh size and number of layers in the finite element model, Figure $4.7 \mathrm{~b}$ shows a comparison of the shell element model (1920 elements) with the experimental

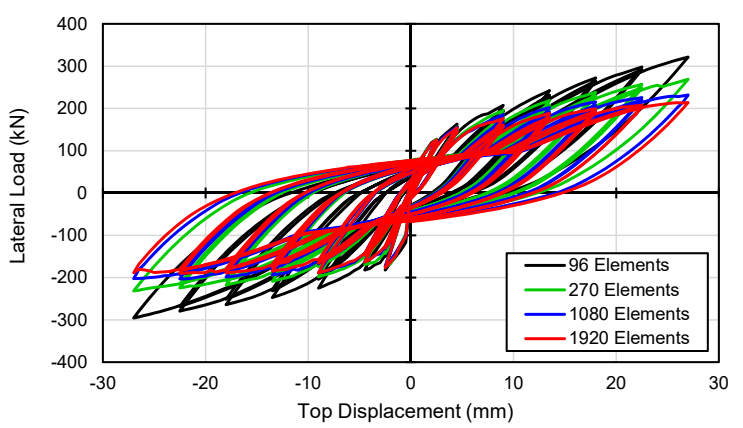

(a) (a)

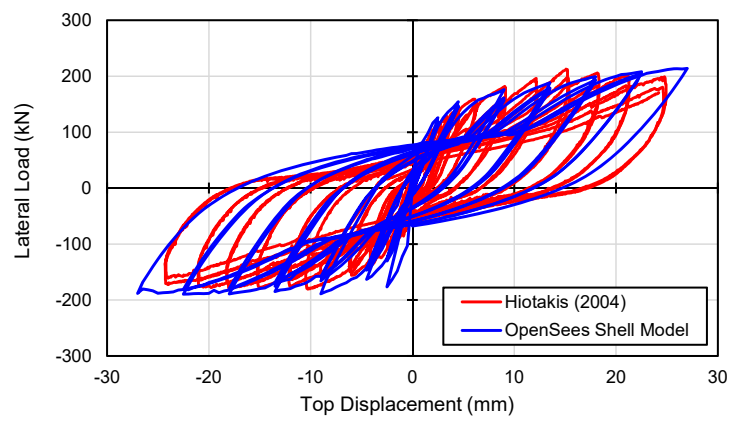

(b) (b)

Figure 4.7 (a) mesh sensitivity; (b) hysteretic response comparison with Hiotakis (2004) 
results from Hiotakis (2004). The finite element results show that the analytical model does a good job at predicting the hysteretic behaviour of the shear wall specimen, adequately capturing the inherent shear behaviour not captured in the previous fibre section model (Fig. 4.4). The finite element model predicts an average ultimate load of $200.5 \mathrm{kN}$ for the shear wall compared with $196.5 \mathrm{kN}$ recorded by Hiotakis (2004). Comparing the hysteretic response, the model also does a good job of capturing the post-yield stiffness, ductility and energy dissipation capacity of the shear wall.

Unfortunately, because of incompatibilities between the current OpenSees version and the 32-bit version of OpenFresco required to run the hybrid simulation, the 3D multi-layer shell element developed by Lu et al. (2015) could not be implemented in the hybrid simulation of the multi-storey RC shear wall. Nonetheless, results demonstrate that it is capable of accurately predicting the nonlinear response of RC shear walls, including the interaction of flexure and shear, if proper care is taken in determining the appropriate material properties, mesh size, and solution algorithms. This element has the potential to be used in future hybrid simulation studies on RC shear wall structures if newer versions of 32-bit OpenSees incorporate the PlaneStressUserMaterial into the source code.

\subsubsection{Multi-Layer 2D Shell Element}

Because of the incompatibility between the current version of OpenSees and the version of 32-bit OpenFresco required to run the hybrid simulation, a second nonlinear finite element model of the Hiotakis (2004) shear wall was developed. The element uses the same multi-layered approach to discretize the different components of a RC shear wall (e.g. core concrete, vertical steel reinforcement, horizontal steel reinforcement etc...) into several layers of varying thickness as shown in Fig. 4.5. In contrast to the previously employed multi-layer shell element, the formulation of this element is based on the original Quad element in OpenSees. The element is a $2 \mathrm{D}$ four node shell element that assumes the element is under a state of plane stress, which is a reasonable assumption for a shear wall element under in-plane loading because the out-of-plane principle stress is zero. The element is defined in a same manner as the previous element in that

Joshua Woods, Department of Civil and Environmental Engineering, Carleton University 
the $\mathrm{RC}$ shear wall is discretized into a series of layers representing the concrete, vertical steel reinforcement, and horizontal steel reinforcement that are assumed to be fully bonded together. As a result of the plane-stress assumption, this element is capable of capturing the in-plane direct shear and coupled bending/shear behaviour but is not able to capture the out-of-plane bending. This is sufficient for this study because the RC shear wall is only loaded in-plane.

The main differences between this element and the previously described element are that it is a $2 \mathrm{D}$ element and the material model that is used in conjunction with the element is based on different parameters. The element is implemented in OpenSees through the same nDMaterial command with a newly developed PlasticDamageConcretePlaneStress, PlaneStressRebarMaterial, and PlaneStressLayeredMaterial to define the concrete and steel material models and sandwich them into layers.

The nonlinear response of the concrete is defined based on its compressive strength, tensile strength, elastic modulus, and four damage parameters $A_{p}, A_{n}, B_{n}$, and $\beta . A_{p}$ governs the tensile fracture energy of the concrete and is related to the ductility of the tensile response of the concrete. The value of $A_{p}$ typically ranges between 0.05-0.2 for conventional strength concrete. The parameter $\beta$ governs the post-yield hardening modulus and the plastic strain rate which controls the unloading branch in the response of the element and typically ranges between 0.2 and 0.8 . Parameters $A_{n}$ and $B_{n}$ control the softening behaviour of the concrete in compression and the ductility of the compressive response and range between 1-4 and 0.6-0.9, respectively. In general, $A_{n}$ has a tendency to effect the ductility of the specimen without significantly altering the peak strength, where $B_{n}$ has an effect on the ductility and the peak strength of the concrete.

The nonlinear behaviour of the steel reinforcement is defined through the use of a uniaxial material model. The Steel02 nonlinear material model for steel reinforcement, which takes into consideration yielding, isotropic hardening, and the Bauschinger effect is once again employed. Because the uniaxial material models cannot be directly assigned to an individual layer of the shell element, it must first be transformed into an nDmaterial using the PlaneStressRebarMaterial for steel reinforcement, which also required the definition of the orientation of the layer. Vertical 
flexural steel reinforcement is assigned a layer direction of 0 degrees while horizontal steel reinforcement is assigned at 90 degrees. Following the definition of the steel and concrete material properties, the materials are combined and the thickness of the individual layers are specified using the PlaneStressLayeredMaterial material which is assigned to the quad two-dimensional shell elements.

To verify the performance of the described modelling approach, the shear wall tested by Hiotakis (2004) is modelled again, this time using the new nonlinear $n D$ material for concrete, the PlaneStressRebarMaterial for the steel reinforcement, and PlaneStressLayeredMaterial to combine them together into a multi-layered plane stress shell element. First, a mesh sensitivity analysis was conducted to determine the effect of the finite element mesh size on the response of the model. Figure $4.8 \mathrm{~b}$ compares the hysteretic response behaviour for three shear wall models with 96, 270, and 1080 elements. The results show that the model is not very sensitive to mesh size, and a reduction in mesh size only has a small impact on the observed hysteretic response behaviour. Figure 4.8a compares the response of the Hiotakis (2004) shear wall and the finite element model. The results show that the finite element model is able to predict the ultimate strength, ductility, and energy dissipation capacity of the RC shear wall specimen.

In addition to a mesh sensitivity analysis, a sensitivity analysis for the parameters of the nonlinear concrete model was also conducted to determine their influence on the modelling results.

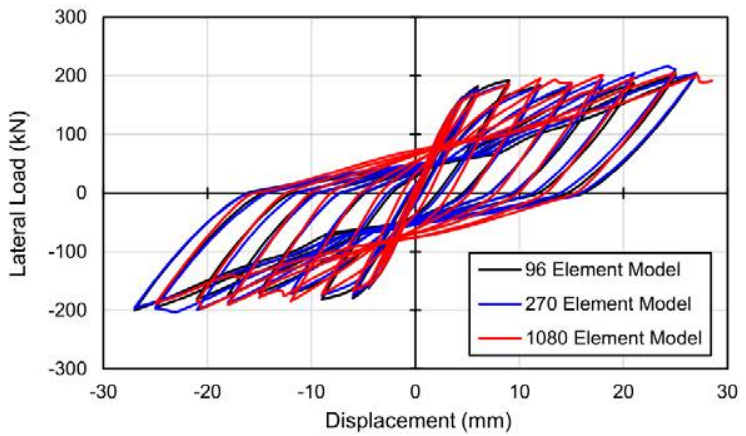

(a)

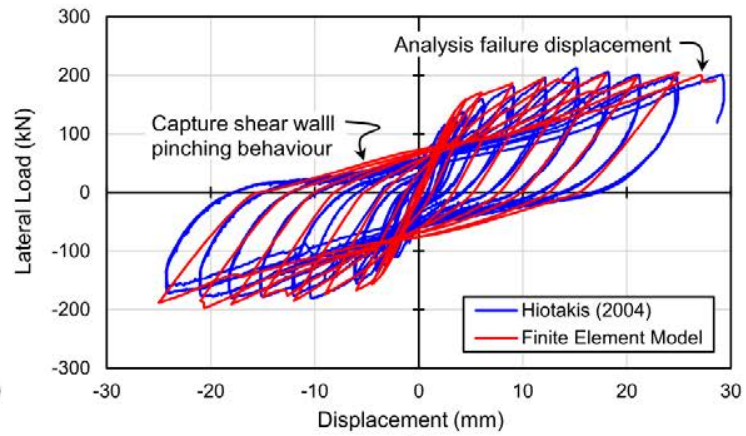

(b)

Figure 4.8 (a) mesh sensitivity; (b) hysteretic response comparison with Hiotakis (2004) 
The developed model of the Hiotakis (2004) shear wall specimen was used for the sensitivity analysis and the nonlinear material parameters were varied independently between their maximum and minimum recommended values. Figure 4.9 shows the results of the sensitivity analysis for the parameters $A_{p}$ for a series of pushover analyses conducted on the shear wall model and $B_{n}$ under reversed cyclic loading. The sensitivity analysis found that the concrete parameters $A_{n}$ and $\beta$ which control the softening behaviour and post-yield hardening modulus did not have a significant impact on the hysteretic response behaviour. Fig. 4.9 shows that the selection of $A_{p}$ effects the ductility of the shear wall response, while $B_{n}$ impacts the strength of the shear wall.

\subsection{Finite Element Modeling of Prototype Wall}

In this study, an analytical model for one of the three-storey shear walls in the N-S direction of the prototype structure (identified in Fig. 3.1) is modelled in the finite element software OpenSees. Although the nonlinear modelling approach using the 3D shell element has been shown to produce reasonable results when compared with experimental results, after discovering a software incompatibility between OpenSees and OpenFresco with the use of the PlaneStressUserMaterial command, the hybrid simulation of the first wall specimen (W1) was conducted using an elastic finite element model for the analytical substructure. The use of an elastic element also simplified the analytical substructure, removing potential complications in the analytical substructure (e.g. mesh sensitivity, model convergence, etc...) and was an opportunity to ensure the

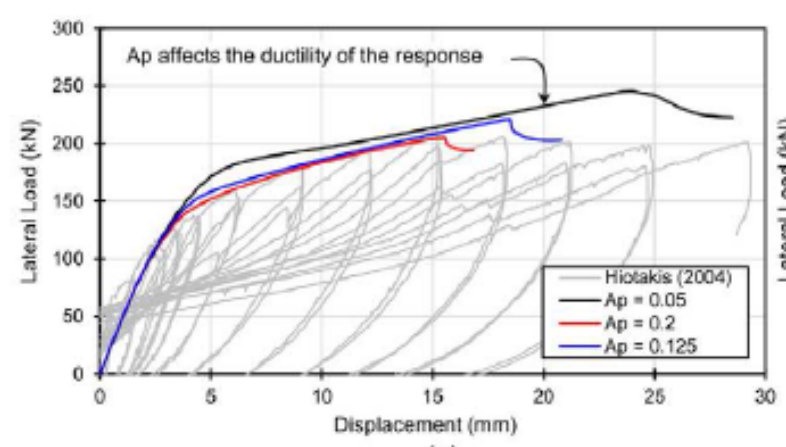

(a)

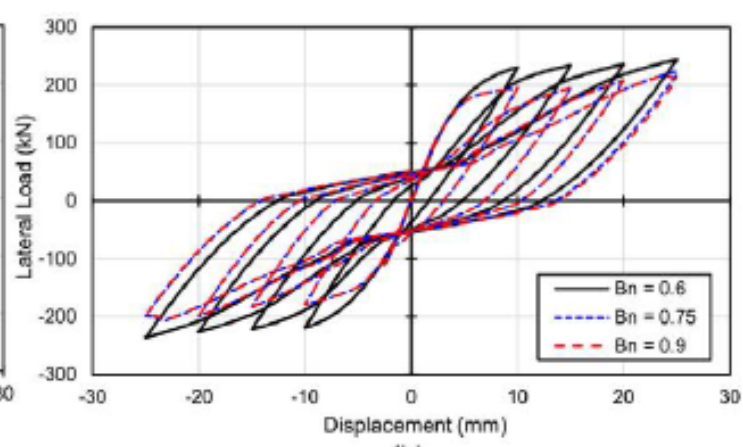

(b)

Figure 4.9 Influence of $A_{p}$ and $B_{n}$ on the shear wall finite element model 
experimental control was working properly before moving on to a more sophisticated finite element modelling approach. The second set of hybrid tests on the second shear wall specimen (W2) used a nonlinear finite element model based on the 2D multi-layer shell element discussed in the previous section. The fact the the hybrid simulations were conducted using both linear and nonlinear analytical substructures also provides the added benefit in being able to compare the differences between the results from the hybrid tests when using different modelling approaches.

In both modelling approaches (elastic and nonlinear shell elements), the RC shear wall model has a total of 2760 elements measuring $125 \mathrm{~mm}$ x $125 \mathrm{~mm}$. This element size corresponds to $3.33 \%$ of the shear walls length, which was found in the verification of the performance of the multi-layered shell element to produce good results. However, another mesh sensitivity analysis was conducted comparing the dynamic time-history analysis results of this 2760 element model with a 690 element model $(250 \mathrm{~mm}$ x $250 \mathrm{~mm})$. The results showed that a satisfactory level of accuracy was obtained when using the larger element size (690 elements), especially when considering the large discrepancy in analysis time (37:25:06s vs. 2:00:39s). For future work requiring more detailed response, the mesh size close to the base of the wall, where significant nonlinearity is expected, can be reduced to improve modelling accuracy. Figure 4.10 shows the proposed threestorey reinforced concrete shear wall model. This element size balances the computational time and accuracy of the finite element results. To impose the appropriate boundary conditions at each floor level, constraints on the lateral displacement are introduced to the nodes at the storey levels to ensure that all the nodes along each floor level have equal lateral displacements. The tributary mass for this shear wall (shaded in Fig. 3.1) is lumped at each storey level. The remaining mass not tributary to the studied RC shear wall is applied as a load on a leaning column to include P-delta effects from the rest of the structure. Figure 4.11 shows a sample nonlinear time-history analysis result for the develop shear wall model subjected to a synthetic ground motion record developed by Atkinson (2005) for Western Canada. 

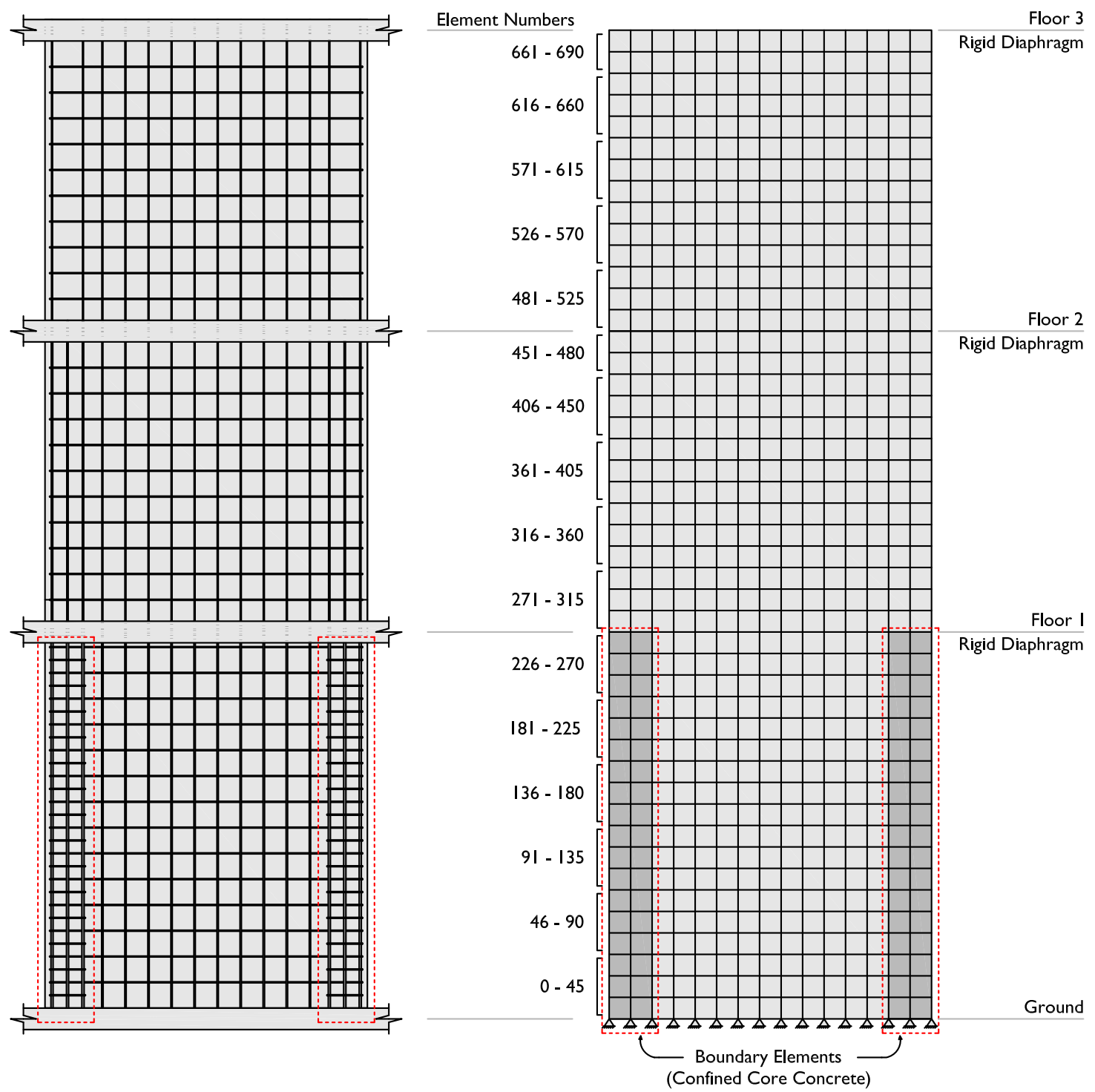

Figure 4.10 Finite element model of prototype RC shear wall 

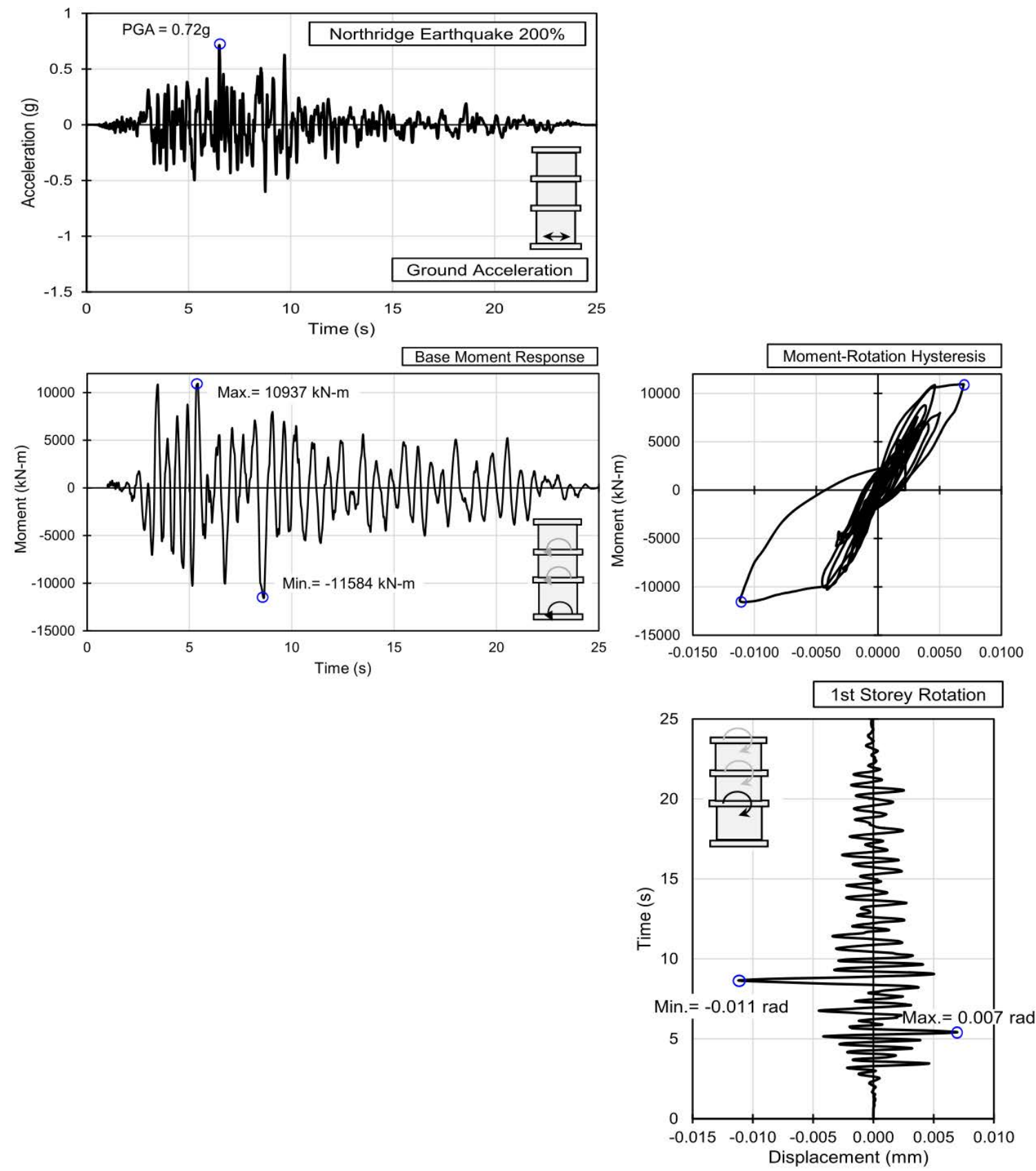

Figure 4.11 Sample earthquake time-history for prototype shear wall model 


\subsection{Model Sensitivity Analysis}

In addition to the size of the mesh in the analytical model, which was discussed previously, other model parameters can also have a significant effect on the performance and accuracy of a nonlinear finite element model subjected to an earthquake record, namely, the type of integration scheme employed, the time-step size for the time-history analysis, as well as the test used to determine if convergence has been achieved at the end of each time step. To independently study the effect of each of these parameters on the analysis result, the previously discussed finite element model of the prototype structural wall is subjected to the Northridge (1994) ground motion with varying dynamic analysis parameters. These dynamic parameters include two different integration schemes, three different time-step sizes, and three different convergence tolerances. The two integration schemes include the Newmark $\beta$ Method and the modified Newmark $\beta$ Method for hybrid simulation developed by (Schellenberg et al., 2009). Figure 4.12 shows the influence of the different parameters on the response of the prototype wall model and Table 4.2 shows the analysis times for the range of dynamic analysis parameters. The results of the sensitivity analysis demonstrate that a time step of $0.005 \mathrm{~s}$ and a NormDisp increment convergence test tolerance of $1 e^{-5}$ when using the Newmark $\beta$ Method are sufficient in accurately capturing the shear wall response while maintaining computational efficiency. For the modified Newmark $\beta$ Method for hybrid simulation, satisfactory results compared with the standard Newmark $\beta$ Method are achieved with a time step of $0.005 \mathrm{~s}$ and a constant 50 iterations per time step. The results in Figure 4.12 demonstrate that a time-step of $0.005 \mathrm{~s}$ sufficiently captures the dynamic response of the three-storey shear wall while also balancing the computational efficiency.

\subsection{OpenSees-OpenFresco Hybrid Model}

In addition to developing a purely numerical finite element model of the analytical structure, a hybrid model must also be developed that integrates the finite element model of the analytical substructure with the experimental substructure in the laboratory. This model established 

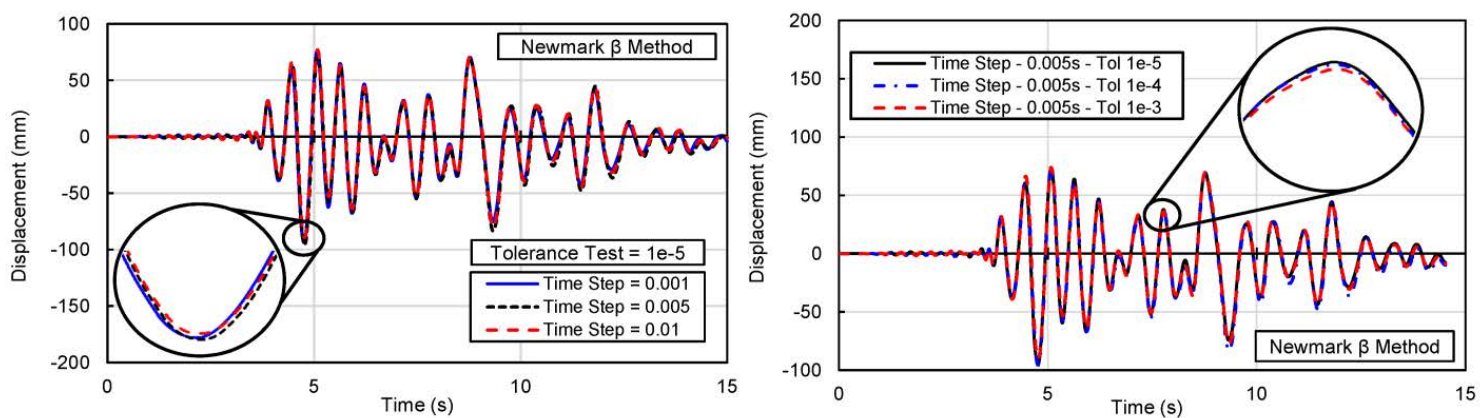

(a)

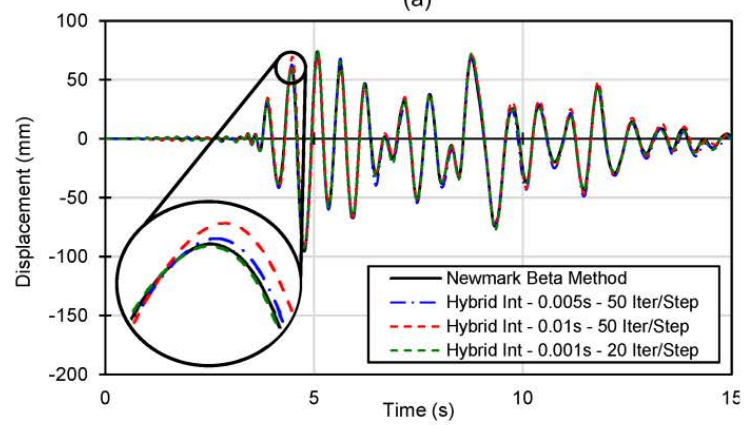

(c)

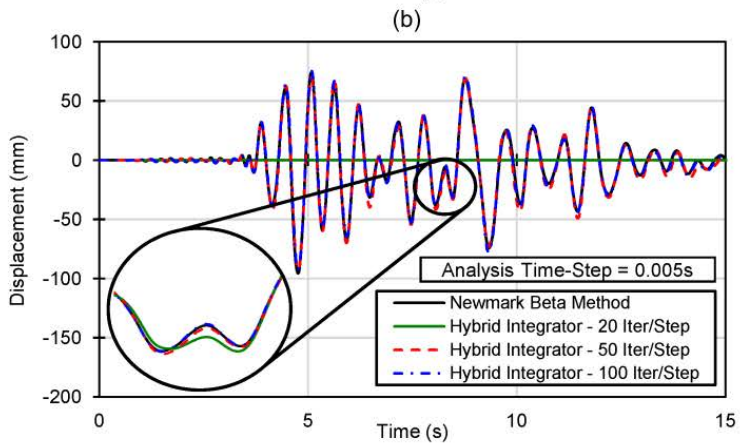

(d)

Figure 4.12 Model sensitivity: (a) time-step (Newmark $\beta$ Method), (b) convergence tolerance; (c) time-step (Newmark HS Method); (d) number of fixed iterations

Table 4.2 Model analysis times under varying dynamic analysis parameters

\begin{tabular}{ccccc}
\hline \hline $\begin{array}{c}\text { Integration } \\
\text { Scheme }\end{array}$ & $\begin{array}{c}\text { Time Step } \\
(\mathrm{s})\end{array}$ & $\begin{array}{c}\text { Convergence } \\
\text { Tolerance }\end{array}$ & $\begin{array}{c}\text { Fixed } \\
\text { Iterations }\end{array}$ & $\begin{array}{c}\text { Analysis Time } \\
\text { (hh:mm:ss) }\end{array}$ \\
\hline Newmark $\beta$ Method & 0.01 & $1.0 e^{-5}$ & - & $00: 44: 03$ \\
Newmark $\beta$ Method & 0.005 & $1.0 e^{-3}$ & - & $00: 29: 30$ \\
Newmark $\beta$ Method & 0.005 & $1.0 e^{-4}$ & - & $00: 44: 29$ \\
Newmark $\beta$ Method & 0.005 & $1.0 e^{-5}$ & - & $01: 03: 37$ \\
Newmark $\beta$ Method & 0.001 & $1.0 e^{-5}$ & - & $02: 48: 48$ \\
Newmark HS Method & 0.01 & - & 50 & $02: 52: 35$ \\
Newmark HS Method & 0.005 & - & 20 & $02: 01: 48$ \\
Newmark HS Method & 0.005 & - & 50 & $04: 47: 18$ \\
Newmark HS Method & 0.005 & - & 100 & $09: 34: 10$ \\
Newmark HS Method & 0.001 & - & 200 & $10: 32: 50$ \\
\hline \hline
\end{tabular}

Notes: NormDisp increment convergence test implemented in fully analytical model

FixedIter fixed iteration convergence test used for hybrid tests 
the required parameters for the communication link between the finite element software and the hydraulic controller in the laboratory. This communication link is used to send command and feedback displacements and forces between the analytical and experimental substructures, facilitating the hybrid simulation. To form this communication link, a middleware is required that is capable of communicating between the equipment controlling the experimental test setup (e.g. hydraulic controller, data acquisition systems etc...) and the analytical model. Generally, a middleware refers to a software that connects one or more software applications together, allowing them to exchange data (Schellenberg et al., 2009).

In this study, the Open Framework for Experimental Setup and Control middleware, commonly referred to as OpenFresco, is utilized to interface between the physical specimen in the laboratory and the numerical model in OpenSees. OpenFresco is an open-source software for hybrid simulation of structural systems developed by the University of California at Berkeley (Schellenberg et al., 2007). OpenFresco is a practical software package that is capable of integrating a wide variety of hybrid simulation algorithms, laboratory and control systems, experimental setups, and computational simulation models for hybrid simulation. Its object-oriented software framework provides flexibility, extensibility and re-usability. Although the original development of the software relied on OpenSees for the computational simulation, more recent releases of the software have integrated a wide variety of existing computer simulation tools commonly used in structural engineering including but not limited to Matlab, LS-DYNA, UI-SimCor, Abaqus and Simulink (Schellenberg et al., 2007). In addition, OpenFrescoExpress has recently been introduced as a simplified version of the OpenFresco inter-ware that can be used for projects involving one or two degrees-of-freedom and includes a useful graphical user interface, although it is not discussed further here.

Figure 4.13 illustrates the software architecture for a local (one experimental site) hybrid simulation. The OpenFresco framework has five key components: the finite element software, experimental element, experimental site, experimental setup and experimental control. As discussed earlier, the analytical model of the structure is defined in the finite element software, including the dynamic properties of the structure such as mass and damping. Currently, OpenFresco supports a 


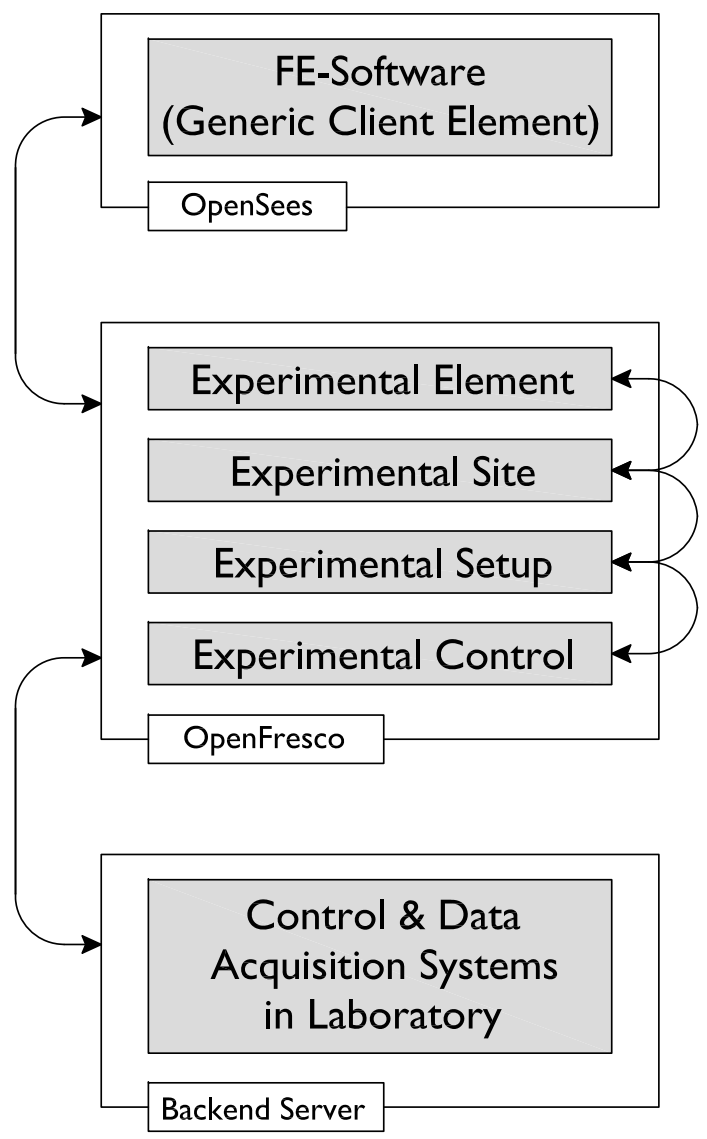

Figure 4.13 OpenFresco software architecture for local hybrid test (adapted from Schellenberg et al., 2007) 
number of commonly employed finite element softwares, the only limitation being that the software must allow for user defined elements. For the prototype three-storey shear wall in this study, the top two storeys of the RC shear wall are modelled analytically while the first storey, which is expected to develop a plastic hinge at its base and exhibit a highly nonlinear response, is tested experimentally. OpenSees and the previously discussed user-defined Quad shell elements with elastic and nonlinear properties are used in the analytical substructure for the hybrid simulation.

In OpenFresco, the experimental element represents the component or portion of the structure that is physically tested (e.g. columns, beams, walls) in the laboratory and provides the interface between the finite element software and the experimental software framework. The experimental element, which is defined in the OpenFresco framework, transforms the prescribed boundary conditions from the global coordinate system of the finite element model to the local coordinate system (referred to as the basic coordinate system in OpenFresco). The basic coordinate system are the degrees of freedom that are suitable for testing. Figure 4.14 shows the transformation between global and local coordinate systems for an experimental beam-column element in OpenFresco. In addition to the coordinate transformation, the experimental element also provides a means for transforming the experimental response quantities such as displacement, velocity, acceleration, and force which are measured by the experimental equipment back to the global coordinate system of the finite element model.

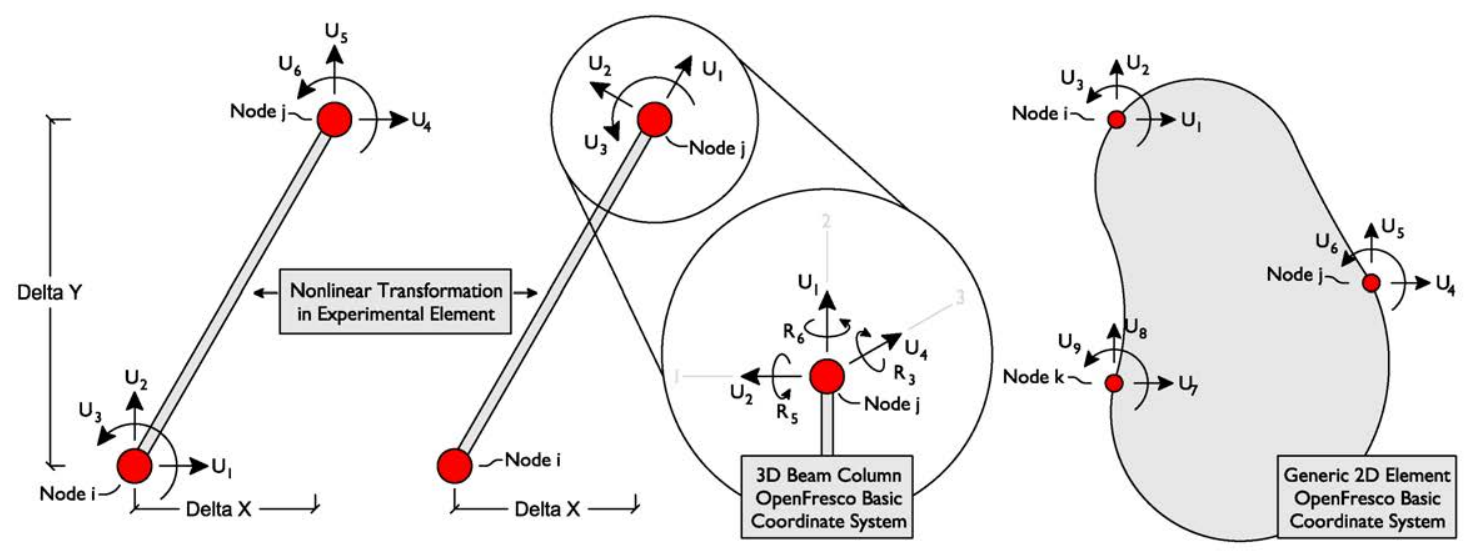

Figure 4.14 Coordinate transformation for OpenFresco experimental element 
In addition to transforming the prescribed boundary conditions between the global and local or basic coordinate systems, the initial stiffness of the experimental test specimen must be provided in the experimental element command. In contrast to an analytical element, whose tangent stiffness matrix can be returned at each step throughout the direct integration scheme, the experimental element is often unable to return a tangent stiffness matrix and needs to instead return its initial stiffness matrix. The inability to return a tangent stiffness matrix for the experimental element stems from the fact that it is generally difficult to calculate the tangent stiffness matrix of the element based solely on the experimental response quantities at the local element degrees of freedom (Schellenberg et al., 2007). In addition, because a physical test specimen is truly history dependent, the experimental element representing the physical test specimen cannot revert to a previous or initial state of analysis once the simulation has begun.

In this study, a Generic experimental element (Fig. 4.14) is used to represent the physical test specimen in OpenFresco. A generic element is capable of representing an element at any number of nodes with any number of degrees-of-freedom (DOFs). The user must specify which nodes are being controlled and the DOFs at those nodes. In this case, there is a single control node at the top of the first storey shear wall with three control DOFs (lateral and axial displacements and rotation). Note that the base of the first storey shear wall is fixed, therefore only the initial stiffness matrix for the three unrestrained DOFs at the interface between the experimental and analytical substructure at the top of the first storey shear wall must be specified by the user. The initial stiffness matrix $\left(K_{\text {initial }}\right)$ according to the basic coordinate system for the generic element is shown in Fig. 4.14, and is defined as follows:

$$
K_{\text {initial }}=\left[\begin{array}{ccc}
12 E I_{x} / L_{w}^{3} & 0 & 6 E I_{x} / L_{w}^{2} \\
0 & E A_{w} / L_{w} & 0 \\
6 E I_{x} / L_{w}^{2} & 0 & 4 E I_{x} / L_{w}
\end{array}\right]
$$

where $E$ is the modulus of elasticity of the concrete, $A_{w}$ is the area of the shear wall, $L_{w}$ is the shear wall height, and $I_{x}$ is the moment of inertia of the shear wall about its strong axis. It is noted that it is good practice to over-estimate the initial stiffness of the experimental element in the 
hybrid simulation. This ensures that the initial command displacement sent to the experimental element is not too large, which could result in instability in the hybrid simulation or accidentally over-damage the experimental substructure. Ultimately, it was found that an assumption of the theoretical initial stiffness based on the geometry and design properties of the RC shear wall $\left(E=5500\left(f_{c}^{\prime}\right)^{1 / 2}\right)$ was sufficiently stiff to produce good results.

The experimental site stores data and provides a communication method if a multi-site distributed hybrid simulation is being conducted. In such a case, the experimental tests are carried out at multiple different locations, which can be anywhere around the world. The experimental objects defined in OpenFresco are responsible for communicating and storing data between sites. To transfer information between sites, different communication protocols are available in OpenFresco, which include but are not limited to TCP/IP and UDP in addition to providing secure data transactions over the internet using cryptographic protocols such as TLS and SSL. Because this test is not a geographically distributed hybrid simulation, the local experimental site command in which no client-server communication is necessary is utilized.

The experimental setup accounts for the distribution of the hydraulic actuators that are responsible for applying the target displacement(s) and measuring the restoring force(s) during the hybrid simulation. Because the shear wall specimen in the laboratory in this study is subject to axial, shear, and overturning moment from the upper portion of the RC shear wall (levels 2 and 3), the initial goal of the project was to use the ThreeActuatorSetup in OpenFresco. Figure 4.15 illustrates the three actuator control setup in OpenFresco which requires user input of actuator lengths, joint offsets, and rigid loading beam lengths. This experimental control object allows for simultaneous control of three actuators, controlling the two translational and single rotational DOFs at the top of the experimental test specimen. As illustrated in Fig. 4.15, the experimental setup is designed to transform between the experimental DOFs and the actuator DOFs in the laboratory. The experimental setup is also responsible for ensuring that the finite element command displacements are scaled and applied to the experimental element according to similitude laws, measuring the loads or restoring forces at each actuator position and scaling them back to the global DOFs in the finite element model. 


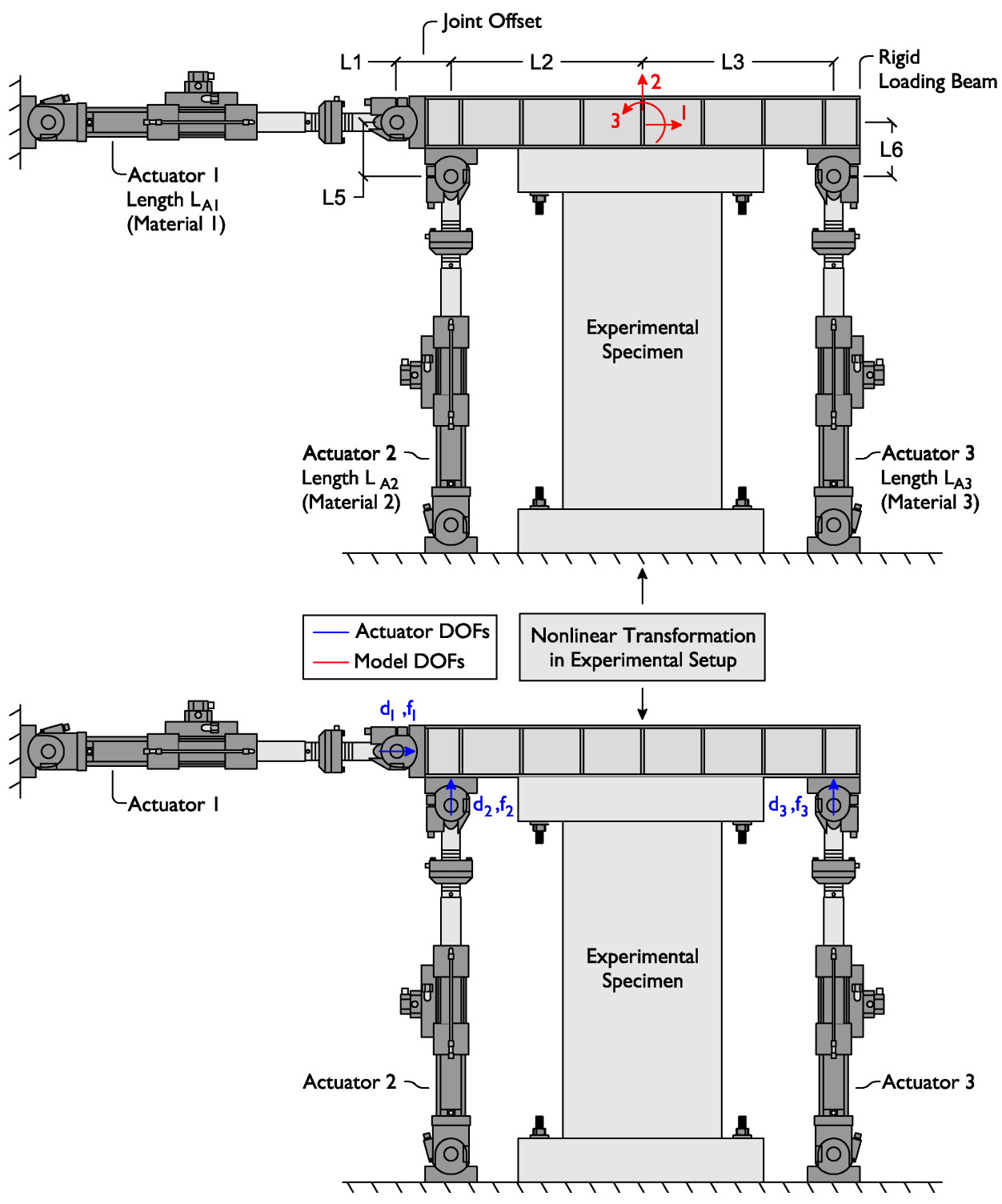

Figure 4.15 Coordinate transformation between global and actuator DOFs 
Ultimately, because of some recent incompatibilities between OpenSees and OpenFresco software versions, the ThreeActuatorSetup could not be employed during the hybrid shear wall tests. Alternatively, to transform between global DOFs and actuators DOFs, MTS degree-offreedom control software was utilized and is discussed in more detail in following chapter. When utilizing MTS DOF, the DOF transformation is handled on the experimental side of the hybrid simulation. This means that a NoTransformation experimental setup could be used in the shear wall hybrid test. This experimental setup does not perform any coordinate transformation on the command and feedback signals, it is only responsible for scaling the command and feedback signal based on similitude laws. Recall that the experimental substructure is scaled to $40 \%$ of the original scale of the structure and as a result, the command $\mathrm{X}$ and $\mathrm{Y}$ displacements and the $\mathrm{Z}$ rotation were scaled by a factor of $0.4,0.4$, and 1.0 respectively before being sent to the experimental site. Upon being sent back to the finite element model, the $\mathrm{X}$ and $\mathrm{Y}$ achieved displacements are inversely scaled by 2.5 (1/0.4) while the rotation remains unscaled. Likewise, feedback X and Y forces and $\mathrm{Z}$ moment are also scaled before being sent back to the finite element software by factors of 6.25 , 6.25, and 15.625, respectively. Recall the scale factors for force and moment from Table 3.6 for force and moment of 0.16 and 0.064 , respectively, which are scaled inversely when fed back to the analytical model.

Finally, the last component of the OpenFresco framework is the experimental control object, which interfaces with the different controllers and data acquisition systems in the laboratory (IP addresses and port numbers). This object was introduced into the OpenFresco framework because of the unique characteristics of experimental control and system hardware used in different laboratories (e.g. control and data acquisition softwares). The use of its own object class for experimental control in OpenFresco means that only those responsible for IT aspects of the control and data-acquisition systems in the laboratory need to be concerned with the experimental control class; while those configuring the actuators need be concerned only with the experimental setup class.

Figure 4.16 shows the combined OpenSees/OpenFresco model used in this study, including analytical and experimental elements. After each of these parameters have been defined in the 
hybrid model, a trial simulation, commonly referred to as a test rehearsal of the hybrid simulation can be completed virtually to ensure that there are no problems with the hybrid model. The results of several hybrid test rehearsals conducted on the shear wall experimental test setup are discussed in the following section.

\subsection{Hybrid Test Rehearsals}

Prior to conducting the hybrid simulation in the laboratory, the results from a fully analytical model are compared with the substructured hybrid OpenFresco-OpenSees model. This is what is commonly referred to as a hybrid test rehearsal and is used to ensure that the substructured hybrid model is working properly and that there are no significant discrepancies between the hybrid and fully analytical models (Schellenberg et al., 2008; You and Schellenberg, 2017). There are two convenient methods that can be used to conduct hybrid test rehearsals depending on the experimental setup and the complexity of the hybrid model. If the experimental setup is a simple system of single actuators used to control uncoupled DOFs, then the SimUniaxial material command may be appropriate to conduct hybrid test rehearsals. In this approach, a uniaxial material model (elastic or nonlinear) is used to represent the force-deformation response of the experimental element at each actuator position (shown in Fig. 4.16). In a simple test configuration, this approach is sufficient to simulate the presence of the experimental element and verify that the hybrid model is working properly. However, this technique will not work properly for experimental setups that use multiple actuators to control coupled DOFs, such as those in this study.

The second approach for conducting a hybrid test rehearsal in an OpenSees-OpenFresco model, which is applicable for coupled systems, uses a SimDomain command in OpenFresco to simulate the hybrid domain and requires a user-defined element to represent the specimen in the experimental substructure. This technique essentially uses one or more user defined element(s) to simulate the behaviour of the experimental element. For example, in the hybrid test rehearsals conducted on the shear wall hybrid model in this study, an elastic beam column element is used 


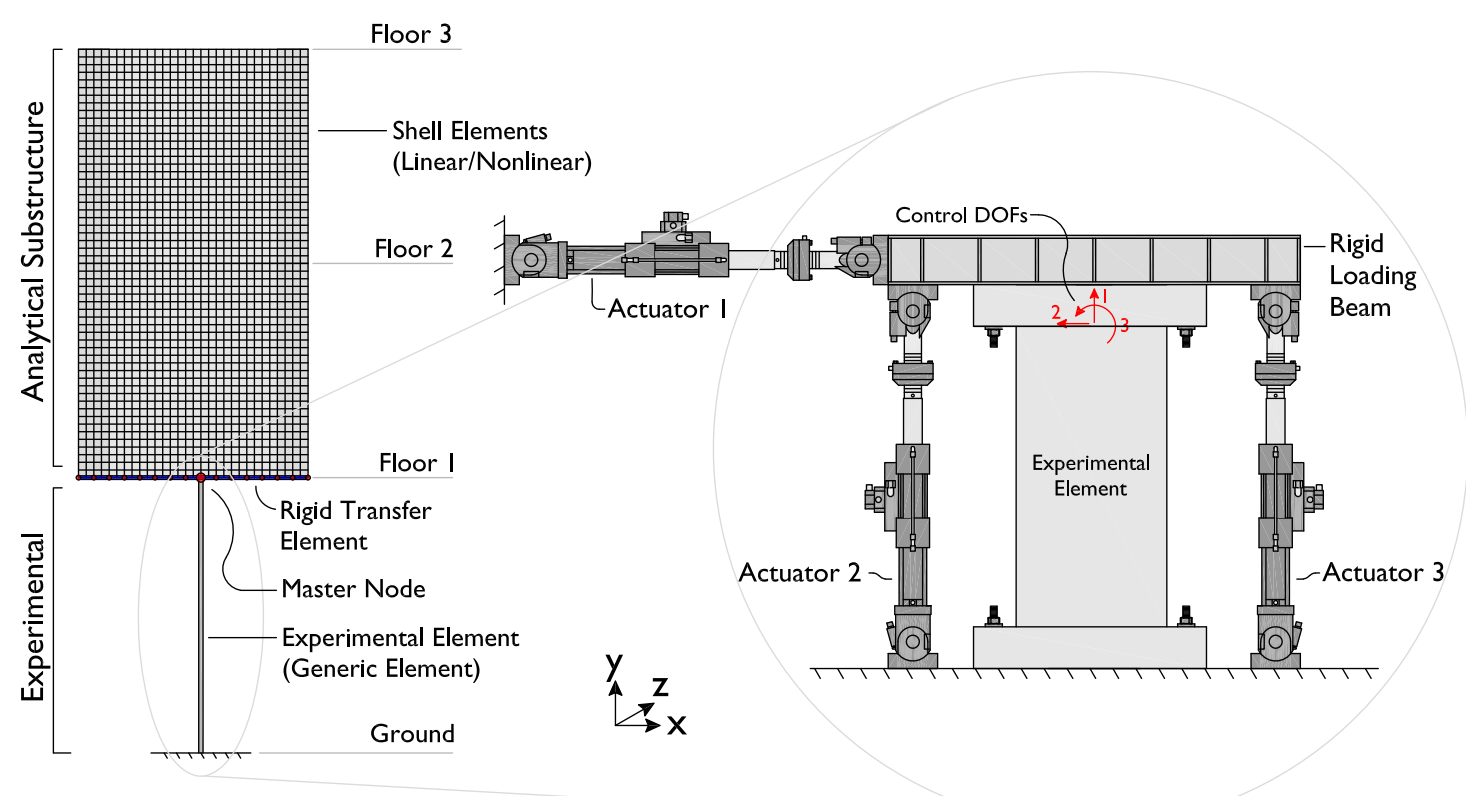

Figure 4.16 Combined OpenSees-OpenFresco hybrid shear wall model

to connect the fixed base node in the middle of the shear wall at its base and the control node at the top of the first storey shear wall. Although a nonlinear element could also be implemented, the purpose of the test rehearsal is to ensure the components of the hybrid model are working properly (e.g. experimental setup, experimental site, experimental control, command/feedback scaling) and not to study the nonlinear response of the model. In this approach, the elastic beam column element mimics the response of the shear wall specimen in the laboratory.

Using the second approach, a hybrid test rehearsal is conducted on the developed shear wall hybrid model. The ground motion record selected for the hybrid test rehearsal is the Canoga Park station record from the 1994 Northridge Earthquake that will be used in the real shear wall hybrid simulations. Figure 4.17 shows the top displacement and base shear results for the duration of the ground motion. The results of the hybrid test rehearsal show good correlation between the fully analytical and hybrid models, suggesting that the components of the hybrid model are defined correctly and functioning as intended. In addition, the fundamental period of vibration between the fully-analytical mode $(0.229 s)$ and substructured model $(0.230 s)$ adequately match, suggesting the stiffness and mass definition in both models is accurate. 

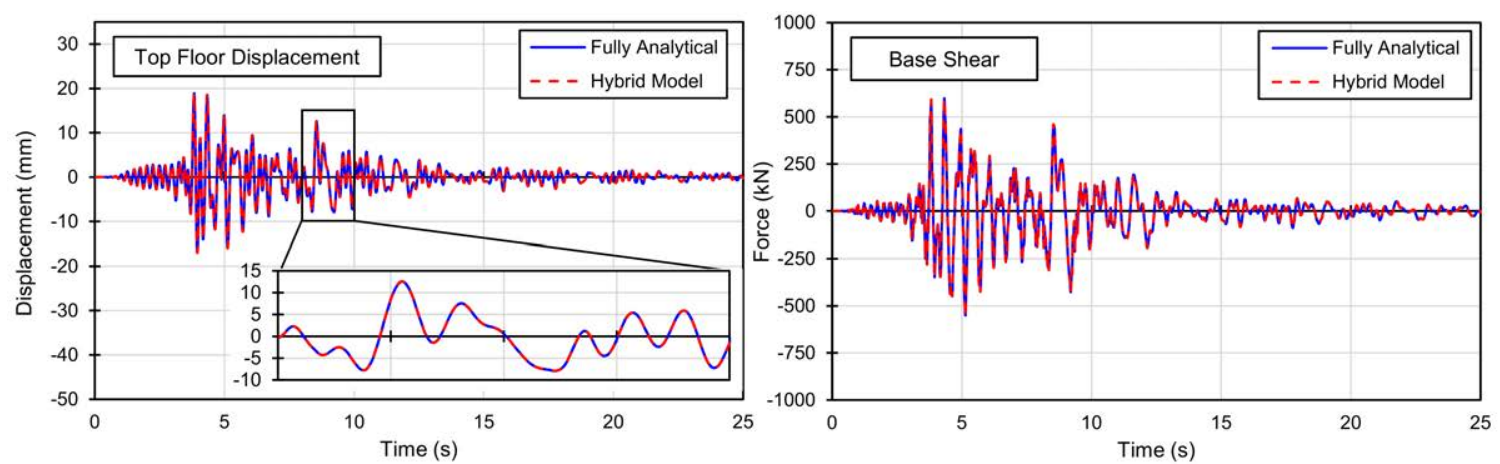

Figure 4.17 Comparison between fully analytical and substrcutured hybrid shear wall models

The importance of rigorous hybrid test rehearsals cannot be understated, as small errors in the definition of the hybrid model or setup of the laboratory equipment can result in damage to the test setup or specimen, resulting in significant time and economic losses. Consequently, additional hybrid test rehearsals were also conducted using the actual signals from the equipment in the laboratory. This approach uses a "demo" mode in combination with calculation editor available in MTS 793 control software (You and Schellenberg, 2017). A sub-component of MTS 793, known as MTS Csi, is used to send and receive command signals from the hydraulic actuators in the laboratory during the hybrid test. The use of demo mode and the calculation editor in MTS 793 allows the laboratory technician to replicate the response of a hydraulic actuator virtually, including the effects of tuning and lag in the control loop. The user must only specify the force-deformation relationship for each control mode in the test (e.g. X, Y, and RZ). The force-deformation relationship for each control channel is implemented using the calculation editor function in MTS 793. After implementing the appropriate elastic stiffness in the X, Y, and RZ directions for the experimental shear wall specimen, the hybrid test can be run virtually, including the laboratory equipment, without moving physically testing the specimen. In this study, hybrid test rehearsals showed satisfactory performance of the hybrid model, including passage of the scaled actuator command signals between the finite element software (OpenSees) and the MTS control software in the lab. With satisfactory performance of the analytical and hybrid models, the next chapter describes construction of the experimental specimens and test setup, as well as the configuration of the hybrid testing system at Carleton University. 


\section{Chapter 5. Experimental Program}

\subsection{Experimental Objectives}

The experimental objectives of this study are to assess the feasibility of using hybrid simulation to replace large-scale experimental testing to study the seismic response of RC shear wall structures. A key component of this objective is the need to develop strategies to overcome some of the challenges associated with hybrid testing of stiff structural elements. Ultimately, the results of the hybrid simulation are intended to be used to study the behaviour of multi-storey RC shear walls under realistic multi-degree-of-freedom deformations during an earthquake. The experimental substructure for the hybrid simulation is a $1 / 2$-scale RC shear wall specimen representative of the first storey of the three-storey RC structure. To maintain compatibility between the analytical and experimental substructures, the lateral and axial displacements and rotation at the top of the experimental shear wall specimen are controlled using three hydraulic actuators. The following section outlines construction details for the shear wall specimens, experimental test setup, and discusses the development, testing, and setup of the new hydraulic control and hybrid testing system in the Civil Engineering Structures laboratory at Carleton University.

\subsection{Test Specimens}

The two shear wall specimens, referred to as W1 and W2, are identical scaled versions of the first storey shear wall from the prototype structure described in Chapter 3. The first storey of the three storey shear wall is scaled to $2 / 5(40 \%)$ of its original size and constructed in the laboratory. Figure 5.1 shows a typical shear wall specimen in this study. The scaled wall specimen measures 
$1500 \mathrm{~mm} \times 1800 \mathrm{~mm} \times 100 \mathrm{~mm}\left(l_{w} \times h_{w} \times t_{w}\right)$. The vertical and horizontal steel reinforcement ratios of the two wall specimens for the hybrid simulation are scaled to match the reinforcement ratios in the prototype structure as closely as possible. Because of limitations on bar sizes and the spacing of the steel reinforcement, the resulting vertical and horizontal reinforcement ratios are $0.4 \%$ in both directions, respectively, compared with $0.43 \%$ and $0.25 \%$, respectively, for a typical wall in the prototype structure. In addition to the shear wall specimens, a large reaction block is also constructed and is used to prevent rotation and translation of the shear wall specimen on the laboratory strong floor. Figure 5.2 shows the geometry and reinforcing scheme for the reaction block. The heavily reinforced reaction block measures $1200 \mathrm{~mm} \times 2000 \mathrm{~mm} \times 650 \mathrm{~mm}\left(l_{w} \mathrm{x} h_{w}\right.$ $\left.\mathrm{x} t_{w}\right)$.

In addition to being appropriately scaled from the prototype structure, the geometry of the shear wall specimens also matches those for walls tested previously at Carleton University by Lombard (1999); Lombard et al. (2000), Hiotakis (2004), and Woods et al. (2016). This will allow for additional response comparisons between the previously conducted static-cyclic tests by Lombard et al. (2000), Hiotakis (2004), and the hybrid simulation test results in this study.

One of the major challenges when conducting hybrid tests on RC structural elements are the consequences of unexpected problems encountered in the experimental test setup or hybrid model. In the event of an error in the design or construction of one or both of these elements, the experimental substructure could be accidentally cracked or more severely damaged, diminishing the value of the experimental results. In an attempt to overcome this challenge, a steel column assembly is also tested in addition to the two shear wall specimens, using the same experimental test setup and hybrid model as the shear wall hybrid tests. The results from the steel column hybrid test are used to verify the performance of the experimental test setup, hydraulic control, communication link between the analytical and experimental substructures, and the substructured finite element model. The goal of testing a steel column first is to improve the likelihood of collecting accurate results from the hybrid tests conducted on both shear wall specimens.

Figure 5.3 shows the steel column assembly and its components. The steel column assembly 


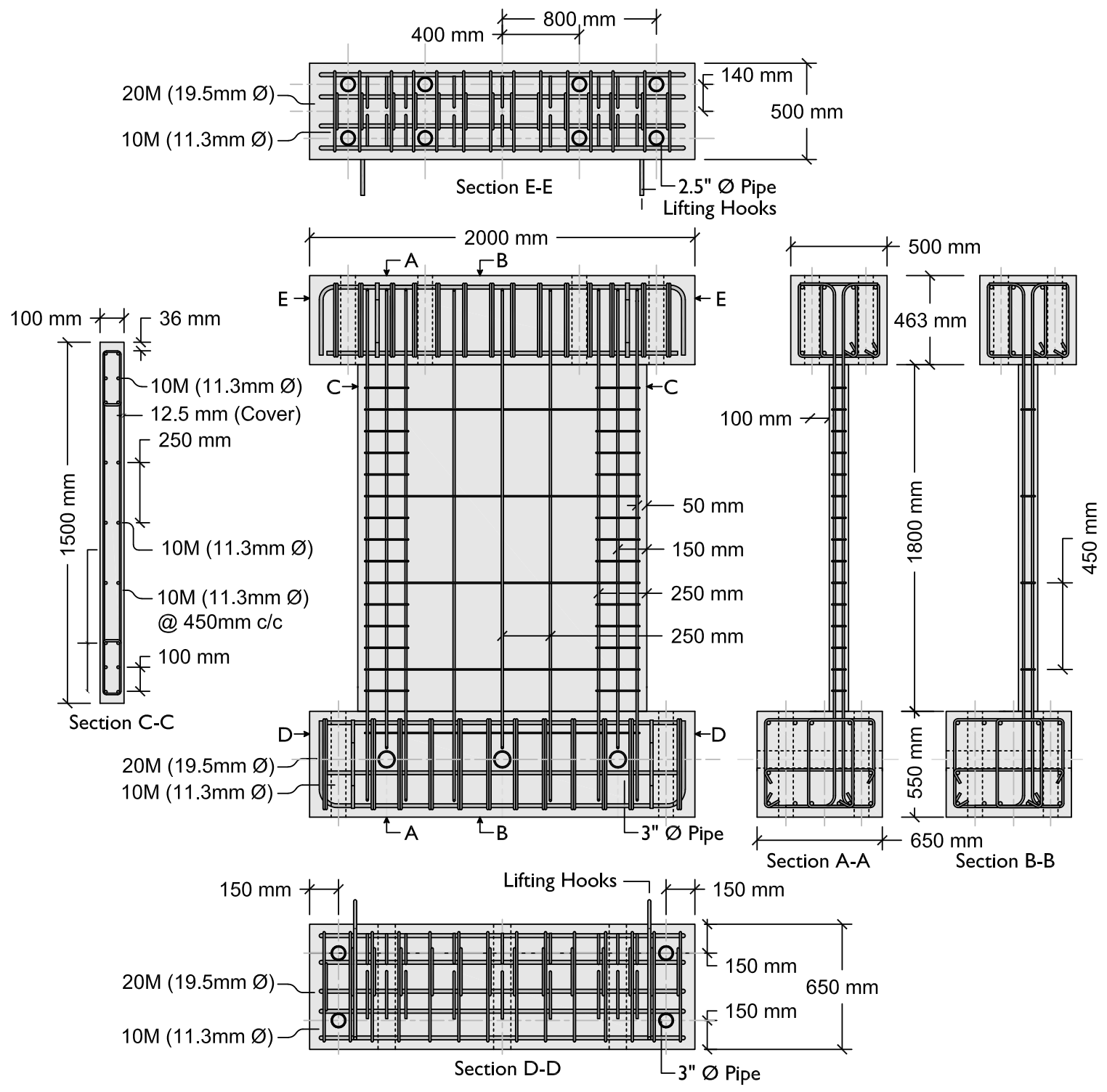

Figure 5.1 Shear wall specimens geometry and steel reinforcement detailing 


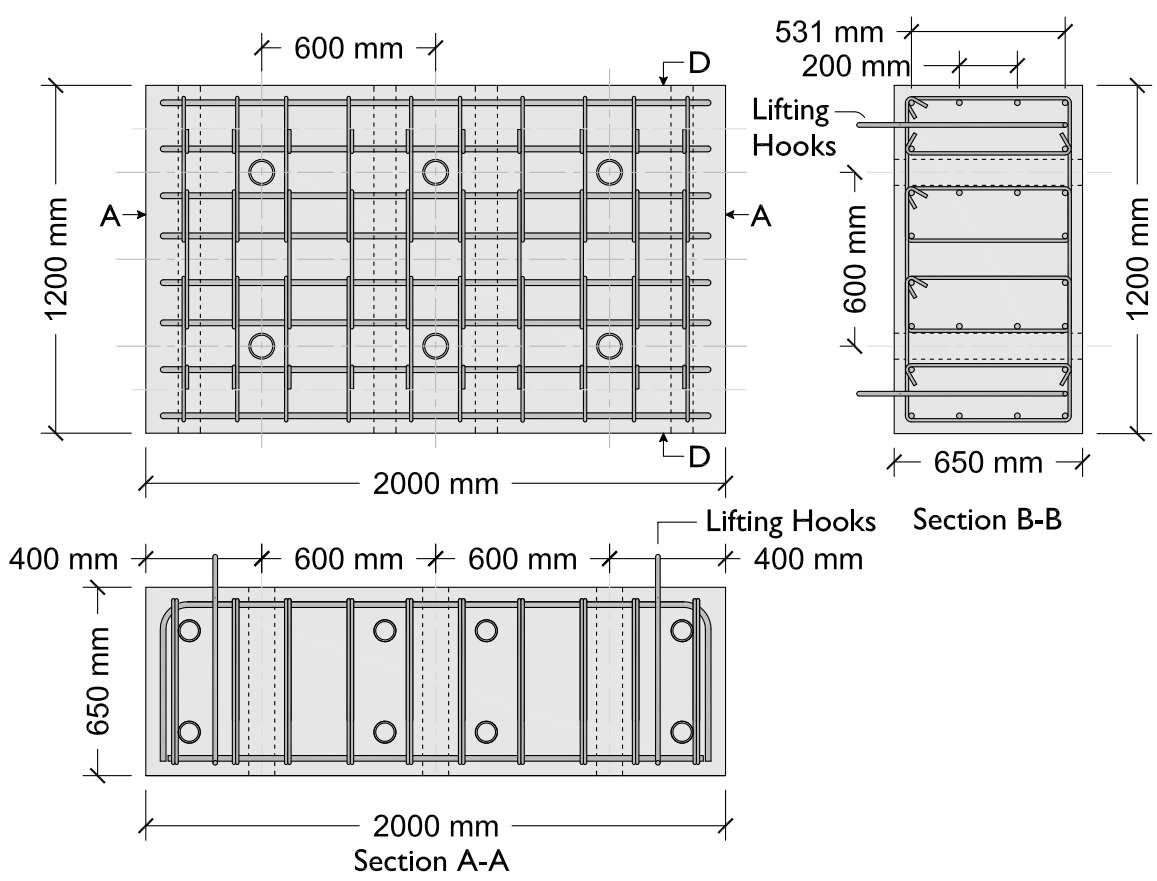

Figure 5.2 Reaction block geometry and steel reinforcement detailing

includes a top and bottom steel section that are designed to remain entirely elastic. They are connected together using a replaceable reduced beam section (RBS), fabricated from a W-section. This section has radial flange cuts to form a 'dogbone' section that is designed to yield at a comparable moment to that of the shear wall specimens. The size of the steel column assembly (top/bottom steel sections and RBS) is selected such that it will have a comparable theoretical axial stiffness as a typical wall specimen in this study. The design of the RBS link follows the guidelines presented in the design standard for Moment Connections in Seismic Applications (CISC, 2014). Design procedure and calculations are available in Appendix B and fabrication drawings for the steel column assembly are available in Appendix C. The use of the RBS link detail allows for more than one trial test to be conducted without the need to replace the entire steel assembly. Ultimately, because of time constraints the steel column assembly was only used to test the functionality of the experimental test setup, hybrid model, and communication between test components, and were not subject to an entire earthquake record. 


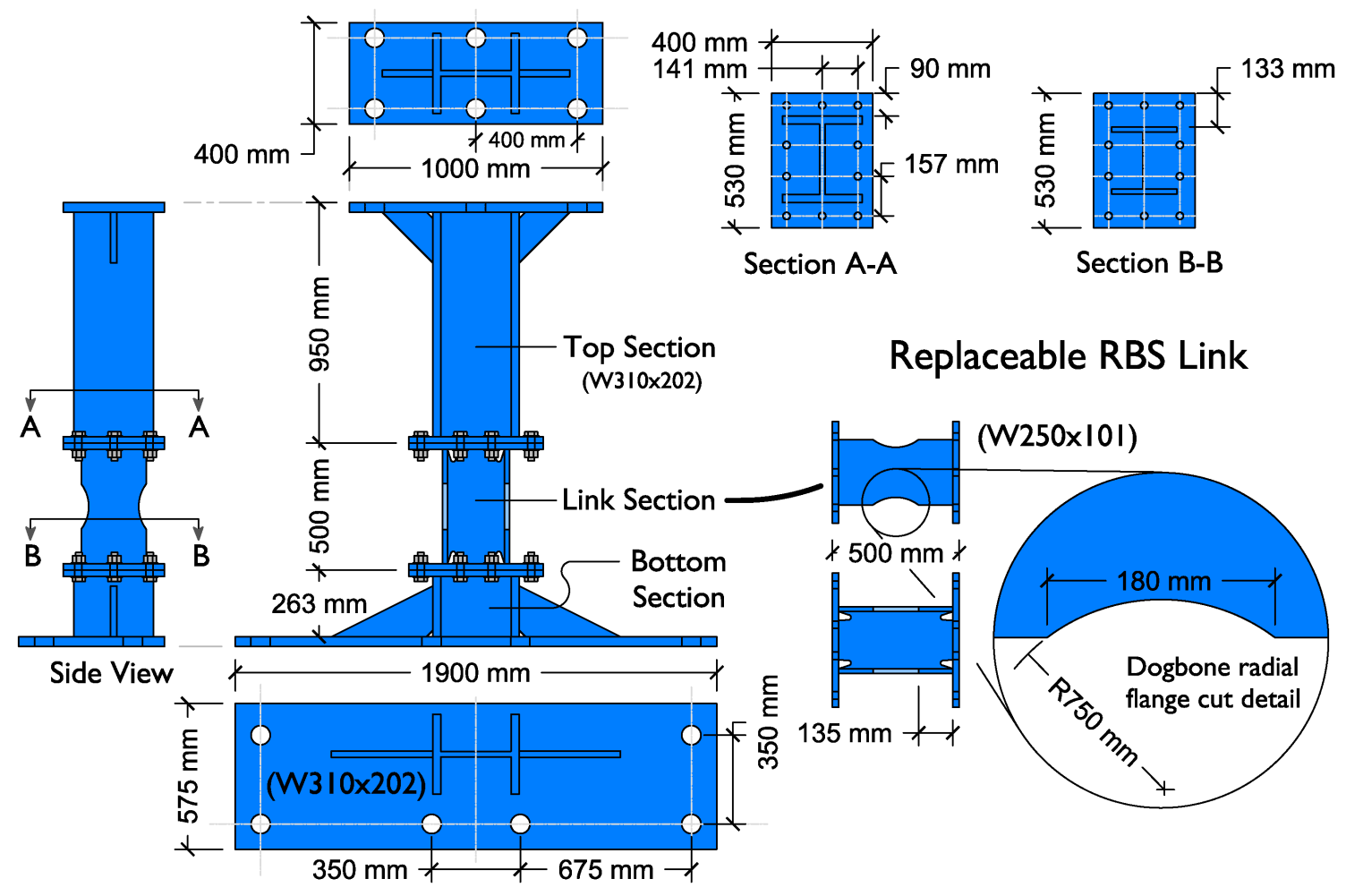

Figure 5.3 Steel column assembly geometry and design details

\subsection{Material Properties}

Tables 5.1 and 5.2 show the material properties of the steel reinforcement and concrete for the shear wall specimens in the hybrid tests, respectively. Material properties are based on a minimum of three repeated rebar coupon or concrete cylinder tests. The wall specimens were cast horizontally in a single stage. Care was taken during vibration of the concrete to ensure that an even aggregate distribution was maintained. The target concrete compressive strength for the wall specimens was $50 \mathrm{MPa}$, although 28-day concrete cylinder strengths for both concrete batches remained below the strength target, and results for the wall panel cylinder strengths range between $30 \mathrm{MPa}$ and $40 \mathrm{MPa}$. Grade 400 reinforcing steel was used for the vertical and horizontal steel reinforcement. 
Table 5.1 Average steel reinforcement material properties

\begin{tabular}{lccc}
\hline \hline & $\begin{array}{c}\text { Vertical } \\
\text { Property }\end{array}$ & $\begin{array}{c}\text { Horizontal } \\
\text { Rebar }(10 \mathrm{M})\end{array}$ & $\begin{array}{c}\text { Cap Beam/Foundation } \\
\text { Rebar }(20 \mathrm{M})\end{array}$ \\
\hline$f_{y}(\mathrm{MPa})$ & 455 & 468 & 455 \\
$\epsilon_{y}(\mathrm{~mm} / \mathrm{mm})$ & 0.00218 & 0.00283 & 0.00247 \\
$E_{s}(\mathrm{GPa})$ & 193 & 190 & 185 \\
$\epsilon_{s h}(\mathrm{~mm} / \mathrm{mm})$ & 0.0125 & 0.0120 & 0.0190 \\
$F_{u}(\mathrm{MPa})$ & 650 & 669 & 594 \\
$\epsilon_{u}(\mathrm{~mm} / \mathrm{mm})$ & 0.273 & 0.292 & 0.334 \\
\hline \hline
\end{tabular}

Table 5.2 Concrete material properties

\begin{tabular}{lcccc}
\hline \hline & \multicolumn{2}{c}{ Wall 1 } & \multicolumn{2}{c}{ Wall 2 } \\
Property & 28-Day & Test Day & 28-Day & Test Day \\
\hline$f_{c}^{\prime}(\mathrm{MPa})$ & 31.6 & 32.5 & 39.2 & 38.3 \\
$\epsilon_{c}^{\prime}(\mathrm{mm} / \mathrm{mm})$ & 0.0011 & 0.0013 & 0.0013 & 0.0019 \\
$E_{c}(\mathrm{~mm} / \mathrm{mm})$ & 27.9 & 25.2 & 31.6 & 26.7 \\
\hline \hline
\end{tabular}

${ }^{a}$ Supporting block cast in same batch with Wall 1
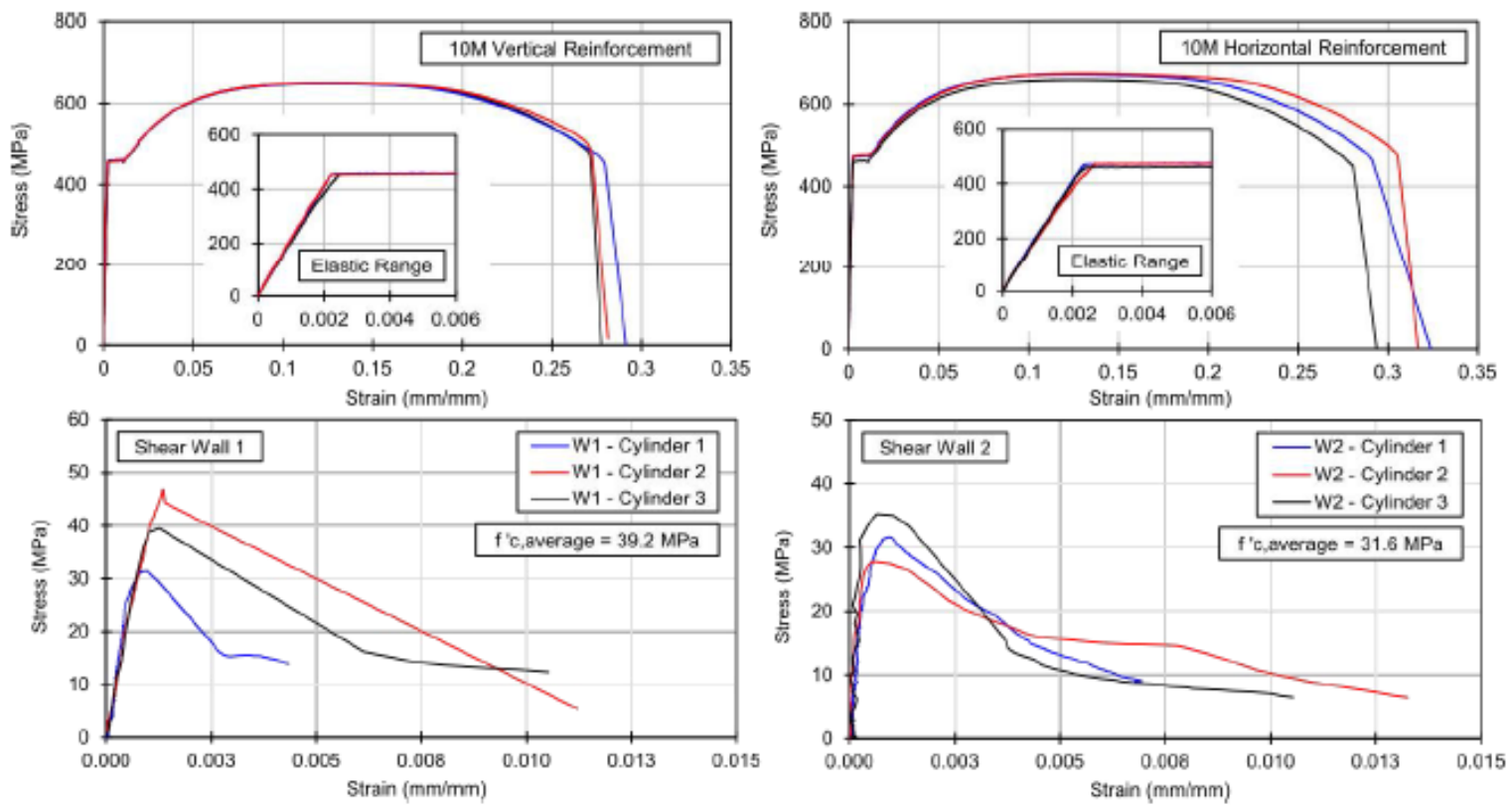

Figure 5.4 Material testing results: (a) concrete; (b) steel 


\subsection{Experimental Test Setup}

Figures 5.5 and 5.6 illustrate the experimental test setup in the Civil Engineering Structures laboratory at Carleton University. Each shear wall specimen has a heavily reinforced cap beam and foundation block. Three hydraulic actuators and a rigid steel loading beam are used to apply the lateral load, axial load, and overturning moment to the top of the wall specimen through the rigid loading beam. The rigid steel loading beam is affixed to the concrete cap beam at the top of the shear wall specimen. The heavily reinforced foundation block is used to secure the base of the shear wall specimen to the laboratory strong floor. To provide additional support and prevent sliding and rotation of the wall specimen during testing, a heavily reinforced reaction block is secured to the laboratory strong floor behind the foundation block. Under in-plane load, the RC shear wall panel slides through an out-of-plane support, identified in Fig. 5.6, and is designed to prevent out-of-plane displacement of the wall specimen and support the self-weight of the cap beam. The three hydraulic actuators are supported by large steel columns secured to the laboratory strong floor. The three actuators include two MTS system series 244.51 hydraulic actuators with $+/-1000 \mathrm{kN}$ (225 kip) capacity and a $+/-500 \mathrm{~mm}(+/-20 \mathrm{in}$.) stroke to control the axial load and overturning moment applied to the specimen. The lateral load is applied using an MTS series linear $243.60 \mathrm{~T}$ hydraulic actuator with a $+1000 \mathrm{kN}-650 \mathrm{kN}$ (225/146 kip) capacity and $+/-150 \mathrm{~mm}$ (6 in.) stroke. Figure 5.7 shows the final constructed shear wall hybrid test setup in the laboratory.

The steel column assembly with the replaceable steel link is designed such that it is tested in the same experimental test setup as the shear wall specimens. Figures 5.8 and 5.9 illustrate the test setup for the steel column assembly and Fig. 5.10 shows the steel column assembly as constructed in the laboratory. To replicate the same load conditions at the top of the steel column assembly when compared to the RC shear wall specimens, the top section of the steel column is connected to a concrete cap beam with the same geometry as those for the wall specimens. The steel column assembly connects directly to the heavily reinforced reaction block at its base, which is shifted 


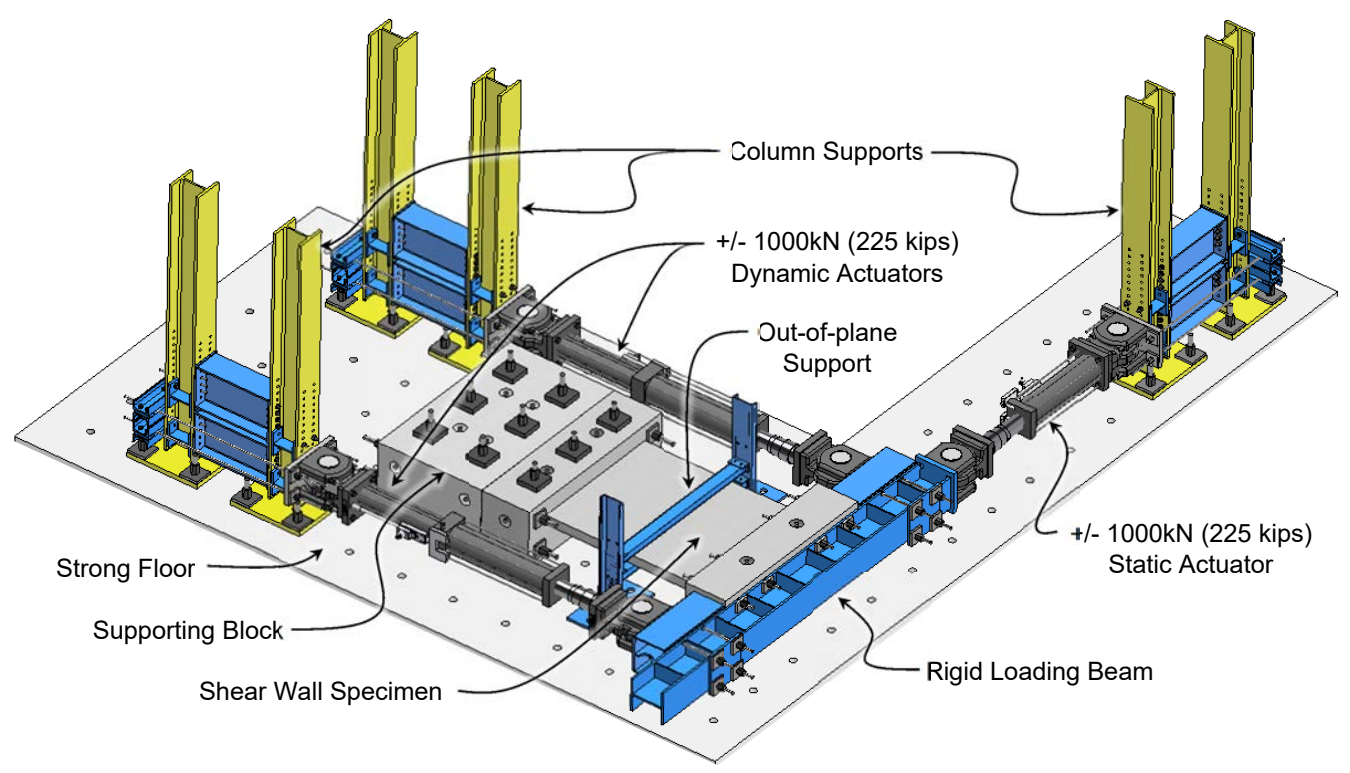

Figure 5.5 3D Experimental test setup for shear wall hybrid simulation

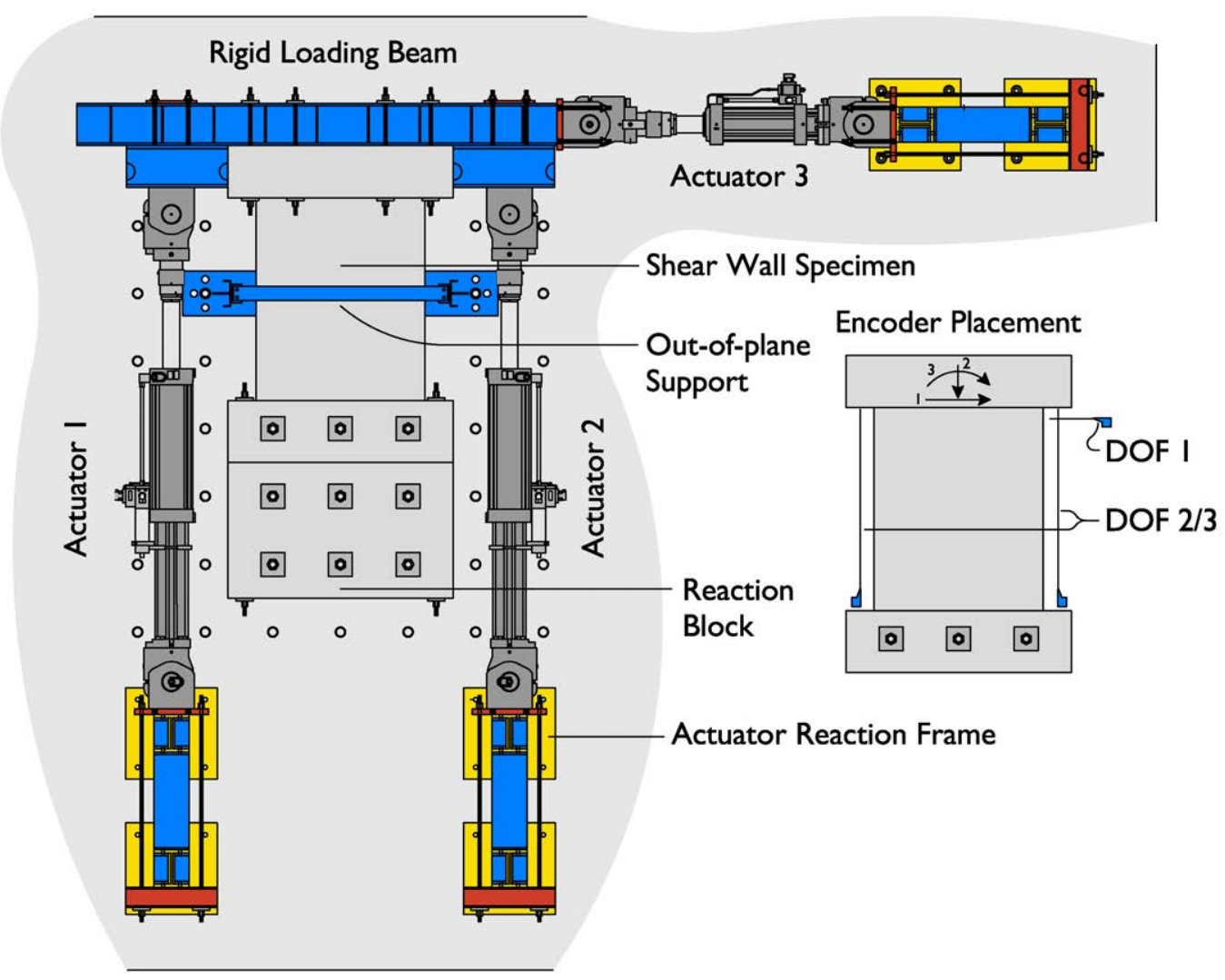

Figure 5.6 2D Experimental test setup for shear wall hybrid simulation 


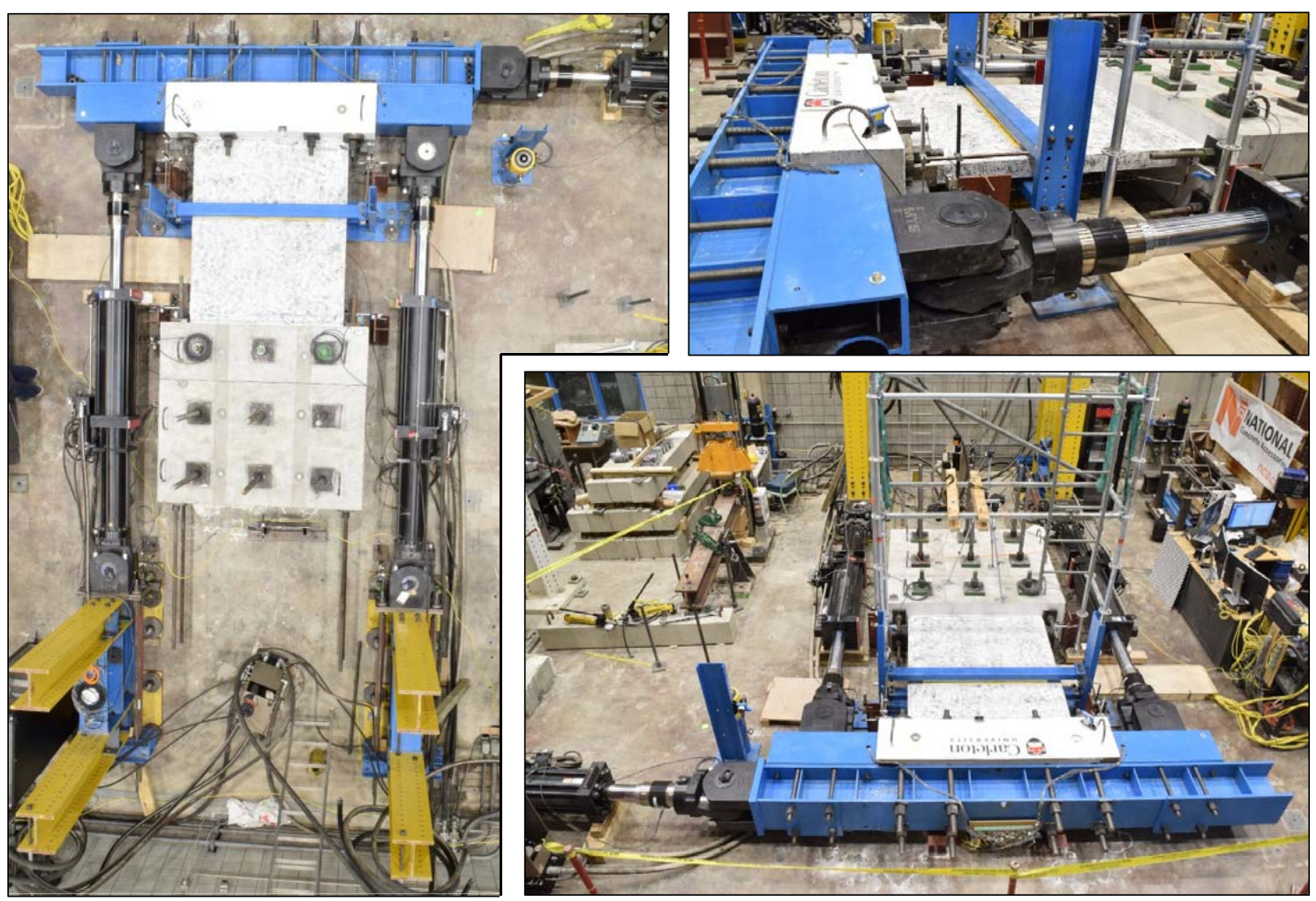

Figure 5.7 Shear wall hybrid test setup in the laboratory 
up to accommodate for the shorter length of the steel column assembly. The same restraint for out-of-plane displacement and rotation used in the shear wall test is used to prevent out-of-plane deformation of the steel column assembly.

\subsubsection{Hydraulic Control and Data Acquisition}

The actuators are controlled in the laboratory using an MTS FlexTest 100 controller. To facilitate the hybrid test, MTS computer simulation interface (MTS Csi), which runs inside the MTS 793 control software, is used to couple the numerical and experimental subassemblies in the laboratory. MTS Csi provides a programming interface to users to connect an MTS controller and communicate with MTS hardware through the MTS CSi application programming interface (API) (Schellenberg et al., 2009). MTS Csi maps specific DOFs in the hybrid model to specific control channels in MTS 793 and has a built-in predictor corrector. Figure 5.11 shows the architecture for each of the components in the hybrid simulation setup at Carleton University. The hybrid simulation setup includes the host computer, predictor/corrector host computer (optional), MTS controller, hydraulic actuators and test specimen. The predictor-corrector host computer is required for real-time hybrid simulation, which ensures that the correct displacement command is sent to the controller every $1 \mathrm{~ms}$ (the controller acquisition rate). This component of the hybrid simulation framework is bypassed in this study because the hybrid tests are not conducted in real-time, and the aforementioned MTS CSi built-in predictor-corrector is used instead. The responsibilities of each component in the hybrid tests architecture are described in more detail below:

1. The numerical analysis host computer is responsible for running the numerical simulation in OpenSees and OpenFresco;

2. If the hybrid simulation is pseudo-static, MTS CSi sends the command displacement(s) directly to MTS793 and then the MTS FlexTest controller in the laboratory which then sends the appropriate voltage(s) to the actuator servo-valve;

Joshua Woods, Department of Civil and Environmental Engineering, Carleton University 


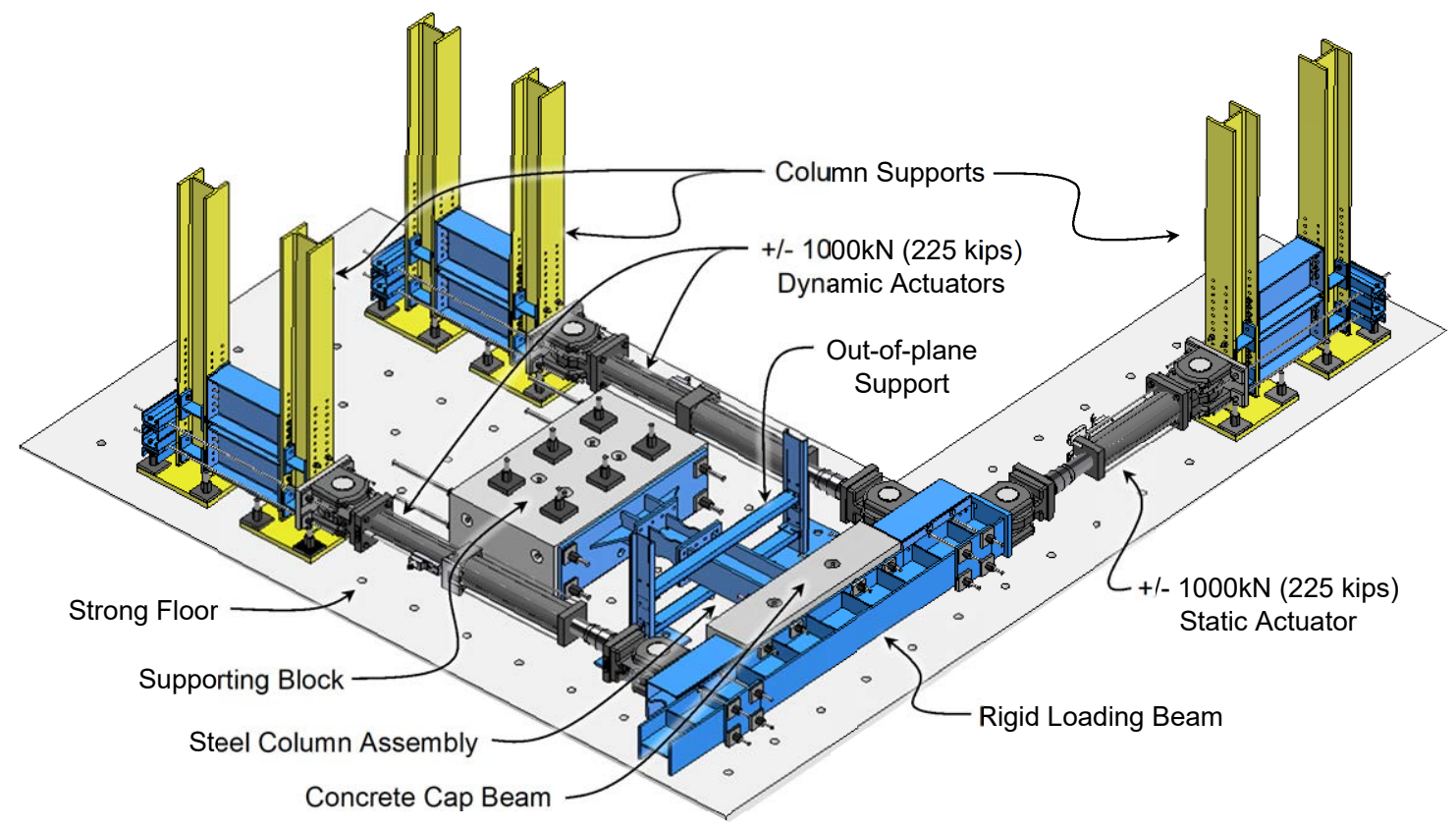

Figure 5.8 3D Experimental test setup for steel column assembly hybrid simulation

3. For real-time hybrid simulation, the numerical analysis host computer is connected to a predictor-corrector algorithm running in a Simulink model on another PC through a TCP/IP channel. The predictor/corrector host computer is responsible for running an algorithm that enables continuous hybrid testing by generating continuous command signals for the actuators regardless of delays in the OpenSees computation. This is critical for applications in real-time hybrid simulation. This means that after reaching the target displacement from OpenSees, the actuators will remain in motion by predicting the next displacement based on an interpolation of the previous target displacement (predictor mode). During this time, OpenSees is completing the computations for the next target displacement. When the integration is complete, the new target displacement is received and using polynomial integration, the algorithm corrects the path of the actuators in order to smoothly reach the target value at the end of the simulation time step (corrector mode). In the current laboratory configuration, the predictor/corrector model can be implemented externally on the predictor/corrector host computer in a simulink model; 


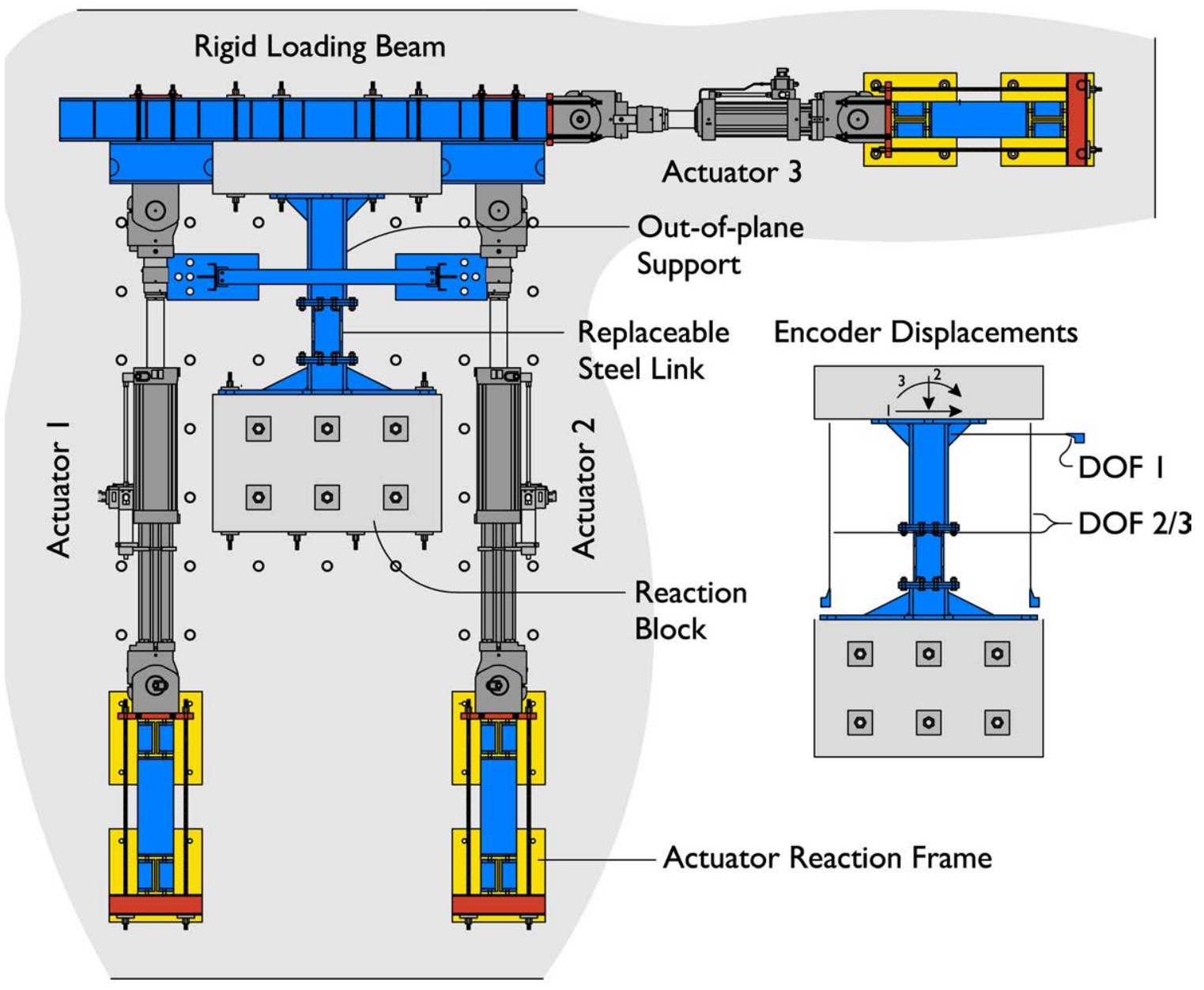

Figure 5.9 Steel column assembly experimental test setup illustration
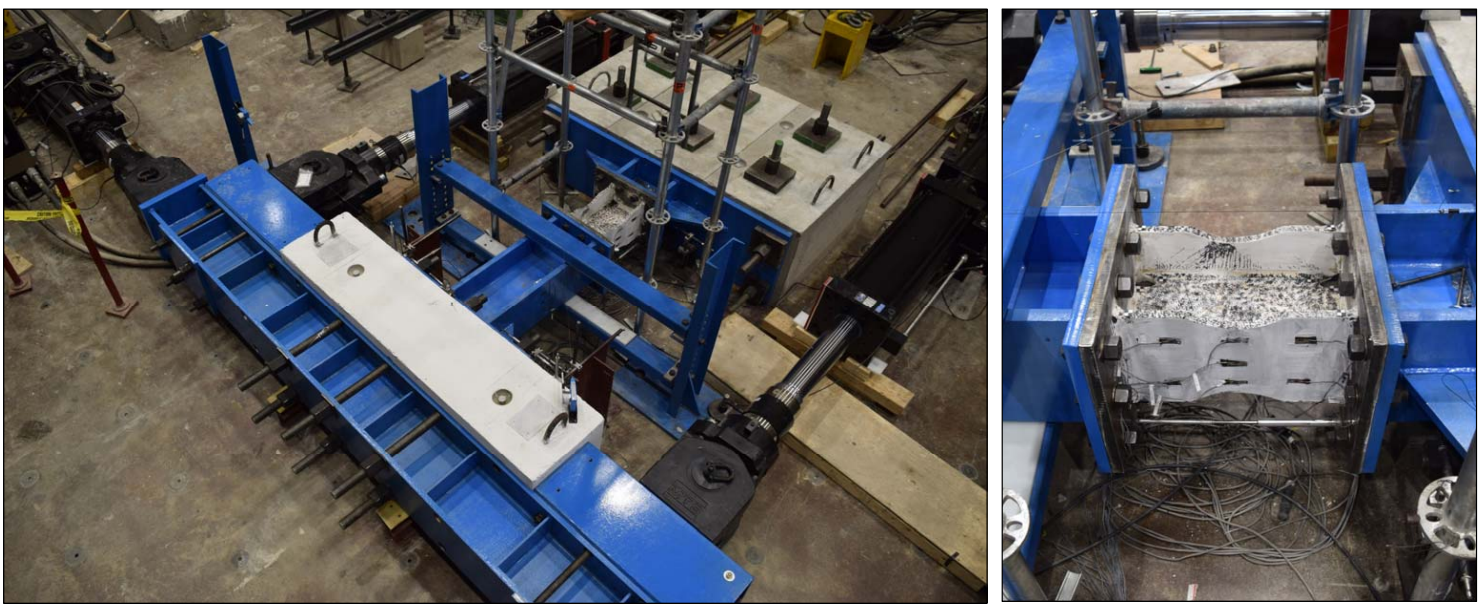

Figure 5.10 Steel column assembly experimental setup in the laboratory 
4. To communicate between the predictor-corrector host computer and the MTS controller for the actuators in a real-time hybrid simulation, a shared memory network is utilized, known as Scramnet. This is a fibre-optic communication link which sends command displacements to the hydraulic controller at the controller clock rate $(1024 \mathrm{~Hz})$;

5. After the command displacements are sent from the hydraulic controller to the hydraulic actuators, the actuator load cells and displacement transducers feed the restoring forces and displacements, referred to as feedback forces and displacements, back to the MTS controller and to the numerical analysis; and

6. This procedure is repeated until the numerical simulation for the full duration of the ground motion is complete.

While the actuator displacement and forces are monitored through the MTS Flextest 100 controller, two 32-channel MTS FlexDAC data acquisition systems are used to record all other signals during the test. Other signals include displacements from the LVDTs used to monitor the deformation of the experimental test specimen and strain gauge reading from the steel reinforcement. The sampling rate for the data acquisition system was set at $5 \mathrm{~Hz}$ for all tests. Data acquisition is executed in a separate application of MTS Multi-Purpose Elite (MPE), which runs parallel and independently from the hybrid simulation.

\subsubsection{High-Precision Encoders for Hybrid Simulation}

Hybrid simulation conducted on a stiff structural element, such as a RC shear wall, presents additional challenges related to hydraulic control and accurate acquisition of command and feedback displacements when compared with hybrid simulation conducted on a flexible experimental substructure. One of the primary challenges is the relationship between the stiffness of the experimental test setup and the stiffness of the experimental substructure. Ideally, the stiffness of the experimental test setup should significantly exceed the stiffness of the experimental element to ensure that the feedback displacement measured by the actuators is the deformation of the experimental specimen and not the deformation of the experimental test setup. Figure 5.12 illustrates 


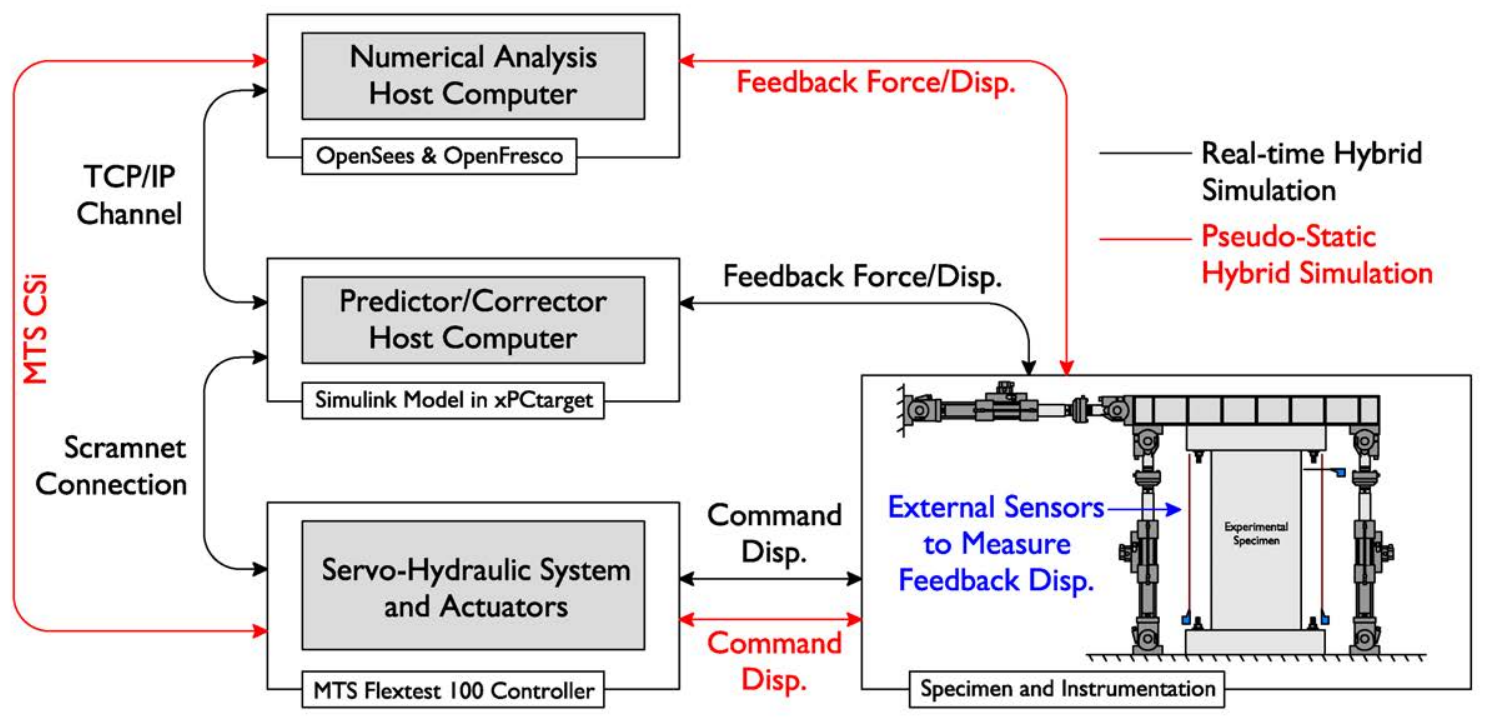

Figure 5.11 Architecture of the hybrid simulation setup at Carleton University

the potential deformation mechanisms of the experimental test setup in this study. It is illustrated that for a stiff RC element, such as a shear wall, it may be difficult or impossible to achieve a sufficiently stiff experimental test setup economically because of the very large axial and in-plane stiffness of wall specimen. A secondary challenge during hybrid testing on a stiff structural element is the accuracy, resolution, and repeatability of the sensor measuring the command and feedback displacements. The accuracy of command and feedback displacement measurement is particularly important for stiff structural elements because a small discrepancy between the command and feedback displacements, in the order of $1 e^{-1} \approx 1 e^{-2} \mathrm{~mm}$, typically corresponds to a large increment in force, which can affect the accuracy of the hybrid simulation results.

To overcome these challenges in this study, high-precision external sensors are used to accurately control the hybrid simulation and effectively bypass any deformation in the experimental test setup to directly measure the deformation of the test specimen. When this external control approach is combined with a high-precision sensor that is capable of accurately measuring very small displacements $1 e^{-1} \approx 1 e^{-2} \mathrm{~mm}$, the exact command and feedback displacement can be applied to the test specimen. Figure 5.11 illustrates how the high-precision external sensors can be integrated into the hybrid simulation control loop. 


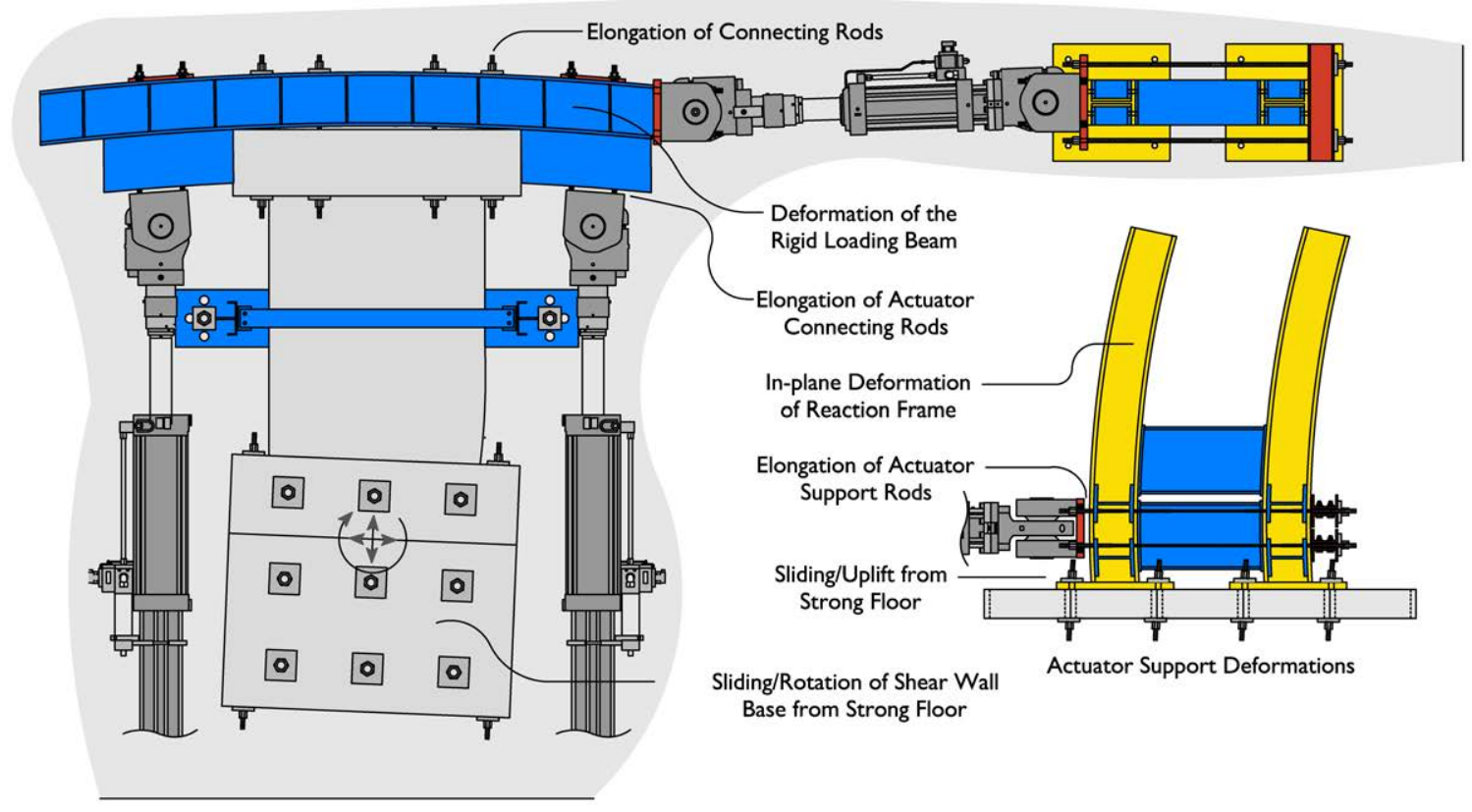

Figure 5.12 Potential deformation mechanisms in the experimental test setup

The displacement sensor used in this study is a Sick AFS/AFM60 SSI absolute encoder. This encoder commonly referred to as a rotary or shaft encoder that converts angular position to a digital signal by passing a light source through a translucent disk. When the light shines through the disk it creates a unique optical pattern that is processed using a detector array to determine the angle of the shaft. To convert the angular rotation of the shaft to displacement, a Sick MRA-G080-103D3 wire draw mechanism is connected to the shaft of the rotary encoder. The wire-draw mechanism spins the shaft on the encoder, which allows it to measure displacement. Figure 5.13 shows the absolute encoder and the wire-draw mechanism. The encoder and wiredraw combination capable of measuring 262,144 steps per shaft revolution, which corresponds to a theoretical displacement resolution of $0.00076 \mathrm{~mm}$ for $230 \mathrm{~mm}$ of wire draw per revolution. However, because of the inherent hysteresis in wire draw mechanisms as a result of slack in the wire-draw cable, the maximum achievable resolution of the encoder and wire-draw combination in this study was found to be $0.027 \mathrm{~mm}$. This proved to be precise enough to enable accurate control in the hybrid simulation. 

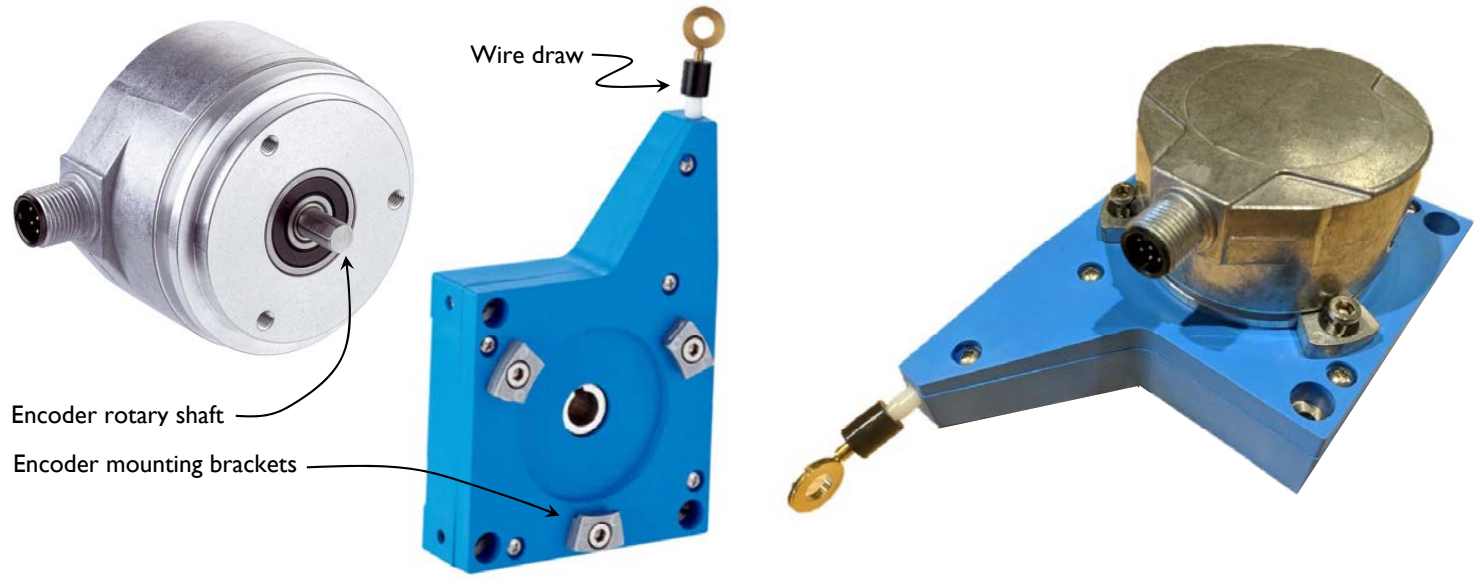

Figure 5.13 High-precision encoder components and assembled device

Prior to conducting a hybrid test using the high-precision encoders to control the hydraulic actuators in the laboratory, the control performance of the encoders is compared with the LVDT mounted inside the body of the hydraulic actuator in addition to a conventional wire-drawn displacement transducer, commonly referred to as a string-pot. Both the string-pot and the encoder were mounted to the body of the hydraulic actuator and a small ramp displacement-time history $(+/-50 \mathrm{~mm}, 2 \mathrm{~mm} / \mathrm{s})$ signal, shown in Fig. $5.14 \mathrm{a}$, was sent to the actuator. In the first test, the displacement command was sent to the actuator LVDT and the corresponding feedback displacements were measured from the string-pot and encoder devices. The test was then conducted two more times, using the external devices (encoder and string-pot) to control the actuator displacement. To quantify the performance of each device for hydraulic control, the absolute displacement error (absolute value of the difference between command and feedback displacements) was measured throughout each of the tests.

Figure 5.14b shows the absolute displacement error for the LVDT-controlled and encodercontrolled tests at a velocity of $2 \mathrm{~mm} / \mathrm{s}$. Note that the string pot is not shown at the $2 \mathrm{~mm} / \mathrm{s}$ velocity because of significant measurement noise in the results at low velocity. The results show an improvement in control and stability when using the high-precision encoders to control the actuator displacements compared to the internal actuator LVDT. The absolute displacement error at a velocity of $2 \mathrm{~mm} / \mathrm{s}$ using encoder control, which is comparable to the expected maximum 
velocity of the actuator during the shear wall hybrid simulation is below $0.1 \mathrm{~mm}$. The tests were also repeated at higher velocities of $4 \mathrm{~mm} / \mathrm{s}$ and $8 \mathrm{~mm} / \mathrm{s}$ to observe the impact, if at all, this would have on the encoder displacement. The results under varying velocity are shown in Fig. $5.14 \mathrm{c}$ and Fig. 5.14d. Test results at higher velocities clearly show the benefit of using the high-precision sensors for actuator control, as the absolute error in the encoder controlled tests is significantly lower when compared to the actuator LVDT. These results show promise for the use of encoders to control the hybrid simulation.

The results of the control test demonstrate the effectiveness of using a high-precision encoder to send and receive displacements for the hybrid simulation. The use of the encoder reduces the displacement error significantly when compared to the actuator LVDT and a conventional string-pot displacement transducer. Figures 5.6 and 5.9 shows the placement of the encoders in the experimental test setup. The encoders are used to measure the axial displacement, rotation, and lateral displacement of the shear wall and steel column test specimens. By mounting two encoders vertically between the concrete foundation block and concrete cap beam, any deformation of the rigid loading beam or associated components (illustrated in Fig. 5.12) are effectively bypassed. Additionally, the placement an encoder horizontally at the top of the test specimen allows for direct measurement of the displacement at the top of the shear wall or steel column, as opposed to the displacement at the location of the actuator applying the lateral load, which could introduce an error in the hybrid simulation because the measured actuator displacement would be larger than the actual displacement at the top of the test specimen.

\subsubsection{MTS DOF Control Software}

During hybrid test rehearsals conducted using the developed OpenSees-OpenFresco hybrid models, it became evident that there were incompatibilities between the current OpenSees and OpenFresco software platforms. As a result, the proposed ThreeActuatorSetup experimental setup in OpenFresco did not function properly when attempting to transform command and feedback signals between global and actuator coordinate systems (illustrated in Fig. 4.15). To overcome this challenge, MTS DOF Control software was used to transform the command and feedback 


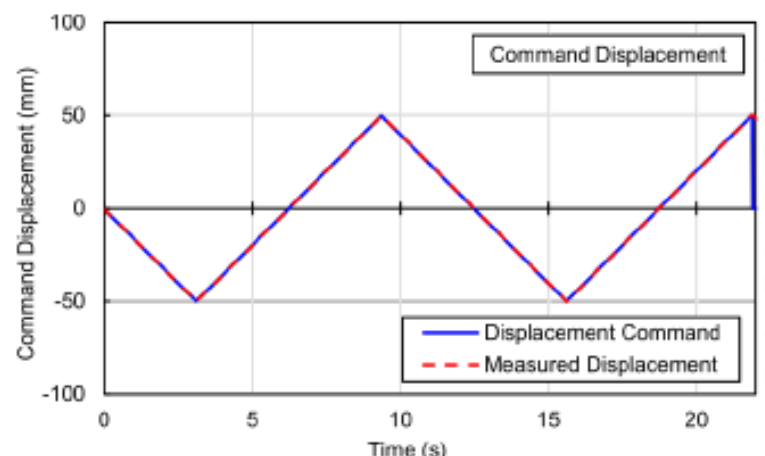

(a)

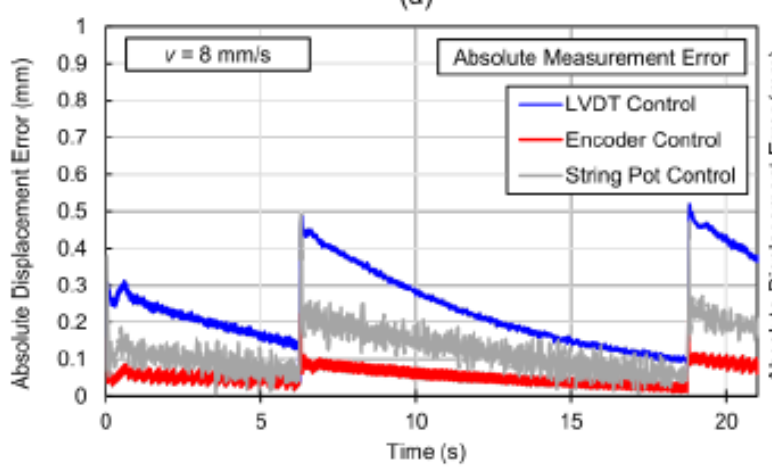

(c)

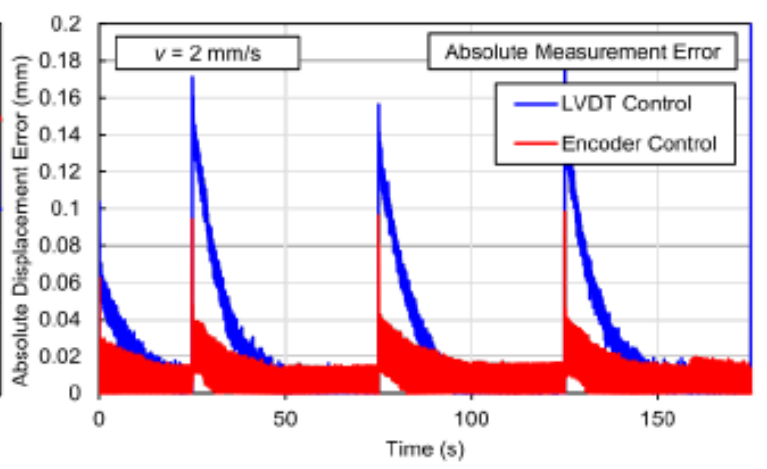

(b)

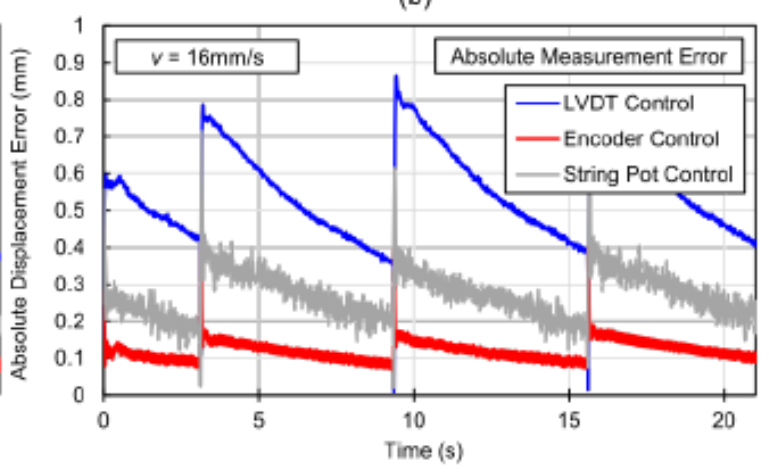

(d)

Figure 5.14 (a) Command displacement; absolute displacement error: (b) $2 \mathrm{~mm} / \mathrm{s}$; (c) $8 \mathrm{~mm} / \mathrm{s}$; (d) $16 \mathrm{~mm} / \mathrm{s}$ 
signals between global and actuator coordinate systems. This control software has been traditionally used in applications for multi-actuator control in shaking tables (e.g. hexapod shake table configuration). The MTS DOF control software is able to control any number of actuators and perform linear or nonlinear (large displacements) transformations to control up to six degrees-offreedom, even in over-constrained systems that have a larger number of actuators compared with the number of degrees-of-freedom.

In the proposed hybrid simulation test setup, three actuators are used to control the three DOFs (axial and lateral displacements $(\mathrm{X} \& \mathrm{Y})$ and rotation $(\mathrm{RZ})$ at the top of of the test specimen. The MTS Csi control program sends and receives the scaled displacements and rotation from OpenFresco and sends them to the appropriate control channels in MTS 793. The MTS DOF Control software is used to create control channels based on the geometry of multiple actuators (length) and their 3D locations (start and end positions). This allows the user to control multiple actuators together simultaneously based on one (or more) global coordinate system(s). Figure 5.15 shows the configuration of the actuators in the MTS DOF Control software for the current experimental test setup. Using this approach, control is no longer based on individual actuator command signals, but on simultaneous signals sent to each actuator to control the $\mathrm{X}$ and $\mathrm{Y}$ displacements and $\mathrm{Z}$ rotations of the test setup. In addition, because the hybrid simulation is controlled using external transducers located in different positions when compared with the hydraulic actuators, a second coordinate system and transformation based on the encoder geometry is required and is shown in Fig. 5.15. In the hybrid simulation, the displacement commands are based on the encoder transformation, while feedback forces are based on the actuator transformation because the load cells are mounted at the ends of the actuators. Because new control channels are established, system level proportional, integral, and derivative (PID) tuning was required prior to conducting the hybrid tests to ensure adequate control of the system could be achieved in each of the three primary directions (X, Y, \& RZ). Figure 5.16 compares command and achieved displacements and rotations in the $\mathrm{X}, \mathrm{Y}$, and $\mathrm{Z}$ directions following system tuning using the both actuator and encoder control and Table 5.3 shows the PID control parameters in $\mathrm{X}, \mathrm{Y}$, and $\mathrm{Z}$ directions for actuator and encoder control, respectively. The results demonstrate 
good control performance of the system, with minimal overshoot and oscillating responds.

\subsubsection{Instrumentation}

To measure the deformation of the test specimens during the hybrid tests, including shear and flexural deformations, a series of linear variable differential transformers (LVDTs) are mounted on the experimental test setup and test specimens. Figure 5.17 shows the locations of the LVDTs for the steel column hybrid test as well as the locations of the strain gauges mounted on the steel column test specimen. In the steel column hybrid test, LVDTs are used to measure the in and out-of-plane deformations as well as the rotations of the link element. Vertically oriented strain gauges are mounted on the upper column section to monitor the axial load after yielding of the steel link and a series of strain gauges are mounted on the flange and web of the steel link to monitor the strain profiles throughout the RBS section during the test.

Figure 5.18 shows the locations of the LVDTs for the shear wall hybrid tests and the locations of the strain gauges on the shear wall steel reinforcement. In the RC shear wall tests, LVDTs are placed over the height of the wall to measure the in-plane displacements of the wall specimen, while LVDTs located on the top cap beam and foundation block measure any out-of-plane deformation or relative slip between the foundation block and the laboratory strong floor. Diagonally mounted LVDTs mounted on the face of the wall specimen measure the shear deformation. Electrical strain gauges are used to monitor any yielding of the vertical or horizontal steel reinforcement throughout the test. More detailed instrumentation drawings are available for the steel

Table 5.3 MTS 793 PID tuning parameters for 3-DOF station setup

\begin{tabular}{lcccccc}
\hline \hline & \multicolumn{2}{c}{$\underline{\text { X-Dir }}$} & \multicolumn{2}{c}{$\underline{\text { Y-Dir }}$} & \multicolumn{2}{c}{$\underline{\text { RZ-Dir }}$} \\
Parameter & Actuator & Encoder & Actuator & Encoder & Actuator & Encoder \\
\hline P Gain & 4.00 & 3.20 & 4.00 & 4.10 & 3.00 & 3.00 \\
I Gain & 0.20 & 0.30 & 0.10 & 0.30 & 0.10 & 0.10 \\
D Gain & 0.08 & 0.08 & 0.00 & 0.00 & 0.00 & 0.00 \\
\hline \hline
\end{tabular}

Joshua Woods, Department of Civil and Environmental Engineering, Carleton University 


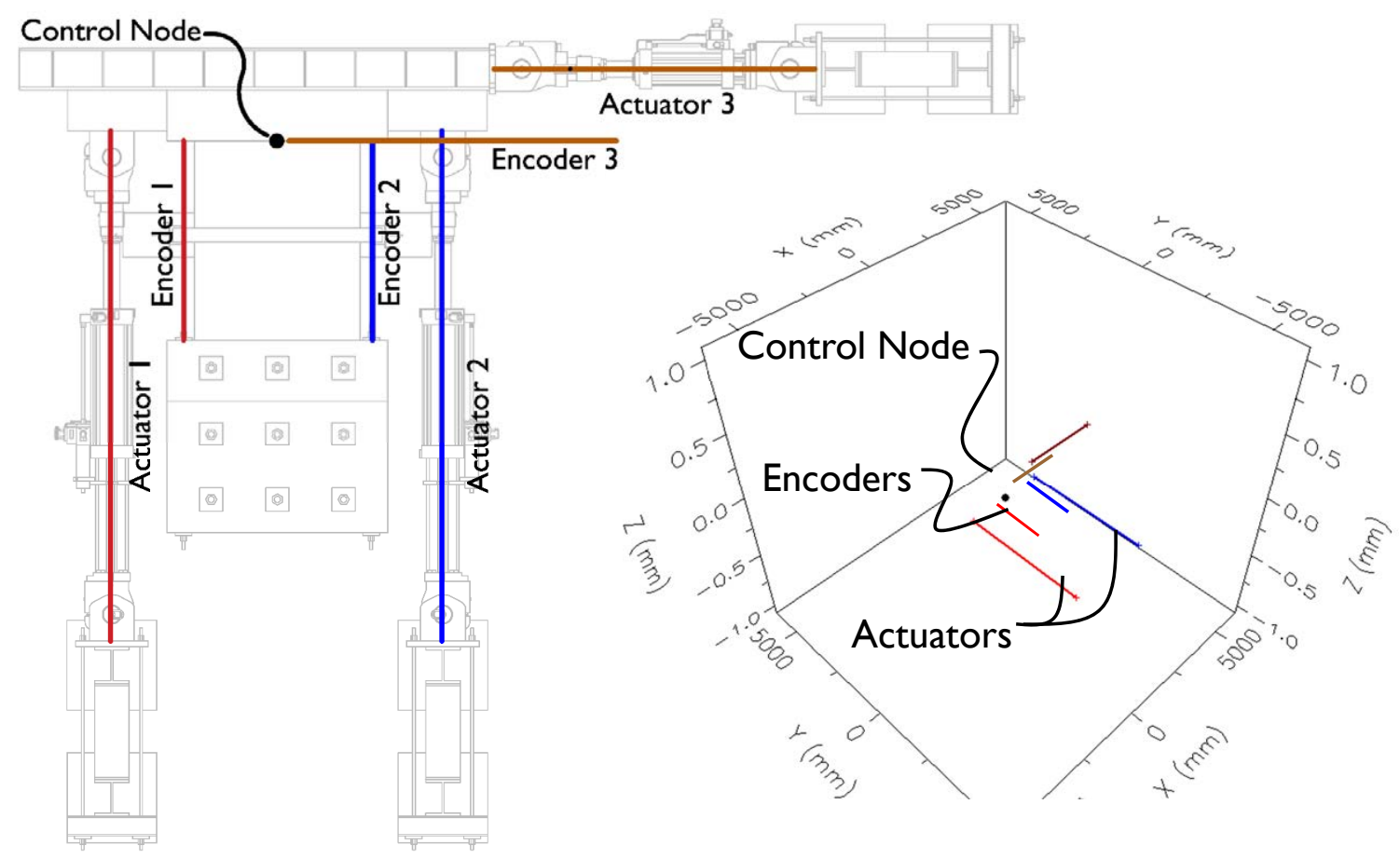

Figure 5.15 3D actuator configuration in MTS DOF control software

column and shear wall specimens in Appendix C.

In addition to traditional instrumentation, image analysis, sometimes referred to as digital image correlation, is used to monitor crack distributions in the surface of the concrete and track strain fields in the plastic hinge region of the wall specimen during the hybrid tests. The images are processed using a freely available image analysis tool called ImPro Stereo, developed by Yang et al. (2014) at the National Taipei University of Technology (NTUT). Additional background information on the development and applications of the ImPro Stereo software are available in Yang et al. (2012, 2015, 2018).

Figure 5.19 shows a typical test setup for the image correlation technique. Before a test is conducted, the measurement region or region of interest $(\mathrm{ROI})$ is painted with a speckle pattern to create a surface with a unique pattern. This pattern ensures the software is able to detect deformation of the test specimen. Two cameras are mounted above the ROI and are calibrated to remove the effects of perspective and lens distortion. The cameras are then used to capture the state of test specimen at many instances throughout the experiment. Following the test, the 

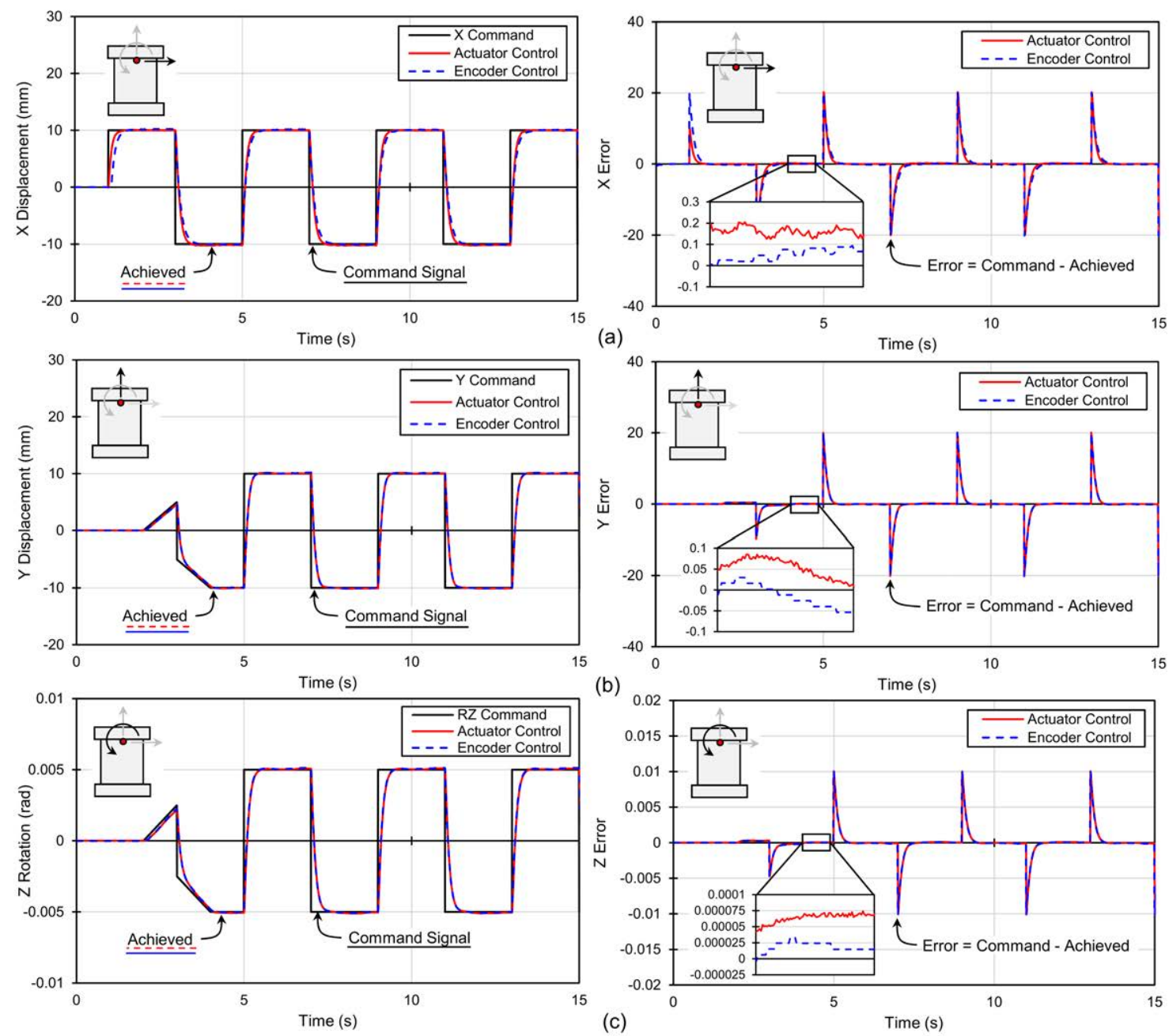

Figure 5.16 Displacement command/feedback and error: (a) X; (b) Y; and (c) RZ 


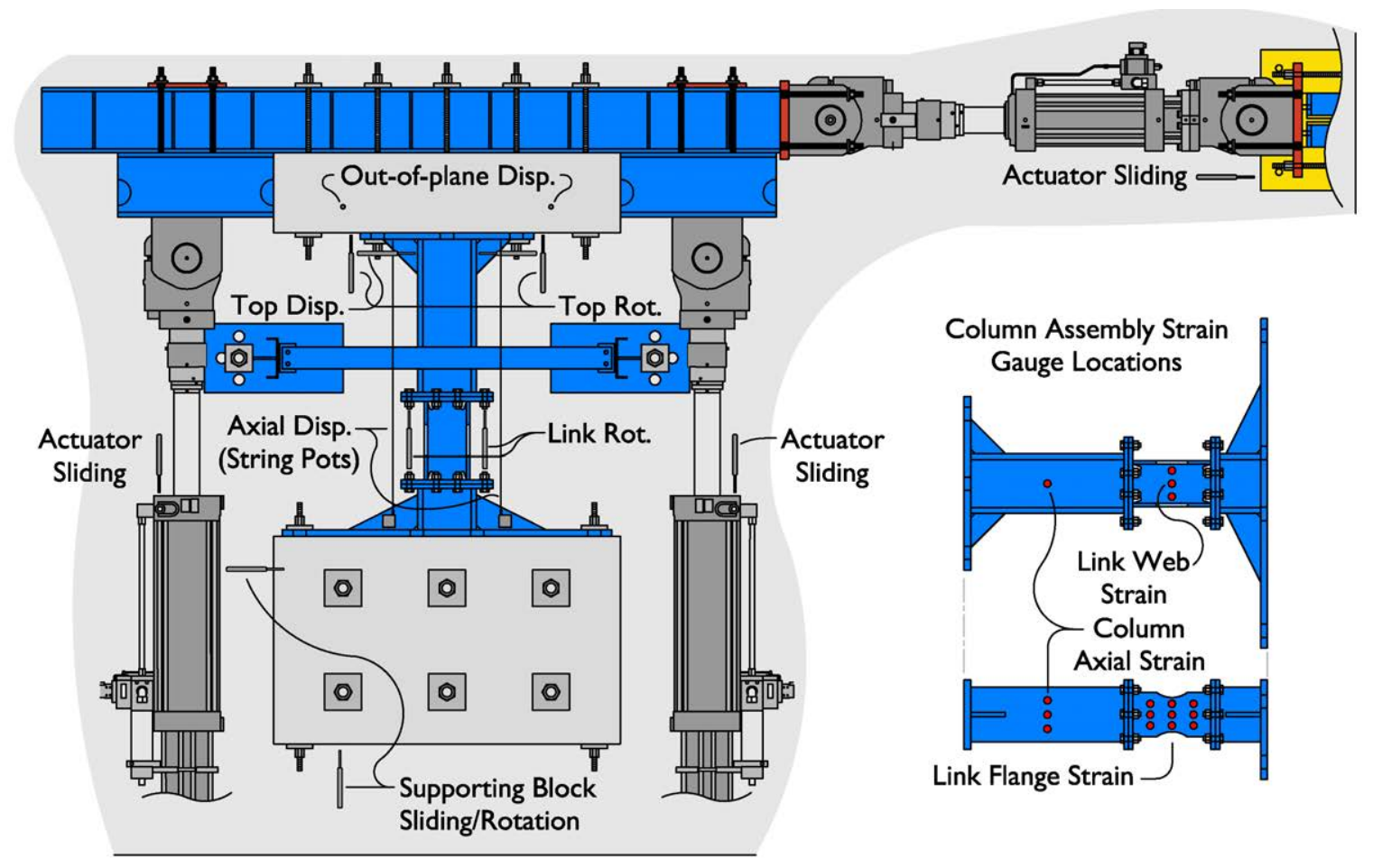

Figure 5.17 LVDT and strain gauge locations for steel column hybrid test

high-definition images are analyzed with respect to the initial undeformed specimen. The ImPro Stereo software is capable of determining the surface displacement, strain fields, and concrete crack distributions throughout the test with sub-pixel accuracy; typically 0.05 pixels or smaller with adequate image quality.

The other benefits of using image analysis technology include its ability to be used in continuous testing. Traditionally, cracks in a concrete test specimen during a component test would be marked manually; by temporarily stopping the test and marking the cracks on the surface of the element using a marker. Although this technique is simple and effective in a traditional static test; during a hybrid simulation there is no opportunity to pause the test, making it difficult to mark and measure cracks on the surface of a concrete specimen. Alternatively, the image analysis technique has the ability to capture the crack distributions throughout the test without stopping and at a higher degree of accuracy when compared to visual measurement techniques. Past experiments conducted at Carleton University have demonstrated the effectiveness of using the image correlation technique in static-cyclic tests of RC shear walls (Woods and Lau, 2016). Additional 


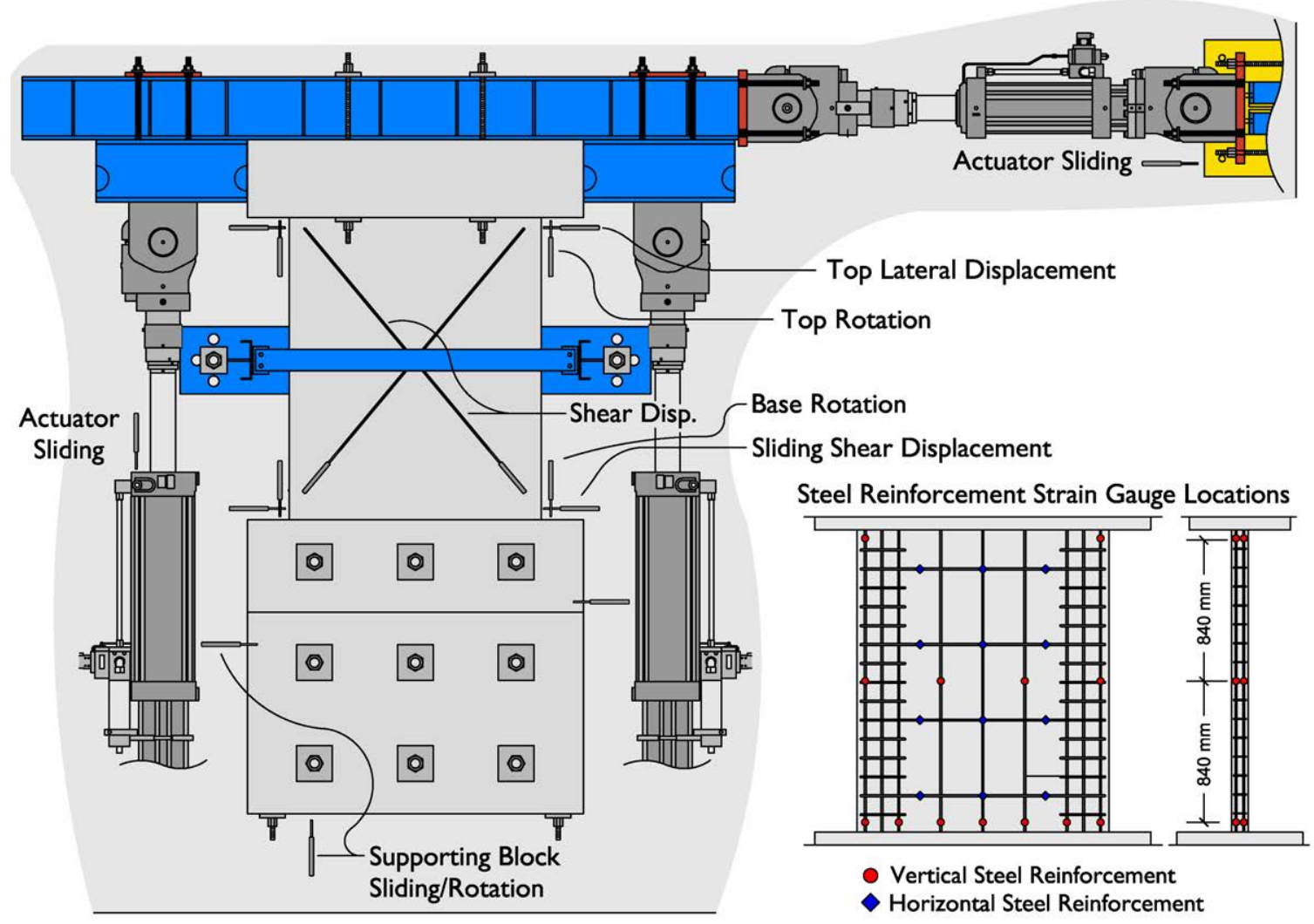

Figure 5.18 LVDT and strain gauge locations for RC shear wall hybrid test 
details on the image analysis techniques are available in publications by Yang et al. $(2012,2015)$ and are not discussed in detail here.

Finally, Figure 5.20 shows the hybrid testing station used to run the hybrid tests in the Civil Engineering Structures lab at Carleton University. The system consists of three stations, responsible for the hydraulic control, data acquisition, and image capture during the hybrid tests. The first station runs the MTS 793 control software, MTS Csi computer simulation interface, and MTS DOF control software, which is an integrated module inside of MTS 793. The first test station communicates directly with the MTS FlexTest 100 controller in the lab. The second station is responsible for running the OpenSees-OpenFresco hybrid model, and collecting data from the MTS FlexDAC units through the MPE software. The third test station is responsible for image acquisition for digital image correlation. The station triggers and stores the images from two DSLR cameras mounted on the test setup at $30 \mathrm{~s}$ intervals throughout each hybrid test.

\subsubsection{Applied Earthquake Ground Motions}

The goal of conducting a hybrid test on a stiff structural element such as a RC shear wall is to eliminate the dependence on large-scale shaking table tests and provide data on the overall system-level performance of these types of structures during an earthquake. In this study, the applied earthquake ground motion records are scaled to different performance levels and applied

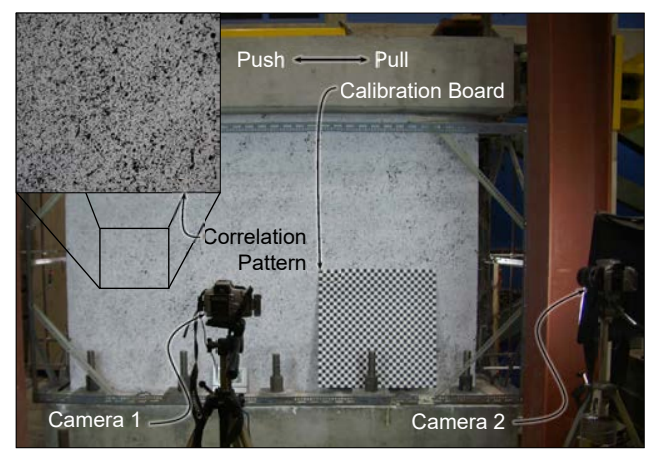

(a)
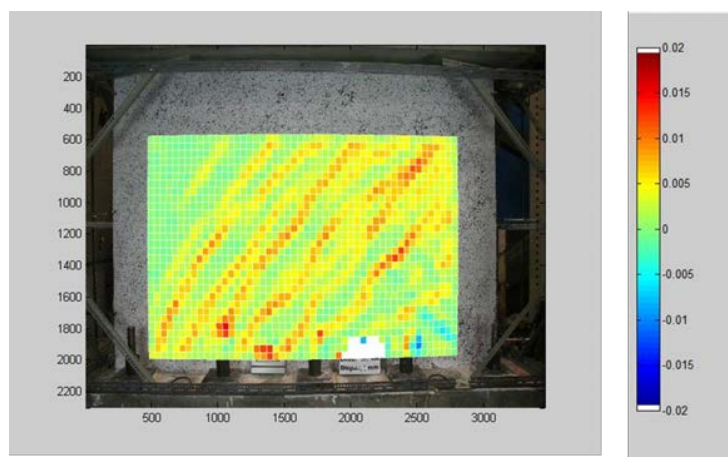

(b)

Figure 5.19 Image correlation: (a) setup; (b) result (adapted from Woods and Lau, 2016) 


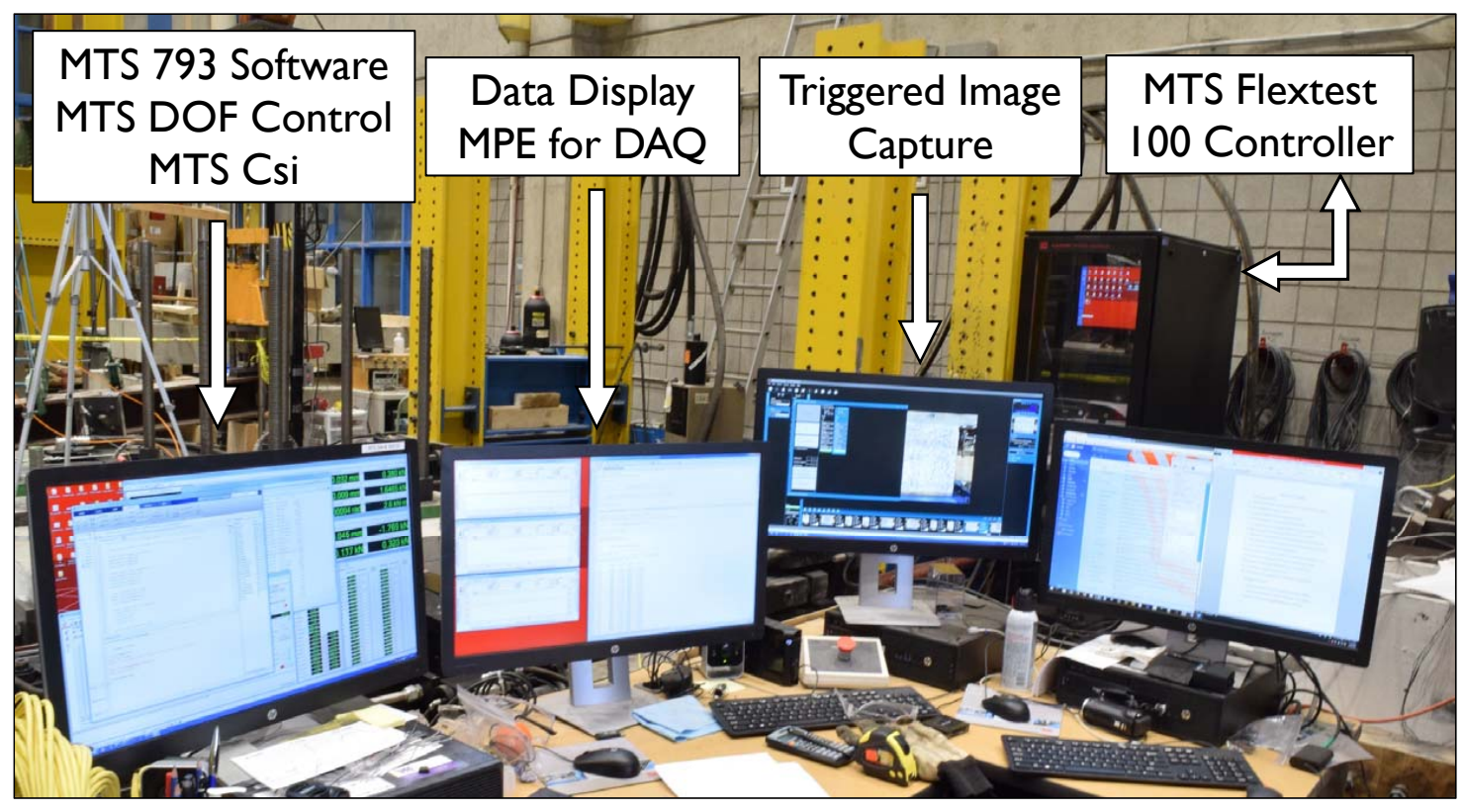

Figure 5.20 Hybrid testing station at Carleton University

to the shear wall specimens to study their performance at different hazard levels. In addition, the application of multiple ground motion records at different intensities to the same shear wall test specimen will be used to assess the influence of multiple earthquake aftershock events on a multistorey shear wall. All of the unscaled ground motion records used in this study were obtained from the NGA West PEER Ground Motion Database (Ancheta et al., 2013).

Figure 5.21 shows the ground motion acceleration-time histories for the earthquake records chosen for the hybrid tests. Although the selected records are not from the site of the prototype structure in Victoria BC; Atkinson (2005) has shown that crustal earthquake ground motions from California are suitable for the B.C. region. Because of the lack of previous hybrid tests on RC shear walls, there is no standard on the scaling or sequence of ground motions that should be used in a hybrid test. However, because the goal of this study is to use hybrid simulation to mimic shaking table results, the same scaling and ground motion selection techniques traditionally used in shaking table tests are employed here. In the past, a number of shaking table tests have been conducted on RC shear walls. Based on a review of recent shake table studies shown in Table 5.4, the large majority of shaking table tests select an unscaled earthquake record and apply the record 
at increasing intensities until failure of the structure. Based on the list of ground motion records used during past studies, the Canoga Park Station record from the 1994 Northridge earthquake in California was ultimately selected for the majority of the shear wall hybrid tests. Two other records, namely, the Gilroy Array \#5 record from the 1989 Loma Prieta earthquake in California and the JR Takatori record from the 1995 Kobe earthquake in Japan were also selected to study the influence of the ground motion record on the response of the structure in the second hybrid shear wall test.

The selected ground motion records were applied in increasing magnitude and compared with traditional earthquake intensity measurements. The commonly used intensity measures include:

- Operational Based Earthquake (OBE) (50\% in 50 years): is a service level motion, is expected to occur frequently over the service life of the structure and the structure is designed to remain elastic throughout;

- Design Based Earthquake (DBE) (10\% in 50 years): is a design earthquake in which the structure is expected to exhibit some nonlinearity, including the potential for crushing of the concrete near the base of the wall and some yielding of the vertical steel reinforcement near the edges of the wall; and

- Maximum Considered Earthquake (MCE) ( $2 \%$ in 50 years): is a severe earthquake in which the structure is expected to sustain significant damage; including crushing of the concrete at the edges of the wall and significant yielding throughout the vertical steel reinforcement
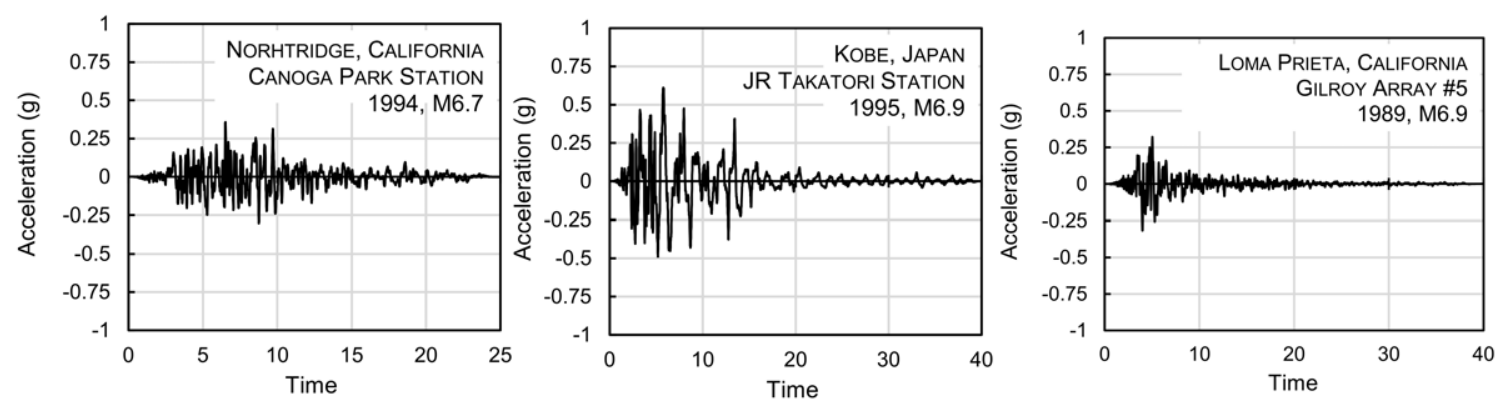

Figure 5.21 Earthquake records: (a) Northridge (1994); (b) Kobe (1995); (c) Loma Prieta (1989) 


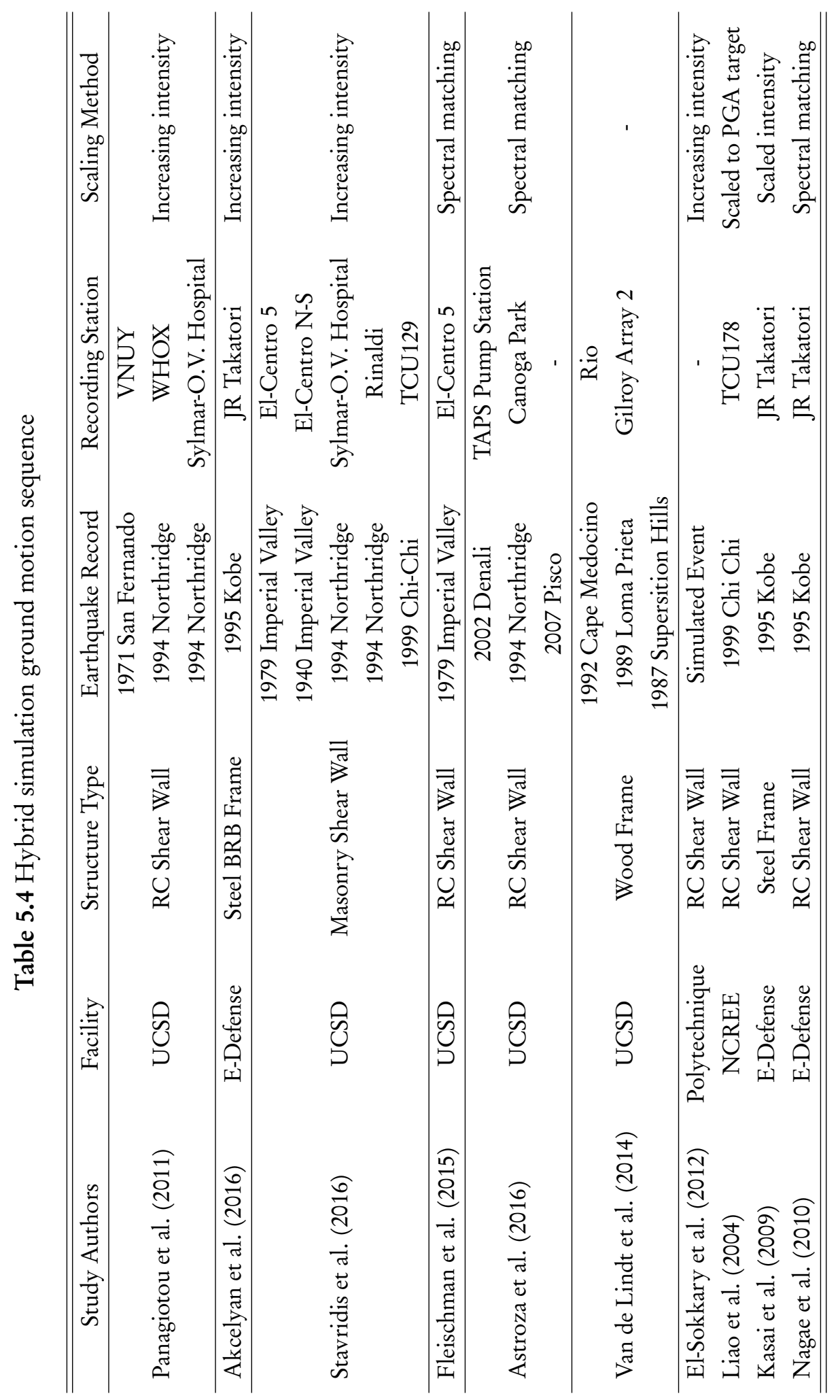


at the base of the wall but collapse is prevented.

For the hybrid tests, scale factors were applied to the Northridge Canoga Park station raw earthquake record to obtain specific behaviours at each of the three hazard levels. In conjunction with recommendations from Whyte and Stojadinovic (2013b), the OBE level earthquake is scaled so it would result in one-third of the yield force on the wall. The DBE level earthquake is scaled to reach the full yield force of the wall and the MCE level earthquake is scaled to three times larger than the DBE event, which is expected to allow the wall to reach post-peak behaviour, however, it may be more common to scale the $\mathrm{DBE}$ event by 1.5 (MCE $\approx 3 / 2 \mathrm{DBE}$ ), which is more common in most design standards, such as ASCE 7-10 ASCE (2013). Table 5.5 shows the order of ground motion records and scaling factors for those motions for each wall specimen. The first wall specimen is first subject to two OBE level motions, followed by a DBE event and two MCE level earthquakes designed to fail the shear wall specimen. Figure 5.22 shows the ground motion sequence for the first series of hybrid tests and the response spectra for earthquake ground motion record. Results from past studies by Whyte and Stojadinovic (2013b) on RC shear walls have shown that significantly different sequences of ground motion intensities (e.g. MCE before the initial DBE motion for the sequence in this test) do not have a significant effect on the failure mechanisms observed during the test. This suggests that the selection of the ground motion sequence is not critical to capture the dominant behaviour and failure mechanism of the test specimen.

Figure 5.23 shows the ground motion sequence for the second series of hybrid tests and the response spectra for earthquake ground motion record. For the second shear wall specimen, the same initial earthquake sequence is applied (OBE, DBE, MCE). This will allow for comparison between the behaviour of the two hybrid test specimens with different analytical substructures (elastic vs. nonlinear finite elements). It is anticipated that as a result of the nonlinear behaviour of the analytical substructure, the demand on the experimental specimen in the lab will be smaller. Therefore, three additional earthquake events will be applied depending on the level of damage 
Table 5.5 Hybrid simulation earthquake ground motion sequence

\begin{tabular}{ccccccc}
\hline \hline $\begin{array}{c}\text { Test } \\
\text { Specimen }\end{array}$ & $\begin{array}{c}\text { Earthquake } \\
\text { Record }\end{array}$ & $\begin{array}{c}\text { Station } \\
\text { Name }\end{array}$ & $\begin{array}{c}\text { Duration } \\
(s)\end{array}$ & $\begin{array}{c}\text { Hazard } \\
\text { Level }\end{array}$ & $\begin{array}{c}\text { Scale } \\
\text { Factor }\end{array}$ & $\begin{array}{c}\text { PGA } \\
(g)\end{array}$ \\
\hline \multirow{6}{*}{ W1 } & Northridge (1994) & Canoga Park & 25 & OBE & 0.20 & 0.07 \\
& Northridge (1994) & Canoga Park & 25 & OBE & 0.50 & 0.18 \\
& Northridge (1994) & Canoga Park & $25.0 s$ & DBE & 1.00 & 0.36 \\
& Northridge (1994) & Canoga Park & 25 & MCE & 2.00 & 0.72 \\
& Northridge (1994) & Canoga Park & 25 & MCE & 3.00 & 1.07 \\
\hline \multirow{6}{*}{ W2 } & Northridge (1994) & Canoga Park & 25 & OBE & 0.20 & 0.07 \\
& Northridge (1994) & Canoga Park & 25 & DBE & 1.00 & 0.36 \\
& Northridge (1994) & Canoga Park & 25 & MCE & 2.00 & 0.72 \\
& Northridge (1994) & Canoga Park & 25 & MCE & 3.00 & 1.07 \\
& Northridge (1994) & Canoga Park & 25 & DBE & 1.00 & 0.36 \\
& Kobe (1995) & JR Takatori & 40 & DBE & 0.50 & 0.50 \\
& Loma Prieta (1989) & & & \\
& Gilroy Array \#5 & 40 & MCE & 1.50 & 0.45 \\
\hline \hline
\end{tabular}

${ }^{a}$ Application depends on assessment of damage to wall specimen following previous tests

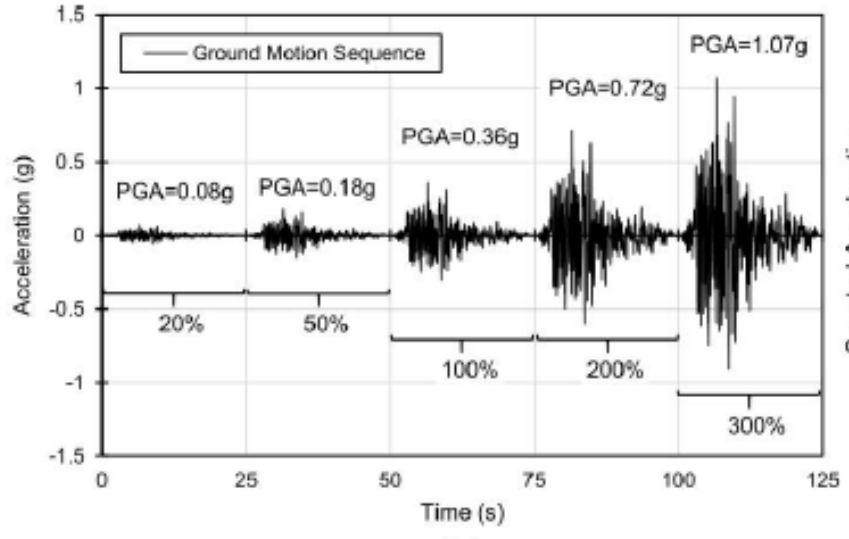

(a)

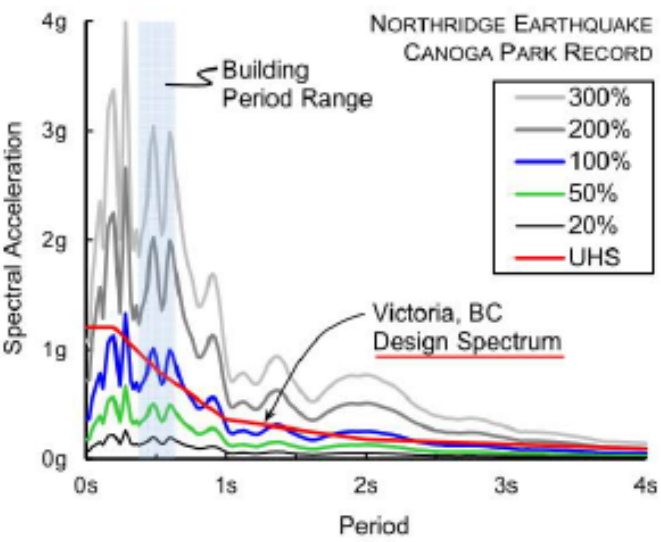

(b)

Figure 5.22 W1 hybrid test: (a) earthquake ground motion sequence; (b) response spectra 
to the shear wall specimens. This includes the same Northridge Canoga Park Station ground motion record at the DBE level, representing a potential aftershock event, followed by two different ground motion records at the DBE level to study the response of the shear wall under ground motions with different frequency content and duration. The application of these ground motions will allow for further comparisons between hybrid test results and additional assessment of the hybrid test system. 

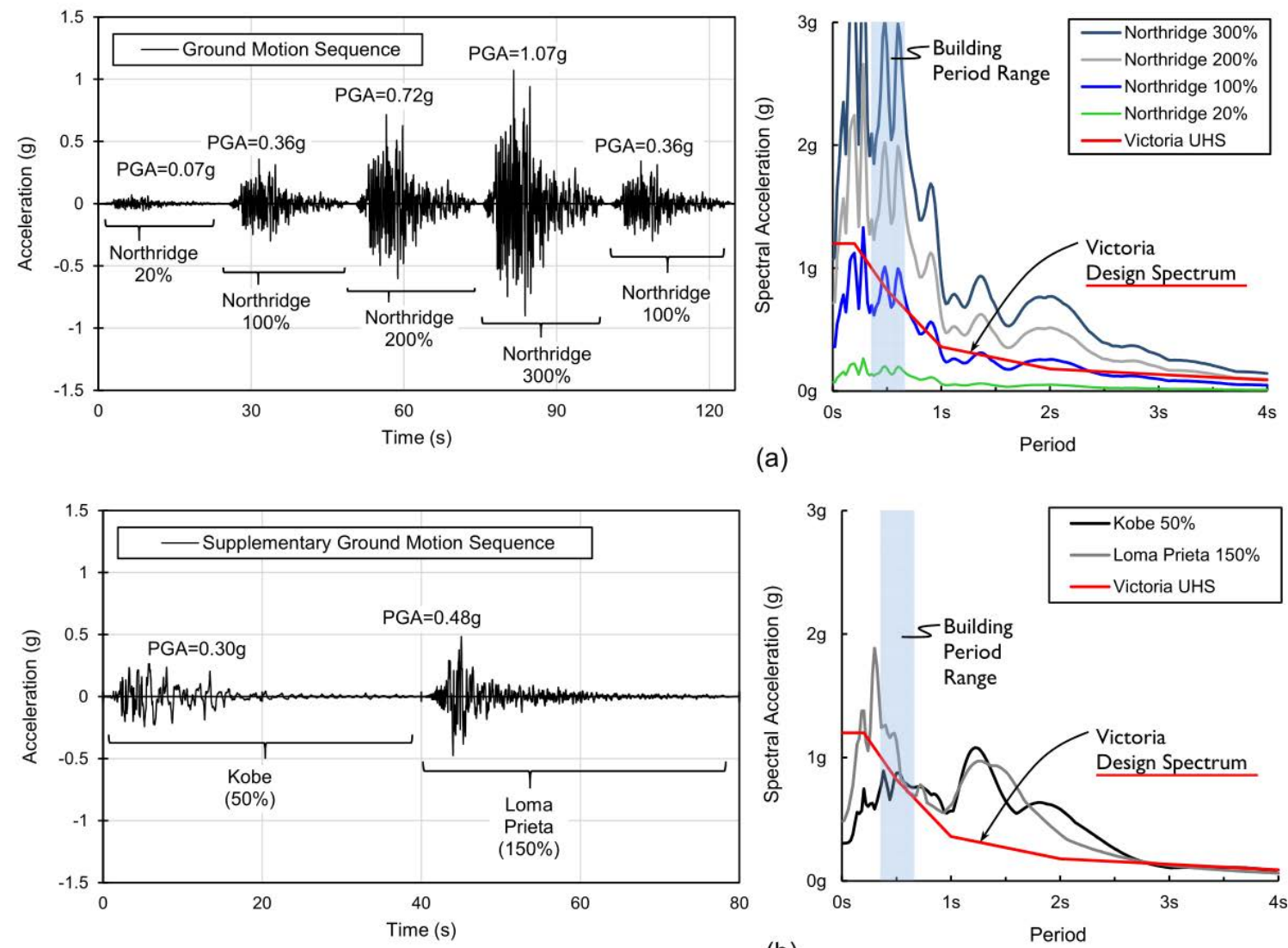

(a)

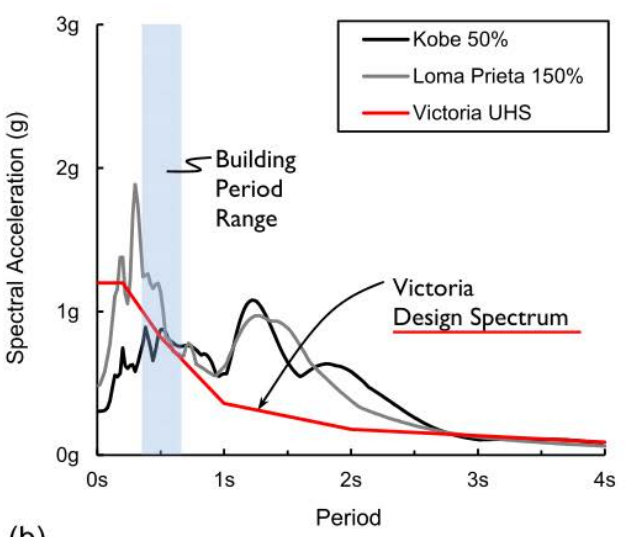

(b)

Figure 5.23 W2 hybrid test: (a) earthquake ground motion sequence and response spectra; (b) supplementary earthquake records and response spectra 


\section{Chapter 6. Results and Discussion}

\subsection{Introduction}

This section presents results from a series of hybrid tests conducted on a three-storey RC shear wall representative of a shear wall taken from a multi-storey reinforced concrete (RC) shear wall building. The hybrid test results are first discussed with respect to the local failure mechanisms observed in the physically tested substructure followed by results from the global response behaviour of the structure. First, a discussion on the preliminary testing of the steel column assembly and the challenges encountered during the experimental test setup are discussed.

\subsection{Preliminary Testing of Steel Column Assembly}

Prior to conducting the hybrid tests on the shear wall specimens, a steel column with a replaceable reduced beam section (RBS) was tested to verify the stiffness of the experimental test setup, performance of the hydraulic control, and the substructured finite element model. Figure 6.1 shows the results of the low-amplitude displacement cycles applied in the $\mathrm{X}$ and $\mathrm{Y}$ directions. Results show that the initial lateral and axial stiffness's of the RBS test specimen are $3.5 \mathrm{kN} / \mathrm{mm}$ and $166 \mathrm{kN} / \mathrm{mm}$, respectively.

The results of these initial tests showed some anomalies compared with the expected results. First, the measured axial stiffness of the steel column assembly was much lower when compared with its theoretical axial stiffness, which was estimated to be approximately $2000 \mathrm{kN} / \mathrm{mm}$. To ac-

count for the discrepancy, a simple elastic finite element model of the test setup (reaction block, 

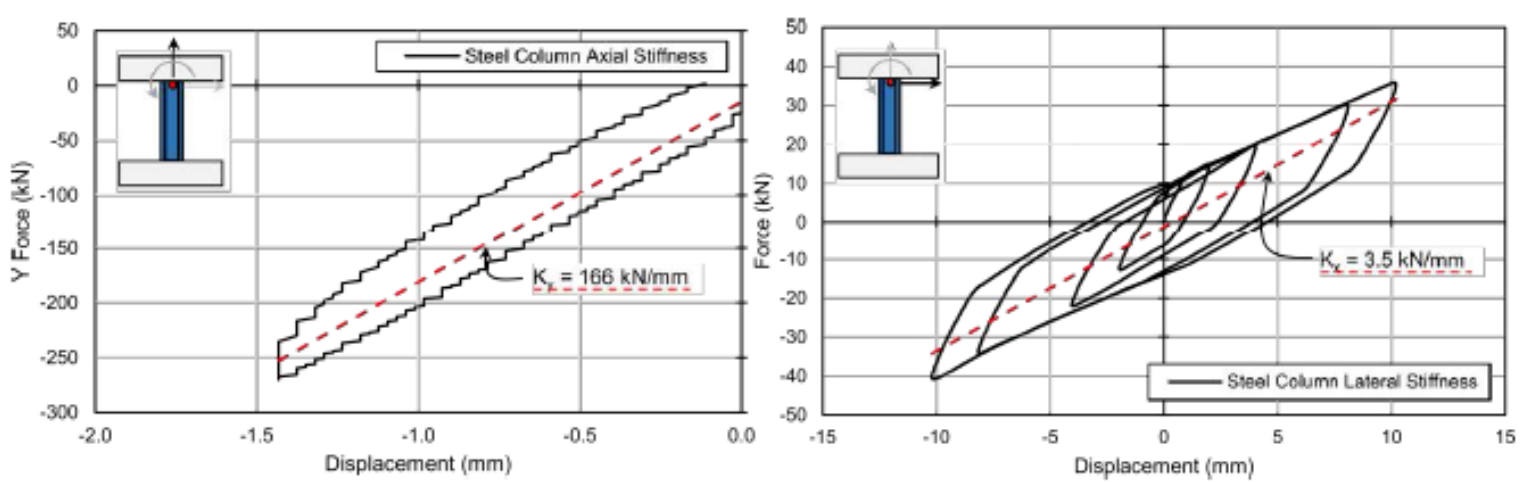

Figure 6.1 Initial stiffness of steel column assembly: (a) axial stiffness; (b) lateral stiffness

loading beam, and cap beam) and the steel column assembly was developed using Abaqus 6.13. Figure 6.2 shows the finite element model used to verify the axial stiffness of the steel column assembly. Results from the Abaqus model estimated a much lower initial stiffness of approximately $650 \mathrm{kN} / \mathrm{mm}$ when compared with the theoretical axial stiffness; much closer to the actual measured initial axial stiffness of the steel column assembly. The majority of the loss in axial stiffness is a result of deformation of the concrete cap beam at the top of the steel column assembly and deformation of the flanges of the reduced beam section (shown in Fig. 6.2), which are not accounted for in the theoretical axial stiffness calculations. The concrete cap beam cracked during axial testing of the column assembly, something that is not captured in the elastic finite element model of the test setup and could account for the lower axial stiffness measured in the laboratory compared to the finite element model. Other factors that could have influenced the axial stiffness of the steel column assembly include out-of-plane deformation. Although out-of-plane deformation was not measured to be significant during testing, even a very small out-of-plane displacement could contribute to a large loss in axial stiffness because the displacement increments are so small ( $<2 \mathrm{~mm})$. In the shear wall hybrid tests, the concrete cap beam is fully restrained across its length (because of the presence of the stiff concrete wall panel), and as a result the loss in axial stiffness because of cap beam deformation was expected to be smaller.

The second observed anomaly in the cyclic displacement tests initially conducted on the steel column assembly was that although the applied $10 \mathrm{~mm}$ displacement was within the elastic range of the steel column assembly, some hysteresis loops were observed in the hysteretic response 


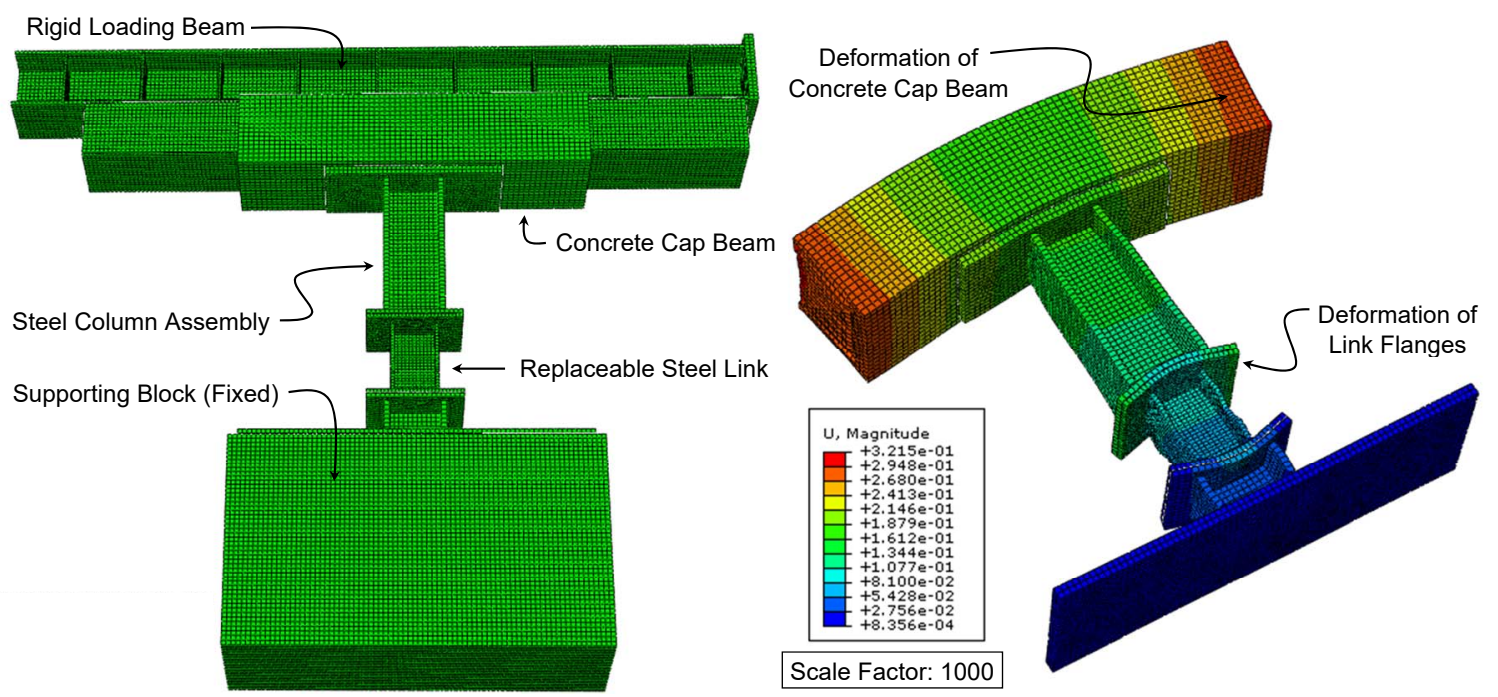

Figure 6.2 Model of column assembly test setup: (a) model mesh; (b) displacement results

behaviour. Ultimately, it was discovered that the swivels on the ends of the hydraulic actuators introduce some friction into the system when the test setup moves back and forth. Figure 6.3 shows the result when the theoretical elastic response of the steel column assembly is combined with an ideal friction force-deformation model. The results show that the friction in the test setup was causing the observed anomaly in the test results. A comparison of the theoretical combined elastic and friction response correlates very well with the measured response of the specimen during the test.

After removing the steel column assembly from the test setup, several tests were carried out on the test setup to characterize the friction response in the test setup by moving the system in a ramp pattern $+/-60 \mathrm{~mm}$ in the $\mathrm{X}$ and $\mathrm{Y}$ directions and +0.04 rad rotation in the $\mathrm{Z}$ directions. Figure 6.4 shows the hysteretic response behaviour of the empty test setup in the $\mathrm{X}, \mathrm{Y}$, and RZ directions. The results show a traditional hysteretic response behaviour associated with friction behaviour, in which the system has an initial stiffness until the static friction force exceeds the static friction force required to overcome the actuator swivel friction and then the test setup moves freely with minimal resistance. The static force required to overcome the swivel friction was found to be approximately $+/-10 \mathrm{kN}$ in the $\mathrm{X}$ and $\mathrm{Y}$ directions and $+/-35 \mathrm{kN}-\mathrm{m}$ moment in the RZ direction. This result also agrees with the developed theoretical friction model shown 

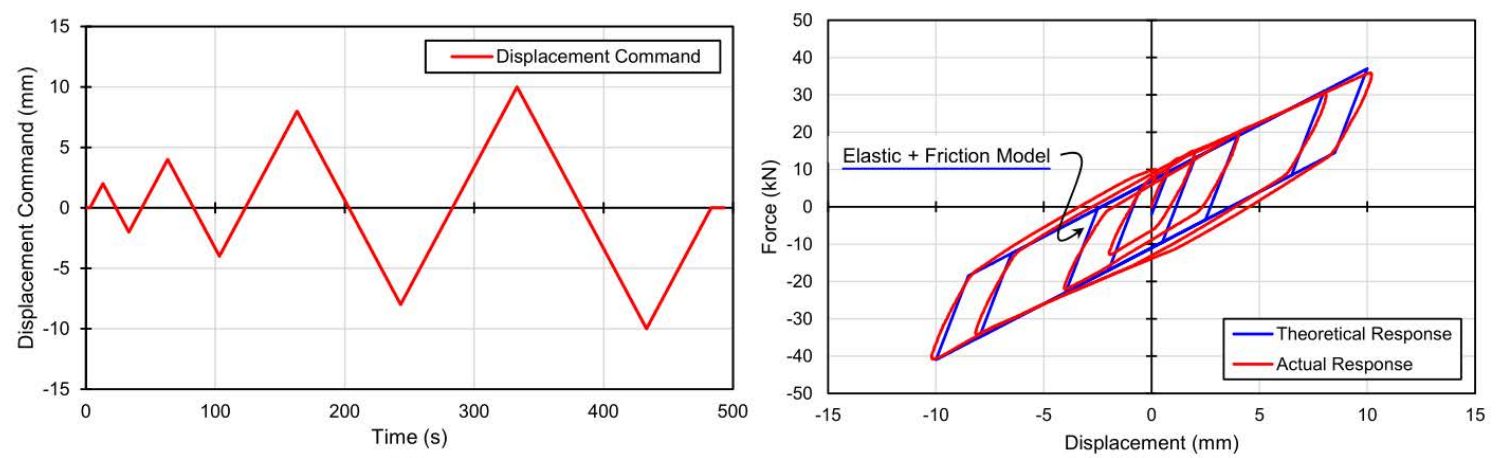

(a)

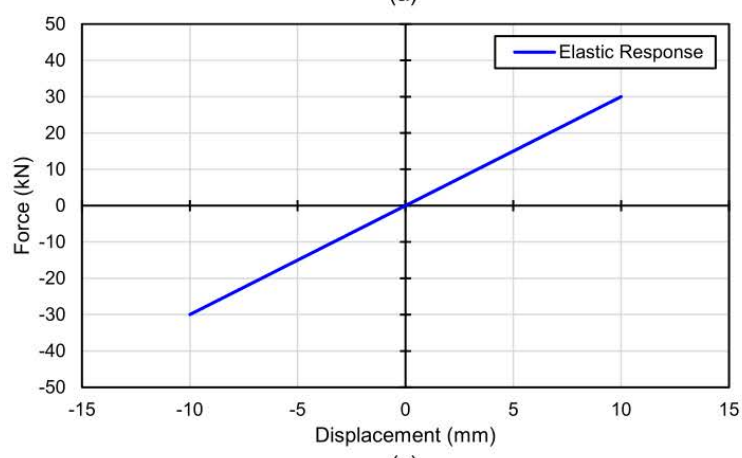

(c) (b)

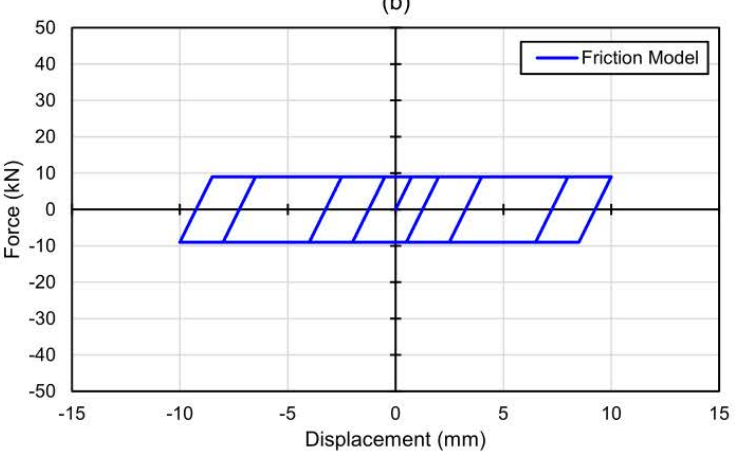

(d)

Figure 6.3 Steel column friction model: (a) cyclic displacement time history; (b) comparison of theoretical and measured hysteretic responses (c) theoretical elastic stiffness; (c) friction model; 
in Fig. 6.3, which predicted a static friction force of approximately $8 \mathrm{kN}-10 \mathrm{kN}$ to match the measured experimental result. Ultimately, because the shear wall specimens are very stiff and the friction forces are small compared to the maximum lateral force and moment capacity of the shear wall specimens, the small force required to overcome the static friction in the actuator swivels is assumed to be negligible, but could potentially pose a challenge if the capacity of the test specimen was smaller but artificially increasing the strength of the test specimen. In such a case, the negative effects of friction in the experimental test setup could be accounted for through the use of a friction compensation scheme, which could remove the additional force in the system resulting from friction. Alternatively, the load cells used to measure the force could be repositioned on the other side of the actuator swivels, effectively bypassing the friction from the experimental test setup.

After conducting the small cyclic displacement tests on the steel column assembly to establish its initial stiffness and verify the performance of the test setup, a hybrid test consisting of a gravity loading phase and a short sinusoidal pulse were conducted on the steel column assembly. The goal of this short test was to verify the communication link between OpenSees, OpenFresco, and the MTS controller in the laboratory and not to study the response or behaviour of the steel column assembly. Figure 6.5 shows the input ground motion, displacement-time history, and force-time history for the short hybrid simulation conducted on the column assembly. In all of the hybrid tests, the gravity load is slowly applied as a ramp function over $200 \mathrm{~s}$ in the laboratory

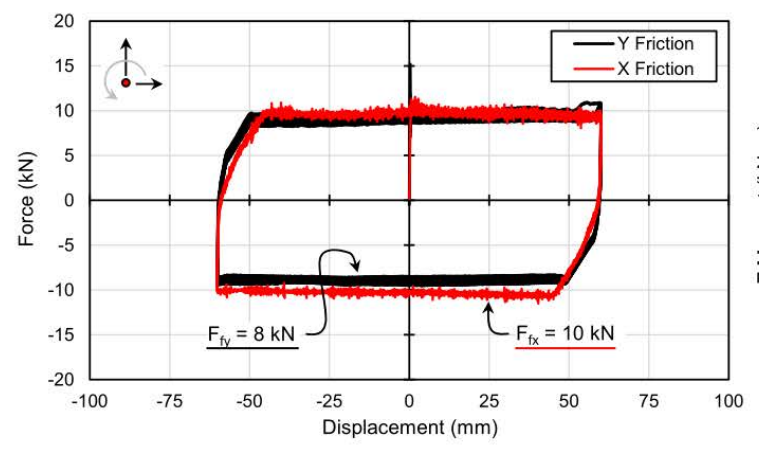

(a)

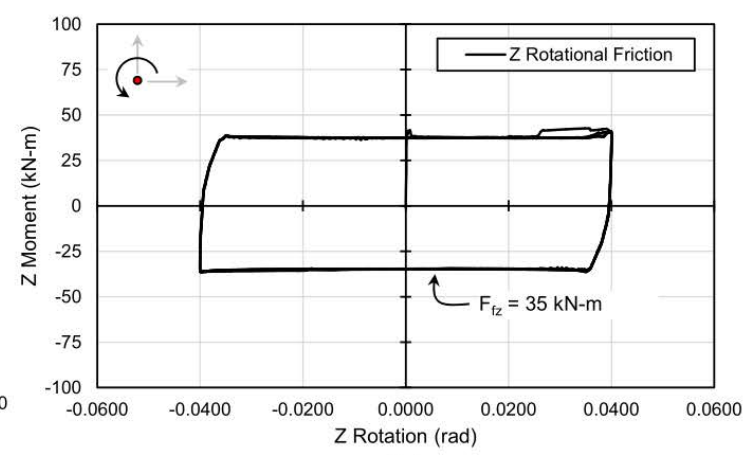

(b)

Figure 6.4 Friction characterization: (a) X/Y direction; (b) RZ direction 
( $2 \mathrm{~s}$ in the finite element model). The gravity load is applied as a transient load in the hybrid model, such that a hybrid integration scheme can be used during the gravity loading phase. Very high damping is applied to the structure during the gravity loading phase to avoid imposing dynamic effects on the structure. Results show that the gravity load reaches the target of $169.5 \mathrm{kN}$. The oscillation in the gravity load response is a result of challenges in controlling such a stiff structural element, nonetheless, the average load is close to the desired gravity load. Although the results from the sinusoidal ground motion do not have significant physical meaning, they do confirm that the communication link between the OpenSees-OpenFresco hybrid model and the MTS controller were working properly and that the scaling of command and feedback displacements and forces in OpenFresco were functioning as intended. Based on satisfactory performance of the experimental test setup and hybrid model, the steel column assembly was removed from the test setup and the hybrid tests were conducted on the RC shear wall specimens.

\subsection{Hybrid Simulation of Wall 1}

\subsubsection{Initial Stiffness of W1}

Prior to conducting a hybrid simulation, one important parameter required as an initial input in the hybrid model is the initial stiffness matrix of the experimental substructure. In the shear wall hybrid simulation, the required initial stiffness input for the experimental element is the $3 \times 3$ stiffness matrix for the three DOFs controlled at the top of the shear wall specimen. The initial stiffness of the experimental substructure is required because unlike in a conventional finite element model where the initial tangent stiffness of an element can be computed directly, in a hybrid simulation the stiffness of the element is not available until the feedback force/displacement is sent to the finite element model at the end of the first time-step. In general, it is considered good practice to overestimate the initial stiffness of the experimental substructure, which results in a smaller target displacement increment during the first time-step. If the initial stiffness is underestimated, a larger target displacement would be sent to the experimental substructure and could potentially damage the test specimen or test setup.

Joshua Woods, Department of Civil and Environmental Engineering, Carleton University 

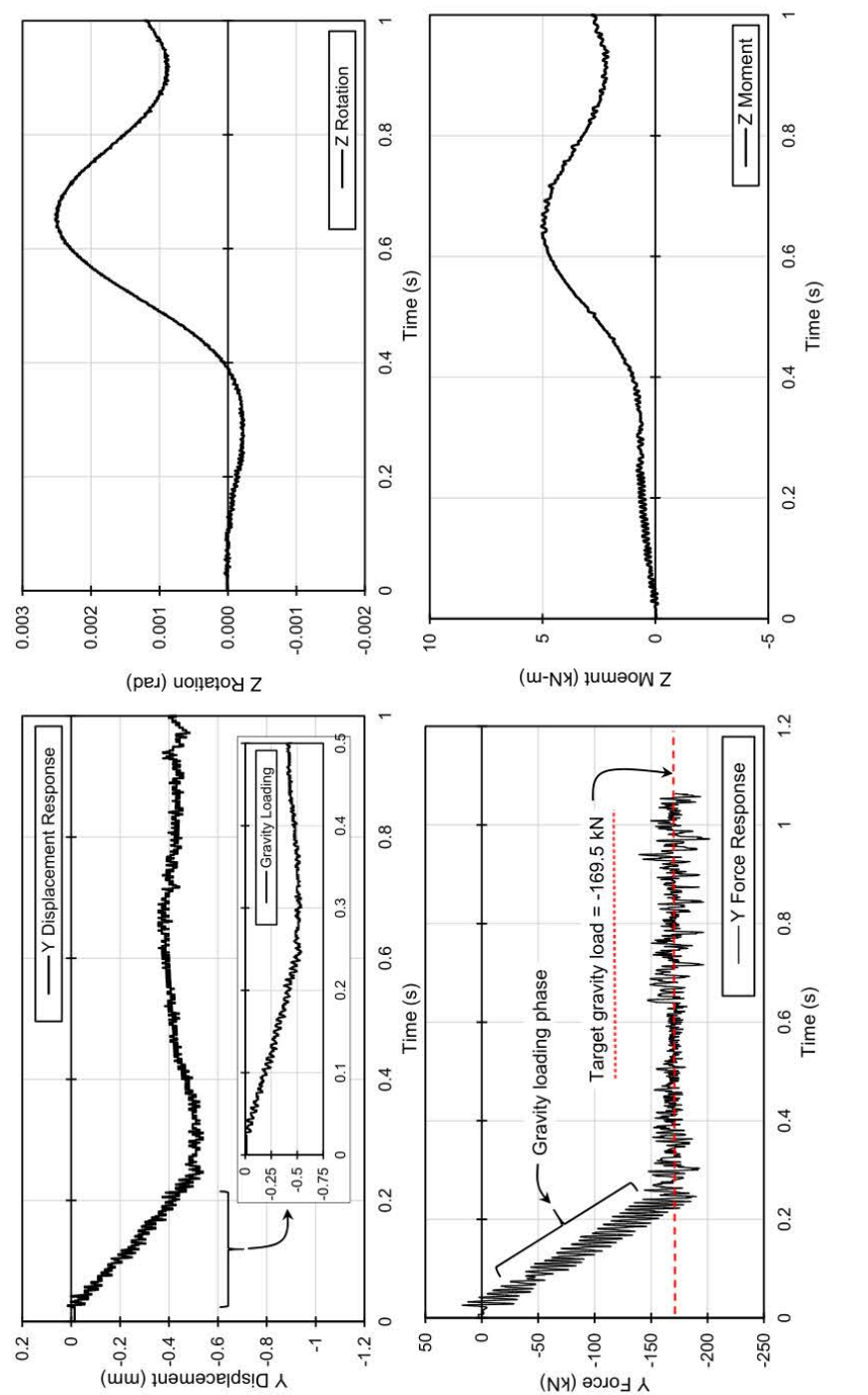

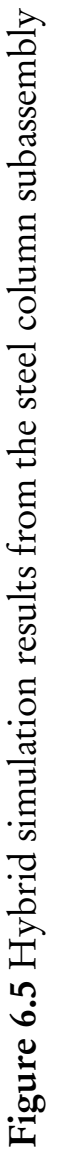
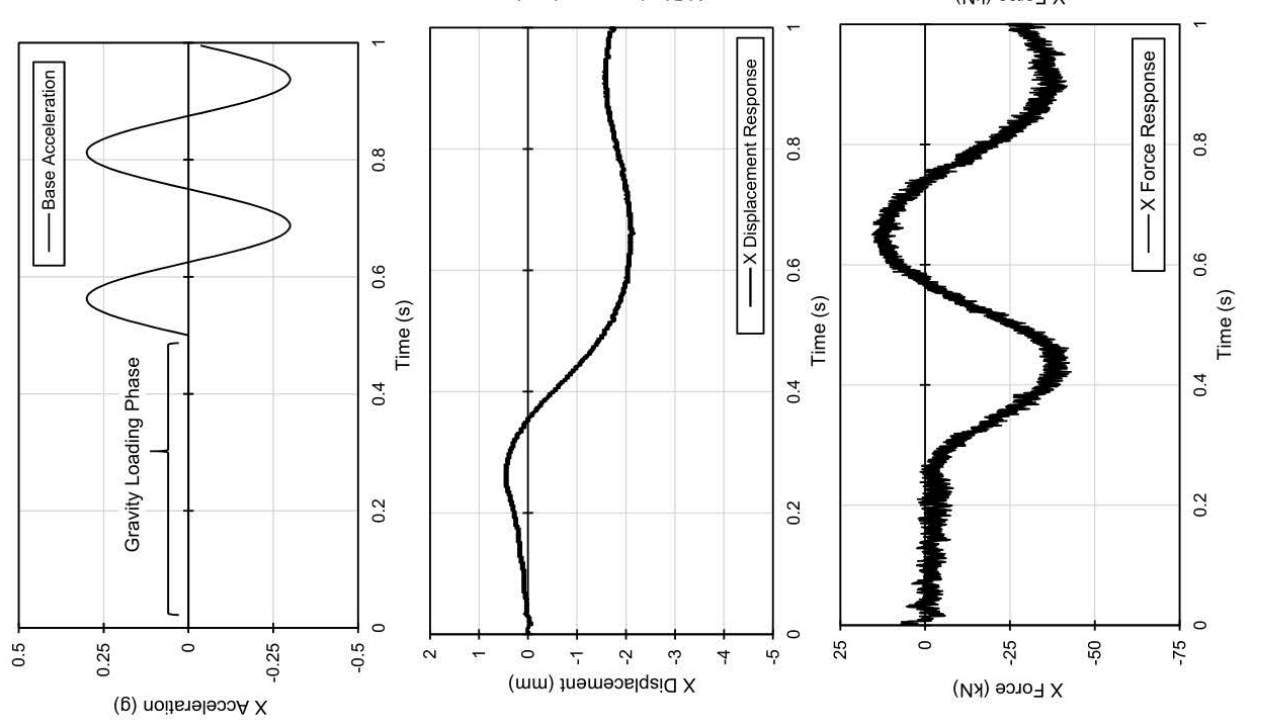

Joshua Woods, Department of Civil and Environmental Engineering, Carleton University 
To determine the initial stiffness matrix of the shear wall specimen, three low-amplitude cycles were applied to the test specimen in each of the three control DOFs (X, Y, and RZ), while holding the displacements and rotation in the other DOFs constant at zero. It is assumed that the low-amplitude cycles are small enough such that they will not significantly damage the shear wall test specimen. Figure 6.6 shows the initial stiffness test force-deformation response in each of the three DOFs and Table 6.1 summarizes the results and compares the measured initial stiffness of the shear wall specimen with theoretical results.

Results show that the initial in-plane, axial, and rotational stiffness of the shear wall specimen are $93.5 \mathrm{kN} / \mathrm{mm}, 750 \mathrm{kN} / \mathrm{mm}$, and $204000 \mathrm{kN}-\mathrm{m} / \mathrm{rad}$, respectively. The hysteresis in the initial stiffness are attributed to the friction in the experimental test setup, as well as the inherent hysteresis that naturally occurs in any test specimen, even at low cyclic displacement amplitudes. The results also show that the shear wall specimen has a much larger axial stiffness compared to the steel column assembly, attributed to the restraint provided by the wall panel, which prevents the cap beam from deforming. In comparison with the theoretical initial stiffness of $12 E I / L^{3}, E A / L$, and $4 E I / L$ in each of the three DOFs, the measured initial stiffness results are much smaller than expected. The differences in initial stiffness are attributed to out-of-plane deformations of the wall specimen, which cannot be appropriately controlled at such low displacement levels. Even a small out-of-plane deformation $(<0.2 \mathrm{~mm})$ can have some influence on the initial stiffness of the test specimen in any of the three DOFs, but other factors not described herein could also have influenced the initial stiffness of the wall specimen. In addition, there were some small cracks noted in the concrete near the base of the shear wall specimen that could have influenced the initial stiffness of the wall specimen. These crack were a result of handling and the post-tensioning process while installing the wall specimen in the experimental test setup. Finally, the slope of the line used to approximate the initial stiffnesses shown in Fig. 6.6 also has an impact on the results.

As noted previously, it is good practice to overestimate the initial stiffness matrix of the physical specimen during the hybrid test to avoid accidentally damaging the test specimen. Consequently, the theoretical initial stiffness matrix based on an initial in-plane stiffness of $12 E I / L^{3}$ was used for all hybrid tests. Ultimately, this variation in initial stiffness was not shown that have 
a dramatic influence on the hybrid simulation response, especially after significant cracking and yielding of the steel reinforcement, which dramatically reduced the stiffness of the specimen over the duration of the hybrid tests.

\subsubsection{Local Hybrid Simulation Results}

This section describes the local response of the experimental shear wall test specimen under increasing ground motion intensity from the earthquake sequence shown in Fig. 5.22. For each ground motion intensity, a complete set of results including $\mathrm{X}, \mathrm{Y}$, and $\mathrm{Z}$ displacement, rotation, force, and moment time-histories as well as force-displacement and moment-rotation hysteretic responses are available in Figures A.1 to A.5 in Appendix A. Tables 6.2 and 6.3 show critical structural response parameters for the shear wall specimen and maximum/minimum local response quantities for each ground motion. The following section briefly describes the local observations made during each hybrid test.

\section{Northridge Earthquake 20\%}

The first shear wall specimen was tested under the Canoga Park Station ground motion record from the 1994 Northridge earthquake scaled to $20 \%$ of its original unscaled intensity. At this intensity, the ground motion represents a very low level OBE earthquake. During this test, it was expected that the shear wall specimen would remain elastic and uncracked, and would serve as a way to verify the performance of the entire hybrid testing setup, including communication

Table 6.1 Shear wall W1 lateral, axial, and rotational initial stiffness

\begin{tabular}{lcc}
\hline \hline \multicolumn{1}{c}{ Loading Direction } & $\begin{array}{c}\text { Experimental } \\
\text { Result }\end{array}$ & $\begin{array}{c}\text { Theoretical } \\
\text { Stiffness }\end{array}$ \\
\hline Lateral, $\mathrm{X}\left(K_{i}, x\right)(\mathrm{kN} / \mathrm{mm})$ & 93.7 & $1736^{a}\left(685^{b}\right)$ \\
Axial, $\mathrm{Y}\left(K_{i}, y\right)(\mathrm{kN} / \mathrm{mm})$ & 750 & 2500 \\
Rotational, $\mathrm{RZ}\left(K_{i}, \mathrm{z}\right)(\mathrm{kN}-\mathrm{m} / \mathrm{rad})$ & $204.2\left(10^{3}\right)$ & $1875\left(10^{3}\right)$ \\
\hline \hline
\end{tabular}

${ }^{a}$ Based on an in-plane stiffness of $12 E I / L^{3}$ assumption;

${ }^{b}$ Based on an in-plane stiffness of $3 E I / L^{3}$ assumption; 

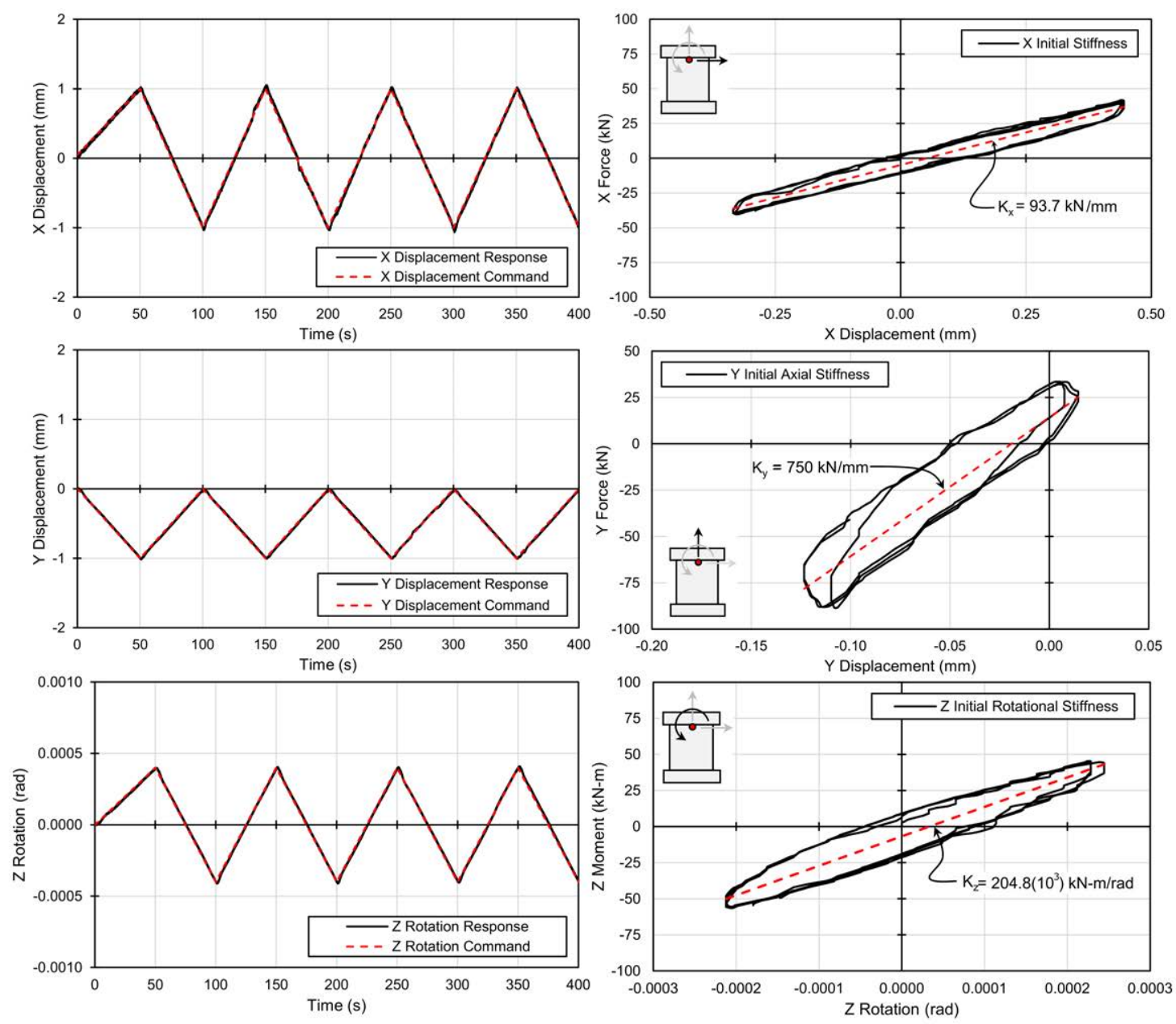

Figure 6.6 Shear wall W1 lateral, axial, and rotational initial stiffness 
links between OpenFresco and the controller, image and data acquisition systems, and to verify command versus achieved displacement histories for the stiff shear wall element. During the hybrid test, the shear wall specimen reached a maximum and minimum displacements and rotations of $+1.20 \mathrm{~mm},-0.97 \mathrm{~mm} 0.0007 \mathrm{rad}$, and $-0.0006 \mathrm{rad}$, respectively. Forces and moments in the $\mathrm{X}$ and $\mathrm{Z}$ directions remained below $+/-85 \mathrm{kN}$ and $+/-150 \mathrm{kN}-\mathrm{m}$ and little-to-no concrete cracking was observed during the test, confirming that the wall remained at or near elastic throughout the earthquake.

Figure 6.7 shows the command and achieved $\mathrm{X}$ displacement and $\mathrm{Z}$ rotation time-histories for the duration of the test. Results show that in general, the hybrid system does a good job at tracking the command displacement history in terms of displacement and rotation, and the tracking error (difference between the command and feedback signals) remained below $0.05 \mathrm{~mm}$ and 0.0005 rad throughout the test. Because of the small command displacements and the stiffness of the shear wall specimen, this low-intensity test represents the most challenging control scenario, but still produces good results. In subsequent hybrid tests under larger ground motion intensities, displacement increments are expected to be larger and the stiffness of the shear wall will be smaller, which produces a more stable control scenario because the displacement increment applied to the shear wall specimen will not correspond to such a large increment in force.

In addition to comparing the command versus achieved displacement over the duration of the hybrid test, Figure 6.8 compares the $\mathrm{X}$ and $\mathrm{Y}$ displacement time-histories based on encoder and actuator displacements. Recall that the externally mounted encoders are used to control the hybrid simulation in an attempt to bypass any deformation in the experimental test setup. Results show that in the X-direction, the experimental test setup is sufficiently stiff, and is able to approximately impose the command displacement to the structure based on actuator displacements with only minor overshoot. This is because in the X-direction, displacement is applied longitudinal to the rigid loading beam, which has a very high axial stiffness. In the Y direction, it is evident that the experimental test setup is not as stiff as the experimental shear wall specimen and the results show that the actuators must apply almost 4 times the command displacement to reach the target axial deformation of the shear wall specimen. In the Y-direction, the displacement is applied 

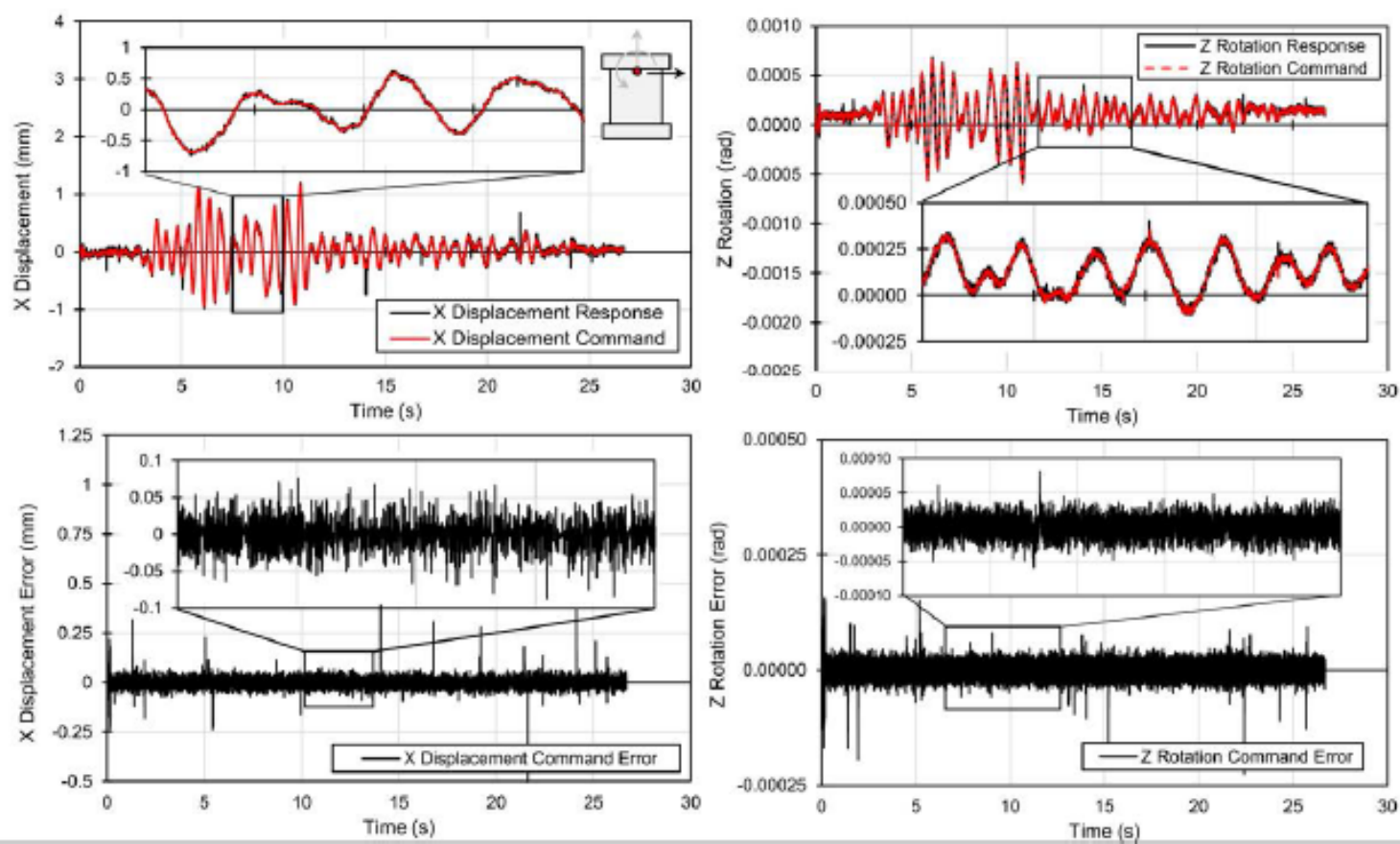

Figure 6.7 W1 Northridge 20\%: Displacement and rotation command/feedback response

transverse to the loading beam, which has a lower stiffness compared to the axial stiffness of the shear wall. The results also show the level of sensor noise in the response of the actuators internal LVDT. These results show the advantages of using externally mounted high-precision sensors to control the hybrid simulation, which can be used to bypass deformations in the experimental test setup and provide stable displacement feedback.
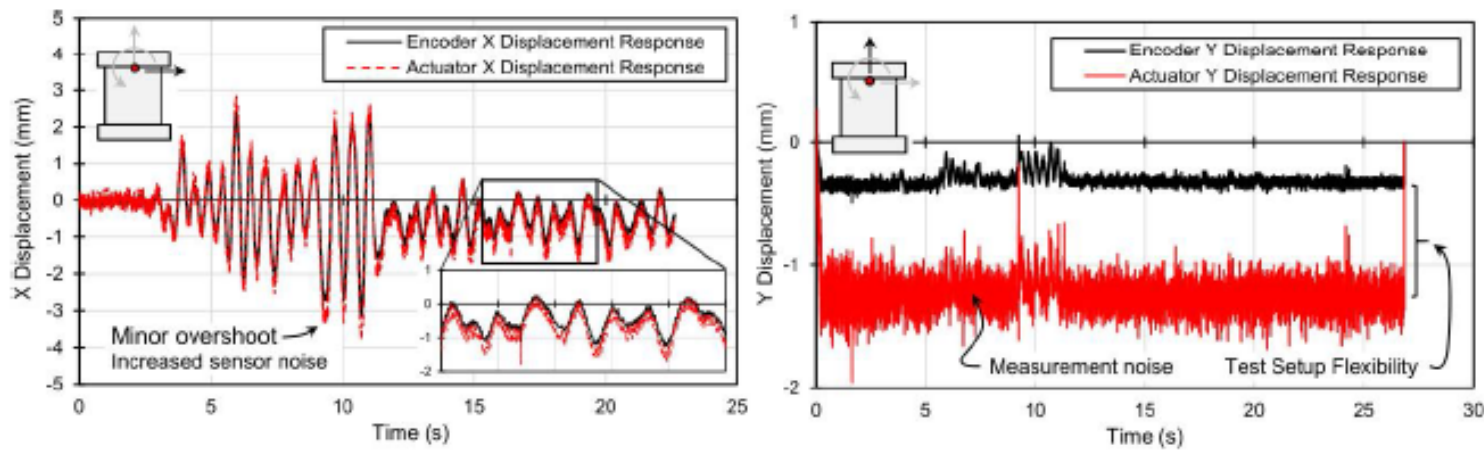

Figure 6.8 W1 Northridge 20\%: Comparison between actuator and encoder displacements 


\section{Northridge Earthquake 50\%}

Following satisfactory performance of the hybrid testing system during the first hybrid test, the Northridge input motion was scaled to $50 \%$ of its original intensity and applied to the three storey RC shear wall. At this intensity, this ground motion is a better representation of an OBE level ground motion, in which a structure may exhibit some minor damage but is expected to remain fully operational following an earthquake.

Figure 6.9 shows the moment-rotation hysteretic response behaviour and maximum vertical steel reinforcement strain profiles at the base of the wall specimen for the second hybrid test. Results show that the wall specimen remains elastic, and the strains in the vertical steel reinforcement close to the edges of the shear wall remain below $1250 \mu_{\epsilon}$. The maximum average moment achieved at the base of the shear wall of $263 \mathrm{kN}-\mathrm{m}$ represents approximately $2 / 5$ of the predicted ultimate moment capacity of the shear wall based on strain-compatibility analysis. The shear wall specimen reached a maximum and minimum displacements and rotations of $+2.39 \mathrm{~mm},-3.15 \mathrm{~mm},+0.002 \mathrm{rad}$, and $-0.0014 \mathrm{rad}$, respectively.

Throughout each hybrid test, high definition images are taken of the test specimen using two Nikon D5300 DSLR cameras. The images are analyzed following each test using the ImPro Stereo software, which is capable of capturing surface strain fields and map concrete crack distributions throughout each test. Figure 6.10 shows the test setup for the image capture system.

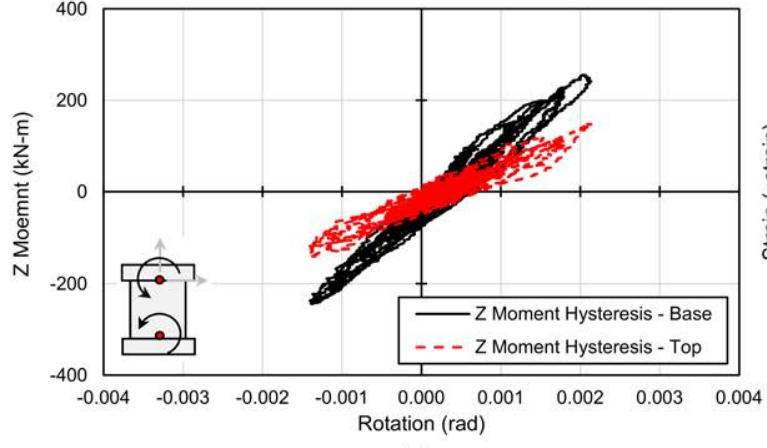

(a)

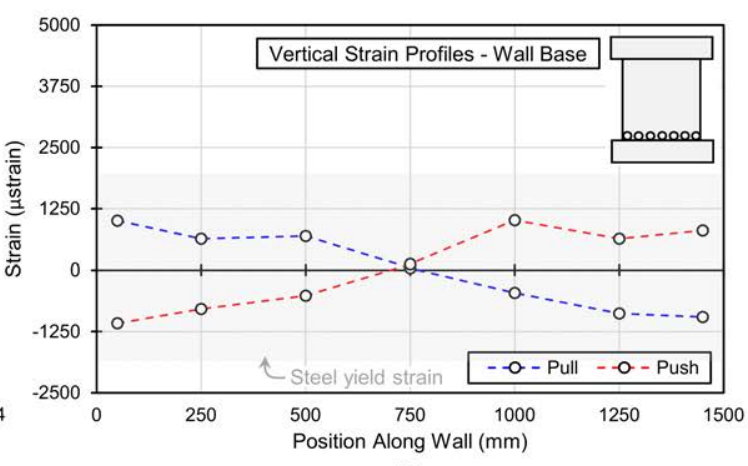

(b)

Figure 6.9 W1 Northridge 50\%: (a) moment-rotation hysteresis; (b) maximum vertical strain profiles 
The test setup consists of the two cameras that are rigidly mounted above the region of interest, which in this case is the plastic hinge region of the shear wall test specimen. Additional lighting is provided over the region of interest to ensure that the changing ambient lighting conditions do not effect the image analysis results. The images are automatically captured using the freely available digiCamControl software at $30 \mathrm{~s}$ intervals throughout each hybrid test.

Figure 6.11 shows the $e_{x y}$ surface strain field at the maximum positive $\mathrm{X}$ displacement during the second hybrid test. Results clearly show the formation of thin diagonal cracks, measuring between $0.1 \mathrm{~mm}$ and $0.25 \mathrm{~mm}$ in the plastic hinge region of the shear wall. These results demonstrate the capabilities of the ImPro Stereo to accurately capture cracks in the concrete that would otherwise be very difficult to identify with the naked eye. It also provides a safe and efficient tool to measure response quantities without the need to approach a loaded specimen or stop the hybrid test.

At the end of the hybrid test, the residual displacement and rotation of the shear wall specimen were negligible. Overall, the observed behaviour is consistent with OBE level performance objectives, which according to the IAEA standards, structural elements should remain elastic and damage can be considered non-structural if crack widths remain below $0.2 \mathrm{~mm}$ (Bin Ibrahim et al., 2002). One observation made during this test was a large fluctuation in the axial force (Y-force) applied to the specimen, shown in Fig. 6.12a. This behaviour is predominantly noted in the Y-force response, although small variation in $\mathrm{X}$ force and $\mathrm{Z}$ moment are also observed during the tests. After filtering the noise from the response using a moving average function, results in Fig. 6.12b show that the Y-force is tracking closely to the target gravity load but there is a significant amount of fluctuation throughout the test. Similar observations have been made by other researchers during hybrid tests, and most suggest the use of filtering to effectively smooth the force response (You and Schellenberg, 2017). 


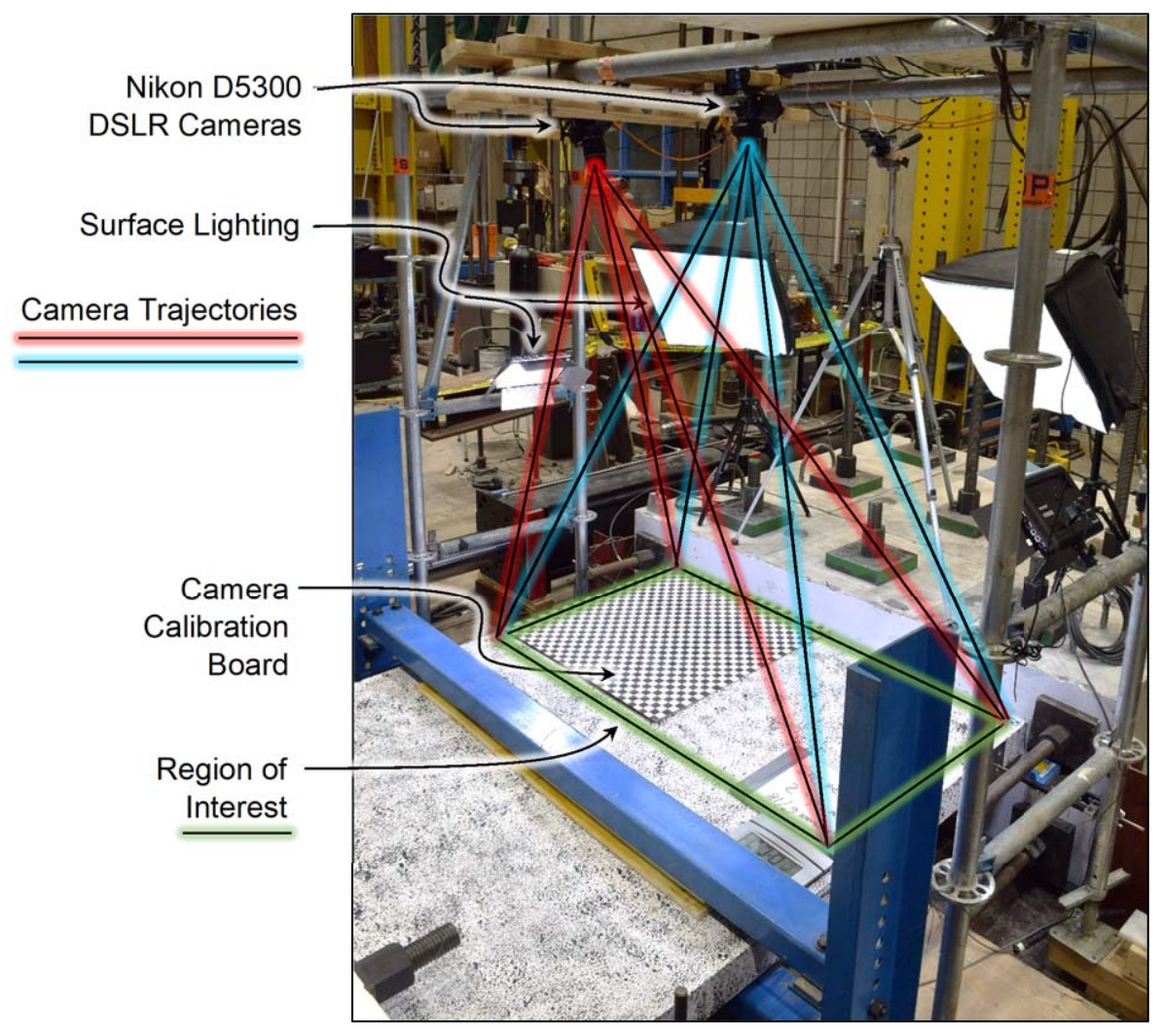

Figure 6.10 Image capture experimental test setup

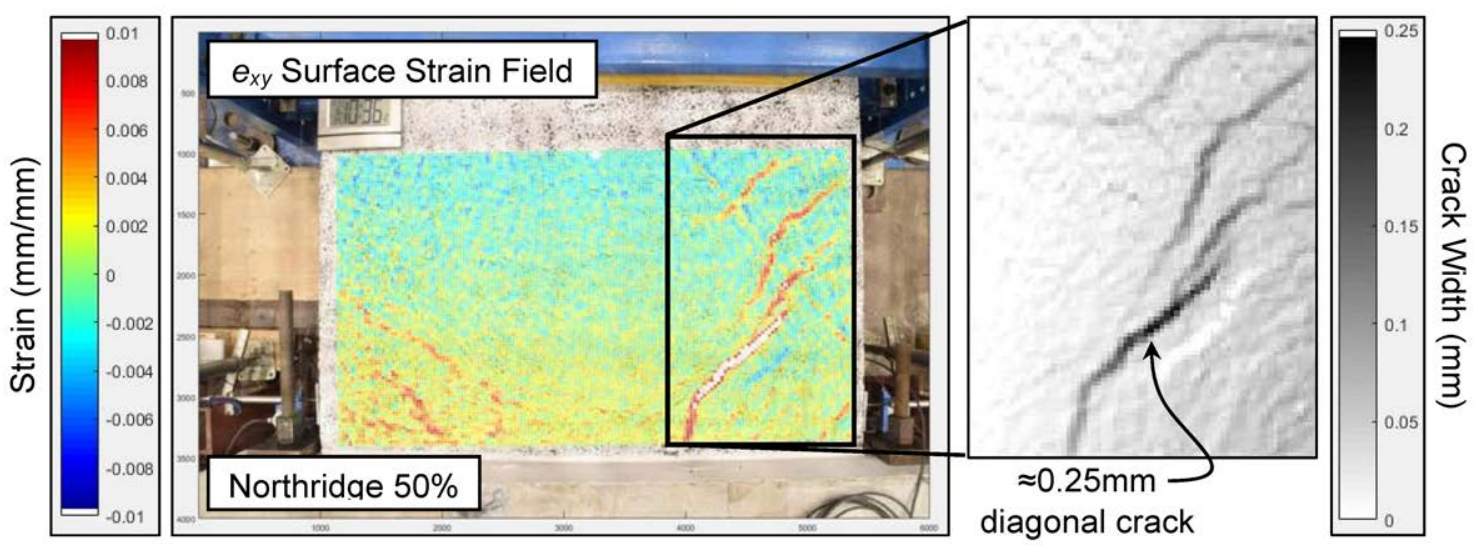

Figure 6.11 W1 Northridge 50\%: $e_{x y}$ surface strain field and crack distribution 


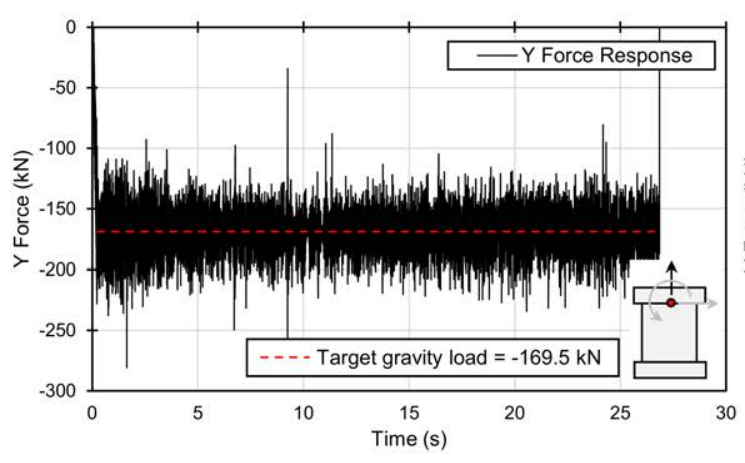

(a)

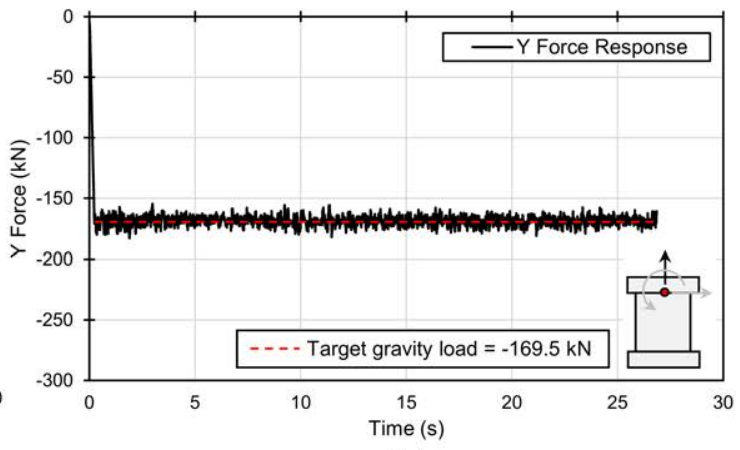

(b)

Figure 6.12 W1 Northridge 50\%: (a) unfiltered Y-force; (b) filtered Y-force

\section{Northridge Earthquake 100\%}

In the third hybrid test on the first shear wall specimen, the full unscaled Northridge Canoga Park Station ground motion record was applied to the multi-storey shear wall. The ground motion at this intensity represents a DBE level seismic event, in which some yielding in the structural elements is expected. Figure 6.13 shows the displacement-time history and momentrotation hysteresis for the test. Results show that the shear wall specimen reached maximum and minimum displacements and rotations of $+/-5.71 \mathrm{~mm},+0.0036 \mathrm{rad}$, and $-0.0032 \mathrm{rad}$, respectively. The moment hysteresis shows some nonlinearity in the response of the wall specimen, characterized by the widening of the hysteretic loops.

Figure 6.14 shows the vertical steel reinforcement strain profiles at the base of the wall. The strain profiles show that the vertical steel reinforcement just exceeds the steel yield strain at
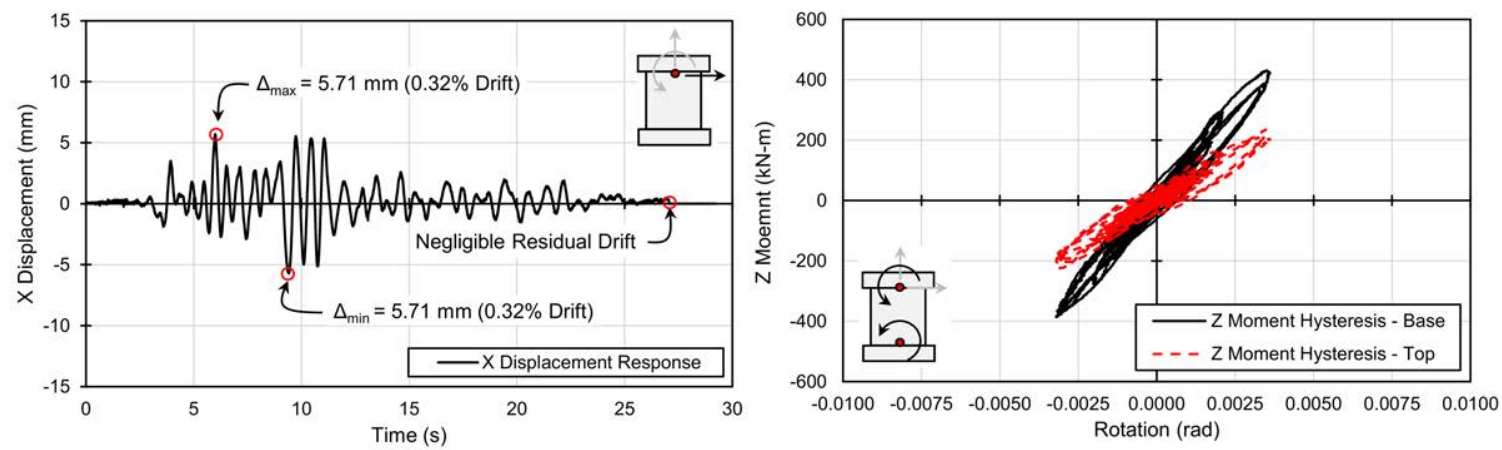

Figure 6.13 W1 Northridge 100\%: (a) top displacement history; (b) moment-rotation hysteresis 
the edges of the shear wall, while the vertical steel reinforcement remains elastic at mid-height of the shear wall. Based on these results, the wall specimen is shown to be at the onset of yielding and in the transition between linear/nonlinear behaviour but did not reach its yield displacement or rotation. Displacement and rotation results also show negligible residual drift and rotation following the test, confirming the wall specimen remained near elastic. Crack widths measured using image analysis ranged between $0.5 \mathrm{~mm}$ and $0.8 \mathrm{~mm}$ at the maximum displacement and rotation. Ultimately, performance of the shear wall specimen is consistent with the design response under a DBE ground motion; in that the specimen experienced some yielding in the vertical steel reinforcement and large cracks in the concrete but exhibited a flexurally ductile response without brittle shear failure or permanent residual drift. This would likely leave the structure fully operational following an earthquake.

\section{Northridge Earthquake 200\%}

The final two ground motions applied to the multi-storey RC shear wall were MCE level events expected to produce significant nonlinearity in the shear wall specimen, including extensive yielding of the vertical steel reinforcement, wide cracks in the concrete, and some residual drift. During the $200 \%$ intensity event, the shear wall specimen reached maximum and minimum displacements and rotations of $+11.7 \mathrm{~mm},-16.5 \mathrm{~mm},+0.0097 \mathrm{rad}$, and $-0.0065 \mathrm{rad}$ resulting in significant nonlinearity in the response of the shear wall specimen. Figure 6.15 shows the top displacement history and the moment-rotation response of the shear wall, which clearly shows
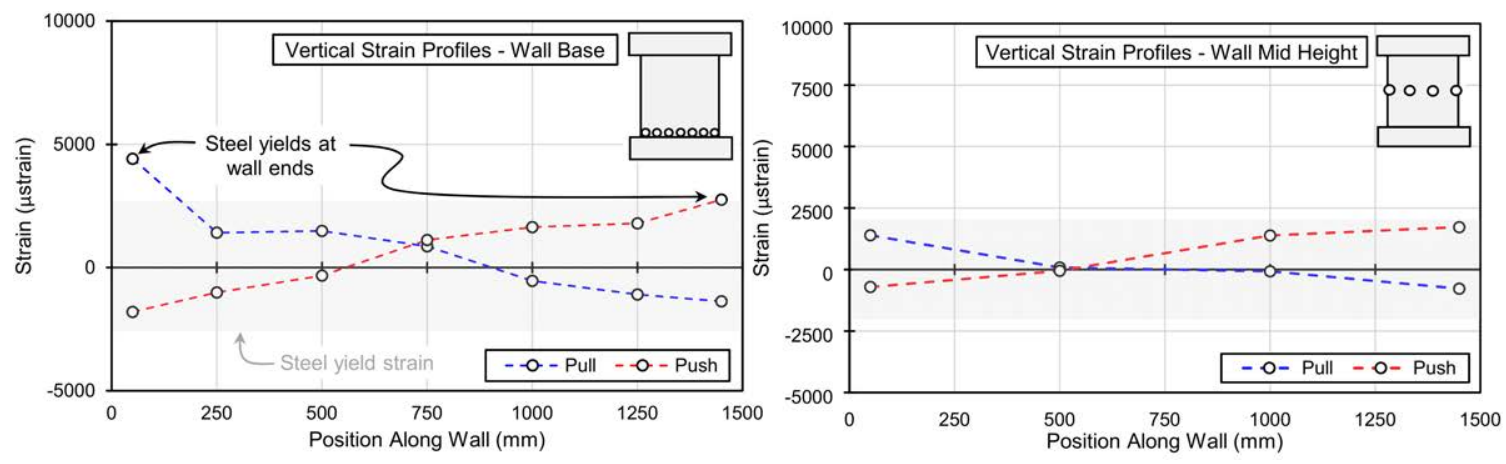

Figure 6.14 W1 Northridge 100\%: strain profiles in the vertical reinforcement 
the nonlinearity in its response. The shear wall specimen yields at lateral displacement and rotation of $8.35 \mathrm{~mm}(0.46 \%$ drift $)$ and $0.004 \mathrm{rad}$, respectively, corresponding to a yield moment and equivalent yield force $\left(F_{y}=M_{y} / h_{w}\right)$ of $491 \mathrm{kN}-\mathrm{m}$ and $272.5 \mathrm{kN}$. The wall specimen reaches a maximum moment of $491 \mathrm{kN}-\mathrm{m}$ and $657 \mathrm{kN}-\mathrm{m}$ at the top and bottom of the shear wall, respectively. The ultimate moment closely matches the predicted ultimate moment capacity of $610 \mathrm{kN}-\mathrm{m}$ according to strain-compatibility analysis. These results also suggest that the steel reinforcement should have been approaching the yield strain at the top of the wall specimen, which is confirmed by strain gauge readings from the vertical steel reinforcement at the top of the shear wall close to its ends, which measured average tensile strains of $2472 \mu \epsilon$, which is roughly the measured yield strain of the steel reinforcement from material test results. Figure 6.16 shows the crack distribution from image analysis at the maximum positive and negative displacement. The results show a large number of horizontal and diagonally oriented cracks in the concrete ranging in width between $0.5 \mathrm{~mm}$ and $1.0 \mathrm{~mm}$.

Table 6.2 summarizes important nonlinear structural response parameters for the shear wall test specimen, including yield displacement, rotation, force, and moment and both displacement and rotational ductility. The displacement ductility $\left(\mu_{\Delta}\right)$ of the wall specimens is calculated using Eq. 6.56:

$$
\mu_{\Delta}=\frac{\Delta}{\Delta_{y}}
$$

where $\Delta$ is the maximum displacement of the test specimen at a particular load cycle and $\Delta_{y}$ is the yield displacement of the wall specimen. The ultimate displacement ductility $\left(\mu_{\Delta}\right)$ is computed using the same equation, but with the ultimate displacement $\left(\Delta_{u}\right)$, which is defined by the displacement when the lateral load carrying capacity of the test specimen drops $20 \%$ from the maximum load reached during the test. In addition, the rotational ductility $\left(\mu_{\theta}\right)$ is computed using Eq. 6.57:

$$
\mu_{\theta}=\frac{\theta}{\theta_{y}}
$$


where $\theta$ is the rotation of the shear wall at a particular load cycle and $\theta_{y}$ is the measured yield rotation. In a similar manner to the ultimate displacement ductility, the ultimate rotational ductility $\left(\mu_{\theta, u}\right)$ can also be computed using the ultimate rotation $\left(\theta_{u}\right)$. During the Northridge earthquake at $200 \%$ of its original intensity, the wall specimen achieves maximum displacement and rotational ductilities of 1.97 and 2.31, respectively. At the end of the test, a small residual displacement and rotation of $1.75 \mathrm{~mm}(0.1 \% \mathrm{drift})$ and $0.0011 \mathrm{rad}$ were observed. Ultimately, the results are once again consistent with the design expectations for a RC shear wall subject to an MCE level ground motion, in which significant damage and nonlinearity are expected but the wall specimen does not collapse, ensuring the life-safety of the structures occupants.

\section{Northridge Earthquake 300\%}

The final ground motion applied to the shear wall specimen was the Canoga Park Station ground motion record at 3 times its original unscaled intensity. This test was expected to cause significant damage to the shear wall specimen, however, because the goal of a parallel study is to repair the shear wall specimens using externally bonded CFRP sheets, the intention was not to test the shear wall to collapse; leaving it in a repairable state. As a result, the test was prematurely stopped prior to completion of the earthquake ground motion record to prevent the shear wall from reaching its ultimate limit state.

Figure 6.17 shows the $\mathrm{X}$ displacement history, $\mathrm{X}$ force-displacement hysteretic response,

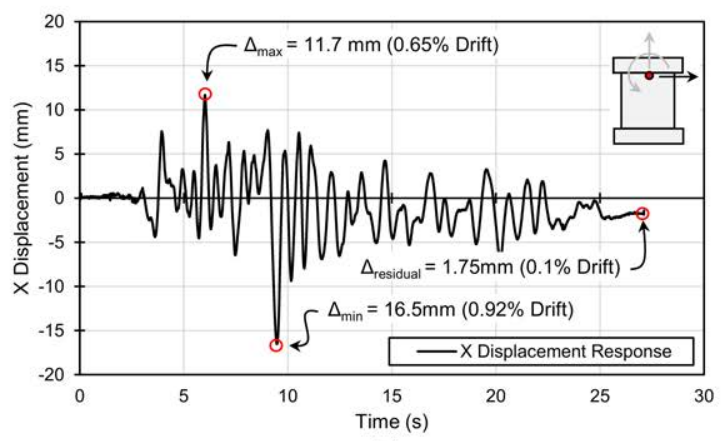

(a)

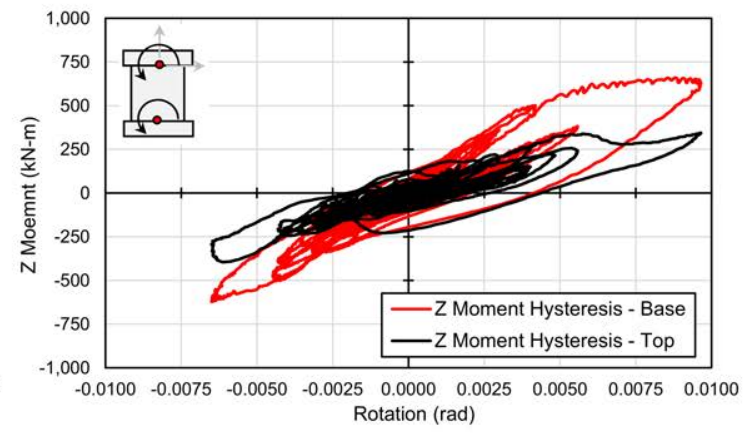

(b)

Figure 6.15 W1 Northridge 200\%: (a) top displacement history; (b) moment-rotation hysteresis 

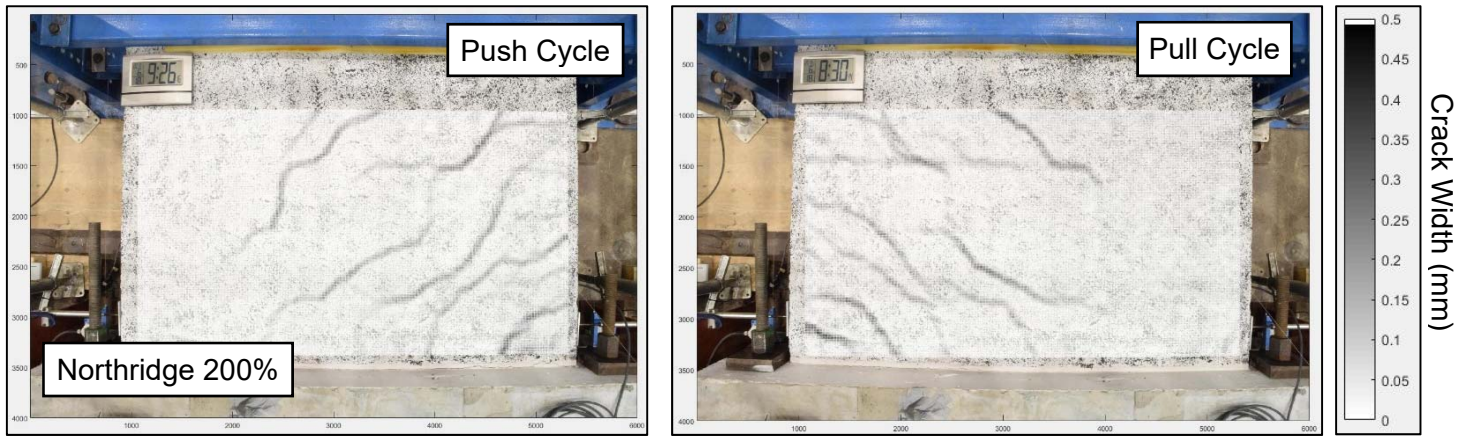

Figure 6.16 W1 Northridge 200\%: maximum crack width distributions

$\mathrm{Z}$ rotation time history, and $\mathrm{Z}$ moment-rotation hysteretic response of the shear wall for the first 10 seconds of the earthquake record. The results show that the wall specimen reached a maximum displacement of $27.1 \mathrm{~mm}(1.5 \% \mathrm{drift})$ and rotation of $0.0159 \mathrm{rad}$. This corresponds to displacement and rotational ductilities of 3.25 and 3.78 respectfully, demonstrating sufficient ductility and good seismic performance from a shear wall specimen designed as a moderately ductile RC shear wall $\left(R_{d} R_{o}=2.5\right)$. Significant residual displacement of $17.6 \mathrm{~mm}(0.98 \% \mathrm{drift})$ and rotation of $0.01 \mathrm{rad}$ were observed following the test.

Vertical strain profiles in the steel reinforcement over the height of the wall specimen shown in Figure 6.18 also show the significant nonlinear behaviour of the shear wall. The results show that the vertical steel reinforcement at the bottom, top, and middle of the wall all exceeded their yield strain, with very large strains in the vertical steel reinforcement near the ends of the shear wall at each location. Figure 6.19 shows the damage to the shear wall specimen. Concrete crushing was observed during the final hybrid test in one of the toes of the wall specimen, suggesting the shear wall exceeded its diagonal compression shear strength $\left(0.83\left(f_{c}^{\prime}\right)^{1 / 2}\right)$ after failing in flexure.

Following the final hybrid test, the shear wall specimen was removed from the experimental test setup and the concrete crack pattern was manually marked on the surface of the shear wall specimen. Figure 6.20 compares the marked crack distribution with those analyzed using ImPro Stereo. The results show that ImPro Stereo is able to capture the distribution of the cracks on 

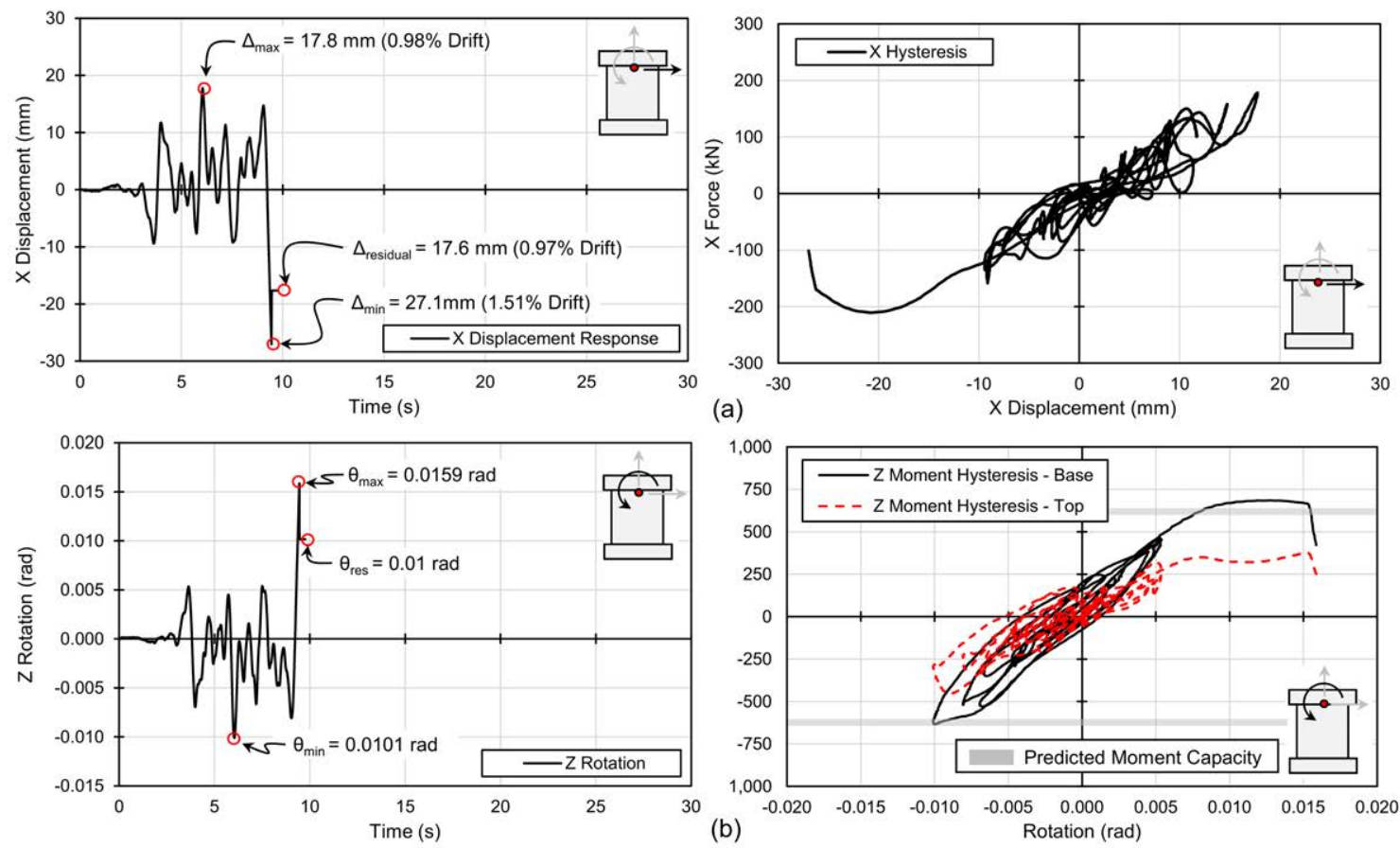

Figure 6.17 W1 Northridge 300\%: (a) X displacement and hysteresis; (b) Z rotation and hysteresis

the surface of the concrete and the results correlate very well with the manually marked crack distribution. Ultimately, the use of the image analysis tool allowed all of the hybrid tests to be conducted without interruption and without the need to approach the loaded test specimen.

Table 6.2 W1 critical structural response parameters

\begin{tabular}{lc}
\hline \hline Response Parameter & Specimen W1 \\
\hline Yield Displacement $\left(\Delta_{y}\right)(\mathrm{mm})$ & -8.35 \\
Yield Rotation $\left(\theta_{y}\right)(\mathrm{rad})$ & $0.0042 /-0.0046$ \\
Yield Moment $\left(M_{y}\right)(\mathrm{kN}-\mathrm{m})$ & $485 /-497$ \\
Equiv. Yield Force $\left(F_{y}\right)(\mathrm{kN})$ & $269 / 276$ \\
Ultimate Displacement $\left(\Delta_{u}\right)$ & $17.8 /-27.1$ \\
Ultimate Rotation $\left(\theta_{u}\right)(\mathrm{rad})$ & $0.0159 /-0.0101$ \\
Maximum Ductility $\left(\Delta_{u} / \Delta_{y}\right)$ & $2.19 / 3.78$ \\
\hline \hline
\end{tabular}



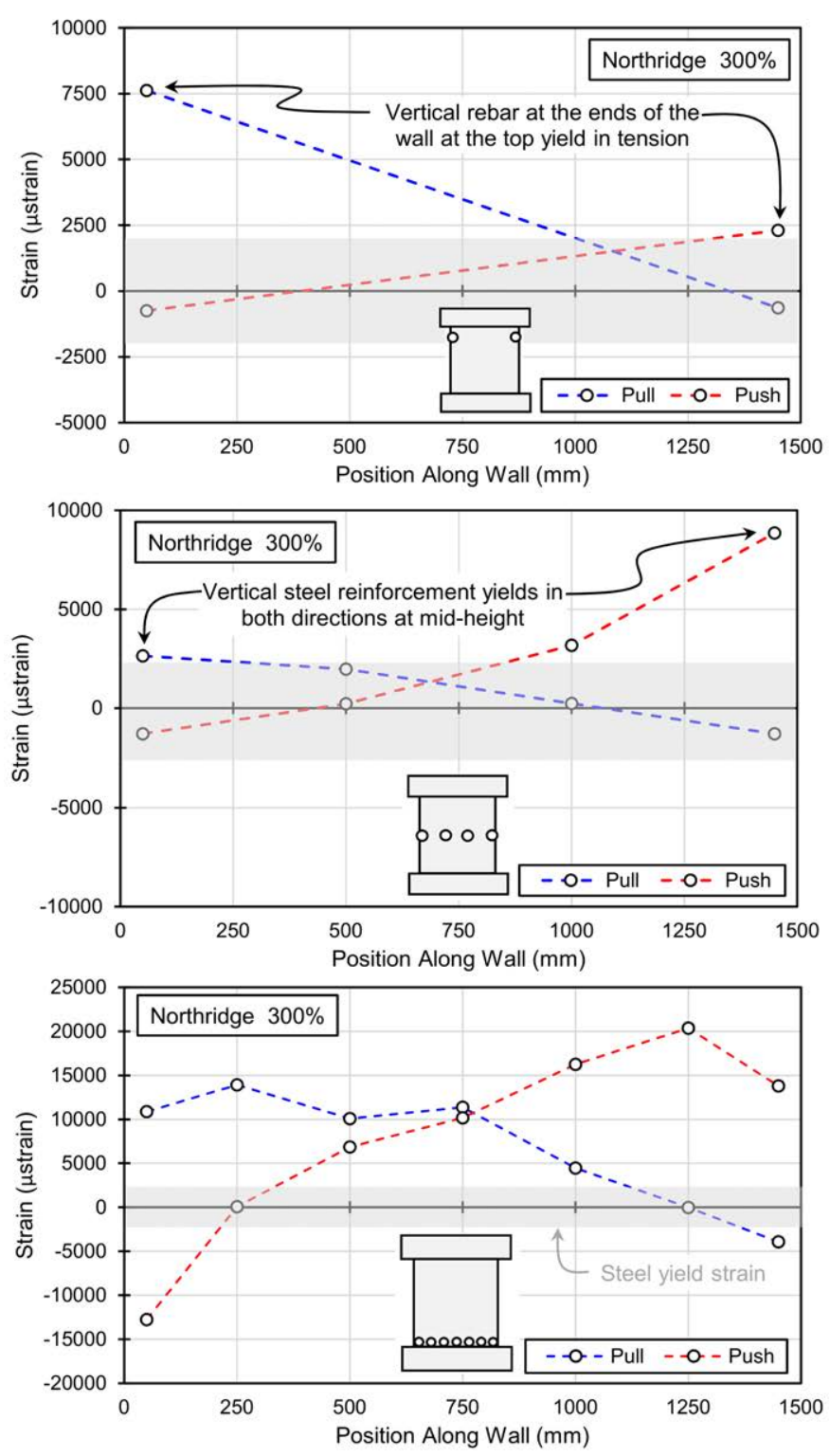

Figure 6.18 W1 Northridge 300\%: vertical strain profiles 


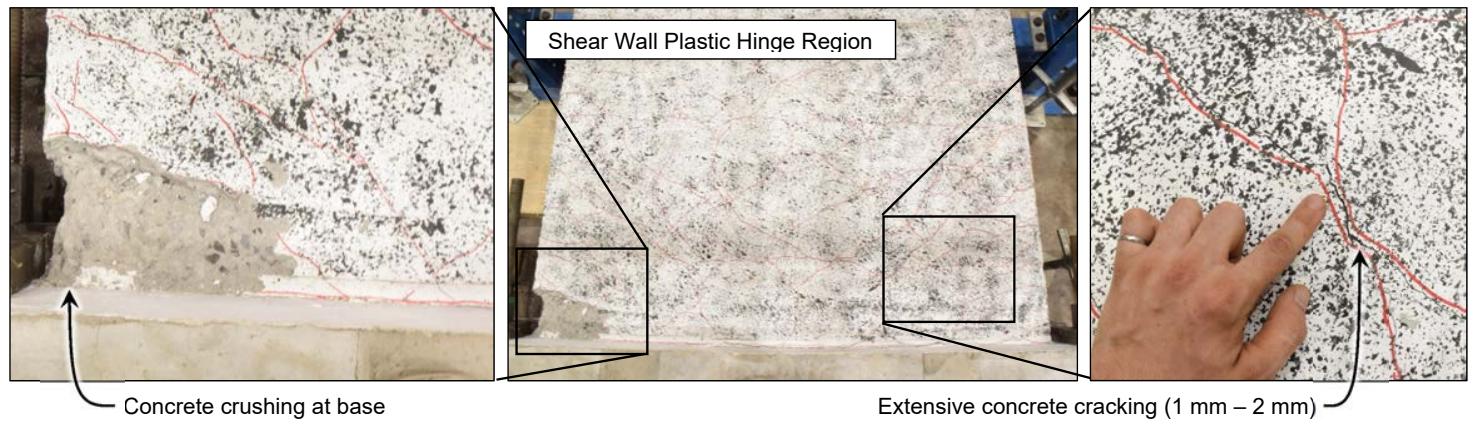

Figure 6.19 Damage to shear wall W1 following the hybrid simulations

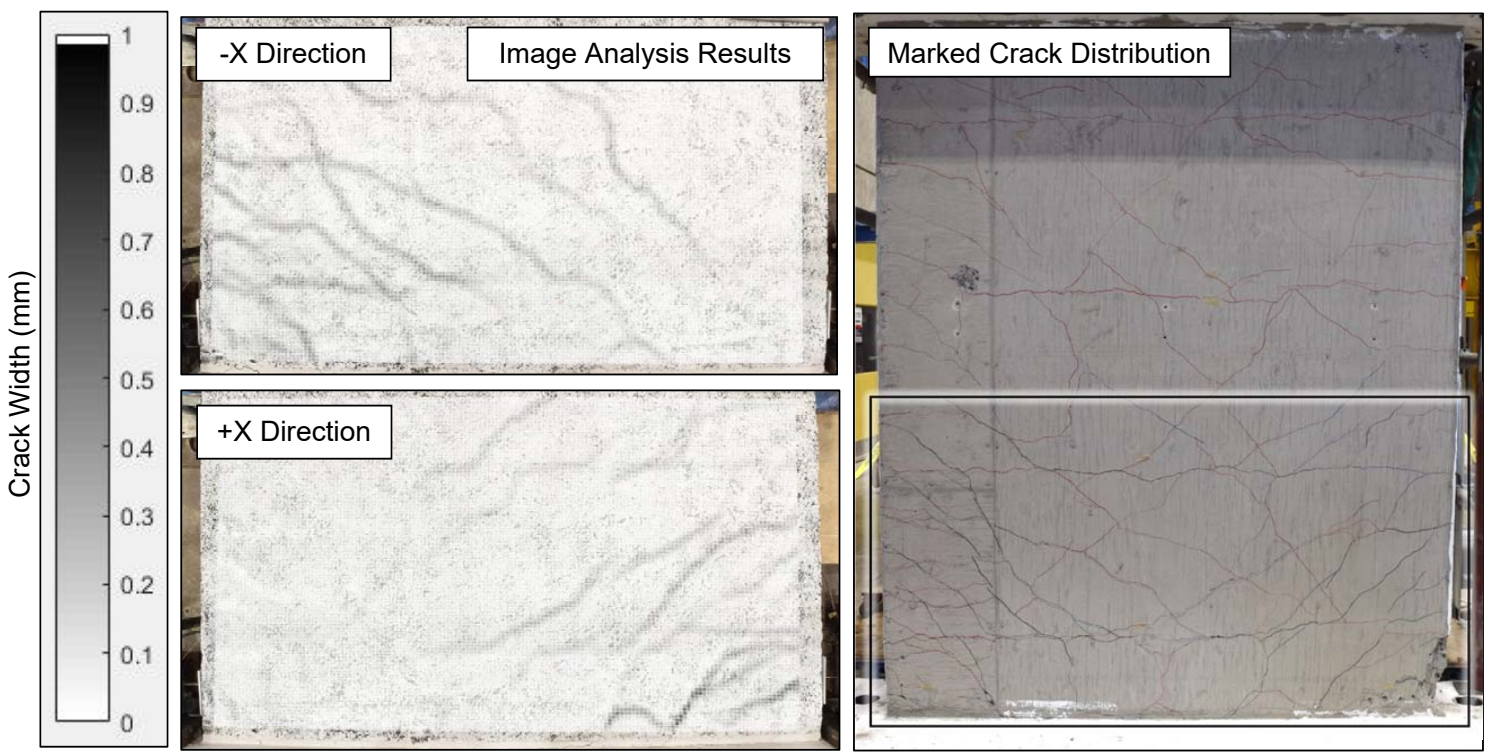

Figure 6.20 Comparison between analyzed and marked crack distributions 


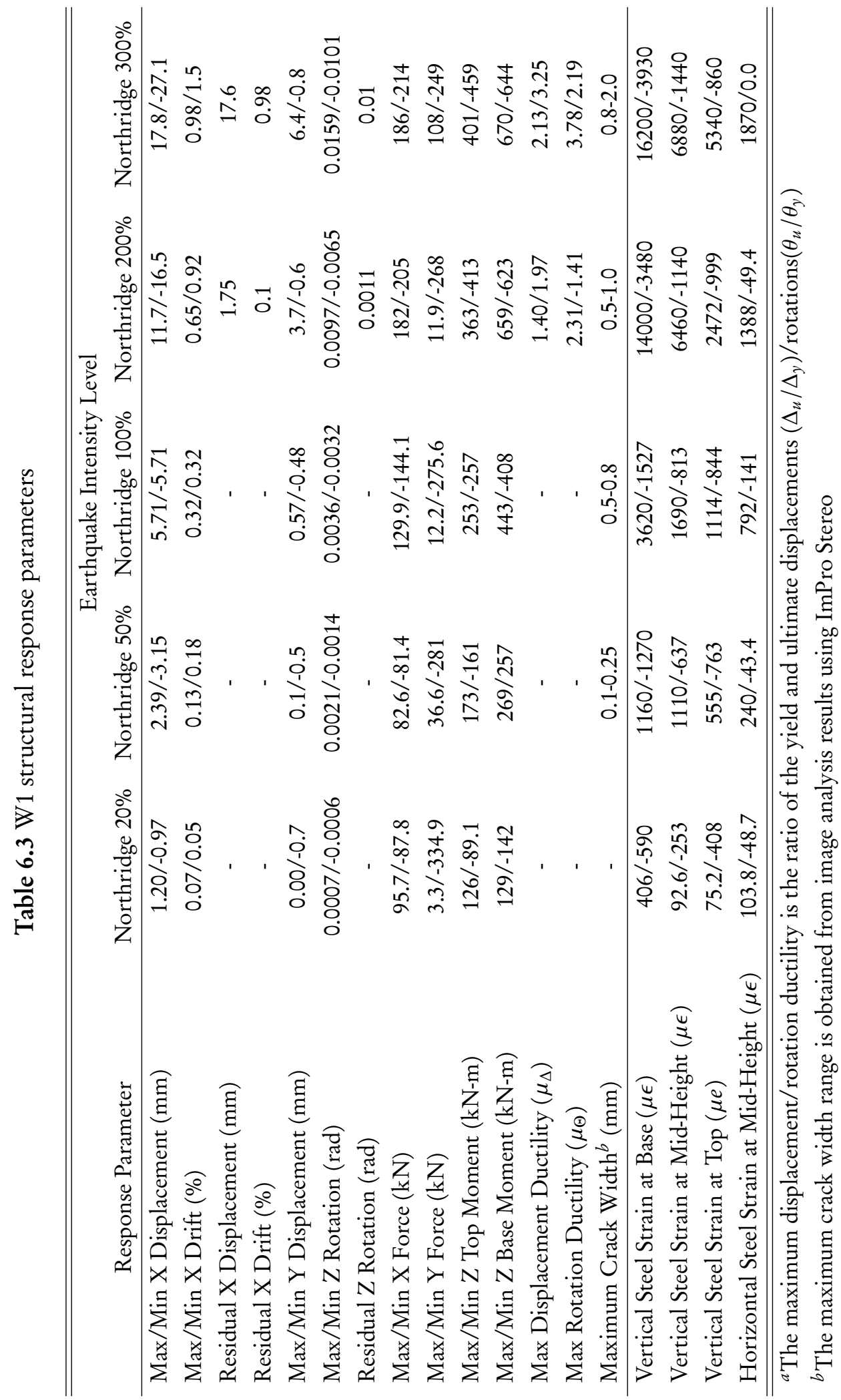




\subsubsection{Global Hybrid Simulation Results}

Figures A.13 to A.17 in Appendix A show the global response of the multi-storey RC shear wall including storey displacement, inter-storey drift, shear, and overturning moment timehistories for each ground motion intensity applied to the shear wall. Tables 6.4 and 6.5 summarize maximum response parameters for the shear wall for each ground motion.

To compare the global response of the three-storey $\mathrm{RC}$ shear wall under increasing ground motion intensity, Figure 6.21 compares the lateral displacement, inter-storey drift, acceleration, velocity, base shear, and overturning moment distribution plotted over the height of the shear wall for each ground motion. Results show that as expected, the top displacement increases as the intensity of the ground motion increases. The inter-storey drift is approximately uniformly distributed over the height of the shear wall with the exception of the two MCE level ground motions at $200 \%$ and $300 \%$ of the Northridge earthquake record, in which the second storey experiences a maximum inter-storey drift just above $2 \%$. The shear and moment distributions over the height of the structure show the influence of the elastic analytical substructure. At the $200 \%$ to $300 \%$ ground motion intensities, the overturning moment distributions show yielding in the first storey shear wall, evident by the change in slope in the moment distributions, which is also shown to limit the shear force to the ultimate in-plane capacity of the shear wall. Note that although the wall specimen yields in the $100 \%$ ground motion intensity, the shear force continues to increase under the $200 \%$ and $300 \%$ ground motion intensities because of the effects of strain hardening on the response of the experimental shear wall specimen.

Overall, the results are consistent with the expected behaviour of the multi-storey shear wall under increasing ground motion intensity. The hybrid test results confirm the feasibility in using hybrid simulation to capture the global response of a full-scale structure. The results also showcase the versatility of hybrid simulation in its ability to provide information related to the local behaviour and failure modes for a critical structural element while at the same time being able to examine the global response of a structure in terms of key structural response parameters.

Joshua Woods, Department of Civil and Environmental Engineering, Carleton University 

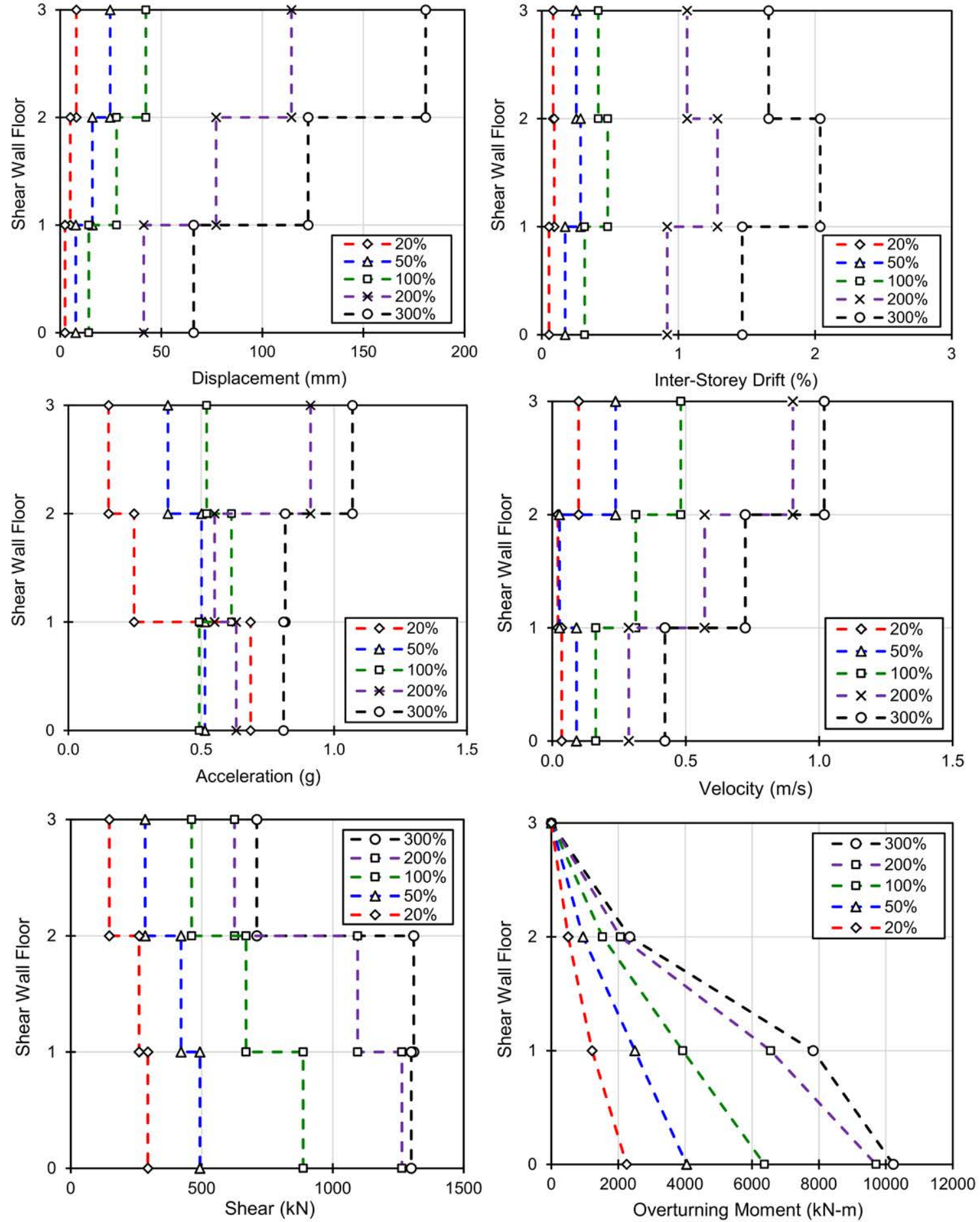

Figure 6.21 Comparison of global shear wall response values 


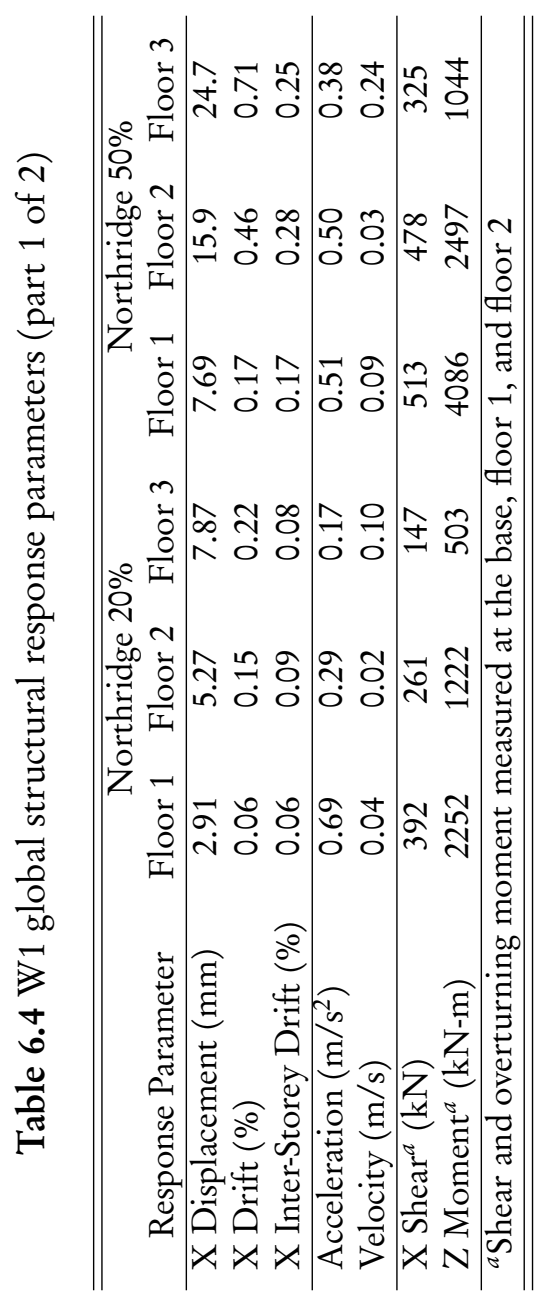




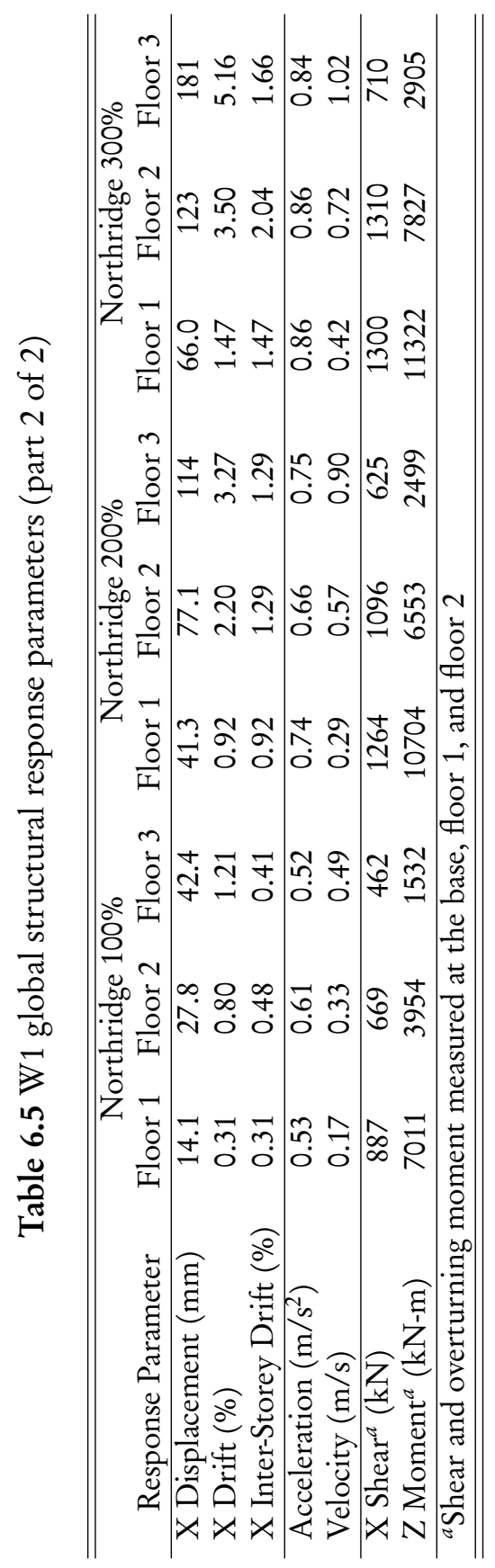




\subsection{Hybrid Simulation of Wall 2}

\subsubsection{Initial Stiffness of W2}

In the same manner as the first shear wall specimen, the initial stiffness matrix of the experimental substructure was determined first by subjecting the wall specimen to three low-amplitude reversed cyclic displacement time histories; one for each of the three degrees-of-freedom (DOFs). Figure 6.22 shows the test results in terms of the force-deformation or moment-rotation response in each of the three DOFs and Table 6.6 summarizes the test results and compares the measured initial stiffnesses with those for wall specimen W1 and the theoretical results. Results show that the initial in-plane, axial, and rotational stiffness of the shear wall specimen are $184 \mathrm{kN} / \mathrm{mm}$, $1093 \mathrm{kN} / \mathrm{mm}$, and $377.8\left(10^{3}\right) \mathrm{kN}-\mathrm{m} / \mathrm{rad}$, respectively. The results show that although the initial stiffness of specimen W2 is comparable to specimen W1, specimen W2 has a higher initial stiffness compared with wall specimen W1 in all three DOFs. The differences in the measured initial stiffnesses of the two wall specimens is attributed to the higher concrete strength measured during cylinder tests conducted on the second batch of concrete and the minor cracks observed near the base of wall specimen W1 prior to testing, which were a result of handling and posttensioning the experimental test setup.

Table 6.6 W2 Initial stiffness and yield displacement

\begin{tabular}{lccc}
\hline \hline & W2 Initial & W1 Initial & Theoretical \\
\multicolumn{1}{c}{ Loading Direction } & Stiffness & Stiffness & Prediction \\
\hline Lateral, $\mathrm{X}\left(K_{i}, x\right)(\mathrm{kN} / \mathrm{mm})$ & 184 & 93.7 & $1736^{a}\left(685^{b}\right)$ \\
Axial, $\mathrm{Y}\left(K_{i}, y\right)(\mathrm{kN} / \mathrm{mm})$ & 1093 & 750 & 2500 \\
Rotational, $\mathrm{RZ}\left(K_{i}, z\right)(\mathrm{kN}-\mathrm{m} / \mathrm{rad})$ & $377.8\left(10^{3}\right)$ & $204.2\left(10^{3}\right)$ & $1875\left(10^{3}\right)$ \\
\hline \hline
\end{tabular}

${ }^{a}$ Based on an in-plane stiffness of $12 E I / L^{3}$ assumption;

${ }^{b}$ Based on an in-plane stiffness of $3 E I / L^{3}$ assumption; 

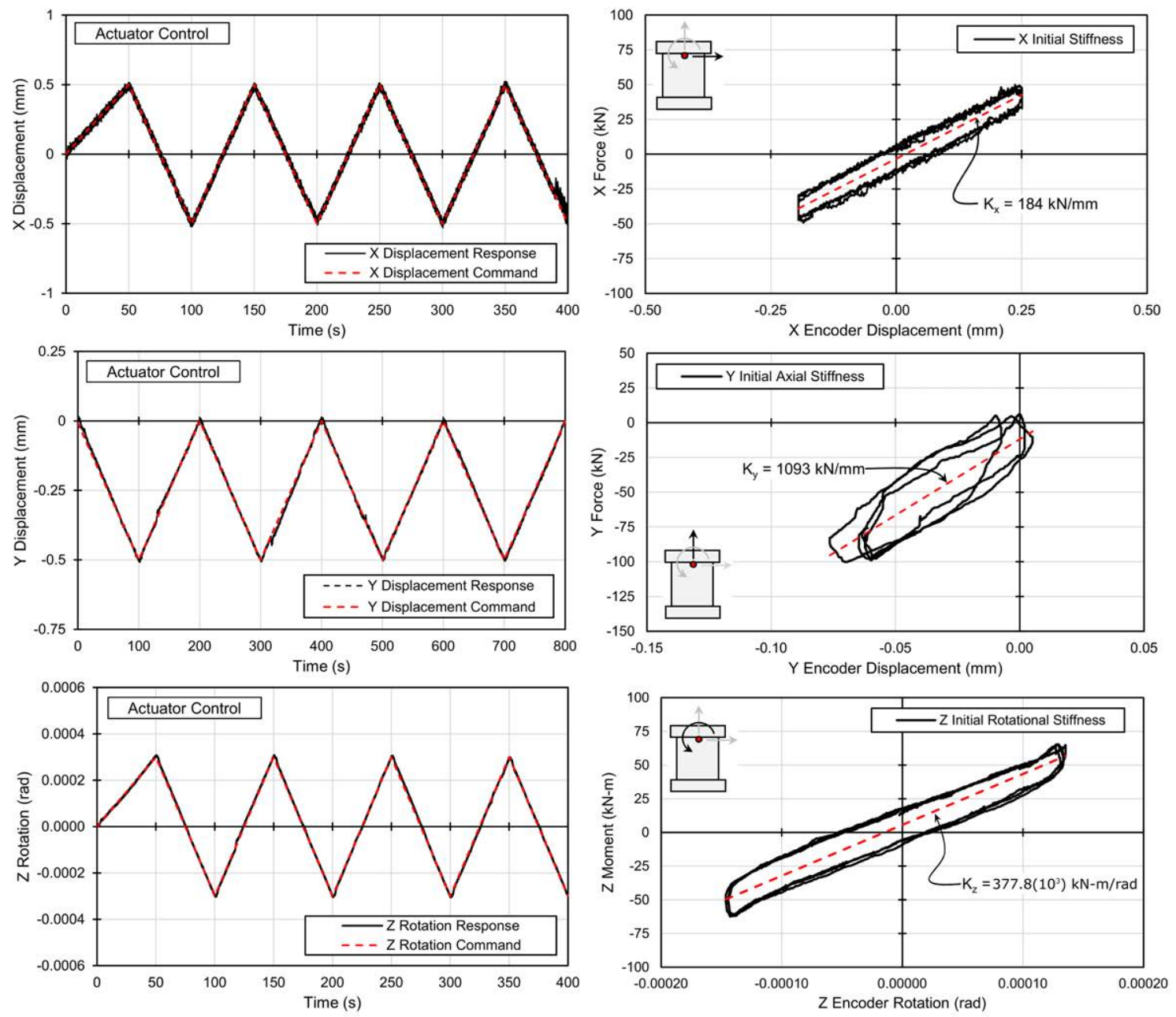

Figure 6.22 Shear wall W2 lateral, axial, and rotational initial stiffness 


\subsubsection{Local Hybrid Simulation Results}

This section describes the local response of the second experimental shear wall specimen, referred to as wall specimen W2. Wall specimen W2 had identical geometry and steel reinforcement ratios compared to wall specimen W1. The only difference between the hybrid tests conducted on shear wall specimens W1 and W2 was the analytical substructure. In contrast with the first set of hybrid tests conducted on specimen W1, the shell elements in the analytical model for the second set of hybrid tests were assigned nonlinear material models for the concrete and steel reinforcement as described in the multi-layer modelling approach discussed in Chapter 5. For each hybrid test on wall specimen W2, a complete set of results including X, Y, and Z displacements, rotations, forces, and moment time-histories as well as force-displacement and moment-rotation hysteretic responses are available in Figures A.6 to A.12 in Appendix A. Tables 6.7, 6.8, and 6.9 show critical structural response parameters for the wall specimen and maximum/minimum local response quantities for each hybrid test. The following subsections describe the local response of the experimental shear wall specimen for each earthquake ground motion record in more detail.

\section{Northridge Earthquake 20\%}

Wall specimen W2 was first tested under the Northridge Canoga Park earthquake record scaled to $20 \%$ of its original unscaled intensity. At this intensity level, this earthquake is representative of a very low magnitude OBE earthquake event. In a similar manner to specimen W1, it was expected that under this ground motion the shear wall would remain elastic and the test would serve as a way to verify the performance of the entire hybrid testing setup; including communication links between OpenFresco and the controller, image and data acquisition systems, and to verify command versus achieved displacement time histories. During the hybrid test, the shear wall specimen reached maximum and minimum displacements and rotations of $+0.78 \mathrm{~mm}$, $-0.86 \mathrm{~mm},+0.0057 \mathrm{rad}$, and $-0.0056 \mathrm{rad}$, respectively. Forces and moments at the base of the shear wall in the $\mathrm{X}$ and $\mathrm{Z}$ directions remained below $+/-90 \mathrm{kN}$ and $+/-200 \mathrm{kN}-\mathrm{m}$, well below the design strength of the wall specimen. No cracks were observed on the surface of the concrete during the test, confirming that the wall remained elastic.

Joshua Woods, Department of Civil and Environmental Engineering, Carleton University 
To gauge the performance of the hybrid system, the command and feedback time-histories and the error between the two are shown in Fig. 6.23. The results show that in general the system tracks relatively well when considering the small command displacements and rotations in combination with the high in-plane,rotational, and axial stiffness of the shear wall specimen. The $\mathrm{X}$ displacement and $\mathrm{Z}$ rotation errors, which are the difference between the command and feedback signals throughout the test remain below $+/-0.0625 \mathrm{~mm}$ and $+/-0.0015 \mathrm{rad}$. Once again, this low-intensity ground motion represents the most challenging control scenario, because the wall specimens has its highest stiffness prior to cracking and the target displacements commands are very small. Nonetheless, the hybrid system shows good tracking performance throughout the test.

To compare the performance of the hybrid system with the same hybrid test conducted on wall specimen $\mathrm{W} 1$, the $\mathrm{X}$ displacement error and $\mathrm{Z}$ rotation error, which are the differences between the command and feedback signals over time are compared for the two wall specimens. Figure 6.24 shows the error results for the $\mathrm{X}$ displacement and $\mathrm{Z}$ rotation feedback signals over the duration of the hybrid test. The results show that the command-feedback errors are larger for wall specimen W2 by an average of $0.025 \mathrm{~mm}$ in the $\mathrm{X}$ direction and $0.00005 \mathrm{rad}$ in the $\mathrm{Z}$ direction. The larger control errors observed in the response of wall W2 are attributed to the higher in-plane and rotational stiffnesses of wall specimen W2. The influence of the stiffness of the wall specimen on the command-feedback error will be compared again following the the final hybrid test conducted on shear wall specimen W2, when the stiffness of the wall specimen is much lower. This comparison will allow for a better understanding of the influence that stiffness has on the ability of the hybrid system to apply the target displacement and rotation to the experimental substructure.

\section{Northridge Earthquake 100\%}

After satisfactory performance of the hybrid testing system during the first hybrid test, the full unscaled Northridge Canoga Park earthquake record was applied to the three-storey shear 

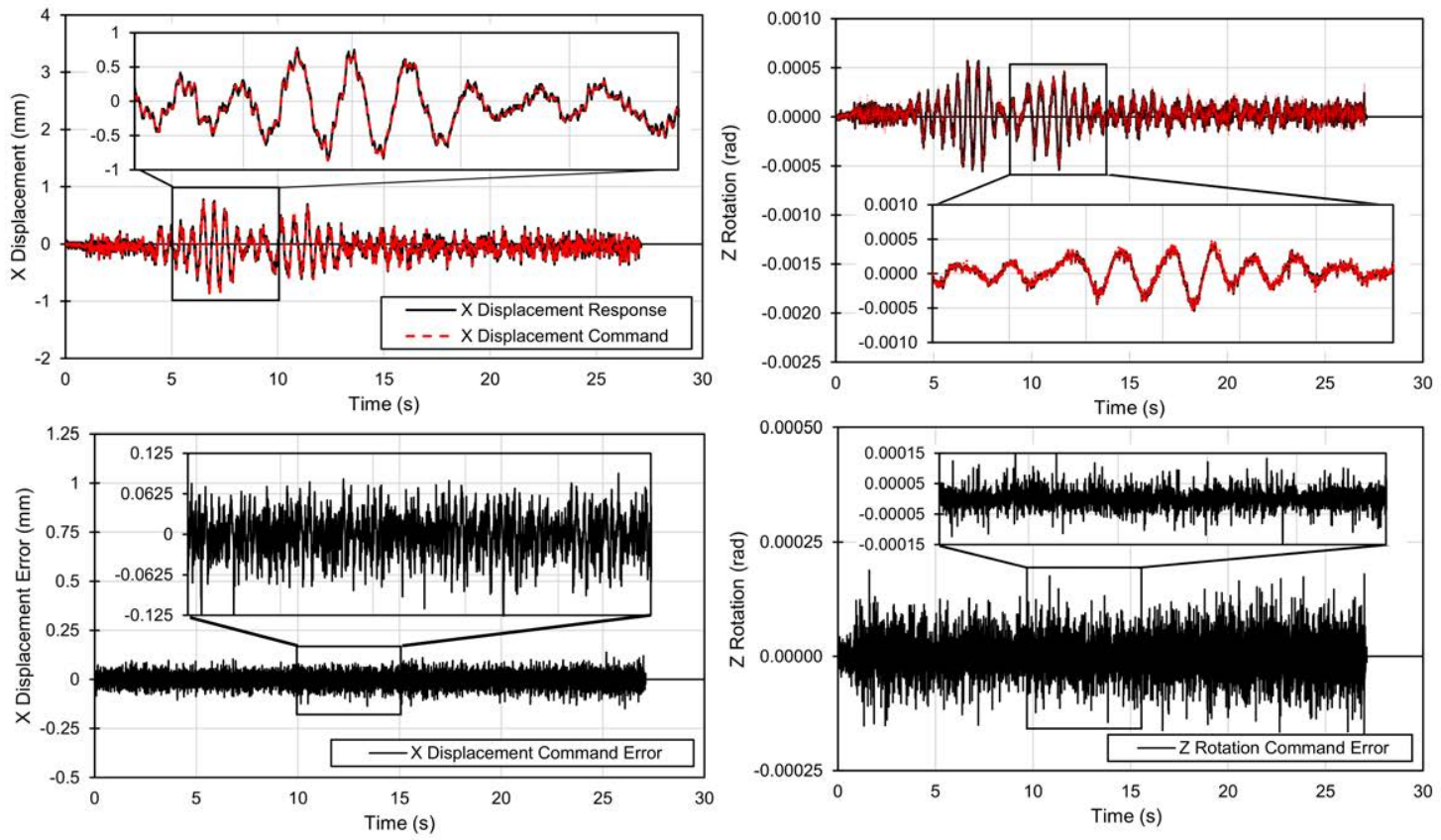

Figure 6.23 W2 Northridge 20\%: displacement and rotation command/feedback response
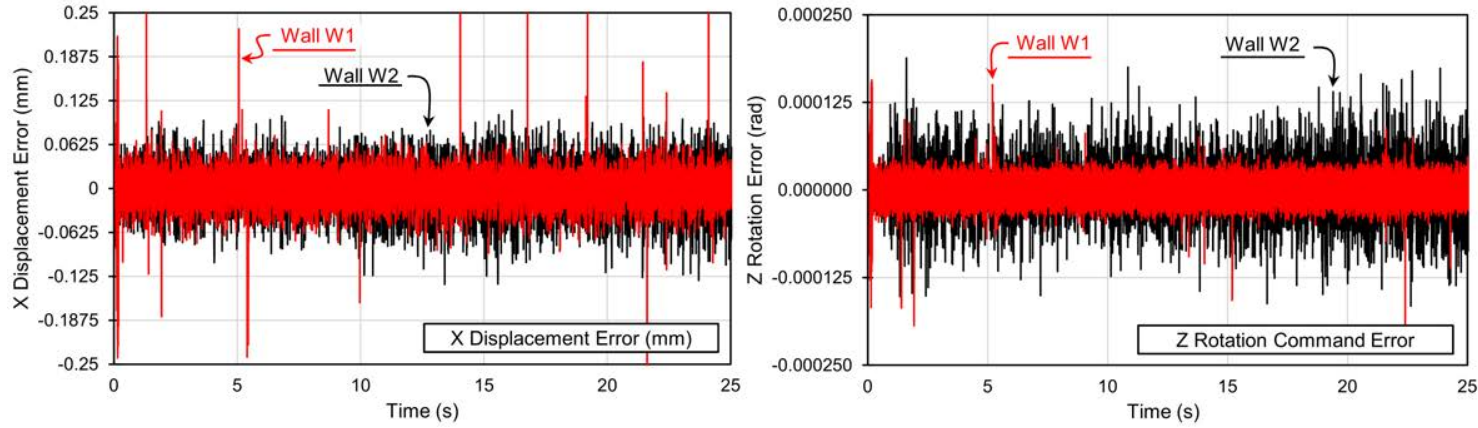

Figure 6.24 Comparison of $\mathrm{X}$ displacement and Z rotation error for specimens W1 and W2 
wall structure. This ground motion once again represents a design level (DBE) ground motion, in which a structure is expected to exhibit some nonlinearity in its response, including cracking of the concrete and yielding of the vertical steel reinforcement.

Figure 6.25 shows the moment-rotation hysteretic response and vertical steel reinforcement strain profiles for the hybrid test. Results show that the wall specimen did not yield during the hybrid test. Figure 6.26 shows the maximum strain profiles in the vertical steel reinforcement at the base of the wall and along the horizontal steel stirrups over the height of the shear wall. The results show that the vertical steel reinforcement along the base of the wall was very close to yielding in tension, reaching maximum tensile strains above $2400 \mu \epsilon$, while the horizontal steel reinforcement remained below its yield strain. The shear wall specimen reached a maximum and minimum displacements and rotations of $+5.04 \mathrm{~mm},-5.57 \mathrm{~mm},+0.004 \mathrm{rad}$, and $-0.0034 \mathrm{rad}$, respectively. Figure 6.27 shows the $e_{x y}$ surface strain field for select regions throughout the shear wall. The strain fields clearly show the formation of thin diagonal cracks, measuring from $0.1 \mathrm{~mm}-0.2 \mathrm{~mm}$ in the plastic hinge region of the shear wall. At the end of the test, the residual displacement was negligible and the residual rotation was small ( $0.00043 \mathrm{rad})$.
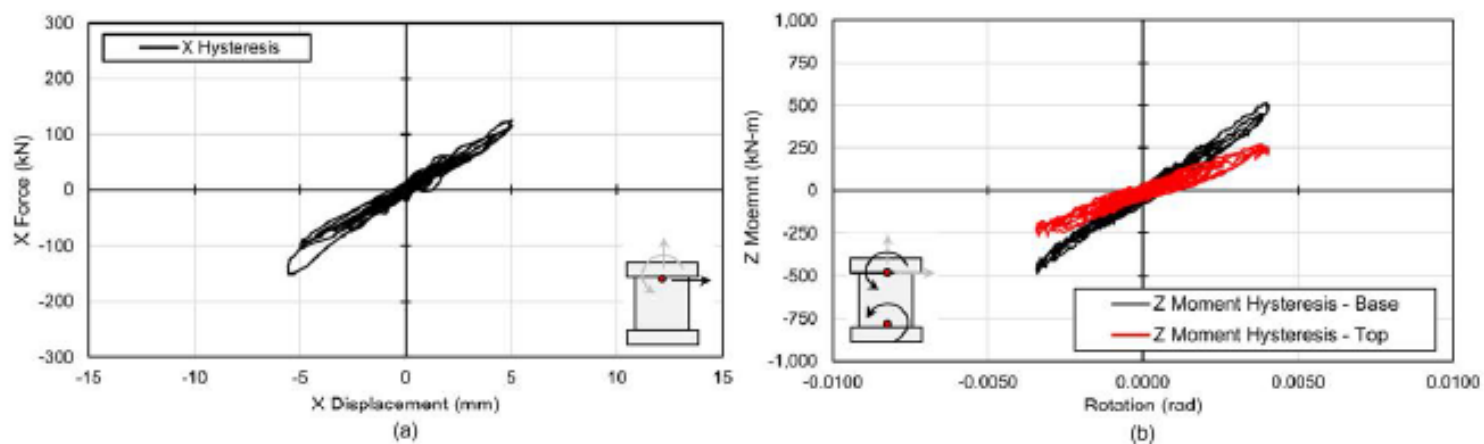

Figure 6.25 W2 Northridge 100\%: (a) force-displacement and (b) moment-rotation hysteretic responses 


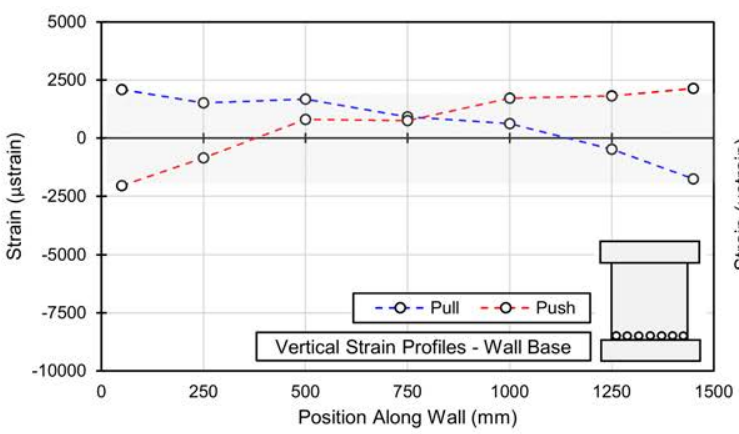

(a)

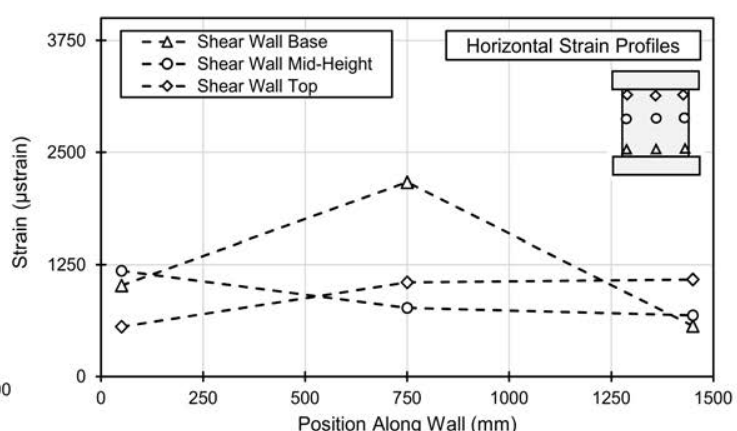

(b)

Figure 6.26 W2 Northridge 100\%: strain profiles: (a) vertical steel reinforcement at wall base; (b) horizontal steel reinforcement over wall height

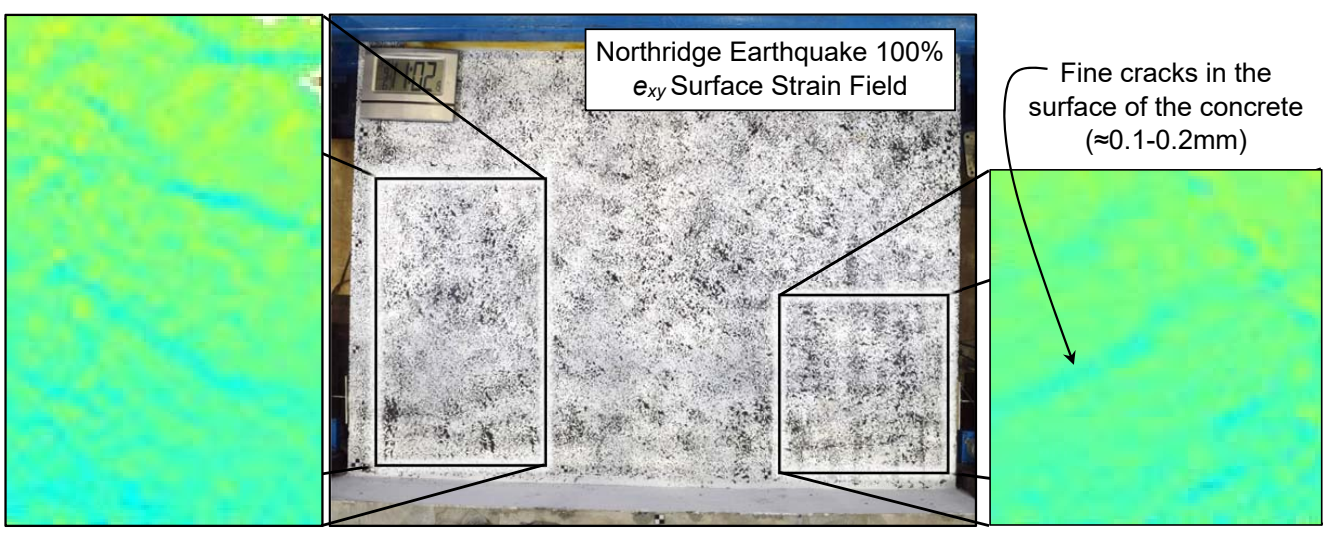

Figure 6.27 W2 Northridge 100\%: maximum crack width distribution 
Northridge Earthquake $200 \%$

In the third hybrid test, the Northridge Canoga Park ground motion was scaled to $200 \%$ of its original intensity and applied to the three-storey shear wall. At this intensity, the ground motion represents an earthquake event between the DBE and MCE hazard levels. Figure 6.28 shows the moment-rotation hysteretic response and the maximum vertical steel reinforcement strain profiles at the base of the wall. The results show that the shear wall specimen reached maximum and minimum displacements and rotations of $+/-7.0 \mathrm{~mm}$ and $+/-0.005 \mathrm{rad}$, which correspond to displacements and rotations very close to the yield displacement and rotation for wall specimen W1. The moment-rotation hysteretic response shows some minor nonlinearity in the response of the wall specimen, characterized by the widening of the hysteretic loops. The maximum vertical steel reinforcement strain profiles in Fig. $6.28 \mathrm{~b}$ show that the layers of vertical steel reinforcement closest to the ends of the shear wall yielded in tension.

\section{Northridge Earthquake $300 \%$}

The fourth earthquake record applied to the shear wall specimen was the Northridge Canoga Park Station ground motion record at 3 times its original unscaled intensity. At this intensity, the ground motion is characterized as an MCE event. This test was expected to cause significant damage to the shear wall specimen in a similar manner to wall specimen W1, however, because of the formation of other plastic hinges at the second and third storeys in the nonlinear

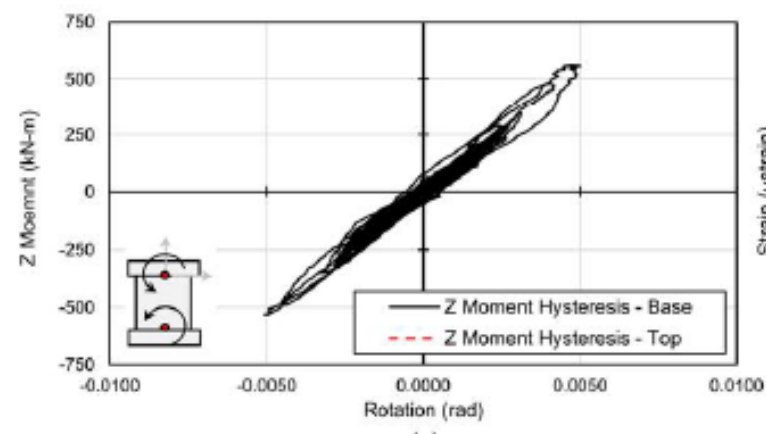

(a)

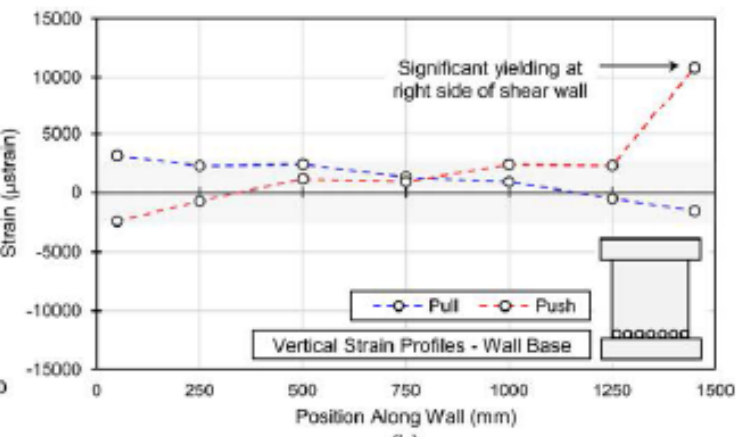

(b)

Figure 6.28 W2 Northridge 200\%: (a) moment rotation hysteresis; (b) vertical steel strain profile at wall base 
finite element model, the demand on wall specimen W2 was lower when compared to wall specimen W1. As a result, the wall specimen maintained significant strength following the hybrid test, which allowed for additional tests to be conducted on the wall specimen. Further discussion on the nonlinear response of the analytical substructure will be discussed in the following section.

Figure 6.29 shows the $\mathrm{X}$ displacement time history, $\mathrm{X}$ force-displacement hysteretic response, $\mathrm{Z}$ rotation time history, and $\mathrm{Z}$ moment-rotation hysteretic response for the duration of the earthquake record. The hysteretic response behaviours show the yielding of the wall specimen at an average $\mathrm{X}$ displacement of $+/-7.99 \mathrm{~mm}$ and average $\mathrm{Z}$ rotation of $+/-0.0044 \mathrm{rad}$. The wall specimen reached a maximum displacement of $10.2 \mathrm{~mm}(0.56 \%$ drift $)$ and rotation of $0.007 \mathrm{rad}$. This corresponds to displacement and rotational ductilities of 1.34 and 1.70 , respectfully. A residual displacement of $0.67 \mathrm{~mm}(0.04 \% \mathrm{drift})$ and rotation of $0.00045 \mathrm{rad}$ were observed following the test. Significant yielding of the vertical steel reinforcement at the top and bottom of the wall specimen were observed during the test, reaching maximum vertical strains of $10800 \mu \epsilon$ at the base of the wall and $4700 \mu \epsilon$ at the top of the wall. The maximum moments at the base of the wall were $+646 \mathrm{kN}-\mathrm{m}$ and $-664 \mathrm{kN}-\mathrm{m}$, respectively, which are close to the design moment capacity of $610 \mathrm{kN}$ - m predicted using strain compatibility analysis.

\section{Northridge Earthquake $100 \%$}

Following the MCE event, the unscaled Northridge Canoga Park record was applied to the hybrid model again. This test was intended to represent a modest aftershock following a significant earthquake. Application of the same Northridge Canoga Park station ground motion record as an aftershock earthquake allowed for comparison with the results from the earlier test at the same earthquake intensity. This comparison would allow for a characterization of the changes in the behaviour of the structure as a result of the cumulative damage to the experimental substructure. Figure 6.30 shows the $\mathrm{X}$ displacement time history, $\mathrm{Z}$ rotation time history, $\mathrm{X}$ forcedisplacement hysteretic response, and $\mathrm{Z}$ moment-rotation hysteretic response behaviours of the shear wall during the earthquake aftershock. The observed behaviour of the wall specimen clearly shows the effects of the $200 \%$ and $300 \%$ earthquake events on the response of the wall specimen, 

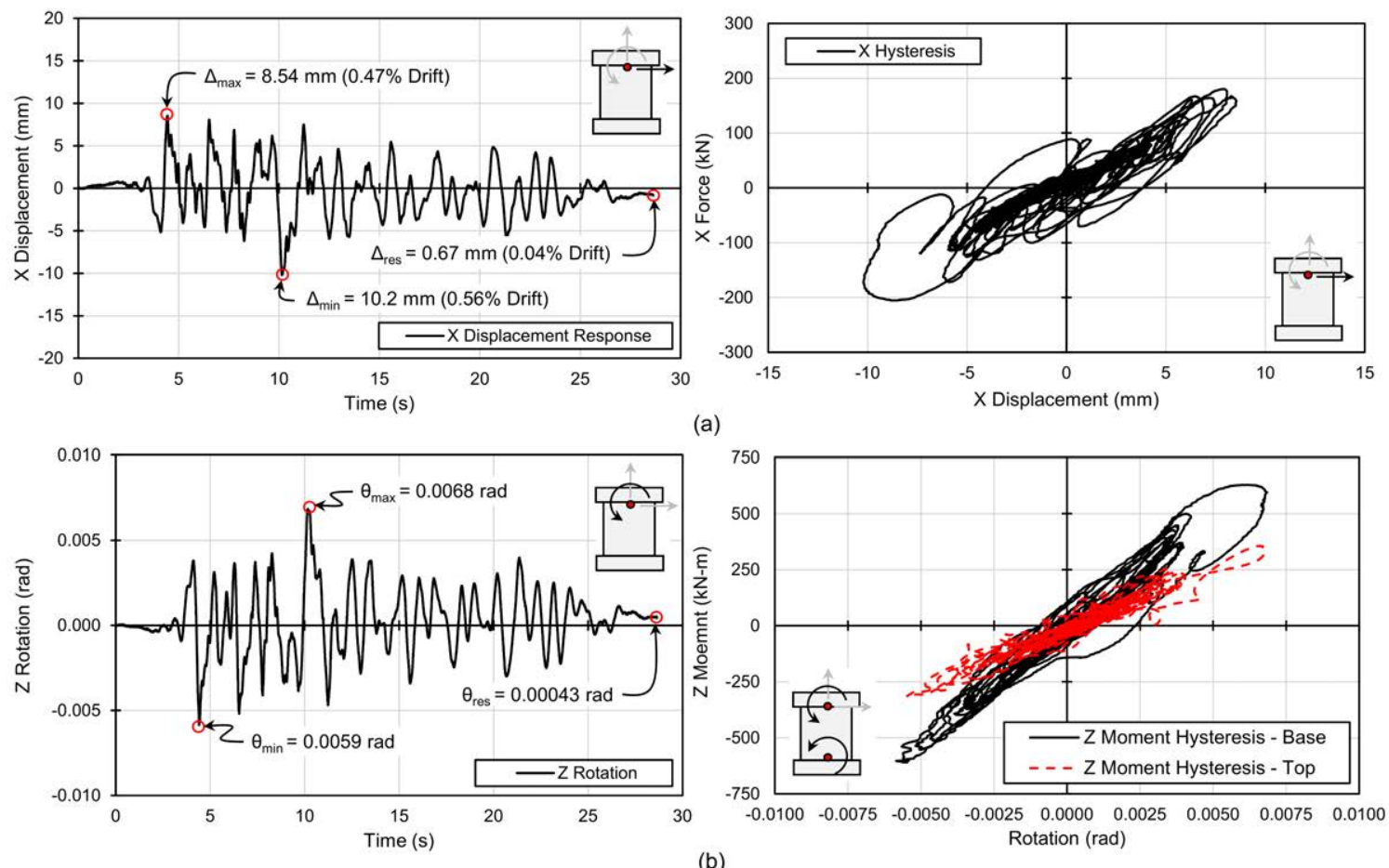

(b)

Figure 6.29 W2 Northridge 300\%: (a) X displacement time history and force displacement hysteresis; (b) Z rotation time history and moment rotation hysteresis

which has lower in-plane and rotational stiffnesses resulting in higher displacements and rotations during the aftershock when compared with the initial event. During the aftershock, the wall specimen reaches an average maximum displacement and rotation of $+/-6.37 \mathrm{~mm}$ and $+/-0.0041 \mathrm{rad}$, respectively, compared with $+/-5.31 \mathrm{~mm}$ and $+0.0037 \mathrm{rad}$ during the initial earthquake. However, because the level of damage to the wall specimen was lower than expected following the MCE (Northridge 300\%), the wall specimens behaviour is comparable to the initial event, and the shear wall survives the earthquake with only minor damage. Nonetheless, the comparison does demonstrate the reproducibility of the test results using the hybrid system.

\section{Kobe Earthquake 50\%}

The final two ground motions applied to the shear wall were DBE events intended to increase the level of damage to the shear wall specimen and study the effect of different ground motion records including different frequency contents and durations on the response of the shear 

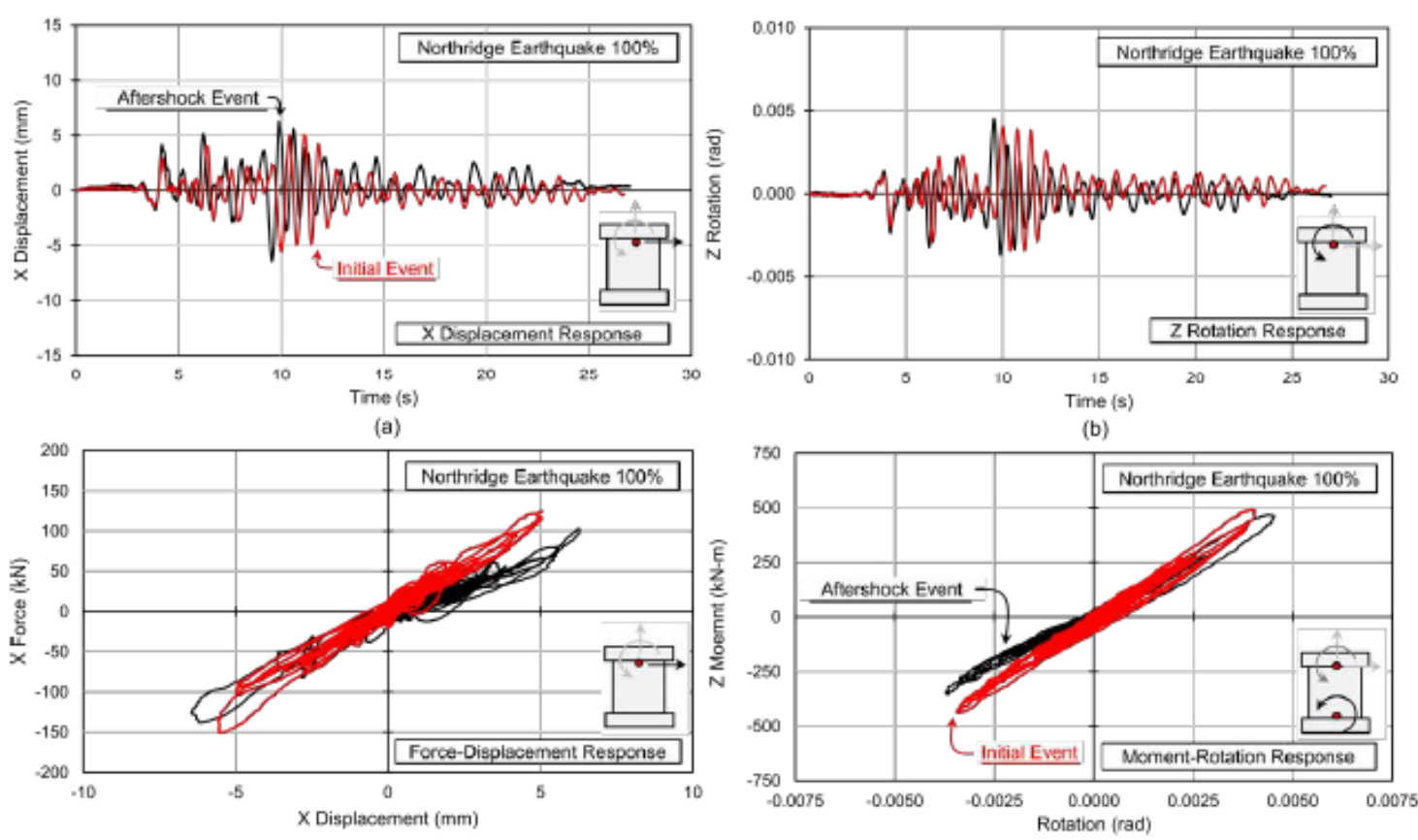

(c)

(d)

Figure 6.30 Northridge 100\%: response comparison for initial and aftershock earthquakes

wall. The first alternative ground motion was the JR Takatori earthquake record from the 1995 Kobe earthquake in Japan scaled to $50 \%$ of its original intensity. Figure 6.31 shows the top displacement, rotation, shear force, top moment and bottom moment time histories for the wall specimen. During the earthquake, the wall specimen reached a maximum moment of $441 \mathrm{kN}-\mathrm{m}$ and $618 \mathrm{kN}-\mathrm{m}$ at the top and bottom of the shear wall, respectively. The ground motion applied to the shear wall has a large pulse, which produced maximum and minimum displacements and rotations of $+8.27 \mathrm{~mm},-8.01 \mathrm{~mm},+0.0054 \mathrm{rad}$, and $-0.0051 \mathrm{rad}$, exceeding the yield moment and rotation of the shear wall. These displacements and rotations correspond to maximum displacement and rotational ductilities of 1.1 and 1.35 , respectively. The large displacement and rotation pulse resulted in a residual displacement of $1.53 \mathrm{~mm}$ ( $0.09 \% \mathrm{drift})$ and rotation of $0.0009 \mathrm{rad}$. Figure 6.32 shows the crack distribution from image analysis at the maximum positive and negative displacement. The results show a large number of horizontal and diagonally oriented cracks in the concrete ranging in width between $0.25 \mathrm{~mm}$ and $0.4 \mathrm{~mm}$. 

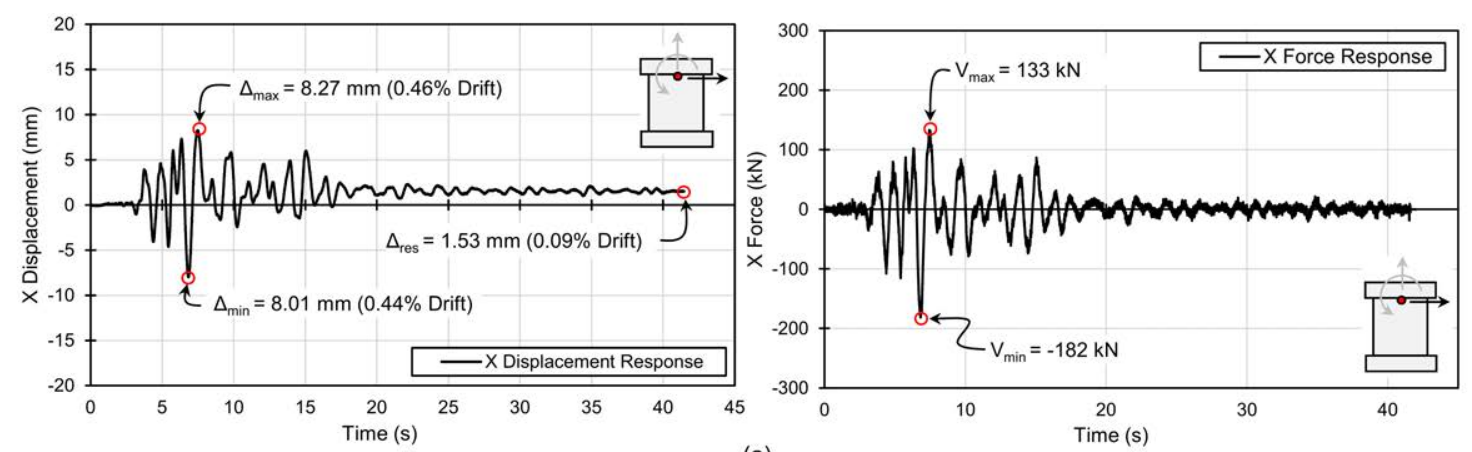

(a)
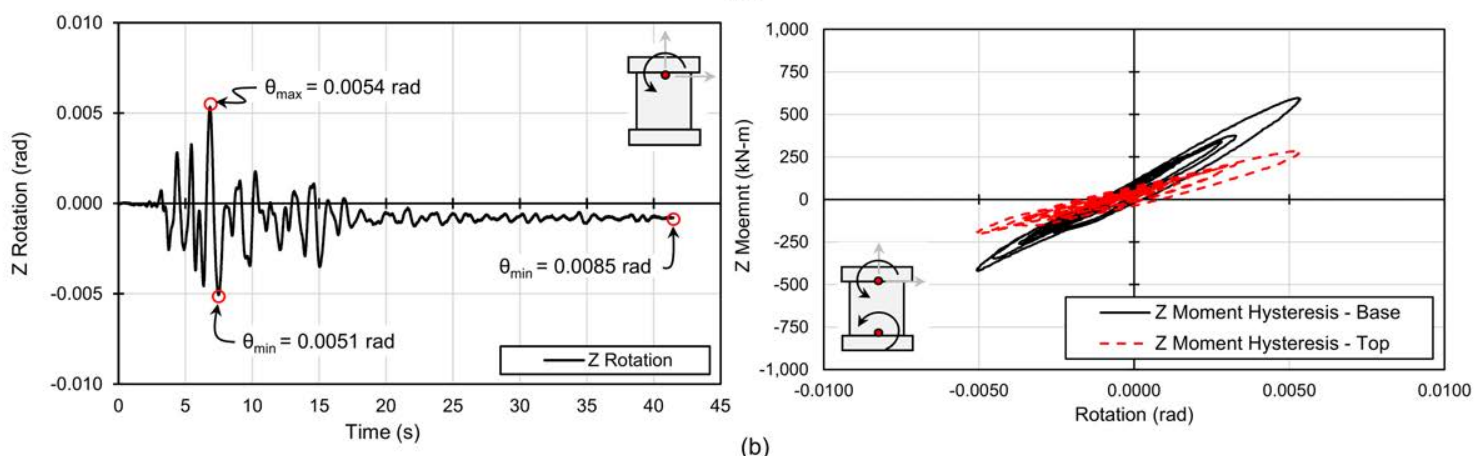

(b)

Figure 6.31 W2 Kobe 50\%: (a) X displacement and shear force time histories; (b) Z rotation and moment-rotation hysteresis
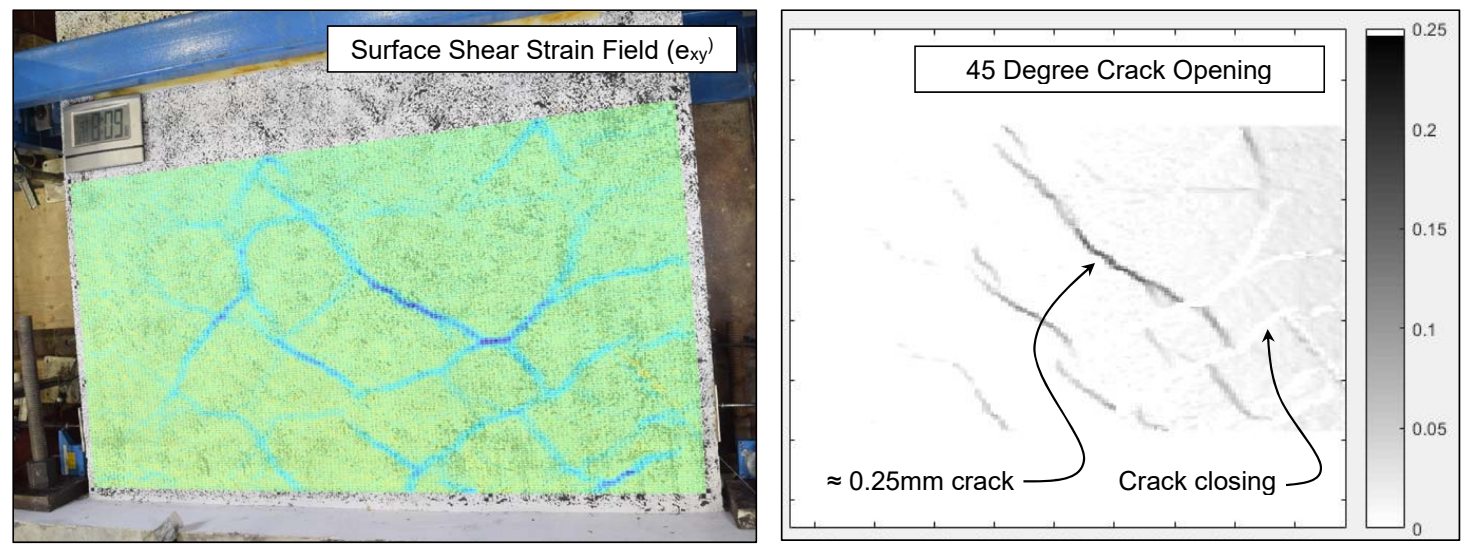

Figure 6.32 W2 Kobe 50\%: maximum crack width distributions 


\section{Loma Prieta $150 \%$}

The final earthquake applied to the wall specimen W2 was the Gilroy Array \#5 ground motion record from the 1989 Loma Prieta earthquake scaled to $150 \%$ of its original intensity. At this intensity level, the ground motion represents an earthquake which falls somewhere between the DBE and MCE hazard levels. Figure 6.33 shows the moment-rotation hysteretic response behaviour and the maximum strain profiles in the vertical steel reinforcement at the base of the shear wall. During the hybrid test, the wall specimen reached a maximum displacement of $10.93 \mathrm{~mm}$ (1.0\% drift) and rotation of $0.007 \mathrm{rad}$. This corresponds to displacement and rotational ductilities of 1.46 and 1.40 respectfully. A residual displacement of $1.28 \mathrm{~mm}(0.07 \%$ drift $)$ and rotation of 0.0005 rad were observed following the test. The maximum vertical steel reinforcement strain profiles at the base of the wall specimen shown in Fig. 6.33 also show the nonlinearity in the response of the wall specimen, as the steel reinforcement at the bottom of the wall reaches a maximum vertical strain of $13000 \mu \epsilon$ near the ends of the wall specimen. At the top and middle of the shear wall, strain gauges show that the steel reinforcement yielded over the height of the wall, reaching maximum strains of $5120 \mu e$ and $5660 \mu \epsilon$, respectively near the edges of the shear wall. Figure 6.34 shows the maximum concrete crack distribution during the Loma Prieta hybrid test. The results show extensive diagonal cracking throughout the surface of the wall specimen. Maximum crack widths measured using image analysis ranged between $0.5 \mathrm{~mm}$ and $0.75 \mathrm{~mm}$ during

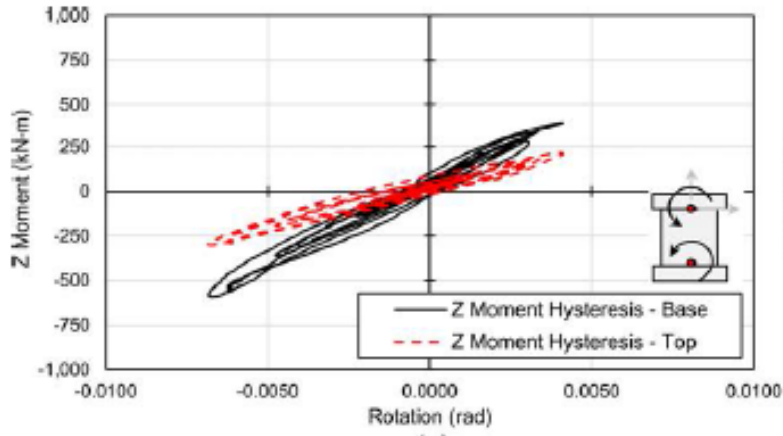

(a)

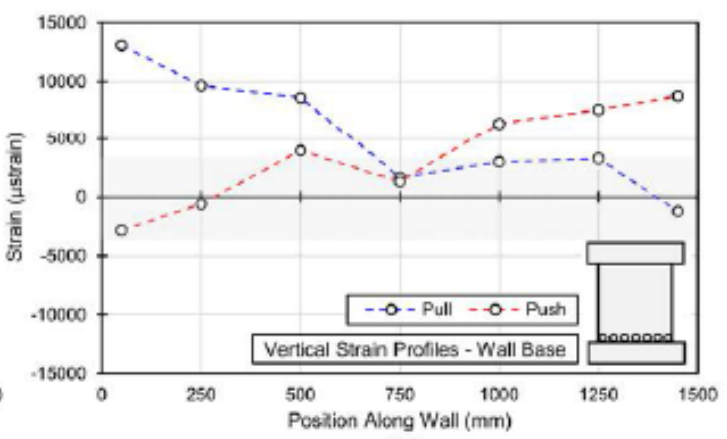

(b)

Figure 6.33 W2 Loma Prieta 150\%: (a) Z moment rotation hysteresis; (b) maximum vertical steel reinforcement strain profiles at shear wall base 

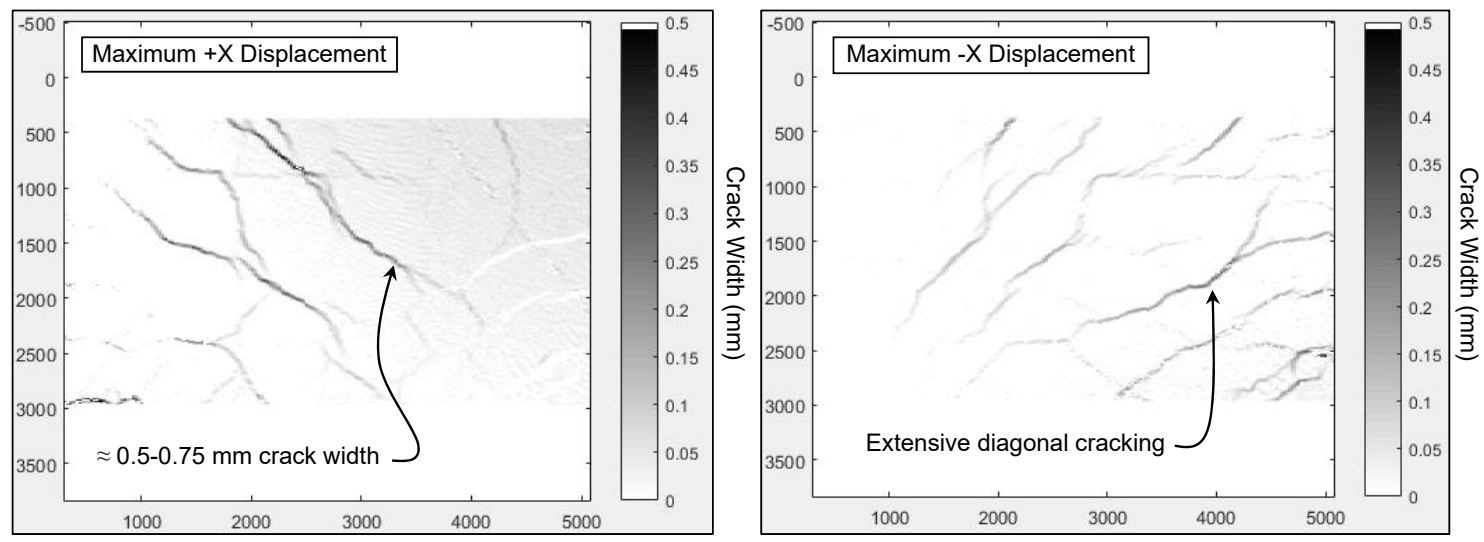

Figure 6.34 W2 Loma Prieta 150\%: concrete crack distribution

the test.

One of the important measures of the performance of the hybrid system is the error between the command and feedback signals sent to the experimental substructure. A previous comparison between the $\mathrm{X}$ displacement and $\mathrm{Z}$ rotation command and feedback signal errors between wall specimens W1 and W2 for the Northridge 20\% earthquake indicated that the stiffness of the experimental substructure could play a role in the level of error between the command and feedback signals. To further study the influence of stiffness on the command-feedback error, Fig. 6.35 compares the command-feedback error for the Northridge $20 \%$ ground motion, when the stiffness of specimen W2 was at its highest, with the command-feedback error during the Loma Prieta $150 \%$ ground motion. At the beginning of the Loma Prieta hybrid test, the wall specimen had approximately $66.1 \%$ lower in-plane stiffness, $80 \%$ lower axial stiffness, and $69.1 \%$ lower rotational stiffness. The results of the comparison show that on average the error between the command and feedback displacements and rotations are significantly smaller when the stiffness of the experimental test specimen was lower. The results show that the command-feedback errors were approximately $25 \%$ and $50 \%$ for $\mathrm{X}$ and $\mathrm{Z}$ directions, respectively during the Loma Prieta $150 \%$ earthquake. These results suggest that the stiffness of the experimental substructure does have an impact on the ability of the hybrid system to achieve target command displacements and rotations during the test. 

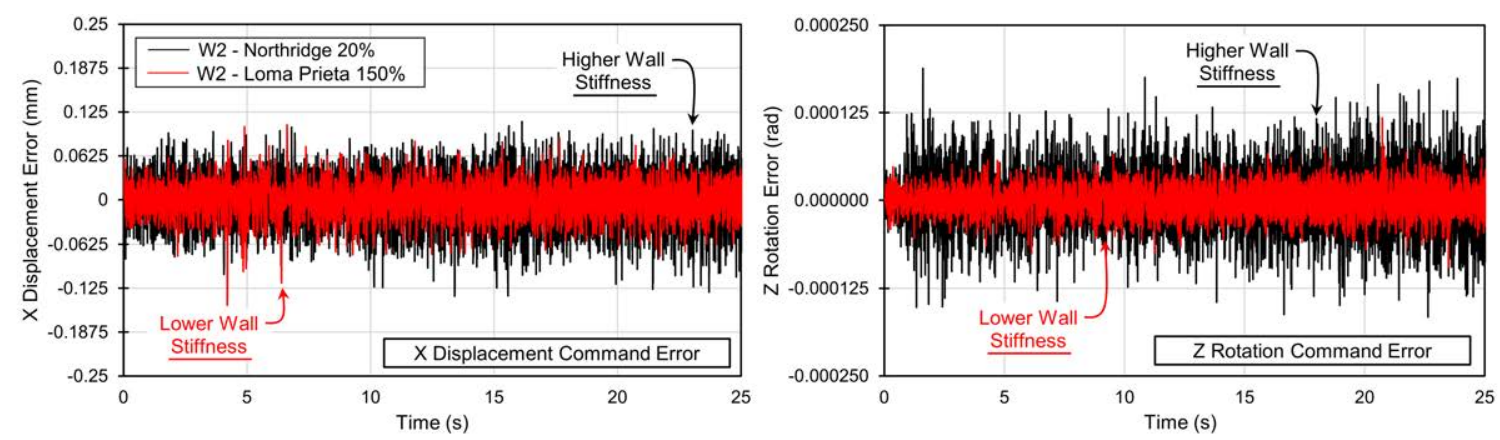

Figure 6.35 Comparison of $\mathrm{X}$ displacement and $\mathrm{Z}$ rotation error for specimen W2

In addition to error between the $\mathrm{X}$ displacement and $\mathrm{Z}$ rotation command and feedback signals, the loss in stiffness also influences the ability of the hybrid system to track the gravity load applied to the wall specimen in the experimental substructure. Figure 6.36 compares the gravity load response for wall specimen W2 during the Northridge 20\% and Loma Prieta 150\% earthquakes. The axial stiffness of the shear wall was approximately $80 \%$ lower compared with the initial axial stiffness during the Northridge 20\% earthquake. The results of the comparison show that the gravity load maintains a more constant load profile when the axial stiffness of the experimental substructure is lower. The average error between the measured and target $\mathrm{Y}$ force is reduced from $10.9 \mathrm{kN}$ to $0.83 \mathrm{kN}$ as a result of the loss in stiffness of the wall specimen. It should be noted that the actuator tuning parameters or actuator servo-valve size could also have an impact on the observed performance of the hybrid test system. The actuator tuning parameters were not modified throughout the testing program to ensure consistency between hybrid tests. The servovalves on both vertical actuators are 90GPM valves, which is very large when compared with the flow demand («30GPM) throughout the hybrid tests. Smaller servo-valves on the two vertical actuators could potentially have produced better control in such a slow hybrid test. Nonetheless, these results confirm that the stiffness of the experimental substructure does influence the ability of the hybrid system to hydraulically control the experimental substructure during the hybrid test. This means that for larger shear wall specimens or stiffer structural elements, additional measures including higher precision sensors may be required to improve the control capabilities of the hybrid system. 

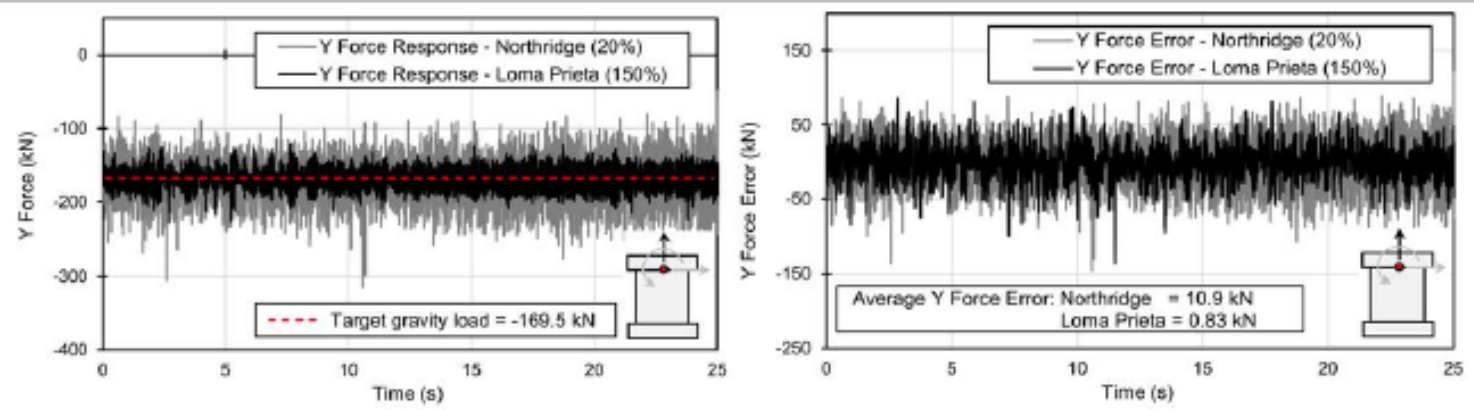

Figure 6.36 Comparison of gravity load (Y-force) response during Northridge $20 \%$ and Loma Prieta $150 \%$ hybrid tests

\section{Cyclic Test}

Following the final hybrid test, the level of damage to specimen W2 was lower when compared to the level of damage to wall specimen W1. This was due in large part to the nonlinearity in the analytical substructure during the Northridge $200 \%$ and $300 \%$ earthquakes, the results of which will be discussed in the following section. To achieve a similar damaged state for the repair and retrofit of the wall specimen using CFRP sheets, planned for a parallel study, a quasi-static reversed cyclic displacement series was applied to the top of wall specimen W2 without gravity load. The loading protocol consisted of displacement cycles at $+/-5 \mathrm{~mm},+/-10 \mathrm{~mm},+/-15 \mathrm{~mm}$, and $+/-20 \mathrm{~mm}$, designed to achieve comparable drift levels to wall specimen W1. The application of a cyclic displacement time history to the top of the wall specimen also allows for characterization of the nonlinear hysteretic behaviour of the wall that can be used to verify analytical models developed in future studies. Figure 6.37 shows the displacement and force time histories and the force-displacement and moment-rotation hysteretic responses measured during the test. The results show the nonlinearity in the response of the wall and wide hysteretic loops associated with the flexurally ductile response of a RC shear wall. During the test, the specimen reaches an average maximum drift and rotation of $1.05 \%$ and $0.01 \mathrm{rad}$, respectively, corresponding to average displacement and rotational ductilities of 2.59 and 2.30 respectively, which are comparable to those achieved during the hybrid tests conducted on wall specimen W1.

Figure 6.38 shows the maximum measured strains in the vertical steel reinforcement at the 

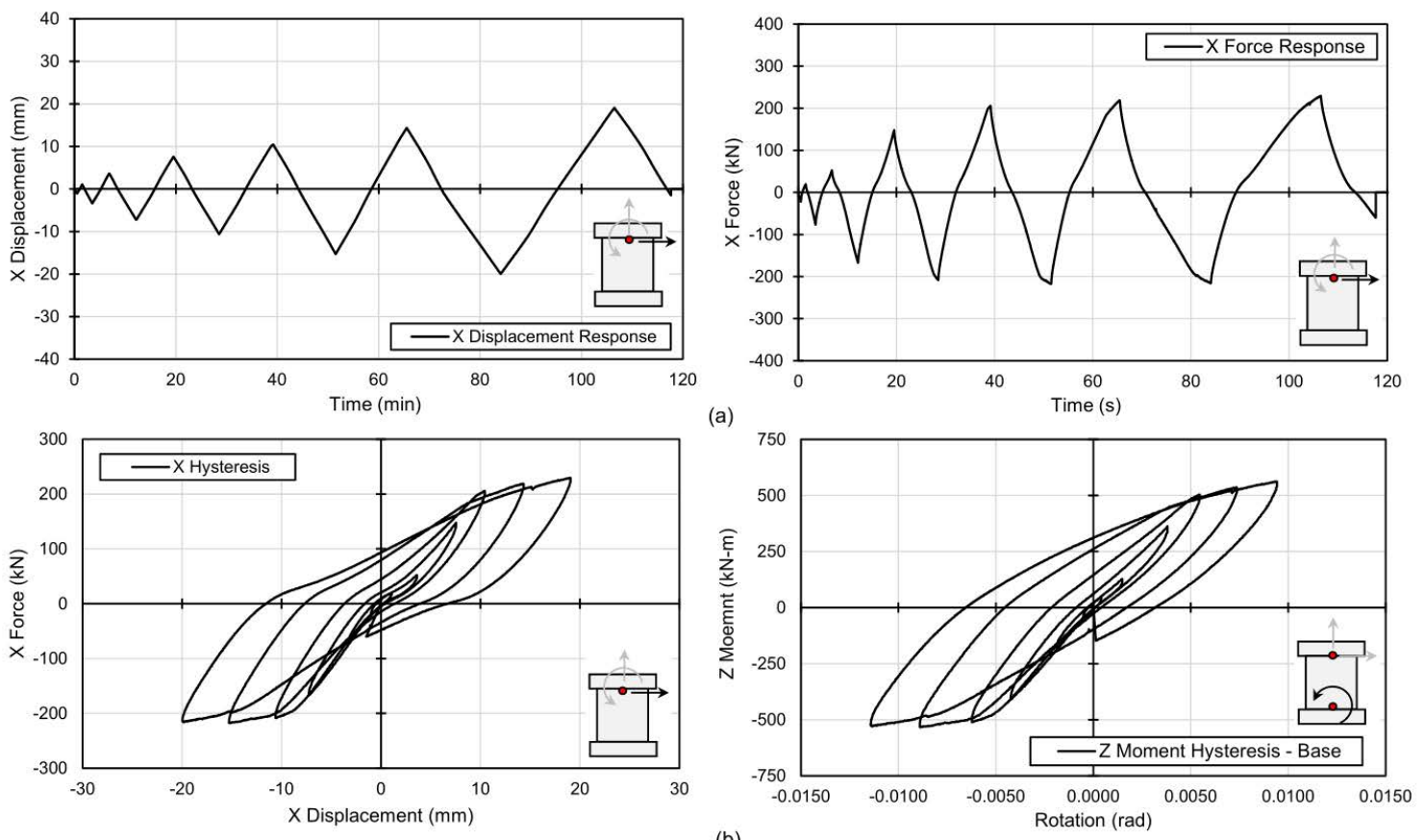

Figure 6.37 (a) lateral displacement and force time histories; (b) force displacement and moment rotation hysteretic responses
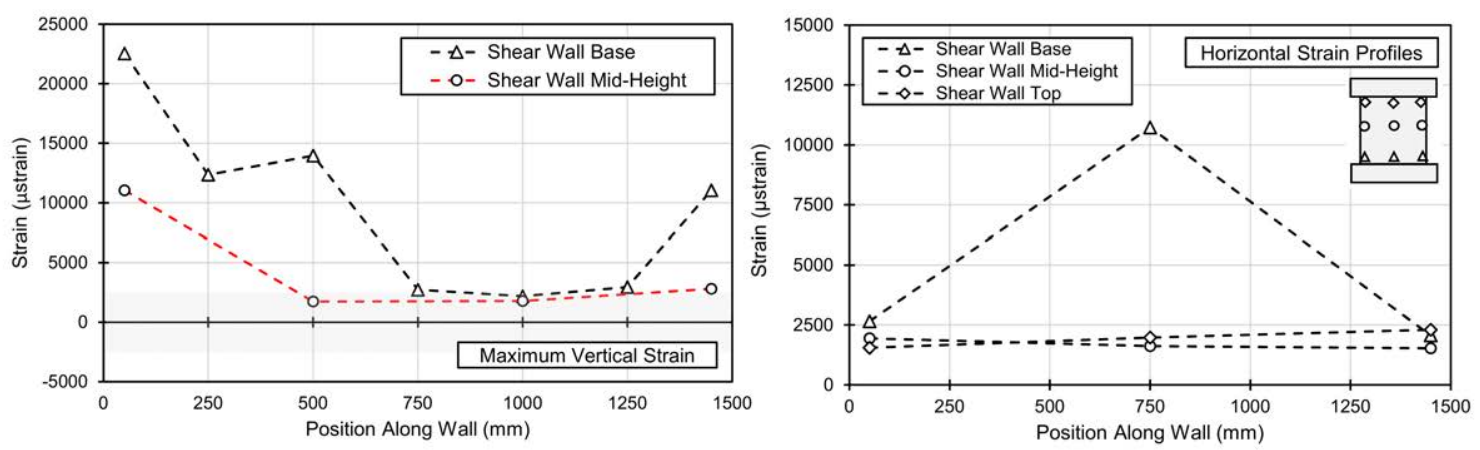

Figure 6.38 Maximum measured strain values: (a) vertical steel reinforcement at wall base and mid-height; (b) horizontal steel reinforcement over wall height 
base and mid-height of the shear wall as well as along the horizontal steel reinforcement at three different locations along the shear wall height. The results show that the strain in the vertical reinforcing bars are above the yield strain across the entire cross section of wall specimen W2 at its base and at mid-height. Yielding of the steel reinforcement is also observed in the stirrups near the base of the wall, where a number of large diagonal cracks were observed in the concrete. Figure 6.39 shows the damage to the shear wall specimen following the cyclic test. In a similar manner to wall specimen W1, crushing of the concrete in one of the toes of the shear wall was observed, suggesting the shear wall exceeded its diagonal compression shear strength after failing in flexure. In addition to crushing at the base, a number of very wide cracks $(>1.5 \mathrm{~mm})$ were observed during the test and confirmed after analyzing the image analysis results. Figure 6.40 compares the manually marked crack distribution on the surface of the concrete shear wall after being removed from the test setup with the analyzed crack distributions using ImPro Stereo. The results once again show good correlation between the marked and analyzed crack distributions.

\subsubsection{Global Hybrid Simulation Results}

In addition to results from the local response of the experimental shear wall element, the use of hybrid simulation also captures the global response of the entire three-storey RC structure. As a result, detailed information related to the dynamic response of the three-storey shear wall

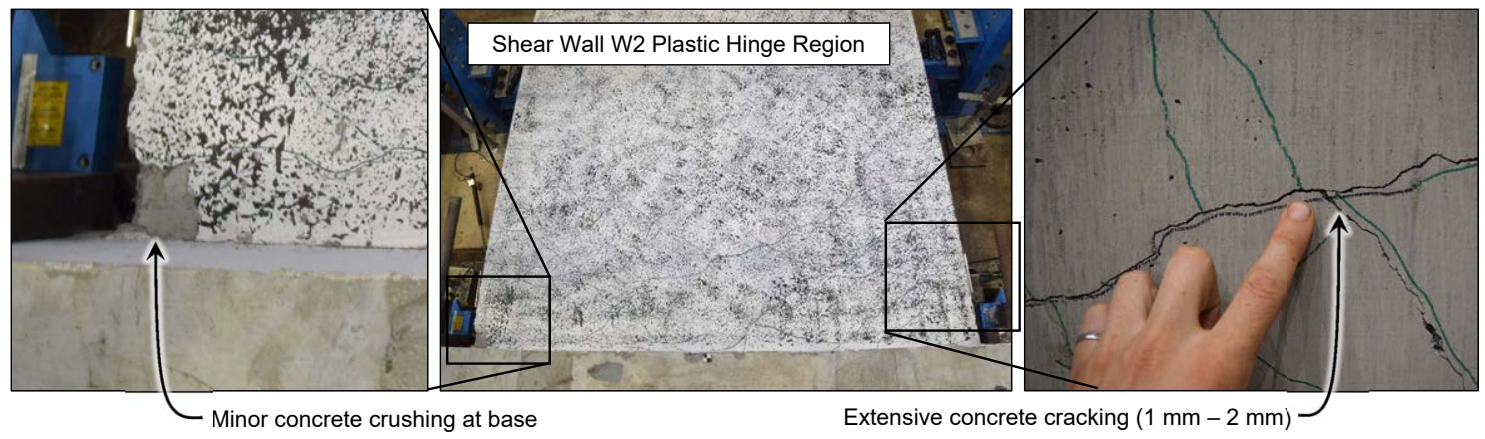

Figure 6.39 Damage to shear wall W2 following the cyclic test 


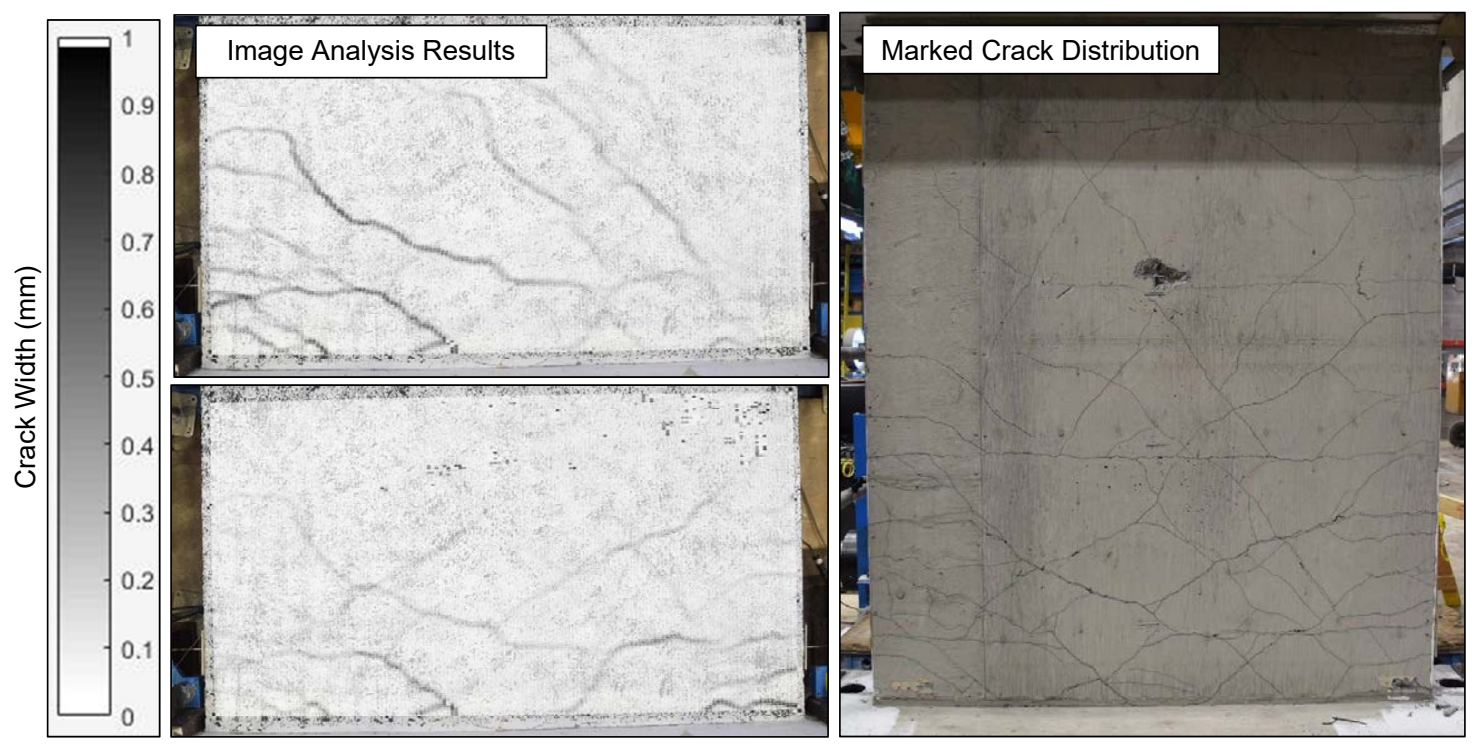

Figure 6.40 Comparison between analyzed and marked crack distributions

Table 6.7 W2 critical structural response parameters

\begin{tabular}{lcc}
\hline \hline Response Parameter & Specimen W2 & Specimen W1 \\
\hline Yield Displacement $\left(\Delta_{y}\right)(\mathrm{mm})$ & $7.51 /-8.46$ & -8.35 \\
Yield Rotation $\left(\theta_{y}\right)(\mathrm{rad})$ & $0.0040 /-0.0049$ & $0.0042 /-0.0046$ \\
Yield Moment $\left(M_{y}\right)(\mathrm{kN}-\mathrm{m})$ & $518 /-553$ & $485 /-497$ \\
Equiv. Yield Force $\left(F_{y}\right)(\mathrm{kN})$ & $287 /-307$ & $269 / 279$ \\
Ultimate Displacement $\left(\Delta_{u}\right)$ & $19.0 /-20.0$ & $17.8 /-27.1$ \\
Ultimate Rotation $\left(\theta_{u}\right)(\mathrm{rad})$ & $0.0094 /-0.011$ & $0.0159 /-0.0101$ \\
Maximum Ductility $\left(\Delta_{u} / \Delta_{y}\right)$ & $2.52 / 2.66$ & $2.19 / 3.78$ \\
\hline \hline
\end{tabular}




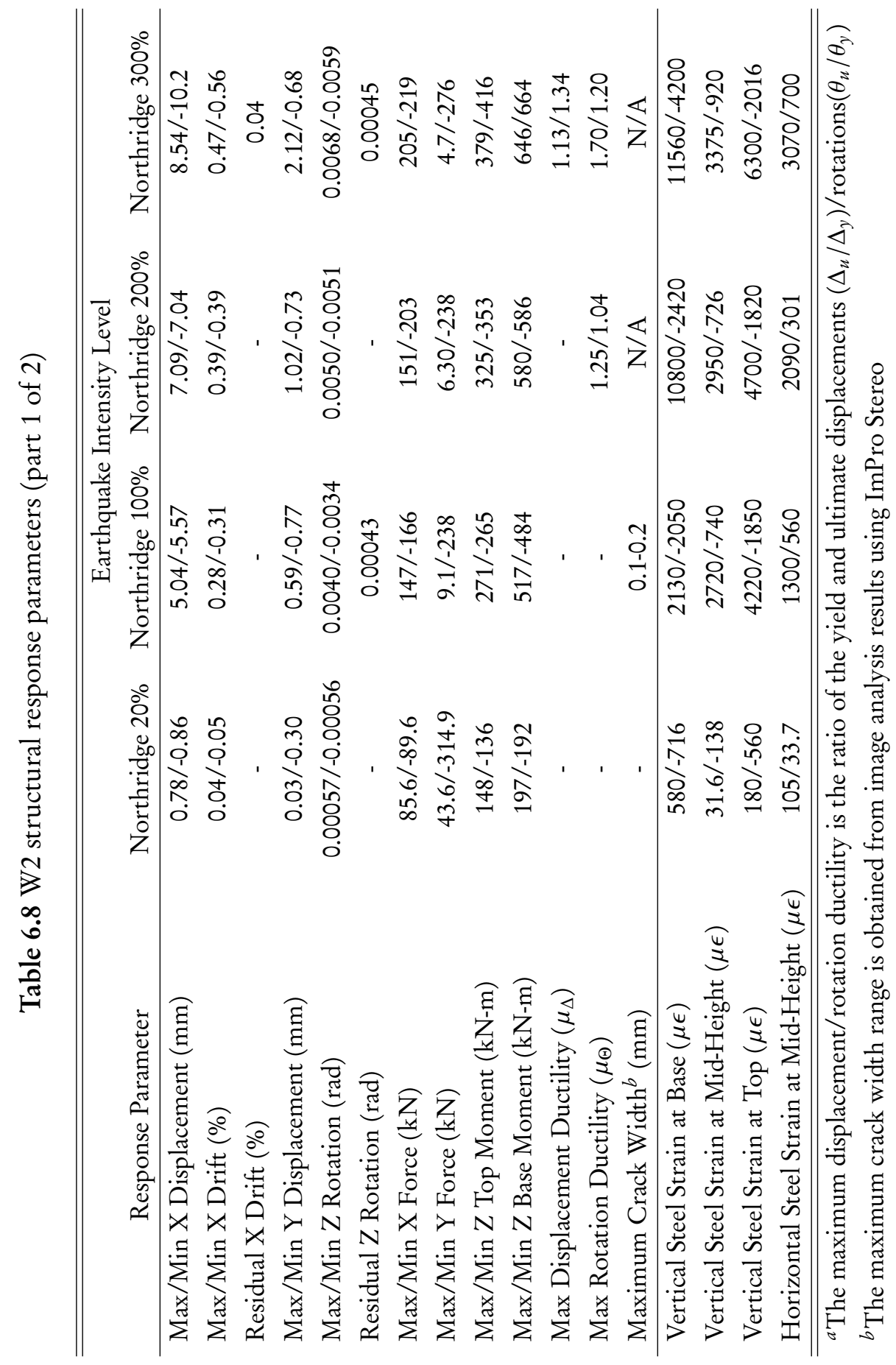




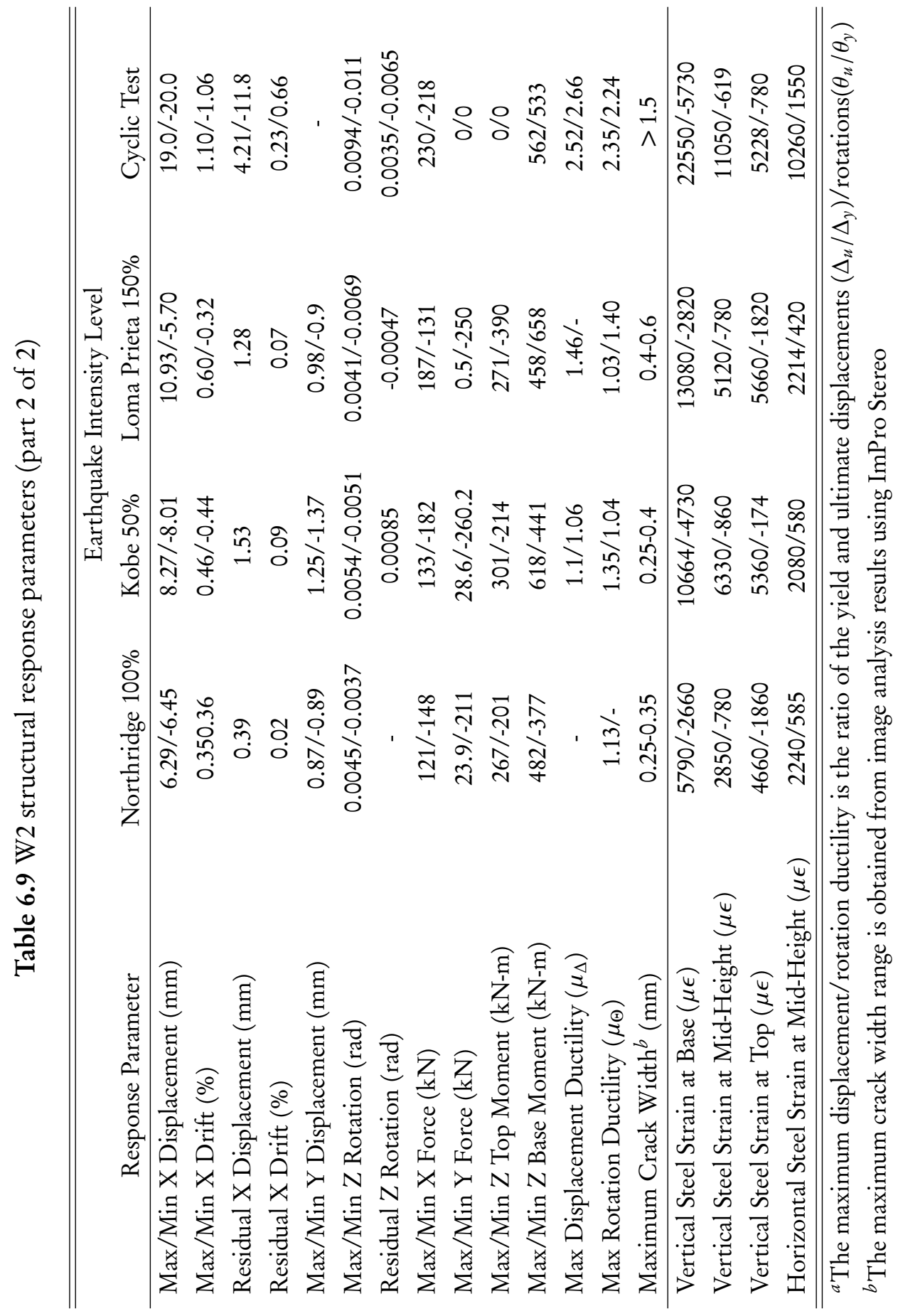


is output in OpenSees, including acceleration, velocity, displacement, and force response of the full-scale shear wall. Figures A.18 to A.24 in Appendix A shows the top displacement, inter-storey drift, shear, and overturning moment time-histories for each of the ground motion intensities applied to the structure. For the applicable ground motions, the global response of the structure is compared with the results from the previous set of hybrid simulations, which were conducted with an elastic finite element model for the analytical substructure. Tables 6.10 and 6.11 summarize the maximum response parameters of the shear wall under each ground motion.

To compare the response of the RC shear wall under increasing ground motion intensity, Figure 6.41 compares the lateral displacement, inter-storey drift, acceleration, velocity, base shear, and overturning moment distribution plotted over the height of the shear wall for each ground motion. The displacement and inter-storey drift results clearly show the highly nonlinear response of the second and third storeys of the shear wall during the Northridge $200 \%$ and $300 \%$ intensity earthquakes. The reasons for the discrepancies between the elastic and nonlinear finite element models are discussed in the following section. The acceleration and velocity distributions show increasing acceleration and velocity in the upper stories of the RC shear wall. The shear and overturning moment distributions over the height of the shear wall show the yielding of the second and third storeys, which effectively limits their shear force and moment under increasing ground motion intensities. Further discussion on the global response of wall specimen W2 is available in the following section, which compares the global and local responses of wall specimens W1 and W2 to get a better understanding of the observed differences in the response and behaviour between the two sets of hybrid tests.

\subsection{Comparison of Hybrid Simulation Results}

In addition to studying the local and global responses of both wall specimens individually, because their ground motion sequence was comparable for the set of Northridge earthquake 

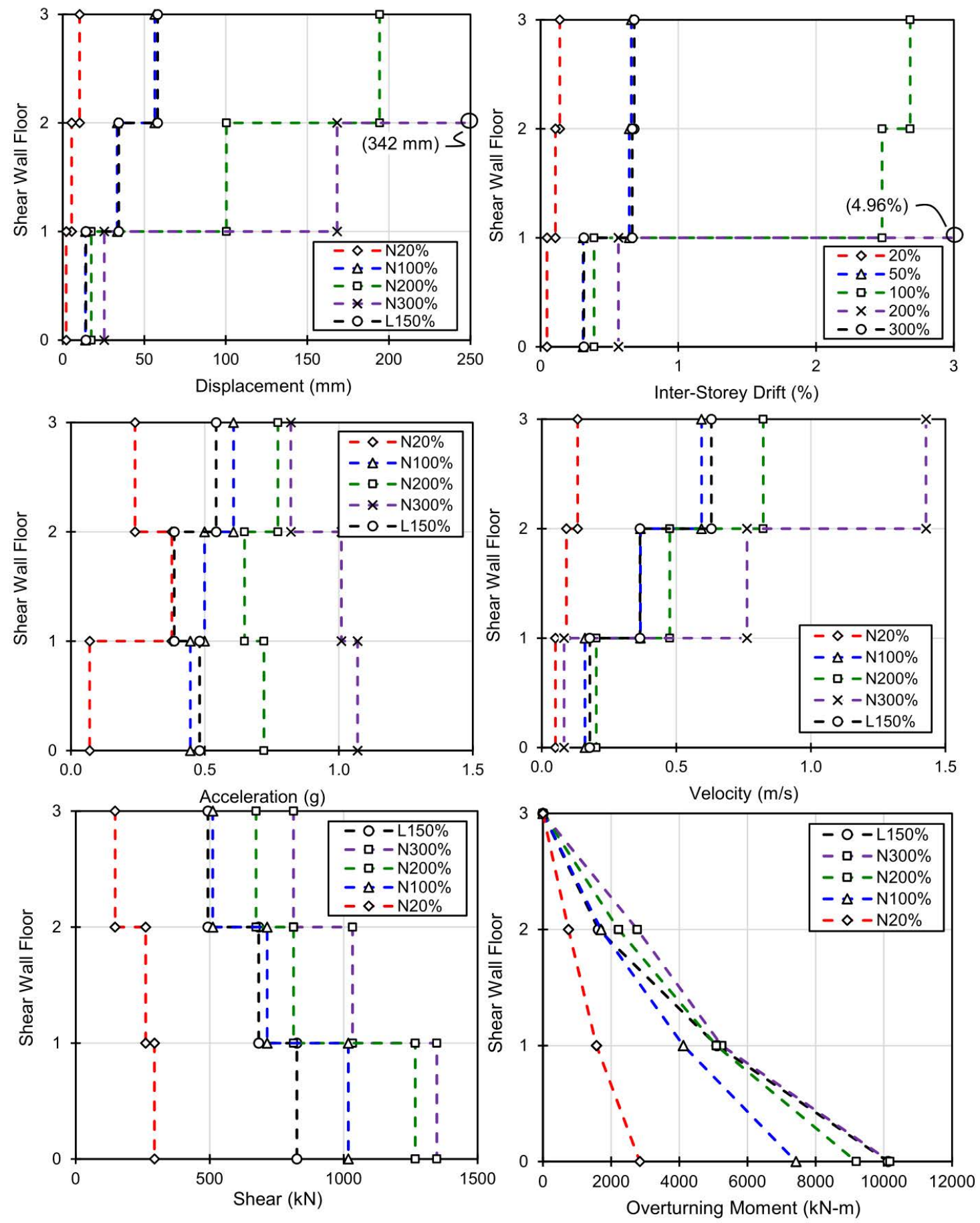

Figure 6.41 Comparison of global shear wall response values 


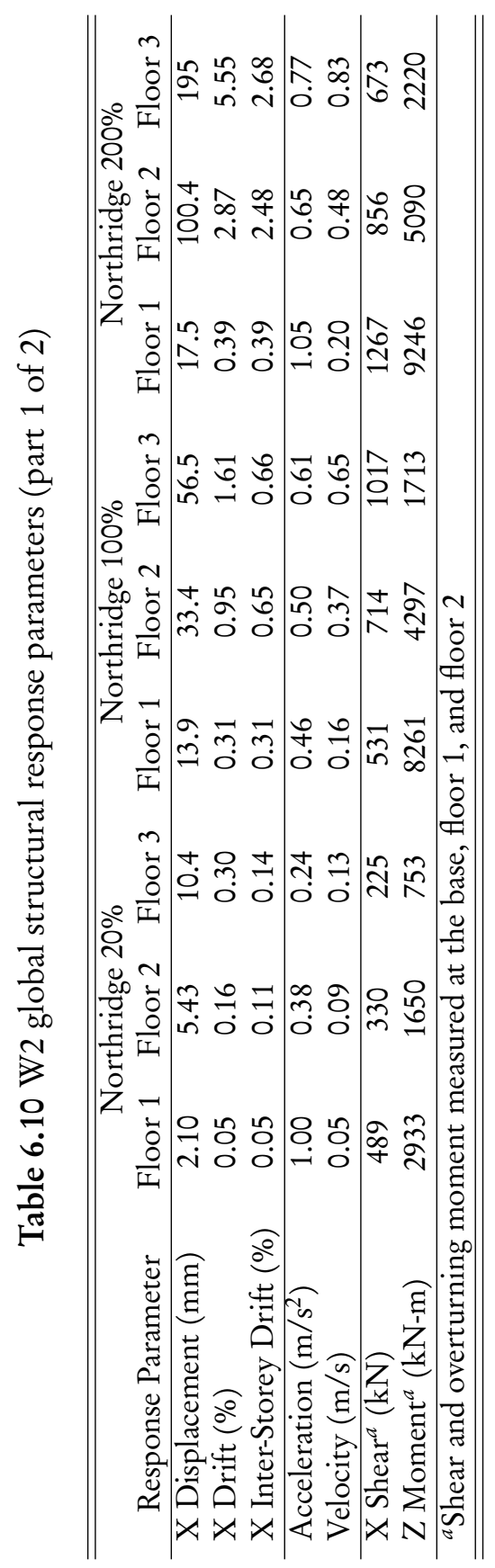




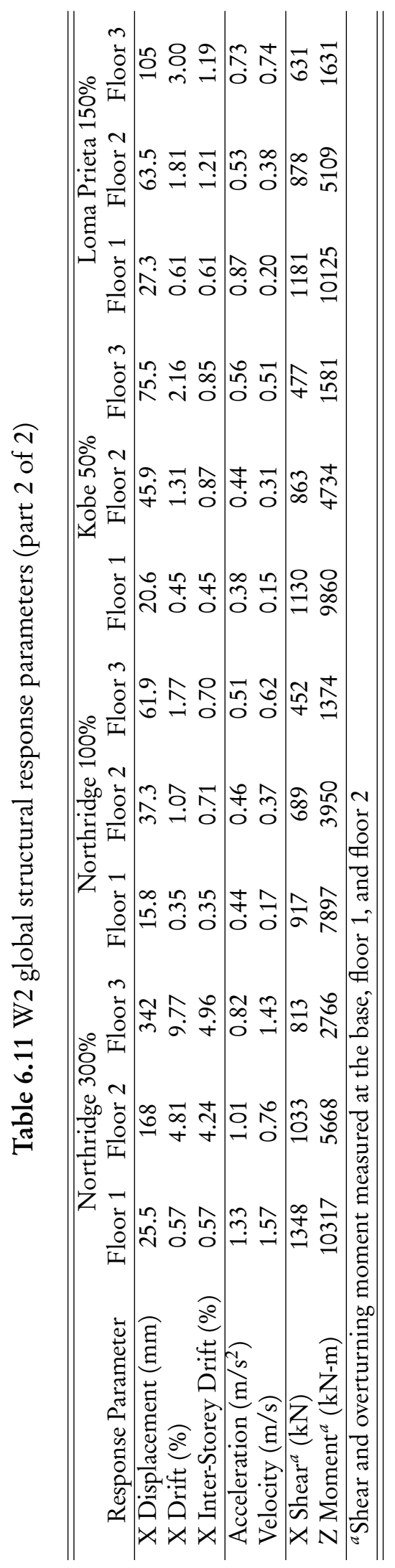


events, this allows for comparison between the hybrid test results, including studying the influence that the finite element model of the analytical substructure had on the seismic behaviour of the multi-storey shear wall.

The first ground motion record applied to both wall specimens was the Northridge Canoga Park ground motion record scaled to $20 \%$ of its original intensity. Figure 6.42 compares the global response of the first storey of the shear wall and the maximum displacement, inter-storey drift, and moment distributions over its height. The results show comparable behaviour between the two hybrid tests. Locally, wall specimen W2 experiences smaller displacements compared with specimen W1, which is reflected in the first storey displacement time-history for the shear wall. The smaller displacements for wall specimen W2 is attributed to the higher initial stiffness measured for specimen W2 in all three degrees-of-freedom. In addition, the nonlinear finite element model of the third storey of the analytical substructure is more flexible compared with the original elastic model, slightly reducing the demand on the experimental substructure while the displacements at the second storey of the shear wall are very comparable. The moment distributions over the height of the shear wall show comparable maximum moments achieved in both tests. Given the small intensity of the earthquake and the small displacements applied to the experimental substructure, the results of the two hybrid tests are very comparable.

At the $100 \%$ intensity level for the Northridge earthquake, the local and global behaviours of both wall specimens is once again shown to be very comparable. Figure 6.43 compares the

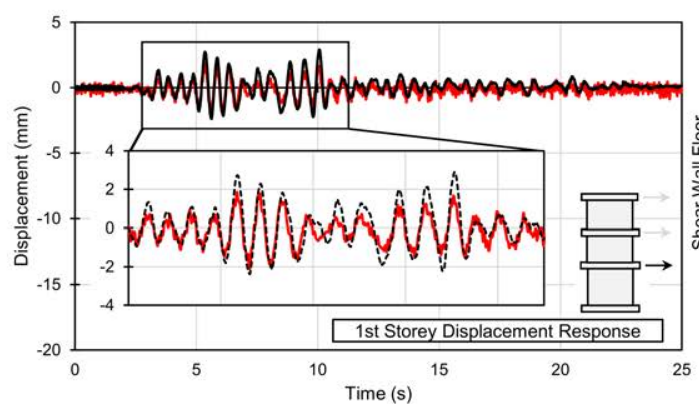

(a)

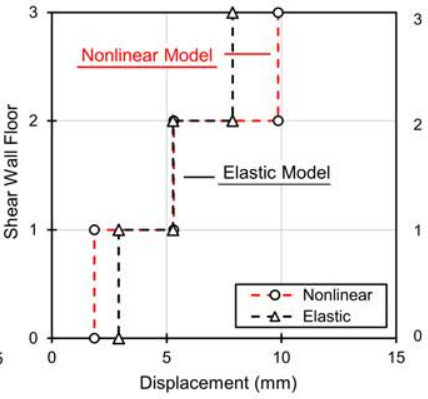

(b)

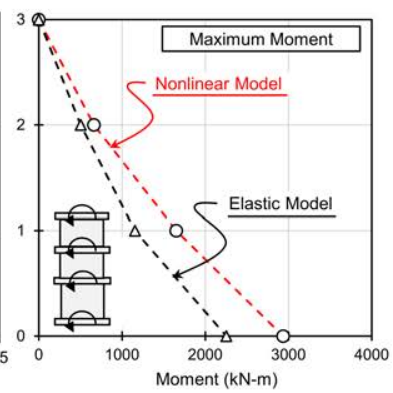

(c)

Figure 6.42 Northridge 20\% global response: (a) first storey displacement time history; (b) maximum displacement profile; (c) maximum overturning moment profile 
local response of specimens W1 and W2 for the two experimental substructures. The results show that the response and behaviour of both wall specimens is very similar, achieving comparable maximum displacements, rotations, and showing comparable hysteretic response behaviour. Figure 6.44 compares the global response of the structures at the $100 \%$ intensity level for the Northridge earthquake. The results show that the global response of both structures is also very comparable, achieving similar displacements and storey shears at the 3rd story level while also having similar shear and overturning moment distributions over the height of the multi-storey shear wall. The major differences lie in the inter-storey drifts of the 2 nd and 3 rd storeys of the structure, which are larger in the nonlinear finite element model when compared to the elastic model, once again because the upper storeys of the analytical substructure in the nonlinear finite element model were softer when compared to the elastic finite element model.

The major differences between the response and observed behaviour of wall specimens W1 and W2 lies in the results from the $200 \%$ and $300 \%$ ground motion intensities for the Northridge earthquake. Figure 6.45 compares the local response of wall specimens W1 and W2 under the Northridge Canoga Park ground motion record scaled to $200 \%$ of its original intensity. The results show that while both specimens show similar response characteristics, including forcedisplacement and moment-rotation hysteretic response behaviour, the demand on specimen W1 when compared with wall W2 is much larger. The reason for the difference in demand is a result of nonlinearity in the second and third storeys of the three-storey shear wall prior to yielding in the first-storey. The moment-rotation response for wall specimen W2 shows that the wall was just on the verge of yielding at the maximum displacement during the test, which would have limited the demand on the second and third storeys of the shear wall. Figure 6.46 shows the global response parameters for the shear wall. The global results clearly show that the second and third storeys of the shear wall yielded, resulting in large inter-storey drifts of up to $2.5 \%$. Nonlinearity in the second storey of the shear wall limited the shear and overturning moment transfered to the first storey shear wall reducing the demand on the wall and causing the discrepancy between the observed behaviours of wall specimens W1 and W2. 

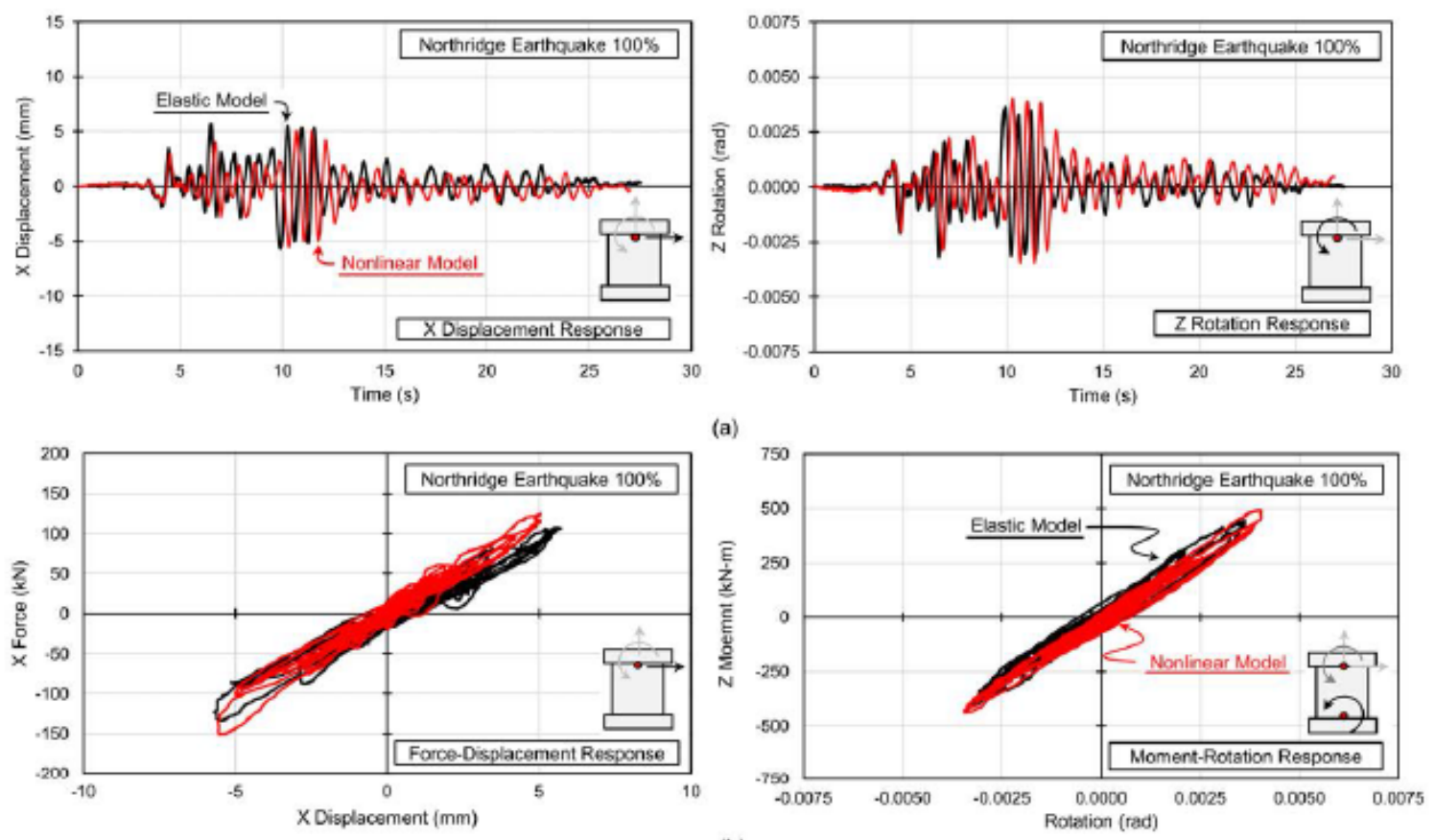

(b)

Figure 6.43 Northridge $100 \%$ local response: (a) X displacement and Z rotation time histories; (b) force displacement and moment rotation hysteresis

Further study into the global response of wall specimen W2 shows that the unintended premature yielding in the second and third storeys came as a result of over-strength in the first storey shear wall. The second and third storey shear walls achieved maximum moments of $6025 \mathrm{kN}-\mathrm{m}$ and $2498 \mathrm{kN}-\mathrm{m}$, respectively during the Northridge $200 \%$ ground motion. These moments are close to the predicted moment capacities of $5480 \mathrm{kN}-\mathrm{m}$ and $2531 \mathrm{kN}-\mathrm{m}$ based on straincompatibility analysis. However, the first storey shear wall, which achieved a maximum moment of $10700 \mathrm{kN}-\mathrm{m}$ during the earthquake, was predicted to yield at a moment of $8900 \mathrm{kN}-\mathrm{m}$ using strain-compatibility analysis, which would have reduced the demand on the second and third storeys of the concrete shear wall, reducing the large inter-storey drifts observed during the test. A design moment of $9400 \mathrm{kN}-\mathrm{m}$ translates into a scaled moment of $600 \mathrm{kN}-\mathrm{m}$ for the experimental substructure based on a scale factor for moment of 15.625 for the shear wall in the experimental substructure at $40 \%$ of full-scale $\left(1 /\left(S_{F} * S_{L}\right)=1 /(0.16 * 0.4)=15.625\right)$. Wall specimen W1 achieved a maximum moment capacity of $657 \mathrm{kN}-\mathrm{m}$ during the first set of hybrid simulations, $15 \%$ higher than the intended design moment. This discrepancy was enough to change the 

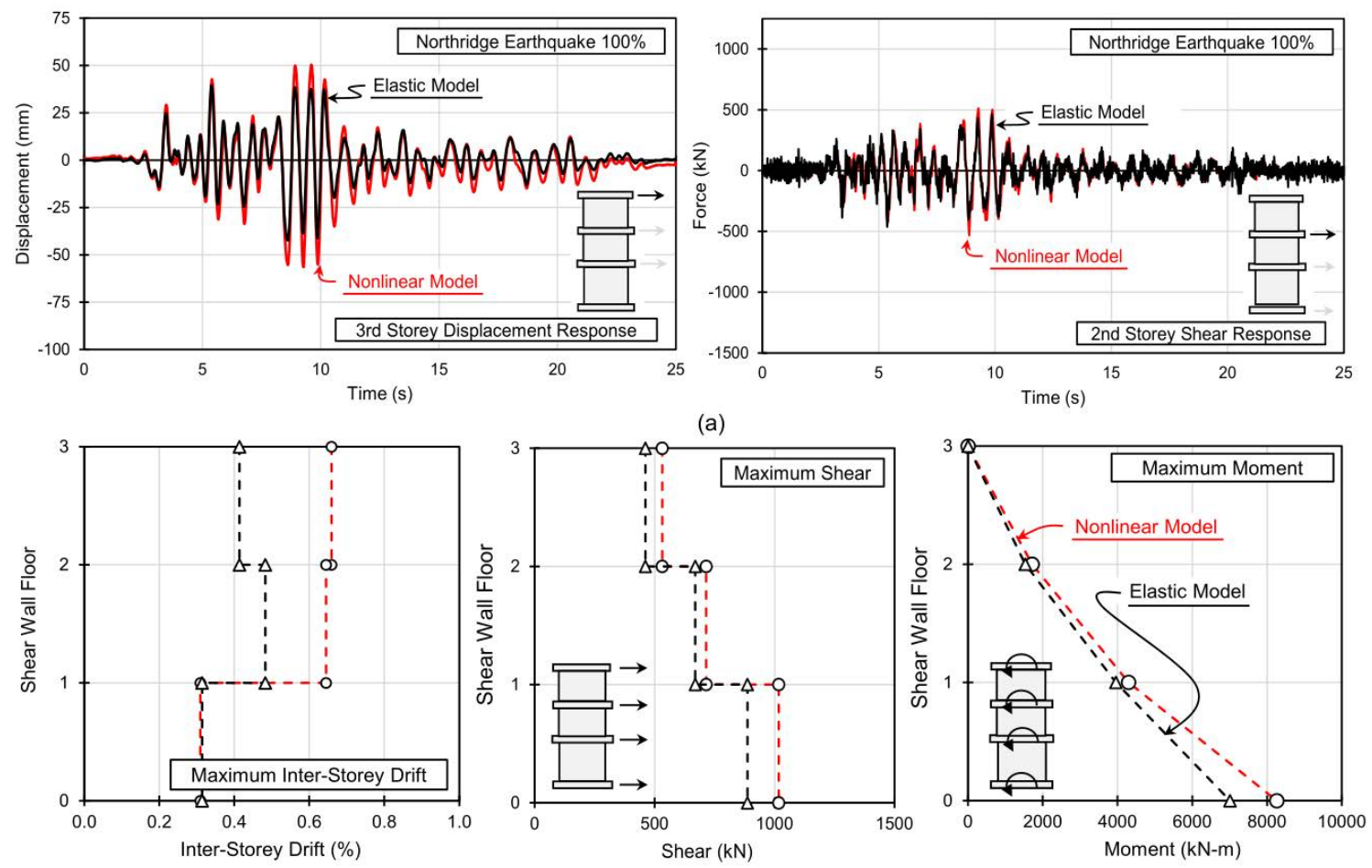

(b)

Figure 6.44 Northridge 100\% local response: (a) X displacement and force time histories; (b) maximum inter-storey drift, shear, and moment distributions 

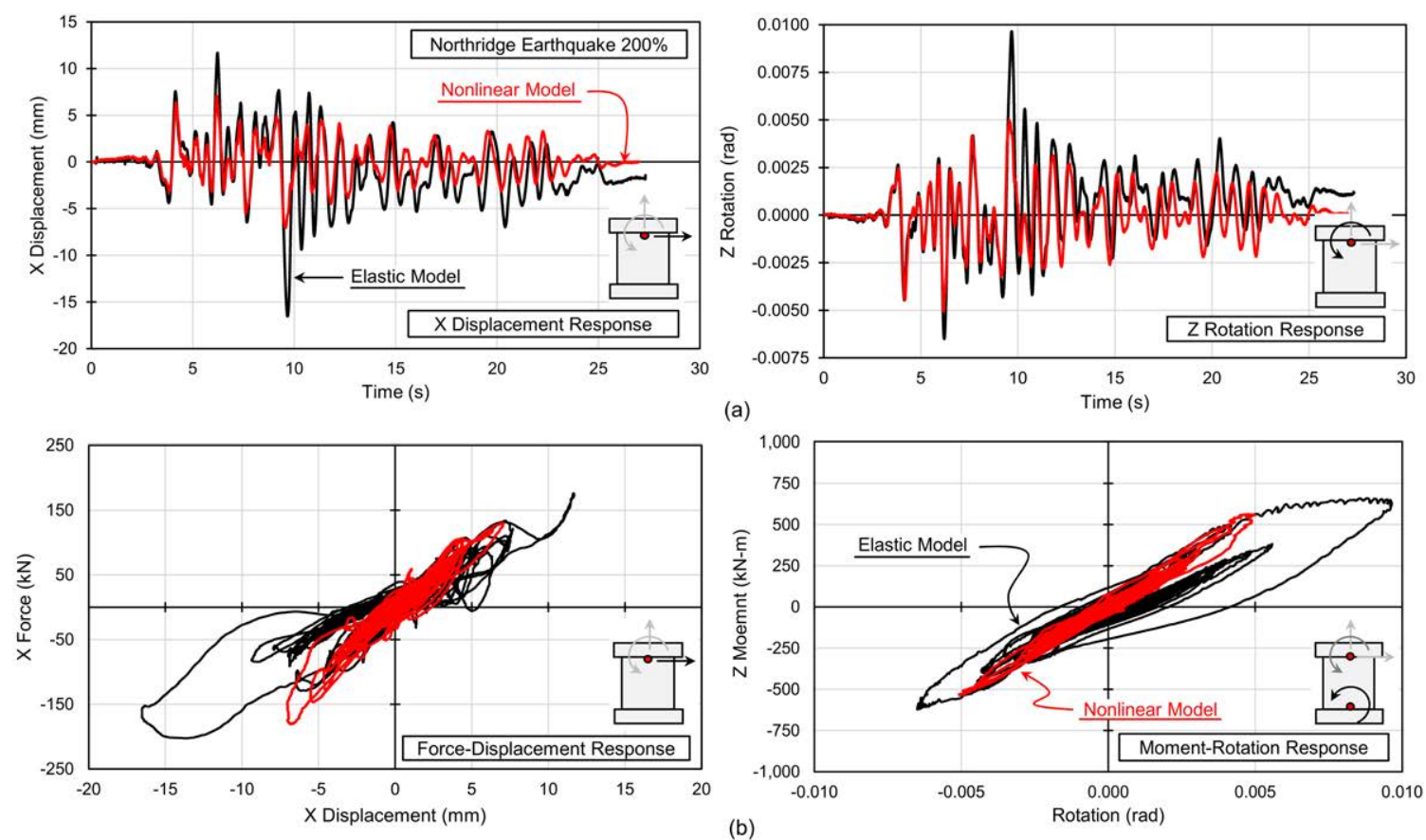

Figure 6.45 Northridge 200\% local response: (a) X displacement and Z rotation time histories; (b) force displacement and moment rotation hysteresis

locations of the plastic hinges throughout the three-storey RC shear wall and ultimately have a dramatic impact on the response of the multi-storey shear wall. It is important to recognize that a small absolute different in the ultimate strength of the scaled specimen corresponds to a large percent change in the strength of the full-scale structure. Reasons for the discrepancies between the predicted and measured ultimate moment capacity of the experimentally tested shear wall could include higher yield strength in the steel reinforcement compared to the assumed design yield strength, which based on tension tests conducted on the vertical steel reinforcement showed the steel had an average yield strength of $455 \mathrm{MPa}$, which is higher than the $440 \mathrm{MPa}$ yield strength used in the design of the wall based on a $10 \%$ overstrength factor. Other factors that could have contributed to the observed increase in strength include a larger thickness of the shear wall, which occurred during casting and resulted in an average wall thickness of $110 \mathrm{~mm}$, compared to the design thickness of $100 \mathrm{~mm}$. These results demonstrate the importance of ensuring that the scaled shear wall specimen has a comparable capacity to the design capacity of the analytical structure to produce accurate hybrid simulation results. 

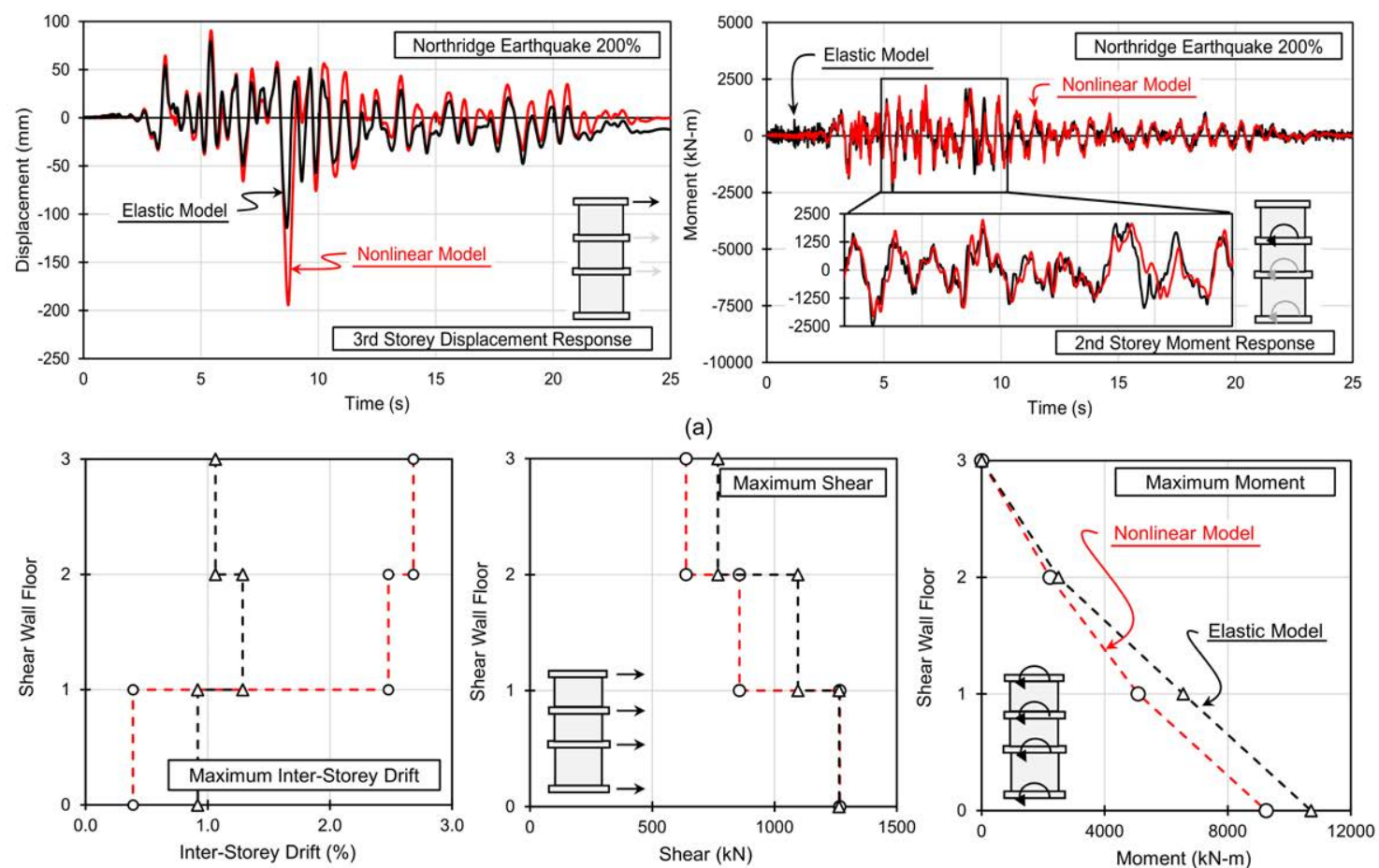

(b)

Figure 6.46 Northridge 200\% local response: (a) X displacement and Z moment time histories; (b) maximum inter-storey drift, shear, and moment distributions 
The hybrid test results for the Northridge earthquake at $300 \%$ of its original intensity is much of the same when compared to the $200 \%$ event. Figure 6.47 compares the local response of the experimental substructure during the Northridge $300 \%$ earthquake. The results show that while wall specimen W1 yields and reaches very high levels of drift, the demand on wall specimen W2 is much smaller. The results also show that as a result of the higher damage to wall specimen W1 during the Northridge 200\% earthquake, the stiffness of wall specimen W1 is lower compared to wall specimen W2 during the Northridge 300\% earthquake. The differences between the response of wall specimens W1 and W2 are once again the result of very large inter-storey drifts $(>3 \%)$ in the second and third storeys of the nonlinear analytical substructure.

As described earlier, one of the goals of a parallel study is to repair the wall specimens using externally bonded CFRP sheets and test them again. Consequently, it was a goal of this study to subject both wall specimens to a comparable level of damage such that the results of the retrofit could be compared with one another. Comparing the demand on wall specimens W1 and W2, wall specimen W1 reached an average ultimate displacement and rotation of $22.5 \mathrm{~mm}$ and $0.013 \mathrm{rad}$, respectively, which is very comparable to the maximum average displacement and rotation of $19.5 \mathrm{~mm}$ and $0.010 \mathrm{rad}$ reached by wall specimen W2. At the end of the hybrid tests, both wall specimens exhibited some concrete crushing at their base and extensive diagonal cracking over the surface of the walls. Figure 6.48 shows the marked cracked distributions for both wall specimens after being removed from the experimental test setup. The results show very similar crack distributions for both wall specimens, with horizontal flexural cracks forming in approximately the same locations over the height of the wall specimens. The results suggest that the level of damage to both specimens following testing is comparable, providing a suitable opportunity to compare their response during future hybrid tests after repair.

Ultimately, although the hybrid test results from the nonlinear model did not behave as designed, the tests nonetheless provided valuable information related to the importance of considerations surrounding scaling and over strength in the design of hybrid tests. The results show that small deviances in the design strength of the experimental substructure can have a dramatic 

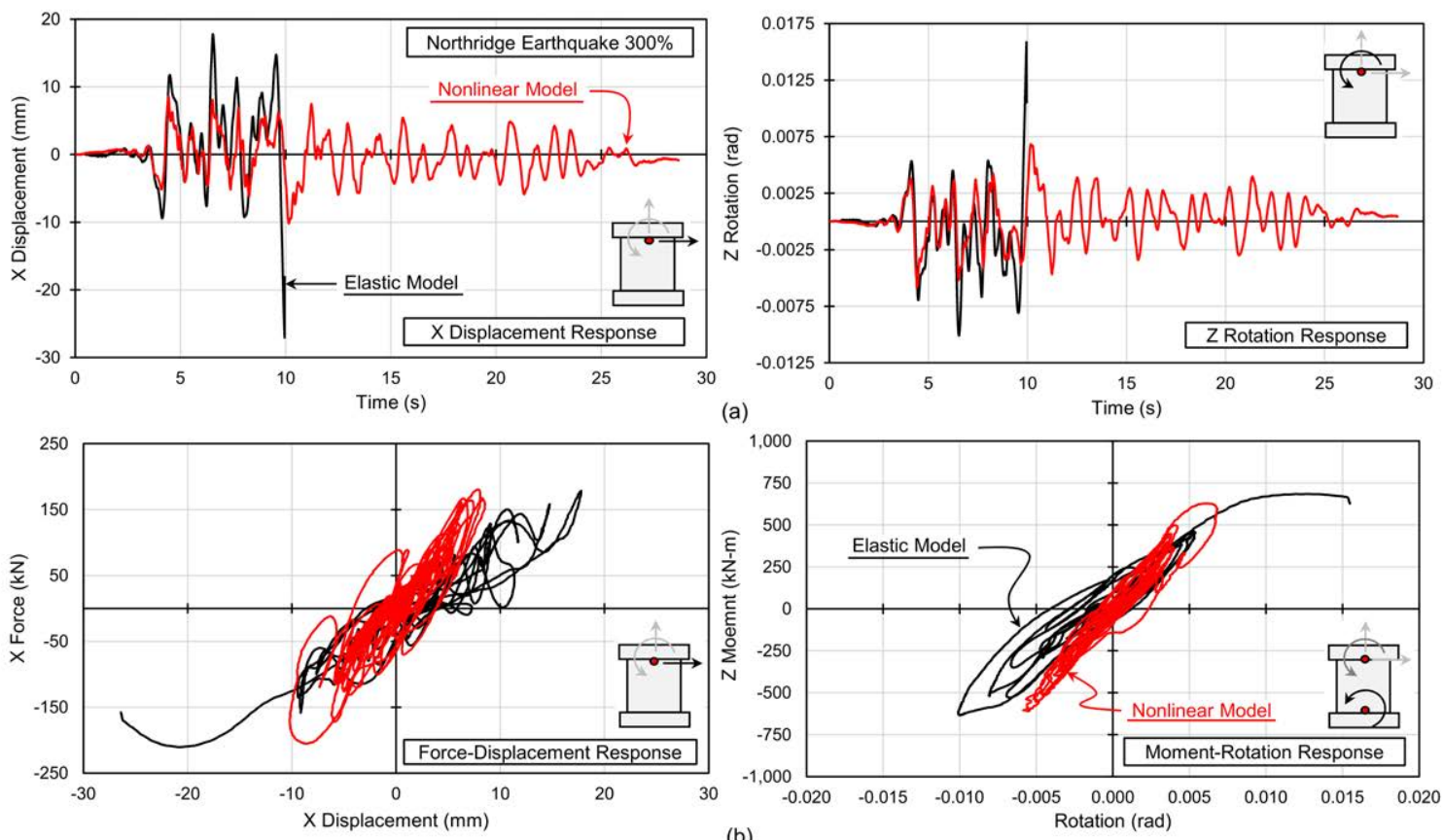

Figure 6.47 Northridge 300\% local response: (a) X displacement and Z rotation time histories; (b) force displacement and moment rotation hysteresis
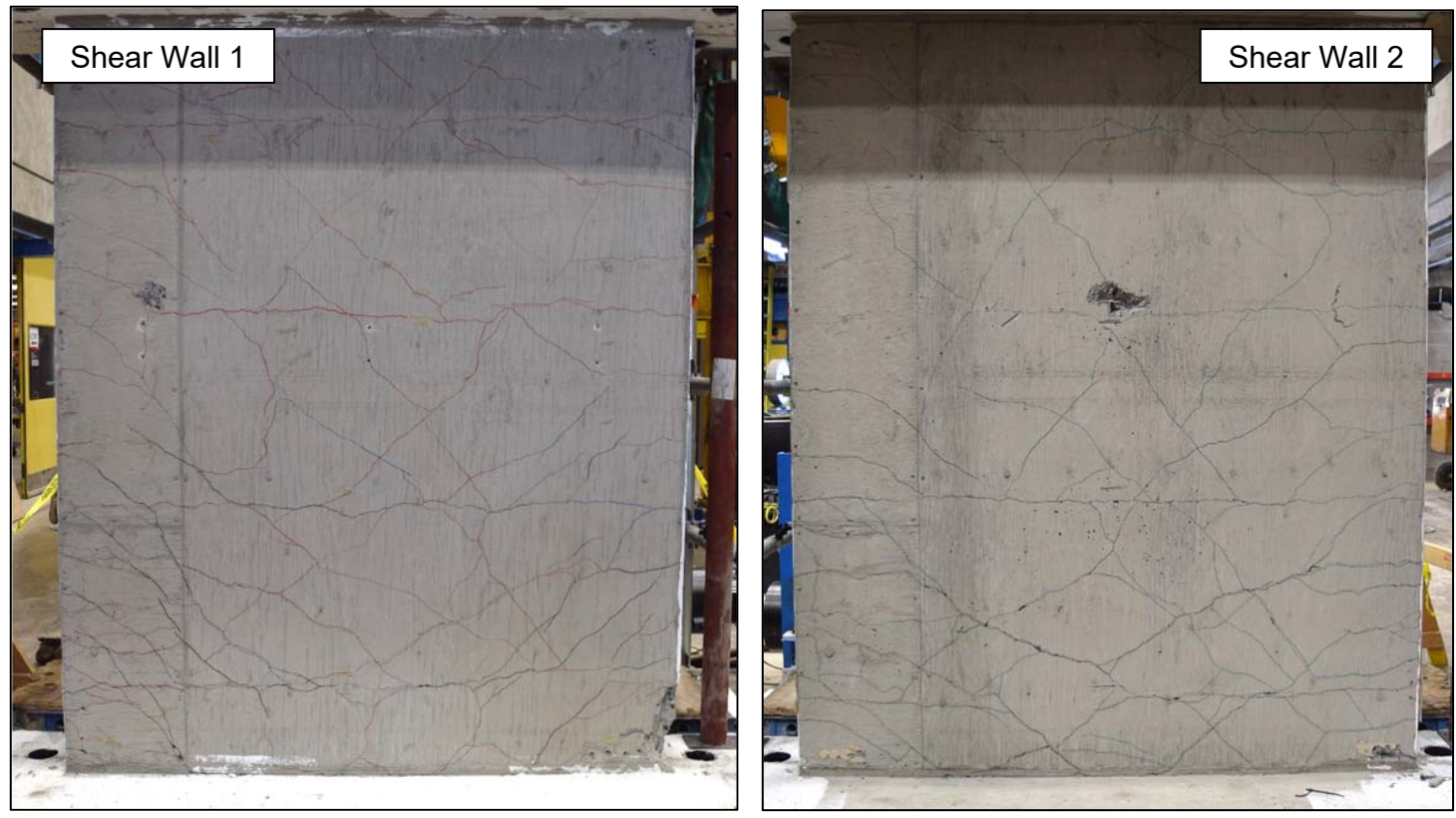

Figure 6.48 Comparison between marked cracked patterns for wall specimens W1 and W2 
influence on the behaviour of the structure and hybrid test results. Nonetheless, the hybrid test results demonstrate the potential for the use of shell elements to capture the shear behaviour of RC elements can be incorporated into the hybrid model, including the influence of nonlinearity in the analytical substructure. Overall, the test results demonstrate the feasibility of using hybrid simulation to study the response of stiff concrete structures.

\subsection{Comparison of Hybrid and Fully Analytical Results}

In addition to comparing between the hybrid test results for wall specimens W1 and W2, the hybrid test results can also be compared with results from a fully analytical finite element model. One of the primary interests in the use of hybrid simulation as a test method is for the development of better analytical modelling approaches that are capable of accurately capturing the nonlinear seismic response of civil engineering structures. Although this has not been a primary focus of this study, comparing the hybrid test results with results from a fully analytical finite element model of the full-scale shear wall provides additional insight into the reproducibility of the hybrid tests and the ability of the multi-layer shell element to accurately capture the nonlinear seismic response of a multi-storey RC shear wall.

\subsubsection{Wall Specimen W1}

The first hybrid test subjected wall specimen W1 to the Northridge Canoga Park station earthquake record scaled to $20 \%$ of its original intensity. Hybrid test results showed that the shear wall remained elastic during the test. Consequently, the hybrid test results are compared with an elastic finite element model of the three-storey RC shear wall. Figure 6.49 compares the first-storey displacement, base shear, first-storey rotation, and base moment time histories for the fully analytical model and the hybrid test. The results show a good correlation between the hybrid test and numerical simulation, which achieve very comparable displacement and base shear time histories while the rotation and base moment time histories are in relatively good agreement. The offset in the rotation time histories between the fully analytical prediction and the hybrid test are a result of uneven loading during the gravity loading phase of the hybrid test. Nonetheless, the 
results validate the elastic finite element model of the three-storey shear wall and also confirm that the hybrid test system is capable of achieving reproducible results in a fully analytical finite element model.

The next hybrid test conducted on wall specimen W1 increased the intensity of the Northridge ground motion to $50 \%$ of its original unscaled intensity. At this earthquake intensity, some cracks were observed in the surface of the concrete during the hybrid test. The formation of these cracks indicate that the wall specimen exceeded its cracking strength, resulting in a loss in stiffness and some minor nonlinearity in its response. These assumptions were confirmed by comparing the hybrid test results with the same elastic fully analytical finite element model. Figure 6.50 compares first-storey displacement and base shear time histories over the duration of the earthquake. The results show the displacements in the hybrid test are larger compared with the elastic model while the forces in the elastic model are higher. This observed behaviour is consistent with what was anticipated, as the wall specimen cracks, a behaviour that is not captured in the elastic finite element model, it becomes softer reducing the forces on the wall while the displacements get larger.

To justify that the elastic model is the reason for the discrepancies between the hybrid and fully analytical results in Fig. 6.50, a nonlinear fully analytical model of the three storey RC shear wall was developed using the multi-layer shell element discussed in Chapter 5. In this model, the strength of the second and third stories of the RC shear wall were increased to ensure they remained elastic (because the hybrid simulation used an elastic finite element model). The first storey shear wall in the fully analytical model is a full-scale model of the physical substructure in the laboratory. Figure 6.51 shows the first-storey displacement and base shear time histories for nonlinear finite element model and compares them with the hybrid test results. The results show that the response of the nonlinear model matches the observed response from the hybrid test results more closely. The displacements in the nonlinear model, which were expected to be higher are closer to the actual observed displacement time history during the hybrid test and the forces are lower, producing a better correlation with hybrid test results. These results show that 

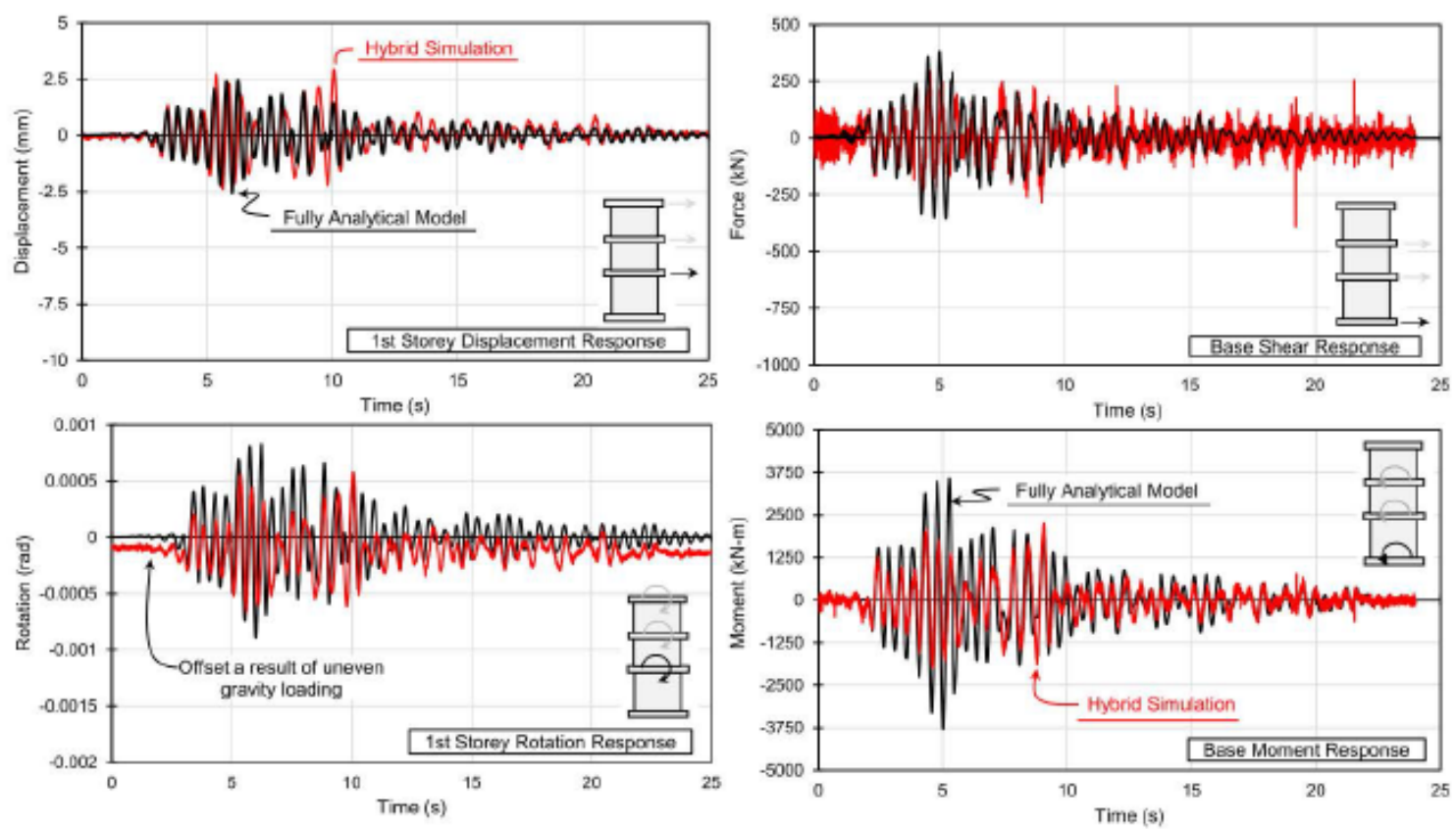

Figure 6.49 Northridge 20\%: comparison between fully analytical and hybrid test results
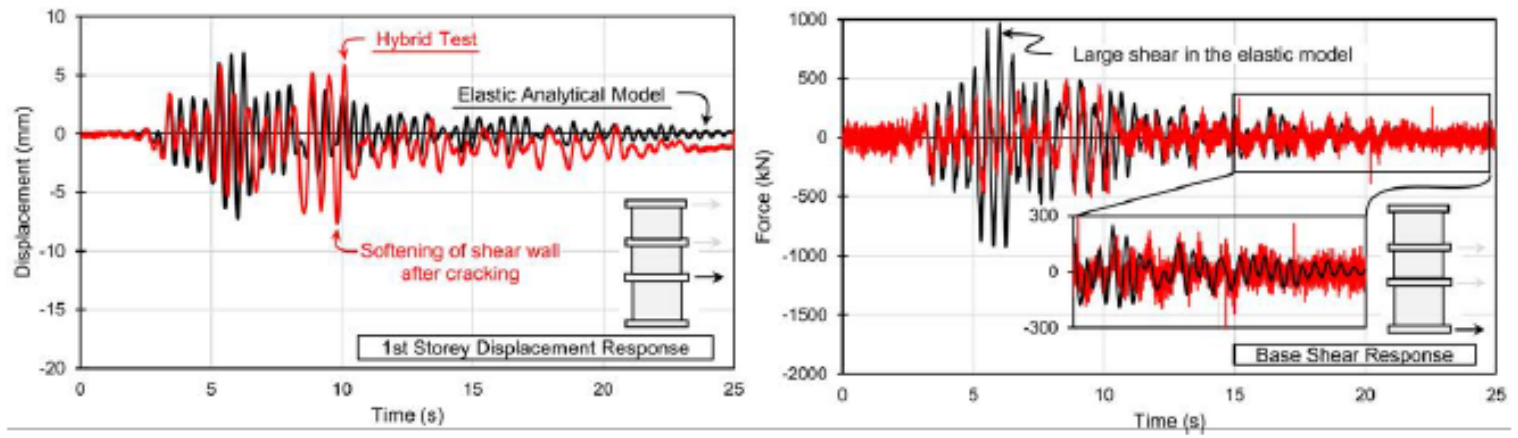

Figure 6.50 Northridge 50\%: comparison between fully analytical elastic wall model and hybrid test results 
the multi-layer shell element does a good job at capturing the response of the wall specimen prior to yield.

Figure 6.52 compares the first-storey displacement time history and moment-rotation hysteretic response behaviour for the hybrid test and fully analytical nonlinear model for the Northridge ground motion at its unscaled intensity (100\%). The results show that once again the results from the nonlinear finite element model correlate relatively well with the hybrid test results, doing a reasonable job at predicting the overall response behaviour of the shear wall, including the maximum top displacement and rotational stiffness of the shear wall.

At the $200 \%$ intensity level for the Northridge earthquake, the nonlinear finite element model matches the hybrid test results even more closely. Figure 6.53 compares the first-storey displacement, first-storey rotation, base overturning moment, and moment-rotation hysteretic response for the hybrid test and fully analytical model. The results show that the analytical model very accurately predicts the maximum displacement and rotation at the first-storey level. The moment rotation hysteretic response behaviour of the fully analytical model also correlates well with the measured moment-rotation hysteretic response during the hybrid test, which have similar rotational stiffness, yield rotation, and ultimate moment capacity. These results suggest that the finite element model also has the ability to accurately capture the yield strength and post-yield behaviour of a multi-storey RC shear wall.
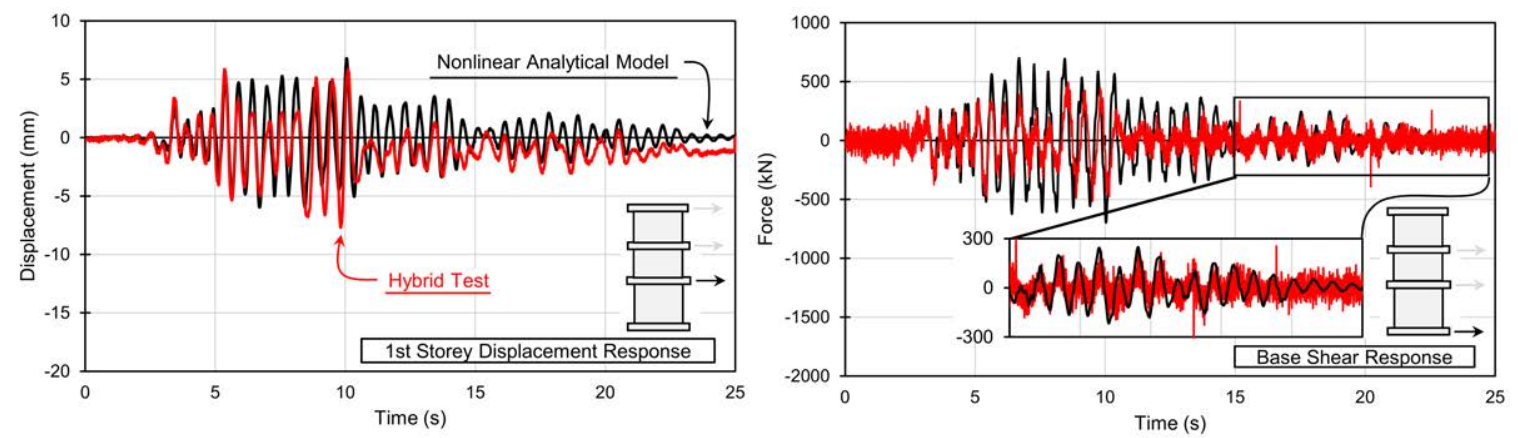

Figure 6.51 Northridge 50\%: comparison between fully analytical nonlinear wall model and hybrid test results 

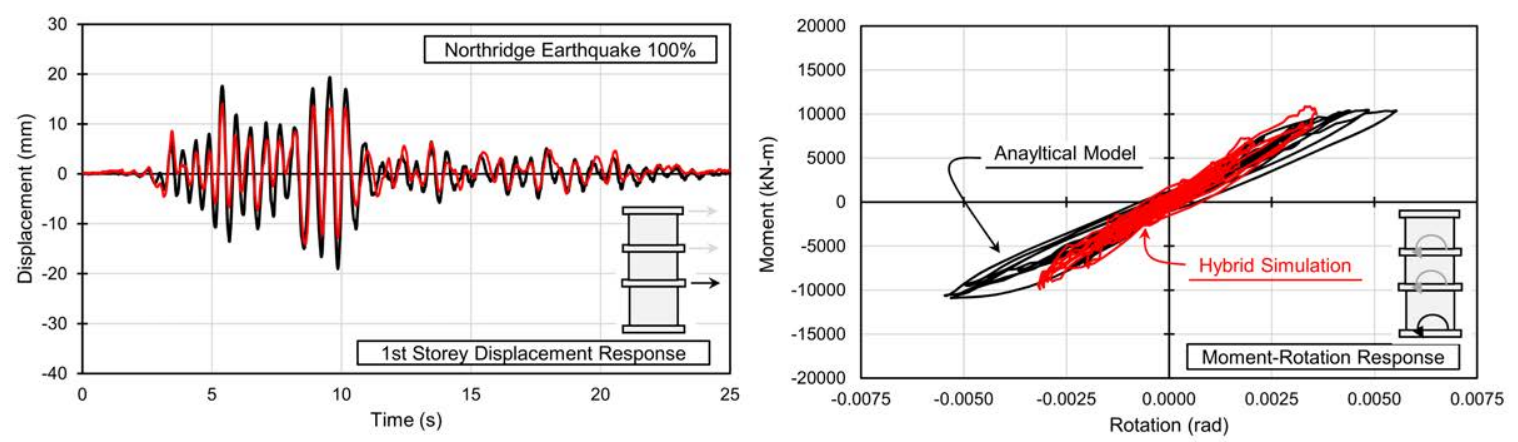

Figure 6.52 Northridge 100\%: comparison between fully analytical and hybrid test results
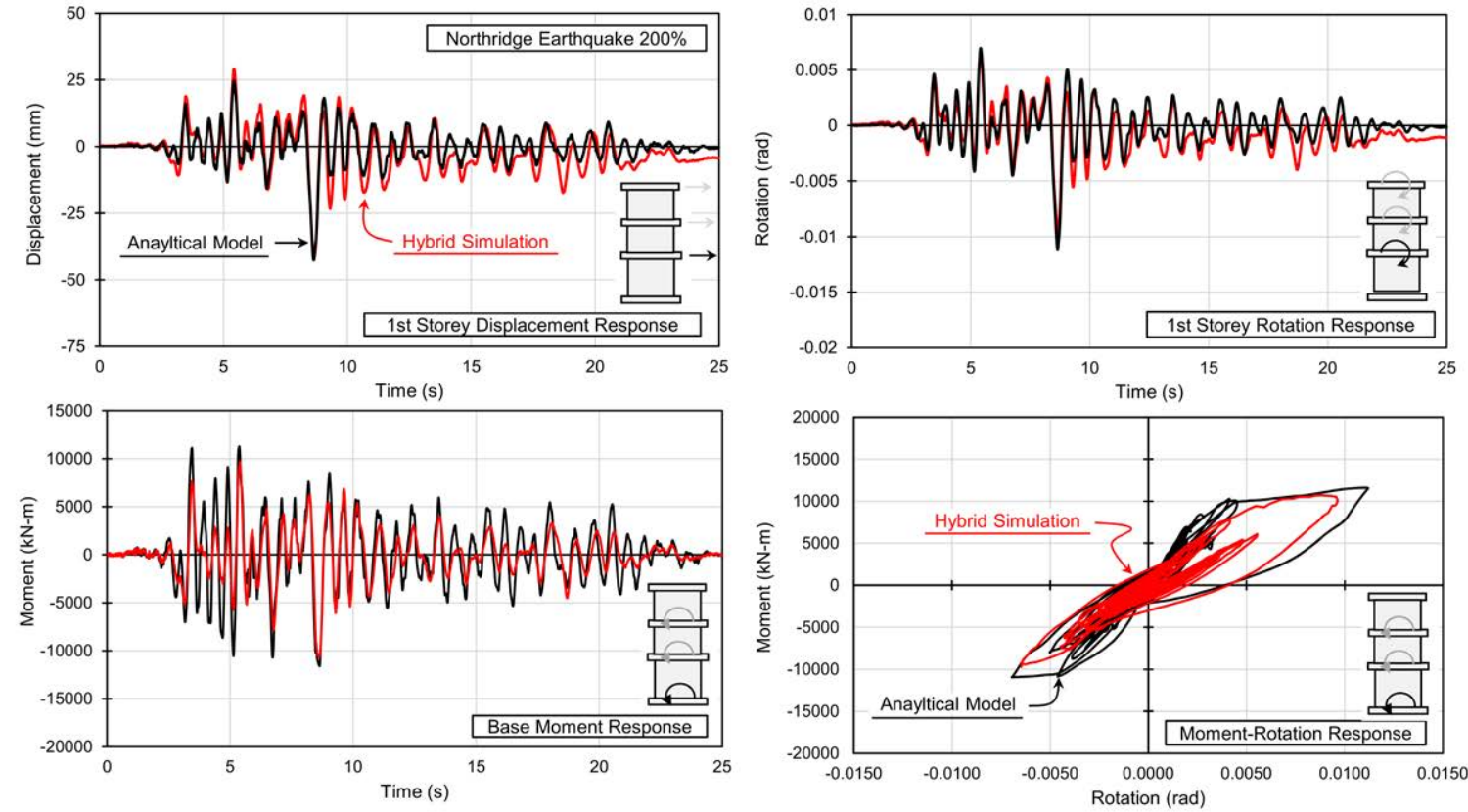

Figure 6.53 Northridge 200\%: comparison between fully analytical and hybrid test results 
Finally, the fully analytical model is also able to capture the collapse of the wall specimen during the Northridge earthquake scaled to $300 \%$ of its original intensity. Figure 6.54 shows the first-storey displacement time history and moment-rotation hysteretic response behaviour for the hybrid test and fully analytical model. The results show that once again the model accurately predicts the ultimate strength of the wall specimen and its collapse. However, the model underpredicts the stiffness of the first-storey shear wall, evident from the lower displacements in the fully analytical model when compared to the hybrid test results and the lower rotational stiffness shown in the moment-rotation response measured during the hybrid test. The discrepancies between the results is likely caused by pre-existing damage to the physical shear wall specimen in the lab, which is not taken into account in the current analytical model. To account for the effects of cumulative damage and stiffness degradation to the shear wall under multiple earthquakes, the fully analytical model was subjected to the Northridge ground motion record scaled to $100 \%$, $200 \%$, and $300 \%$ sequentially to mimic the loading protocol during the hybrid test. Figure 6.54 compares the same results as before for the model subjected to the earthquake sequence as opposed to a single earthquake record. The fully analytical results show better agreement with the hybrid test results when the wall is subjected to the full earthquake sequence. The first-storey displacement response correlates well with the hybrid test result because the stiffness of the specimen is more accurately represented. This is also evident from the moment-rotation response, which shows very comparable results to the hybrid test, including rotational stiffness, yield rotation, and ultimate moment capacity.

Ultimately, these results demonstrate that the multilayer shell element is able to accurately capture the nonlinear response of a RC shear wall and reproduce the hybrid test results. The fully analytical model is shown to be able to accurately capture the stiffness, strength, and energy dissipation capacity of the full-scale shear wall. The results demonstrate the potential for using hybrid test results to develop accurate nonlinear finite element models of full-scale civil engineering structures. 

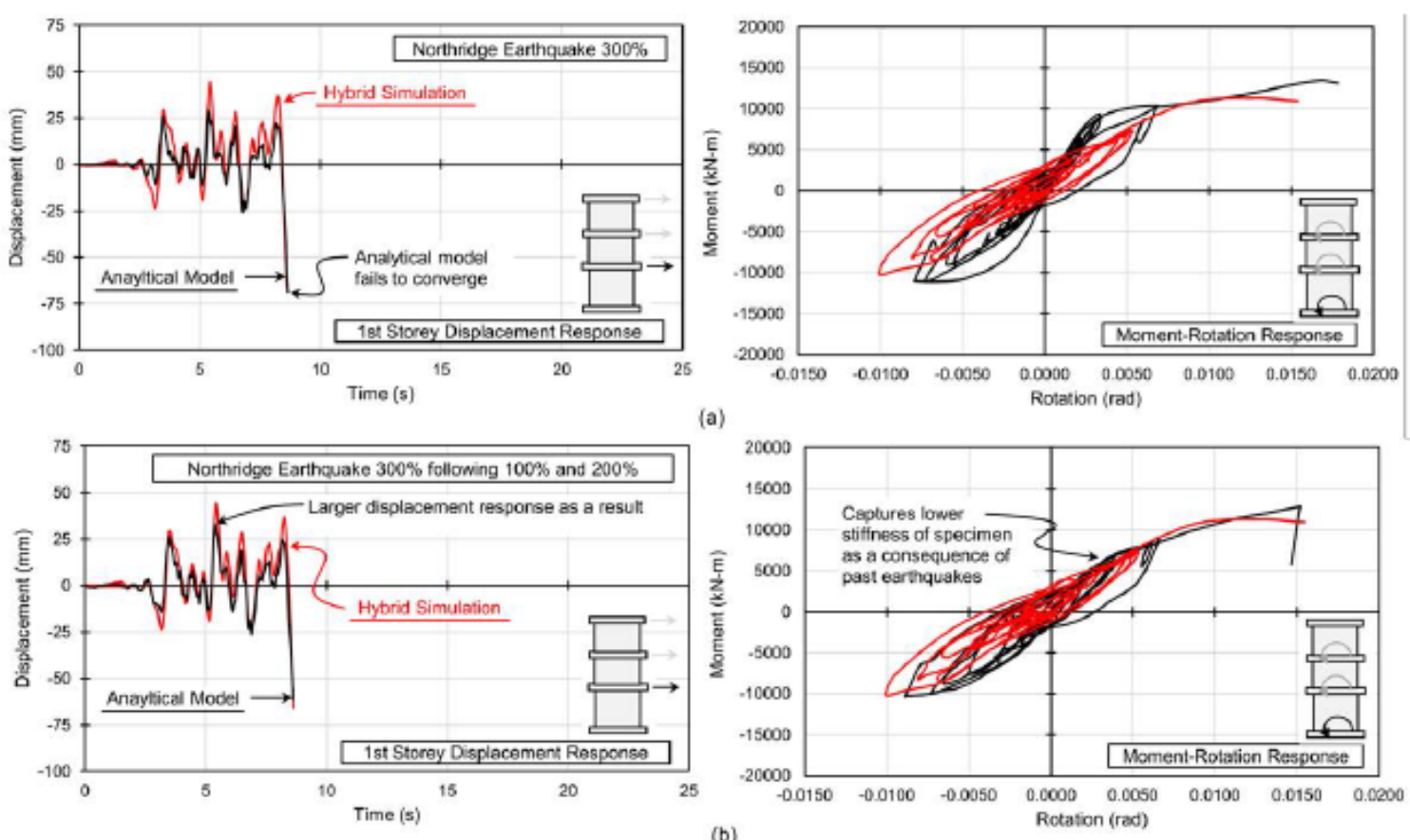

Figure 6.54 Comparison between fully analytical and hybrid test results: (a) Northridge 300\% (b) Northridge $100 \%, 200 \%$, and $300 \%$ earthquake sequence

\subsubsection{Wall Specimen W2}

In a similar manner to wall W1, wall W2 was first subjected to the Northidge earthquake scaled to $20 \%$ of its original intensity. At this intensity level the wall specimen remained entirely elastic, including the second and third storeys of the multi-storey shear wall, which were modelled using nonlinear material properties for this set of hybrid tests. Consequently, the hybrid test results are once again compared with an elastic finite element model of the RC shear wall structure. Figure 6.55 compares the first and third storey displacement, base overturning moment, and firststorey rotation response of the elastic finite element model with the hybrid test results. The results confirm that once again the elastic finite element model does a good job of predicting the response behaviour of the shear wall prior to cracking, accurately predicting the ultimate displacement, rotation, and moment over the height of the shear wall.

During the subsequent earthquake events, the wall specimen behaved in a nonlinear manner and its response could not be accurately reproduced using an elastic finite element model. To 

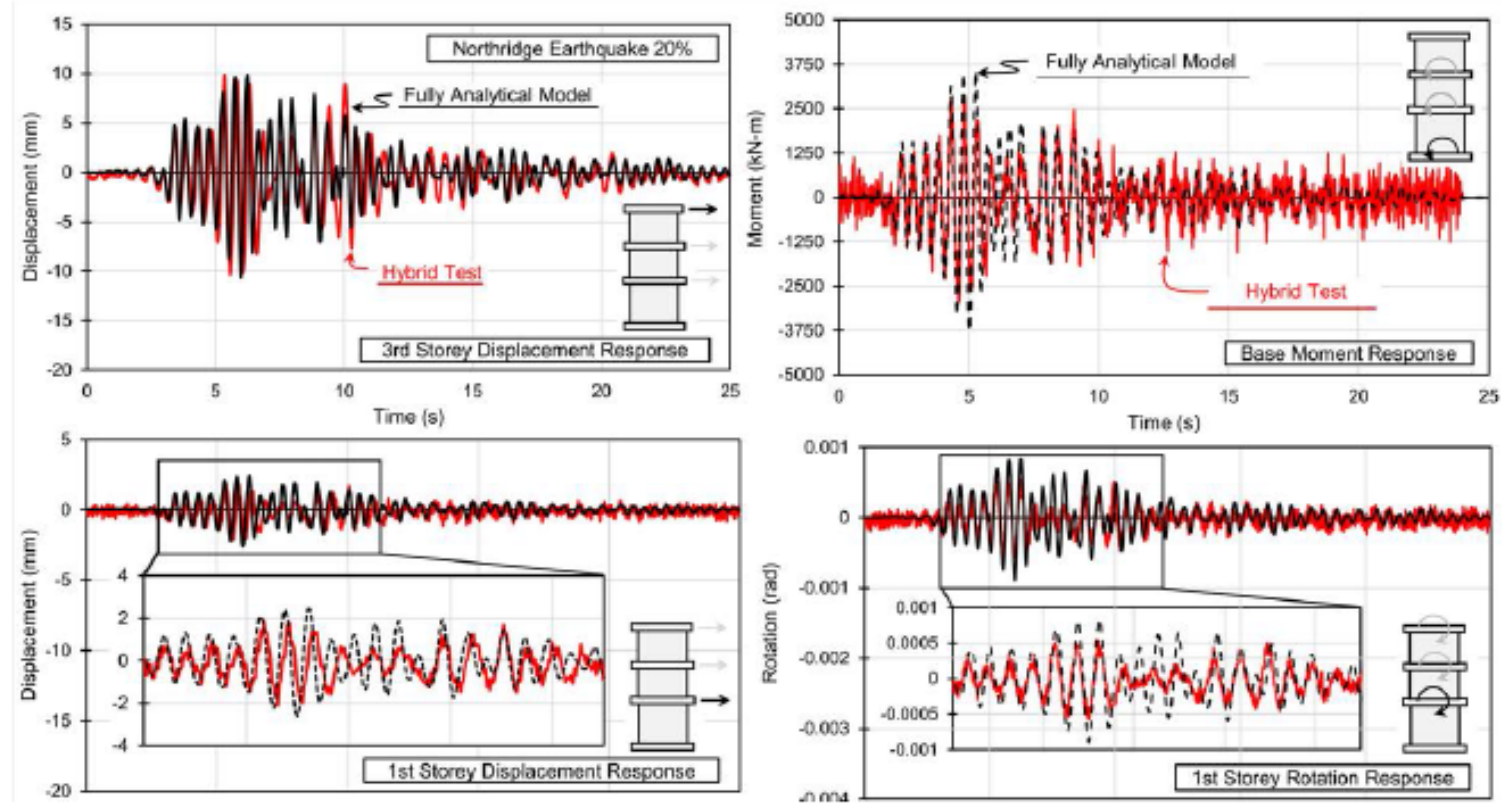

Figure 6.55 Northridge 20\%: comparison between fully analytical and hybrid test results

model the nonlinear response of the second shear wall specimen (W2), the same analytical model used to capture the response of the first wall specimen (W1) was employed. The main differences between the models for wall W1 and W2 were the strengths of the second and third storeys of the shear wall, which were increased to ensure they remained elastic during the fully analytical simulations of wall specimen W1. For wall specimen W2, the strengths of the second and third storeys were the same as those used during the hybrid test, in an attempt to reproduce the hybrid test results as closely as possible. Figure 6.56 compares the displacement time-history at each storey level for the hybrid test with the results from the fully analytical model for the Northridge earthquake scaled to $200 \%$. Recall that the demand on wall specimen W2 during the second set of hybrid tests was much smaller when compared with wall specimen W1 because of nonlinearity in the second and third storeys of the RC shear wall. The results in Fig. 6.56 show that the fully analytical model predicts this result, accurately capturing the large displacements in the second and third storeys of the RC shear wall observed during the hybrid test.

At the $300 \%$ intensity level, the fully analytical model is once again able to reproduce the hybrid test results and accurately capture the response of the shear wall. Figure 6.57 compares 

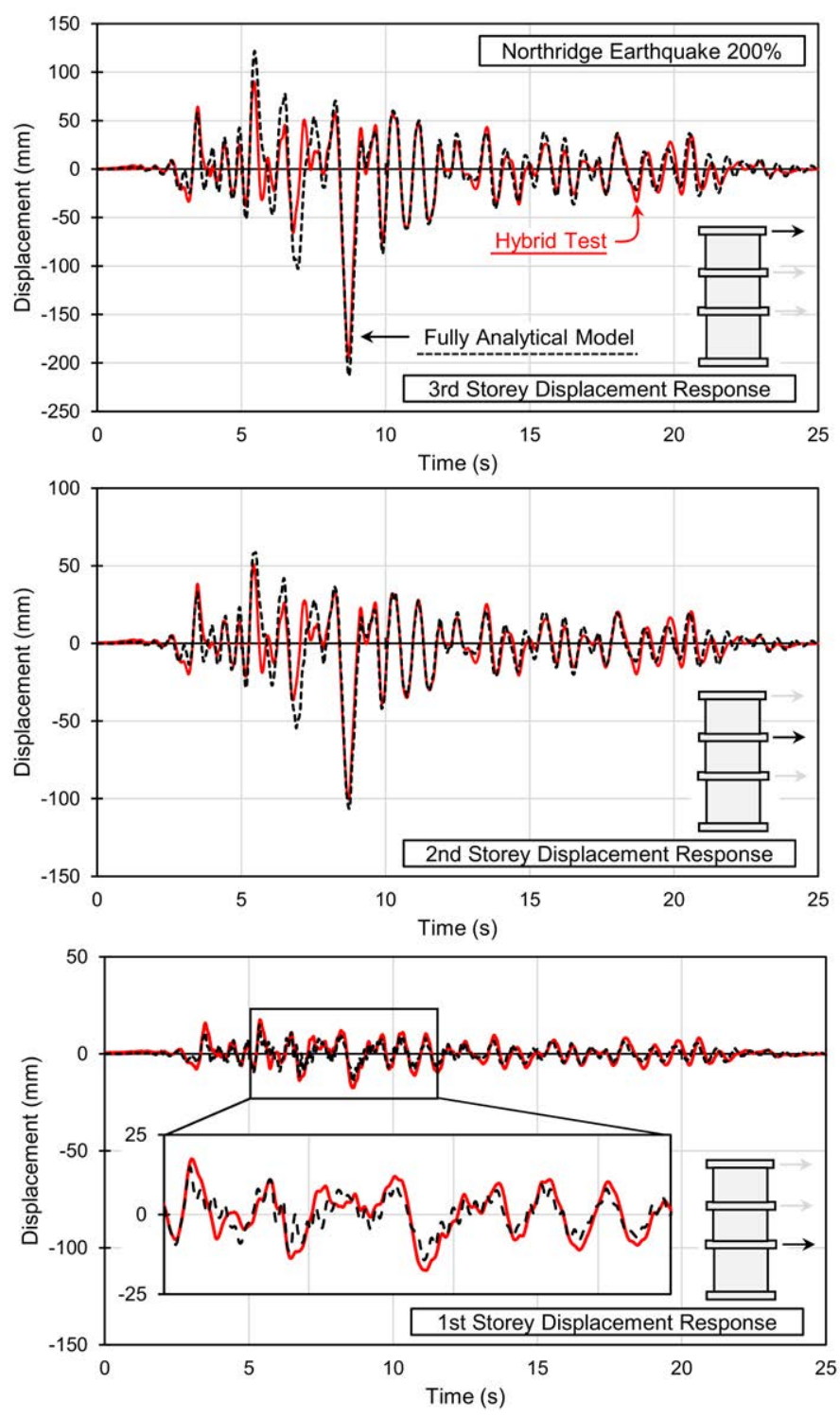

Figure 6.56 Northridge 200\%: comparison between fully analytical and hybrid test results 
the third storey displacement, base shear, and overturning moment time-histories as well as the moment-rotation response for the hybrid test and fully analytical model. The results show that the fully analytical moment-rotation response correlates well with the hybrid test results and the fully analytical wall achieves very comparable maximum overturning moments, base shear, and maximum displacement.

Ultimately, the results from wall specimen W2 once again confirm the finite element model is capable of accurately predicting the hybrid test results. The developed finite element model can now be used as a tool for future hybrid tests to estimate the shear wall response prior to hybrid testing. The correlation between the fully analytical model and the hybrid test results also demonstrates that the hybrid test is capable of achieving reproducible results, even with the measured error between target and feedback displacements, fluctuation in the gravity load on the physical test specimen, and friction in the experimental test setup. This once again demonstrates that hybrid testing is a feasible tool to capture the seismic response of a full-scale RC shear wall. 

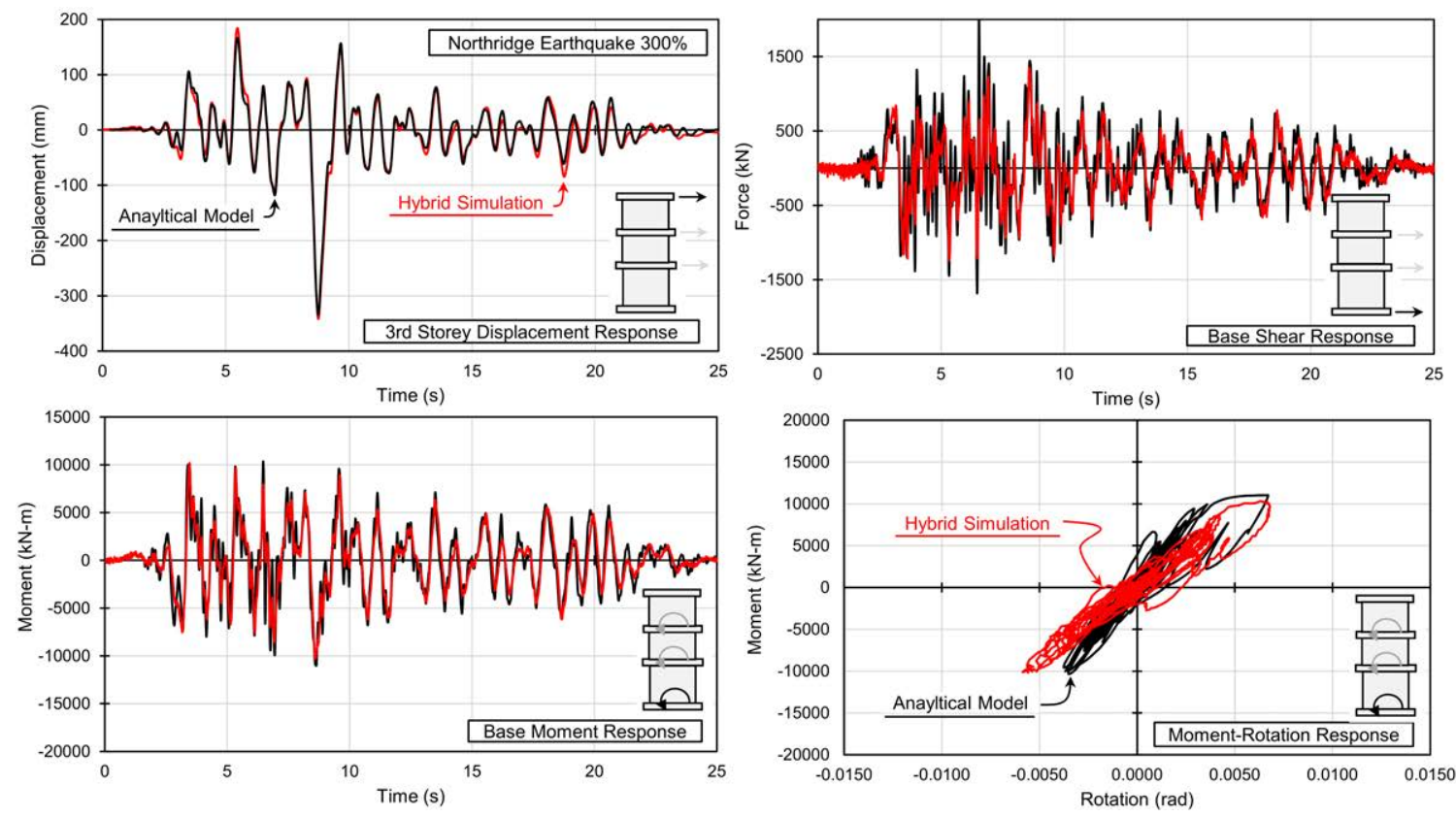

Figure 6.57 Northridge 300\%: comparison between fully analytical and hybrid test results 


\section{Chapter 7. Conclusions and Future Research}

\subsection{Conclusions}

Traditionally, component level structural testing has been used to validate the seismic performance of individual structural elements. Although this testing technique has been shown to be effective in capturing the ductility, energy dissipation capacity, and failure mechanisms in these elements, it may not accurately depict the load distribution on a structural element during an earthquake and does not provide any information related to the overall system-level behaviour. At the opposite end of the experimental testing spectrum, researchers have traditionally used large-scale shaking tables to test the dynamic performance of full-scale structures and obtain overall system-level performance. However, these experiments are time consuming and expensive, requiring a significant investment in facilities and infrastructure.

Some of the major challenges addressed in this study revolve around hybrid simulation conducted on stiff structural elements. Traditionally, this has posed a challenge with respect to hydraulic control, because a small target displacement translates into a large increment in force, so any discrepancies between the two can result in errors in the hybrid test results. To address these concerns, this study has shown that using high-precision measurement devices to externally control the hybrid simulation can provide accurate hybrid test result, including very small command and feedback errors (less than $0.05 \mathrm{~mm}$ on average). In addition, through proper consideration for potential deformation mechanisms in the experimental test setup, the use of external control provides an opportunity to bypass these deformations, preventing errors in the command-feedback displacement control loop. With respect to hydraulic control, MTS DOF, MTS Csi, OpenFresco, 
and OpenSees were shown to be capable of sending, receiving, and transforming command and feedback displacements, rotations, forces, and moments consistently throughout the experiments.

Ultimately, hybrid simulation has been shown to be a feasible alternative to traditional test methods used to study the full-scale response of multi-storey RC shear wall structures. As a test method for stiff RC structures, hybrid simulation has the potential to bridge the gap between component-level and full-scale structural testing, providing a cost-effective and innovative testing solution. In this study, hybrid simulation has been shown to provide detailed information about the local response of a RC shear wall specimen; including ductility, energy dissipation capacity, and failure mechanisms while at the same time capturing the global response of the structure. The results have shown that hybrid simulation is a test method that is able to capture the complex nonlinear behaviour of critical structural elements while also capturing the overall response of a structural system, without the need for large experimental test facilities or construction of a complete full-scale structure in the lab.

Other conclusions drawn from this research include:

- The Carleton University hybrid simulation testing facility is capable of conducting slow hybrid simulations using the developed hybrid test architecture. The hybrid control software used to map laboratory control channels to model DOFs has been shown to be reliable and accurate for a 3-DOF experimental test setup. In addition, the use of a predictor-corrector algorithm has once again been shown to provide accurate and efficient continuous hybrid testing;

- The use of two different coordinate transformations to convert between model DOFs and experimental DOFs based on actuator and encoder coordinate systems allows for the integration of independent force and displacement feedback loops in the hybrid test. This presents the ability to effectively bypass any deformations in the experimental test setup and measure the exact response of the physical substructure;

- The use of high-precision digital encoders for external control are effective control devices for stiff structural elements, even when the command and feedback displacements are very 
small $(<0.01 \mathrm{~mm})$.

- To transform between global DOFs in the finite element model and actuator or encoder DOFs in the laboratory, a separate control software has been integrated in the hybrid control loop. This DOF control software is shown to be an effective and versatile tool to define multiple co-existing coordinate transformations in the same experimental test setup;

- A relationship exists between the stiffness of an experimental substructure and the command-feedback error in a hybrid system. In stiffer structural elements, the commandfeedback error has been shown to be larger when compared with a less-stiff test specimen. The level of command-feedback error also depends on the accuracy and resolution of the sensor used for displacement feedback.

- The incorporation of multi-layered shell elements into the hybrid simulation has the ability to capture the inherent shear behaviour commonly observed in the cyclic and dynamic response of RC structural elements;

- In a fully-analytical finite element model, the multi-layer modelling approach is shown to be able to accurately capture the nonlinear response behaviour of a RC shear wall, including accurately predicting the strength, ductility, and energy dissipation capacity when compared with hybrid test results;

- The freely available image analysis tool ImPro Stereo has the ability to capture concrete crack distributions and surface strain fields over a large area of a structural element, eliminating the need to manually mark cracks or pause a test;

\subsection{Recommendations for Future Research}

This research has demonstrated that hybrid simulation can be a feasible test method to study the response of a full-scale RC shear wall. However, the development of hybrid simulation as a test technique for stiff structural systems is still ongoing, but it has the potential to be used 
to study a wide range of civil engineering structures under different loading conditions. The following include several topics/initiatives suggested for further investigation into the use of hybrid simulation to study the response of RC structures:

1. Study the response of RC shear walls taller than 3-stories using hybrid simulation, with the potential for more than one experimental substructure at different levels;

2. Study the complete 6 degree-of-freedom response of a RC shear wall using hybrid simulation to understand the influence of out-of-plane behaviour on the local and global shear wall response;

3. Investigate the use of high-performance computing to increase the size of the analytical substructure, creating a more realistic finite element model of an entire RC shear wall structure;

4. Examine the feasibility and challenges associated with real-time hybrid testing of stiff structural elements to potentially include rate dependent effects;

5. Extend the use of hybrid simulation to more than one natural hazard (e.g. earthquake, fire, blast) and study the response of a RC shear wall structure under those hazards;

6. Supplement the existing OpenSees source code to allow the user to load a damage state for an analytical substructure. The would allow for damage to the analytical substructure to be transferred between hybrid tests; and

7. Incorporate a pause feature into the MTS Csi hybrid control software to allow for examination of the experimental test specimen, such as crack patterns or damage to the physical substructure, during a hybrid test. In addition, this would allow for multiple earthquake ground motions to be applied to a physical substructure in series (without restarting the hybrid test each time), which is not feasible in slow hybrid simulation because of the time required.

Joshua Woods, Department of Civil and Environmental Engineering, Carleton University 


\section{List of References}

Abbiati, G., Bursi, O. S., Cazzador, E., Ceravolo, R., Mei, Z., Paolacci, F., and Pegon, P. (2015). Pseudo-dynamic testing based on non-linear dynamic substructuring of a reinforced concrete bridge. In Experimental Research in Earthquake Engineering, pages 83-98. Springer.

ACI (2008). Guide for the design and construction of externally bonded FRP systems for strengthening concrete structures. In ACI440.2R-08, page 76. ACI, Farmington Hills, MI.

ACI (2014). Building code requirements for structural concrete. In ACI318-14, page 520. American Concrete Institute, Farmington Hills, MI.

Adebar, P., Ibrahim, A., and Bryson, M. (2007). Test of high-rise core wall: Effective stiffness for seismic analysis. ACI Structural Journal, 104:549-559.

Ancheta, T. D., Darragh, R. B., Stewart, J. P., Seyhan, E., Silva, W. J., Chiou, B. S., Wooddell, K. E., Graves, R. W., Kottke, A. R., Boore, D. M., et al. (2013). PEER NGA-West2 database.

Antoniades, K. K., Salonikios, T. N., and Kappos, A. J. (2003). Cyclic tests on seismically damaged reinforced concrete walls strengthened using fiber-reinforced polymer reinforcement. ACI Struct. J., 100(4).

Antoniades, K. K., Salonikios, T. N., and Kappos, A. J. (2005). Tests on seismically damaged reinforced concrete walls repaired and strengthened using fiber-reinforced polymers. Journal of Composites for Construction, 9(3):236-246.

ASCE (2013). Minimum Design Loads for Buildings and Other Structures. American Society of Civil Engineers, Reston, VA, asce/sei 7-10 edition.

ASTM (American Society for Testing and Materials) (2013). Standard test method for tensile properties of polymer matrix composite materials. In ASTM D3039/D 3039M, page 13. ASTM, West Conshohocken, PA.

Astroza, R., Ebrahimian, H., Conte, J. P., Restrepo, J. I., and Hutchinson, T. C. (2016). System identification of a full-scale five-story reinforced concrete building tested on the NEES-UCSD shake table. Structural Control and Health Monitoring, 23(3):535-559. 
ATC (1996). Seismic evaluation and retrofit of concrete buildings - volume 1. Applied Technology Council for the Federal Emergy Management Agency, Redwood City, California.

Atkinson, G. M. (2005). Ground motions for earthquakes in southwestern british columbia and northwestern washington: Crustal, in-slab, and offshore events. Bulletin of the Seismological Society of America, 95(3):1027-1044.

Balendra, T., Lam, K.-Y., Liaw, C.-n., and Lee, S.-L. (1987). Behavior of eccentrically braced frame by pseudo-dynamic test. Journal of Structural Engineering, 113(4):673-688.

Bin Ibrahim, A., Bin Ismail, P., Forde, M., Gilmour, R., Kato, K., Khan, A., Ooka, N., Siong, S., Terada, K., and Wiggenhauser, H. (2002). Guidebook on non-destructive testing of concrete structures. International Atomic Energy.

Bouguet (2008). Calibration toolbox for matlab [computer software]. In Available online.

Bousias, S. N. (2014). Seismic hybrid simulation of stiff structures: Overview and current advances. Journal of Structures, 2014.

Campbell, S. and Stojadinovic, B. (1998). A system for simultaneous pseudodynamic testing of multiple substructures. In Proceedings, Sixth US National Conference on Earthquake Engineering.

Carney, P. and Myers, J. J. (2003). Shear and flexural strengthening of masonry infill walls with FRP for extreme out-of-plane loading. In Architectural Engineering 2003: Building Integration Solutions, pages 1-5.

Carrion, J. E. and Spencer, B. (2006). Real-time hybrid testing using model-based delay compensation. In Proceedings of the 4th International Conference on Earthquake Engineering, volume 299.

Ceroni, F., Pecce, M., Matthys, S., and Taerwe, L. (2008). Debonding strength and anchorage devices for reinforced concrete elements strengthened with FRP sheets. Composites Part B: Engineering, 39(3):429-441.

Chae, Y., Kazemibidokhti, K., and Ricles, J. M. (2013). Adaptive time series compensator for delay compensation of servo-hydraulic actuator systems for real-time hybrid simulation. Earthquake Engineering E Structural Dynamics, 42(11):1697-1715.

Chang, S.-Y. (2002). Explicit pseudodynamic algorithm with unconditional stability. Journal of Engineering Mechanics, 128(9):935-947.

Chang, Y.-Y. (2008). Multi-site network hybrid testing of a multi-span bridge system. PhD thesis, Carleton University. 
Charlet, A. (2007). Hybrid simulation and its application to the gravity load collapse of reinforced concrete frames. Master's thesis, University of British Columbia, Vancouver, BC, Canada.

Chen, C. and Ricles, J. M. (2010). Tracking error-based servohydraulic actuator adaptive compensation for real-time hybrid simulation. Journal of Structural Engineering, 136(4):432-440.

Chen, C., Ricles, J. M., Marullo, T. M., and Mercan, O. (2009). Real-time hybrid testing using the unconditionally stable explicit CR integration algorithm. Earthquake Engineering $\mathcal{E}$ Structural Dynamics, 38(1):23-44.

Chen, P.-C. and Tsai, K.-C. (2013). Dual compensation strategy for real-time hybrid testing. Earthquake Engineering E Structural Dynamics, 42(1):1-23.

Chung, J. and Hulbert, G. (1993). A time integration algorithm for structural dynamics with improved numerical dissipation: The generalized-alpha method. Journal of Applied Mechanics, 60:371-375.

CISC (2014). Moment connections for seismic applications. In Canadian Institute of Steel Construction. Canadian Institute of Steel Construction, Markham, Canada.

Computers and Structures Inc. (2015). Etabs 2015 [computer software]. In Berkeley, California.

Cruz-Noguez, C., Hassan, A., Lau, D., Woods, J., and Shaheen, I. (2014a). Seismic retrofit of deficient RC shear walls with FRP tow sheets. In Proceedings, Tenth U.S. National Conference on Earthquake Engineering, Anchorage, Alaska.

Cruz-Noguez, C., Lau, D., Sherwood, E., , S., Lombard, J., Foo, S., and Cheung, M. (2014b). Seismic behavior of RC shear walls strengthened for in-plane bending using externally bonded FRP sheets. Journal of Composites for Construction, 19(1):04014023.

Cruz-Noguez, C., Lau, D., and Sherwood, E. (2014c). Seismic behavior of RC shear walls with externally bonded frp sheets: Analytical studies. Journal of Composites for Construction, 18(5):04014011.

CSA (2002). Design and construction of building components with fibre-reinforced polymers. In CSAS806-02, page 189. CSA, Rexdale, Canada.

CSA (2014a). Design of concrete structures. In CSAA23.3-14. Canadian Standards Association, Rexdale, Canada.

CSA (2014b). Handbook of steel construction. In CSAS16-14. Canadian Standards Association, Rexdale, Canada.

Darby, A., Blakeborough, A., and Williams, M. (1999). Real-time substructure tests using hydraulic actuator. Journal of Engineering Mechanics, 125(10):1133-1139. 
Darby, A., Blakeborough, A., and Williams, M. (2001). Improved control algorithm for realtime substructure testing. Earthquake engineering E structural dynamics, 30(3):431-448.

Darby, A., Williams, M., and Blakeborough, A. (2002). Stability and delay compensation for real-time substructure testing. Journal of Engineering Mechanics, 128(12):1276-1284.

Dassault Systemes (2013). ABAQUS v6.13 [computer software]. In Waltham, Massachusetts.

Dong, B., Sause, R., and Ricles, J. M. (2015). Accurate real-time hybrid earthquake simulations on large-scale MDOF steel structure with nonlinear viscous dampers. Earthquake Engineering E Structural Dynamics, 44(12):2035-2055.

Dorka, U. and Heiland, D. (1991). Fast online earthquake simulation using a novel pc supported measurement and control concept. In Proceedings of the 4th Int. Conf. Structural Dynamics, pages 636-645.

Dvorkin, E. N. and Bathe, K.-J. (1984). A continuum mechanics based four-node shell element for general non-linear analysis. Engineering computations, 1(1):77-88.

Eatherton, M. R. and Hajjar, J. F. (2010). Large-scale cyclic and hybrid simulation testing and development of a controlled-rocking steel building system with replaceable fuses" newmark structural engineering laboratory report. University of Illinois at Urbana-Champaign (UIUC) Report.

Ehsani, M. and Peña, C. (2009). Blast loading retrofit of unreinforced masonry walls with carbon fiber reinforced polymer (CFRP) fabrics. Structure Magazine, April, pages 16-20.

El-Sokkary, H., Galal, K., Ghorbanirenani, I., Léger, P., and Tremblay, R. (2012). Shake table tests on FRP-rehabilitated rc shear walls. Journal of Composites for Construction, 17(1):79-90.

Elkhoraibi, T. (2007). Generalized bybrid simulation framework for structural systems subjected to seismic loading. University of California, Berkeley.

Elkhoraibi, T. and Mosalam, K. M. (2007). Towards error-free hybrid simulation using mixed variables. Earthquake engineering E structural dynamics, 36(11):1497-1522.

Elnady, M. (2008). Seismic rehabilitation of RC structural walls. PhD thesis, McMaster University, Hamilton, ON, Canada.

FEMA (1997). Nherp guidelines for the seismic rehabilitation of buildings. Federal Emergy Management Agency, Redwood City, California.

FEMA (2000). Prestandard and commentary for the seismic rehabilitation of buildings. Federal Emergy Management Agency, Redwood City, California.

FEMA (2005). Improvement of nonlinear static seismic analysis procedures. Federal Emergy Management Agency, Redwood City, Californiaty. 
Filiatrault, A., Tinawi, R., and Leger, P. (1992). The use of energy balance in nonlinear seismic analysis. In Proceedings of the 10th world conference on earthquake engineering, pages 4111-4116.

Filiatrault, A., Tremblay, R., Christopoulos, C., Folz, B., and Pettinga, D. (2013). Elements of earthquake engineering and structural dynamics. Presses inter Polytechnique.

Filippou, F. C., Popov, E. P., and Bertero, V. V. (1983). Effects of bond deterioration on hysteretic behavior of reinforced concrete joints. Earthquake Engineering Research Centre Report.

Fintel, M. (1995). Performance of buildings with shear walls in earthquakes of the last thirty years. PCI journal, 40(3):62-80.

Fleischman, R., Restrepo, J., Nema, A., Zhang, D., Shakya, U., Zhang, Z., Sause, R., Tsampras, G., and Monti, G. (2015). Inertial force-limiting anchorage system for seismic resistant building structures. In Structures Congress 2015, pages 1302-1313.

Forouzan, A., Shaloudegi, K., and Nakata, N. (2017). Force-based hybrid simulation: concept, numerical algorithm, and experimental validation. In 13th International Workshop on Advanced Smart Materials and Smart Structures Technology. Tokyo, Japan.

Ghannoum, W. M. and Moehle, J. P. (2012). Shake-table tests of a concrete frame sustaining column axial failures. ACI Structural Journal, 109(3):393.

Ghobarah, A. and Khalil, A. (2004). Seismic rehabilitation of reinforced concrete walls using fibre composites. In Proceedings of the 13th World Conference on Earthquake Engineering, Vancouver, Canada.

Ghorbanirenani, I., Tremblay, R., Léger, P., and Leclerc, M. (2011). Shake table testing of slender rc shear walls subjected to eastern North America seismic ground motions. Journal of Structural Engineering, 138(12):1515-1529.

Grelle, S. V. and Sneed, L. (2011). An evaluation of anchorage systems for fiber-reinforced polymer (FRP) laminates bonded to reinforced concrete elements. In Structures Congress 2011, pages $1157-1168$.

Hall, J., Schuman, P., and Hamilton III, H. (2002). Ductile anchorage for connecting FRP strengthening of under-reinforced masonry buildings. Journal of Composites for Construction, $6(1): 3-10$.

Hassan, A. (2015). Modelling of debonding mechanisms in externally bonded FRP sheets in $\mathrm{RC}$ shear walls for earthquake resistance. Master's thesis, Carleton University, Ottawa, ON, Canada.

Hassan, A., Cruz-Noguez, C., and Lau, D. (2013). Influence of FRP-concrete debonding mechanisms on the seismic response of FRP-reinforced shear walls. In Proceedings of the Canadian Society for Civil Engineering General Conference, pages 1-11. 
Hassan, A. and Lau, D. (2016). Modelling debonding mechanisms in FRP strengthened reinforced concrete shear walls for improved seismic performance. In 7th International Conference on Advanced Composite Materials in Bridges and Structures. Vancouver, Canada.

Hassan, A., Lau, D., Cruz-Noguez, C., Woods, J., and Shaheen, I. (2015). Modelling of debonding mechanisms in externally bonded FRP sheets in RC shear walls for earthquake resistance. In 11th Canadian Conference on Earthquake Engineering. Victoria, Canada.

Hilber, H. M., Hughes, T. J., and Taylor, R. L. (1977). Improved numerical dissipation for time integration algorithms in structural dynamics. Earthquake Engineering E Structural Dynamics, $5(3): 283-292$.

Hiotakis, S. (2004). Repair and strengthening of reinforced concrete shear walls for earthquake resistance using externally bonded carbon fibre sheets and a novel anchor system. Master's thesis, Carleton University, Ottawa, ON, Canada.

Hiotakis, S., Lau, D., and Londono, N. (2004). Research on seismic retrofit and rehabilitation of reinforced concrete shear walls using FRP materials. In Proceedings of the Joint National Science Council Taiwan - National Research Council of Canada NSC-NRC Workshop on Construction Technologies, pages 17-26.

Horiuchi, T., Inoue, M., Konno, T., and Namita, Y. (1999). Real-time hybrid experimental system with actuator delay compensation and its application to a piping system with energy absorber. Earthquake Engineering E Structural Dynamics, 28(10):1121-1141.

Horiuchi, T., Nakagawa, M., Sugano, M., and Konno, T. (1996). Development of a real-time hybrid experimental system with actuator delay compensation. In Proceedings of the 11th World Conference on Earthquake Engineering.

Igarashi, A., Seible, F., and Hegemeier, G. (1990). Development of the pseudodynamic technique for testing a full scale 5-story shear wall structure. In US-Japan Seminar, Development and Future Dimensions of Structural Testing Techniques. Honolulu, HI.

Jacques, E. (2011). Blast retrofit of reinforced concrete walls and slabs. PhD thesis, Universite d'Ottawa/University of Ottawa.

Jeong, S.-H. and Elnashai, A. S. (2005). Analytical assessment of an irregular RC frame for fullscale 3D pseudo-dynamic testing part ii: Condition assessment and test deployment. Journal of Earthquake Engineering, 9(02):265-284.

Kalfat, R., Al-Mahaidi, R., and Smith, S. T. (2013). Anchorage devices used to improve the performance of reinforced concrete beams retrofitted with FRP composites: State-of-the-art review. Journal of Composites for Construction, 17(1):14-33. 
Kam, W. Y., Pampanin, S., and Elwood, K. (2011). Seismic performance of reinforced concrete buildings in the 22 February Christchurch (Lyttleton) earthquake. Bulletin of the New Zealand Society for Earthquake Engineering, 44(4).

Kammula, V., Erochko, J., Kwon, O., and Christopoulos, C. (2012). Performance assessment of the self centering energy dissipative (SCED) bracing system using hybrid simulation. In Proceedings of the 15th world conference on earthquake engineering, Lisbon.

Kammula, V., Erochko, J., Kwon, O.-S., and Christopoulos, C. (2014). Application of hybridsimulation to fragility assessment of the telescoping self-centering energy dissipative bracing system. Earthquake Engineering E Structural Dynamics, 43(6):811-830.

Kanakubo, T., Aridome, Y., Fujita, N., and Matsui, M. (2000). Development of anchorage system for CFRP sheet in strengthening of reinforced concrete structures. Strain, 50:15.

Karavasilis, T. L., Ricles, J. M., Sause, R., and Chen, C. (2011). Experimental evaluation of the seismic performance of steel MRFs with compressed elastomer dampers using large-scale real-time hybrid simulation. Engineering Structures, 33(6):1859-1869.

Khalifa, A., Alkhrdaji, T., Nanni, A., and Lansburg, S. (1999). Anchorage of surface mounted FRP reinforcement. Concrete International, 21:49-54.

Kim, H. (2009). Extending hybrid simulation methods in OpenFresco software framework. CE299 Report.

Kim, H., Stojadinovic, B., Yang, T., and Schellenberg, A. (2011). Alternative control strategies in hybrid simulation. In Proceedings of the 2nd EFAST Workshop E 4th International Conference on Advances in Experimental Structural Engineering. JRC, ELSA.

Kuang, J. and Ho, Y. (2008). Seismic behavior and ductility of squat reinforced concrete shear walls with nonseismic detailing. ACI Structural Journal, 105(2).

Kwon, O.-S., Nakata, N., Elnashai, A., and Spencer, B. (2005). A framework for multi-site distributed simulation and application to complex structural systems. Journal of Earthquake Engineering, 9(05):741-753.

Lam, L. and Teng, J. (2001). Strength of rc cantilever slabs bonded with GFRP strips. Journal of Composites for Construction, 5(4):221-227.

Lamarche, C.-P., Tremblay, R., Léger, P., Leclerc, M., and Bursi, O. (2010). Comparison between real-time dynamic substructuring and shake table testing techniques for nonlinear seismic applications. Earthquake Engineering E Structural Dynamics, 39(12):1299-1320.

Lau, D. T. and Woods, J. E. (2017). A concentric tube anchor system for FRP retrofit of RC structural walls under extreme loads. International Journal of Protective Structures. 
Layssi, H., Cook, W. D., and Mitchell, D. (2012). Seismic response and CFRP retrofit of poorly detailed shear walls. Journal of Composites for Construction, 16(3):332-339.

Lefas, I. D., Kotsovos, M. D., and Ambraseys, N. N. (1990). Behavior of reinforced concrete structural walls: strength, deformation characteristics, and failure mechanism. Structural Journal, 87(1):23-31.

Lin, P.-C., Tsai, K.-C., Wang, K.-J., Yu, Y.-J., Wei, C.-Y., Wu, A.-C., Tsai, C.-Y., Lin, C.-H., Chen, J.-C., Schellenberg, A. H., et al. (2011). Seismic design and hybrid tests of a full-scale three-story buckling-restrained braced frame using welded end connections and thin profile. Earthquake Engineering E Structural Dynamics, 41(5):1001-1020.

Lin, Y. and Christenson, R. (2009). Comparison of real-time hybrid testing with shake table tests for an mr damper controlled structure. In Proceedings of the of the American Control Conference.

Lombard, J. (1999). Seismic strengthening and repair of reinforced concrete shear walls using externally bonded carbon fibre tow sheets. Master's thesis, Carleton University, Ottawa, ON, Canada.

Lombard, J., Lau, D. T., Humar, J. L., Foo, S., and Cheung, M. (2000). Seismic strengthening and repair of reinforced concrete shear walls. In Proceedings of the 12th World Conference on Earthquake Engineering, Auckland, New Zealand, pages 1-8.

Lowes, L. N., Lehman, D. E., Birely, A. C., Kuchma, D. A., Marley, K. P., and Hart, C. R. (2012). Earthquake response of slender planar concrete walls with modern detailing. Engineering Structures, 43:31-47.

Lu, X., Xie, L., Guan, H., Huang, Y., and Lu, X. (2015). A shear wall element for nonlinear seismic analysis of super-tall buildings using opensees. Finite Element Analysis and Design, 98(1):14-25.

Magonette, G. and Negro, P. (1998). Verification of the pseudodynamic test method. European Earthquake Engineering, 12:40-50.

Mahin, S. A. and Shing, P.-S. B. (1985). Pseudodynamic method for seismic testing. Journal of Structural Engineering, 111(7):1482-1503.

Mahin, S. A., Shing, P.-S. B., Thewalt, C. R., and Hanson, R. D. (1989). Pseudodynamic test method-current status and future directions. Journal of Structural Engineering, 115(8):21132128 .

Mahin, S. A. and Williams, M. E. (1980). Computer controlled seismic performance testing. In Dynamic Response of Structures: Experimentation, Observation, Prediction and Control, pages 616-630. ASCE.

Joshua Woods, Department of Civil and Environmental Engineering, Carleton University 
Massone, L. M. and Wallace, J. W. (2004). Load-deformation responses of slender reinforced concrete walls. ACI Structural Journal, 101(1).

McCrum, D. and Williams, M. (2016). An overview of seismic hybrid testing of engineering structures. Engineering Structures, 118:240-261.

McKenna, F., Fenves, G., Scott, H., and Jeremic, B. (2000). Open system for earthquake engineering simulation (opensees) [computer software]. Pacific Earthquake Engineering Research Center, University of California at Berkeley, Available from http://opensees.berkeley.edu/.

Menegotto, M. and Pinto, P. (1973). Method of analysis for cyclically loaded rc frames including changes in geometry and non-elastic behaviour of elements under combined normal force and bending. In IABSE Congress Reports of the Working Commission, volume 13.

Molina, F., Verzeletti, G., Magonette, G., Buchet, P., and Geradin, M. (1999). Bi-directional pseudodynamic test of a full-size three-storey building. Earthquake engineering $E$ structural dynamics, 28(12):1541-1566.

Molina, F., Verzeletti, G., Magonette, G., Buchet, P., Renda, V., Geradin, M., Parducci, A., Mezzi, M., Pacchiarotti, A., Federici, L., et al. (2002). Pseudodynamic tests on rubber base isolators with numerical substructuring of the superstructure and strain-rate effect compensation. Earthquake engineering $\mathcal{E}$ structural dynamics, 31(8):1563-1582.

Mosqueda, G. (2003). Continuous hybrid simulation with geographically distributed substructures. PhD thesis, University of California at Berkeley, California, USA.

Mosqueda, G., Stojadinovic, B., Hanley, J., Sivaselvan, M., and Reinhorn, A. (2006). Fast hybrid simulation with geographically distributed substructures. In Proceedings of the 17th Analysis and Computation Specialty Conference.

Mosqueda, G., Stojadinovic, B., and Mahin, S. A. (2007). Real-time error monitoring for hybrid simulation. part i: methodology and experimental verification. Journal of Structural Engineering, 133(8):1100-1108.

Nakashima, M., Kaminoso, T., Ishida, M., and Kazuhiro, A. (1990). Integration techniques for substructure online test. In Proceedings of the 4th U.S. National Conference on Earthquake Engineering.

Nakashima, M., Kato, H., and Takaoka, E. (1992). Development of real-time pseudo dynamic testing. Earthquake Engineering E Structural Dynamics, 21(1):79-92.

Nakashima, M. and Masaoka, N. (1999). Real-time on-line test for MDOF systems. Earthquake engineering E structural dynamics, 28(4):393-420. 
Nakata, N., Spencer Jr, B. F., and Elnashai, A. S. (2007). Multi-dimensional mixed-mode hybrid simulation control and applications. Technical report, Newmark Structural Engineering Laboratory. University of Illinois at Urbana ....

Negro, P., Mola, E., Molina, F. J., and Magonette, G. E. (2004). Full-scale psd testing of a torsionally unbalanced three-storey non-seismic RC frame. In Proceedings of the 13th World Conf. on Earthquake Engineering.

Neild, S., Stoten, D., Drury, D., and Wagg, D. (2005). Control issues relating to real-time substructuring experiments using a shaking table. Earthquake engineering E structural dynamics, 34(9):1171-1192.

Newmark, N. M. (1959). A method of computation for structural dynamics. Journal of Engineering Mechanics Division, 85:67-94.

NRC (2010). National building code of canada. In National Building Code of Canada. NRC (National Research Council), Ottawa, ON.

Oesterle, R., Aristizabal-Ochoa, J., Fiorato, A., Russell, H., and Corley, W. (1979). Earthquake resistant structural walls-tests of isolated walls-phase ii. Report to the National Science Foundation, Portland Cement Association.

Oesterle, R., Fiorato, A., Johal, L., Carpenter, J., Russell, H., and Corley, W. (1976). Earthquake resistant structural walls-tests of isolated walls. Report to the National Science Foundation.

Palermo, D., Vecchio, F. J., and Solanki, H. (2002). Behavior of three-dimensional reinforced concrete shear walls. ACI Structural Journal, 99(1):81-89.

Panagiotou, M., Restrepo, J. I., and Conte, J. P. (2010). Shake-table test of a full-scale 7-story building slice. phase i: Rectangular wall. Journal of Structural Engineering, 137(6):691-704.

Paterson, J. and Mitchell, D. (2003). Seismic retrofit of shear walls with headed bars and carbon fiber wrap. Journal of Structural Engineering, 129(5):606-614.

Pilakoutas, K. and Elnashai, A. (1995). Cyclic behavior of reinforced concrete cantilever walls. part i: Experimental results. ACI Structural Journal, 92(3):271-281.

Priestley, M. and Paulay, T. (1992). Seismic design of reinforced concrete and masonry buildings. New York: John Wiley E Sons, Inc.

Ramos, M. D. C., Mosqueda, G., and Lignos, D. G. (2013). Hybrid simulation of the seismic response of a steel moment frame building structure through collapse. $\mathrm{PhD}$ thesis, State University of New York at Buffalo.

Rocks, J., Luna, B., and Whittaker, A. (2011). Seismic response of low aspect ratio reinforced concrete shear walls. In Proceedings of the of the SMiRT conference, volume 21. 
Saatcioglu, M., Mitchell, D., Tinawi, R., Gardner, J., Gillies, A., Ghobarah, A., Anderson, D., and Lau, D. (2001). The August 17, 1999, Kocaeli (Turkey) earthquake damage to structures. Canadian Journal of Civil Engineering, 28(4):715-737.

Salonikios, T., Kappos, A., Tegos, I., and Penelis, G. (1999). Cyclic load behavior of lowslenderness reinforced concrete walls: design basis and test results. ACI Structural Journal, 96(4):649-660.

Saouma, V., Haussmann, G., Kang, D.-H., and Ghannoum, W. (2013). Real-time hybrid simulation of a nonductile reinforced concrete frame. Journal of Structural Engineering, 140(2):04013059.

Sarebanha, A., Schellenberg, A., Schoettler, M., Mosqueda, G., and Mahin, S. (2017). Hybrid simulation of full scale seismic isolation bearings using the caltrans srmd test facility. In Proceedings of the of 16th World Conference on Earthquake Engineering.

Schellenberg, A., Huang, Y., and Mahin, S. A. (2008). Structural FE-software coupling through the experimental software framework, openfresco. In Proceedings, 14th World Conference on Earthquake Engineering, Beijing, China.

Schellenberg, A., Mahin, S., and Fenves, G. (2007). Software framework for hybrid simulation of large structural systems. In Proceedings, Structures Congress, ASCE, Long Beach, CA.

Schellenberg, A., Mahin, S., and Fenves, G. (2009). Advanced implementation of hybrid simulation. Technical report, PEER Report 2009/104, Pacific Earthquake Engineering Research Center, University of California, Berkeley.

SEAOC (1995). Performance based seismic engineering of buildings, vision 2000 report, volumes i and ii. Structural Engineers Association of California, Sacramento, California.

Seible, F., Hegemier, G., and Igarashi, A. (1996). Simulated seismic laboratory load testing of full-scale buildings. Earthquake Spectra, 12(1):57-86.

Seo, C.-Y., Karavasilis, T. L., Ricles, J. M., and Sause, R. (2014). Seismic performance and probabilistic collapse resistance assessment of steel moment resisting frames with fluid viscous dampers. Earthquake Engineering E Structural Dynamics, 43(14):2135-2154.

Shaheen, I. (2014). Seismic retorfit and strengthening of deficient reinforced concrete shear walls using externally bonded fibre reinforced polymer sheets. Master's thesis, Carleton University, Ottawa, ON, Canada.

Shing, P., Spacone, E., and Stauffer, E. (2002). Conceptual design of fast hybrid test system at the university of colorado. In Proceedings of the 7th US National Conference on Earthquake Engineering, Boston, Massachusetts. 
Shing, P. B., Bursi, O. S., and Vannan, M. T. (1994). Pseudodynamic tests of a concentrically braced frame using substructuring techniques. Journal of Constructional Steel Research, 29(13):121-148.

Shing, P. B., Nakashima, M., and Bursi, O. S. (1996). Application of pseudodynamic test method to structural research. Earthquake Spectra, 12(1):29-56.

Shing, P.-S. B., Vannan, M. T., and Cater, E. (1991). Implicit time integration for pseudodynamic tests. Earthquake Engineering E Structural Dynamics, 20(6):551-576.

Sivaselvan, M. V., Reinhorn, A. M., Shao, X., and Weinreber, S. (2008). Dynamic force control with hydraulic actuators using added compliance and displacement compensation. Earthquake Engineering E Structural Dynamics, 37(15):1785-1800.

Stojadinovic, B., Mosqueda, G., and Mahin, S. A. (2006). Event-driven control system for geographically distributed hybrid simulation. Journal of Structural Engineering, 132(1):68-77.

Takanashi, K. and Nakashima, M. (1987). Japanese activities on on-line testing. Journal of Engineering Mechanics, 113(7):1014-1032.

Takanashi, K., Udagawa, K., Seki, M., Okada, T., and Tanaka, H. (1975). Nonlinear earthquake response analysis of structures by a computer-actuator on-line system. Bulletin of Earthquake Resistant Structure Research Center, 8:1-17.

Teng, J. G., Chen, J.-F., Smith, S. T., and Lam, L. (2002). FRP-strengthened RC structures. Front. in Phys.

Thewalt, C. R. and Mahin, S. A. (1995). Non-planar pseudodynamic testing. Earthquake engineering Estructural dynamics, 24(5):733-746.

Tsai, K., Yeh, C., Yang, Y., Wang, K., Wang, S., and Chen, P. (2003). Seismic hazard mitigation: Internet-based hybrid testing framework and examples. In Proceedings of the International Colloquium on Natural Hazard Mitigation: Methods and Applications.

Tsai, K.-C., Hsiao, P.-C., Wang, K.-J., Weng, Y.-T., Lin, M.-L., Lin, K.-C., Chen, C.-H., Lai, J.W., and Lin, S.-L. (2008). Pseudo-dynamic tests of a full-scale CFT/BRB frame-part i: Specimen design, experiment and analysis. Earthquake Engineering and Structural Dynamics, 37(7):10811098.

Vecchio, F. J. and Collins, M. P. (1986a). The modified compression-field theory for reinforced concrete elements subjected to shear. ACI Structural Journal, 83(2):219-231.

Vecchio, F. J. and Collins, M. P. (1986b). The modified compression-field theory for reinforced concrete elements subjected to shear. ACI Structural Journal, 83(2):219-231. 
Vecchio, F. J. and Collins, M. P. (1988). Predicting the response of reinforced concrete beams subjected to shear using modified compression field theory. ACI Structural Journal, 85(3):258268.

Vision, O. S. C. (2016). Open source computer vision [computer software]. In Avaialble from: http://opencr.org/.

Wagg, D. and Stoten, D. (2001). Substructuring of dynamical systems via the adaptive minimal control synthesis algorithm. Earthquake Engineering E Structural Dynamics, 30(6):865-877.

Wallace, J. (2015). Performance of structural walls in recent earthquakes and test and implications for US building codes. In Proceedings of the 15th World Conference on Earthquake Engineering, Lisboa, Portugal.

Wallace, M., Wagg, D., and Neild, S. (2005). An adaptive polynomial based forward prediction algorithm for multi-actuator real-time dynamic substructuring. In Proceedings of the Royal Society of London A: Mathematical, Physical and Engineering Sciences, volume 461, pages 38073826. The Royal Society.

Whyte, C. A. and Stojadinovic, B. (2013a). Effect of ground motion sequence on response of squat reinforced concrete shear walls. Journal of Structural Engineering, 140(8):A4014004.

Whyte, C. A. and Stojadinovic, B. (2013b). Hybrid simulation of the seismic response of squat reinforced concrete shear walls. Pacific Earthquake Engineering Research Center Report.

Wiradinata, S. (1985). Behaviour of squat shear walls subject to load reversals. PhD thesis, University of Ottawa, Ottawa, ON, Canada.

Wood, S. (1990). Shear strength of low-rise reinforced concrete walls. ACI Structural Journal, 87(1).

Woods, J. (2014). Seismic retrofit of deficient reinforced concrete shear walls using fibrereinforced polymer sheets: experimental study and anchor design. Master's thesis, Carleton University, Ottawa, ON, Canada.

Woods, J. and Lau, D. (2016). Image analysis method used in the seismic rehabilitation of squat reinforced concrete shear walls using CFRP sheets. In Proceedings of the 24th Australasian conference on mechanics of structures and materials, pages 1-6.

Woods, J. E., Lau, D. T., and Cruz-Noguez, C. A. (2016). In-plane seismic strengthening of nonductile reinforced concrete shear walls using externally bonded CFRP sheets. Journal of Composites for Construction, page 04016052.

Yang, T., Tung, D. P., Li, Y., Lin, J. Y., Li, K., and Guo, W. (2017). Theory and implementation of switch-based hybrid simulation technology for earthquake engineering applications. Earthquake Engineering E Structural Dynamics, 46(14):2603-2617. 
Yang, Y., Huang, C., and Wu, C. (2014). Impro stereo [computer software]. In ImPro Stereo Website: http://sites.google.com/site/improstereon.

Yang, Y.-S., Huang, C.-W., and Wu, C.-l. (2012). A simple image-based strain measurement method for measuring the strain fields in an RC-wall experiment. Earthquake Engineering $\mathcal{E}$ Structural Dynamics, 41(1):1-17.

Yang, Y.-S., Yang, Wu, C.-1., Hsu, T. T., Yang, H.-C., and Lu, H.-J. (2018). Image analysis method for crack distribution and width estimation for reinforced concrete structures. $A u$ tomation in Construction, 91:120-132.

Yang, Y.-S., Yang, C.-M., and Huang, C.-W. (2015). Thin crack observation in a reinforced concrete bridge pier test using image processing and analysis. Advances in Engineering Software, 83:99-108.

Yassin, M. H. M. (1994). Nonlinear analysis of prestressed concrete sructures under monotonic and cyclic loads. University of California, Berkeley.

You, S. and Schellenberg, A. (2017). Test rehearsal methods for quasi static and real-time hybrid simulations. In Proceedings of the 16th World Conference on Earthquake Engineering.

Zhang, H. and Smith, S. T. (2012). FRP-to-concrete joint assemblages anchored with multiple FRP anchors. Composite Structures, 94(2):403-414.

Zhong, W. (2005). Fast hybrid test system for substructure evaluation. Master's thesis, University of Colorado at Boulder, Boulder, CO, USA. 


\section{Appendix A. Hybrid Simulation Results}

This section provides detailed results for each hybrid test conducted in this study. For each hybrid test, the results presented include the global and local displacement, rotation, force, and moment time-histories and hysteresis. The local hybrid test results are presented first, followed by the global results. 

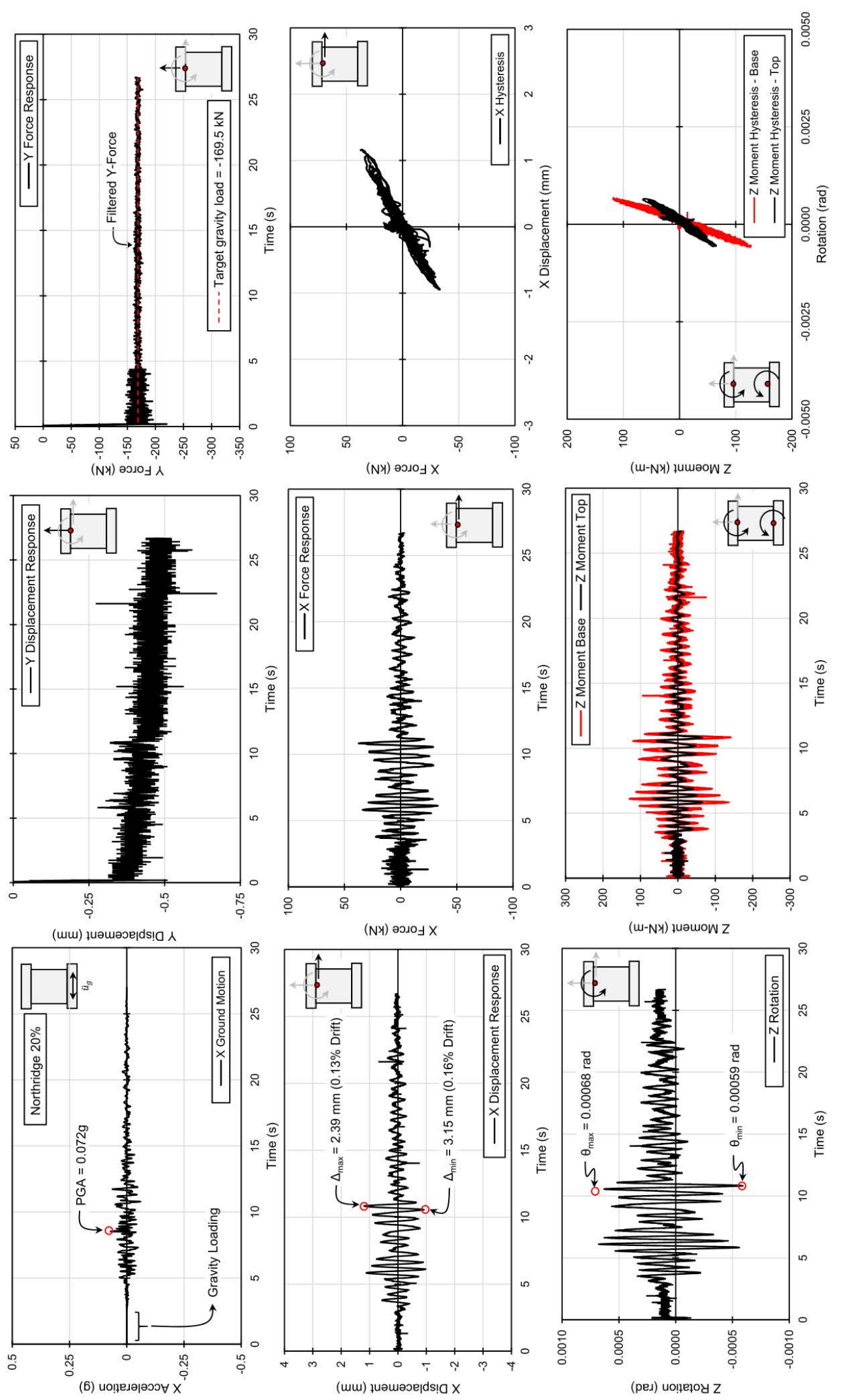

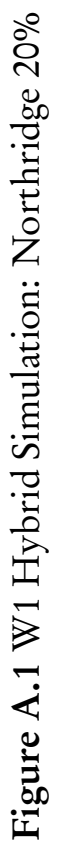



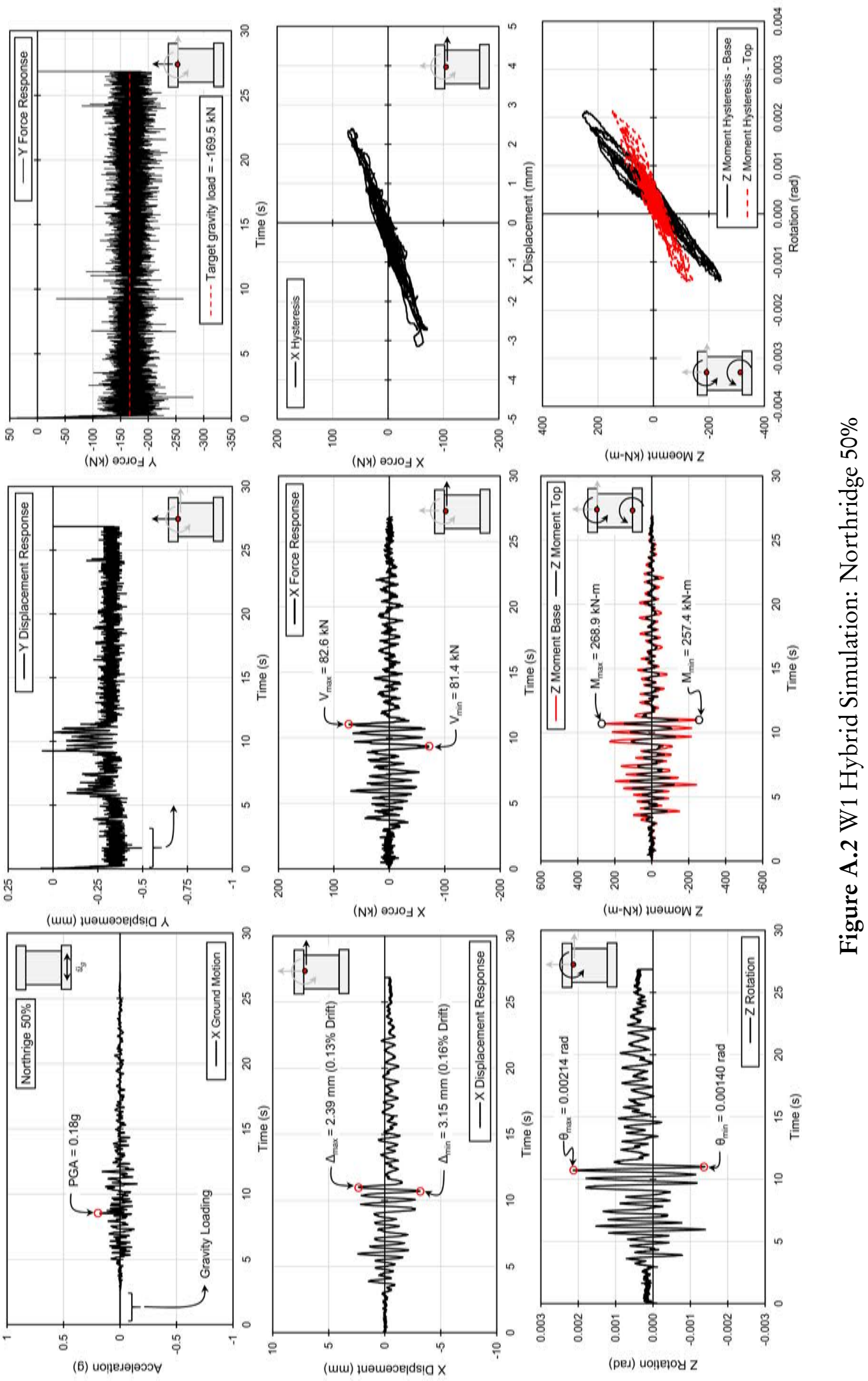

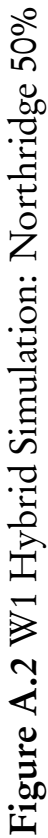



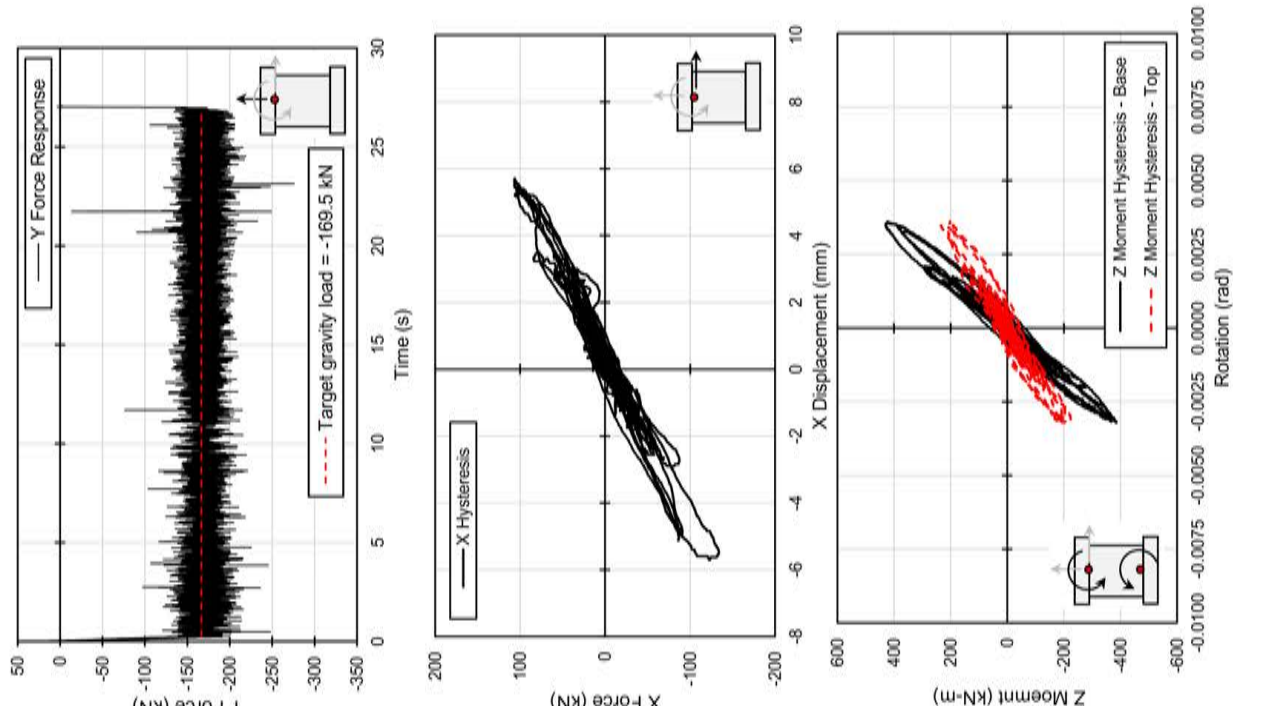

$\stackrel{\circ}{\circ}$
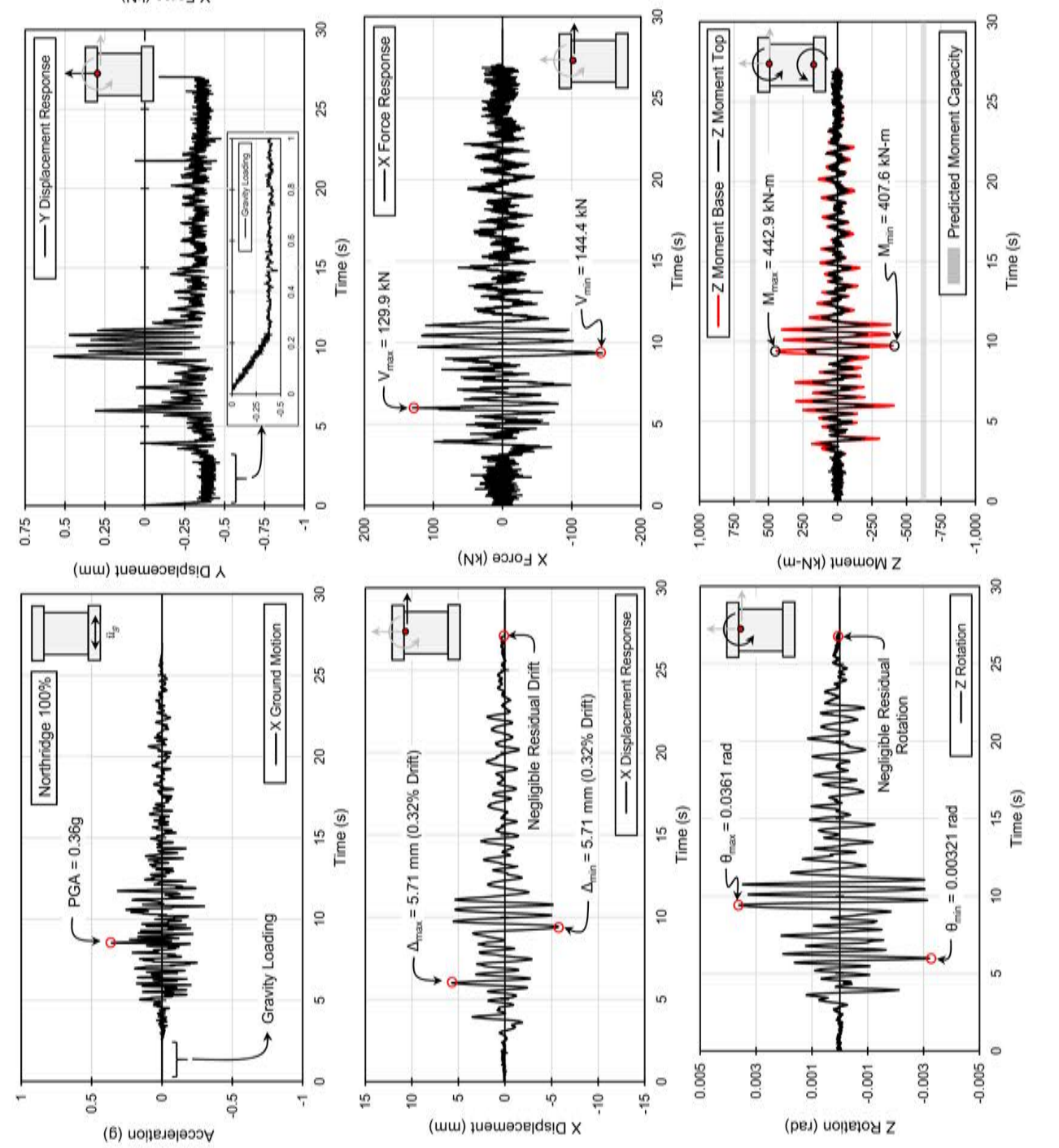

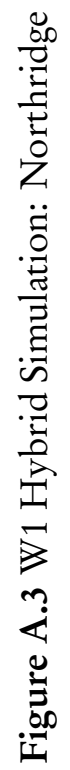



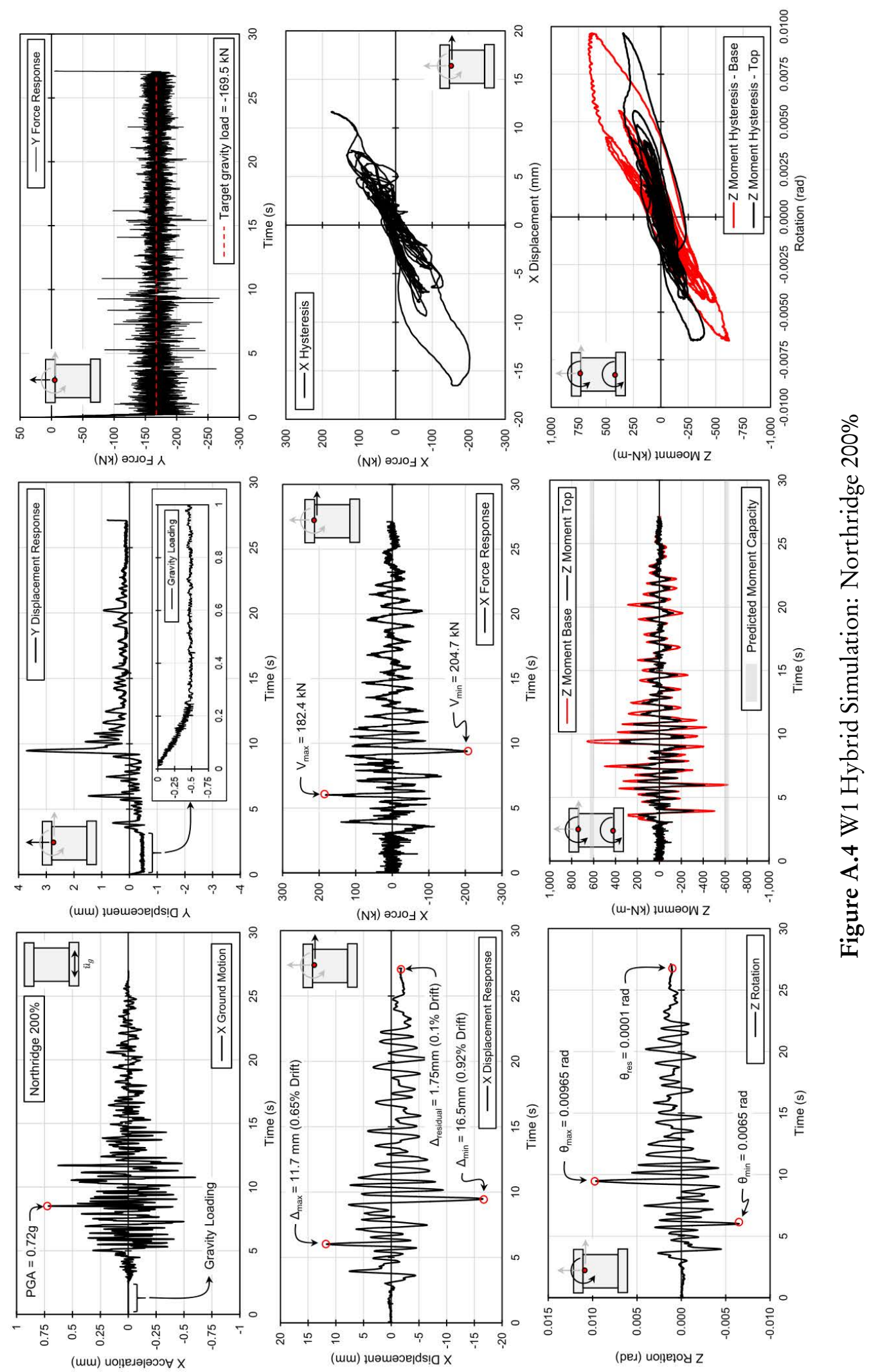

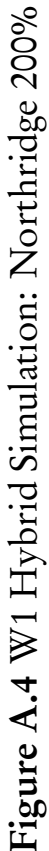



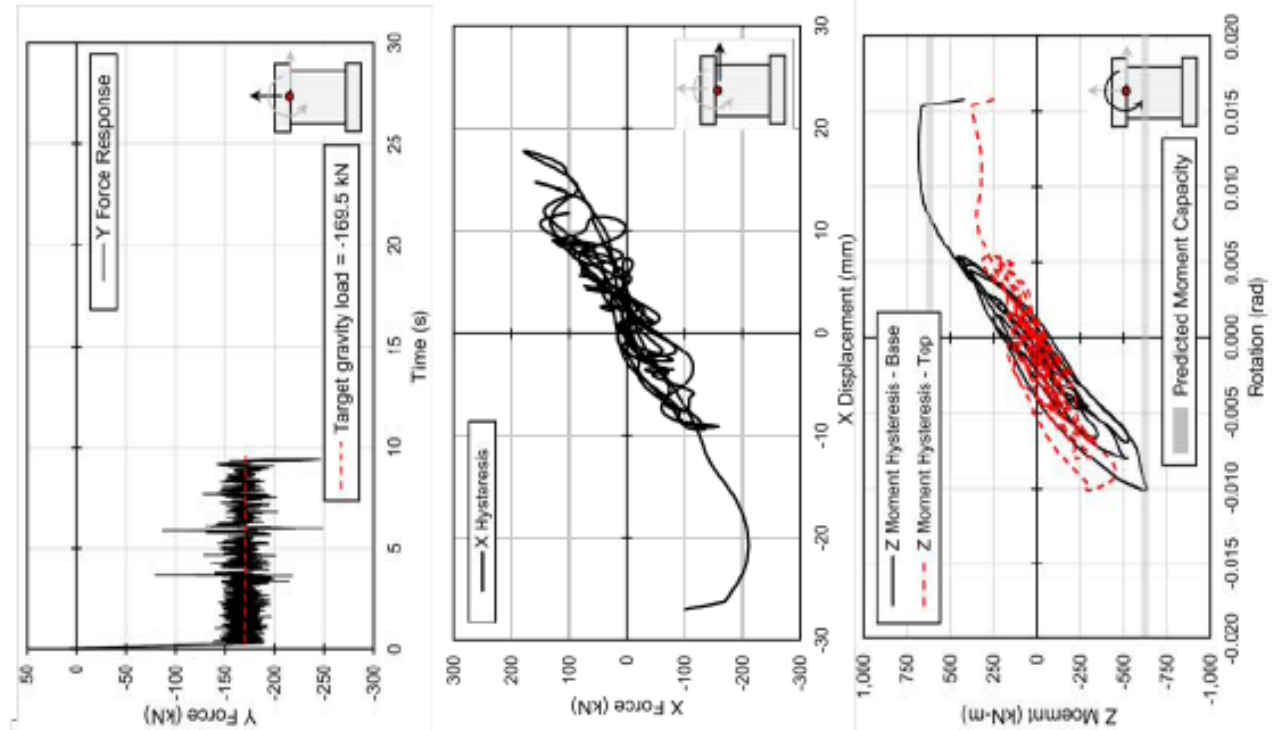

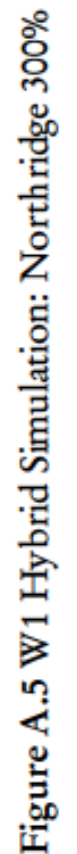
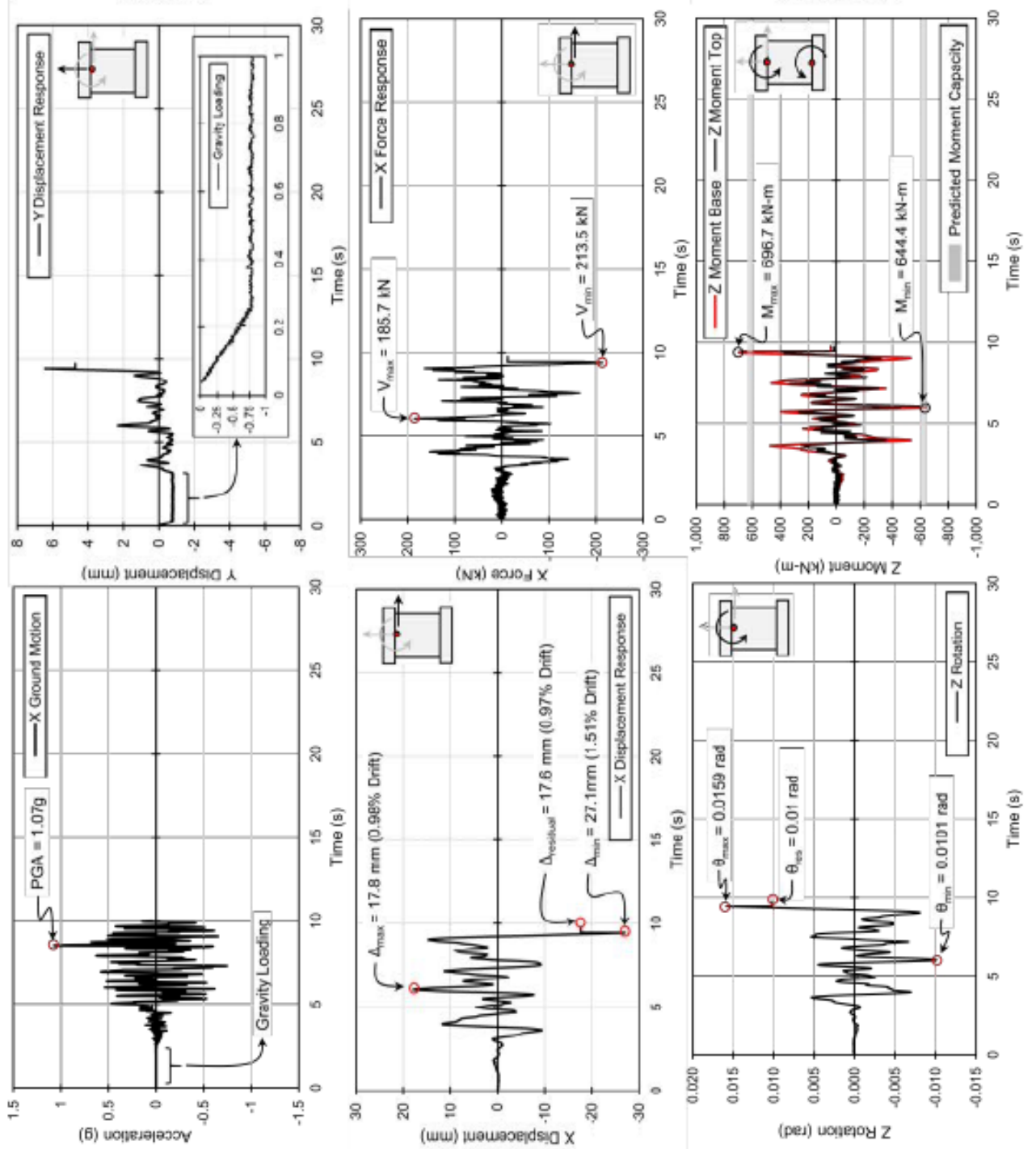

Joshua Woods, Department of Civil and Environmental Engineering, Carleton University 

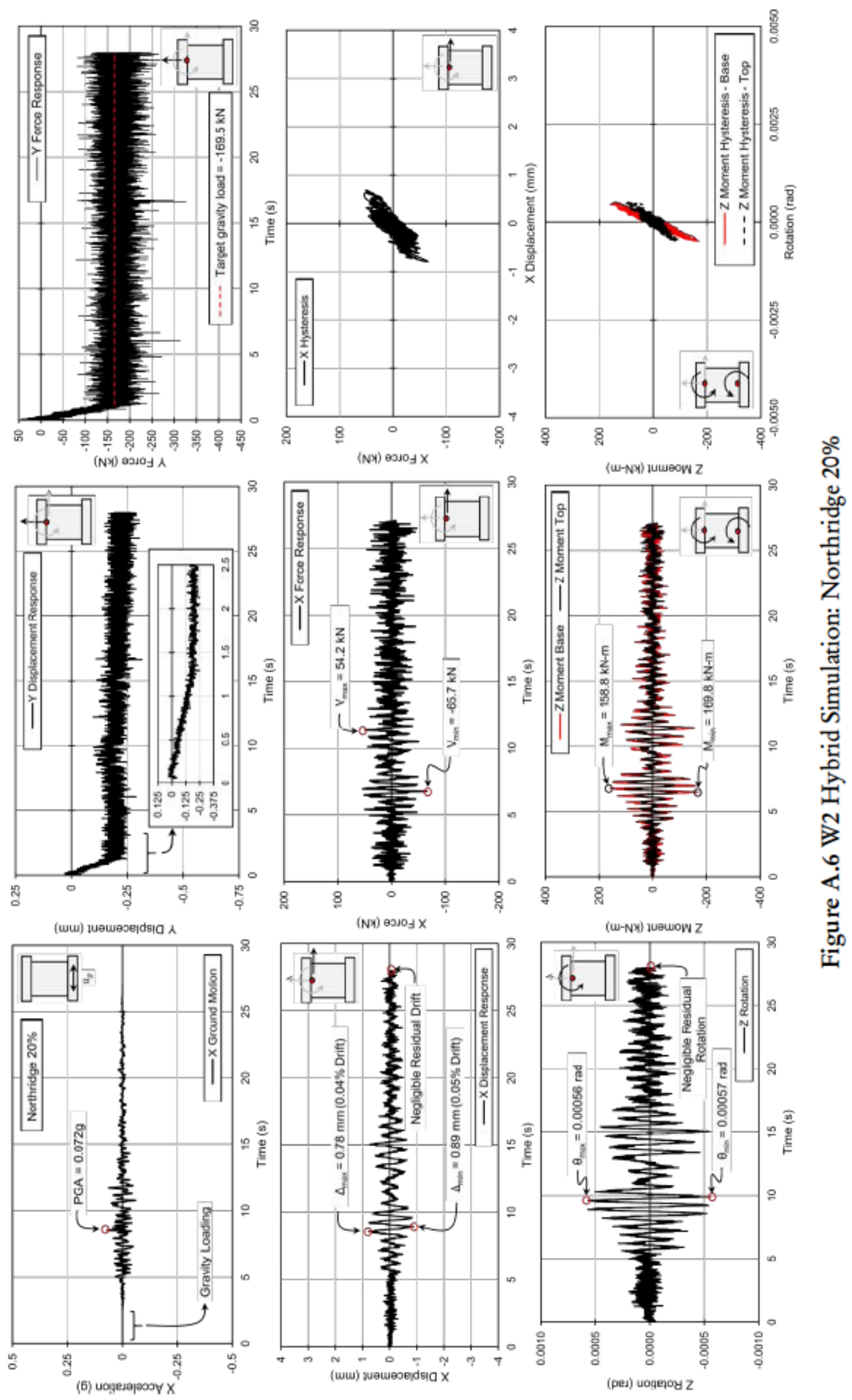

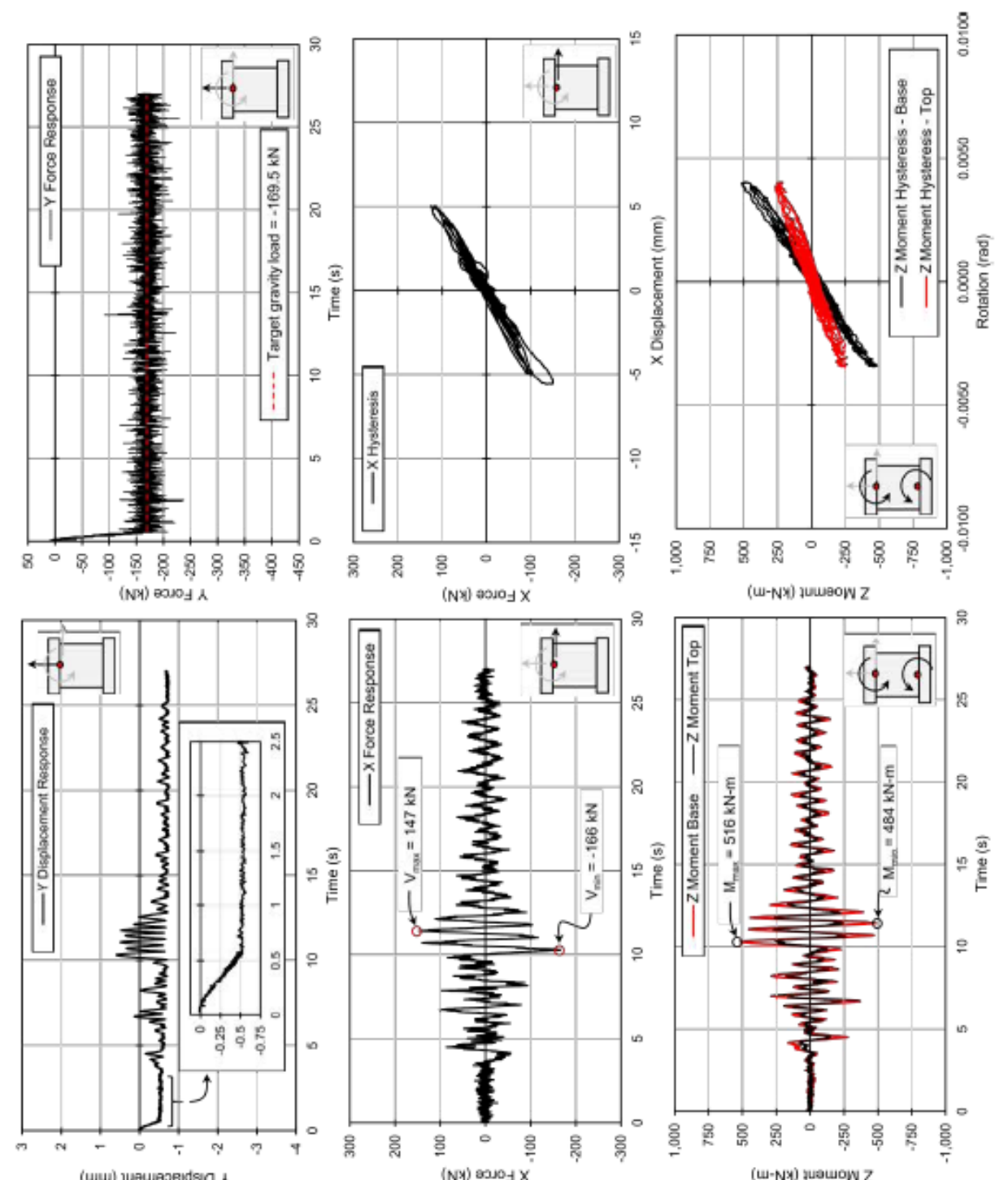

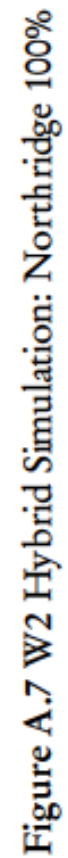
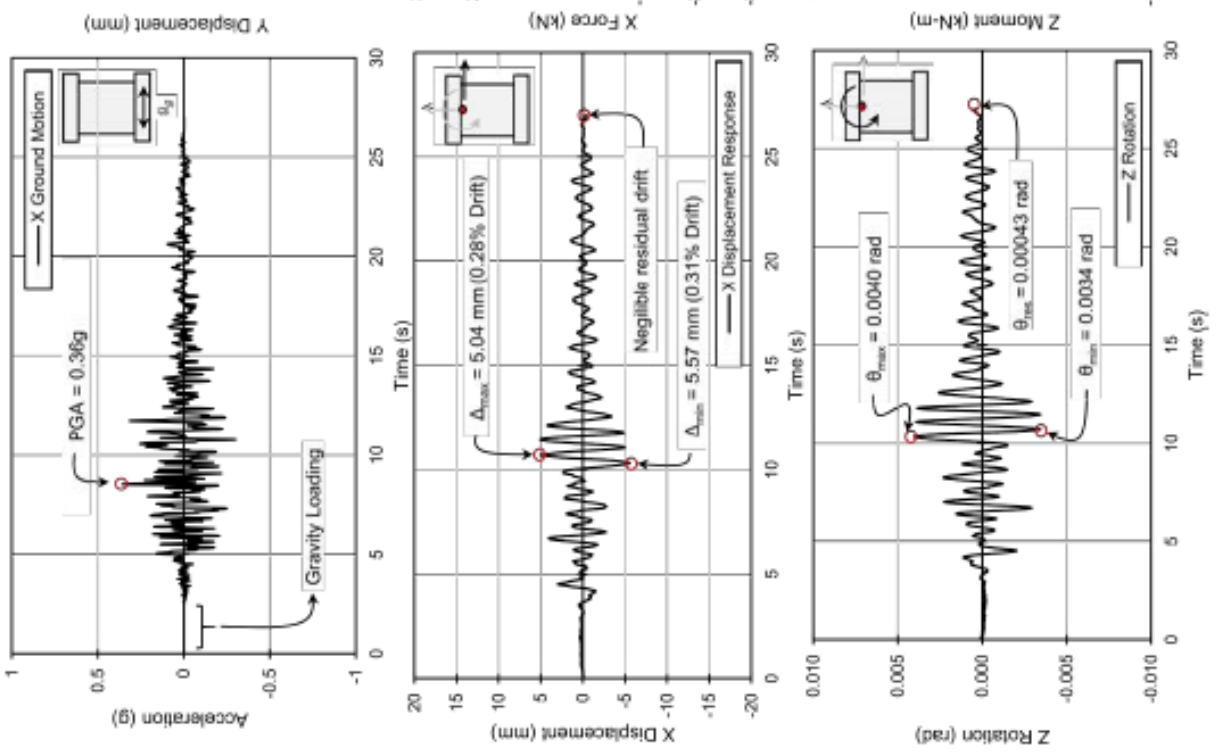

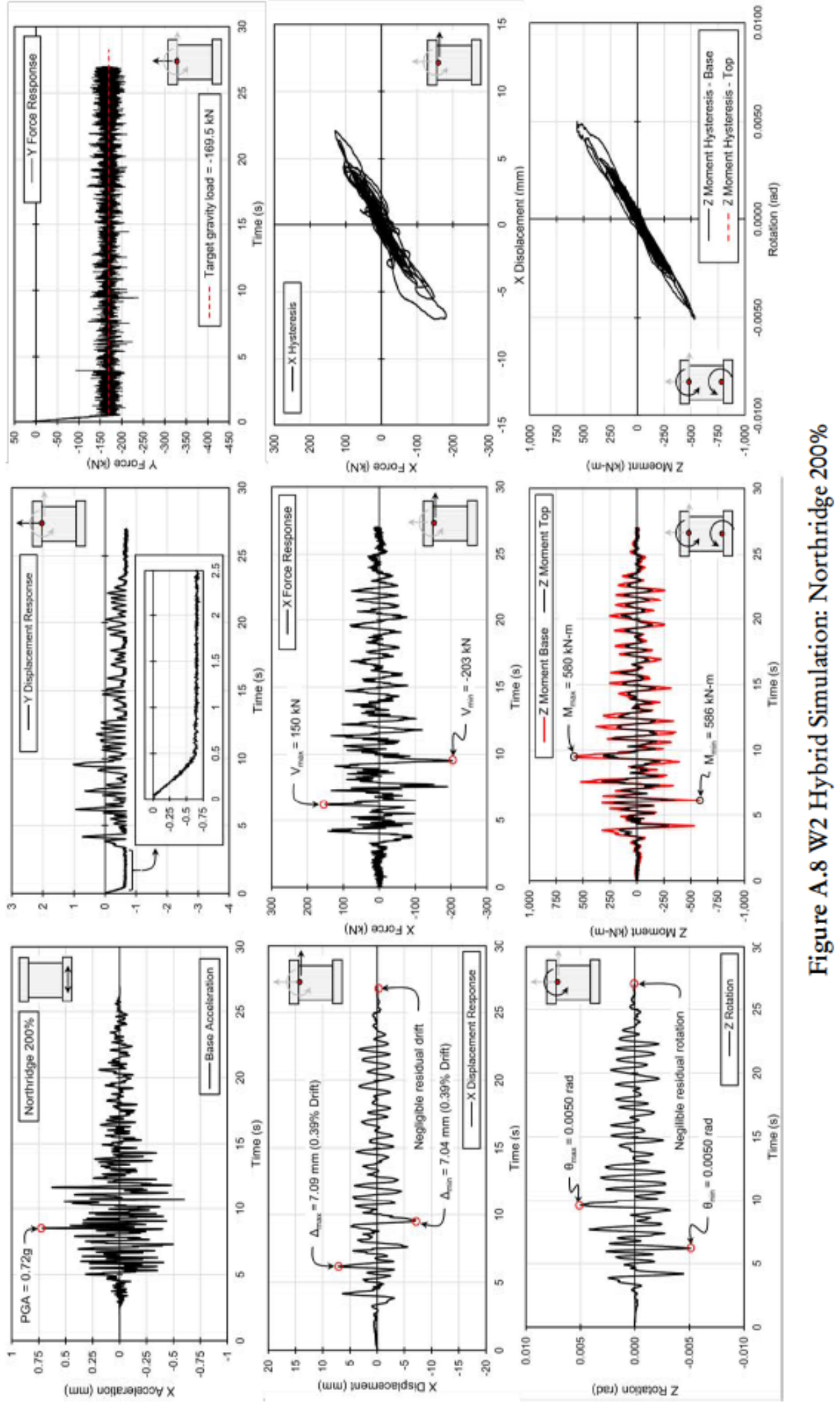

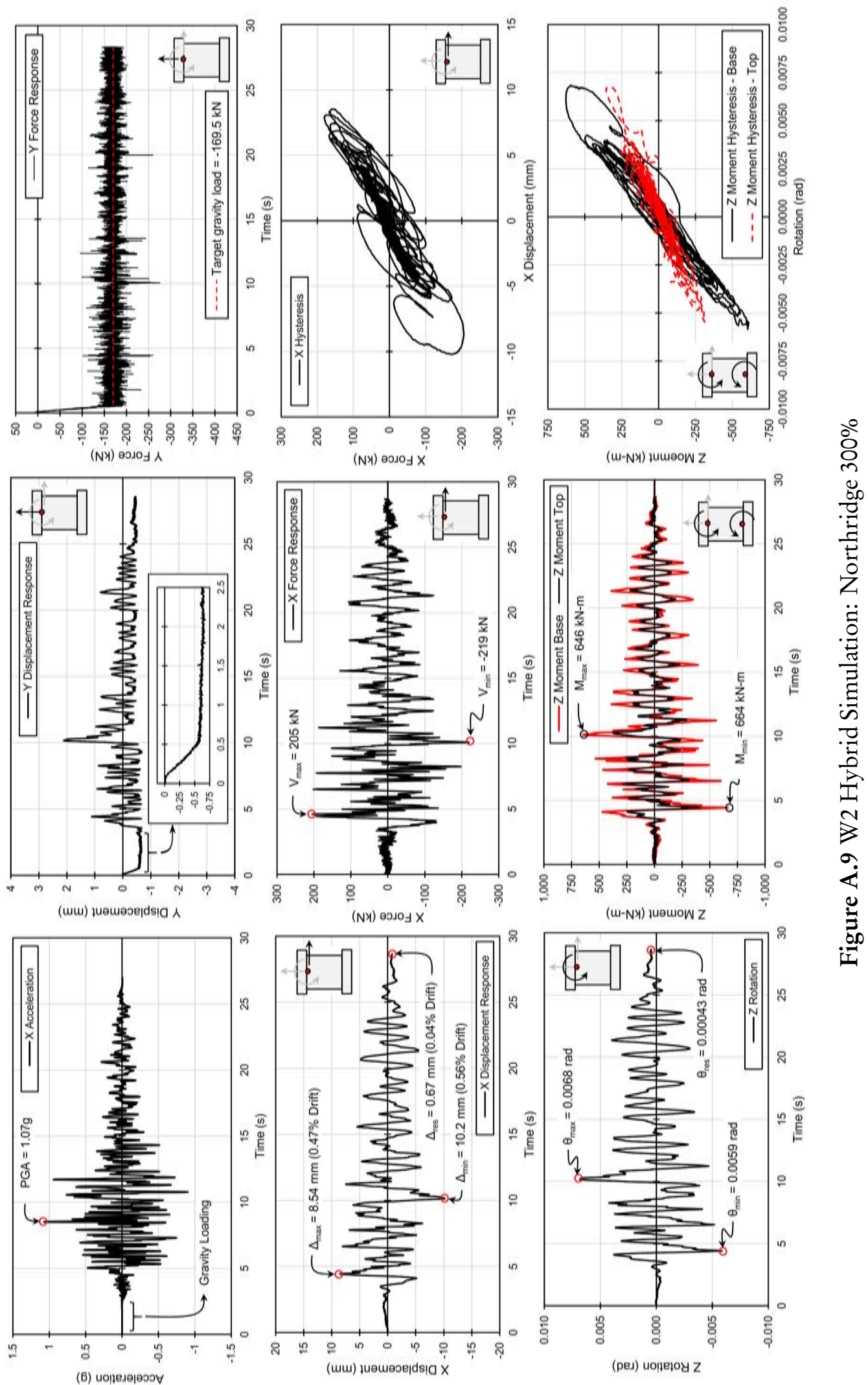

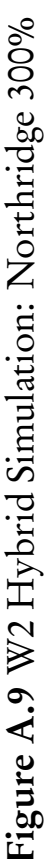



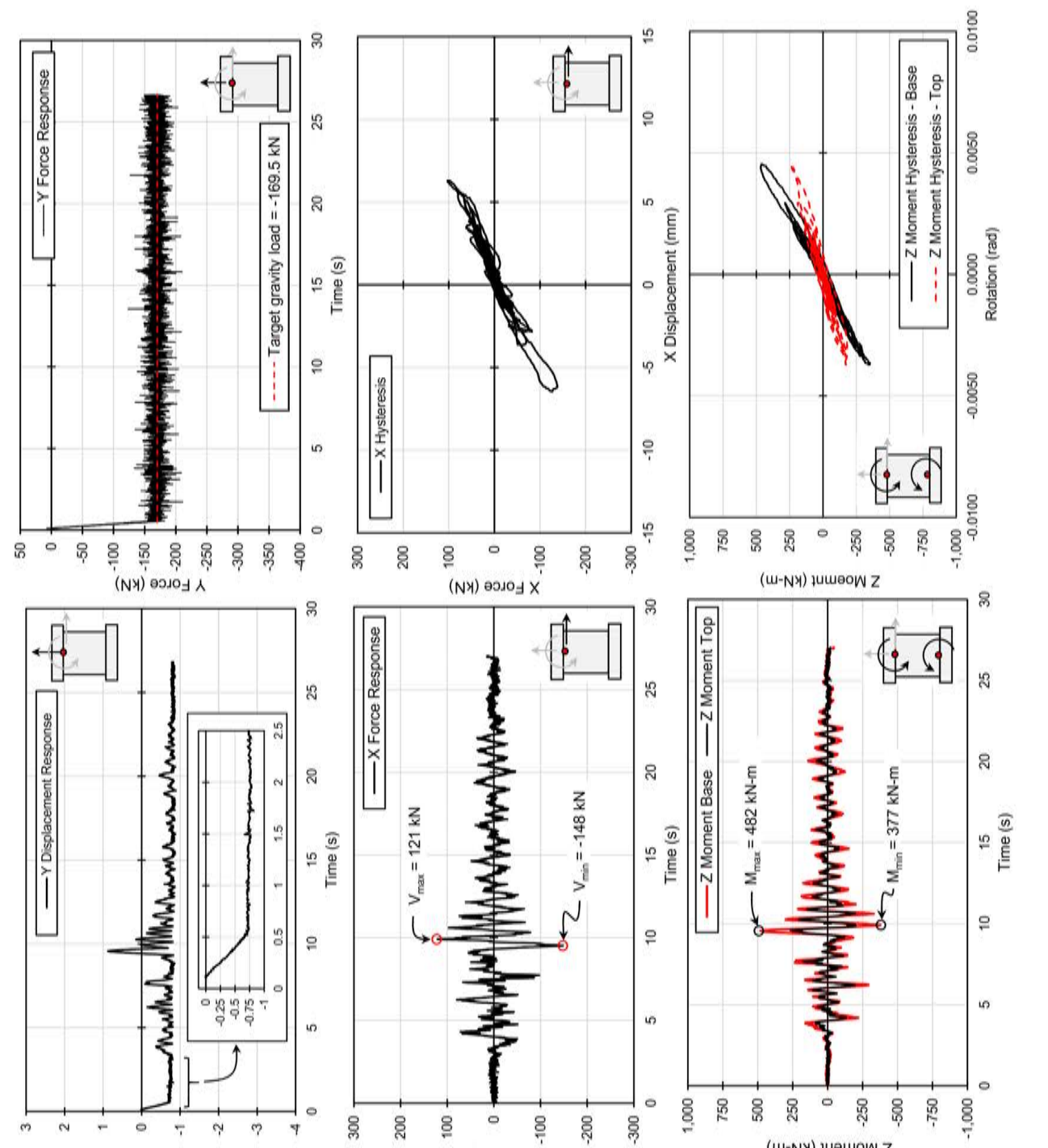

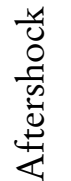
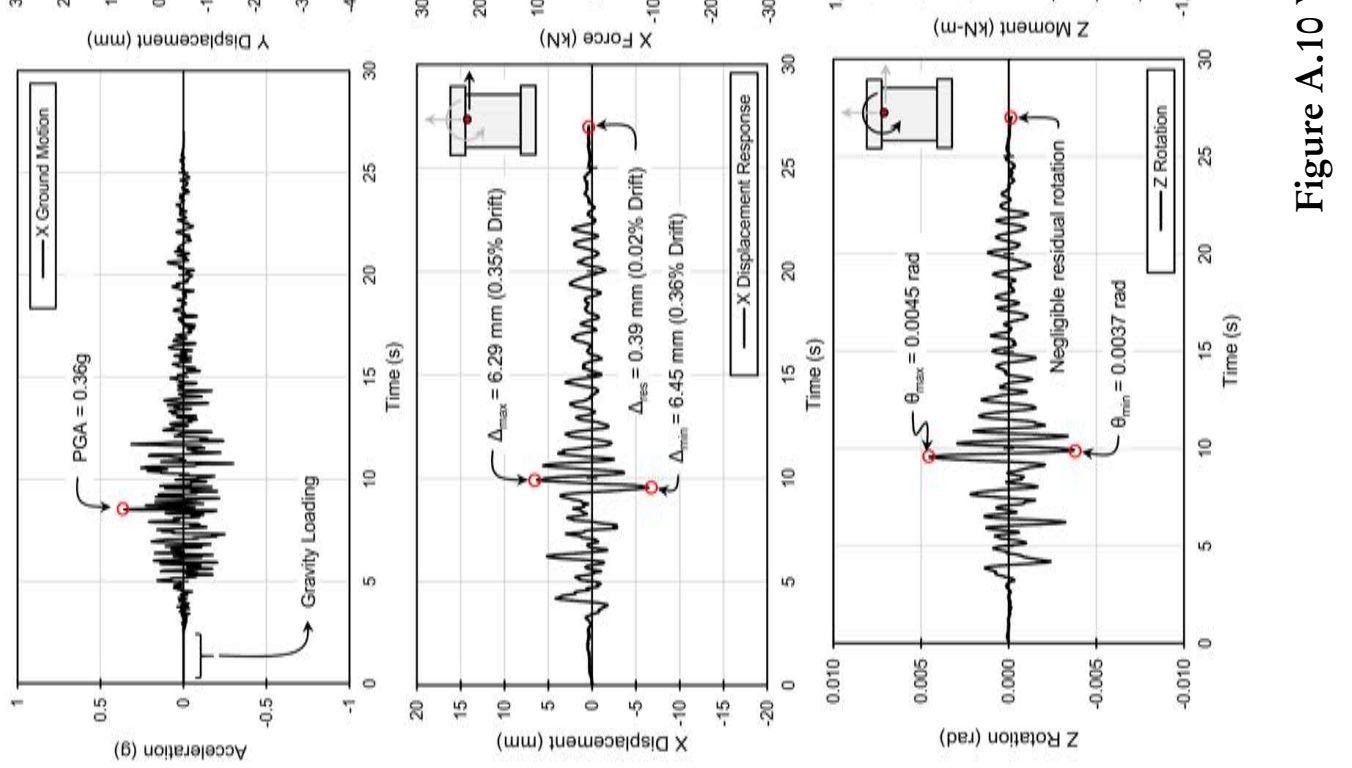

Joshua Woods, Department of Civil and Environmental Engineering, Carleton University 

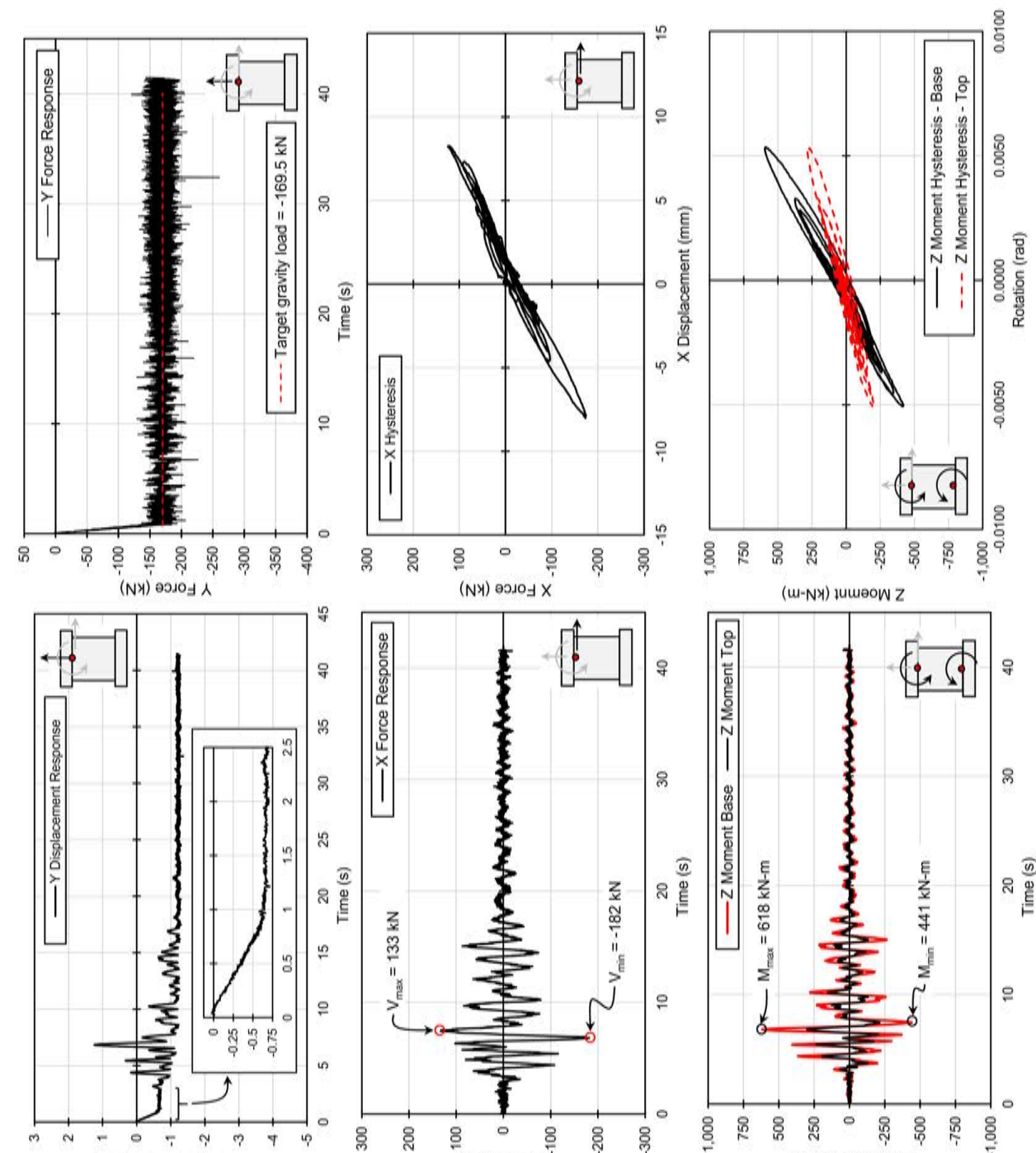

(ww) juøuвoㄹds!a $A$
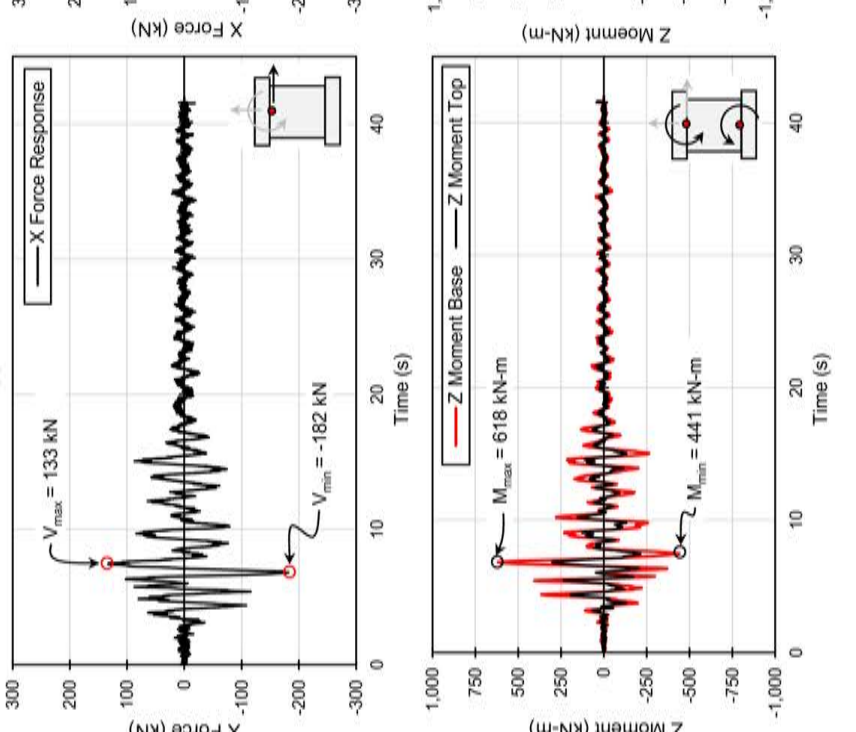

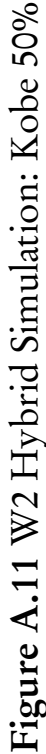
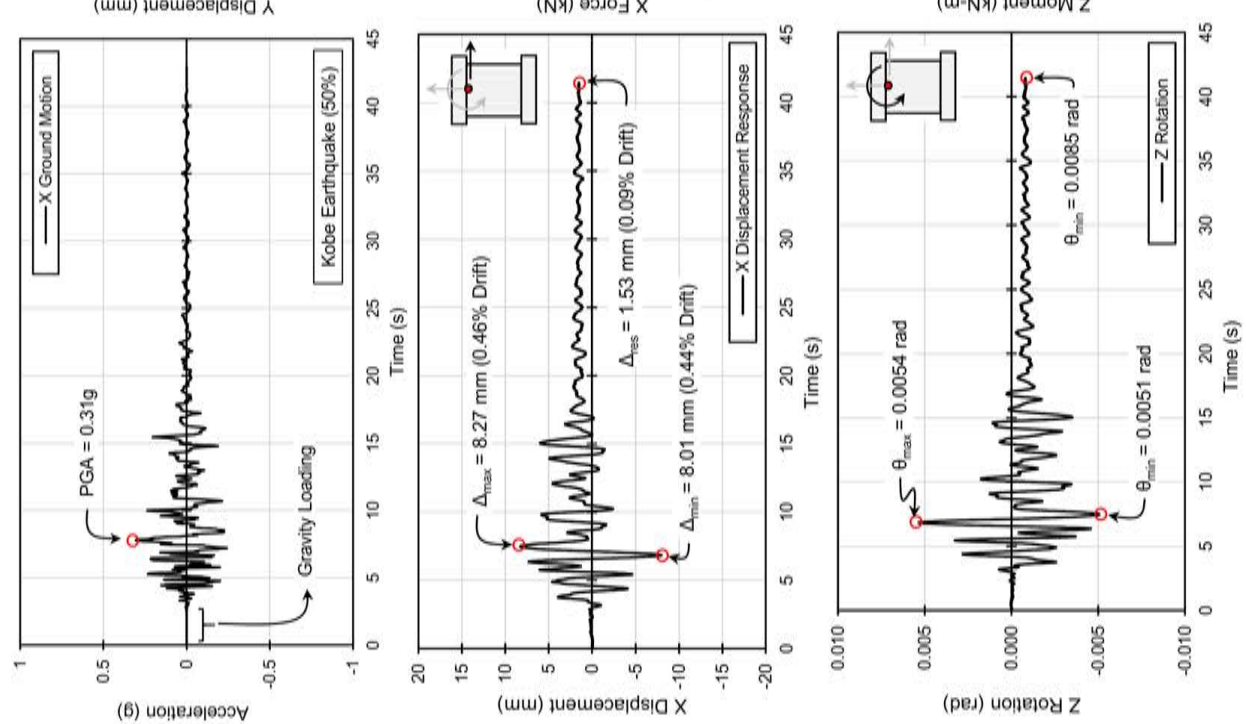

Joshua Woods, Department of Civil and Environmental Engineering, Carleton University 

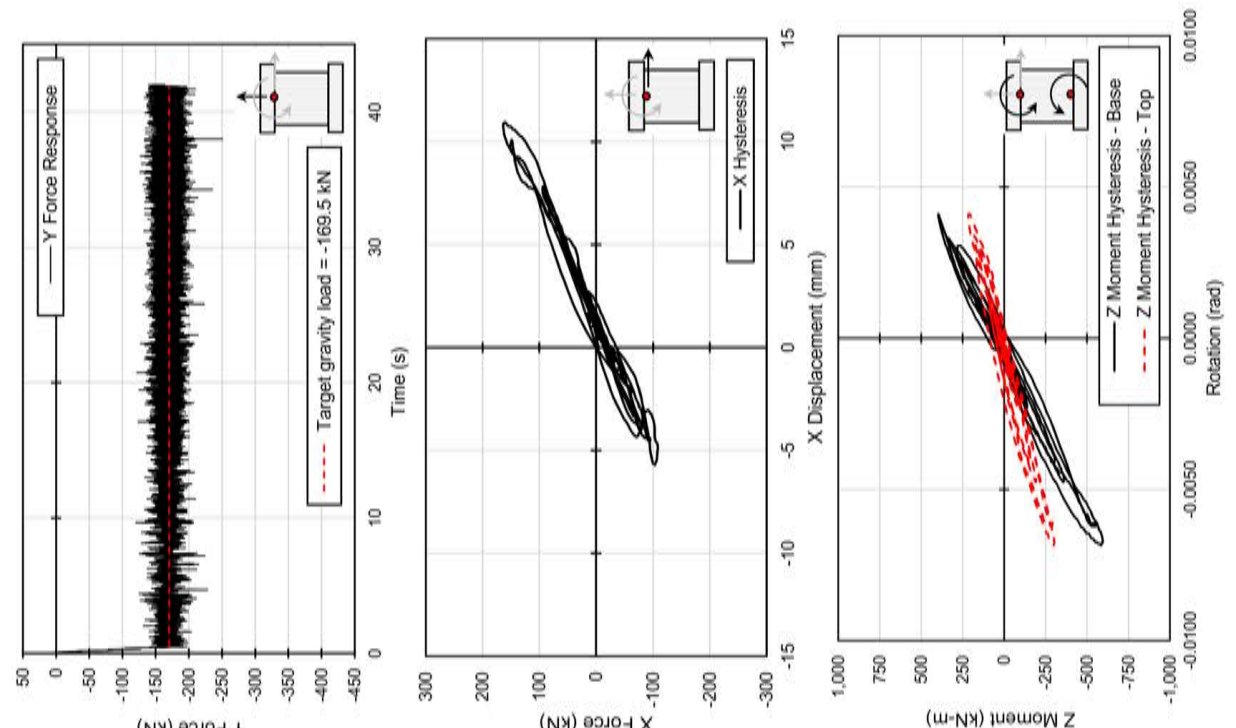

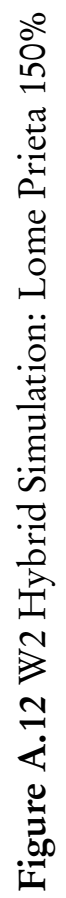
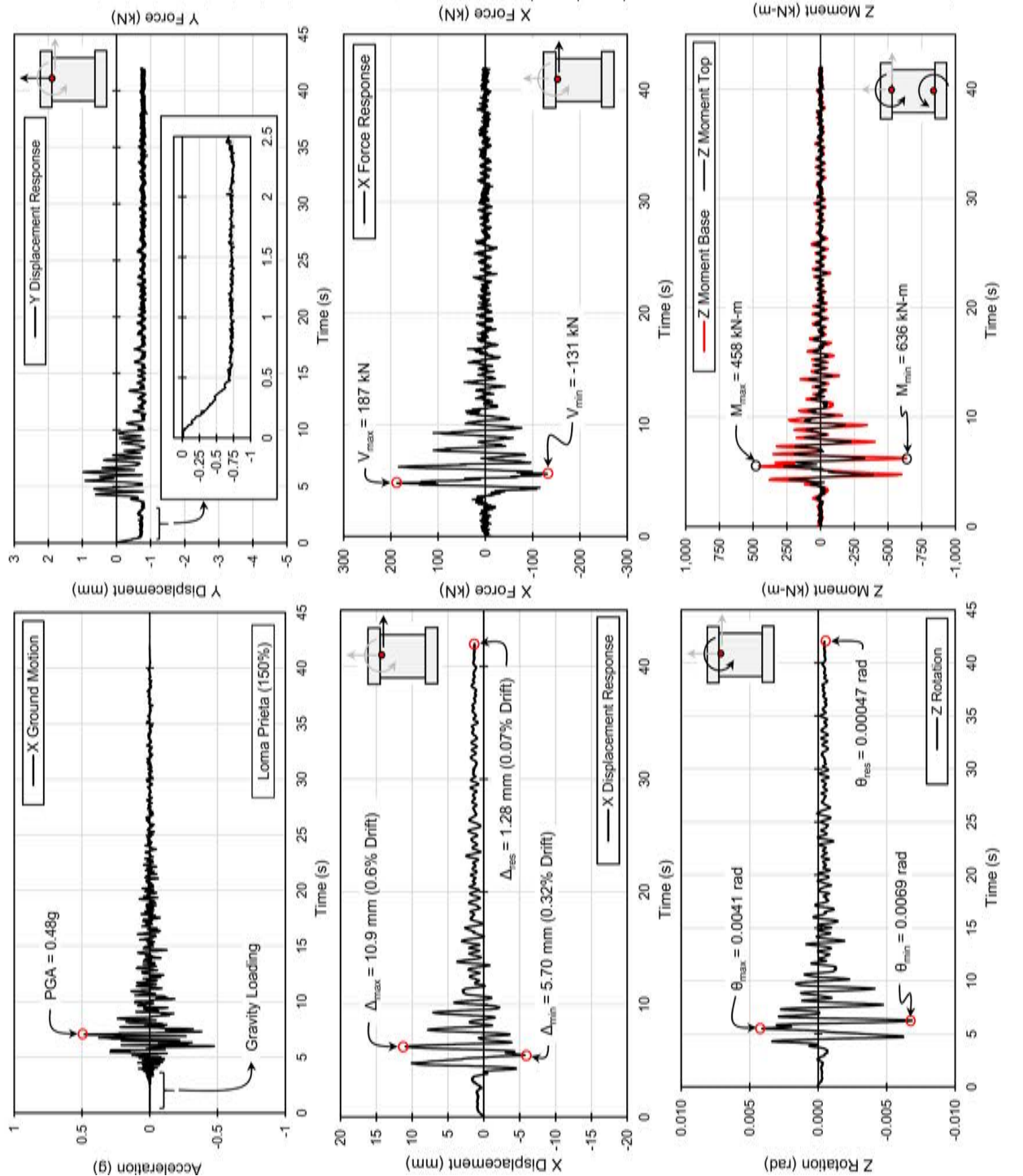

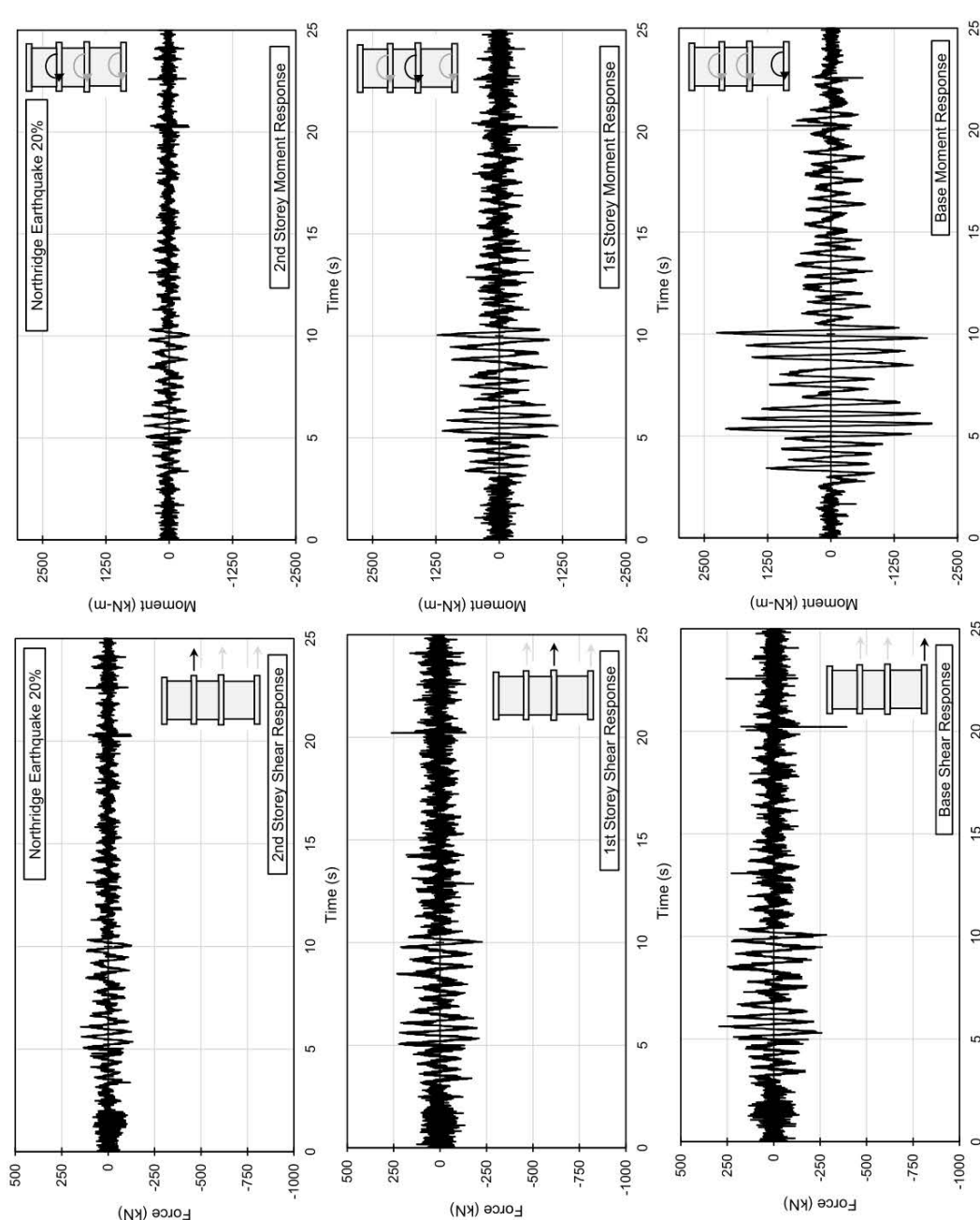

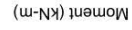

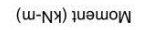
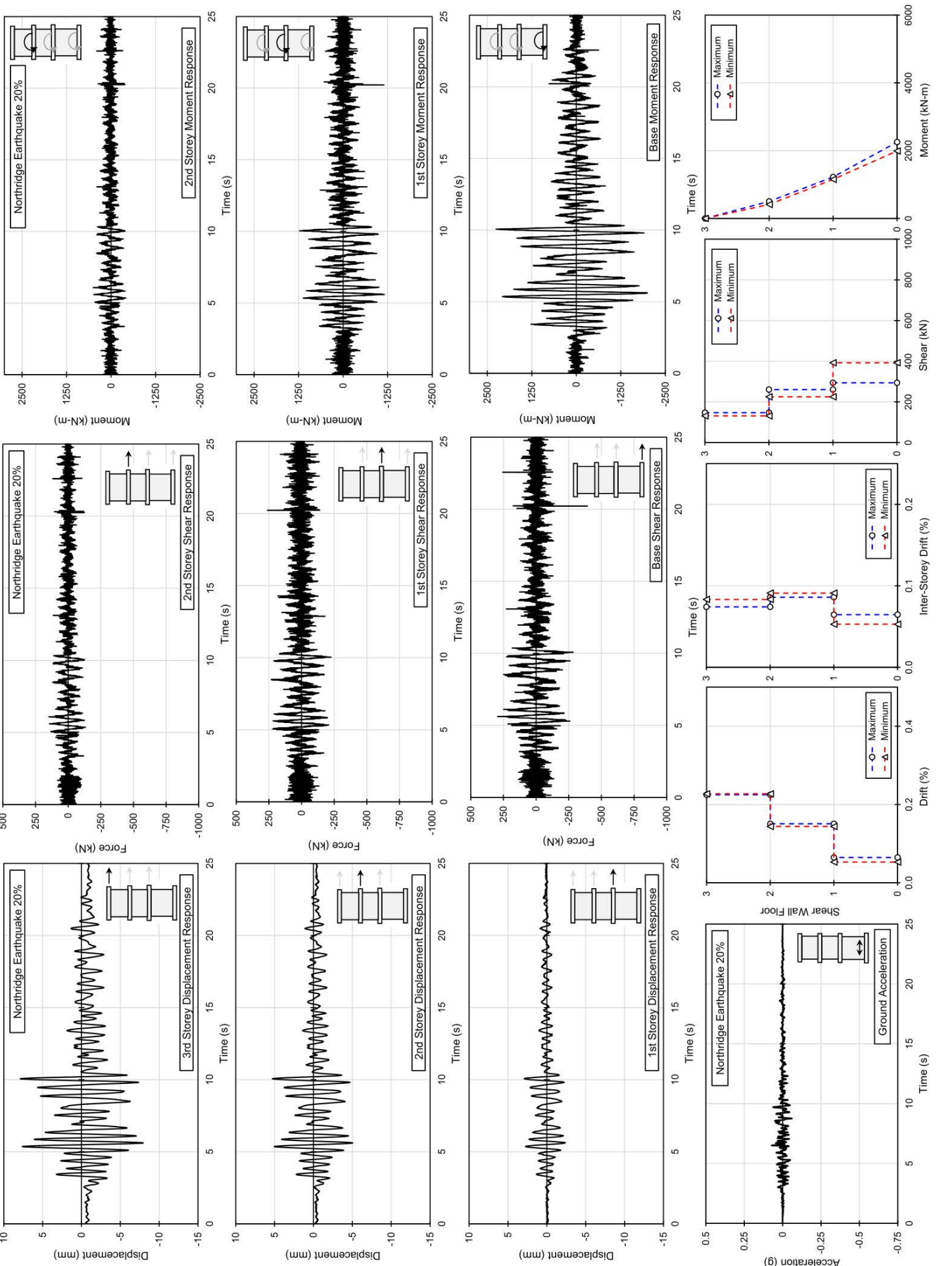

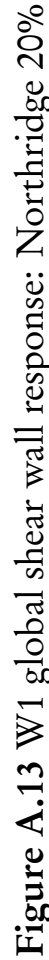

Joshua Woods, Department of Civil and Environmental Engineering, Carleton University 

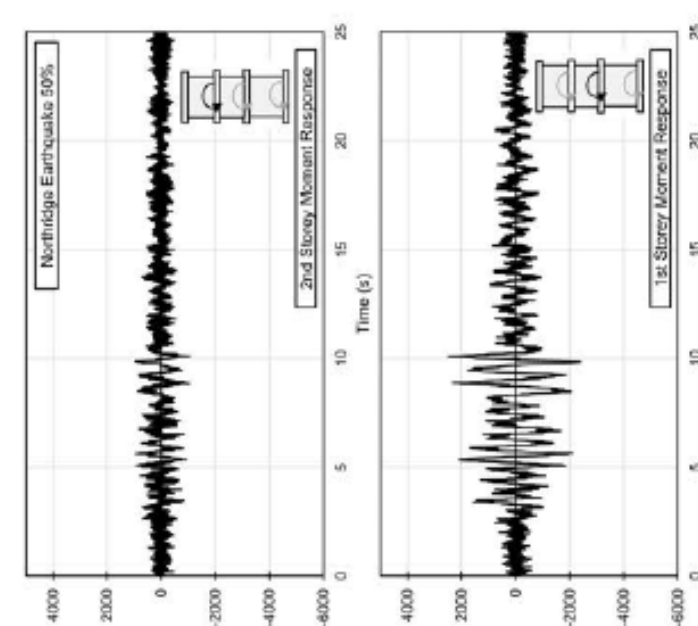

(ui-Now) yeuay
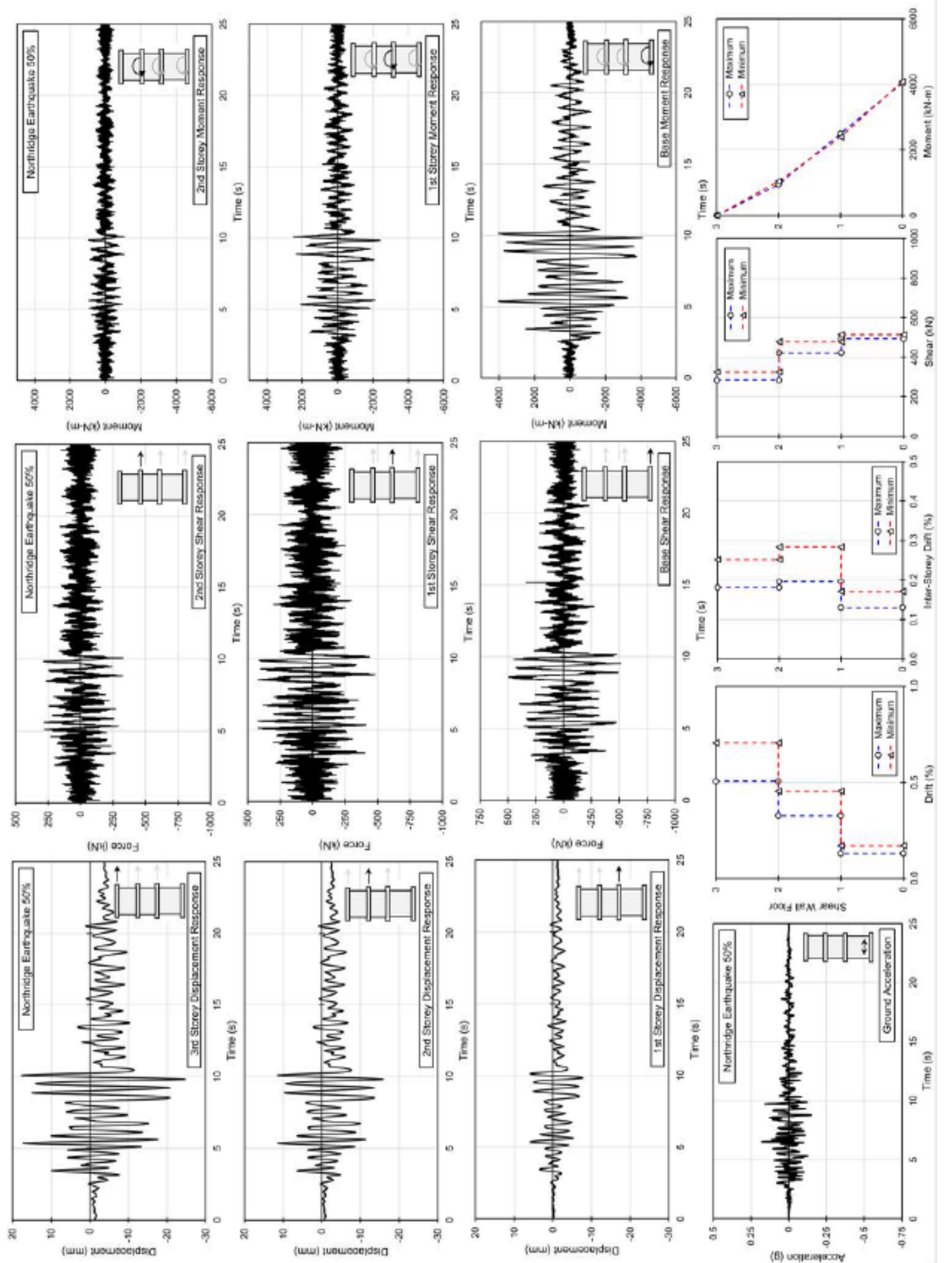

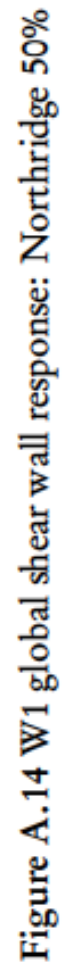



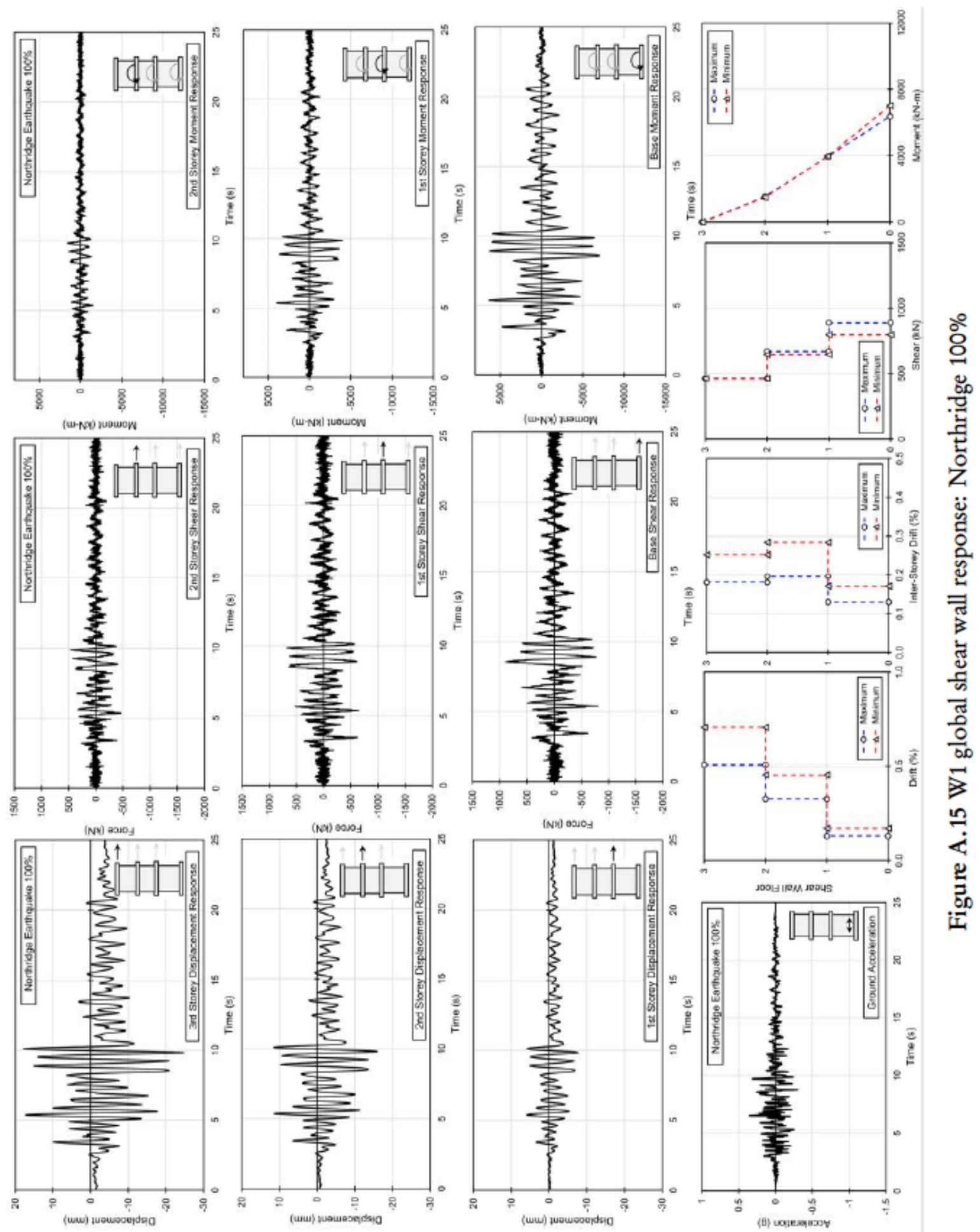

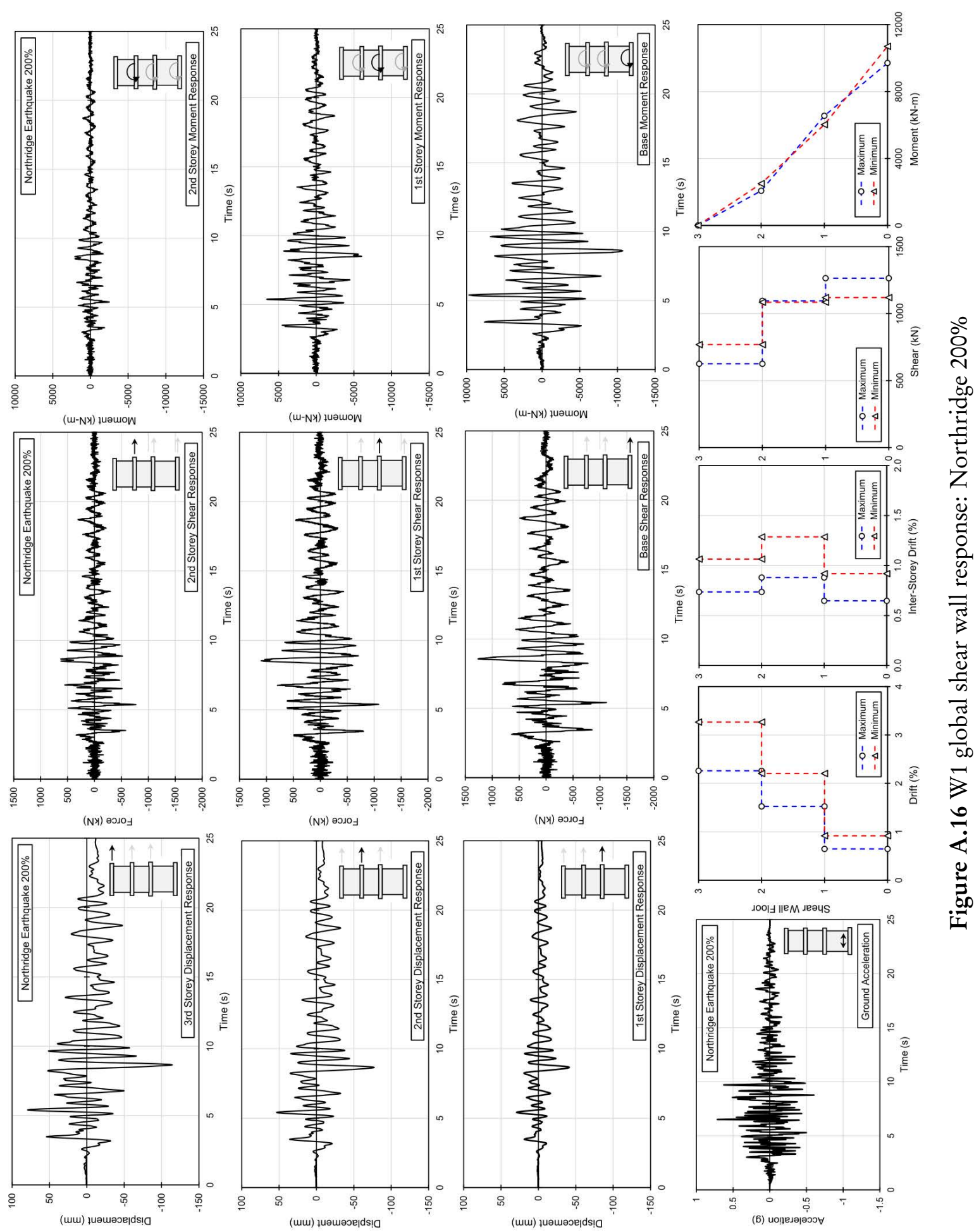

Joshua Woods, Department of Civil and Environmental Engineering, Carleton University 

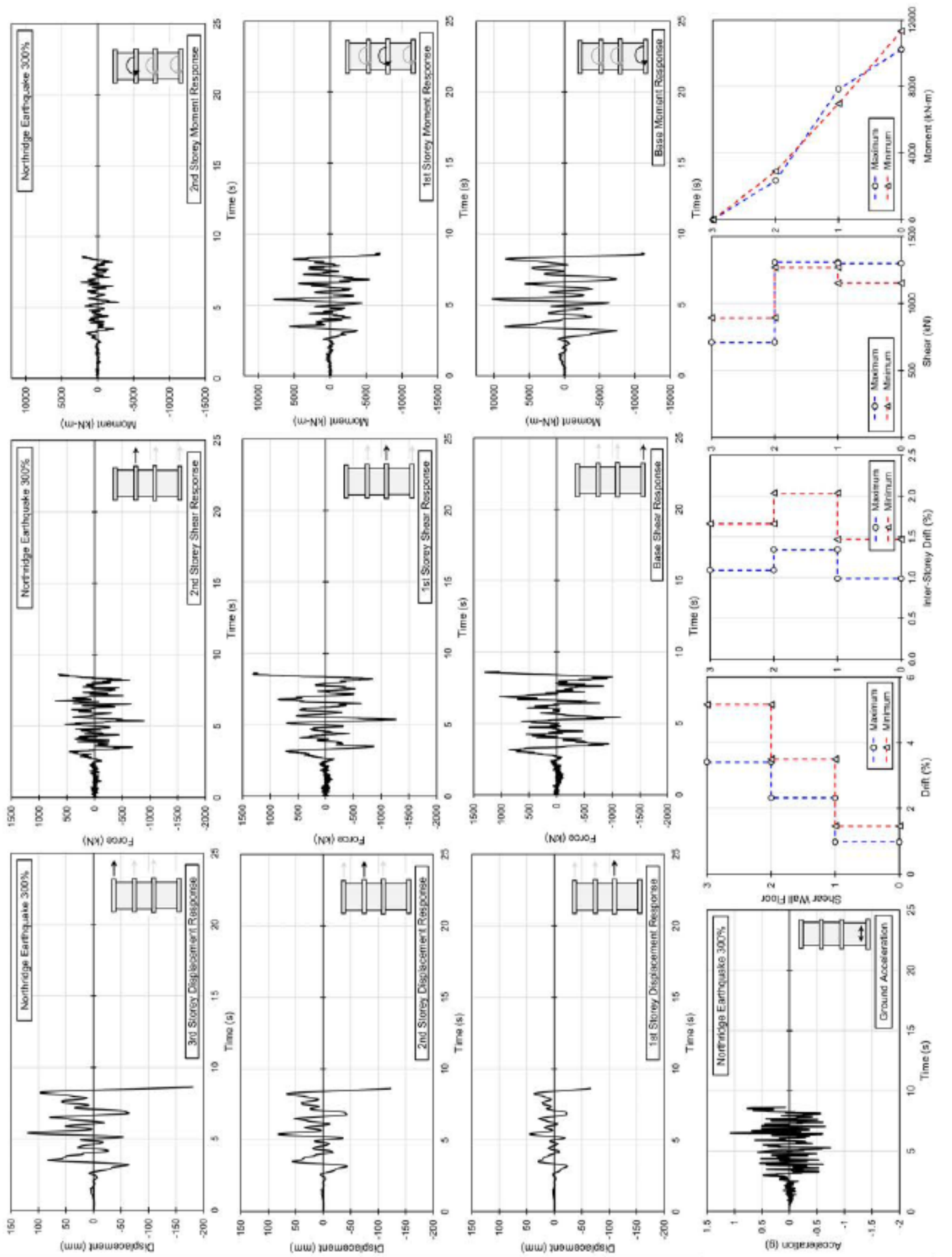

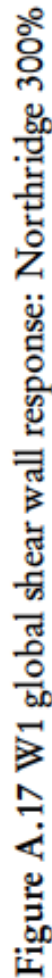



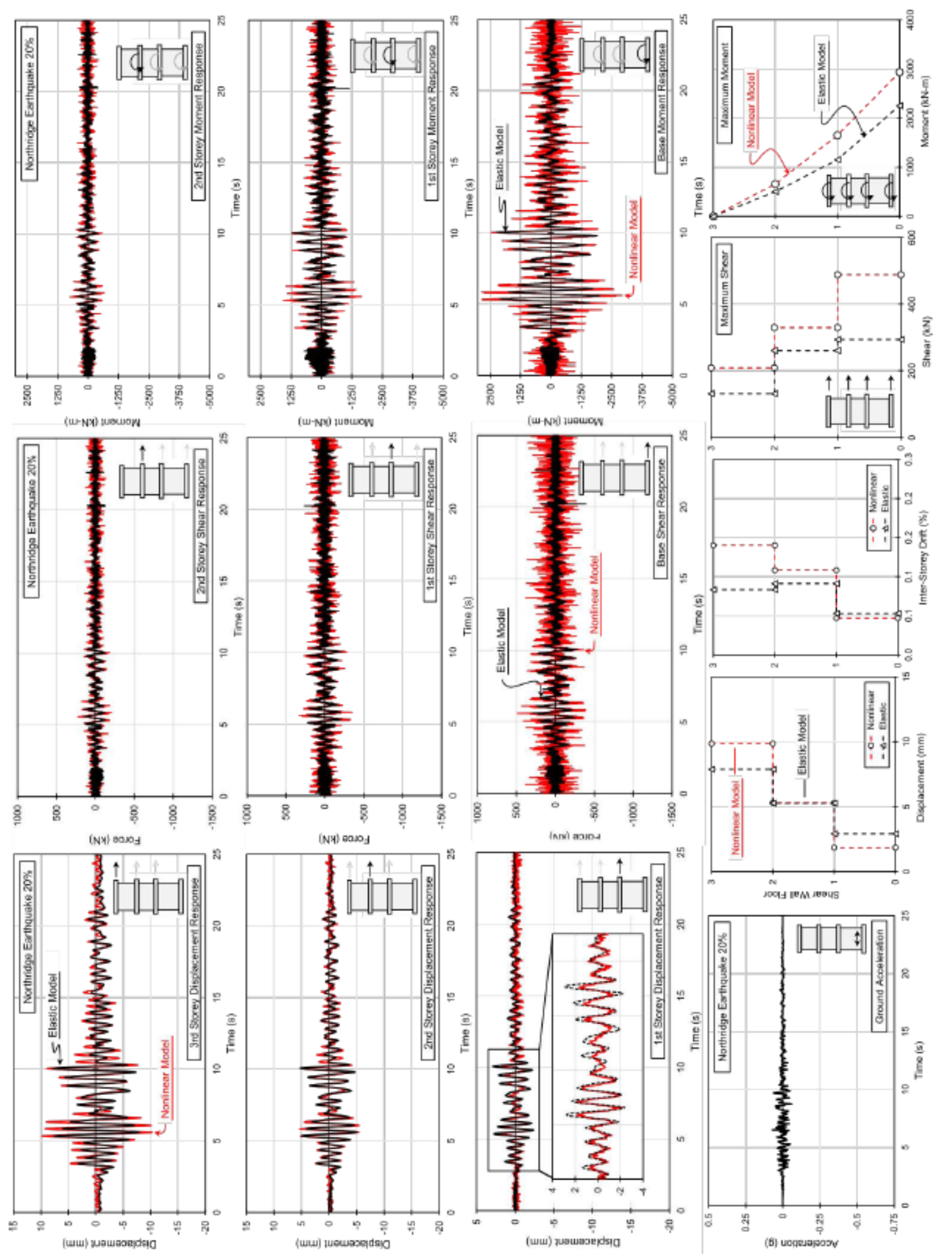

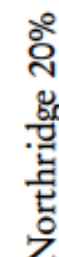

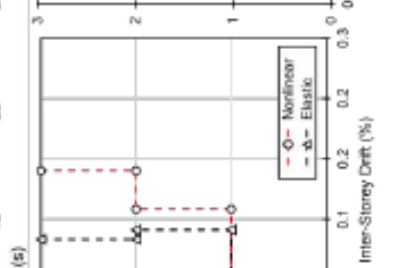

ชั.

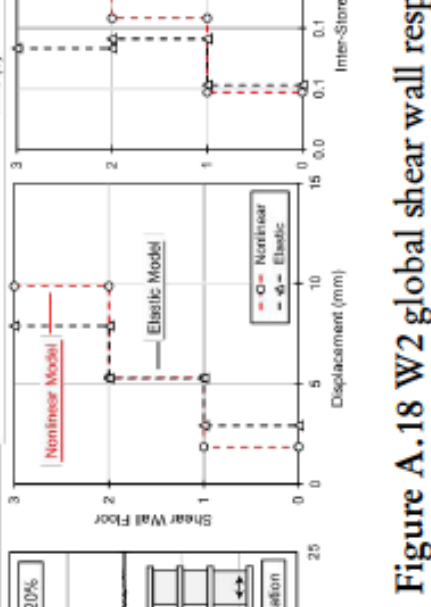



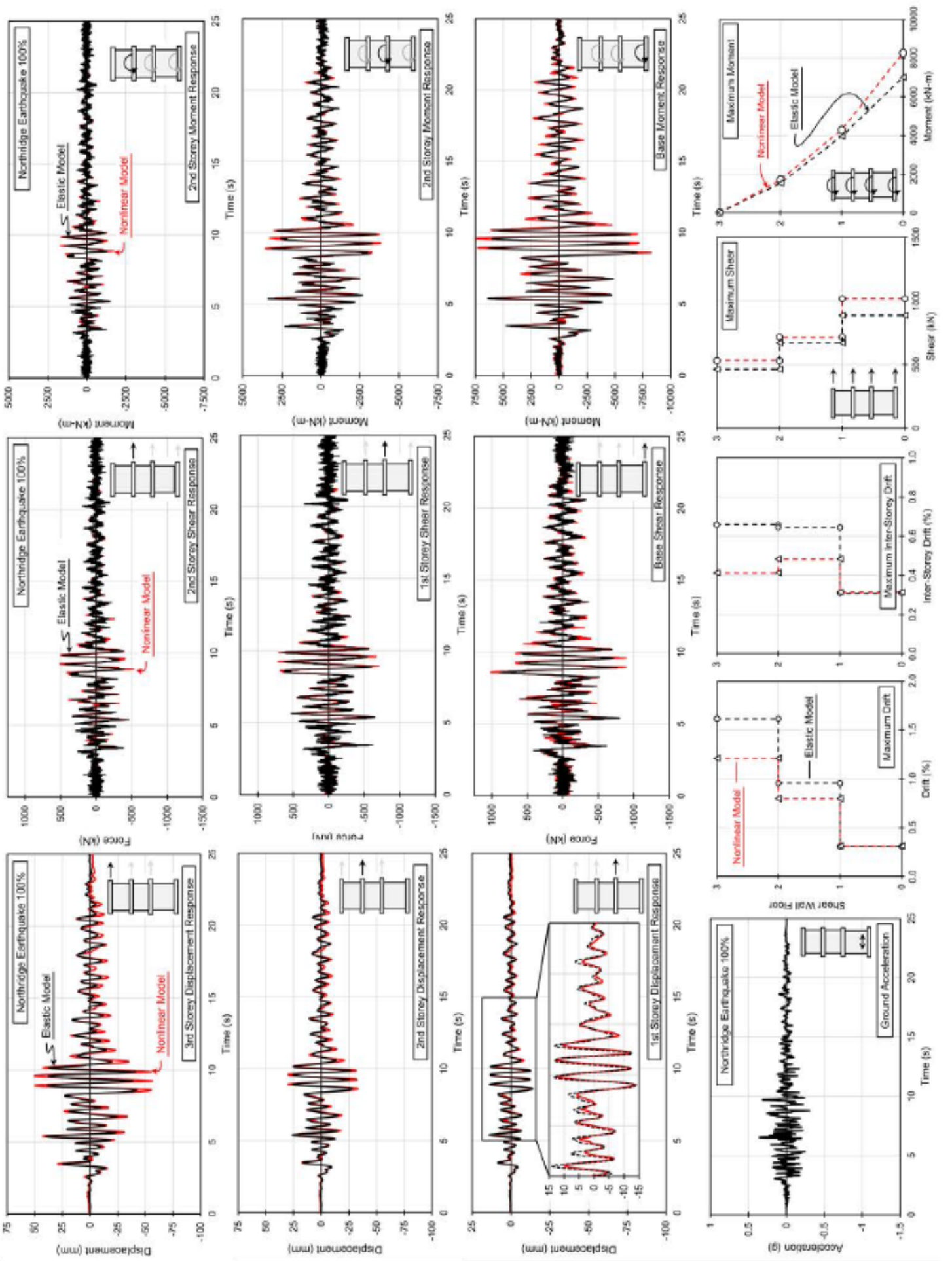

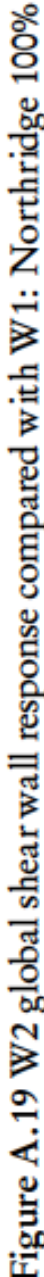



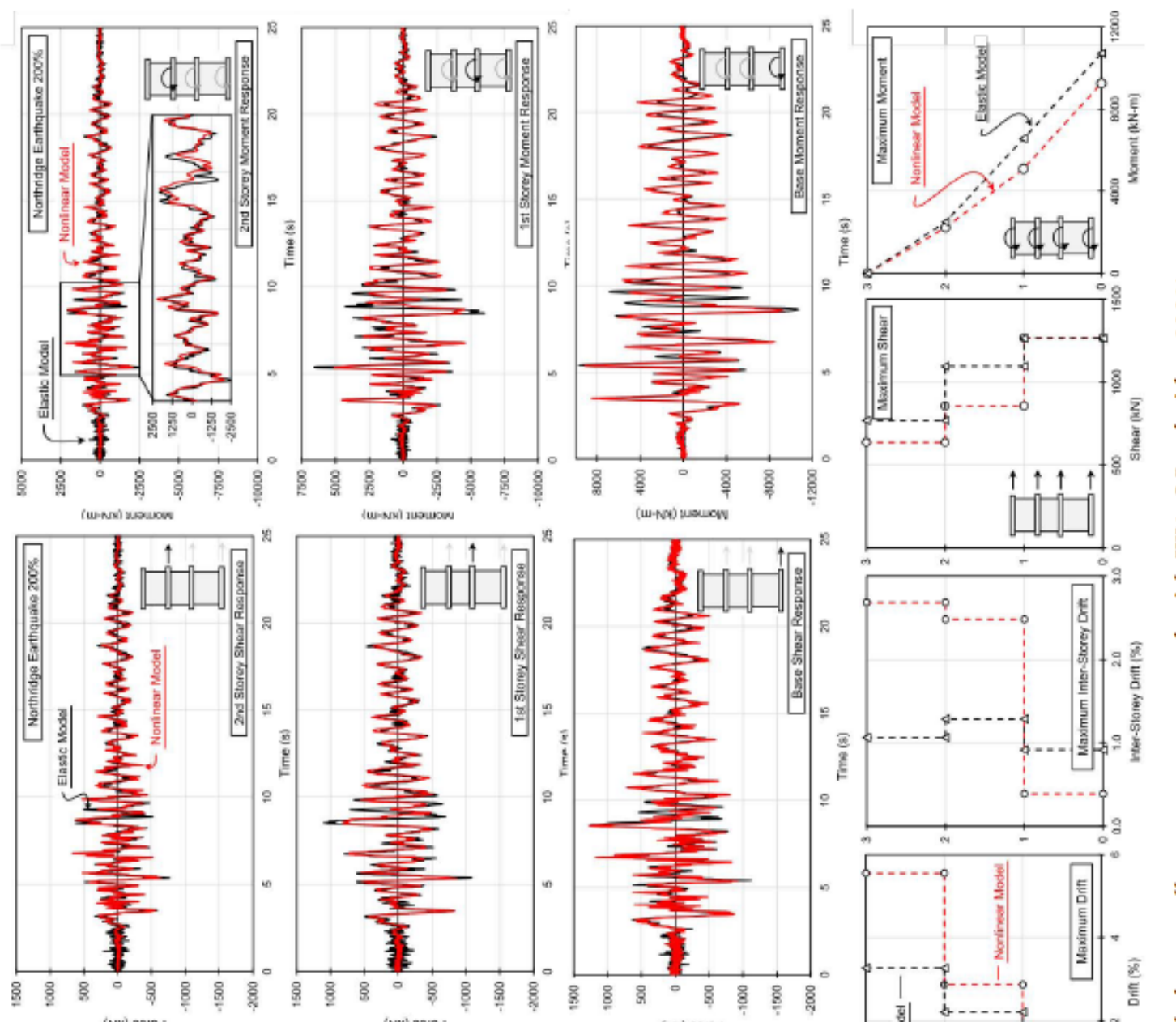

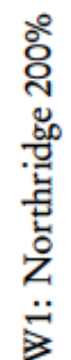
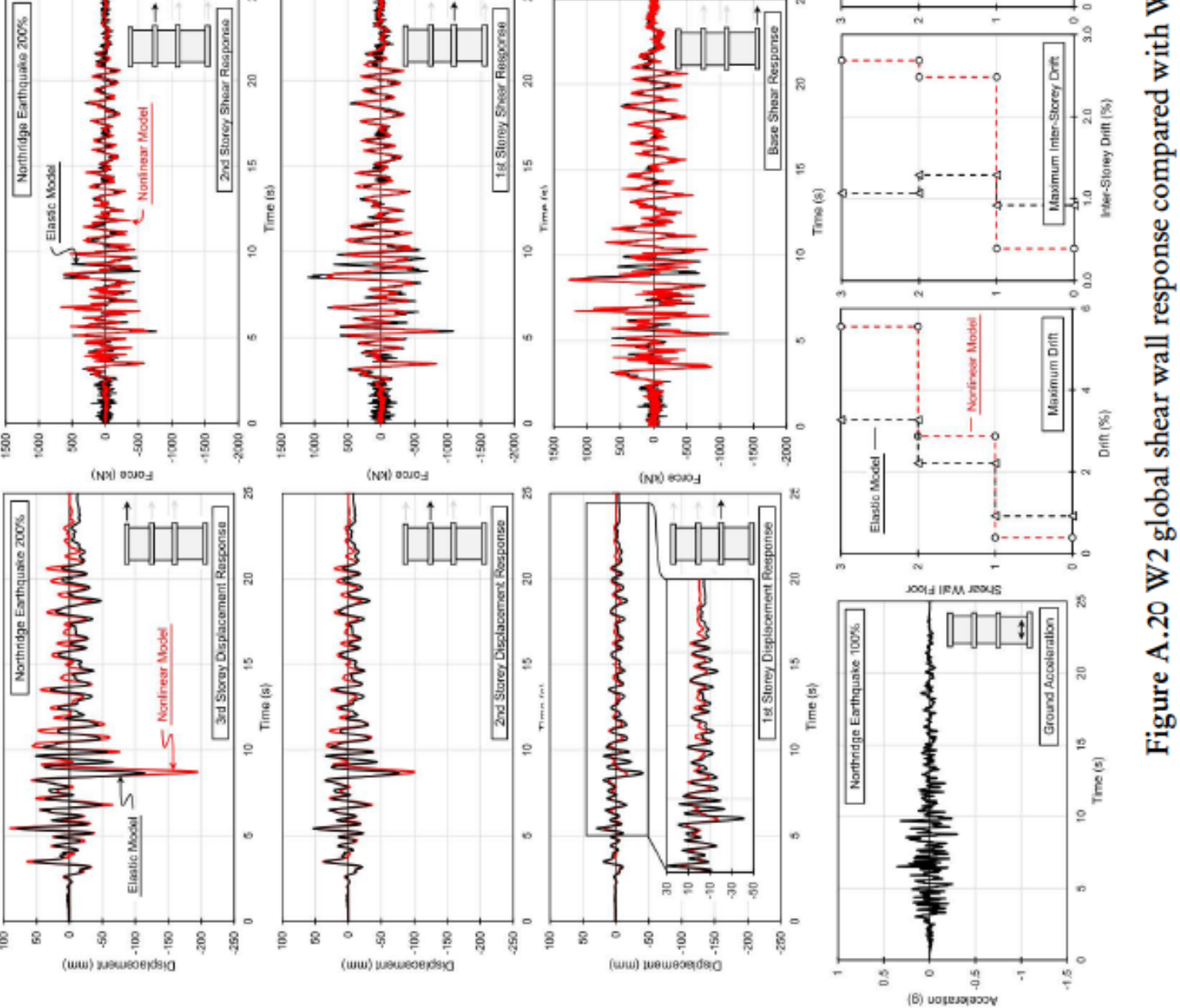

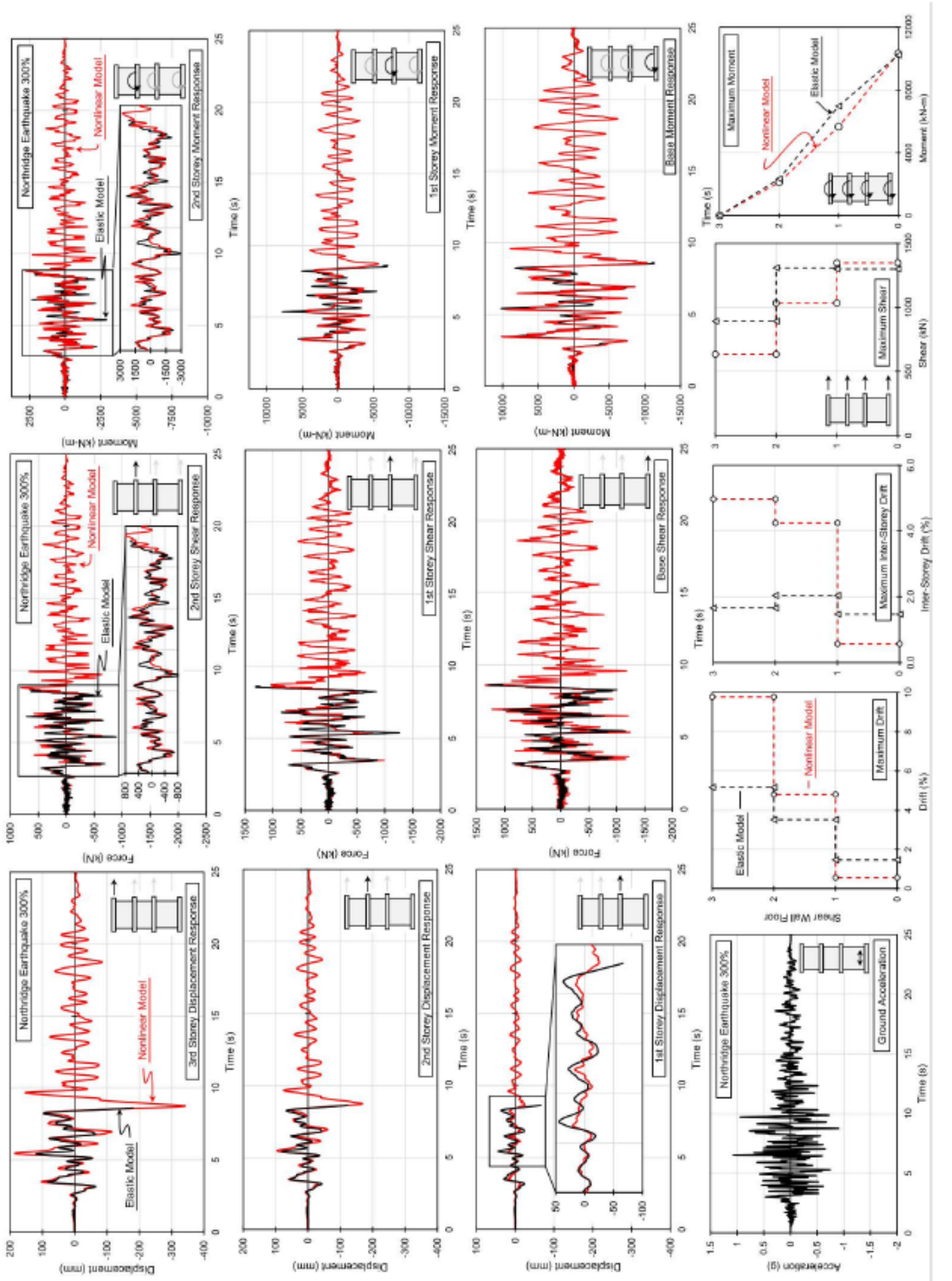

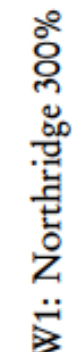
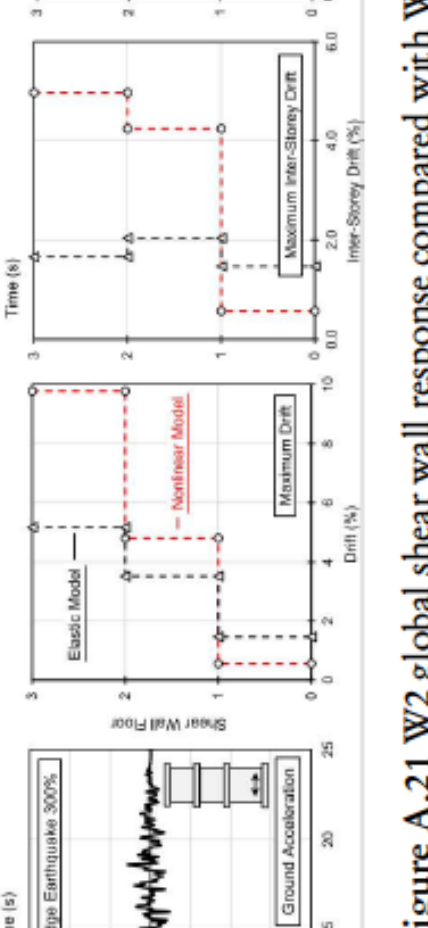

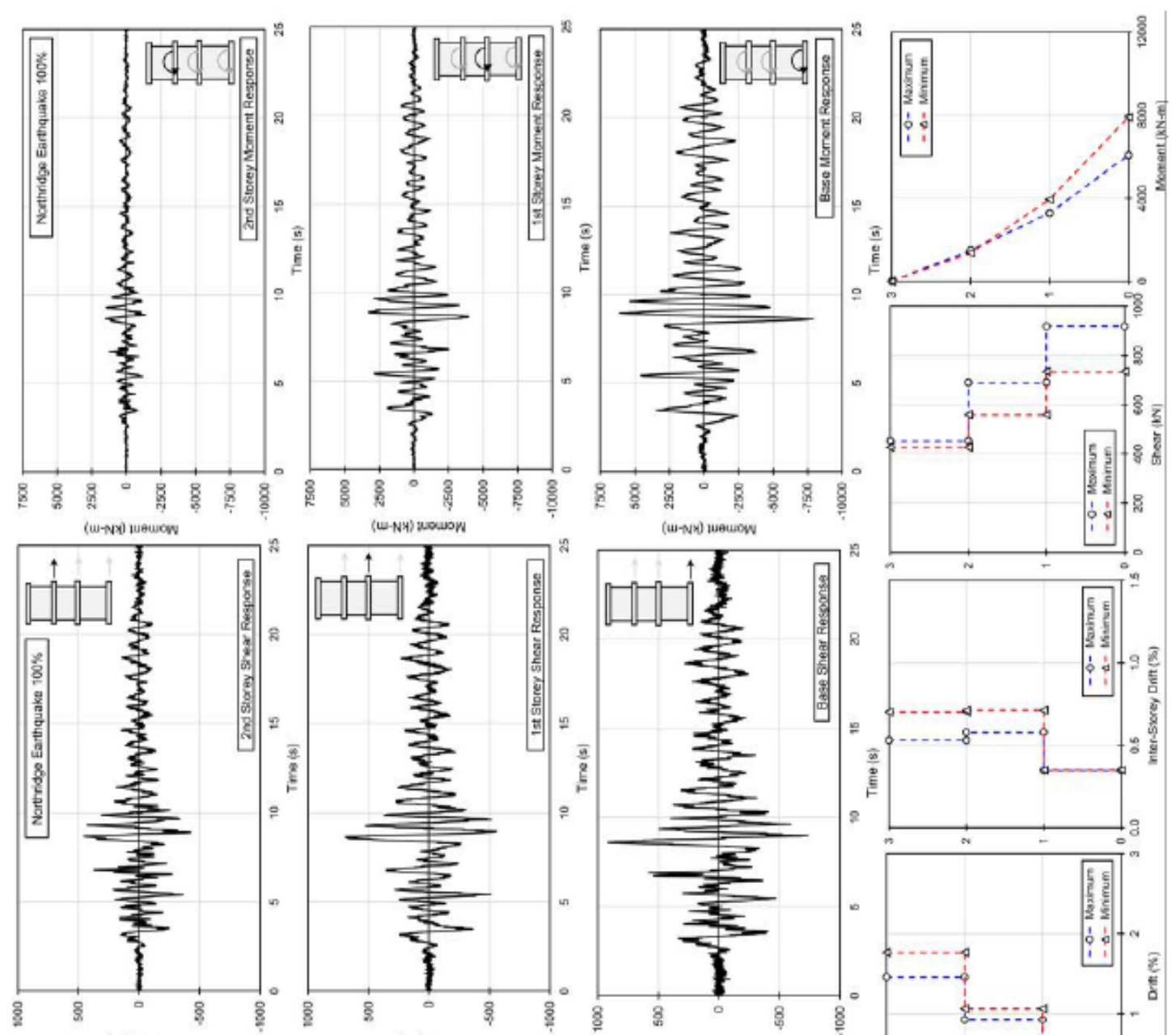

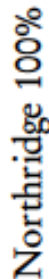
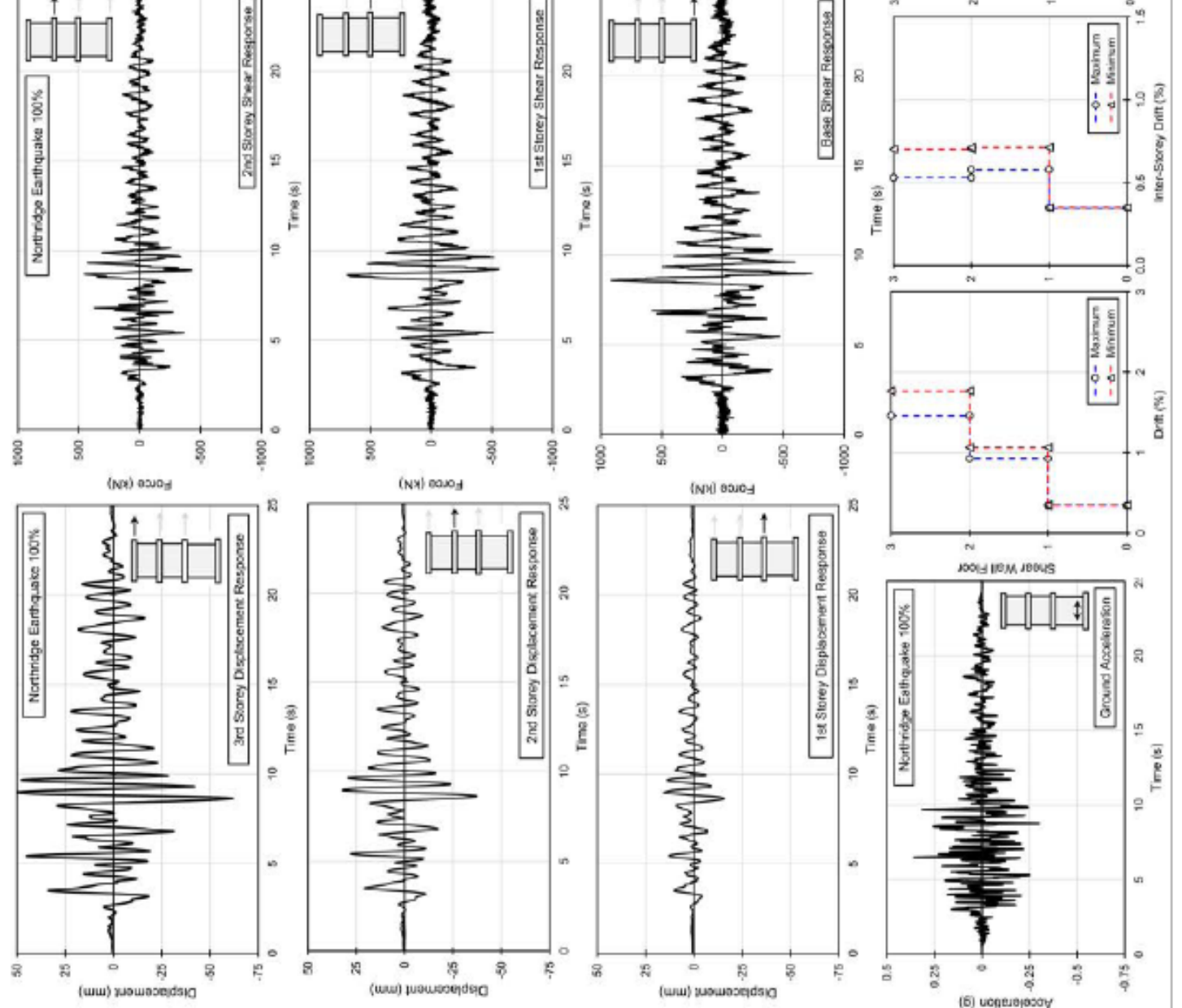

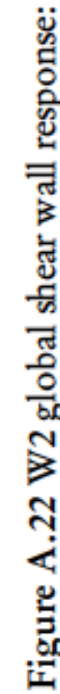



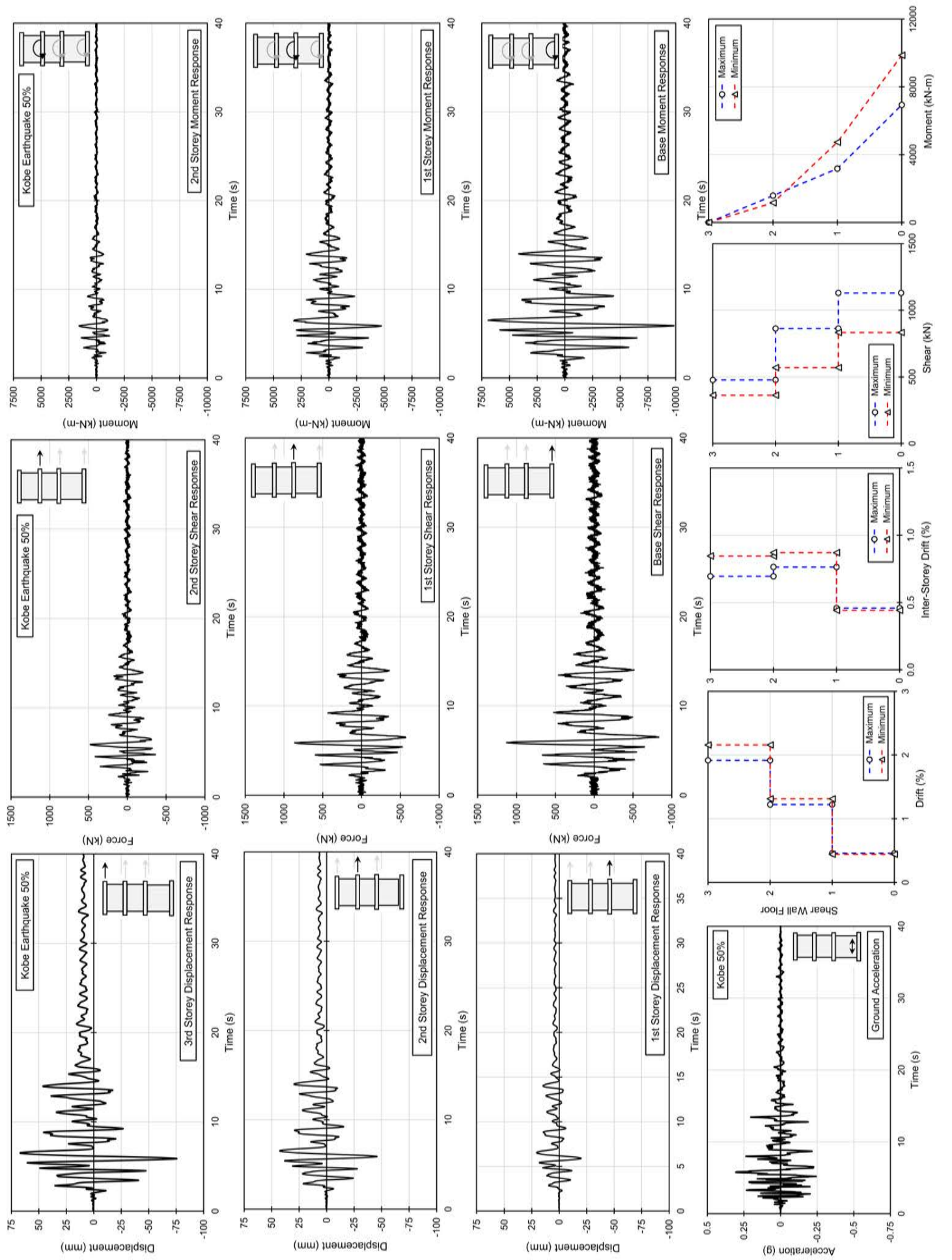

80
8
0
0
0
1
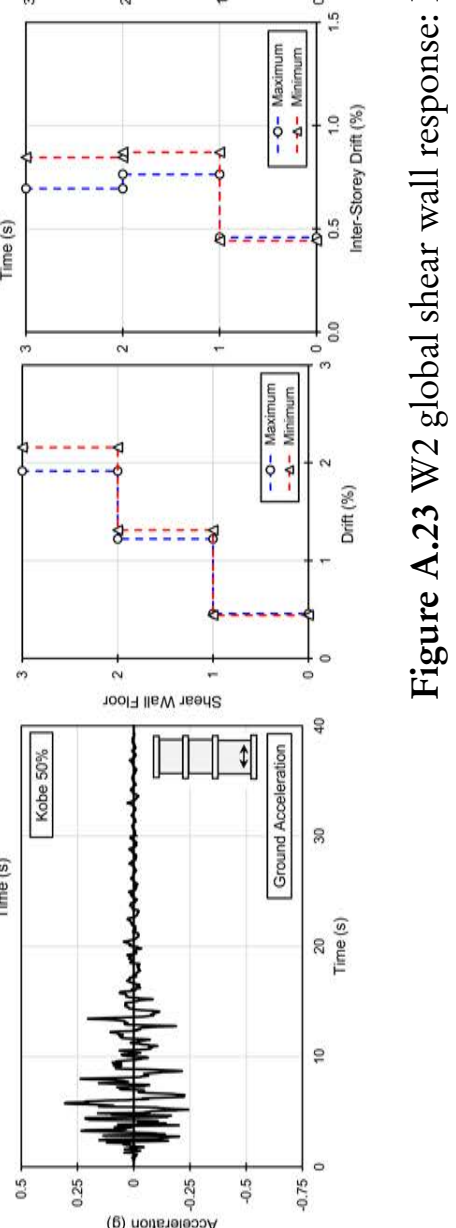

Joshua Woods, Department of Civil and Environmental Engineering, Carleton University 


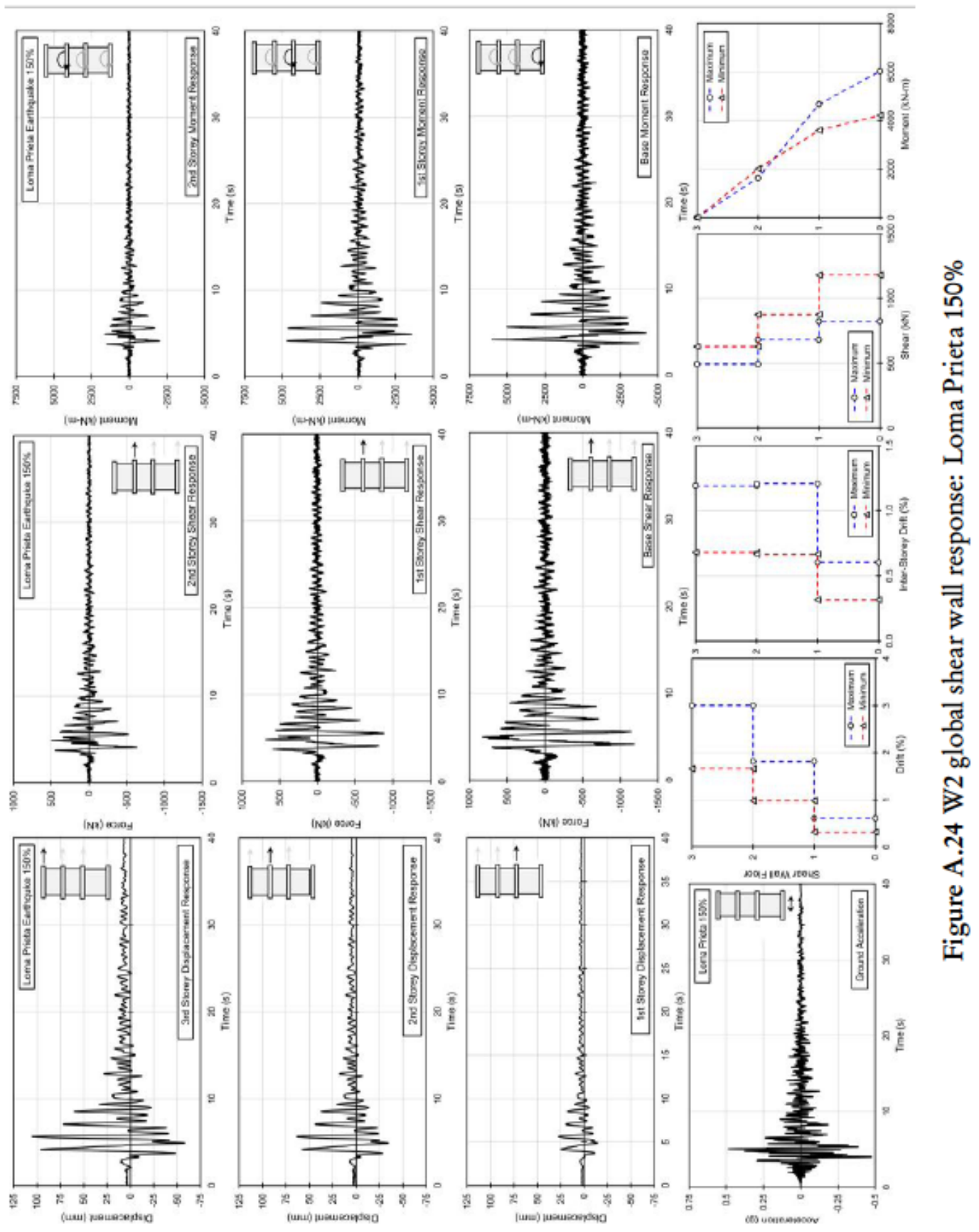




\section{Appendix B. Additional Design Details}

\section{B.1 Design Loads}

This appendix outlines the assumptions and procedures used in the structural analysis and design of a multi-storey reinforced concrete (RC) building in Victoria, BC. The primary gravity load resisting system in the structure consisted of reinforced concrete columns and slabs, while the lateral load resisting structural system consisted of reinforced concrete shear walls and a reinforced concrete core. The objective of the design is to develop a realistic geometry and estimation of the seismic design forces on an actual structure. To assist in the design process, the structure is modelled in three-dimensions using the finite element software ETABS Computers and Structures Inc. (2015). The structure is designed in accordance with relevant Canadian design standards. This included the National Building Code of Canada NRC (2010) to determine the seismic design forces expected on the structure. The concrete components of the structure are designed in accordance with the Canadian Design Standards for Reinforced Concrete Structures CSA (2014a). The gravity load design is completed for the structure based on the NBCC (2010) requirements, and includes allowance for structural (eg. slabs, columns, and walls) and non-structural components, such as roofing material, partitions, and other finishes. The structure is assumed to have a two-way flat plate slab floor system and the majority of the gravity load is carried by the interior concrete columns. The structure is designed as a typical office building of normal importance (importance factor of 1.0 for ultimate limit states design). The self-weight of the concrete slab is assumed to be $4.8 \mathrm{kPa}$ based on a uniform slab thickness of $200 \mathrm{~mm}$. The corresponding dead loads not including the structural elements and live loads used in the design of the structure are shown 
in Table B.1. The design snow load is determined from the NBCC design requires for Victoria, BC. The specified snow ground snow load in Victoria is $1.1 \mathrm{kPa}$ with a 1 -in-50 probability of exceedence per year and an associated rain load of $0.2 \mathrm{kPa}$. The basic snow exposure factor is taken as 0.8 based on the roofs total characteristic length of $42 \mathrm{~m}$. The wind exposure value, roof slope factor, and shape are all taken as 1.0 assuming a flat roof configuration, which would result in a higher snow load distribution compared to a roof at an angle. The size of the roof and a $0.5 \mathrm{~m}$ parapet around the exterior of the structure results in a maximum snow load of $1.14 \mathrm{kPa}$ around the outer perimeter that gradually reduced to $1.08 \mathrm{kPa}$ at a distance of $3 \mathrm{~m}$ from the edge of the roof. Given that the structure is expected to have a fundamental period less than $1 \mathrm{~s}$, its height is less than $60 \mathrm{~m}$, the ratio of its height to its effective width is less than 4 , then the static procedure applies for the calculation of wind effects on the building according to the NBCC (2010). Results from the static procedure result in a specified external wind pressure of $0.57 \mathrm{kPa}$. The structure is assumed to be located in rough terrain, such that its exposure factor $\left(C_{e}\right)$ is $0.7(h / 12)^{0.3}$. For lowrise structures, the gust effect factor is taken as 2.0 and the external pressure coefficient, averaged over the surface considered is taken from Figure I-7 in NBCC (2010). Table B.2 shows the wind loads acting over the surface of the structure.

\section{B.2 Equivalent Static Force Procedure}

The seismic design of the three-storey reinforced concrete structures is initially carried out using the equivalent static force procedure as described in Part 4 of Division B of the NBCC (2010). Although a full dynamic analysis of the structure is required because of the high seismicity in the location of the building $\left(I_{e} F_{a} S_{a}(0.2)>0.35\right)$, results from the simplified equivalent static force procedure place limitations on the results from the dynamic analysis. Spectral ground acceleration values are taken from the NBCC (2010) and are based on a $2 \%$ in 50 years probability of exceedence ( 2450 year return period) and a 5\% damping ratio. Given the local site conditions in Victoria, BC it is assumed that the building is situated on site class C soil, representing very dense soil or soft rock. These ground conditions results in a soil amplification factors $\left(F_{a} \& F_{v}\right)$ 
Table B.1 Specified dead and live loads

\begin{tabular}{ccc}
\hline \hline Load & $\begin{array}{c}\text { Floor } \\
(k P a)\end{array}$ & $\begin{array}{c}\text { Roof } \\
(k P a)\end{array}$ \\
\hline Roofing & - & 0.24 \\
Partitions & 1.00 & 0.00 \\
Insulations & - & 0.10 \\
Fire Proofing & 0.10 & 0.10 \\
Ceiling & 0.24 & 0.24 \\
Flooring & 0.10 & - \\
Mech./Elec. & 0.48 & 0.48 \\
\hline Dead Load & 1.92 & 1.16 \\
\hline Live Load & 2.40 & 1.00 \\
\hline \hline
\end{tabular}

Table B.2 Specified wind loads

\begin{tabular}{ccccc}
\hline & \multicolumn{2}{c}{ N-S Direction } & \multicolumn{2}{c}{ E-W Direction } \\
& Windward Side & Leeward Side & Windward Side & Leeward Side \\
Region & $(k P a)$ & $(k P a)$ & $(k P a)$ & $(k P a)$ \\
\hline End Zone & 1.31 & -0.91 & 1.31 & -0.91 \\
Interior Zone & 0.86 & -0.63 & 0.86 & -0.63 \\
\hline \hline
\end{tabular}

Pressure values are expressed in terms of $C_{e}$

E-W: $V_{\text {base,wind }}=397 k N$ N-S: $V_{\text {base,wind }}=665 k N$ 
of 1.0 from Tables 4.1.8.4B and 4.1.8.4C of the NBCC (2010) and the site specific design spectral response acceleration values $\left[S_{a}(T)\right]$ are shown in Table B.3. The code-based fundamental period of the structure is calculation according to section 4.1.8.11(3)(c) which results in a code based period of $0.312 \mathrm{~s}$. However, results have demonstrated that the code based equations are often provide a conservative estimate of the actual fundamental period of vibration of a structure, the NBCC (2010) places an upper limit on the amount that the code-based fundamental period can be increased based on the results from a dynamics analysis. Assuming the the dynamic analysis will result in a natural period for the structure, thus reducing seismic design forces, the fundamental period calculated according to Eq. 3.38 is amplified by the maximum value of 2.0 as specified in section 4.1.8.11(3)(d)(ii) of the NBCC (2010), resulting in a natural period of 0.624s. Given that the period of the structure falls within the short period range of the design spectrum, the higher mode factor $\left(M_{v}\right)$ and base overturning reduction factor $(J)$ as described in Tables 4.1.8.11 in the NBCC (2010) are taken as 1.0. The seismic force resisting system is designed as a moderately ductile shear wall. The NBCC (2010) recommends ductility $\left(R_{d}\right)$ and overstrength $\left(R_{o}\right)$ factors of 2.0 and 1.4, respectively for this type of SFRS. The equivalent static force procedure approximates the elastic base shear and reduces it by a response modification factor. The total area and weight of each storey of the structure is shown in Table B.4. Based on the code-based fundamental period and the total seismic weight shown in Table B.4 this results in a total seismic base shear of $9086 \mathrm{kN}$, which is governed by the upper bound of Eq. 3.39. The total base shear is distributed over the height of the building and shear walls assuming the concrete slab will act as a rigid diaphragm. Table B.5 shows the seismic weight, lateral force, and overturning moment for each level of the

Table B.3 Uniform hazard spectrum for Victoria, British Columbia

\begin{tabular}{ccc}
\hline \hline $\mathrm{T}(\mathrm{s})$ & $\mathrm{S}(\mathrm{T})$ & $S_{a}(T)$ \\
\hline 0.2 & $1.2 \mathrm{~g}$ & $1.2 \mathrm{~g}$ \\
0.5 & $0.82 \mathrm{~g}$ & $0.82 \mathrm{~g}$ \\
1.0 & $0.38 \mathrm{~g}$ & $0.38 \mathrm{~g}$ \\
2.0 & $0.18 \mathrm{~g}$ & $0.18 \mathrm{~g}$ \\
4.0 & $0.09 \mathrm{~g}$ & $0.09 \mathrm{~g}$ \\
\hline \hline
\end{tabular}

Joshua Woods, Department of Civil and Environmental Engineering, Carleton University 
prototype building.

\section{B.3 Response Spectrum Analysis}

To optimize the design of the structure and get a better understanding of the seismic performance of the building, a response spectrum analysis is conducted. The response spectrum analysis method is an approximation for multi degree-of-freedom systems and gives an estimation of the maximum peak response without the need for conducting a full nonlinear time history analysis. Response spectrum analysis is an iterative dynamic analysis method that uses a three-dimensional linearly elastic model of the structure to optimize the design. The results from the equivalent static force procedure are used as a initial condition for the construction of the three-dimensional model. The User's Guide to NBCC (2010) contains details information on the modal response spectrum analysis method. According to this document, the analysis begins with the development of a two-dimensional or three-dimensional model. The analysis is then performed independently along pairs of orthogonal directions.

Table B.4 Seismic weight of prototype structure

\begin{tabular}{cccccc}
\hline \hline & $\begin{array}{c}\text { Floor Area } \\
\left(m^{2}\right)\end{array}$ & $\begin{array}{c}\text { Snow Load } \\
(\mathrm{kPa})\end{array}$ & $\begin{array}{c}\text { Structural } \\
\text { Elements }(\mathrm{kPa})\end{array}$ & $\begin{array}{c}\text { Superimposed } \\
\text { Dead Load }(\mathrm{kPa})\end{array}$ & $\begin{array}{c}\text { Total Weight } \\
\mathrm{kN}\end{array}$ \\
\hline Roof $(3)$ & 1500 & 1.08 & 5.11 & 1.16 & 9809 \\
2 & 1500 & - & 5.51 & 1.92 & 11139 \\
1 & 1500 & - & 5.62 & 1.92 & 11310 \\
& & & & Total & 32260 \\
\hline \hline
\end{tabular}


Table B.5 Equivalent static force procedure results

\begin{tabular}{ccccc}
\hline \hline & $h_{x}$ & $W_{x}$ & $W_{x} h_{x}$ & $F_{x}$ \\
Level, $i$ & $(\mathrm{~m})$ & $(\mathrm{kN})$ & $\mathrm{kN}-\mathrm{m}$ & $\mathrm{kN}$ \\
\hline Roof (3) & 11.5 & 9809 & 112805 & 4210 \\
2 & 8.0 & 11140 & 89119 & 3326 \\
1 & 4.5 & 11310 & 50895 & 1900 \\
& & & Total & 9436 \\
\hline \hline
\end{tabular}

The following steps are repeated until an optimized design that meets both the strength and drift requirements are achieved (Filiatrault et al., 2013):

1. Determine the periods and mode shapes for the structure $\left(T_{i}, \phi_{i}\right)$ for each orthogonal direction while the motion in the opposite direction is restrained

2. Determine the design spectral acceleration value for each mode $S\left(T_{i}\right)$ from the uniform hazard spectra as defined by the NBCC (2010)

3. Subject the three-dimensional model to the design spectral accelerations and obtain the elastic base shear $\left(V_{e}\right)$, using an appropriate combination rule (SRSS) to include the effects of each mode together. In addition, the maximum response quantities from each mode can be determined, including storey shears and lateral displacements

4. The design elastic base shear $\left(V_{e d}\right)$ is set equal to the elastic base $\operatorname{shear}\left(V_{e}\right)$ from the previous step. However, for short period structures designed with $R_{d}>1.5$ located in a site class other than site class $\mathrm{F}$, the base shear $V_{e}$ is multiplied by the coefficient $\left(\frac{2 S(0.2)}{3 S\left(T_{a}\right)} \leq 1.0\right)$ to obtain the design elastic base shear $\left(V_{e d}\right)$ accounting for the upper limit on design seismic loads which is permitted in NBCC for short period structures

5. The minimum lateral earthquake force $(V)$ is determined using the ESFP where the period $\left(T_{a}\right)$ is the smaller of the period obtained from the modal analysis in Step 1 and the applicable upper limits on the period specified in NBCC 2010.

6. Determine the design base shear $\left(V_{d}\right)$ by multiplying the design elastic base shear by $\left(I_{e} / R_{d} R_{o}\right)$. Note that if the design base shear $\left(V_{d}\right)$ is less than $80 \%$ of the base shear from the equivalent static force procedure $(V)$, then limit set $V_{d}=V$ 
7. Accidental in-plane torsional effects can now be taken into account in the analysis. Given that the ratio $B_{x}$ is less than 1.7 at each level of the structure, torsional effects can be accounted for by displacing the centre of mass at every level by $+/-0.05 D_{n} x$ in the structural model

8. Multiply the forces and deflections by the ratio of $V_{d} / V_{e}$ to obtain design values

9. To obtain realistic values of anticipated deflections and drifts, the design values need to be multiplied by $\left(R_{d} R_{o}\right) / I_{E}$ to account for the inelastic response of the SFRS

The steps listed above are repeated to obtain an optimized design of the structure. In using the method described above, regular structures such as the 3-storey RC structure described herein can benefit from a $20 \%$ reduction in design seismic forces using the response spectrum analysis method. A complete set of results of the response spectrum analysis are presented in Table B.6, including storey shears, overturning moment and inter-storey drifts. The design storey drifts are compared with the drift limits imposed in NBCC (2010), which limits inter-storey drifts for buildings of normal importance to $2.5 \%$ drift to control damage to structural and nonstructual components. Although the drifts are below the requirements of the NBCC (2010), this is common for RC shear wall structures which are typically governs by strength design as opposed to drift limitations.

\section{B.4 Design of Gravity Load Resisting System}

While the RC shear walls are responsible for resisting the in-plane lateral loads due to wind or earthquake, modern design standards also recognize the importance of accounting for deformation of the SFRS when designing the gravity load resisting system. This is a result of the fact that as the building deforms laterally during an earthquake, the gravity load resisting system must also be capable of achieving the same level of deformability. This is paramount for the structure to be able to meet the life-safety performance objectives of modern seismic design standards used around the world. For designing the gravity load resisting system, the load combination shown 
Table B.6 Response spectrum analysis results

\begin{tabular}{|c|c|c|c|c|}
\hline & \multicolumn{4}{|c|}{ E-W Direction } \\
\hline Level & $\begin{array}{l}\text { Storey Shear } \\
(\mathrm{kN})\end{array}$ & $\begin{array}{l}\text { Overturning Moment } \\
\qquad(\mathrm{kN}-\mathrm{m})\end{array}$ & $\begin{array}{l}\text { Displacement } \\
(\mathrm{mm})\end{array}$ & $\begin{array}{c}\text { Inter-Storey Drift } \\
(\%)\end{array}$ \\
\hline 3 (Roof) & 4546 & 15910 & 171.8 & 2.02 \\
\hline 2 & 7075 & 45217 & 101.2 & 1.78 \\
\hline \multirow[t]{2}{*}{1} & 8583 & 79293 & 38.9 & 0.86 \\
\hline & \multicolumn{4}{|c|}{ N-S Direction } \\
\hline Level & $\begin{array}{c}\text { Storey Shear } \\
(\mathrm{kN})\end{array}$ & $\begin{array}{l}\text { Overturning Moment } \\
(\mathrm{kN}-\mathrm{m})\end{array}$ & $\begin{array}{c}\text { Displacement } \\
(\mathrm{mm})\end{array}$ & $\begin{array}{c}\text { Inter-Storey Drift } \\
(\%) \\
\end{array}$ \\
\hline 3 (Roof) & 4759 & 16656 & 159.5 & 1.87 \\
\hline 2 & 7442 & 47461 & 93.9 & 1.65 \\
\hline 1 & 8995 & 83178 & 36.2 & 0.80 \\
\hline
\end{tabular}

in Eq. B.58 applies for earthquake scenarios:

$$
1.0 D+0.5 L+E+0.25 S
$$

Under this load combination, gravity load carrying columns are required to have sufficient ductile rotational capacity to sustain the design inelastic rotations and cyclic demands. To do so, the design of gravity load carrying columns must consider the axial load demand, moment demand and shear demands and check if these demands exceed the column capacities under all gravity load combinations. When columns are subject to particularly high axial load ratios, this reduces the potential curvature ductility capacity, so additional transverse reinforcement is required to improve concrete confinement and thus the ductility of the column. The cross-sectional area of the longitudinal steel reinforcement $\left(A_{s}\right)$ must be between $1 \%$ and $6 \%$ of the gross cross sectional area of the column $\left(A_{g}\right)$. The resulting factored axial capacity of a column can be determined using Eq. B.59:

$$
P_{r}=0.80 \alpha_{1} \phi c f_{c}^{\prime}\left(A_{g}-A_{s}\right)
$$

where $A_{g}$ is the total gross area of the column cross-section and $A_{s}$ is the total area of longitudinal steel reinforcement. The capacity of the column under uniaxial or biaxial bending as a result of 
eccentricity in the axial load can be determined based on a section analysis to construct a column $(P-M)$ interaction diagram. To ensure the column exhibits a ductile response, particularly in potential plastic hinge regions near the base of the columns, the area of the rectangular steel hoops in each direciton must meet the minimum requirements shown in Eq. B.60 and Eq. B.61:

$$
\begin{gathered}
A_{s h} \geq 0.2 k_{n} k_{p} \frac{A_{g} f_{c}^{\prime}}{A_{c h} f_{y h}} s_{h} h_{c} \\
A_{s h} \geq 0.09 \frac{f_{c}^{\prime}}{f_{y h}} s_{h} h_{c}
\end{gathered}
$$

where $A_{c} h$ is the cross-section area of the column; $s_{h}$ is the hoop or tie spacing; $h_{c}$ is the crosssection dimension of the concrete core perpendicular to the direction of the transverse steel being evaluated; $k_{n}=n_{1} /\left(n_{1}-2\right)$ where $n_{1}$ equals the number of longitudinal bars laterally supported by transverse ties or hoops corners. The spacing of transverse must also meet minimum spacing requirements to prevent buckling of longitudinal reinforcement between transverse hoops. According to CSA A23.3-04, the tie spacing $\left(s_{x}\right)$ is the smallest of:

- $1 / 4$ of the minimum member dimension $(b$ or $h)$;

- 6 times the diameter of the smallest longitudinal reinforcing bar; or

- $s_{x}=100+\left(\frac{350-h_{x}}{3}\right)($ in $m m)$

where $h_{x}$ is the dimension of the column cross-section thqt is perpendicular to the transverse steel under consideration. The additional confinement is provided in consideration with the axial force on the column and the location of potential plastic hinge regions. For first floor columns, the additional confinement must be provided over the entire height of the column, while for stories above the first level, the additional confinement must be provided over a distance $\left(l_{o}\right)$, which when $P_{f}<0.5 \phi_{c} f_{c}^{\prime} A_{g}$, is the larger of:

- 1.5 times the depth of the column at the face of the joint; and

- $1 / 6$ of the column's unsupported height.

Outside the confined regions, the transverse reinforcement must sustain the factored shear forces, the design procedure for which meets the same requirements as those disucssed in the previous 
section for the shear strength of shear walls.

\section{B.5 Experimental Test Setup Design}

This section outlines the capacity-based design of the experimental test setup for the hybrid simulation. This includes verification of the capacity of the rigid loading beam, as well as design of the steel column test specimen for hybrid test rehearsals.

\section{B.5.1 Rigid Loading Beam}

The rigid loading beam is responsible for acting as a stiff element used to apply the target deformations to the test specimen. Properties for the rigid loading beam are shown in Table B.7. The section class for the rigid loading beam is classified using Table 5 of CSA (2014b), which defines limits for the flange and web of the section. Section class limits for the flange and web of the beam, respectively, are shown below:

$$
\begin{aligned}
& \frac{b}{2 t} \leq \frac{170}{\sqrt{F_{y}}} ; \\
& \frac{h}{w} \leq \frac{1100}{\sqrt{F_{y}}} ;
\end{aligned}
$$

Table B.7 Dimensions of the rigid steel loading beam

\begin{tabular}{cc}
\hline \hline Property & Value \\
\hline $\mathrm{d}(\mathrm{mm})$ & 375 \\
$\mathrm{~b}(\mathrm{~mm})$ & 355 \\
$\mathrm{t}(\mathrm{mm})$ & 22.0 \\
$\mathrm{w}(\mathrm{mm})$ & 19.0 \\
$\mathrm{~h}(\mathrm{~mm})$ & 330.2 \\
$\mathrm{~A}\left(\mathrm{~mm}^{2}\right)$ & 21894 \\
$\mathrm{I}_{x}\left(\mathrm{~mm}^{4}\right)$ & $552.1 \times 10^{6}$ \\
$\mathrm{a}(\mathrm{mm})$ & 460 \\
\hline \hline
\end{tabular}

Joshua Woods, Department of Civil and Environmental Engineering, Carleton University 
Therefore, the rigid loading beam is a Class 1 section. The moment resistance of the beam $\left(M_{r}\right)$, assuming that the flange is fully laterally supported, is computed using Eq. B.64:

$$
M_{r}=Z_{x} F_{y}
$$

The shear strength of the beam $\left(V_{r}\right)$ is computed using the following set of equations, according to CSA (2014b), using Eq. B.65 and Eq. B.66:

$$
\begin{gathered}
\text { if } \frac{h}{w} \leq \sqrt{\frac{k_{v}}{(a / h)^{2}}} \text { where } k_{v}=5.34+\frac{4}{\left(1.4^{2}\right)} ; \\
V_{r}=F_{s} A_{v}=F_{s} d w ;
\end{gathered}
$$

Table B.8 summarizes the results of the strength analysis of the rigid loading beam.

\section{B.5.2 Steel Column Assembly Design}

To facilitate preliminary hybrid simulations on a stiff structural element prior to conducting the hybrid simulation on the shear wall elements in this study, a steel column with a removable steel yielding link is designed. This will allow for repeated trial hybrid simulations on a nonlinear structural element with a comparable axial stiffness and moment capacity of the RC shear wall specimens. Selection of the size of the steel beam is based on the axial stiffness of the shear wall

Table B.8 Moment capacity and shear strength of the rigid loading beam

\begin{tabular}{cc}
\hline \hline Property & Value \\
\hline$b / 2 t$ & 8.52 \\
$h / w$ & 16.4 \\
$M_{r}(\mathrm{kN}-\mathrm{m})$ & 1033 \\
$a / h$ & 1.40 \\
$k_{v}(\mathrm{MPa})$ & 7.38 \\
$F_{s}(\mathrm{MPa})$ & 231 \\
$V_{r}(\mathrm{kN})$ & 1402 \\
\hline \hline
\end{tabular}

Joshua Woods, Department of Civil and Environmental Engineering, Carleton University 
specimens $\left(k_{\text {axial }}\right)$, computed using Eq. B.67:

$$
k_{\text {axial }}=\frac{E A}{L}=\frac{30000 \times(1500 \times 100)}{1800}=2500 \mathrm{kN} / \mathrm{mm}
$$

For a proposed column length of $2275 \mathrm{~mm}$, this allows for the determination of a target area for the steel column $\left(A_{\text {target }}\right)$, which is computed using Eq. B.68:

$$
A_{\text {target }}=\frac{k_{\text {axial }} L}{E}=\frac{2500 * 2275}{200000}=28450 \mathrm{~mm}^{2}
$$

The most efficient steel W-section that meets these requirements is a W310x202, Table B.9 shows the properties for proposed section. In addition to matching the axial stiffness of the shear wall specimen, it is also a goal of this preliminary study to approximately match the lateral load corresponding to yielding of the shear wall specimen to ensure the test setup is sufficiently stiff, and that sufficient tie-down force is provided at the approximate maximum lateral load to prevent sliding during the hybrid simulation. Based on a strain compatibility analysis of the shear wall cross section, the shear wall specimens have a yield moment capacity $\left(M_{\text {target }}\right)$ of approximately $492.7 \mathrm{kN}-\mathrm{m}$, which for a lever arm of $2.45 \mathrm{~m}$ from the centre of the lateral actuator, results in a maximum lateral load of $201 \mathrm{kN}$. Using the estimated ultimate moment capacity of the shear wall specimens, a target plastic modulus $\left(Z_{x}\right)$ can be computed for the removable steel yielding link, computed using Eq. B.69:

$$
Z_{x, \text { target }}=\frac{M_{\text {target }}}{1.1 F_{y}}
$$

The calculation of $Z_{x, \text { target }}$ includes a factor of 1.1 to account for strain hardening in the steel yielding link. Based on the required plastic section modulus, a W250x101 was selected for the RBS detail. Although this section has a slightly higher plastic section modulus, this will be reduced after modifications are made to the section flange. Based on the selected top and bottom steel column sections and RBS, the combined axial stiffness of the system can be estimated. Because these sections are connected in series, the total equivalent axial stiffness of the assembly $\left(k_{e q}\right)$ can 
Table B.9 Dimensions of the rigid steel column

\begin{tabular}{ccc}
\hline \hline Property & Rigid Column & RBS Link \\
\hline Section Size & $310 \times 202$ & $250 \times 101$ \\
$\mathrm{~d}(\mathrm{~mm})$ & 341 & 264 \\
$\mathrm{~b}(\mathrm{~mm})$ & 315 & 257 \\
$\mathrm{t}(\mathrm{mm})$ & 31.8 & 19.6 \\
$\mathrm{w}(\mathrm{mm})$ & 20.1 & 11.9 \\
$\mathrm{~h}(\mathrm{~mm})$ & 277 & 225 \\
$\mathrm{~A}\left(\mathrm{~mm}^{2}\right)$ & 25800 & 12900 \\
$\mathrm{I}_{x}\left(\mathrm{~mm}^{4}\right)$ & $520 \times 10^{6}$ & $1640^{6}$ \\
$\mathrm{Z}_{x}\left(\mathrm{~mm}^{3}\right)$ & $3510 \times 10^{3}$ & $1440^{3}$ \\
Web Class & 1 & 1 \\
Flange Class & 1 & 1 \\
\hline \hline
\end{tabular}

be approximated using Eq. B.70:

$$
\frac{1}{k_{e q}}=\frac{1}{k_{1}}+\frac{1}{k_{2}}+\ldots \frac{1}{k_{i}}\left(\text { where } k=\frac{E A}{L}\right)
$$

Adding the axial stiffness of each of the three segments of the column results in a total axial stiffness of $2200 \mathrm{kN} / \mathrm{mm}$, which closely matches the axial stiffness of the shear wall specimens, which have an approximate axial stiffness of $2430 \mathrm{kN} / \mathrm{mm}$.

\section{B.5.3 RBS Detail Design}

The replaceable moment connection for the column test is designed according to the CSIC standard Moment Connections for Seismic Applications. The standard provides the following design procedure for reduced beam section (RBS) details. First, choose trial values for the beam sections, column sections, and RBS dimensions $a, s$, and $c$, which are subject to the limits shown in Eq. B.71:

$$
0.5 b \leq a \leq 0.75 b ; 0.65 d_{b} \leq s \leq 0.85 d_{b} ; 0.1 b \leq c \leq 0.25 b
$$

where $b$ is the width of the beam flange, $a$ is the horizontal distance from the face of the column flange to the start of an RBS cut, $s$ is the length of an RBS cut, $c$ is the depth of the cut at the centre of the RBS, and $d_{b}$ is the depth of the beam. After preliminary limits have been selected, 
compute the plastic section modulus $\left(Z_{e}\right)$ at the centre of the RBS using Eq. B.72:

$$
Z_{e}=Z_{b}-2 c t_{b}\left(d_{b}-t_{b}\right)
$$

where $Z_{b}$ is the plastic section modulus of the beam, and $t_{b}$ is the thickness of the beam flange. After computing the section modulus, compute the probable maximum moment $\left(M_{p r}\right)$ at the centre of the RBS using Eq. B.73:

$$
M_{p r}=C_{p r} R_{y} F_{y} Z_{e}
$$

where $R_{y}$ is the ratio of the probably yield stress to the specified minimum yield stress, $C_{p r}$ is a factor to account for plastic hinging effects at large storey drifts, strain hardening, and other connection conditions taken as 1.1 for Type D and Type MD frames and 1.0 for Type LD frames. Compute the shear force at the centre of the RBS at each end of the beam, taking $M_{p r}$ as the moment at the centre of the RBS. Using this shear force, the probable moment at the column face $\left(M_{c f}\right)$ can be computed using Eq. B.74:

$$
M_{c f}=M_{p r}+V_{h} x \leq R_{y} F_{y} Z_{b}
$$

where $x$ is the distance from the assumed location of the plastic hinge to the column face. The moment at the column face is due to the effects of beam hinging is not permitted to exceed the moment resistance of the gross beam section based on the expected yield stress, shown in the right hand side of the inequality in Eq. B.74. If this inequality is not satisfied, then the appropriate adjustments should be made to the values of $c, a$, and $s$ and the design procedure can be repeated. Table B.10 shows the dimensions for the RBS detail. In addition to determining the details of the RBS, weld access holes to facilitate welding of the RBS to the steel end plates is also required. CISC (2014) recommends the following for the weld access detail:

- The length of the weld access hole be the larger of $1.5 w$ and $38 \mathrm{~mm}$;

- The height of the weld access hole be the larger of $1.5 w$ and $38 \mathrm{~mm}$ but not greater than $50 \mathrm{~mm}$;

Joshua Woods, Department of Civil and Environmental Engineering, Carleton University 
Table B.10 Design dimensions for the RBS detail

\begin{tabular}{cc}
\hline \hline Property & Value \\
\hline $\mathrm{a}(\mathrm{mm})$ & 135 \\
$\mathrm{~s}(\mathrm{~mm})$ & 180 \\
$\mathrm{c}(\mathrm{mm})$ & 30 \\
$\mathrm{Z}_{e}\left(\mathrm{~mm}^{3}\right)$ & $1112 \times 10^{3}$ \\
$\mathrm{M}_{p r}(\mathrm{kN}-\mathrm{m})$ & 471 \\
$\mathrm{M}_{c f}(\mathrm{kN}-\mathrm{m})$ & 1350 \\
\hline \hline
\end{tabular}

- The radius of the weld access hole must be larger than $10 \mathrm{~mm}$; and

- The slopes formed on either side of the radius shall provide a smooth transition from web to flange.

CISC (2014) also specifies design recommendations for bolted end plate connections, which are shown in Table B.11. where $b_{p}$ is the width of the end-plate, $d_{b}$ is the depth of the beam, $g$ is the horizontal spacing of the bolts, $p_{f i}$ is the vertical distance from the inside of a beam flange to the nearest inside bolt row; $p_{f_{o}}$ is the vertical distance from the outside of a beam flange to the nearest outside bolt row; $t_{p}$ is the thickness of the end-plate. The design of the end plate bolts is based on the moment at the face of the column, $\left(M_{c f}\right)$, computed previously. Following selection of the preliminary connection geometry $\left(g, h_{i}, p_{f i}, p_{f_{o}}\right.$, and $\left.p_{b}\right)$, the required bolt diameter for bolt tension $\left(d_{\text {bolt }}\right)$ can be computed using Eq. B.75:

$$
d_{\text {bolt }} \geq \sqrt{\frac{2 M_{c f}}{0.75 \pi \phi_{n} F_{u}\left(h_{0}+h_{1}\right)}} ;
$$

Table B.11 Bolted end plate details for RBS connection

\begin{tabular}{cccc}
\hline \hline Property & Maximum & Minimum & Design \\
\hline$b_{p}(\mathrm{~mm})$ & 400 & 150 & 400 \\
$d_{b}(\mathrm{~mm})$ & 1400 & 250 & 264 \\
$g(\mathrm{~mm})$ & $b_{p}$ & 100 & 282 \\
$p_{f i}(\mathrm{~mm})$ & 115 & 38 & 42 \\
$P_{f_{o}}(\mathrm{~mm})$ & 115 & 38 & 42 \\
$t_{p}(\mathrm{~mm})$ & 57 & 13 & 25.4 \\
\hline \hline
\end{tabular}

Joshua Woods, Department of Civil and Environmental Engineering, Carleton University 
where $F_{u}$ is the minimum tensile strength of the bolts, $\phi_{n}$ is a strength reduction factor of $0.9, h_{0}$ and $h_{1}$ are the vertical distances between the flange of the beam in tension and the outer and inner rows of bolts, respectively. Once a trial bolt diameter has been selected, the required end-plate thickness $\left(t_{p}\right)$ can be determined using Eq. B.76:

$$
t_{p} \geq \sqrt{/ f r a c 1.11 M_{c f} \phi_{d} F_{y p} Y_{p}}
$$

where $F_{y p}$ is the yield strength of the end plate material, the end-plate yield line mechanism parameter $\left(Y_{p}\right)$, can be determined using Eq. B.77:

$$
Y_{p}=\frac{b_{p}}{2}\left[h_{1}\left(\frac{1}{p_{f i}}+\right)+h_{0}\left(\frac{1}{p_{f_{o}}}\right)-\frac{1}{2}\right]+\frac{2}{g}\left[h_{1}\left(p_{f i}+s\right)\right]
$$

where

$$
s=\frac{1}{2} \sqrt{b_{p} g}
$$

After selection of an appropriate end-plate thickness, the probable beam flange force $\left(P_{c f}\right)$ can be computed using Eq. B.79:

$$
P_{c f}=\frac{M_{c f}}{d_{b}-t_{b}}
$$

Check shear yielding and shear rupture of extended portion of the bolted end-plate connection due to the pull exerted by the tension beam flange using Eq. B.80 and Eq. B.81, respectively:

$$
\begin{aligned}
& \frac{P_{c f}}{2} \leq \phi_{d} 0.6 F_{y p} b_{p} t_{p} ; \\
& \frac{P_{c f}}{2} \leq \phi_{n} 0.6 F_{u p} A_{n} ;
\end{aligned}
$$

where $A_{n}$ is the net area of the end-plate, equal to $t_{p}\left(b_{b}-2 d_{h}\right)$, and $d_{h}$ is the bolt diameter plus $2 \mathrm{~mm}$. If the connection meets the requirements for shear yielding and shear rupture, the factored bolt shear resistance of the connection $\left(V_{c f}^{a}\right)$ is taken as the resistance provided by the bolts at 
one (compression) flange, computed using Eq. B.82:

$$
V_{c f}^{a} \leq 0.60 \phi_{n} n A_{b} F_{u}
$$

where $\mathrm{n}$ is the number of bolts at the compression flange. In addition to the shear strength, the bolt-bearing/tear-out failure of the end-plate $\left(V_{c f}^{b}\right)$ can be computed using Eq. B.83:

$$
V_{c f}^{b} \leq \phi_{n}\left(N_{i}\right) r_{n i}+\phi_{n}\left(n_{o}\right) r_{n o}
$$

where $n_{i}$ is the number of inner bolts, taken as $2, n_{o}$ is the number of outer bolts, also taken as 2, $r_{n i}$ is $1.2 L_{c} t_{p} F_{u} \leq 2.4 d_{b t} t F_{u}$ for each inner bolt, and $r_{n o}$ is $1.2 L_{c} t F_{u} \leq 2.4 d_{b t} t_{p} F_{u}$ for each outer bolt, and $L_{c}$ is the clear distance between the edge of the hole and the edge of the adjacent hole. 


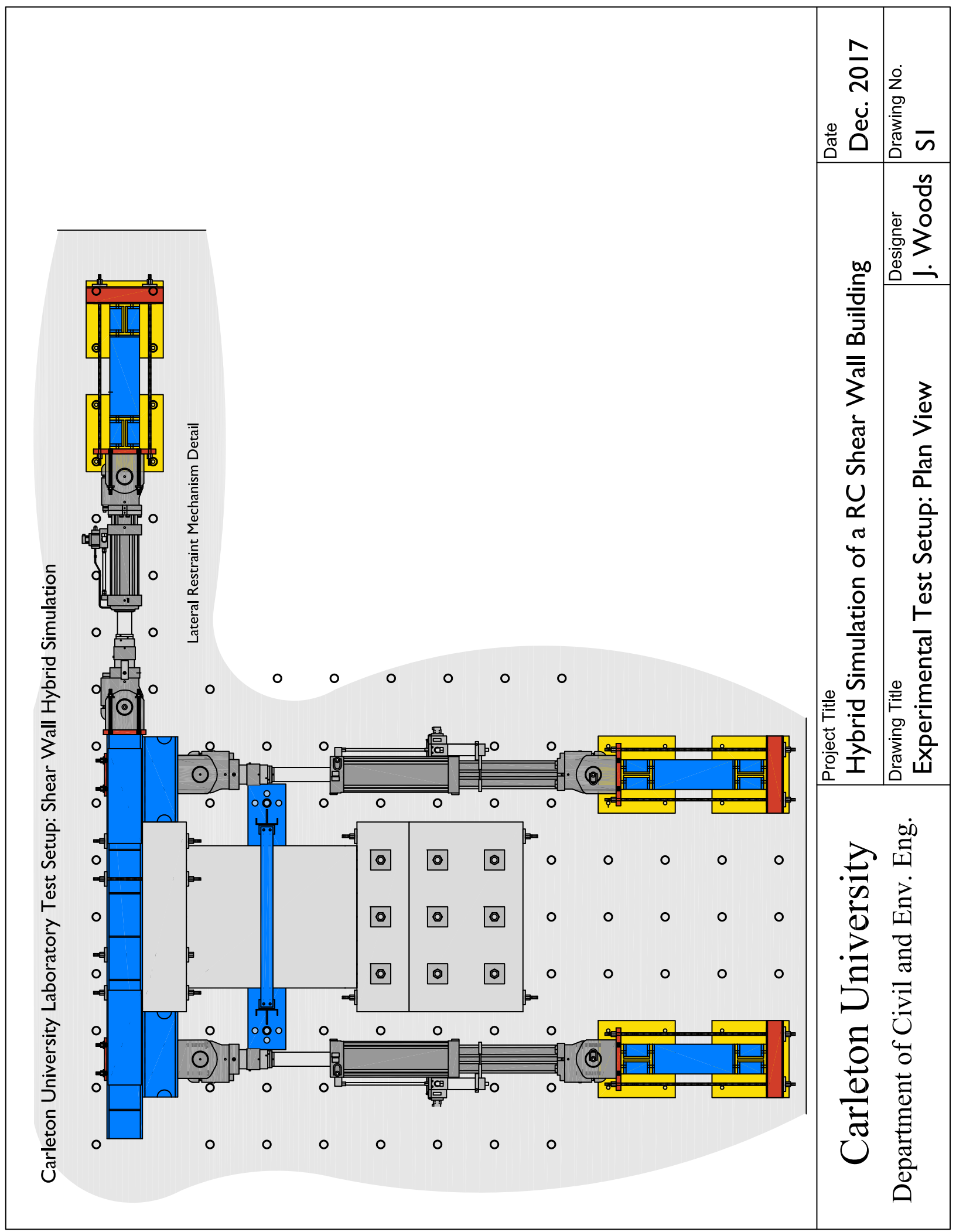




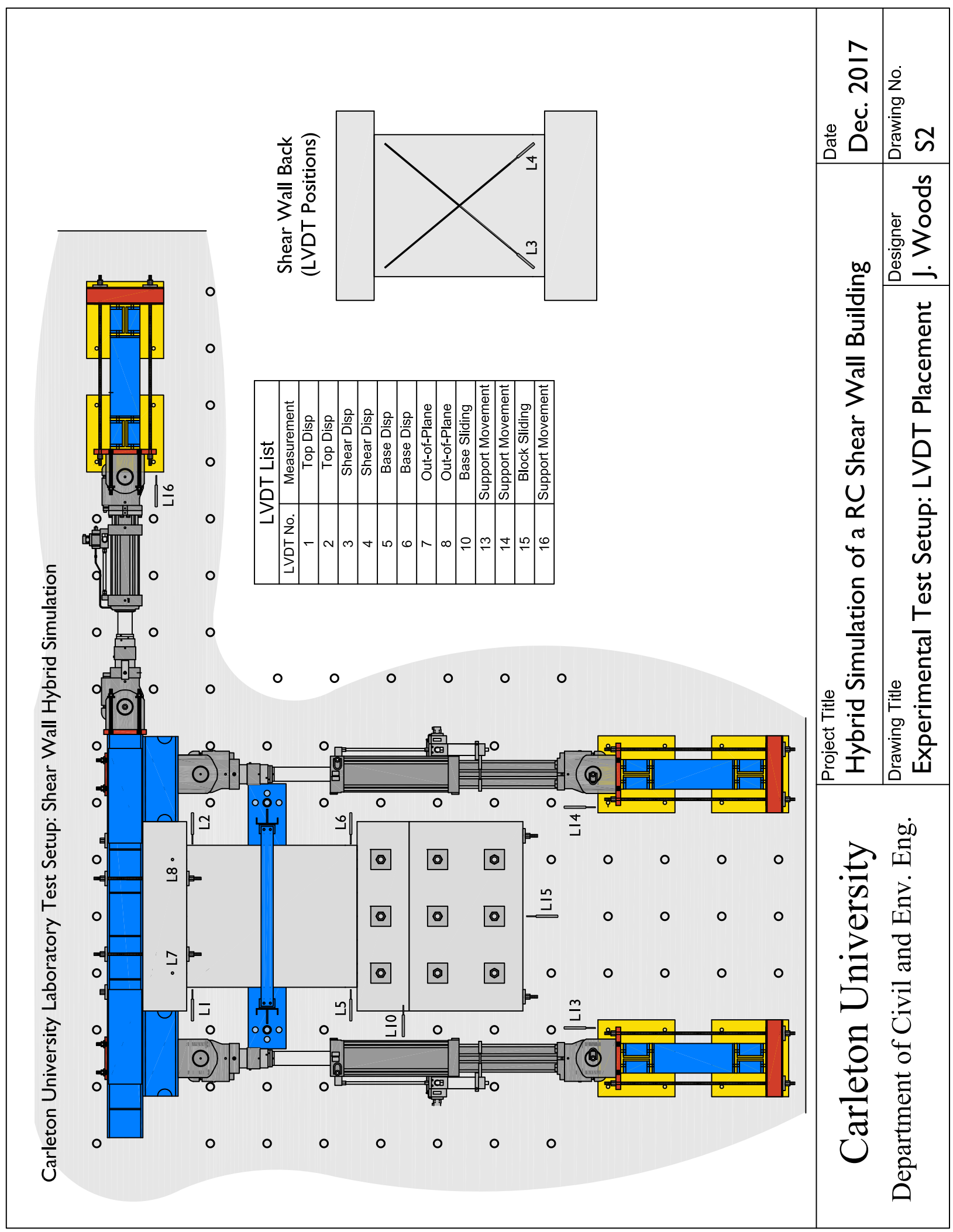




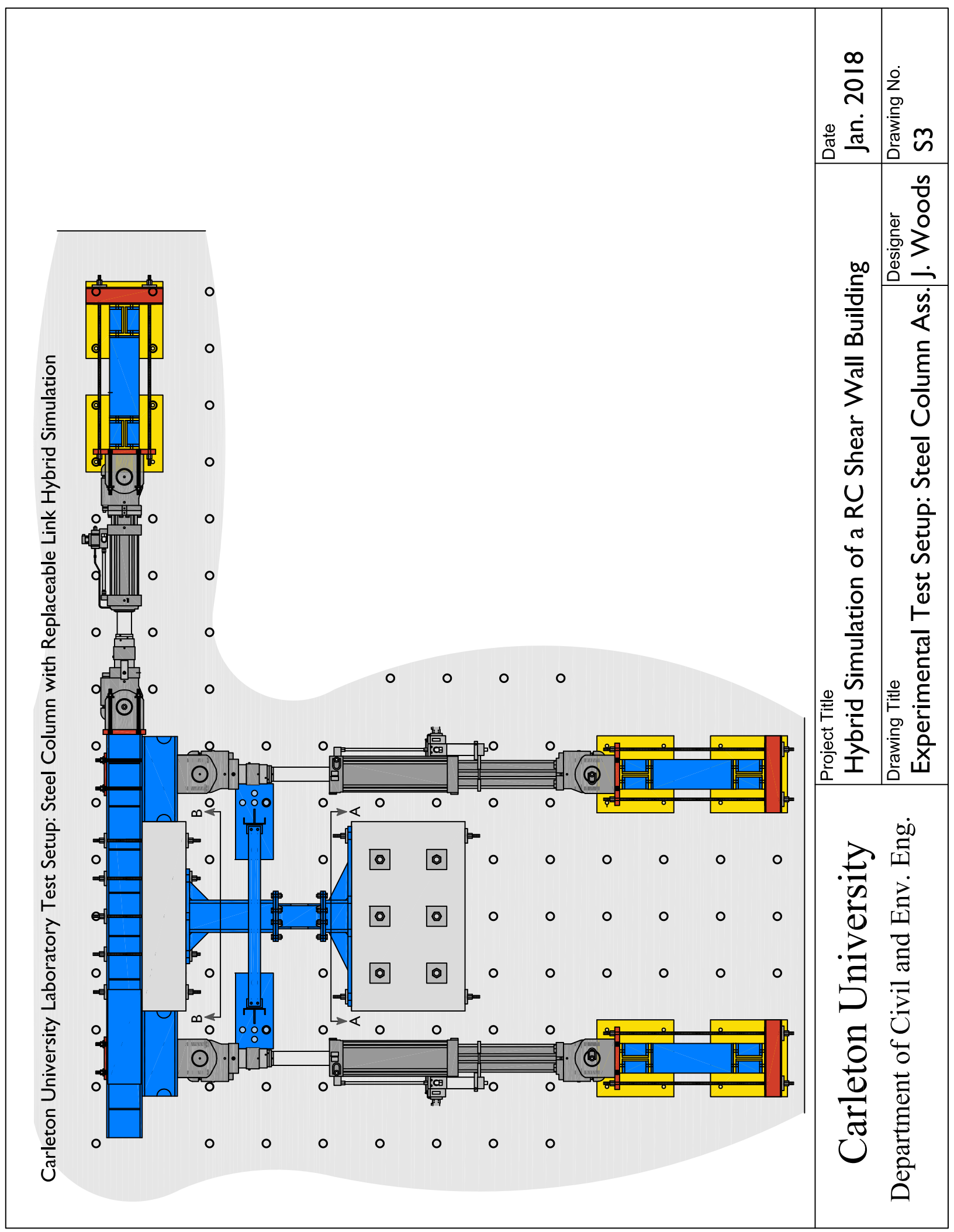




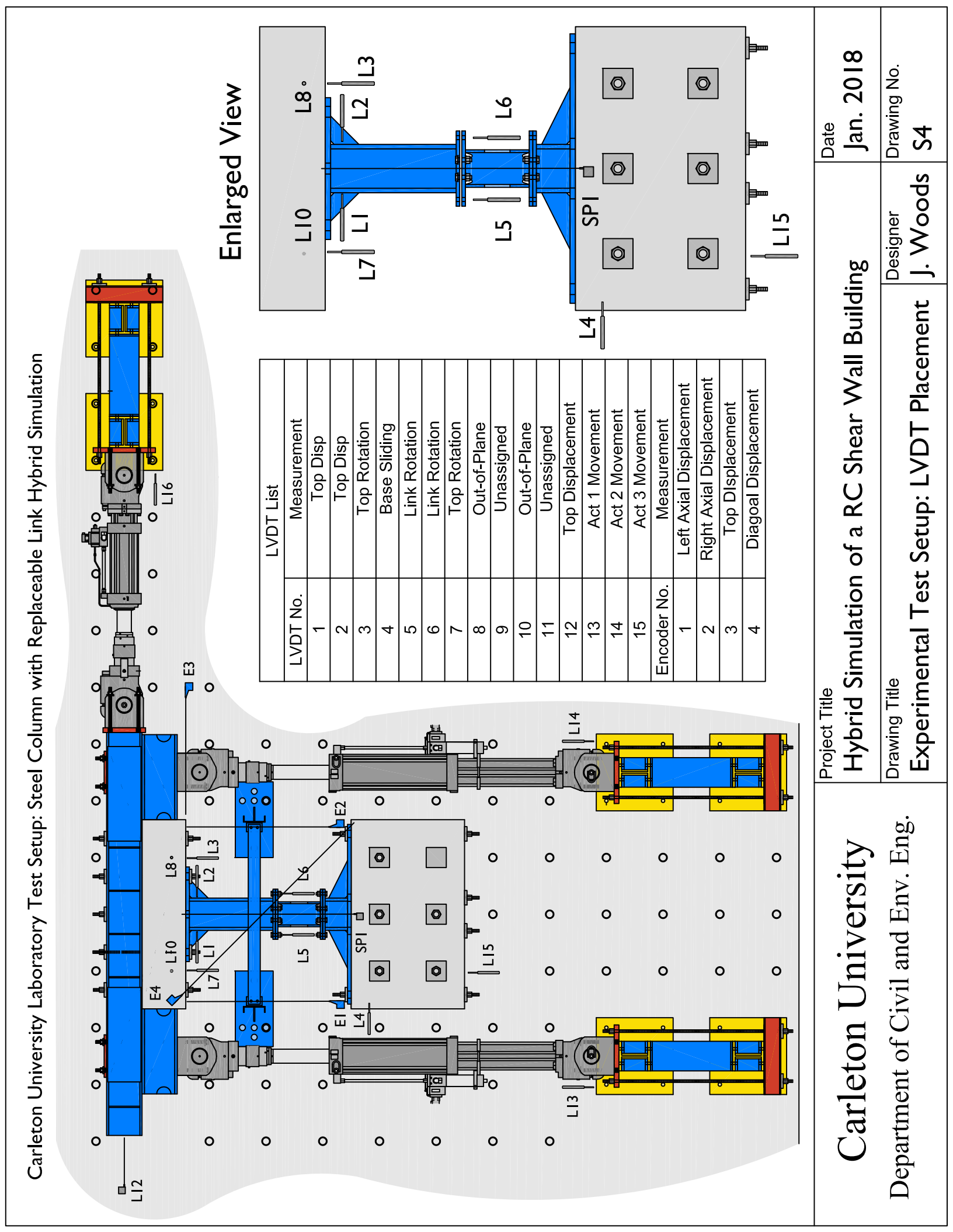




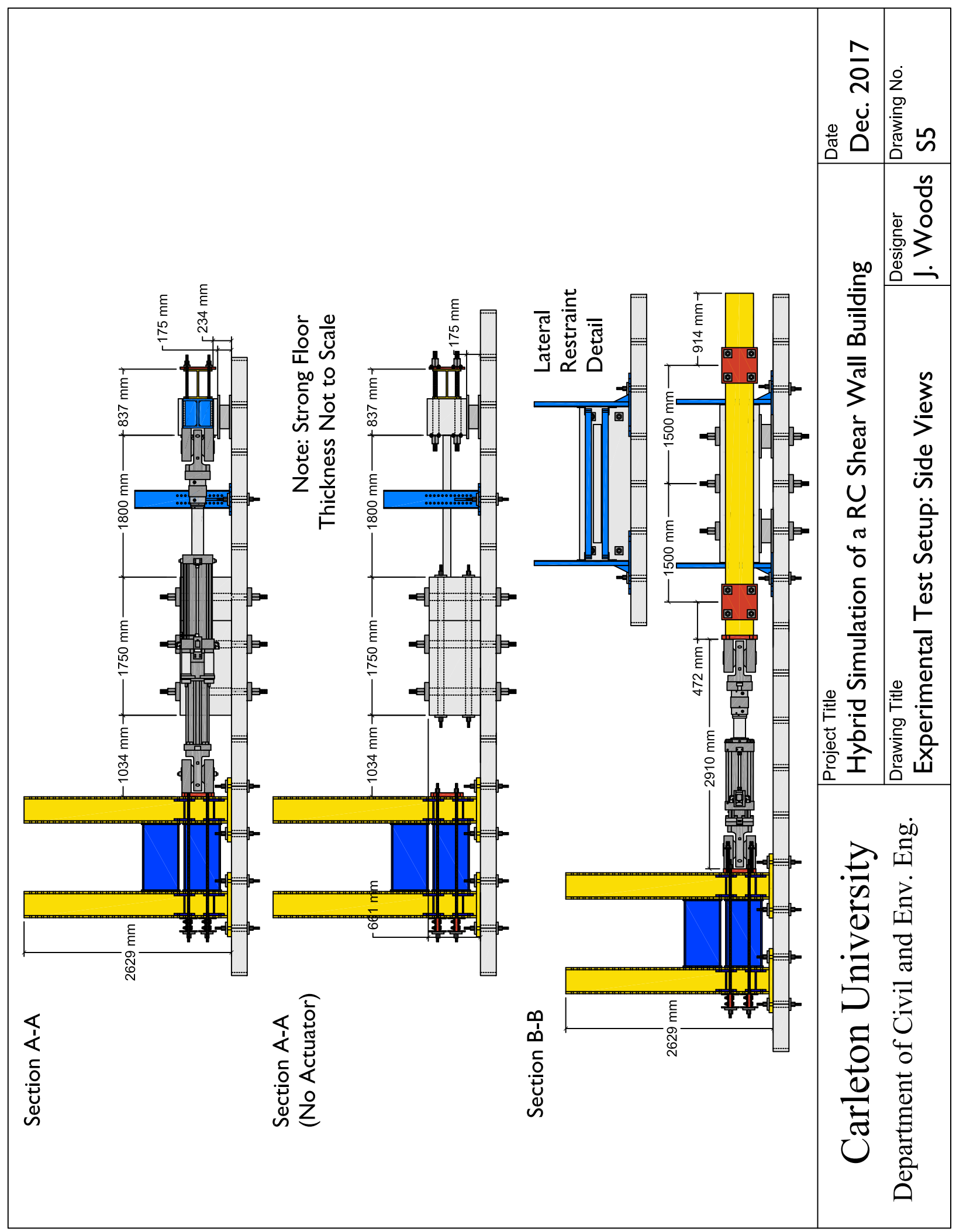




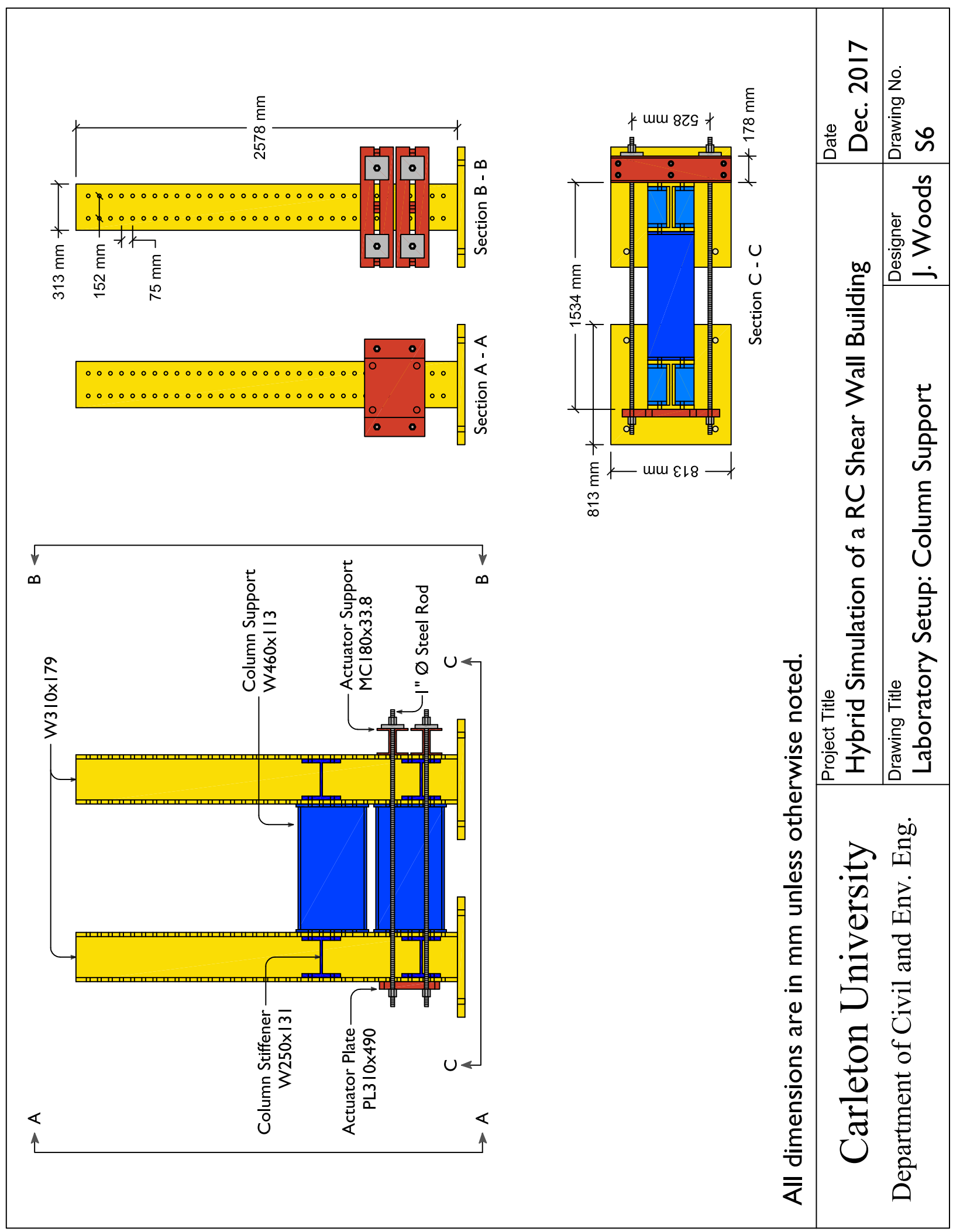

Joshua Woods, Department of Civil and Environmental Engineering, Carleton University 


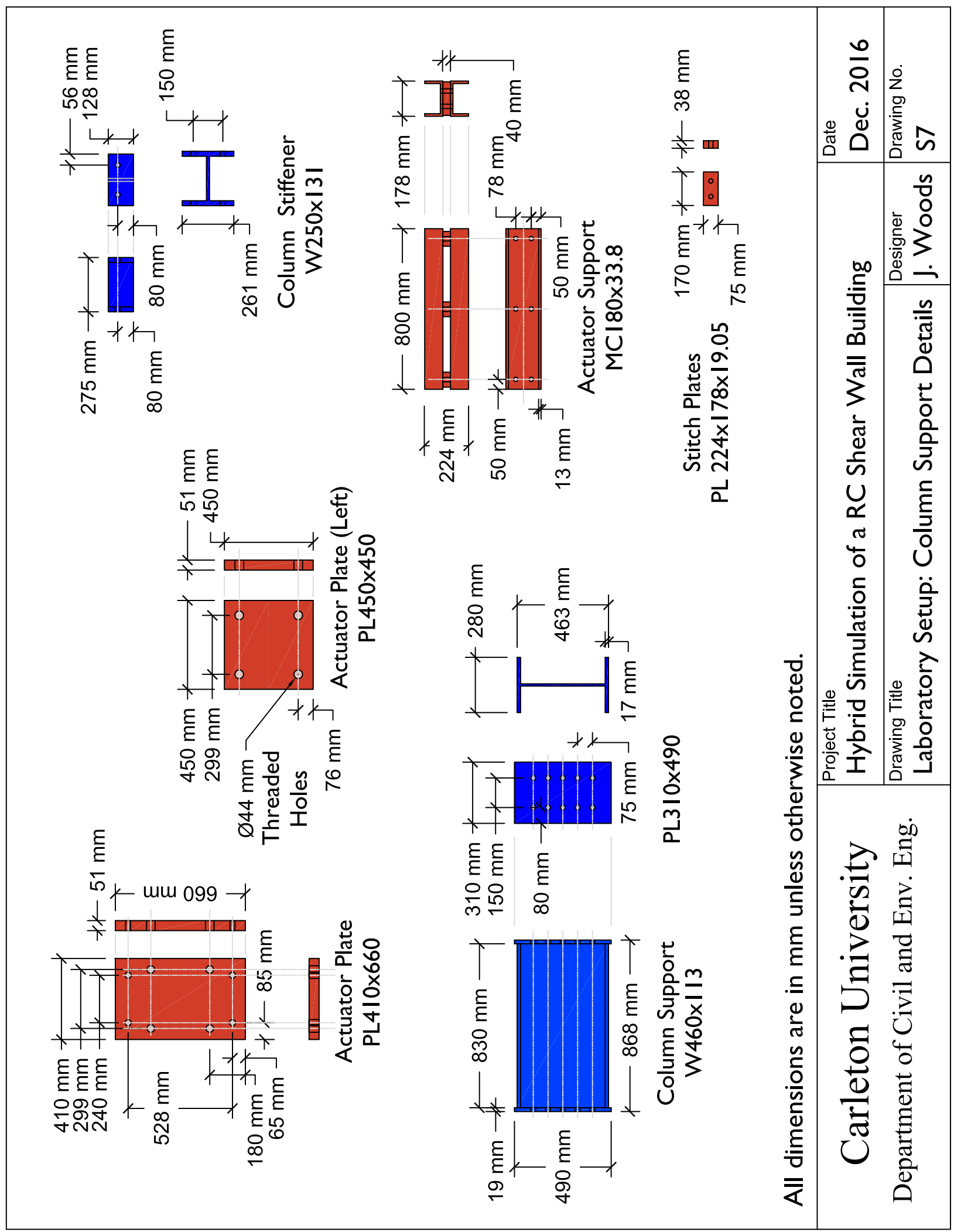




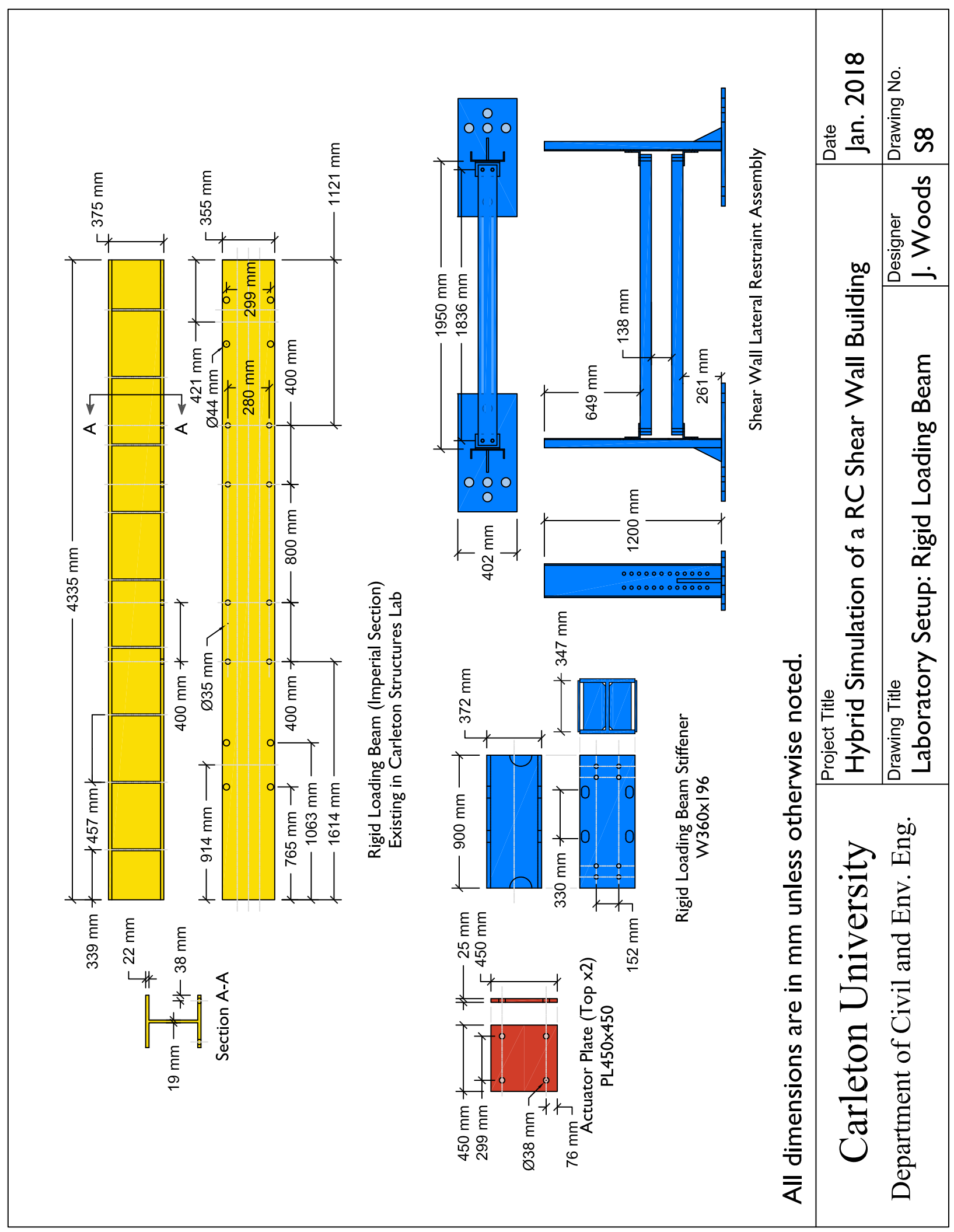




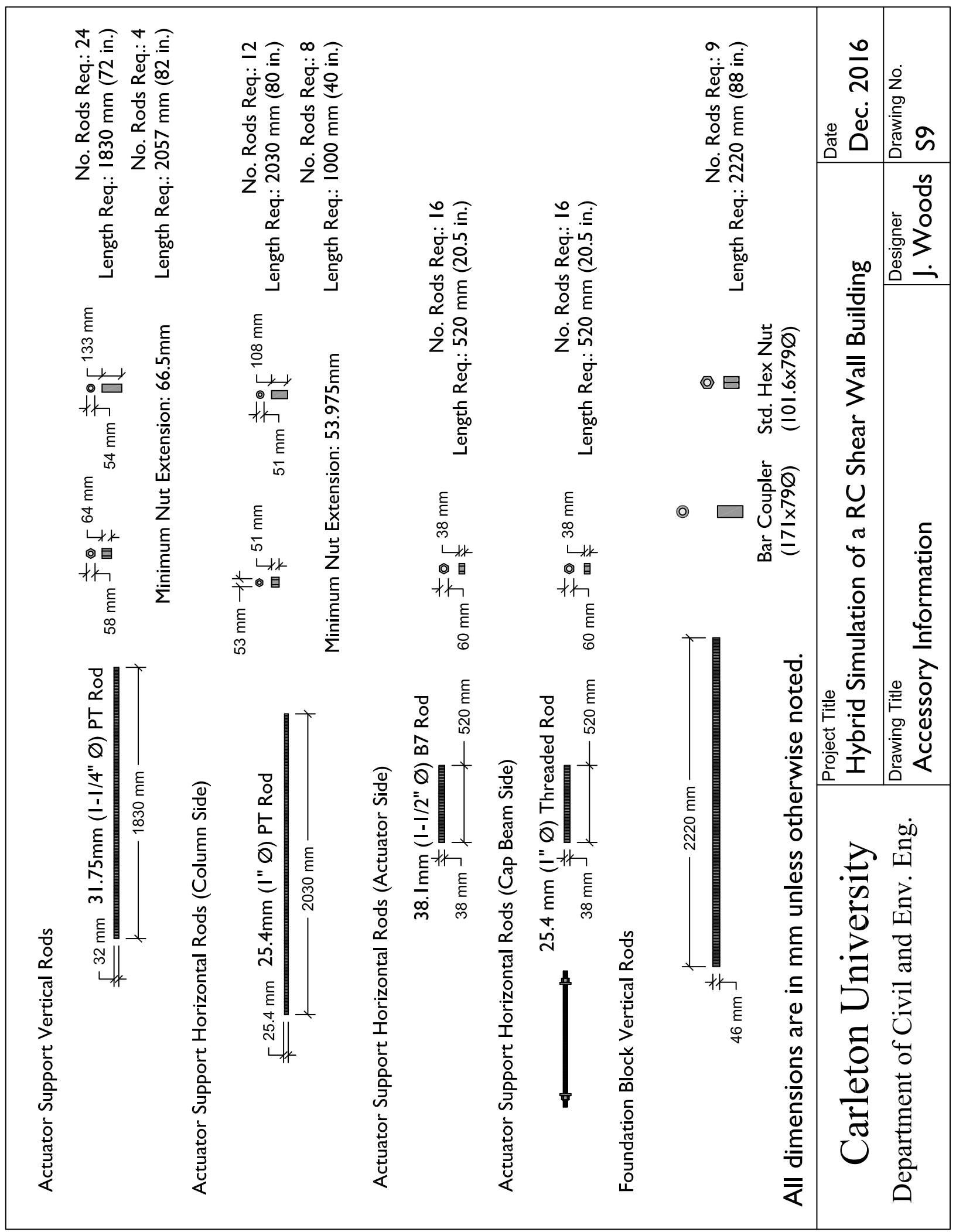




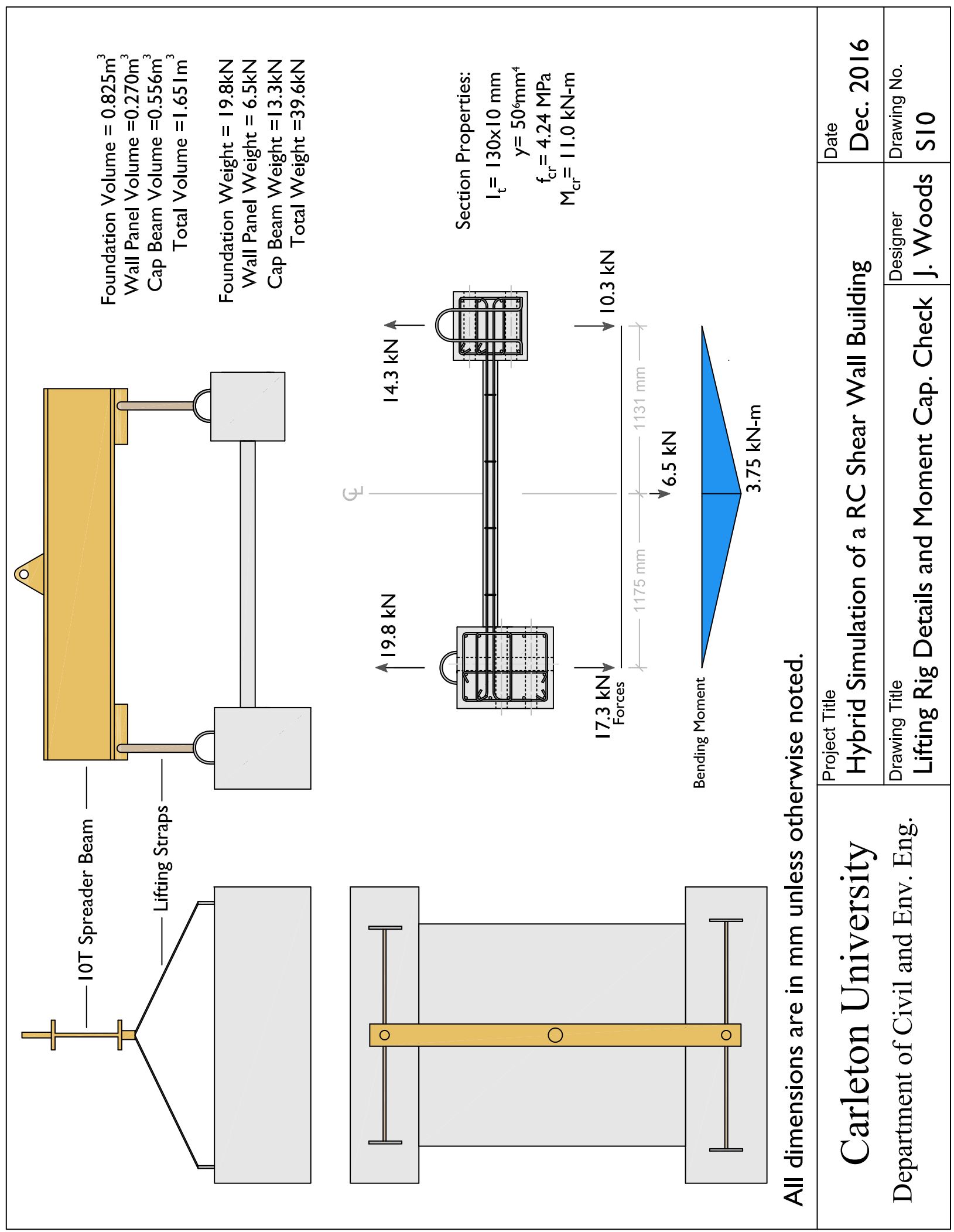

Joshua Woods, Department of Civil and Environmental Engineering, Carleton University 


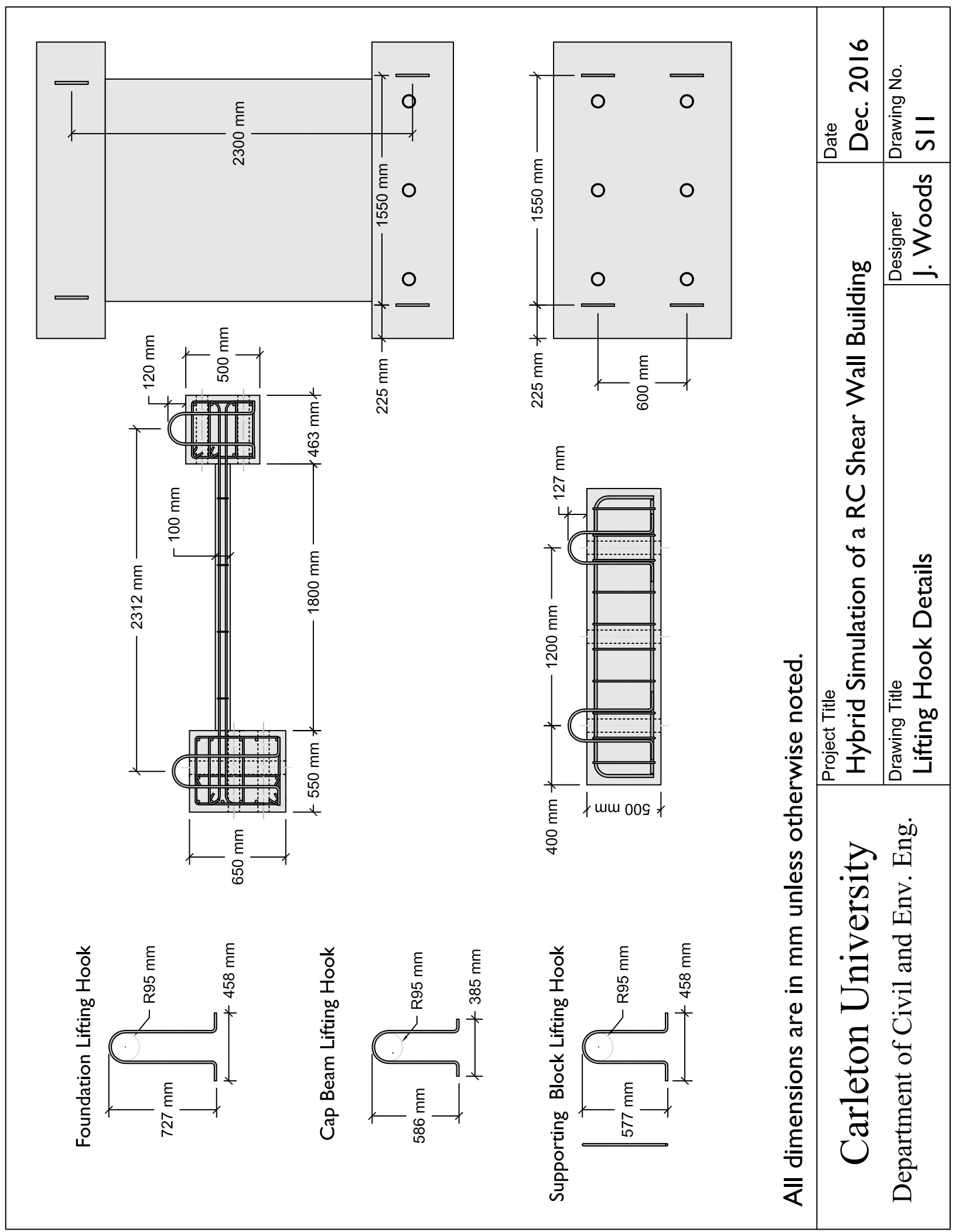




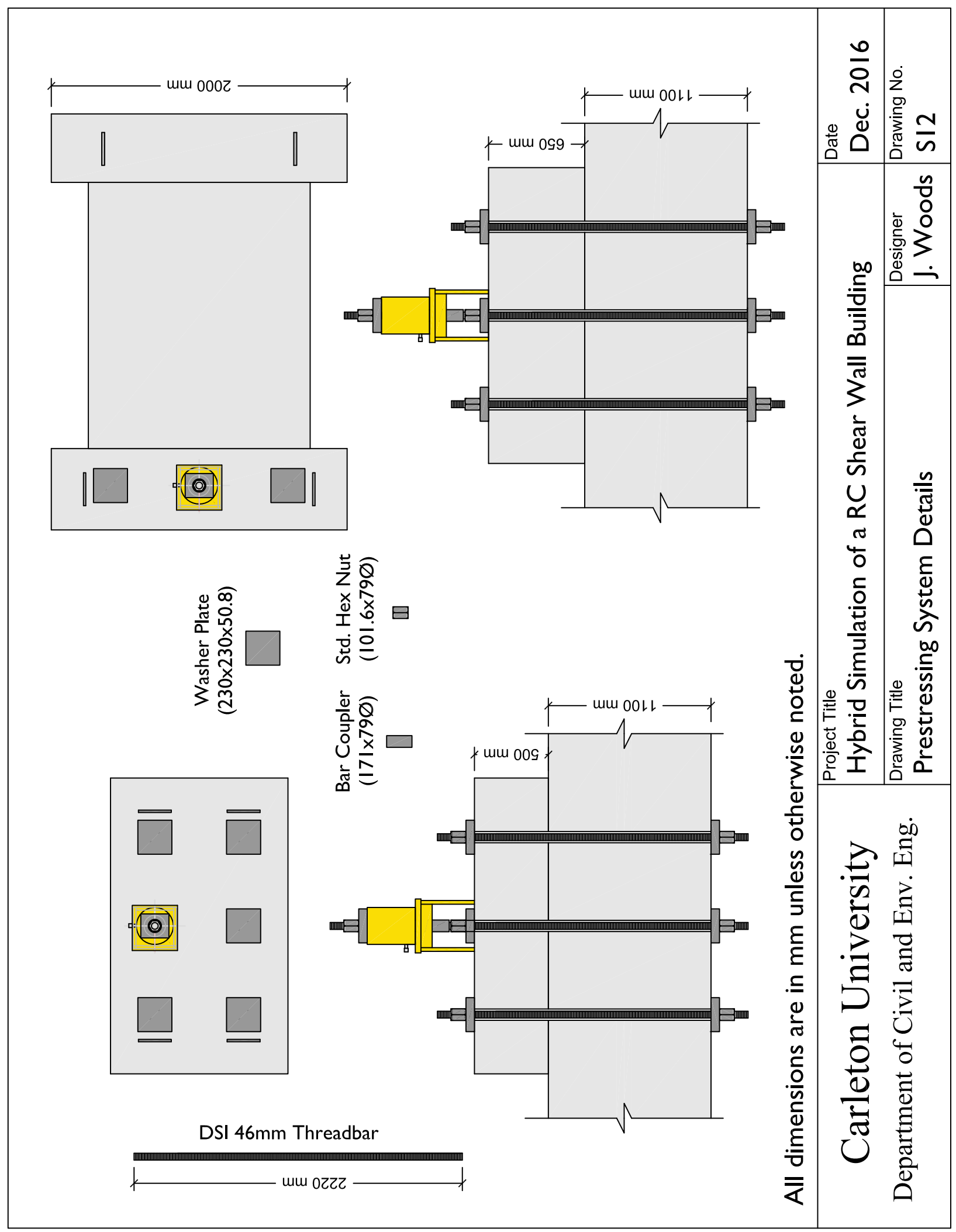




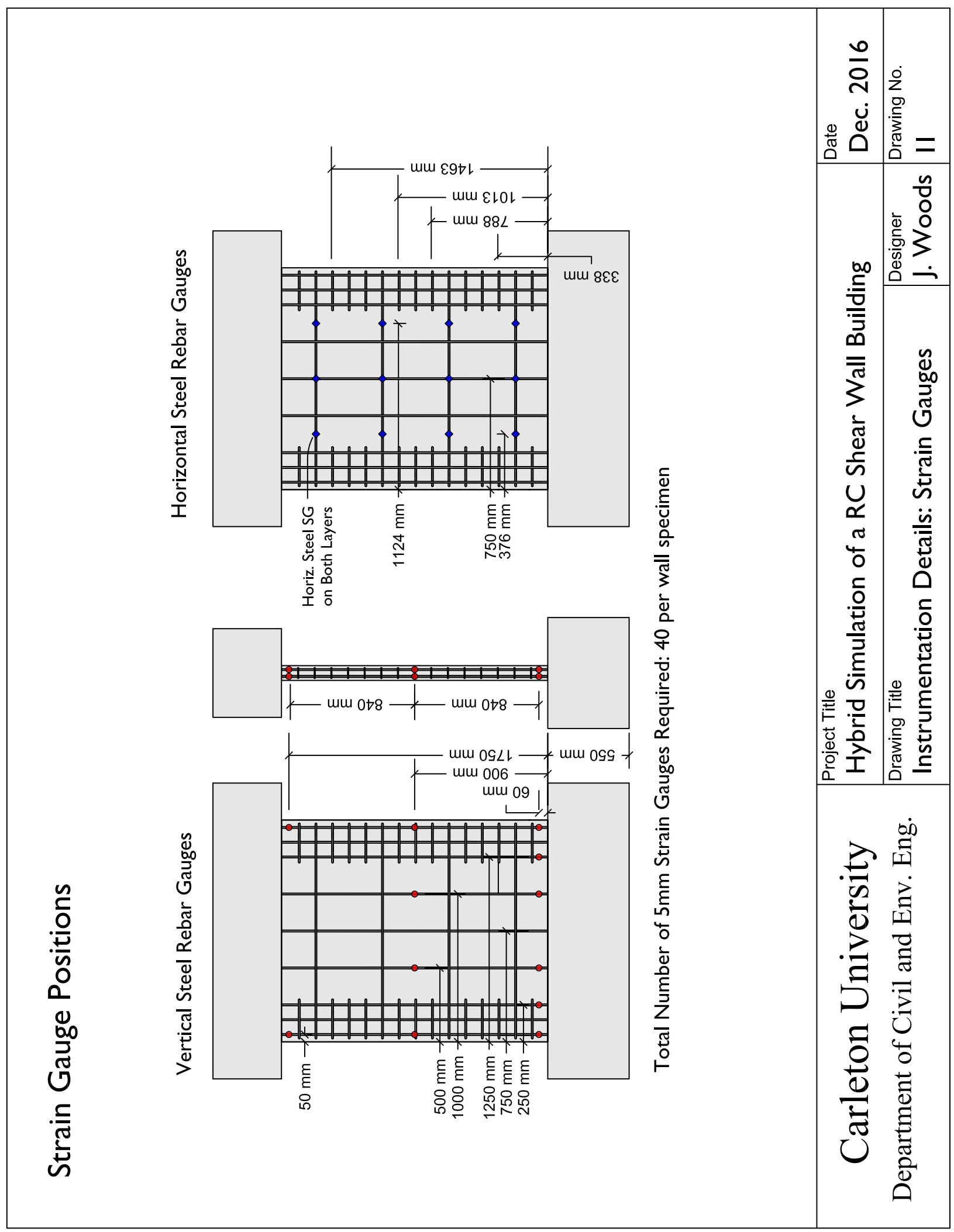




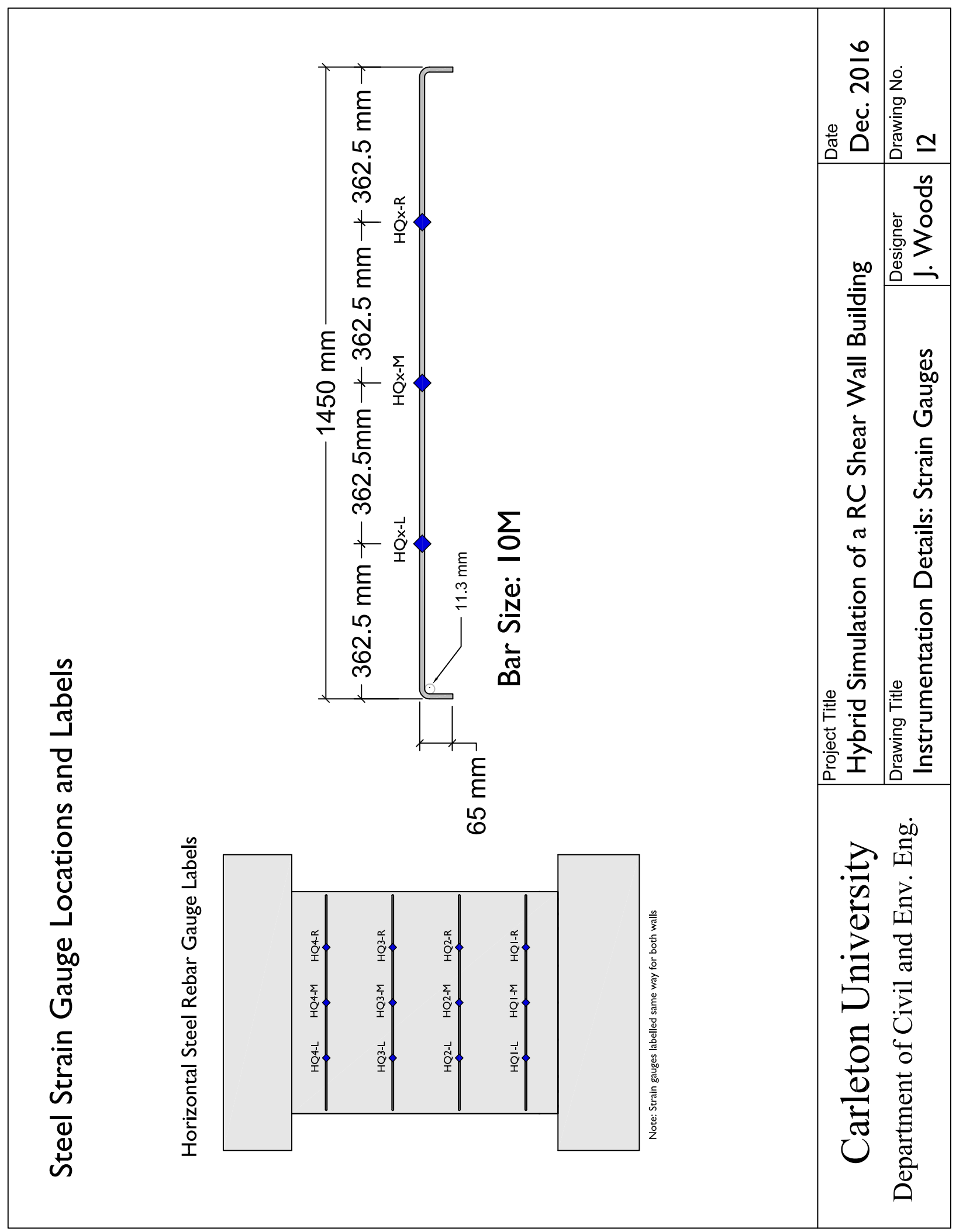

Joshua Woods, Department of Civil and Environmental Engineering, Carleton University 


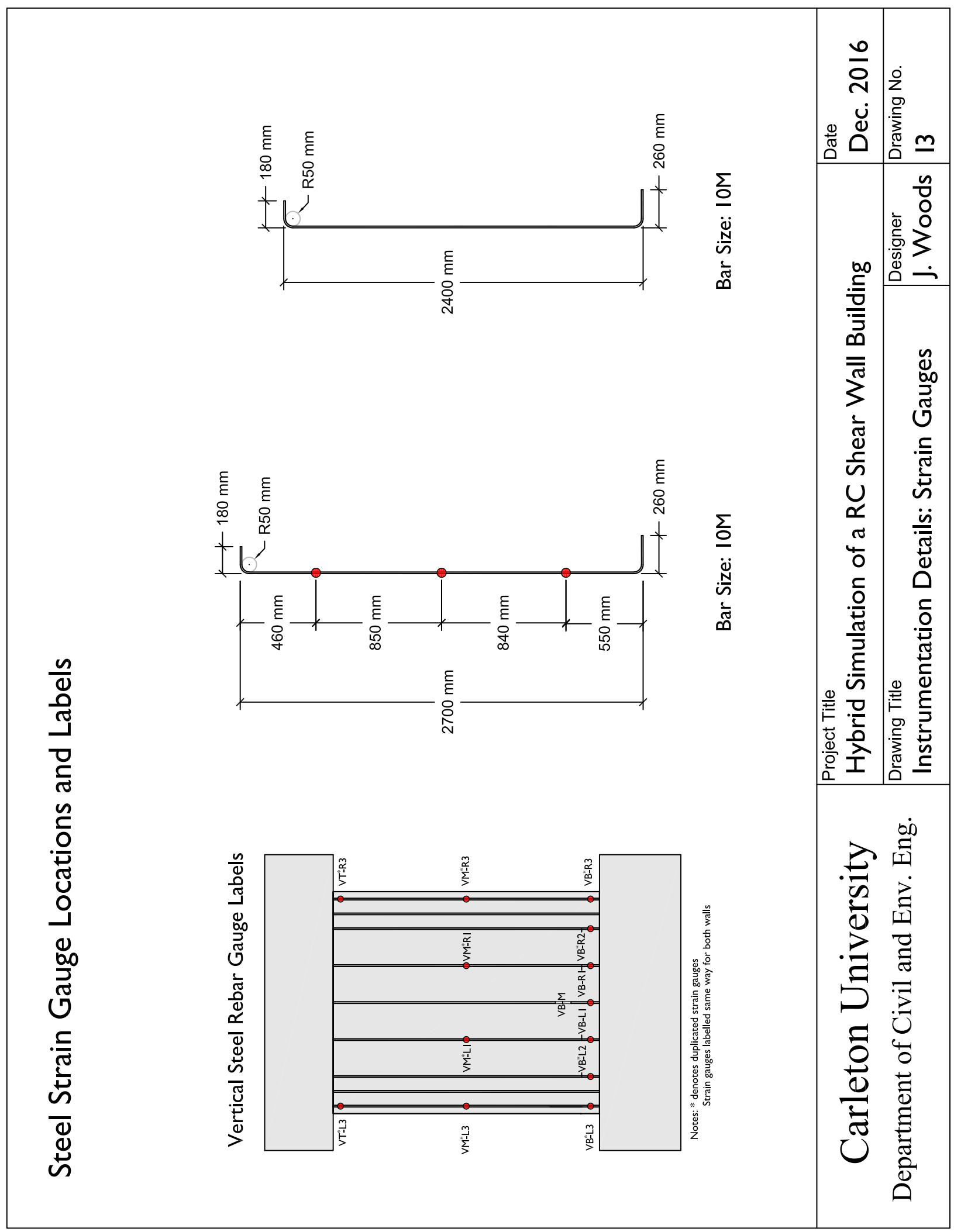




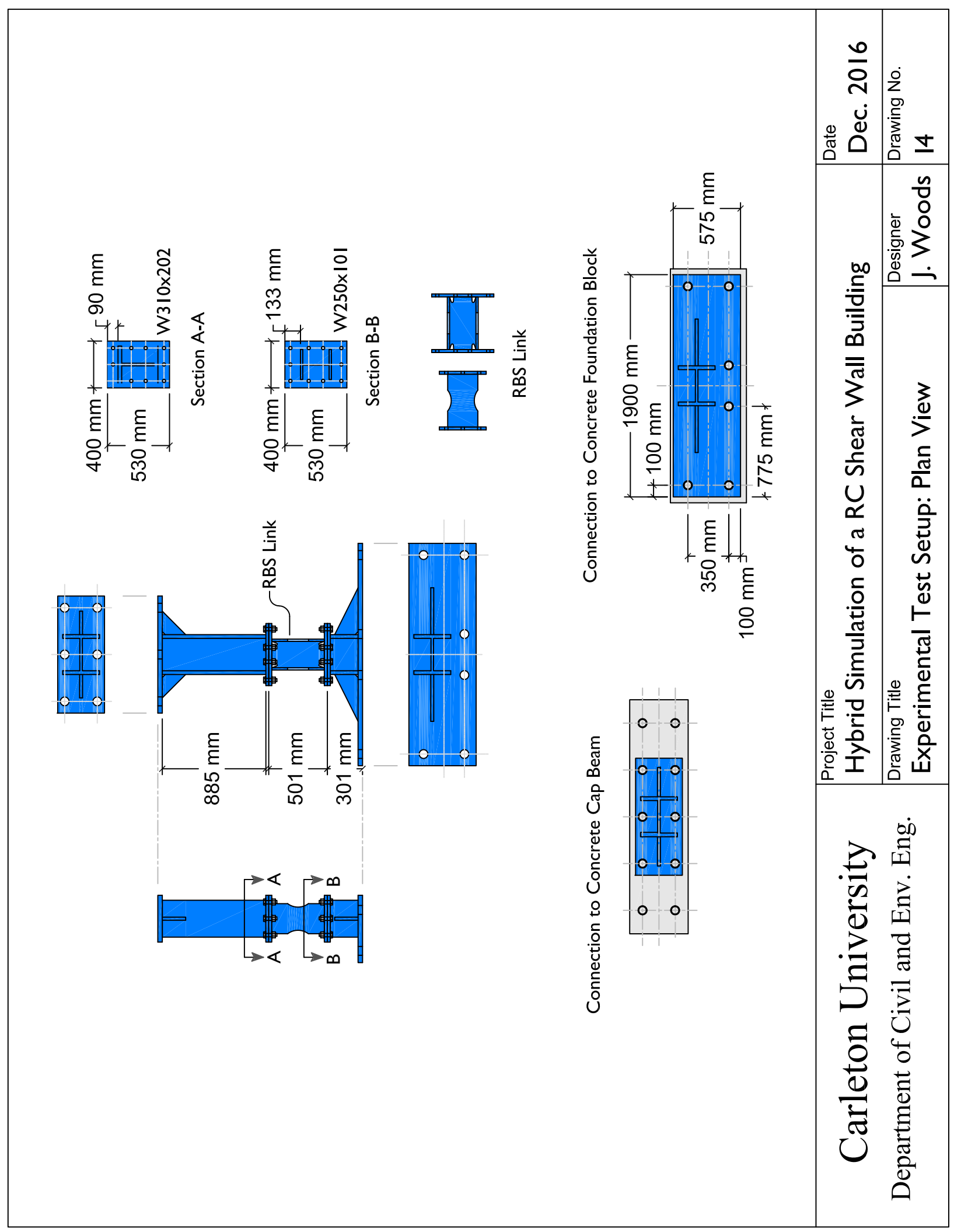




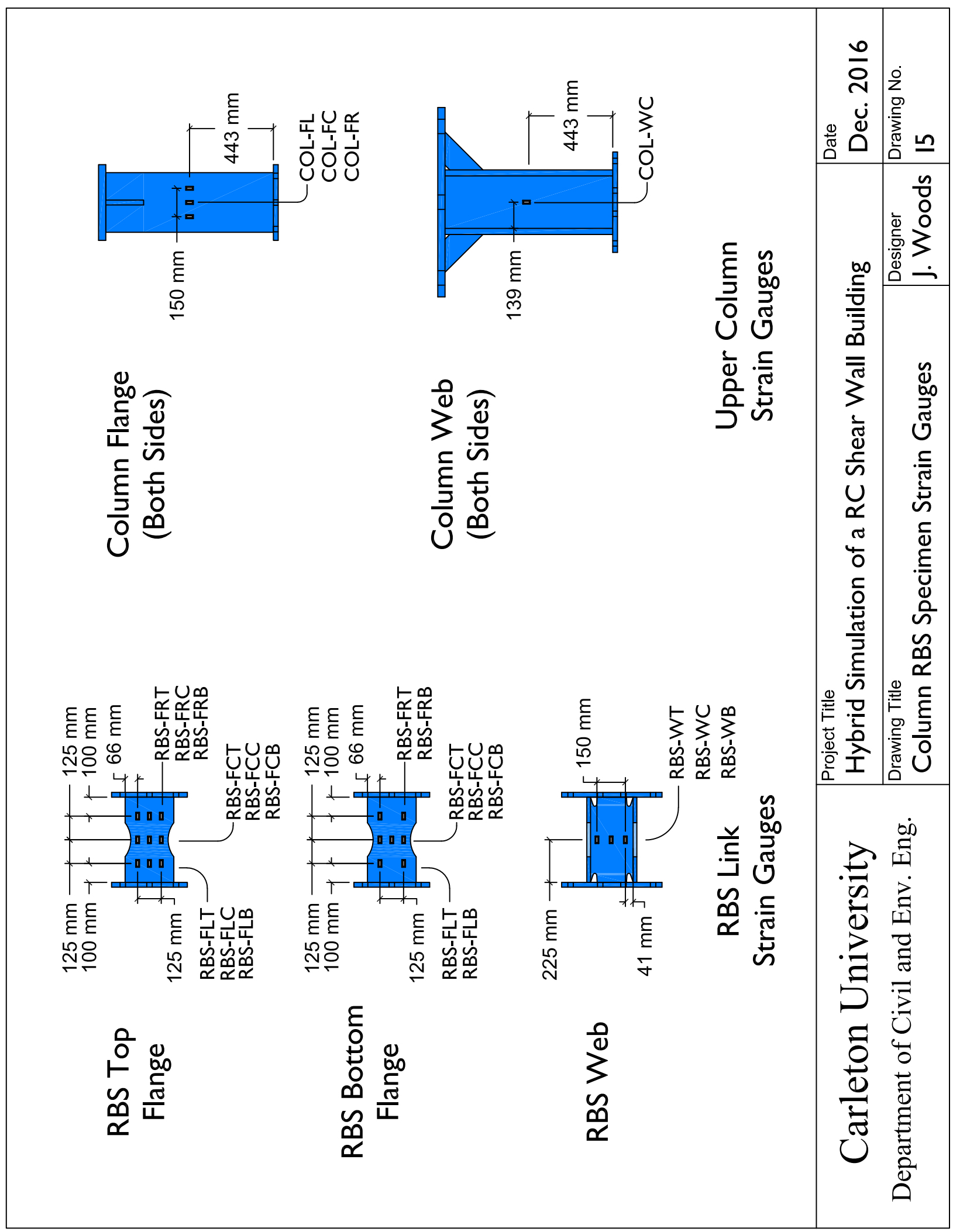



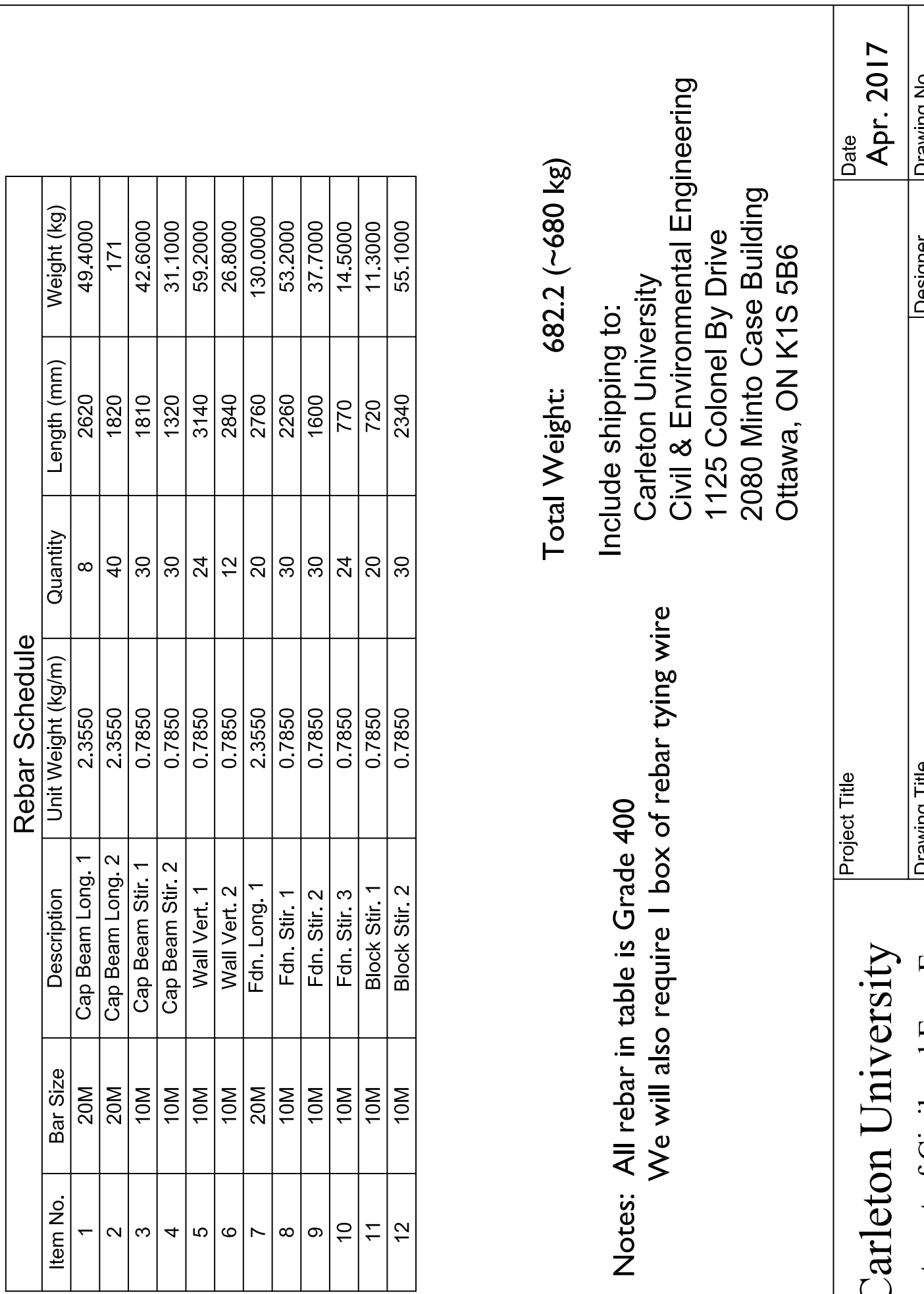

๑ँ
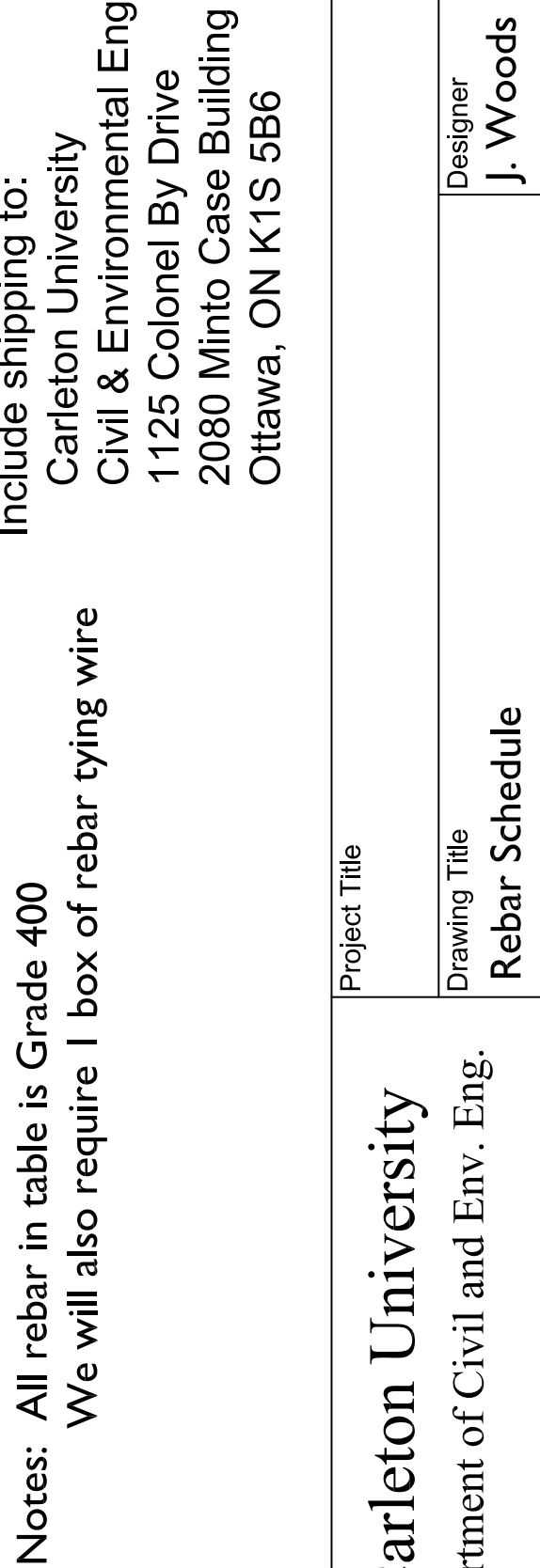


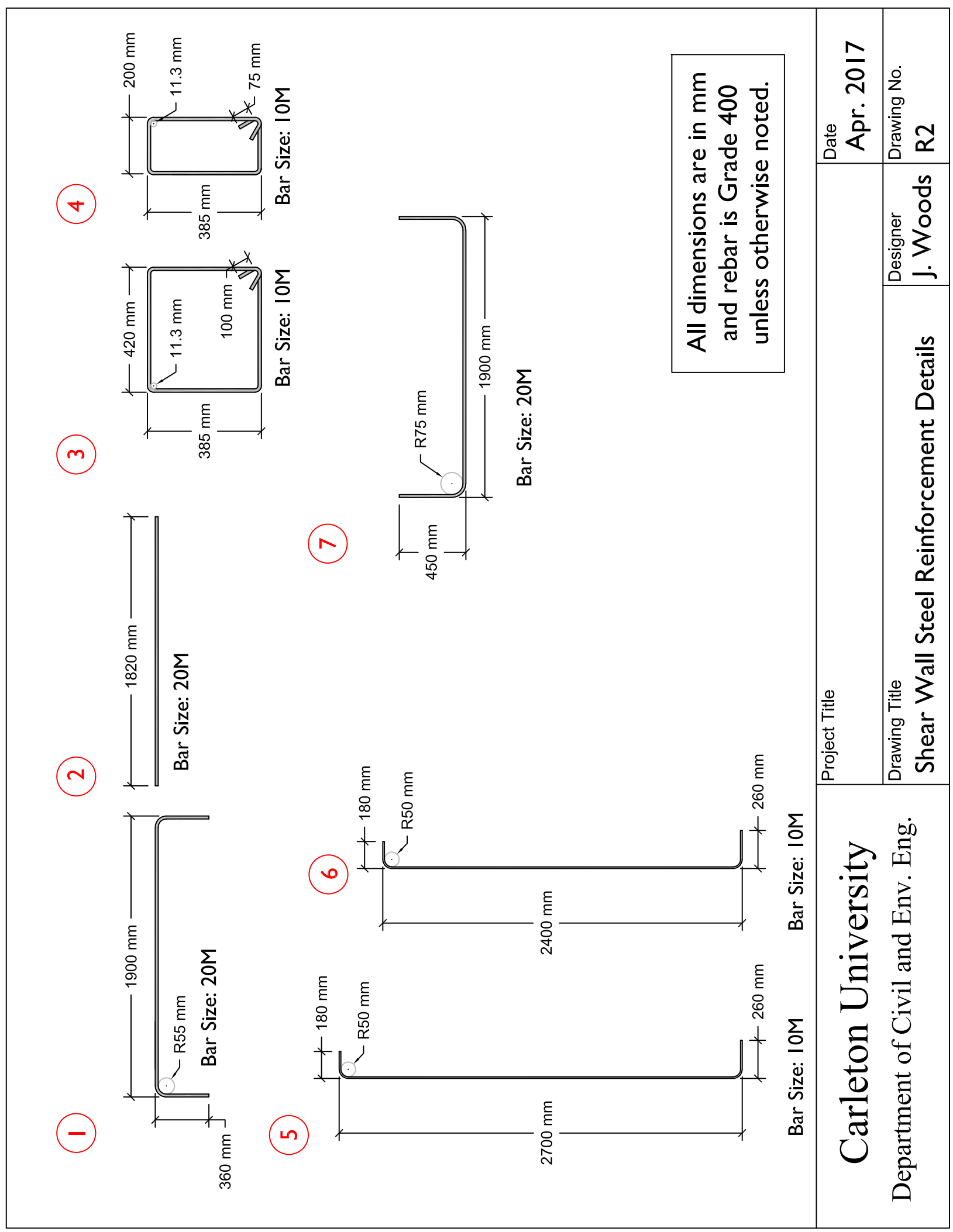




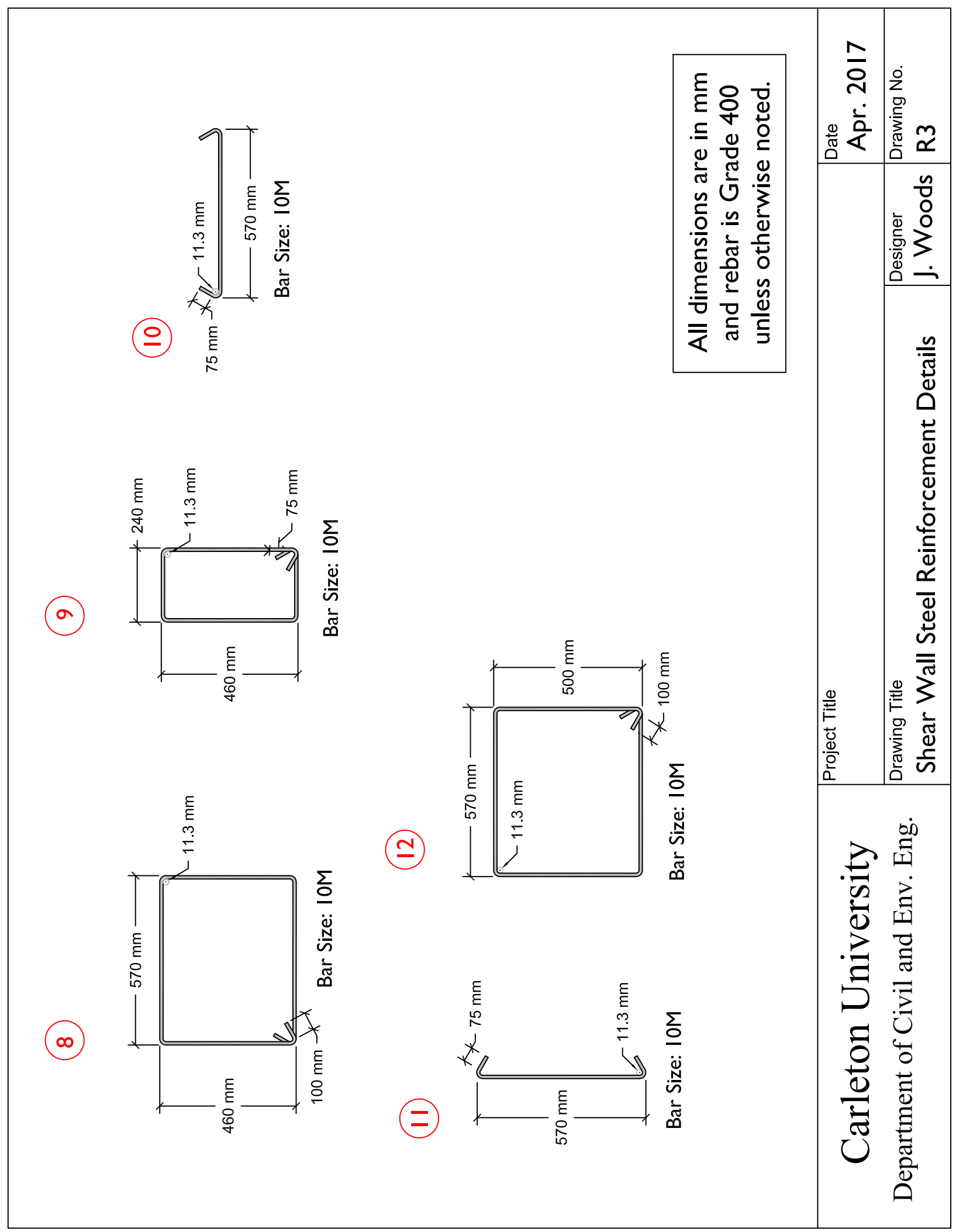




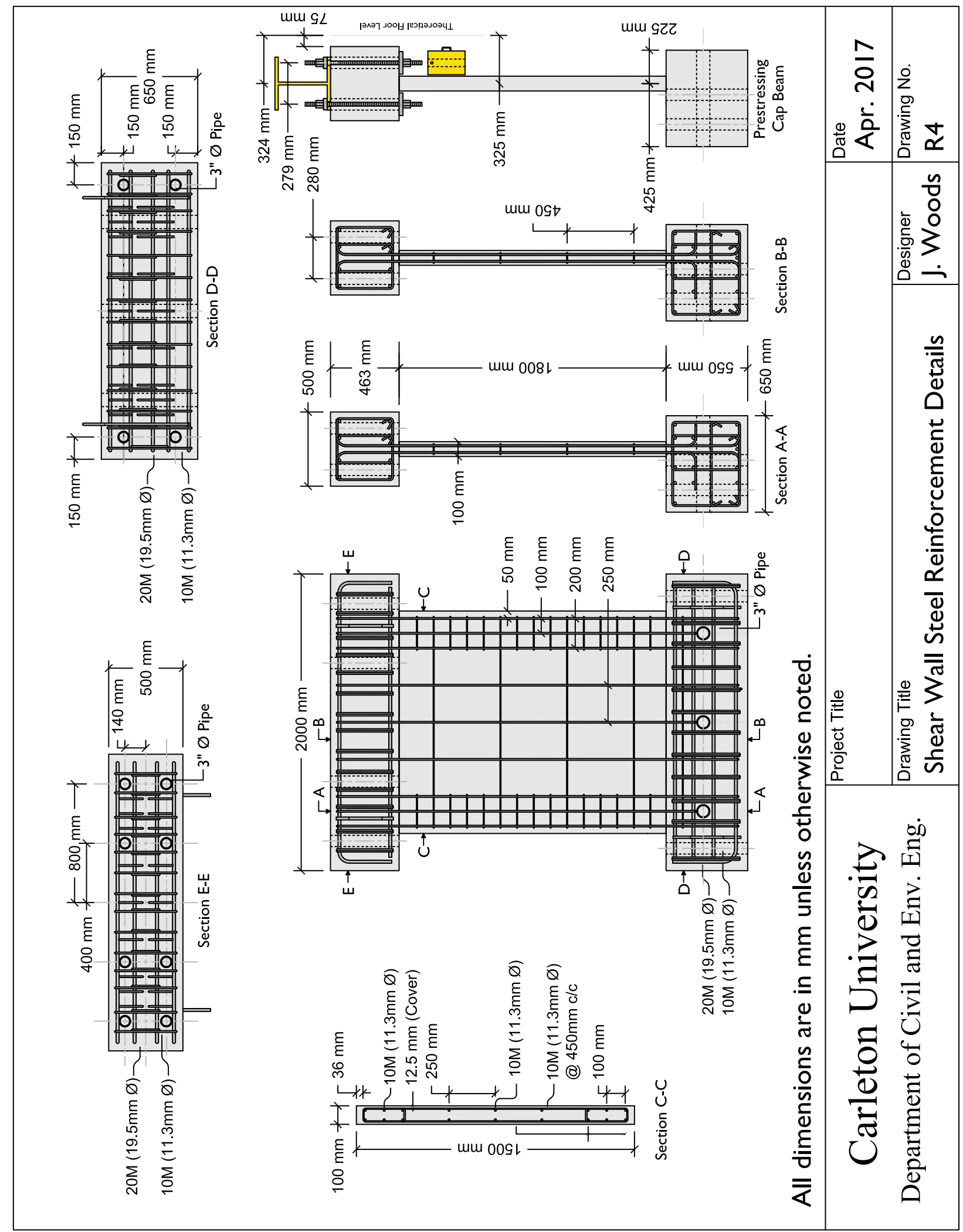




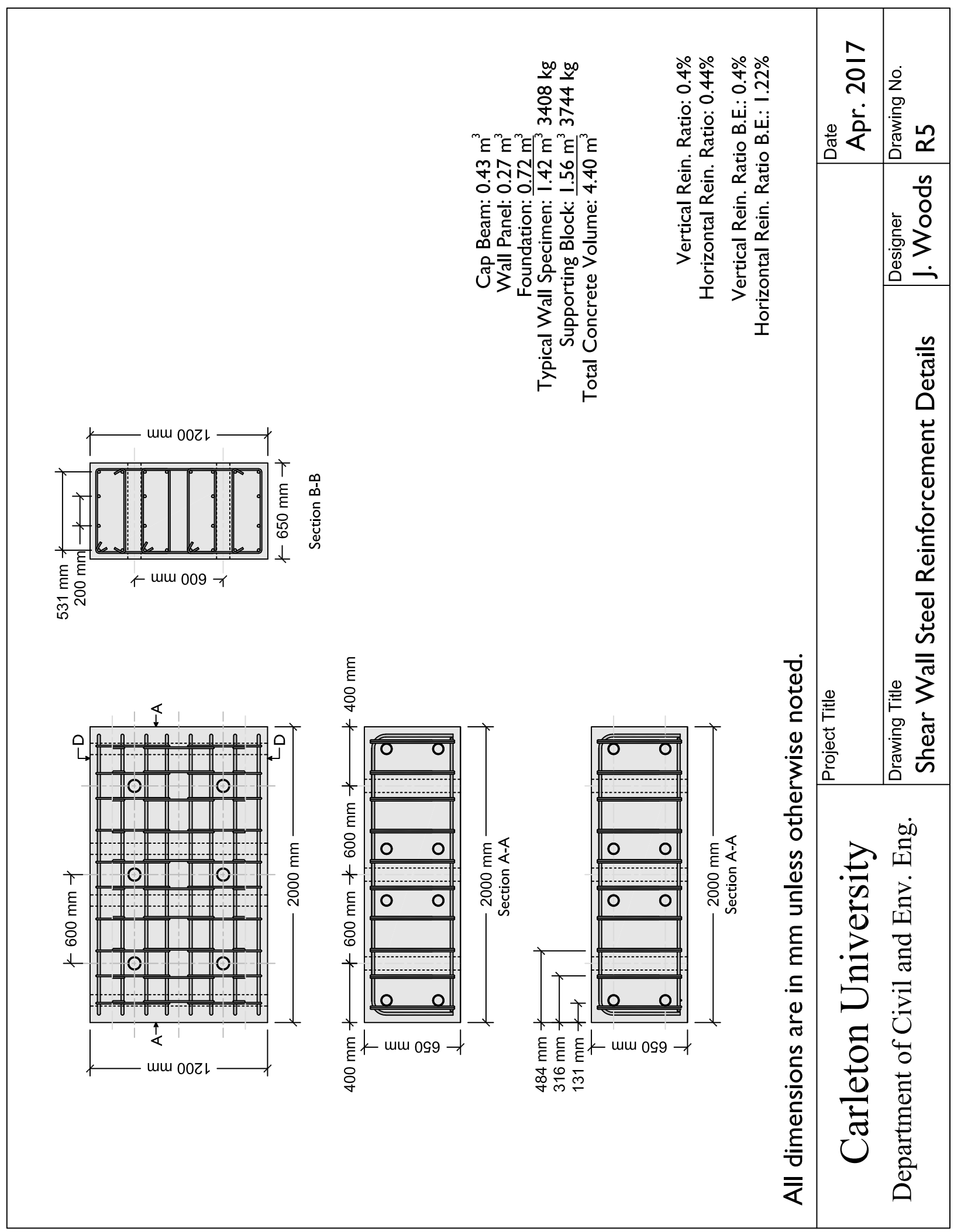




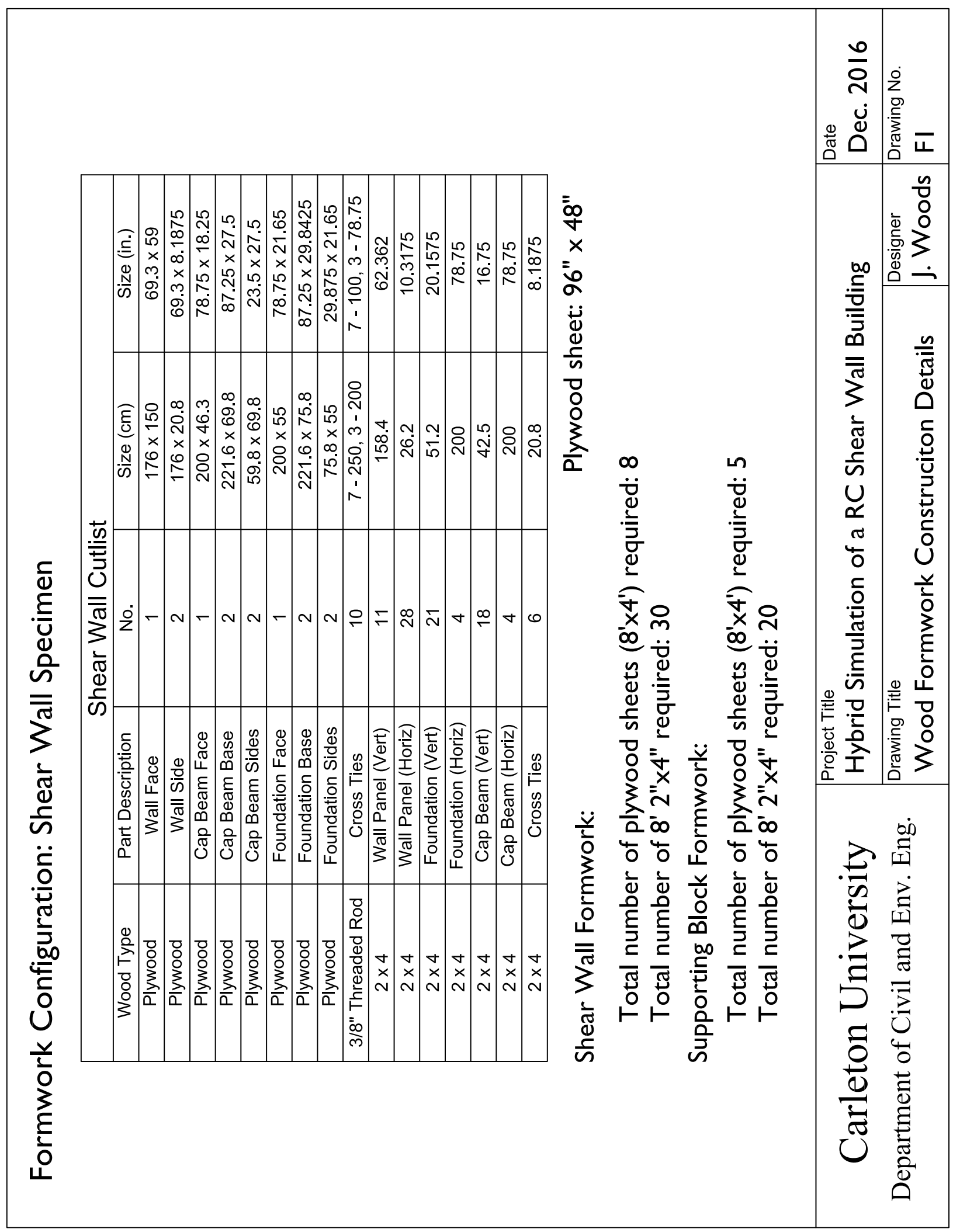




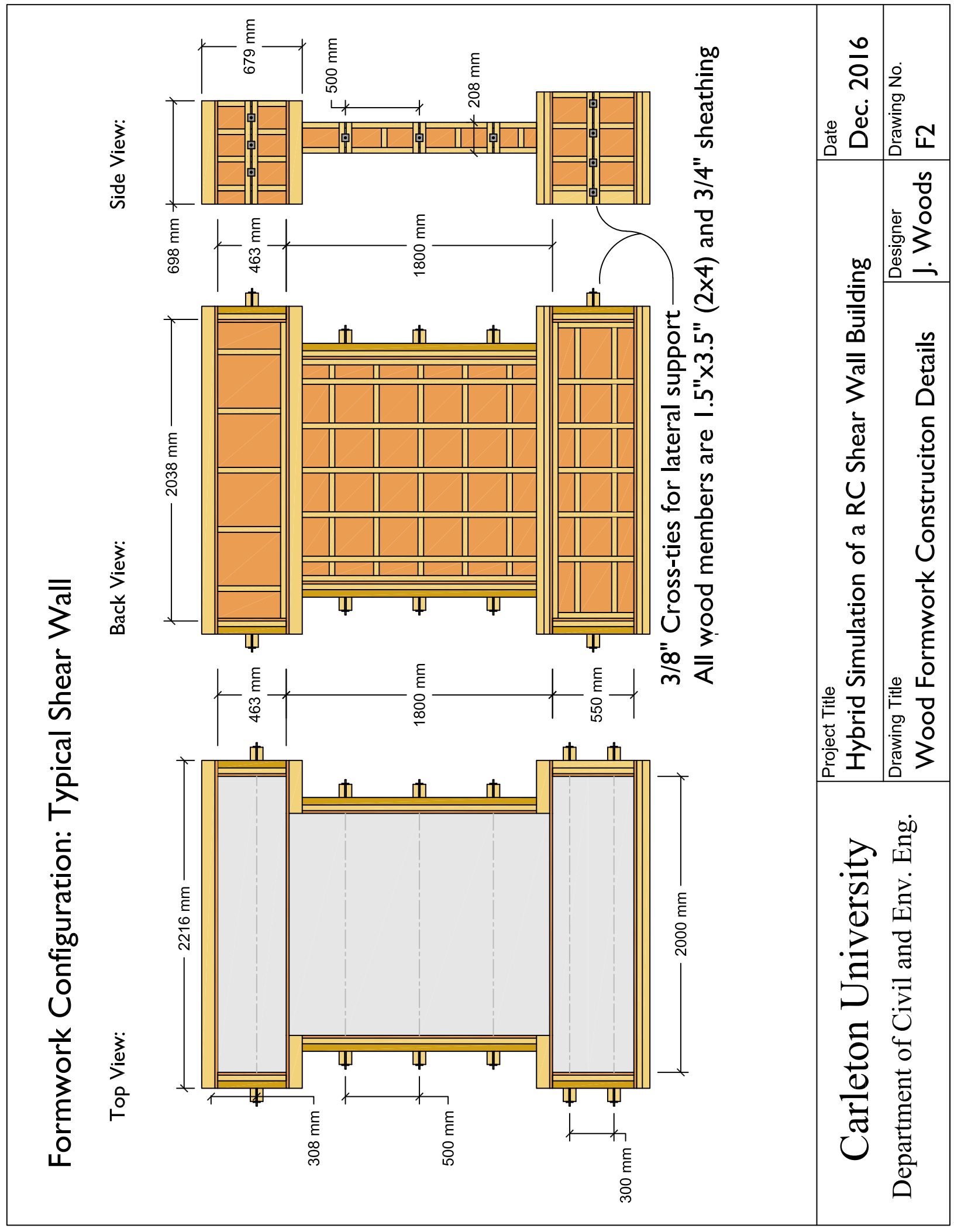




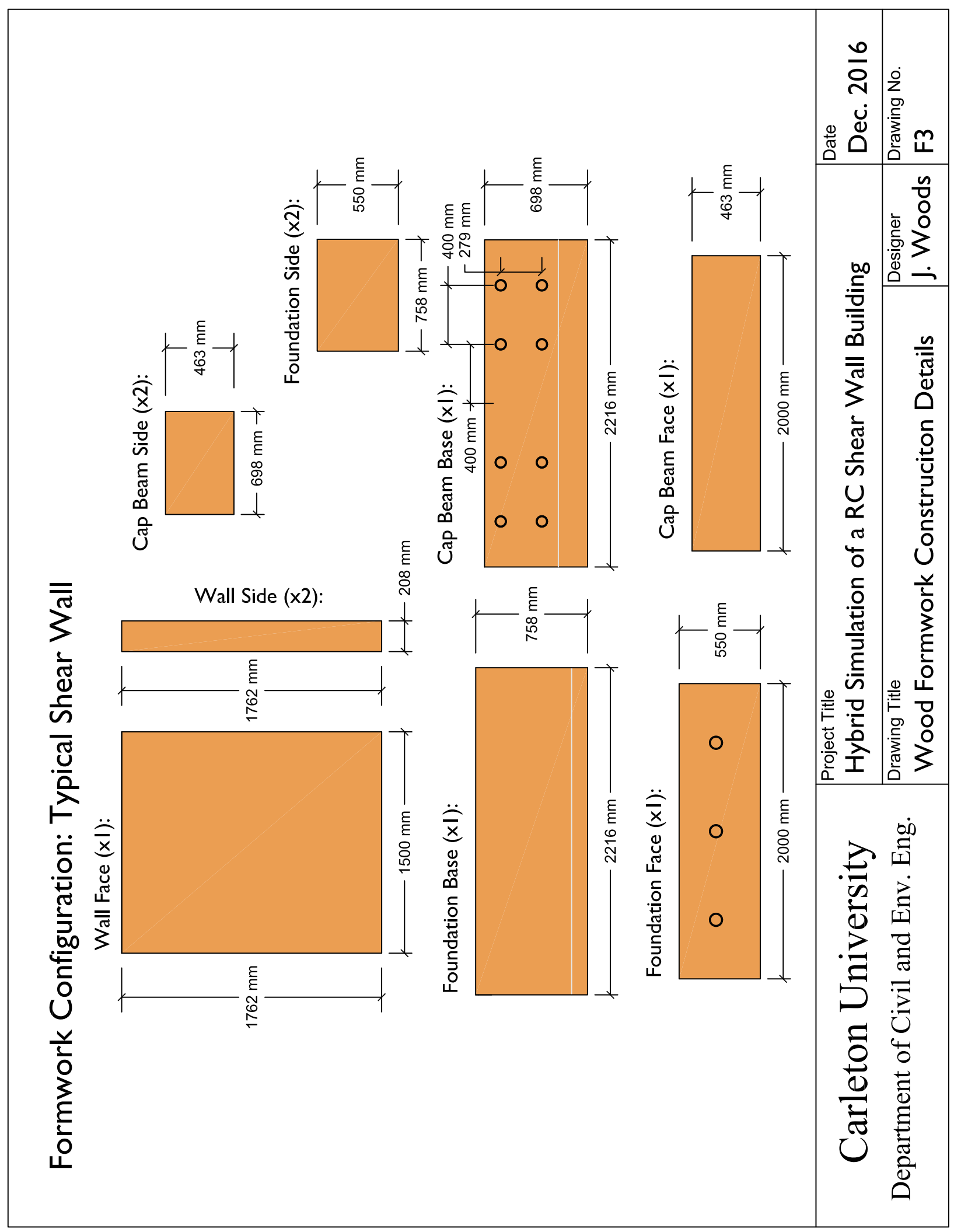




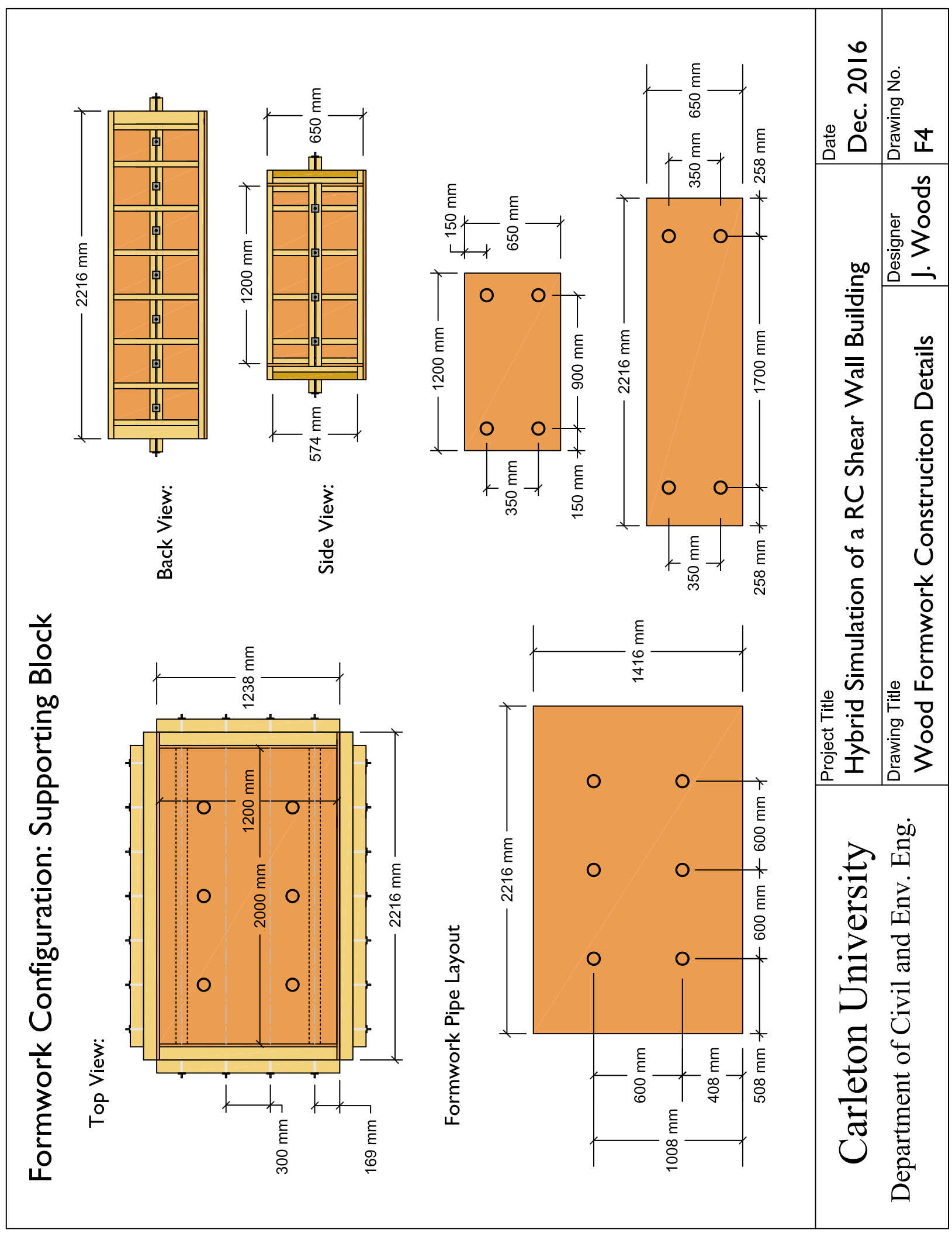




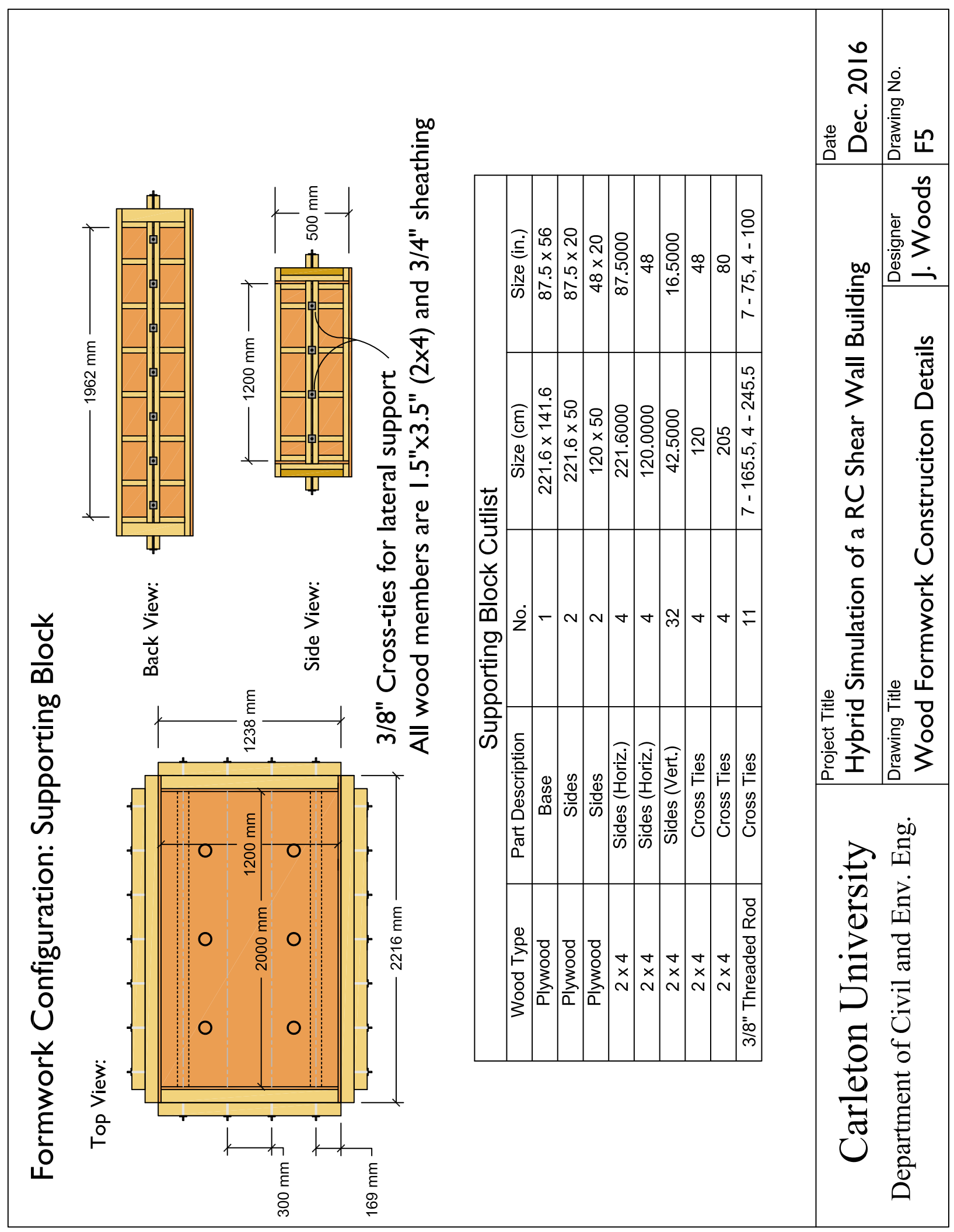




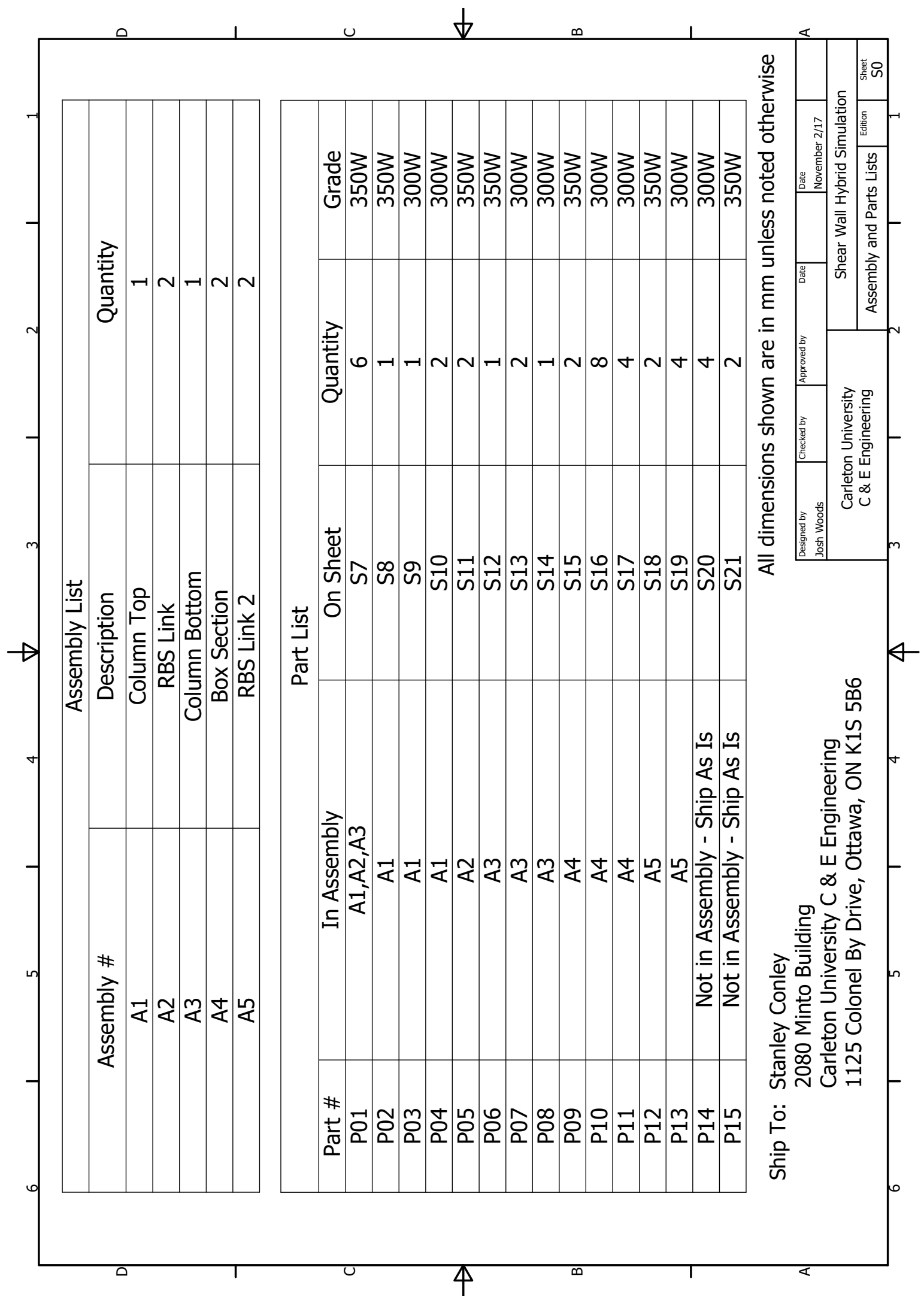

Joshua Woods, Department of Civil and Environmental Engineering, Carleton University 


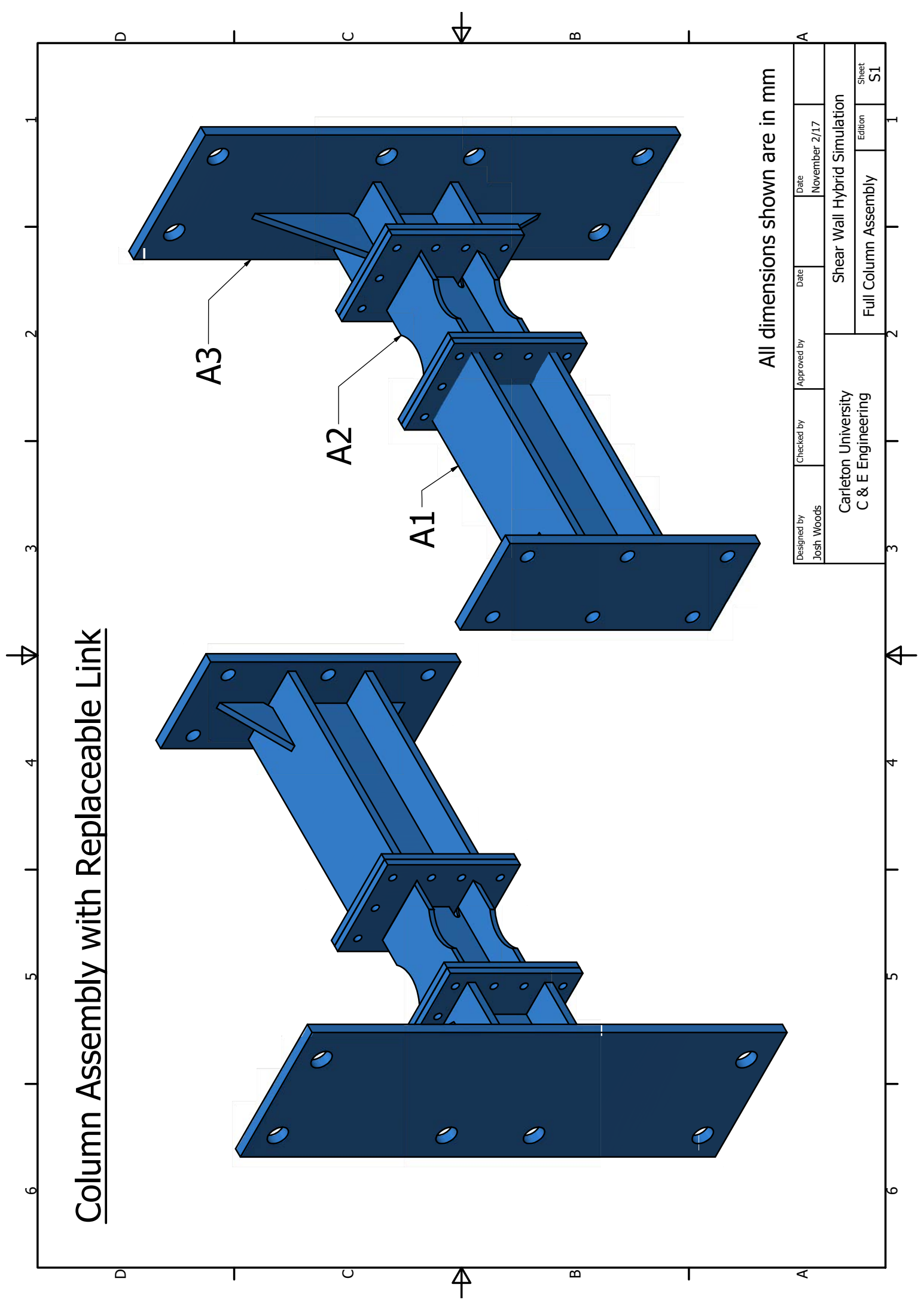

Joshua Woods, Department of Civil and Environmental Engineering, Carleton University 


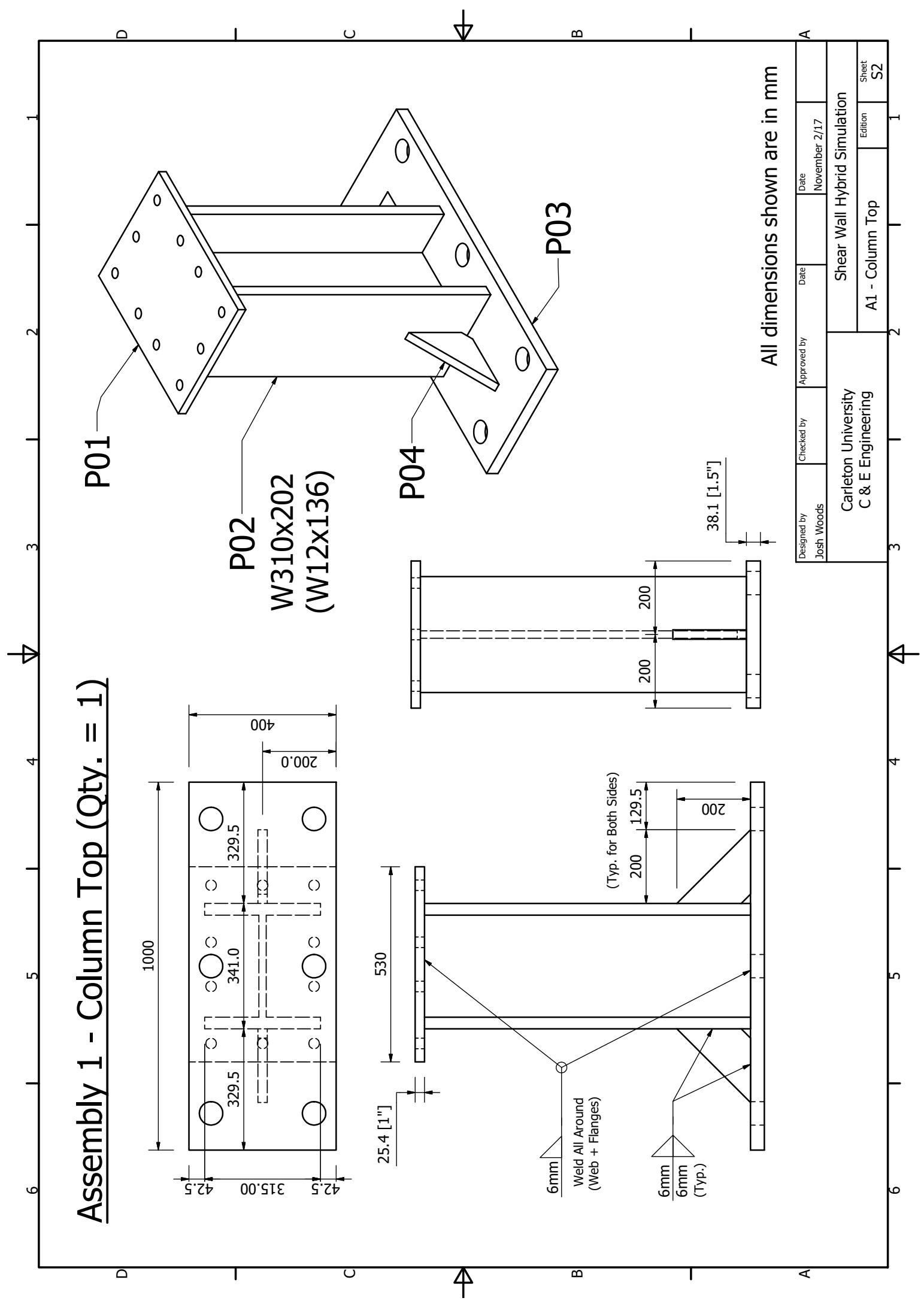

Joshua Woods, Department of Civil and Environmental Engineering, Carleton University 


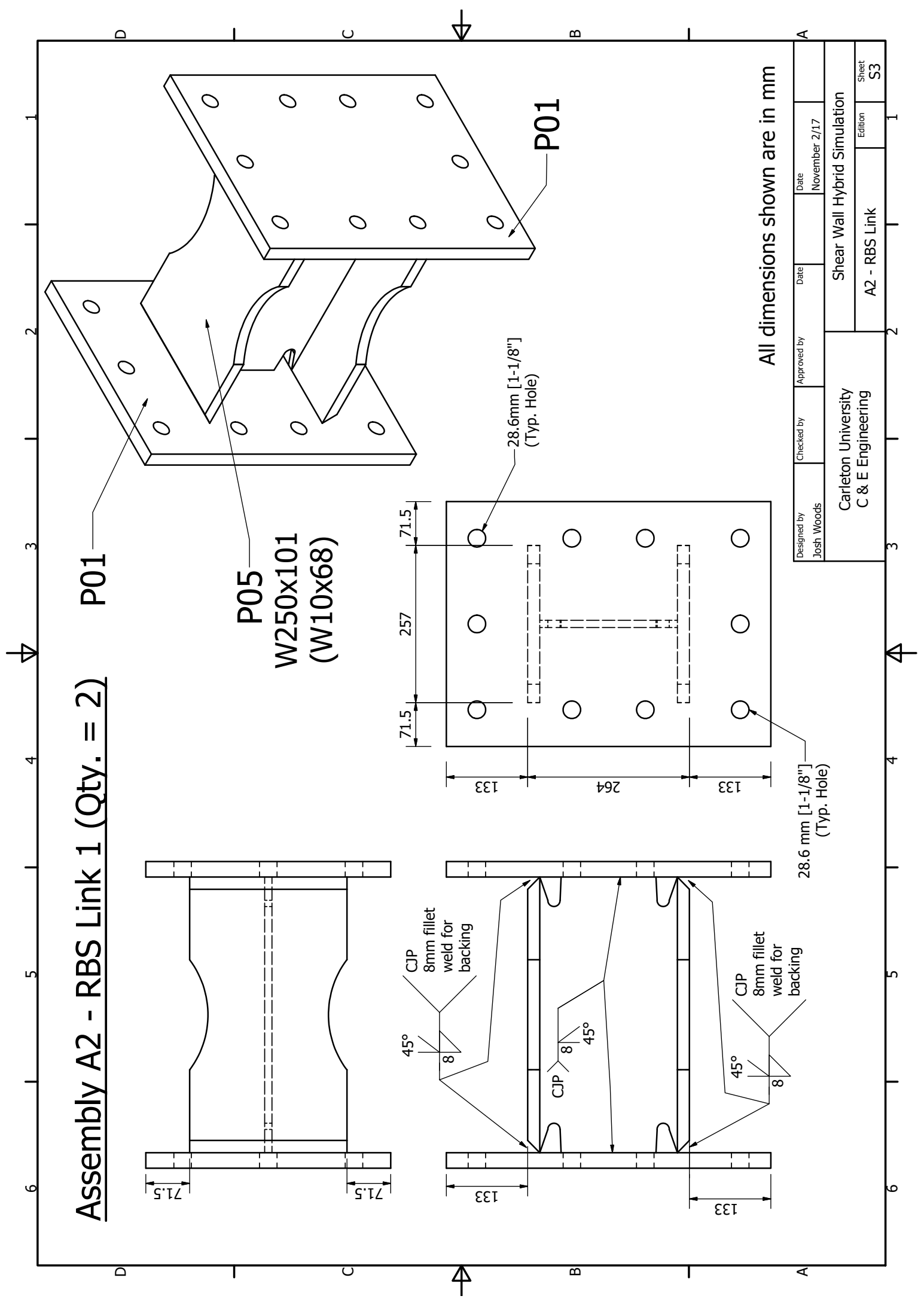

Joshua Woods, Department of Civil and Environmental Engineering, Carleton University 


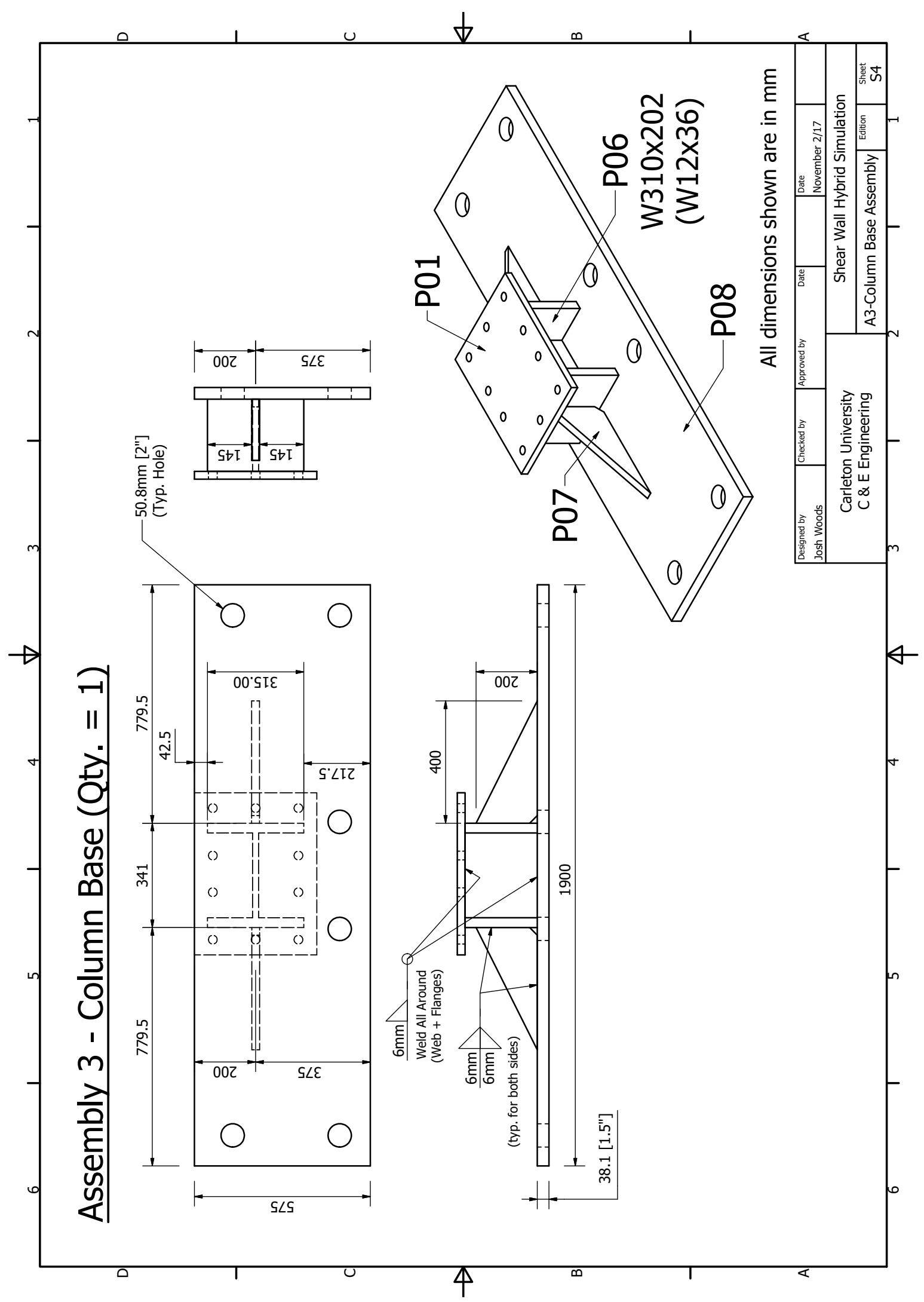

Joshua Woods, Department of Civil and Environmental Engineering, Carleton University 


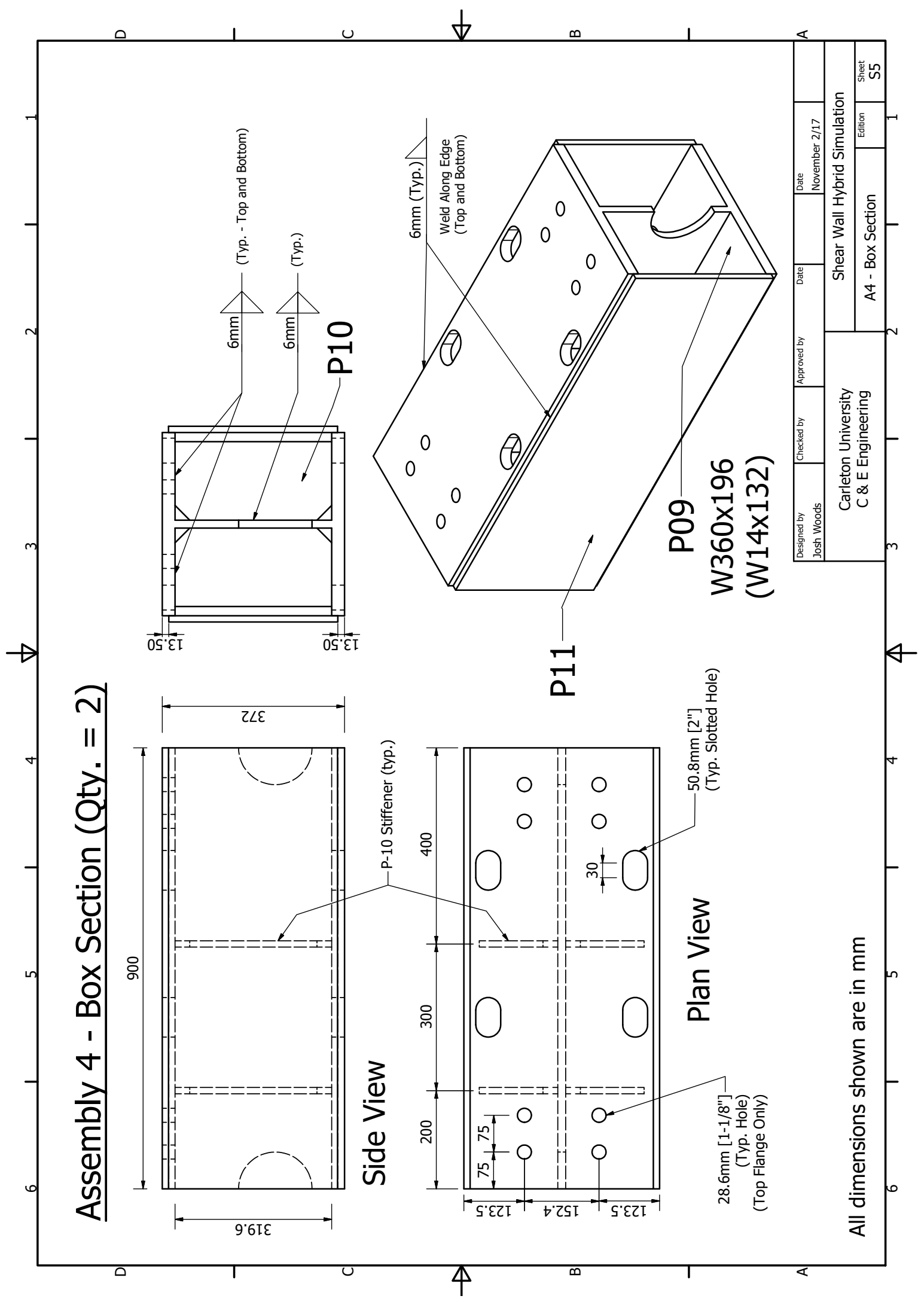

Joshua Woods, Department of Civil and Environmental Engineering, Carleton University 


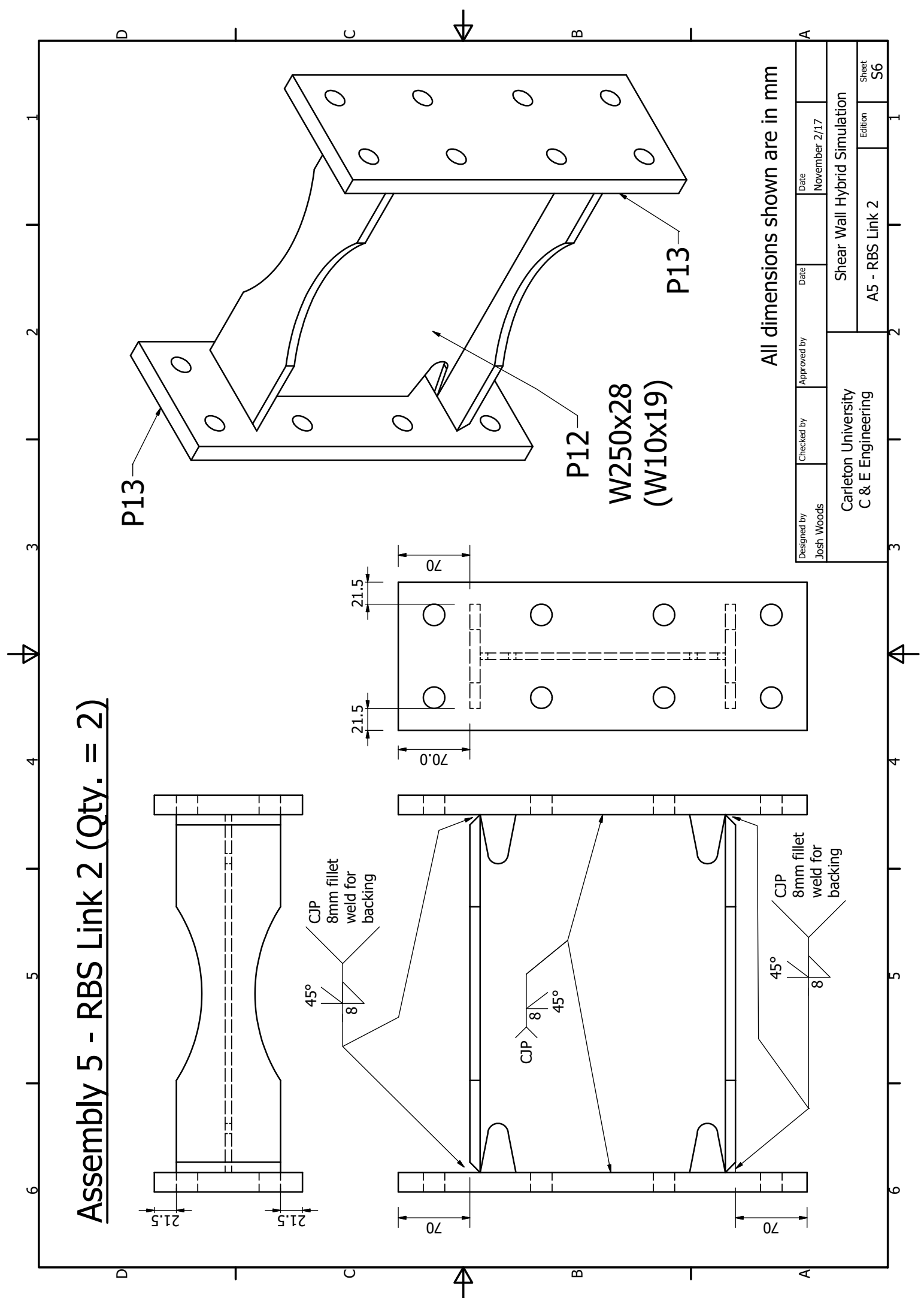

Joshua Woods, Department of Civil and Environmental Engineering, Carleton University 


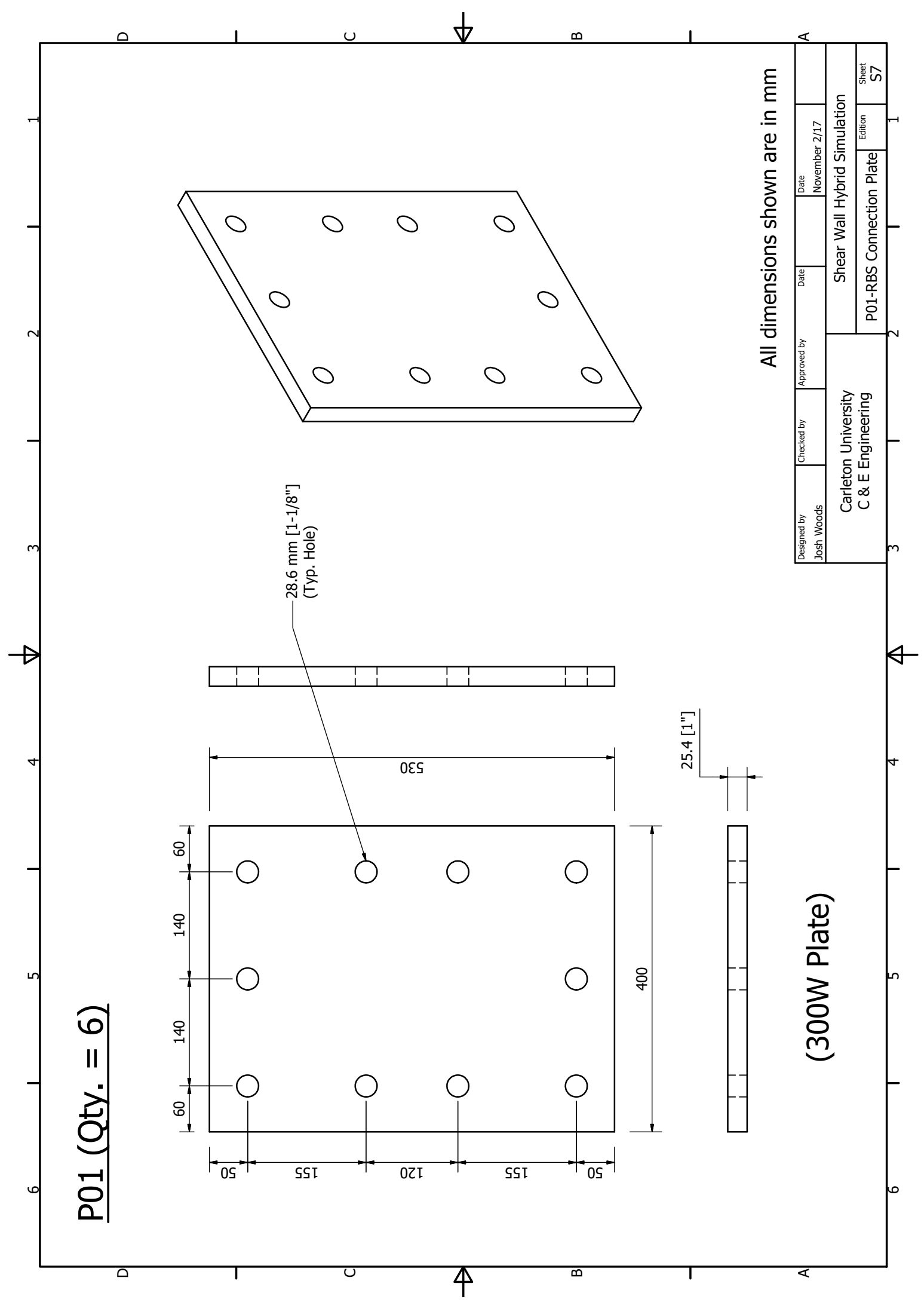

Joshua Woods, Department of Civil and Environmental Engineering, Carleton University 


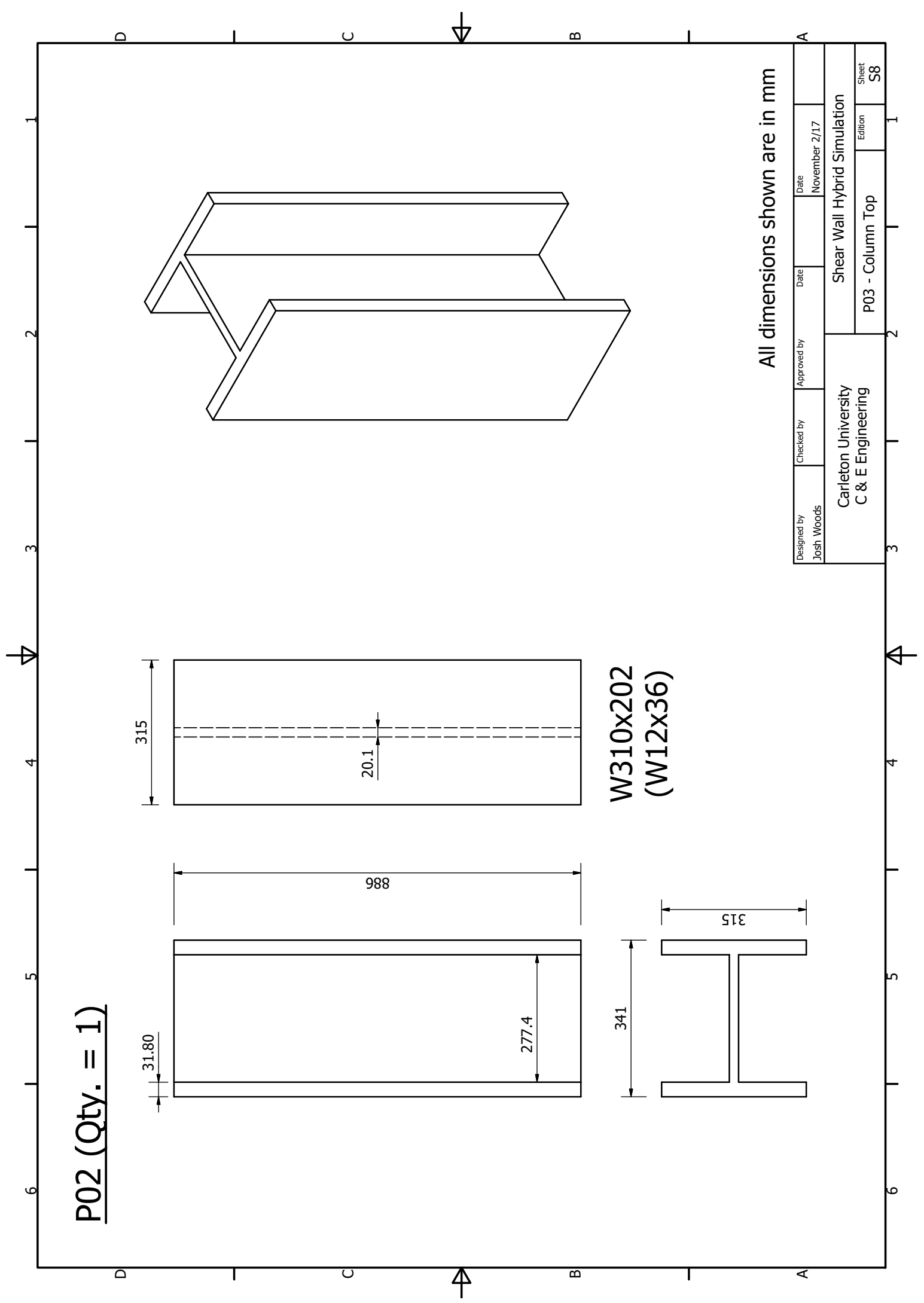

Joshua Woods, Department of Civil and Environmental Engineering, Carleton University 


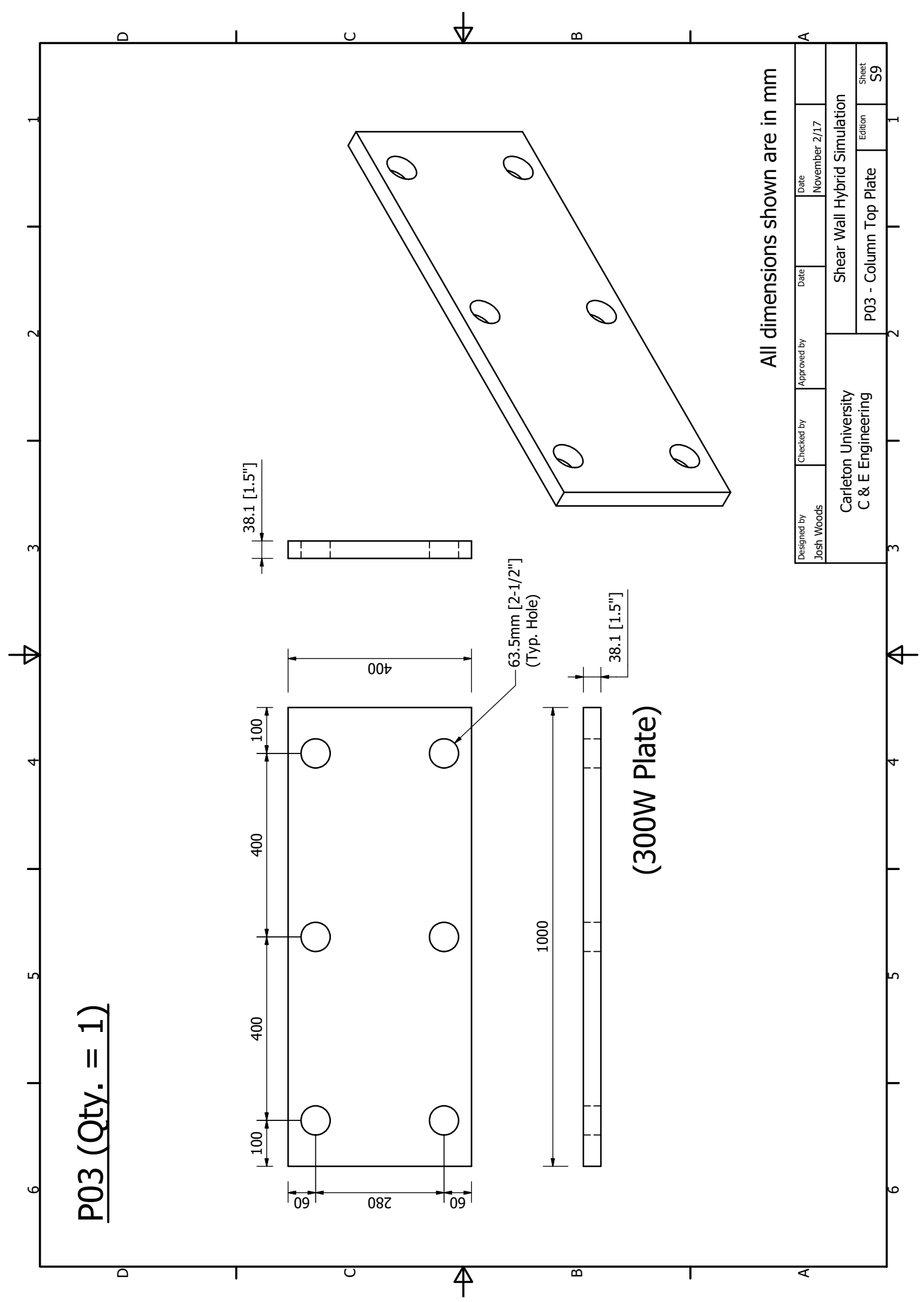

Joshua Woods, Department of Civil and Environmental Engineering, Carleton University 


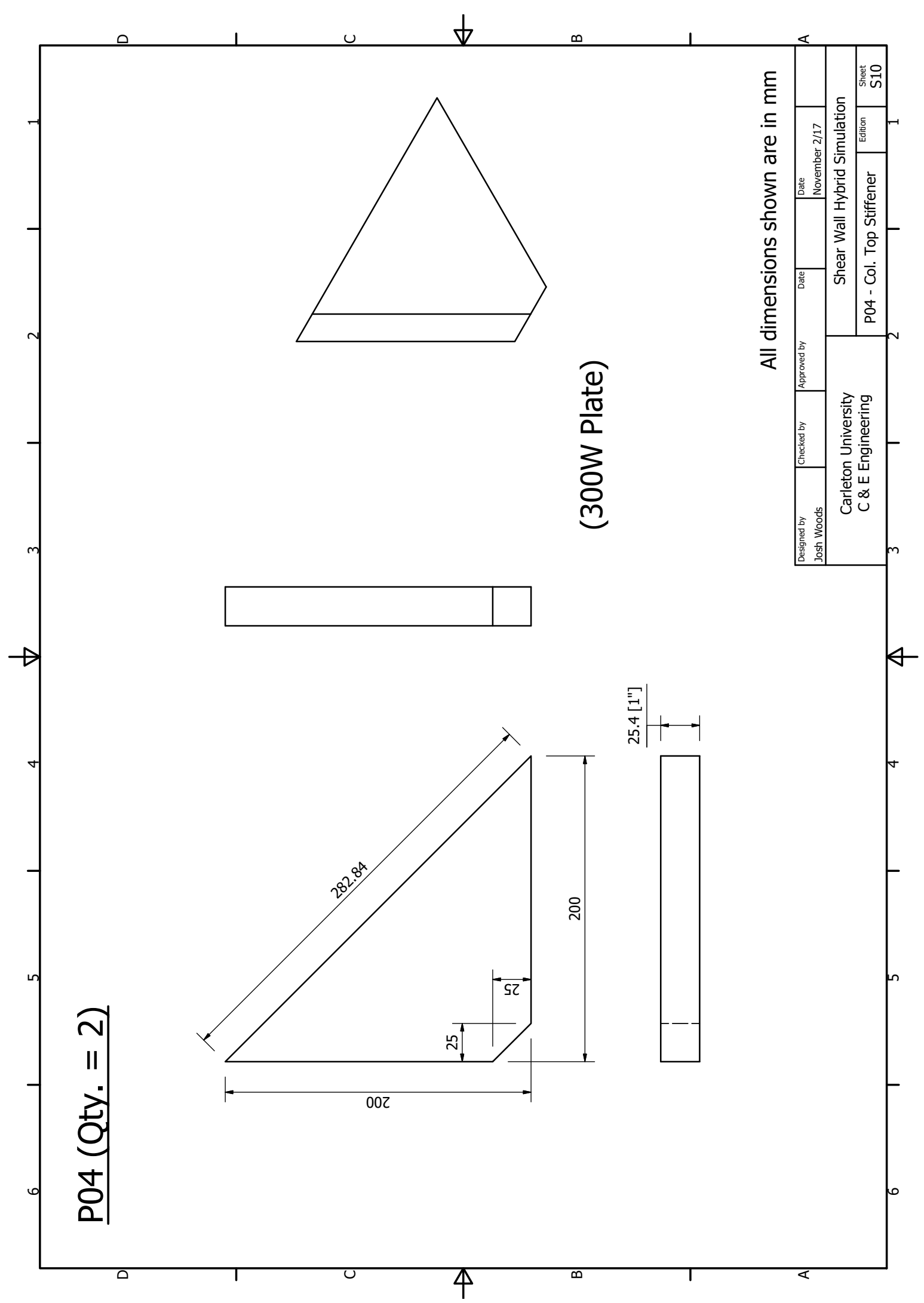

Joshua Woods, Department of Civil and Environmental Engineering, Carleton University 


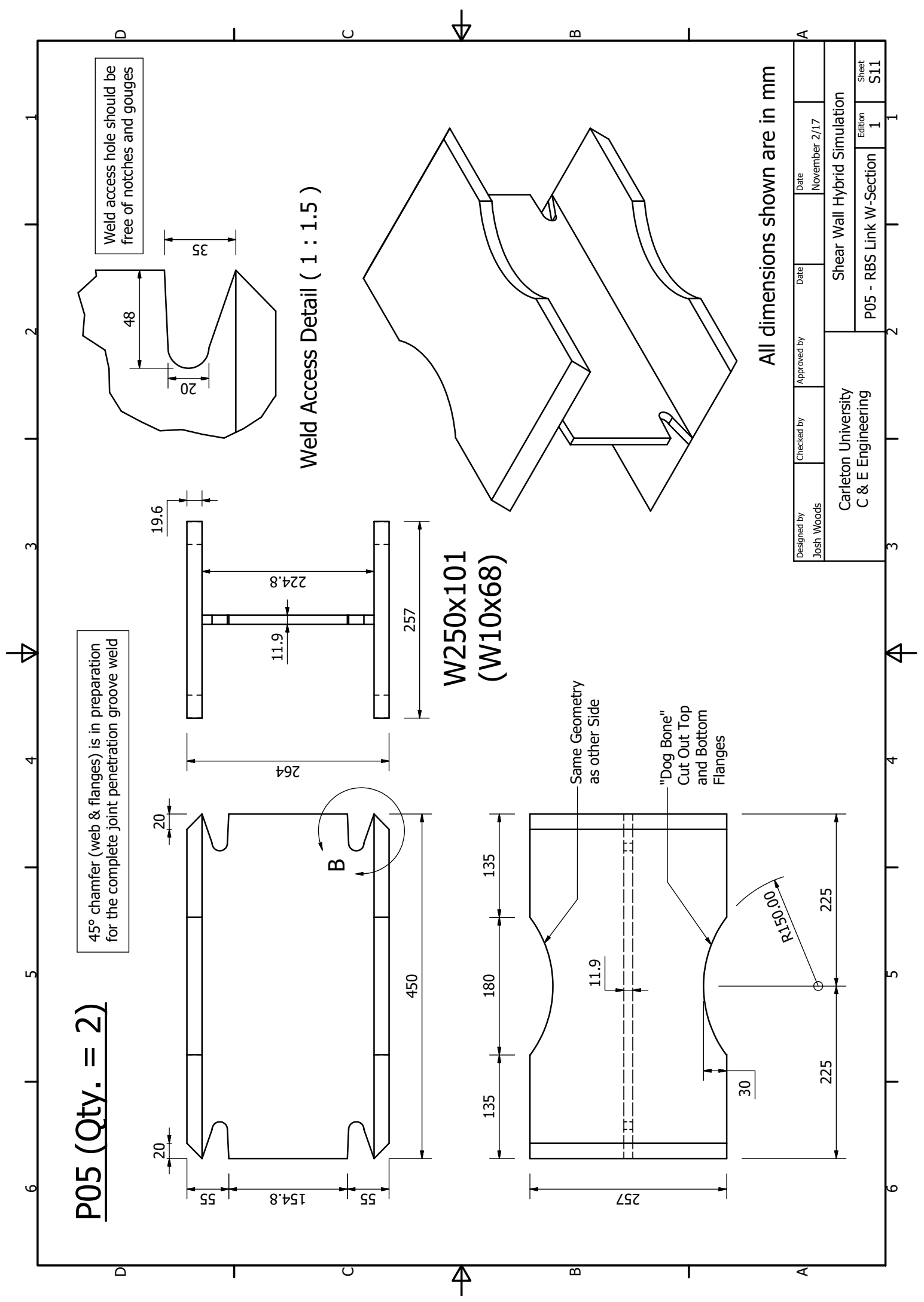

Joshua Woods, Department of Civil and Environmental Engineering, Carleton University 


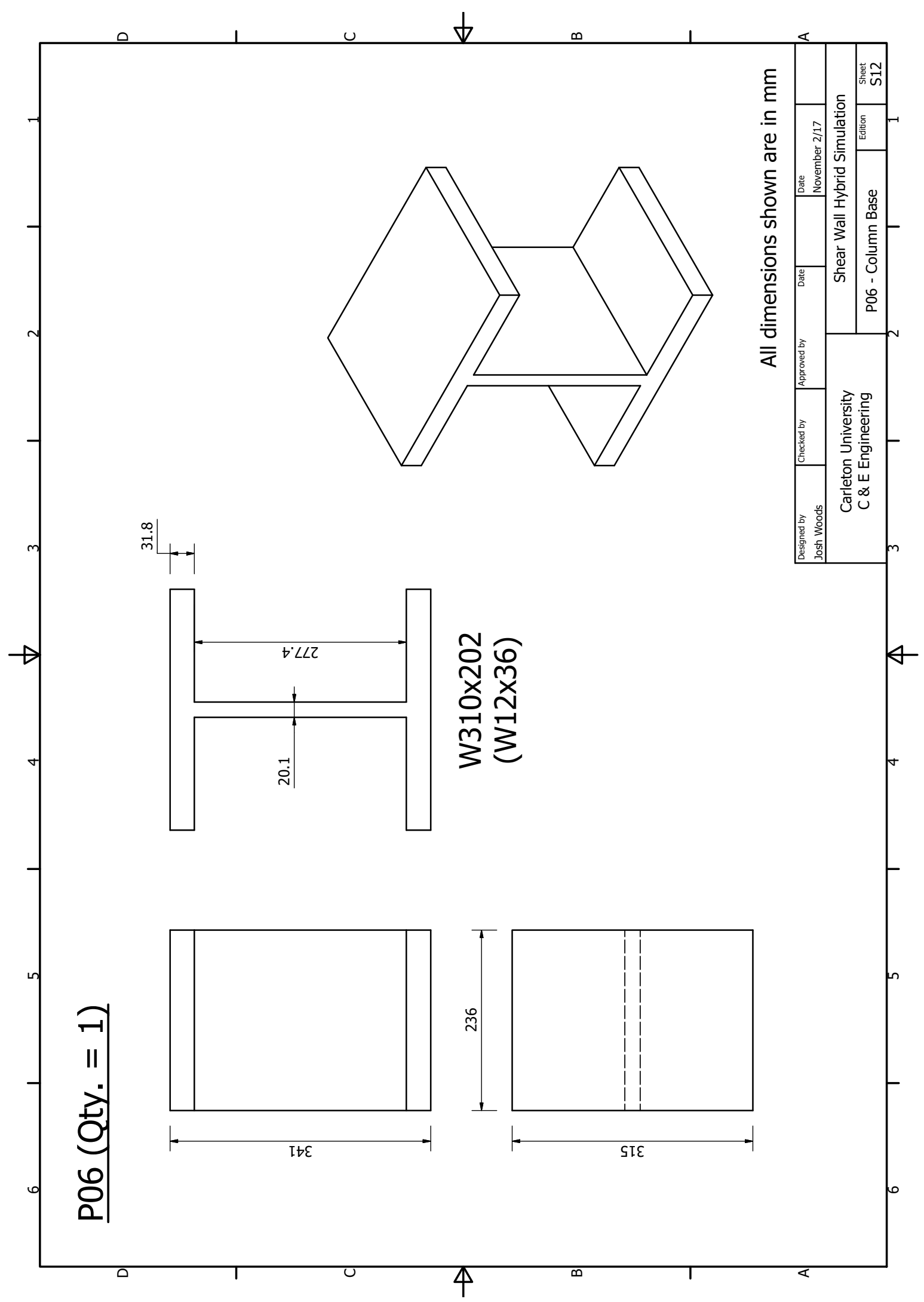

Joshua Woods, Department of Civil and Environmental Engineering, Carleton University 


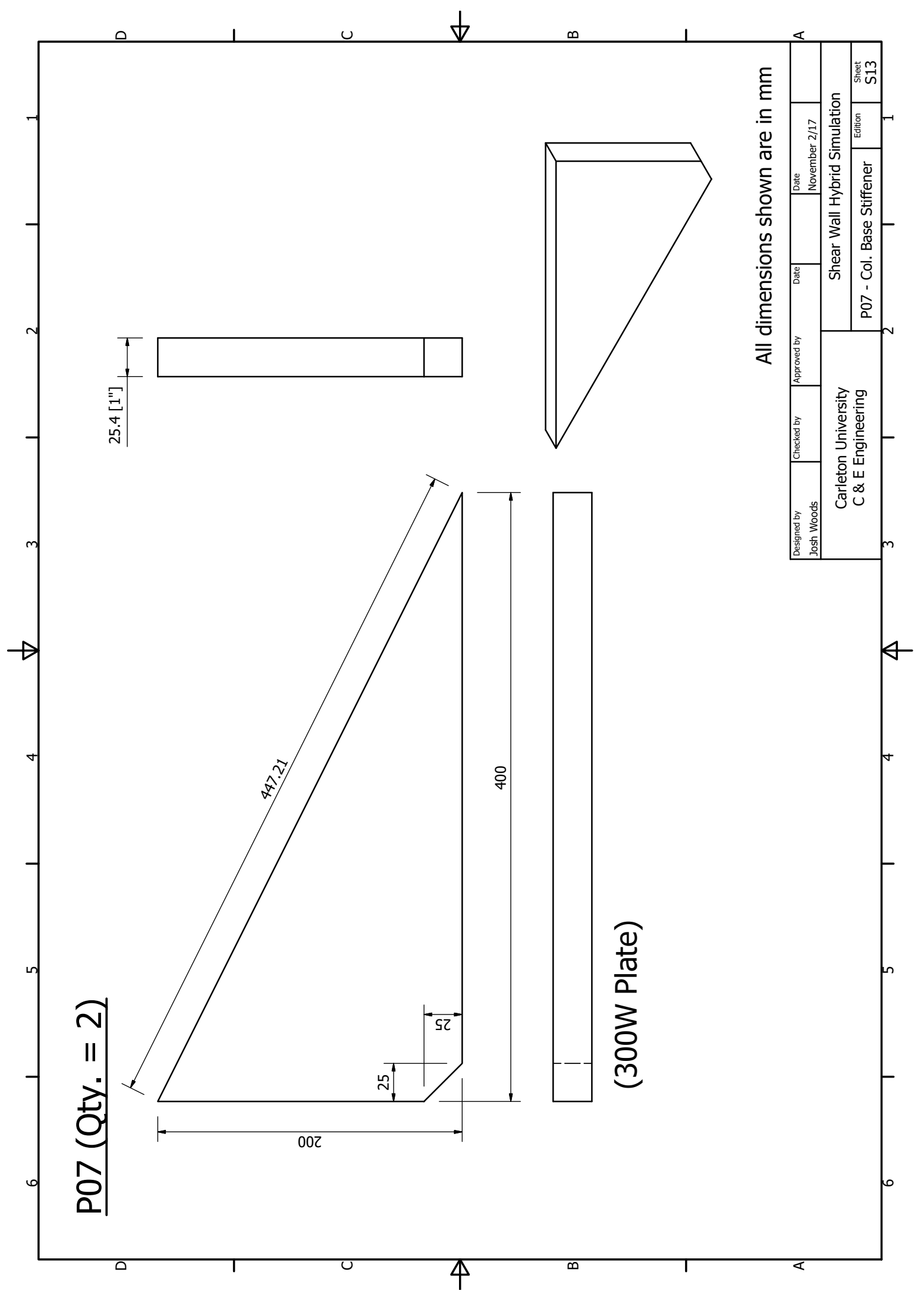

Joshua Woods, Department of Civil and Environmental Engineering, Carleton University 


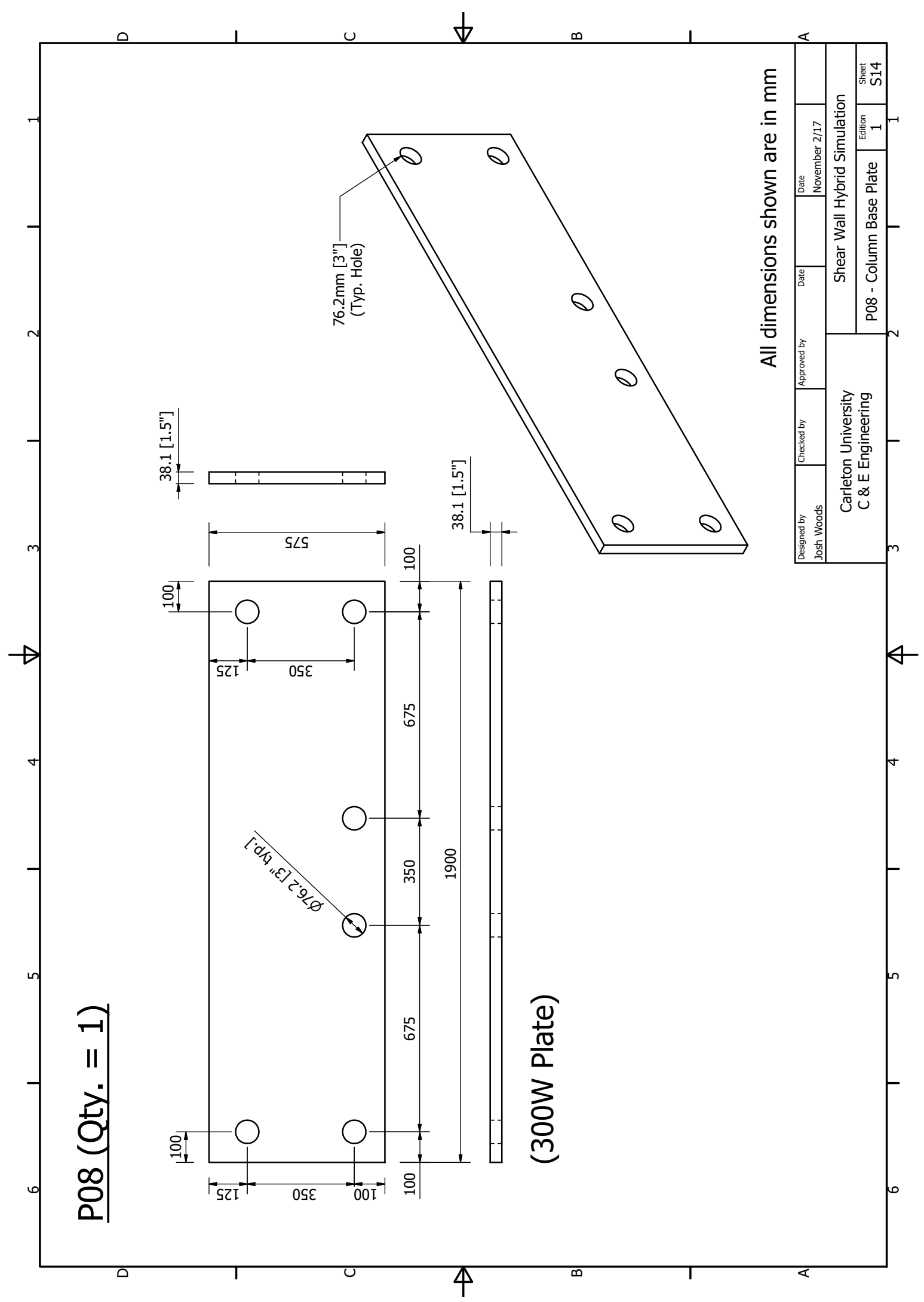

Joshua Woods, Department of Civil and Environmental Engineering, Carleton University 


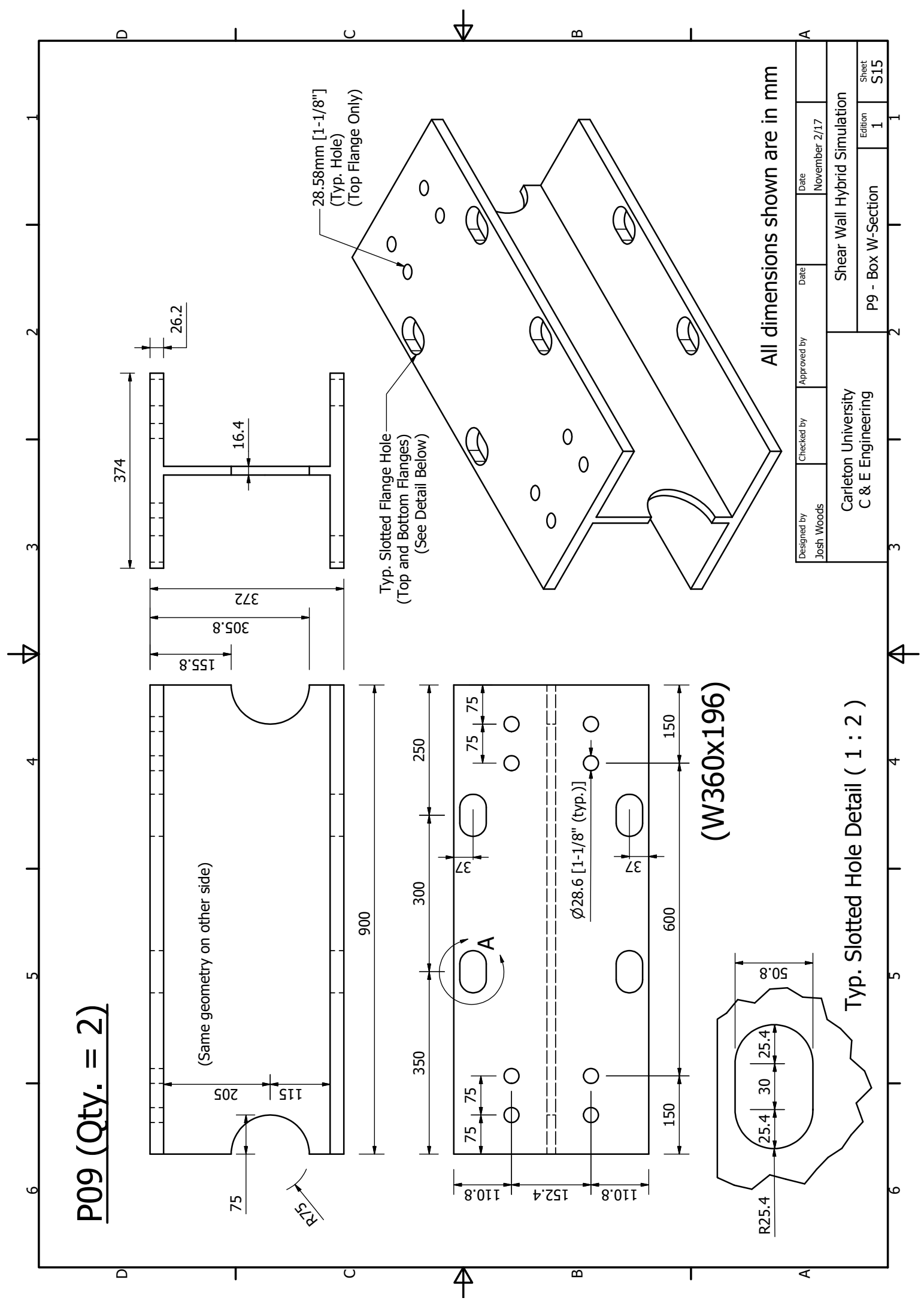

Joshua Woods, Department of Civil and Environmental Engineering, Carleton University 


\section{Appendix C. Design Drawings}




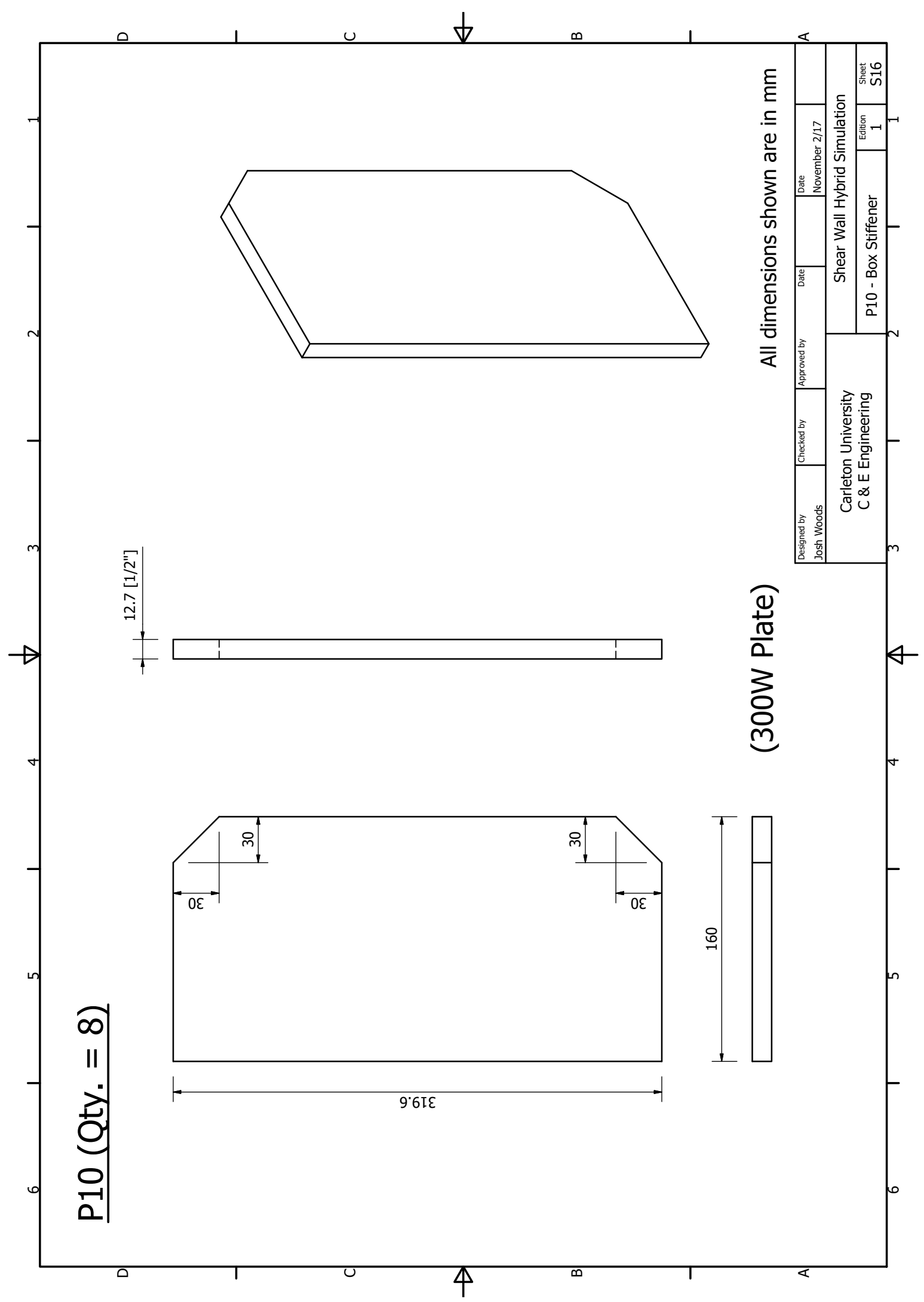

Joshua Woods, Department of Civil and Environmental Engineering, Carleton University 


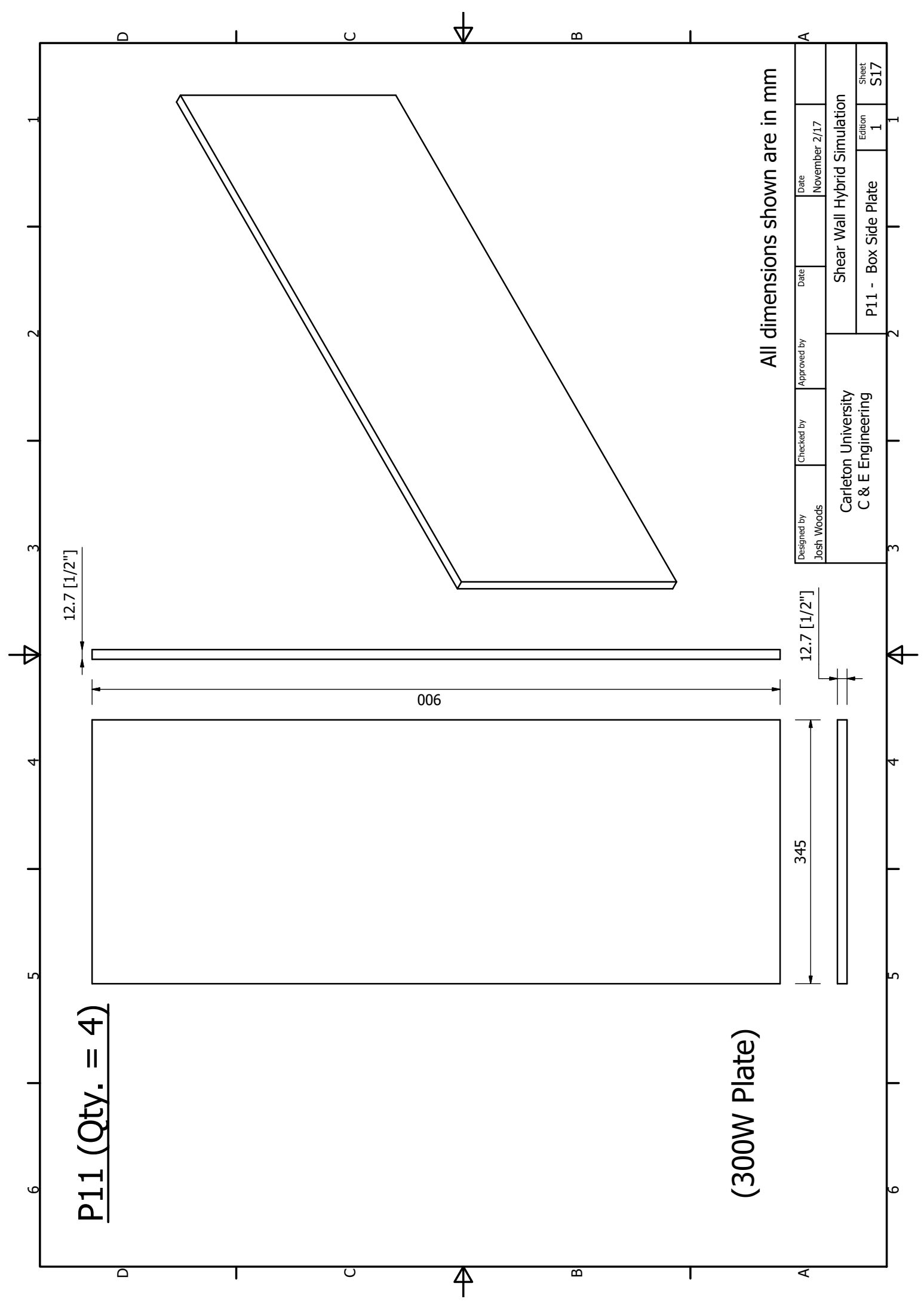

Joshua Woods, Department of Civil and Environmental Engineering, Carleton University 


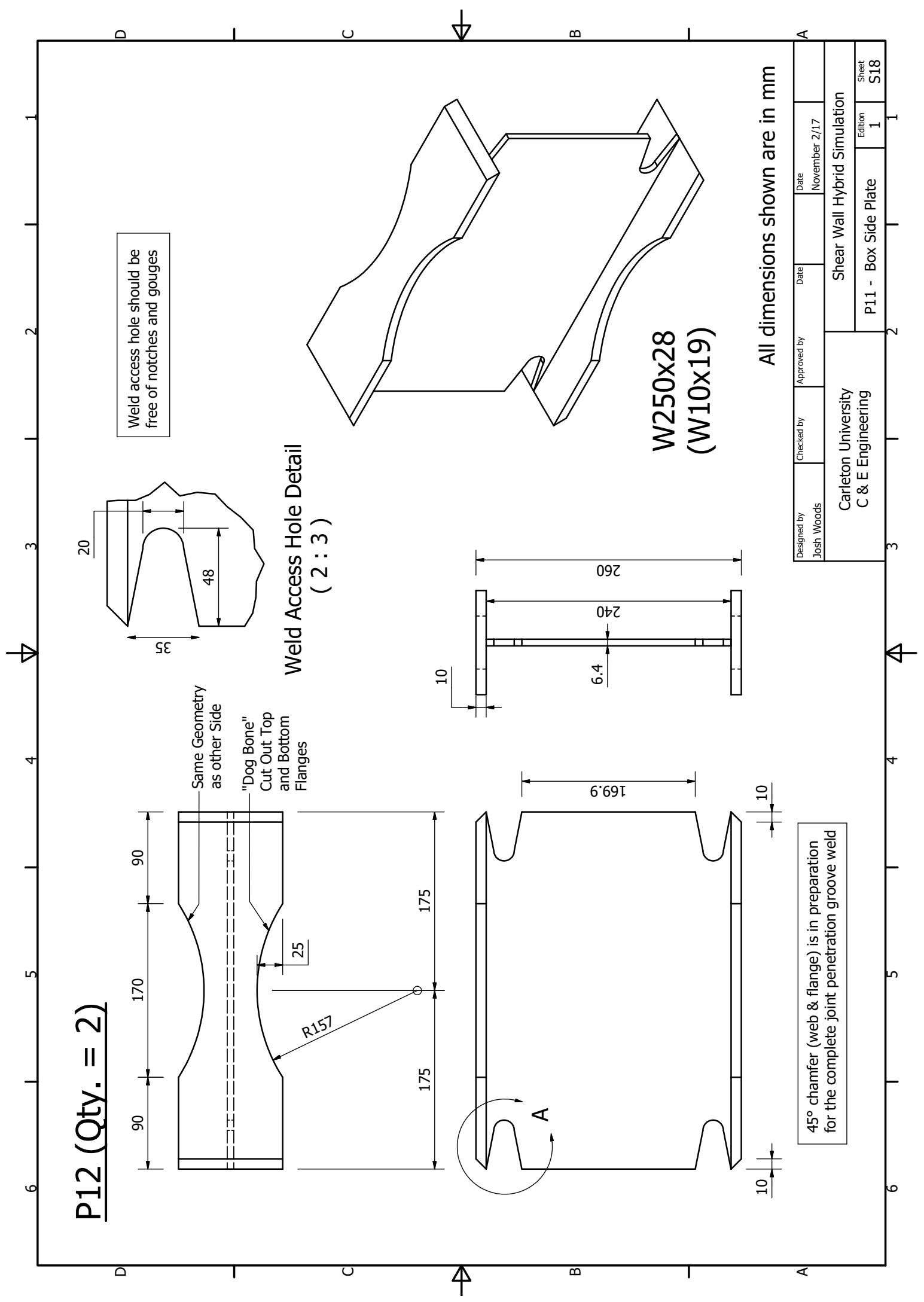

Joshua Woods, Department of Civil and Environmental Engineering, Carleton University 


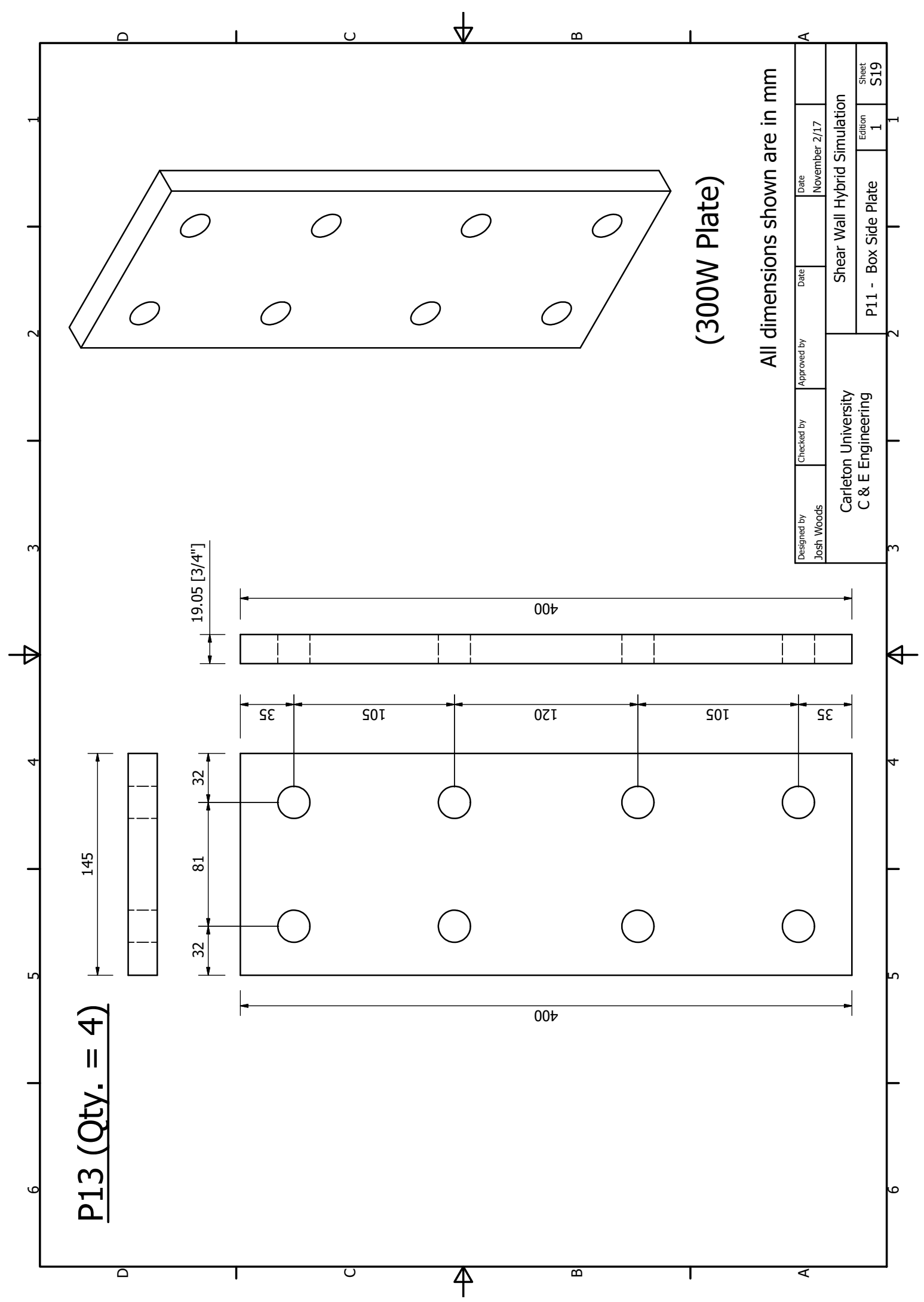

Joshua Woods, Department of Civil and Environmental Engineering, Carleton University 


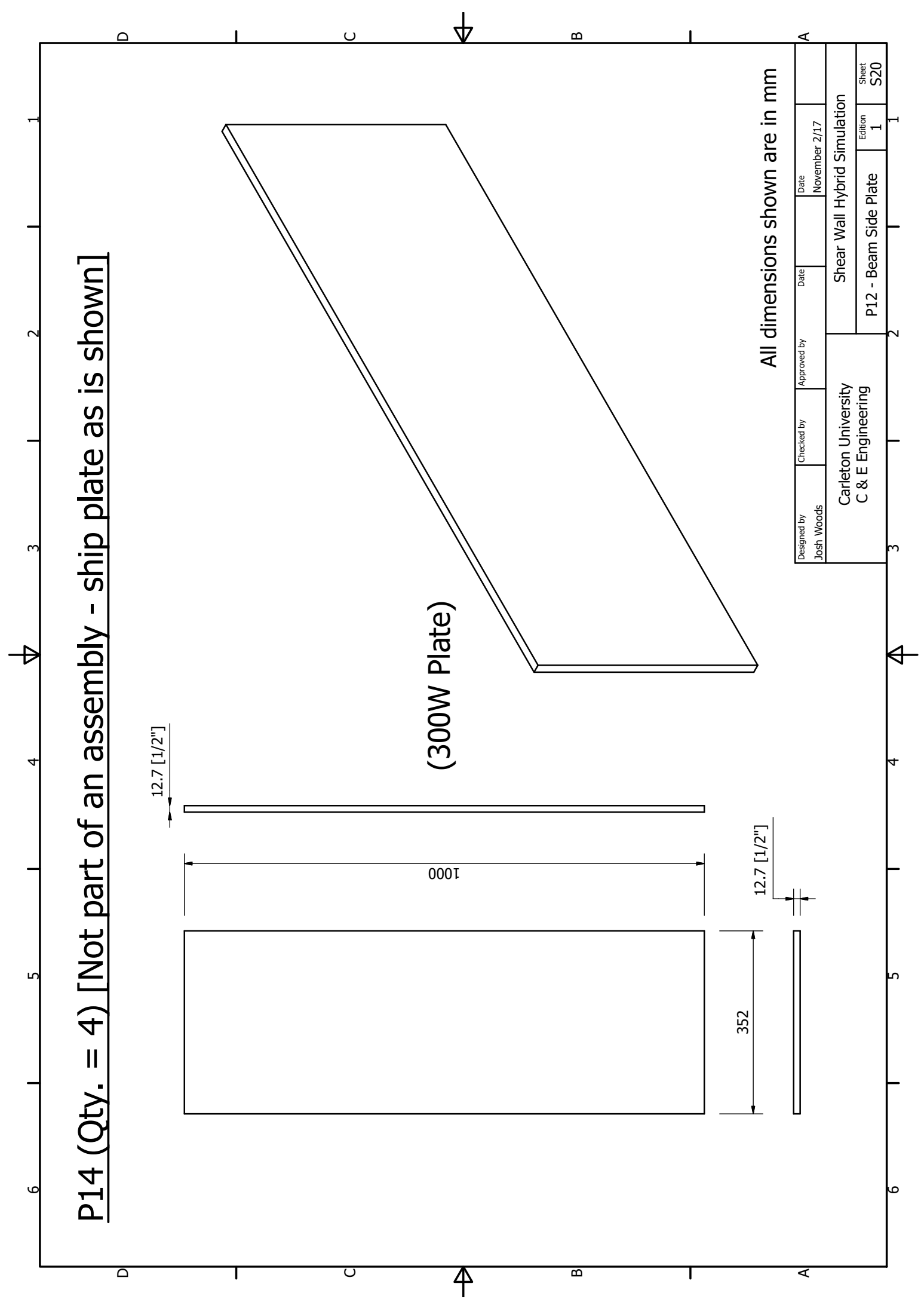

Joshua Woods, Department of Civil and Environmental Engineering, Carleton University 


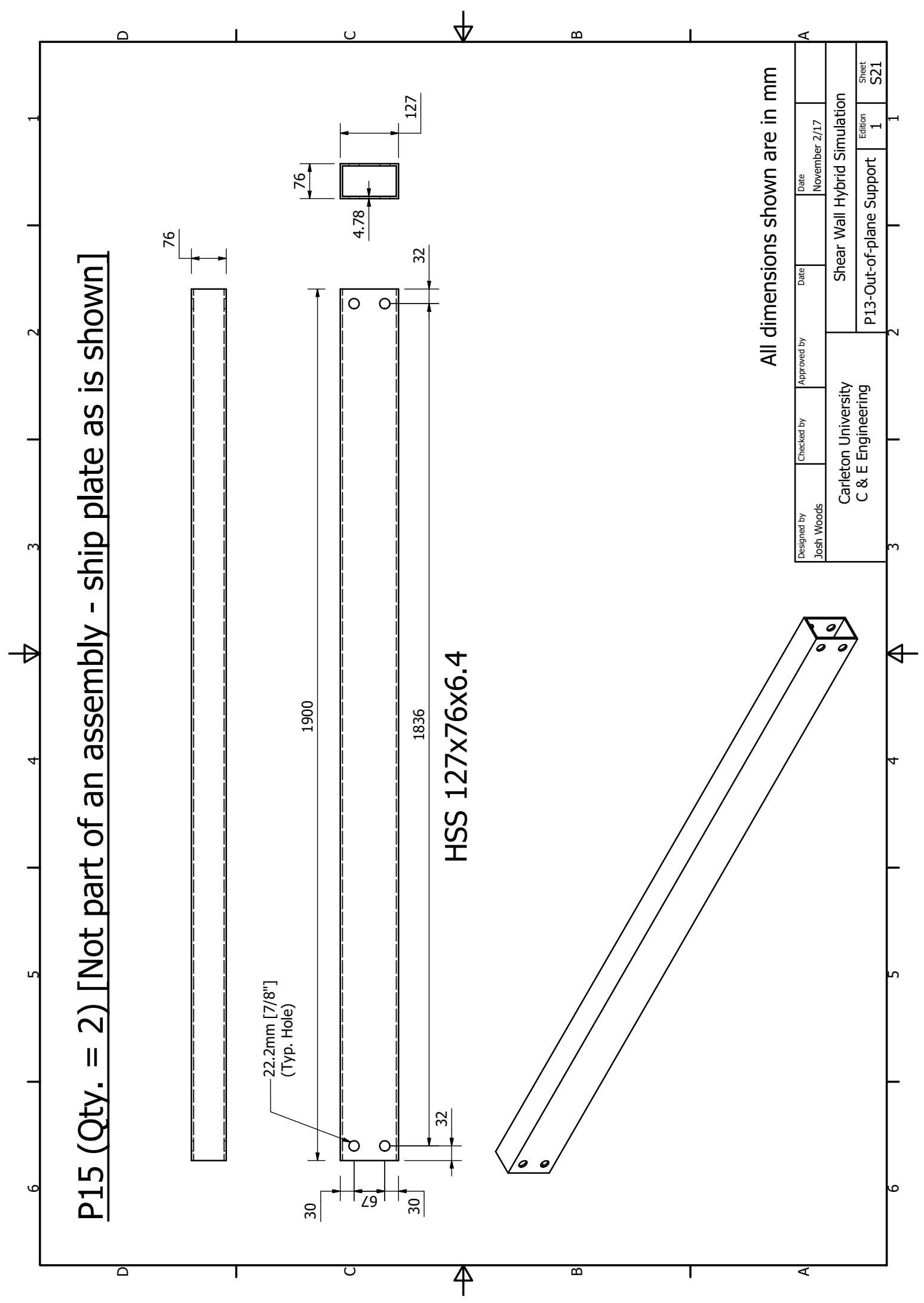

Joshua Woods, Department of Civil and Environmental Engineering, Carleton University 